University of Massachusetts Amherst

ScholarWorks@UMass Amherst

Doctoral Dissertations 1896 - February 2014

$1-1-1976$

\title{
What is memory aging the aging of?
}

Marion. Perlmutter

University of Massachusetts Amherst

Follow this and additional works at: https://scholarworks.umass.edu/dissertations_1

\section{Recommended Citation}

Perlmutter, Marion., "What is memory aging the aging of?" (1976). Doctoral Dissertations 1896 - February 2014. 1478.

https://doi.org/10.7275/8tvw-6a68 https://scholarworks.umass.edu/dissertations_1/1478

This Open Access Dissertation is brought to you for free and open access by ScholarWorks@UMass Amherst. It has been accepted for inclusion in Doctoral Dissertations 1896 - February 2014 by an authorized administrator of ScholarWorks@UMass Amherst. For more information, please contact scholarworks@library.umass.edu. 


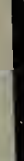

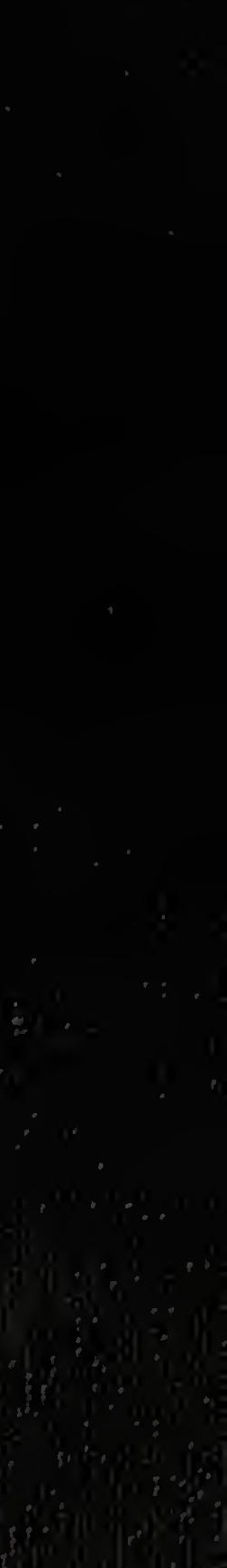


WHAT IS MEMORY AGING THE AGING OF?

A Dissertation Presented

by

Marion Perlmutter

Submitted to the Graduate School of the University of Massachusetts in partial fulfillment

of the requirements for the degree of

DOCTOR OF PHILOSOPHY

August 1976

Psychology 
Abstract

This dissertation examined memory phenomena in male and female, high school and Ph.D. educated, 20 and 60 year olds. Sizable age decrements in episodic recall and recognition performance were observed. The age differences in memory performance could not be attributed to mediational overload, mediational capacity deficit, selector impairment, or any of several strategy deficits. An associative procrssing production deficiency at acquisition, and mediational inefficiency at acquisition and/or retrieval, probably contributed to the age deficits. The adults demonstrated a wide range of generaj.ized-abstracted knowledge about memory, as well as considerable competence in two memory monitoring skills, memory prediction and memory confidence rating. No systematic age differences were observed in these types of metamemory. It was considered unlikely that cohort effects, at least in their most obvious interpretation, accounted for aging effects. Restricted disuse and expectation of decay hypotheses of memory aging remained tenable, and a biological explanation received some support. 
'HAT IS MIRY AGING THE AGING OF?

A Dissertation Presented

E.

Marion Perl mutter

Approved as to style and content by:

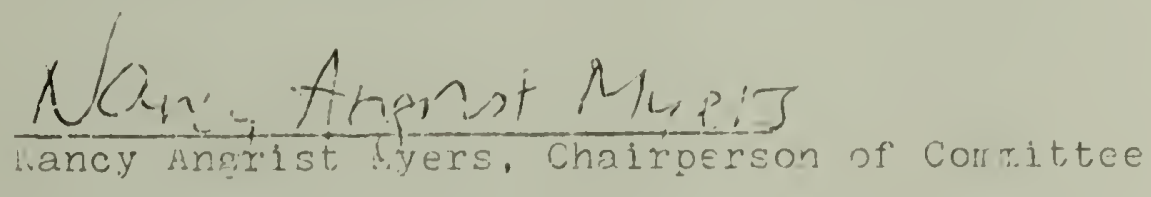

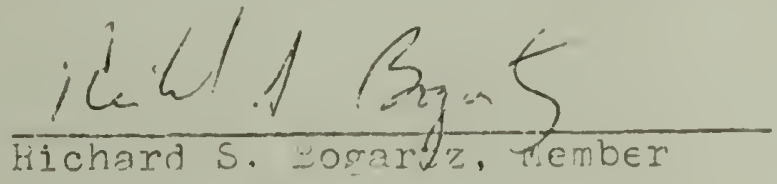

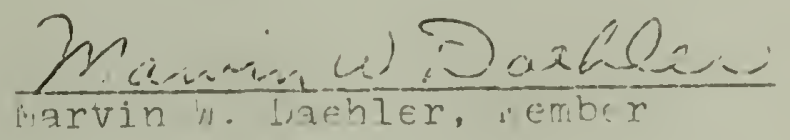

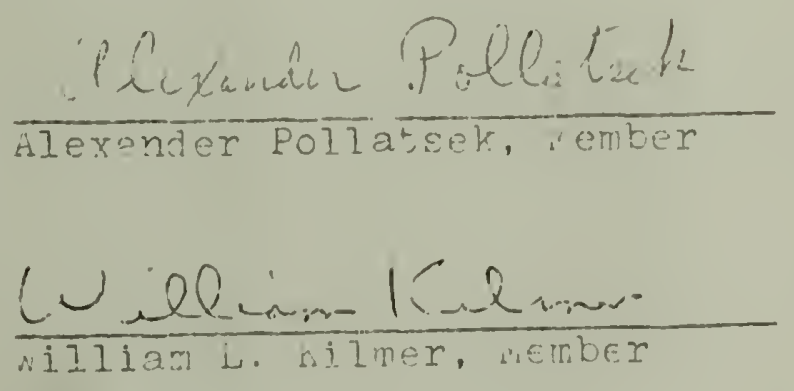

$\frac{1}{\text { bonnie Strickland, ienutment }}$

Feycinolory 
Abstract........................... i

Table of Contents.......................ii

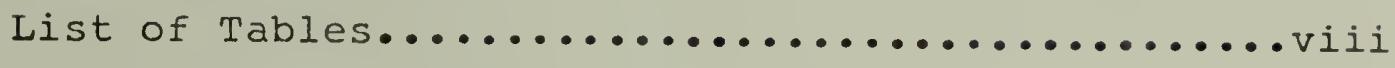

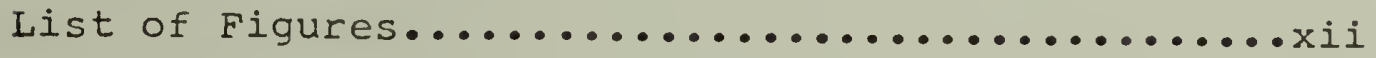

I. Introduction....................... I

A. What is Memory Aging the Aging of?......... I

1. Sensation and Perception............... I

2. Attention........................ 3

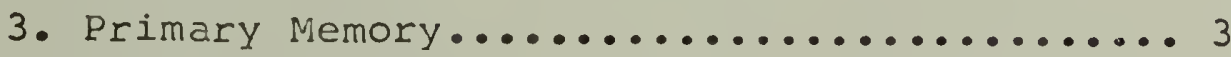

4. Secondary Memory................... 6

a. Short-Term Retention.............. 8

1. Acquisition................... 8

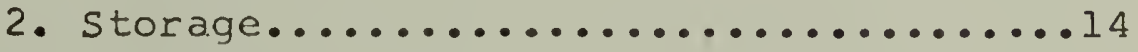

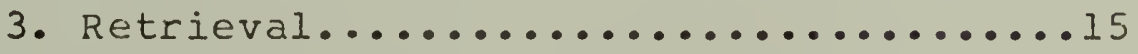

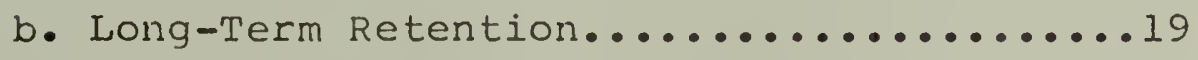

c. Episodic-Semantic Distinction.........20

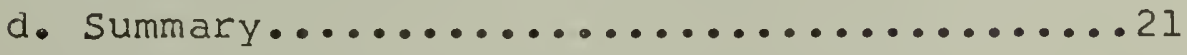

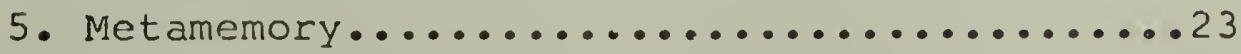

a. Generalized-Abstracted Knowledge.......27

1. Organismic Factors................28 a. Static Aspects: Memory Capacity.....28

b. Dynamic Aspects: Memory Strategies...28

2. Environmental Factors................... 30

a. Static Aspects: Characteristics of Stimulus Materials................30

b. Dynamic Aspects: Use of Externals

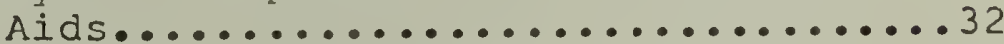

b. Specific-Concrete knowledge...........33

1. Static Aspects: Memory Monitoring of

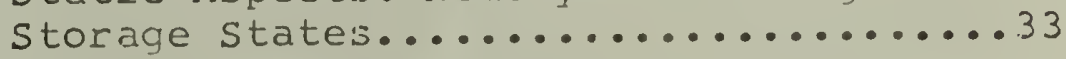

2. Dynamic Aspects: Differentiating Mnemonic Functions from other Func-

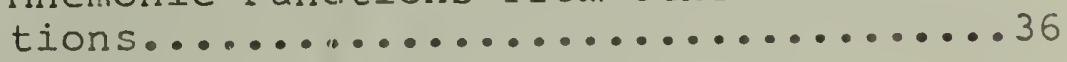


c. Coordination of Various Metamemory

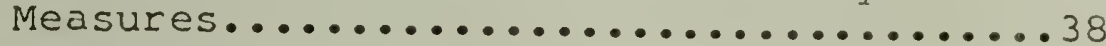

d. Coordination of Metamemory and Memory....39

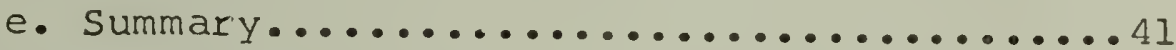

B. Methodological Issues in Studying Memory

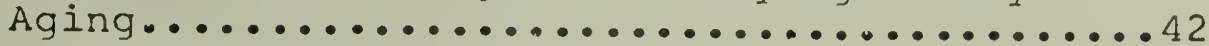

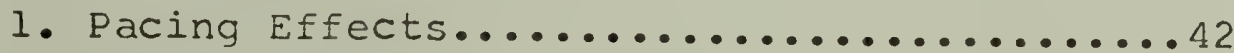

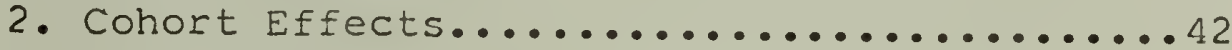

II. Statement of Problem...................47

A. Processing Differences Contributing to Age Differences in Memory...............47

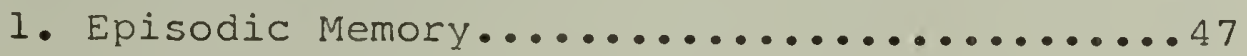

a. Processing Overload Hypothesis..........48

1. Mediator Overload.................48

2. Selector Impairment................50

b. Processing Deficit Hypothesis...........5I

1. Mediator Deficiency................51

2. Production Deficiency...............52

3. Mediator Inefficiency...............52

c. Strategy Deficit Hypothesis...........55

2. Semantic Memory.......................54

B. Metamemory Knowledge in Adulthood..........55

1. Generalized-Abstracted Knowledge:

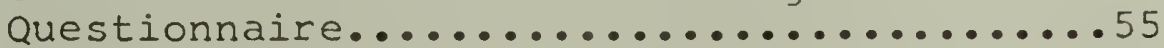

2. Specific-Concrete Knowledge: Memory

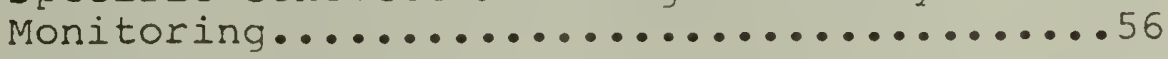

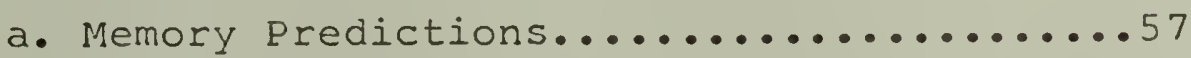

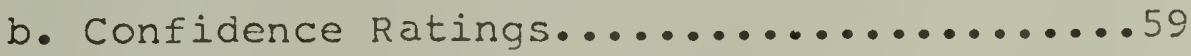

C. Explanations of Aging Effect..............60

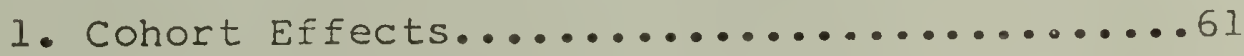

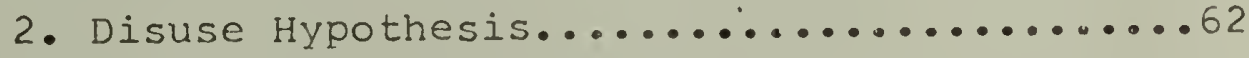

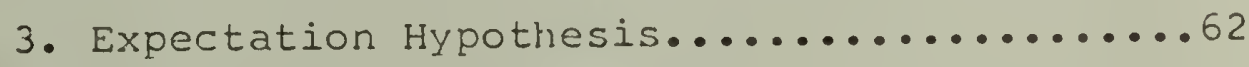

4. Biological Hypothesis...................63

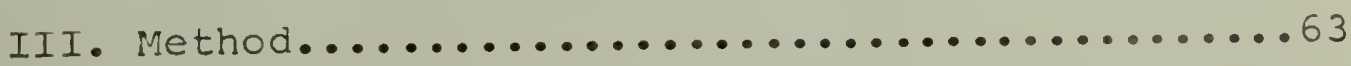

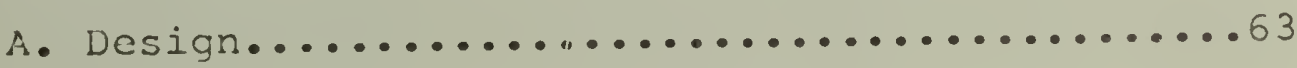




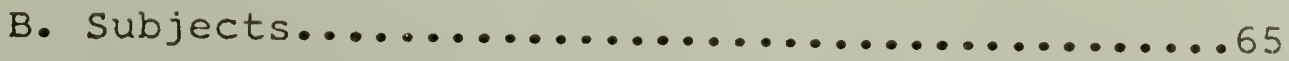

C. Materials and Procedure...............66

1. Background Questionnaire..............66

2. Metamemory Questionnaire.............68

3. Tests..........................68

a. Preliminary Tasks................68

1. Incidental Association Task.........68

2. Intentional Memory Task............68

b. Recall Prediction..................69

c. Recall Tasks, Recall Confidence Ratings, and Rerognition Predictions............69

1. Incidental Association Items........69

2. Intentional Memory Items............69

3. General Information Items...........70

d. Recognition Tasks and Recognition

Confidence Ratings.................70

IV. Results.........................

A. Associations...........................

B. Memory Performance..................... 11

1. Episodic Memory....................

a. Percent Remembered on Recall and Recognition Tests..................74

b. Number of Responses in Recall........866

c. Intrusions in Recall..................101

d. Percent Correct in Recognition...........12

e. Signal Detection Analysis of Recognition.ll8

2. Semantic Memory.....................121

a. Percent known on Recall and Recognition

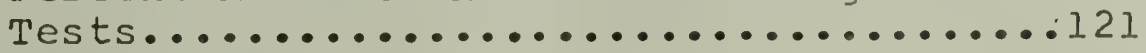

b. Number of Responses in Recall..........129

c. Percent Correct in Recognition..........140

d. Signal Detection Analysis of Recognition.153

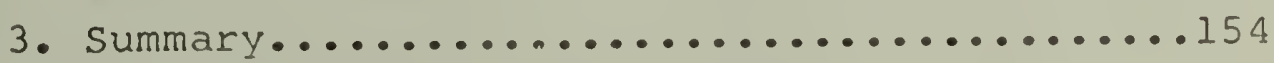

a. Episodic Memory Performance............154

h. Semantic Memory Performance............158 


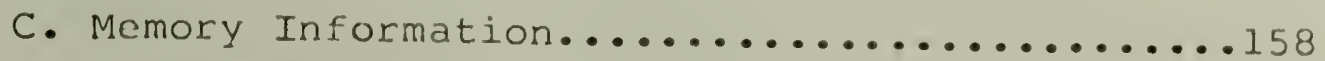

1. Metamemory Questionnaire................158

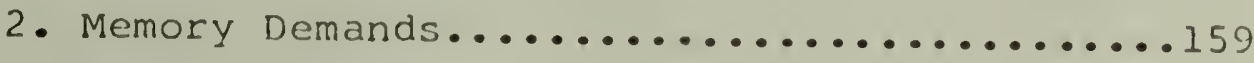

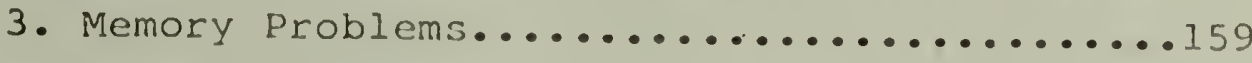

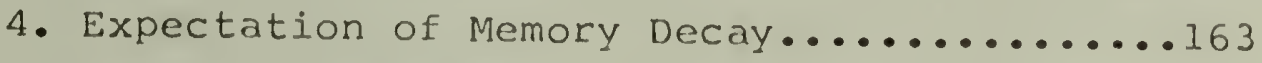

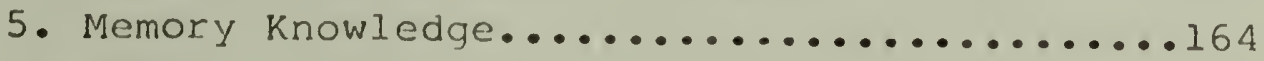

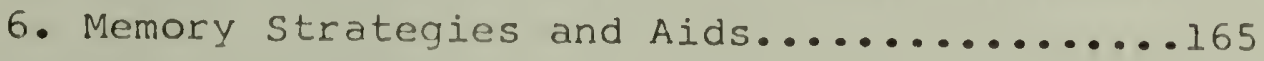

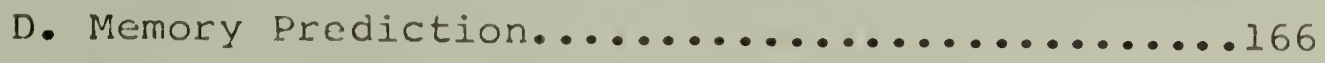

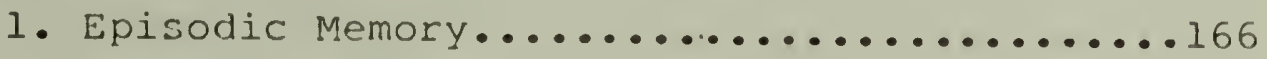

a. Recall and Recognition Predictions.......166

b. Differences Between prediction and

Performance......................174

c. Absolute Differences Between Prediction

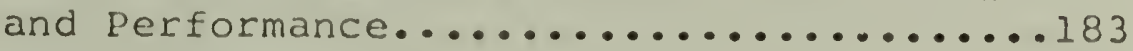

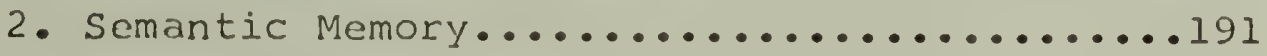

a. Recognition predictions................191

b. Differences Between prediction and

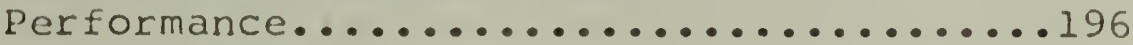

c. Absolute Difference Between Prediction

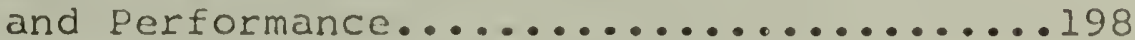

d. Probability Correct Responding Given Positive and Negative Feeling of Knowing

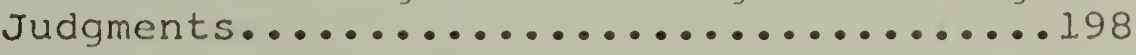

e. Differences in Probability Correct Responding Given Positive and Negative Feeling of Knowing Judgments..........204

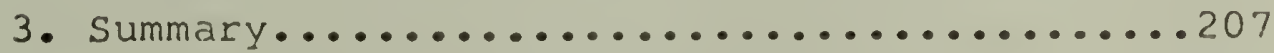

a. Episodic Memory Predictions............207

b. Semantic Memory Predictions.............210

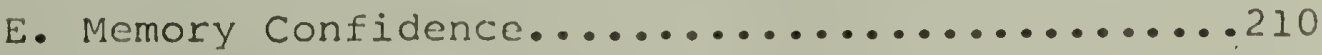

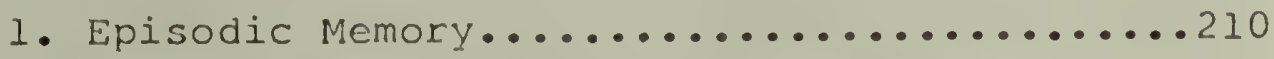

a. Confidence Ratings on Hits and False

Alarms on Recall and Recognition Tests...210

b. Difference Scores for Confidence Ratings on Hits and False Alarms on Recall and

Recognition Tests..................216

c. Confidence Ratings on Old and New Recognition Items..................222

d. Difference Scores for Confidence Ratings on Old and New Recognition Items........222 
2. Semantic Memory........................228

a. Confidence Ratings on Hits and False Alarms on Recall and Recognition Tests...228

b. Difference Scores for Confidence Ratings on Hits and False Alarms on Recall and

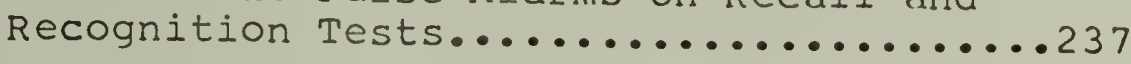

c. Confidence Ratings on True and False Recognition Items.....................237

d. Difference Scores for Confidence Ratings on True and False Recognition Items.....240

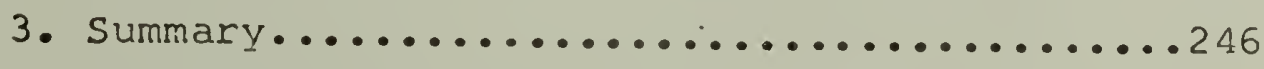

a. Episodic Memory Confidence............246

b. Semantic Memory Confidence............252

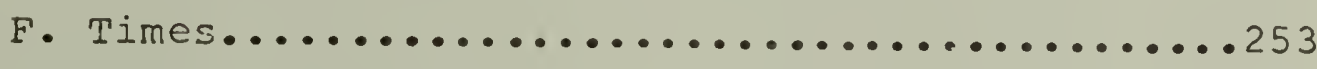

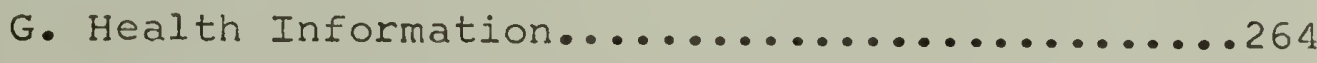

a. Objective Physical Health.............264

b. Subjective Physical Health.............266

c. Subjective Mental Health..............269

H. Relationships Between Measures............269

1. Health and Memory Information...........269

2. Memory Prediction, Confidence, and Performance.......................270

3. Predictors of Memory Performance........273

4. Regression Analyses..................274

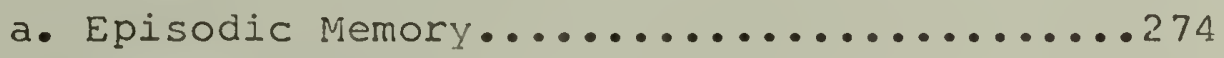

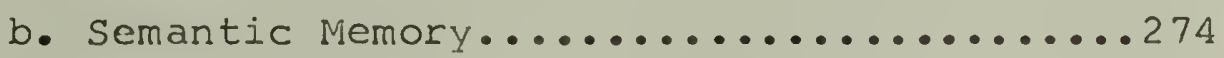

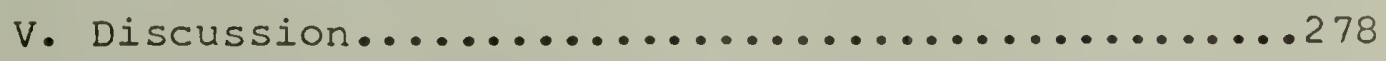

A. Processing Differences Contributing to Age

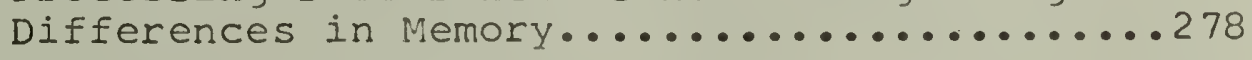

1. Episodic Memory....................278

a. Processing Overload Hypothesis.........279

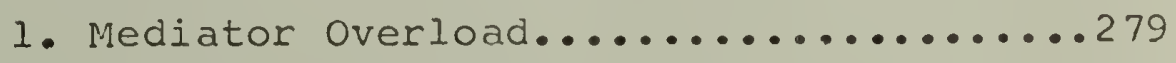

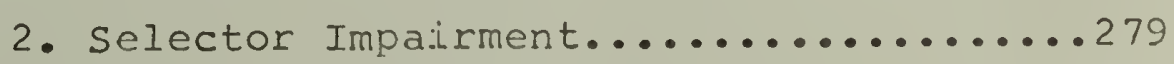

b. Processing Deficit Hypothesis..........281

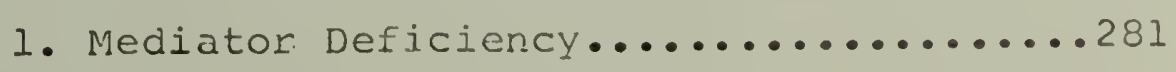

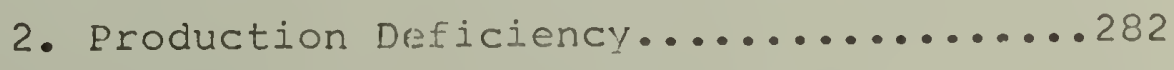

3. Mediator Inefficiency...............282 
c. Strategy Deficit Hypothesis..........283

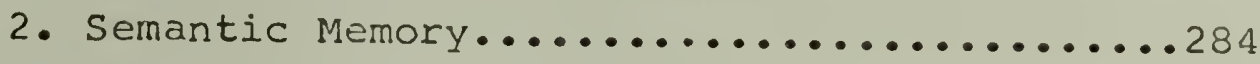

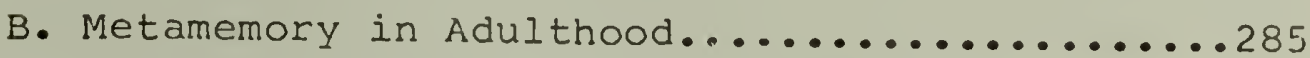

1. Generalized-Abstracted Knowledge:

Questionnaire.......................286

2. Specific-Concrete Knowledge: Memory

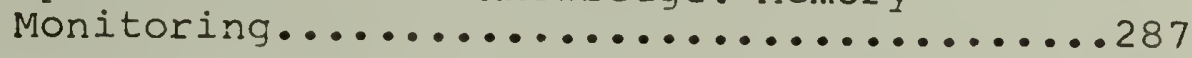

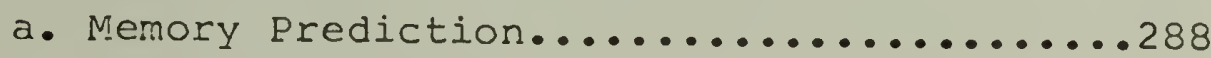

1. Episodic Memory..................288

2. Semantic Memory..................290

b. Memory Confidence...................291

1. Episodic Memory..................291

2. Semantic Memory..................291

c. Coordination of Various Metamemory

Measures.........................292

d. Coordination of Metamemory and Memory....292

e. Age Differences in Metamemory..........294

C. Explanation of Aging Effects..............294

a. Cohort Effects.....................295

b. Disuse Hypothesis......................297

c. Expectation Hypothesis...............298

d. Biological Hypothesis.................299

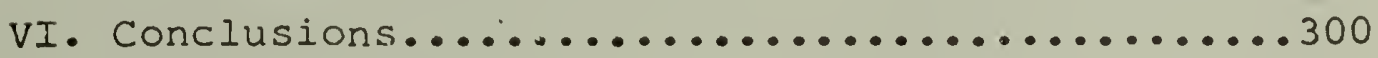

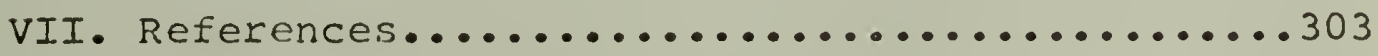

VIII. Appendix A: Sample Data Sheet............313

IX. Appendix B: Metamemory Questionnaire Re-

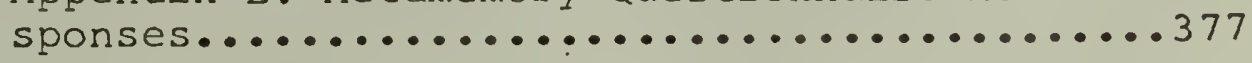

X. Appendix C: Prediction, Confidence, Per-

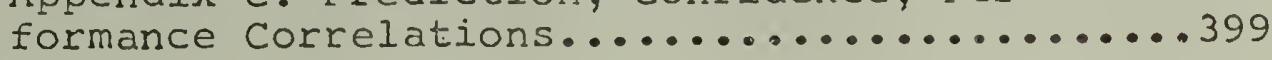




\section{List of Tables}

Table 1. Hypothesized Processing Differences Contributing to Age Differences in Memory Performance.

Table 2. Summary of Procedure..............67

Table 3. Mean Number of Associations Generated by Each Age $x$ Education $x$ Sex Group............ 72 Table 4. Summary ANOVA for Associations........ 73 Table 5. Mean Percentage of Incidental and Intentional Words Correctly Recalled and Recognized by Each Age x Education $x$ Sex Group....... 75 Table 6. Mean Number of Correct and Incorrect Responses on Incidental and Intentional Recall Tests for Each Age x Education x Sex Group......8 87 Table 7. Mean Number of List, Associate, and Miscellaneous Intrusions on Incidental and Intentional Recall for Each Age $x$ Education $x$

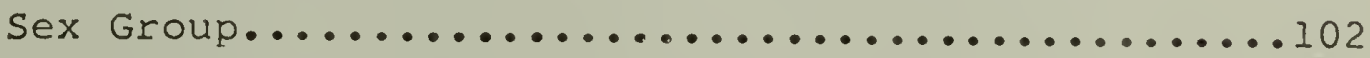
Table 8. Mear Percentage of Correct Recognition of Incidental, Intentional, and New Items by Each Age $x$ Education $x$ Sex Group..............113 Table 9. Mean Percentage of Items From Each Time Period Correctly Recalled and Recognized by Each

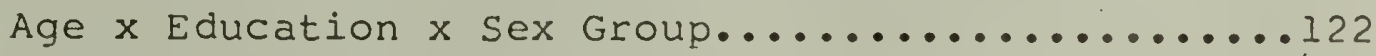
Table 10. Mean Number of Correct and Incorrect Recall Responses for Each Time Period by Each Age

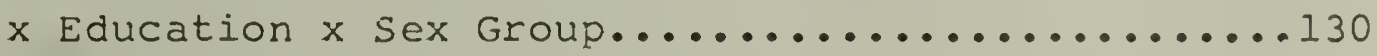
Table 11. Mean Percentage of True and False Items From Each Time Period Correctly Recognized by Each

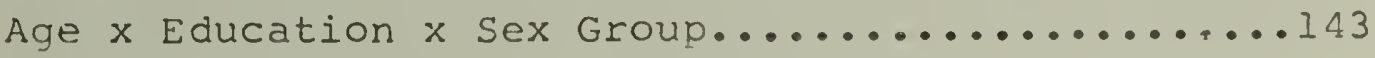


Table 12. Summary ANOVAs for Episodic and

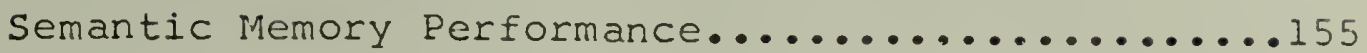

Table 13. Summary ANOVAs for Memory Information

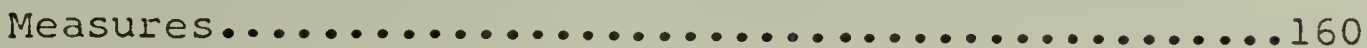

Table 14. Mean Predictions of the Number of Incidental and Intentional Items Correctly Recalled and Recognized by Each Age x Education $x$ Sex Group. 167

Table 15. Mean Difference Scores of Prediction and Performance on Recall and Recognition of Incidental and Intentional Items by Each Age $x$

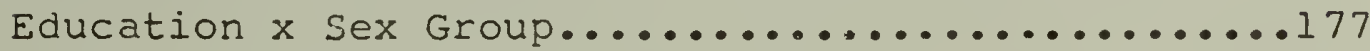

Table 16. Mean Prediction Accuracy Scores on Recall and Recognition of Incidental and Intentional Items by Each Age $x$ Education $x$ Sex Group..185 Table 17. Mean Predictions of the Number of General Information Knowledge Facts to be Correctly Recognized by Each Age $x$ Education $x$ Sex

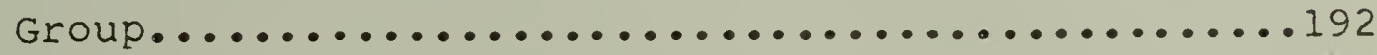
Table 18. Mean Difference Scores of Prediction and Performance on General Information Knowledge Recognized by Each Age x Education x Sex Group....197 Table 19. Mean Prediction Accuracy Scores on General Information Knowledge Recognition by Each Age $x$ Education $x$ Sex Group..................199 Table 20. Mean Probability of a Correct Response on True and False Items Given Positive and Negative Feeling of Knowing Judgments for Each Age $x$

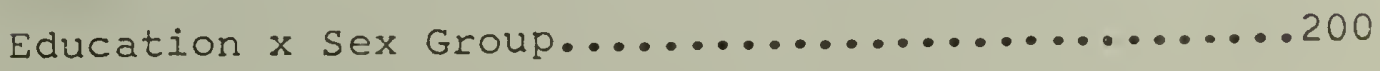
Table 21. Mean Difference of Probability Correct Responding on True and False Items Given Positive Or Negative Feeling of Knowing Judgments.........205 
Table 22. Summary ANOVAs for Episodic and

Semantic Prediction Accuracy.................208

Table 23. Mean Confidence Ratings for Hits and

False Alarms on Recall and Recognition Memory

Tests for Each Age x Education x Sex Group.......212

Table 24. Mean Difference Scores for Confidence

Ratings on Hits and False Alarms on Recall and Recognition Memory Tests for Each Age x Education

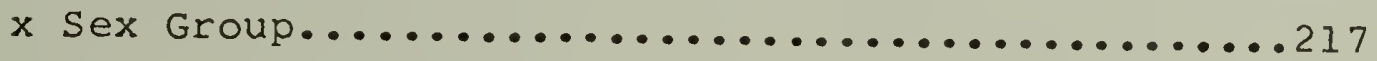

Table 25. Mean Confidence Ratings on Correct and Incorrect $\mathrm{Old}$ and $\mathrm{N} \in \mathrm{W}$ Items on Recognition Memory Test for Each Age x Education x Sex Group..223

Table 26. Mean Difference Scores for Confidence Ratings on Correct and Incorrect old and New Items Recognition Memory Tests for Each Age $x$ Education $x$ Sex Group.......................229

Table 27. Mean Confidence Ratings for Hits and False Alarms on General Information Recall and Recognition Tests for Each Age $x$ Education $x$ Sex

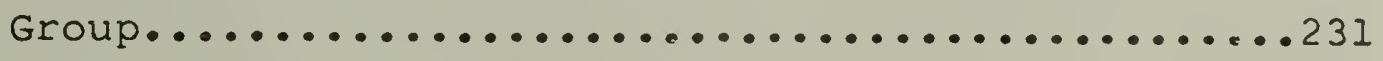
Table 28. Mean Difference Scores for Confidence Ratings on Hits and False Alarms on General Information Recall and Recognition Tests for Each

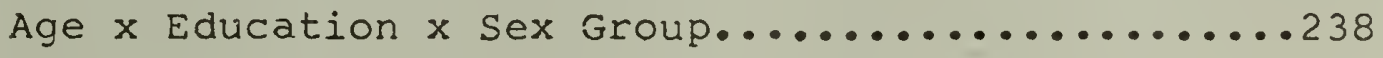
Table 29. Mean Confidence Ratings on Correct and Incorrect True and False Items on General Information Recognition Tests for Each Age $\mathrm{x}$

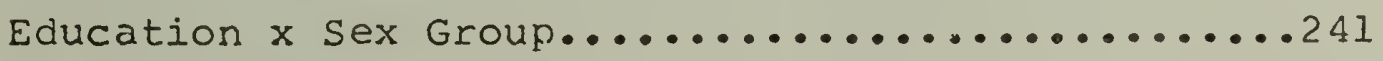
Table 30. Mean Difference Scores for Confidence Ratings on Correct and Incorrect True and False Items on General Information Recognition for Each Age $x$ Education $x$ Sex Group..................247 
Table 31. Summary ANOVAS for Episodic and Semantic Confidence Accuracy.................249

Table 32. Mean Time Spent on Each Task by Each Age $x$ Education $x$ Sex Group..................254 Table 33. Summary ANOVA for Preparation Time....265 Table 34. Summary ANOVAs for Health Information

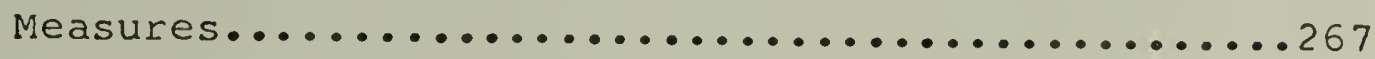

Table 35. Correlations Between Health and Memory Measures for Entire Sample............271 Table 36. Correlations for Average Episodic and Sernantic Memory Performance, Prediction, and Confidence for the Entire Sample..........272 Table 37. Correlation of Episodic and Semantic Memory Performance with Health and Memory Information Measures, Preparation Time, and Number of Associations, for the Entire Sample...........275

Table 38. Sumary of Multiple Regression for Episodic and Semantic Memory Performance.........276 Table 39. Summary of Data Most Relevant to Hypothesized Processing Differences Contributing to Age Difference in Episodic Memory Performance..280 
List of Figures

Figure 1. A modal model of memory..............

Figure 2. A schematization of metamemory.........25

Figure 3. Memory - metamemory relationships.....40

Figure 4. Hypothetical developmental functions

of three cchort groups...................44

Figure 5. Hypothetical functions from two crosssectional and one longitudinal assessment of age

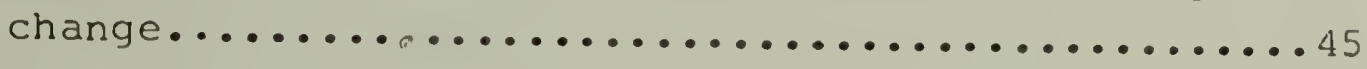

Figure 6 . Design plan......................

Figure 7. Mean percentage of items correctly remembered by males and females in each Education

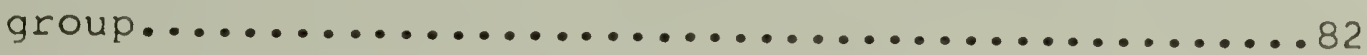

Figure 8. Mean percentage of items correctly remembered by males and females in each Age group..83 Figure 9. Mean percentage of incidental and intentional items correctly recalled and recognized by each Age group.........................

Figure 10. Mean number of recall responses by males and females in each Education group.......96 Figure 11. Mean number of correct and incorrect recall responses by males and females in each Age

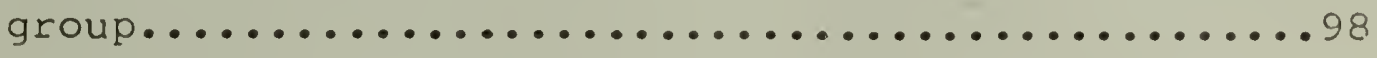

Figure 12. Mean number of correct and incorrect recall responses by males and females in each

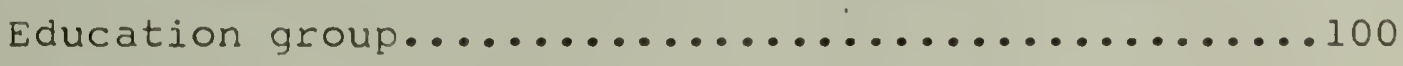
Figure 13. Mean number cf list, associate, and miscellaneous intrusions on incidental and inten-

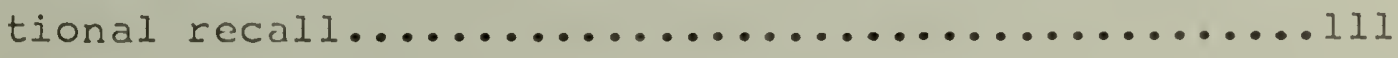


Figure 14. Mean percent correct recognition by males and females in each Education group.........117 Figure 15. Mean percent correct responses for incidental, intentional, and new items, by each

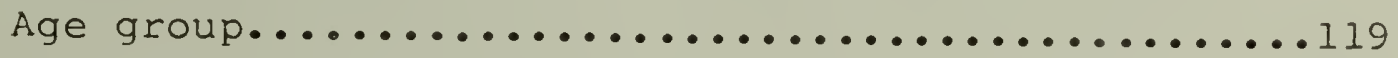

Figure 16. Mean number of correct and incorrect responses to general information questions by each

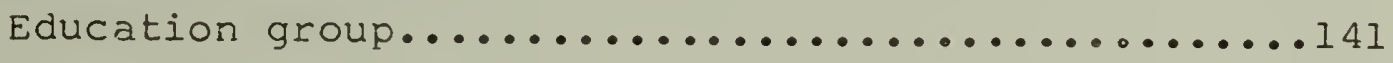
Figure 17. Mean predictions of memory performance by males and females in each Age $x$ Education group. 172 Figure 18. Mean predictions for incidental and intentional memory performance by each Education

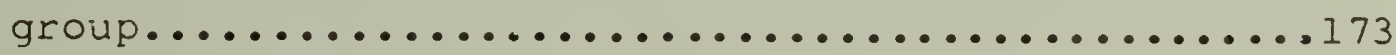

Figure 19. Mean predictions of recall and recognition memory performance by males and females in

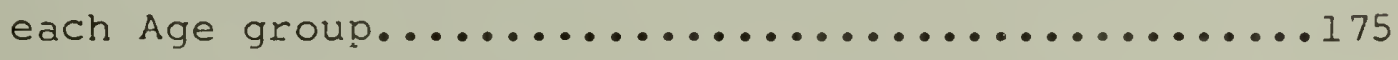
Figure 20. Mean predictions of recall and recognition memory performance by males and females in

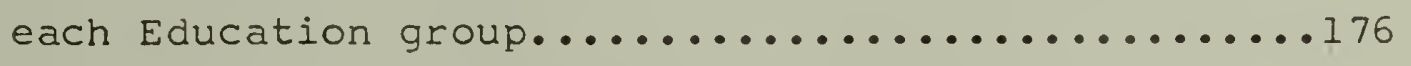
Figure 21. Mean differences in prediction and performance on incidental and intentional memory

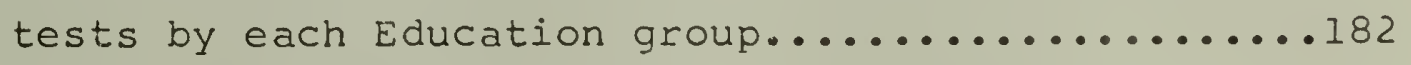
Figure 22. Mean differences in prediction and performance on incidental and intentional recall

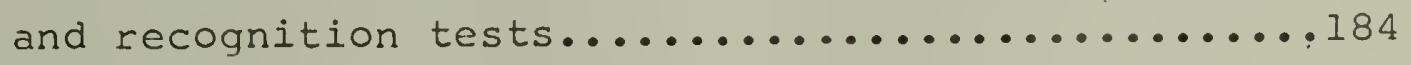
Figure 23. Mean prediction accuracy scores on episodic memory tasks for males and females in

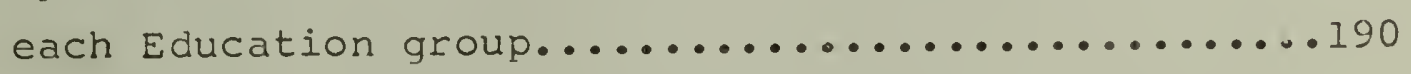
Figure 24. Mean predictions for general information recognition by males and females in each Age

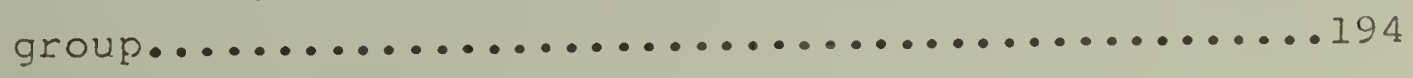


Figure 25. Mean predictions of general information recognition by males and females in each Age $x$

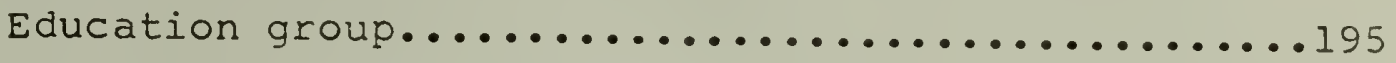
Figure 26. Mean confidence ratings on episodic memory tests for each Age $x$ Education group.......215 Figure 27. Mean difference scores between confidence ratings on hits and false alarms on memory tests for each Age $x$ Education group.............220 Figure 28. Mean difference scores for confidence ratings on hits" and false alarms on episodic memory

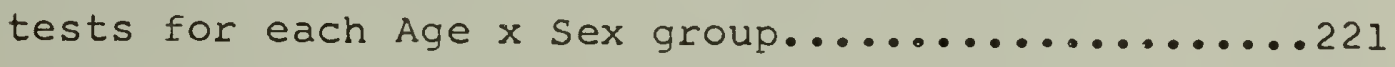
Figure 29. Mean confidence ratings on correct and incorrect old and new recognition items......227 Figure 30. Mean confidence ratings for hits and false alarms on general information knowledge

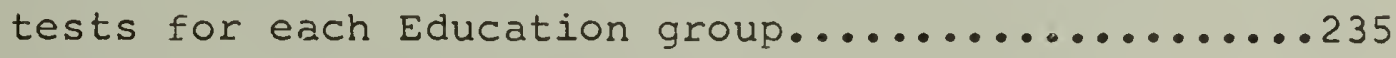
Figure 31. Mean confidence ratings for hits and false alarms on general information recall and

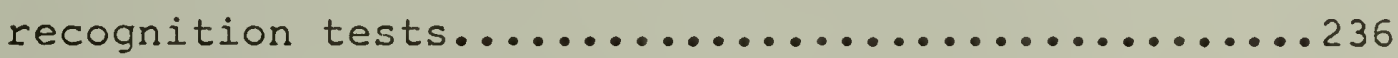
Figure 32. Mean confidence ratings for correct and incorrect true and false items on general

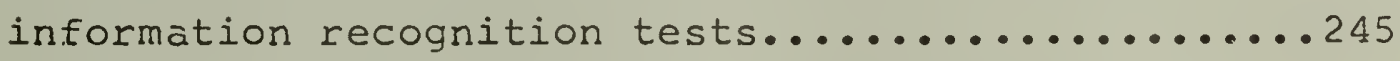
Figure 33. Mean times spent on each task by the

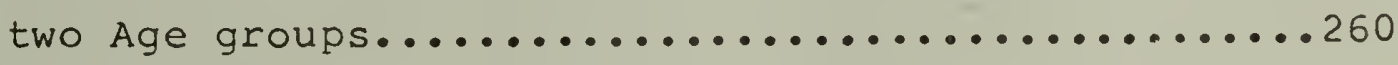
Figure 34. Mean times spent on each task by the

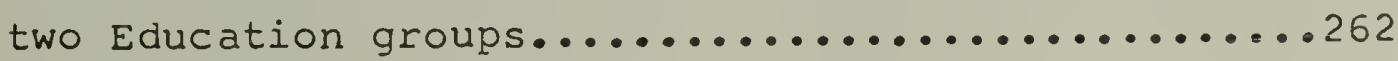


I. Introduction

It is often assumed "common knowledge" that memory declines with age. Yet, the nature of this decline is still not clearly delineated. This dissertation is concerned with age-related memory differences in normal adults, an attempt is made to more clearly describe aging effects on a variety of memory phenomena.

First, an underlying conceptualization of memory will be explicated. Figure 1 depicts a modal model of memory. This general type of model underlies most present information processing conceptualizations, and will serve as a framework throughout this dissertation. The following four sections will review literature elucidating adult age differences in memory components outlined in Figure 1.

A. What is Memory Aging the Aging of?

1. Sensation and Perception

An analysis of sensory and perceptual changes in normal adult development is beyond the scope of this dissertation. However, it should be noted that older subjects show deficiencies in all five senses: vision, audition, taste, smell, and touch (see Kimmel, 1974). Also, this aging effect is probably important, since higher thresholds of stimulation are required for older subjects to perceive. Some apparent memory deficits in older subjects may thus be more appropri- 

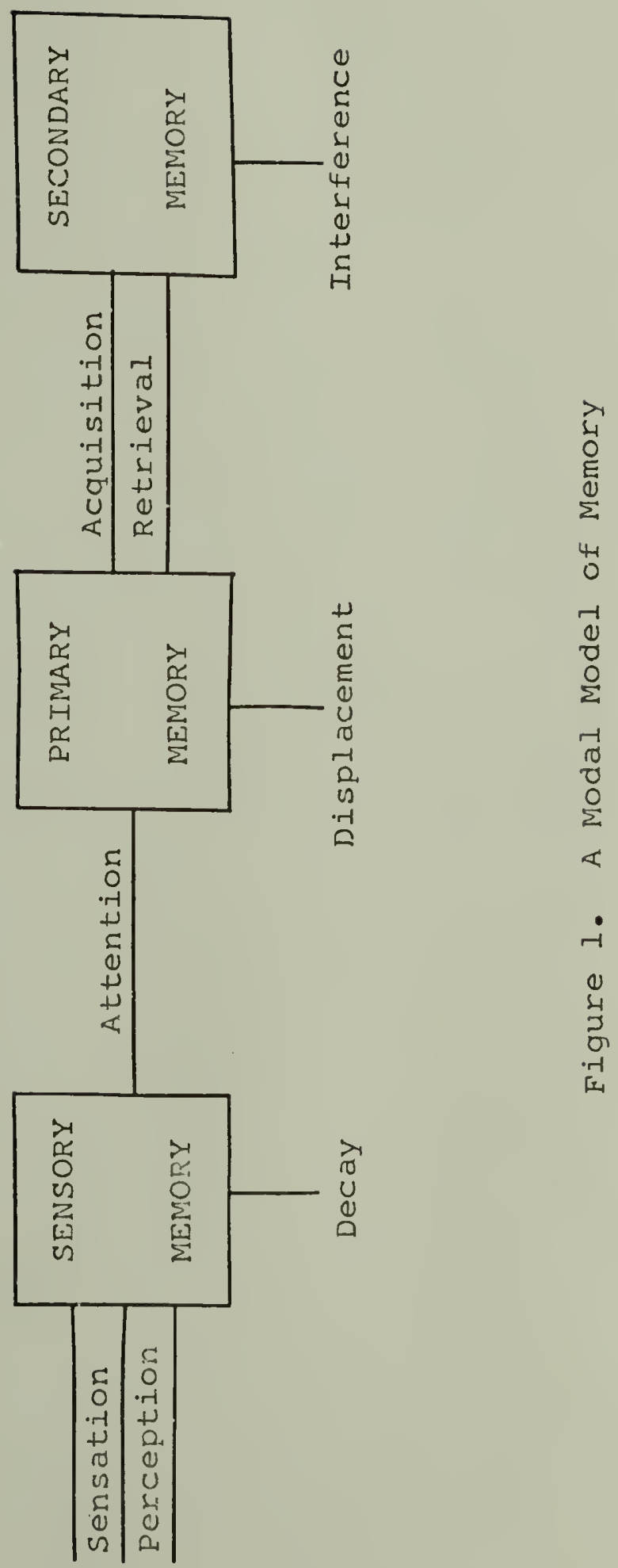
ately attributed to failures in sensory registration.

2. Attention

A thorough analysis of attention is also beyond the scope of this dissertation. However, it should be noted that age-related difficulties in dealing with situations requiring division of attention, may contribute to memory deficits. Additionally, it is well documented that older subjects are more penalized than younger subjects, when they must jointly attend to two input sources, an input source and memory, or memory and response execution (see Kay, 1953). In a series of divided attention studies, Craik (1973) concluded that more of older subjects' processing capacity is taken up organizing or programming division of attention, and this leaves less capacity to process stimuli. If older subjects' capacity is differentially reduced in this way, they are forced to process information less deeply, and this in turn, may account for memory deficits.

\section{Primary Memory}

At least two types of memory can be distinguished. One involves representation of the present, and the other, knowledge about the past. Memory investigators generally speak of primary memory when referring to the first, and of secondary memory when referring to the second (e.g., Waugh \& Norman, 1965). The validity of 
this conceptual distinction is at least threefold. First, these memory stages have different functions; while primary memory temporarily holds or organizes information, secondary memory is a permanent knowledge store. Second, while some specific processes may be critical for primary memory, others, for example, retrieval, are more central to secondary memory. Finally, the course of development of these two sorts of memory may be different. For instance, it appears that primary memory is unimpaired in the elderly, but that there are age decrements in secondary memory. Primary memory may be considered the number of items that can be retained in consciousness. One measure of this capacity is the recercy effect. Indeed, Watkins (1974) defined primary memory as "the mechanism underlying the recency effect in free recall" (p. 695). It is probably of considerable significance that most experiments have demonstrated no age difference in the recency portion of recall. That is, even when age differences are observed in overall level of recall, no differences are found for the last few serial positions (e.g., Bromely, 1958; Craik, 1968). Another procedure used to evaluate primary memory is the memory span task. This procedure involves determination of the number of items in the longest string that can be reported in correct serial order. 
Most people can correctly recall, in serial order, seven digits, or five words. However, purer estimates of primary memory range from about two to four items (see Watkins, 1974). Thus, the memory span measure largely reflects primary memory, but it probably also partially reflects secondary memory. Many investigators find no significant age differences in digit spans of twenty to sixty-five year olds (e.g., Bromely, 1958; Craik, 1968), although others report slight but reliable age decrements (e.g•, Botwinick \& Storandt, 1974; Taub, 1973).

A similar task, backward span, requires subjects to repeat strings of items, but in reverse serial order. Generally, larger age decifits are found with backward, than forward, span tasks (e.g.. Botwinick \& Storandt, 1974; Bromely, 1958). Apparently, if reorganization is added to the retention requirement, older subjects become more disadvantaged. In summary, it appears that older subjects' performance is unimpaired when only primary memory capacity is tapped, however, deficits are observed when additional cognitive activity, such as organization, is required.

Of course, this summarizes performance measured by number of items recalled, and that may not be the most sensitive index of mnemonic functioning. Anders, Fozard, \& Lillyquist (1972) used the sternberg (1966) 
reaction time paradigm to assess age differences in primary memory more precisely. Subjects were presented sequences of 1 to 7 items, and then required to decide whether a test item had appeared on the list. The dependent measure of interest was the time required to make decisions for items from various length lists. For all age groups $(20,38$, and 68 year olds) decision latencies increased linearly. Morecver, the change in slope of these functions suggested that search speed decreases with age. Additionally, the intercepts of the latency functions increased with age, suggesting an age ralated slowing of other basic operations involved in the task, possibly decision or response execution processes. Thus, although the number of items retrieved from primary memory may remain essentially stable throughout adulthood, the speed of search and retrieval probably declines.

\section{Secondary Memory}

Remembering more items than can be held in primary memory is indicative of secordary memory functioning. It is important to note that this primary--secondary. memory distinction is independent of retention interval. That is, even when retention is tested immediately, if primary memory span has been exceeded, secondary memory processes contribute to performance. Furthermore, it is apparently the secondary memory system that is most 
impaired by aging (e.g., Craik, 1975; Horn, 1975).

If a model such as depicted in Figure 1 is assumed, the locus of secondary memory deficits may be in one or several different memory components. That is, secondary memory implies at least three things. First, acquisition processes transfer information to secondary memory. Second, information is retained in the storage capacity of secondary memory. And third, retrieval processes transfer information from a passive state in secondary memory to an active state in consciousness. Moreover, our knowledge about the development of memory suggests quite a different course of growth for these various mnemonic components. For example, while habituation and recognition in young infants indicates basic retentional capacities quite early in development, other memory components do not appear mature until a later age. For instance, one important mnemonic feat, probably not accomplished until the end of the sensorimotor period ( 18 months), is the ability to represent non-present experience. But considerable refinement and control of this critical memory capacity, representation, is evident throughout childhood. Furthermore, several aspects of this important memory ability can be delineated. There are relatively automatic, semantic, or logically based operations, that organize, or conceptualiy tie information in memory. 
And, there is a large repertoire of voluntary mnemonic strategies that may be deliberately deployed to more effectively acquire or retrieve information.

Thus, three secondary memory stages have been specified: 1) acquisition, 2) storage, and 3) retrieval. Furthermore, two sorts of mnemonic operations have been delineated: 1) automatic constructive and organizational processes, and 2) deliberate acquisition and retrieval strategies. It has been argued that evidence of secondary memory implies processing at each of these stages. Yet, experimental manipulations do permit examination of separate contributions of each stage, although of course, each can never be entirely isolated from the others. The following three sub-sections will review research which has begun to delineate the locus and nature of age deficits in secondary memory, as outlined above.

\section{a. Short-Term Retention}

1. Acquisition. If age deficits in secondary memory are to be attributed to deficiencies in acquisitional processes, not to decrements in storage capacity or retrieval processes, then no age difference in retention should be observed when initial learning has been equated. Several studies have reported such a finding (e.g., Moenster, 1972; Hulicka \& Weiss, 1965; Wimer \& Wigdor, 1958), and the one carried out by 
Hulicka \& Weiss (1965) will serve as an example. They had subjects learn paired associates under three conditions: equal number of training trials, learning to criterion, and overlearning. Older subjects learned less with equal exposures, and required more trials to criterion, but once having learned the material, they retained it as well as the younger subjects. At least in this paired associate task, age deficits in retention can apparently be eliminated by providing older subjects with extra exposure to the stimulus materials.

However, these paired associate learning studies do not explain why older subjects require additional exposure to remember information. Several theorists hypothesize various mechanisms to account for acquisition of superspan amounts of information. Miller (1956) suggested that items must be chunked for recall, Mandler (1967) considered categorical organization important, Flavell (1970) talked about verbal rehearsal, Paivio (1971) emphasized imagery, and Craik \& Lockhart (1972) proposed that depth of processing determines retention. Several studies have examined age changes in use of these acquisitional mechanisms.

Hulicka \& Grossman (1967) investigated age differences in use of verbal and imaginal mediators, and 
their effect on recall. They compared younger ( 16 year olds) and older ( 74 year old) subjects' paired associate learning performance under a control and three mediational instruction conditions. In the control condition no special instructions were given, in a verbal instructions condition subjects were provided with a word or phrase which linked the words of the pair, in an experimenter image condition they were provided with the connector and told to form an image of the scene suggested by the phrase, and in a self image condition they were simply instructed to attempt to form an image which included both items of the pair. Performance of all subjects improved under mediational instructions, both when merely given the mediational technique, and when given the mediators as well as the technique. When no instructions were given, younger subjects reported use of mediators much more often than older subjects. (Moreover, when instructions were given to use mediators, the old subjects showed relatively more improvement; the overall age effect was, greatly attenuated by mediational instructions. Thus, older subjects appear to benefit more from production instructions. Apparently, they are able to perform the appropriate mnemonic operations, but they typically fail to do so.

These results were essentially confirmed by 
Canestrari (1968). He used a paired associate task, two age levels ( 20 and 62 year olds), and three instructional conditions (standard, verbal mediators, and pictorial mediators). He found that mediators improved performance, and that younger subjects' performance was superior to older subjects, regardless of condition. However, he also found that providing mediators resulted in greater improvement for older subjects.

Another deficiency possibly limiting acquisition in older subjects, may involve a decrement in their use of organization. Clustering analyses assess the extent to which words from the same category are recalled together. Although this might merely reflect organization at retrieval, it is generally also considered to reflect organization imposed on incoming. material at acquisition. Denney (1974) examined recall and clustering of middle aged ( 42 year olds) and elderly ( 81 year olds) subjects, on two stimulus lists. A complementary list was composed of eight pairs of words related in a complementary fashion, and a similarity list was composed of eight pairs of words that shared a similarity relationship. The older subjects recalled fewer words, and clustered less than younger subjects. Additionally, while the younger subjects clustered more on the similarity than 
similarity than complementary list, oider subjects demonstrated comparable non-significant levels of clustering on both lists. These results indicate that there is an age-related decline in use of organization, and this decline may, at least in part, account for the memory decrement observed in the elderly.

Hiltsch (1969) investigated age differences in organization and recall by manipulating instructions. He gave three age levels $(17,35$, and 49 year olds) a multitrial free recall task. A control group received standard free recall instructions, an organization instructions group were told to try to organize the lists in some way, and an alphabetic instructions condition were told to try to organize the words alphabetically. The results indicated both instructions to organize stimulus materials disproportionately benefited older subjects, suggesting again that there may well be age-related deficits in organization. Apparently, older subjects were not spontaneously using organization, although the evidence indicates that this could indeed improve their performance. In a subsequent study, Hultsch (1971) utilized a free classification task, which permitted more direct assessment of age differences in organization and recall. His design included three age levels $(24,46$, 
and 62 year olds), and two experimental conditions (sortirg and nonsorting). There were no significant age differences in free classification performance in the sorting condition, although the level of recall of all subjects was higher than in the sorting than nonsorting condition. An age-related decrement in recall was also observed, but perhaps more interesting, there was a significant age by condition interaction which indicated that a greater age decrement under the nonsorting than sorting condition. Age differences were attenuated when subjects were encouraged to meaningfully organize stimulus materials. This study thus indicates again that at least a portion of the memory deficit observed in older subjects can be attributed to their failure to effectively organize material at acquisition. Moreover, instruction and sorting manipulations are effective in reducing, although not eliminating, age differences. An apparent organizational production deficiency may account for part, but probably not all, of the memory impairment associated with aging.

If production deficiencies are indeed a major source of age decrement, then equating acquisition, by controlling a wide range of encoding operations, should attenuate age differences in recall. Eysenck (1974) used an incidentil learning paradigm to test 
this notion. Two age levels ( $18-30$ and 55-65 year olds) were tested in five experimental conditions designed to manipulate level of encoding processing. Two conditions required relatively shallow processing (counting letters or generating rhymes), two required deeper levels of semantically based processing( generating adjectives or images), and one served as a control (intentional learning). In general, younger subjects recalled more than older subjects, and deeper levels of processing resulted in better performance than shallower levels. More interesting was the age by condition interaction. The largest age effect was obtained in the intentional learning' condjtion, a diminished but significant age difference was observed with deeper levels of processing, and no age difference was found with shallow levels of processing. Thus, these results again indicate that manipulations that equalize acquisitional processing attenuate age differences in retention. It is interesting too, that shallow processing tasks completely eliminated age differences, but deeper processing only diminished them; younger subjects apparently maintain some advantage when semantic processing is incorporated in a task, thlis suggesting some real limitation in older subjects' automatic semantic processing.

2. Storage. No good evidence of age changes 
in storage capacity seems to exist (e.g., Wickelgren, 1975), and indeed present theoretical formulations of memory seem to assume that if material is registered in secondary memory, it is not lost, although it may become iraccessible (e.g., Atkinson \& Shiffrin, 1970).

3. Retrieval. One method used to evaluate the relative importance of deficiencies in acquisition versus retrieval, is to compare recall and recognition. While recall involves both acquisition and retrieval, recognition is generally assumed to involve mainly acquisition. That is, if memory is conceptualized as the creation of a trace, and recollection is determined by appropriateness of information in the retrieval environment, then the difference between recall and recognition resides in differences in the retrieval environment (Watkins \& Tulving, 1975). For recognition, a copy of the encountered stimulus is physically present, while for recall, it must be cognitively retrieved. Thus, if it is assumed that retrieval plays a minimal role in recognition, then age deficits in recognition can be cautiously interpreted as reflecting deficits in acquisition or storage. On the other hand, greater magnitude recall deficits can be attributed to retrieval difficulties.

Empirical work has generally demonstrated more severe age decrements in recall than in recognition. 
All investigators seem to find fairly large age differences in recall tasks (e.g., Bromely, 1958). On the other hand, most investigators find either small, though reliable, age differences (e.g., Botwinick \& Storandt, 1974; Gordon \& Clark, 1974), or no significant age differences (Craik, 1971) in recognition. In a single study Erber (1974) examined age decrements in both recall and recognition. While she found older subjects ( 60 years old) performed significantly worse than younger subjects (23 year olds) on both tasks, age accounted for $25 \%$ of the variance in the recall task, but only $10 \%$ of the variance in the recognition task. Thus, when retrieval demands are minimized, as in recognition, memory disadvantages of older subjects are reduced; but probably not eliminated.

Several recall studies also support this notion, demonstrating that older subjects are aided more than younger subjects when good retrieval support is provided. Laurence (1967a) examined recall of 12 item lists, which were either all from a single conceptual category, or all from different categories. While performance of. older subjects was considerably worse than younger subjects on unrelated lists, it was only slightly worse on related lists; there was a highly significant age by list type interaction. It is possible that when items from a single conceptual. category were to be remembered, 
the category concept served as an effective retrieval cue, although of course, it could be argued that the effect resulted from acquisitional differences. In a subsequent cued recall study by Laurence (1967b), age decrements were eliminated when category names of items were provided at retrieval. Thus, this adds to the interpretation that deficits in effective retrieval contribute importantly to older subjects' recall disadvantage.

Craik (1968) also examined the effects of retrieval information on recall. He tested 22 and 62 year olds, and manipulated the size of the pool from which items were drawn (digits, counties, animals, and unrelated words). The results revealed a tendency for the age decrement in recall to be attenuated for small word pools. Thus, when adequate retrieval information is available, either by providing recall cues, or by using a linited set of items, older subjects' retention is less impaired.

There is another index which has been used for estimating relative retrieval versus arquisition deficits on categorized recall lists. This entails separate analyses of the number of chunks recalled, and the number of words recalled per chunk. The number of chunks recalled has been taken as an index of retrieval effectiveness, while the number of words re- 
called per churk has been considered an index of encoding efficiency. Craik \& Masani (1969) found that older subjects ( 72 year olds) recalled fewer chunks than the younger subjects (22 year olds), but did not diffex in the number of words recalled per chunk. Because the older subjects retrieved fewer chunks than younger subjects, the author concluded that aging had a detrimental effect on retrieval, but because there were no differences in the number of words recalled per chunk, they concluded that aging did not affect encoding. However, it is not entirely clear that the number of words recalled per chunk is a pure index of encoding efficiency, uninfluenced by retrieval effectiveness. Furthermore, Hultsch (1975) found age deficits in both these measures. Thus, the conclusion that aging did not affect encoding should be interpreted with reservation.

Hultsch (1975) employed similar analyses, but, perhaps more appropriately, interpreted the number of words recalled per category, as retention or accessibility of stored event information, and the number. of categories recalled, as a measure of accessibility of higher order memory units. Thus, both measures probably reflect retrieval and acquisition, but of different sorts of information. Three age levels 120 , 59, and 70 year olds), and two recall conditions (free 
and cued) were used. The results indicated significant age differences in number of words recalled, categories recalled, and words recalled per category. Thus, this study indicate that adult age differences in retention probably reflect deficits in accessibility of higher order units, as well as availability of elementary units.

An additional indication of retrieval deficits in old subjects comes from a repeated recall study carried out by Buschke (1974). In his task, subjects were given repeated recall trials of a 20 word list which was only presented once. The results indicated greater variability in the pool of words that older subjects consistently recalled from trial to trial. Apparently, many of the words were adequately acquired and stored, but on some trials, there was retrieval failure for some of them.

b. Long-Term Retention

No qualitative distinction has been assumed between short and long term retention. However, laboratory investigations most often assess retention after short intervals, and anecdotes seem to perpetuate the notion that memory for remote events is unimpaired in the elderly. Long-term memory studies are thus also of interest. Bahrick, Bahrick, \&. Wittlinger (1975) investigated recall and recognition of names and faces of high school classmates, statistically chrolling for effects 
of conditions that influence original learning such as class size, and conditions that influence rehearsal, such as attendance at class reunions. Their results indicate fairly stable recognition of names and faces, as well as name-face matching until about 35 years of age, but some deterioration past that age. Recall declined earlier, and more steadily. Thus, older subjects were poorer than younger subjects at recognizing, and especially at recalling, events from the past; the notion that memory for remote events is unimpaired in the.elderly must be rejected.

\section{c. Episodic-Semantic Distinction}

All of the laboratory studies thus far reviewed have examined memory for fairly specific information, usually lists of memorized words. But this type of memory is hardly characteristic of memory demands ordinarily placed on people. That is, people often make use of more generalized abstracted information that does not have a single specific referent. Several memory theorists (i.e., Collins \& Quillian, 1969; Rumelhardt, Lindsay \& Norman, 1972; Anderson \& Bower, 1973; Tulving, 1972; Piaget \& Inhelder, 1973) have found it useful to differentiate two such forms of memory; and Tulving's episodic-semantic distinction is a prototype of this notion. He characterizes episodic memory as a fairly literal, based upon single autobiographical 
referents, and semantic memory as a more schematized sort of memory, based upon cognitive referents. AIthough it is often difficult, if not impossible, to dichotomize information as belonging to one or the other of these memory types, the distinction may still be useful. For example, Botwinick \& Storandt (1974), examined 20 to 70 year olds recall of words (episodic memory), and general information (semantic memory) and found that older subjects recalled significantly fewer words than younger subjects, but nonsignificantly more general information. It is possible then that aging affects retention of more general information.

\section{d. Summary}

Several processing deficits have thus far been demonstrated to underlie memory deterioration in adulthood. Early paired associate learning research found that elderly subjects suffer from acquisitional deficits. When level of original learning was equated, age-related retentional differences were eliminated (e.g., Moenster, 1972; Hulicka \& Weiss, 1965; Wimer \& Wigdor, 1958). Subsequent research has helped to delineate the nature of these acquisitional deficits. Hulicka \& Grossman (1967), as well as Canestari (1968), found that instructing subjects to use mediators diminishes age decrements in paired associate learning. Denney (1974) found little clustering by the elderly, and Hultsch found that 
instructions to organize (Hultsch, 1969), and sorting tasks (Hultsch, 1971), disproportionally benefited older subjects. Eysenck (1974) studies effects of various incidental learning procedures on retention, and found that tasks which controlled acquisitional processing, attentuated age differences in recall. Moreover, his study suggested that younger and older subjects could be equated with respect to shallow levels of processing, but younger subjects maintain some advantage on tasks which involve deeper semantic processing. Thus, considerable evidence points to age-related deficits in acquisition. Many findings suggest that elderly suffer from production deficiencies (c.f., Flavell, 1970), i.e., their diminished retention could be improved if they engaged in appropriate acquisitional processes. However, several other findings point to a more complex deficiency, which may not be susceptible to subjects' control.

Several other lines of research have indicated further mnemonic deficits in the aged. Investigations of recall and recognition have found greater age-related decline in recall than recognition $(e \cdot g \cdot$, Botwinick \& Storandt, 1974; Craik, 1971; Erber, 1972), and this has been taken to indicate retrieval problems in the elderly. Also, recall studies that manipulate retrieval support (e.g., Laurence, 1967ab; Craik, 1968), find that 
retrieval deficits contribute importantly to age differences; when adequate retrieval cues are provided, age differences are diminished. Likewise, measures of the number of categories recalled, and the number of words recalled per category on related lists (e.g., Craik \& Masani, 1969; Hultsch, 1975), indicate agerelated retrieval deficits of higher order information, as well as elementary information. Finally, a repeated trials experiment, carried out by Buschke (1974), also demonstrated retrieval deficits in the elderly; older subjects evidenced greater variability than younger subjects, in the pool of words they recalled consistently from trial to trial. Considerable evidence has thus also accumulated showing age-related deficits in retrieval-assuciated mechanisms.

\section{Metamemory}

We cannot help but feel that if there is ever going to be a genuine breakthrough in the psychological study of memory....it will, among other things, relate the knowledge stored in an individual's memory to his knowledge of that knowledge. (Tulving \& Madigan, 1970 , p. 477)

An additional menmonic phenomenon is metamemory. This is a newly born conceptualization (c.f., Kruetzer, Leonard, \& Flavell, 1975), referring to knowledge ar individual has about information stored, and operations 
utilized, in menory. Metamemory is to memory per se, as cognition is to behavior. Research investigating this memory phenomenon has barely begun, and to my knowledge, no work has yet examined the character of metamemory throughout adulthood, nor the role it plays in memory decline. Moreover, researchers still need to formulate a more precise definition of this vague concept, and further work also reeds to focus on developing better techniques for assessing metamemory.

Several distinct questions can be proposed about metamemory, and they will be framed in a developmental perspective. First, what metamemory knowledge do variously aged subjects possess? second, at each age level, what role does metamemory play in mnemonic functioning? And third, what is the relationship between the development of metamemory and development of other memory skills?

While relatively little research has yet addressed these questions, some, though largely with children, is relevant. A preliminary framework may be helpful for organizing existing metamemory data, as well as for considering new ways to analyze metamemory. One possible schematization of metamemory is presented in Figure 2. Metamemory mày be characterized in terms of several continiums. One of these might be described as running from specific, relating to concrete task-defined memory 
Figure 2. A Schematization of Metamemory

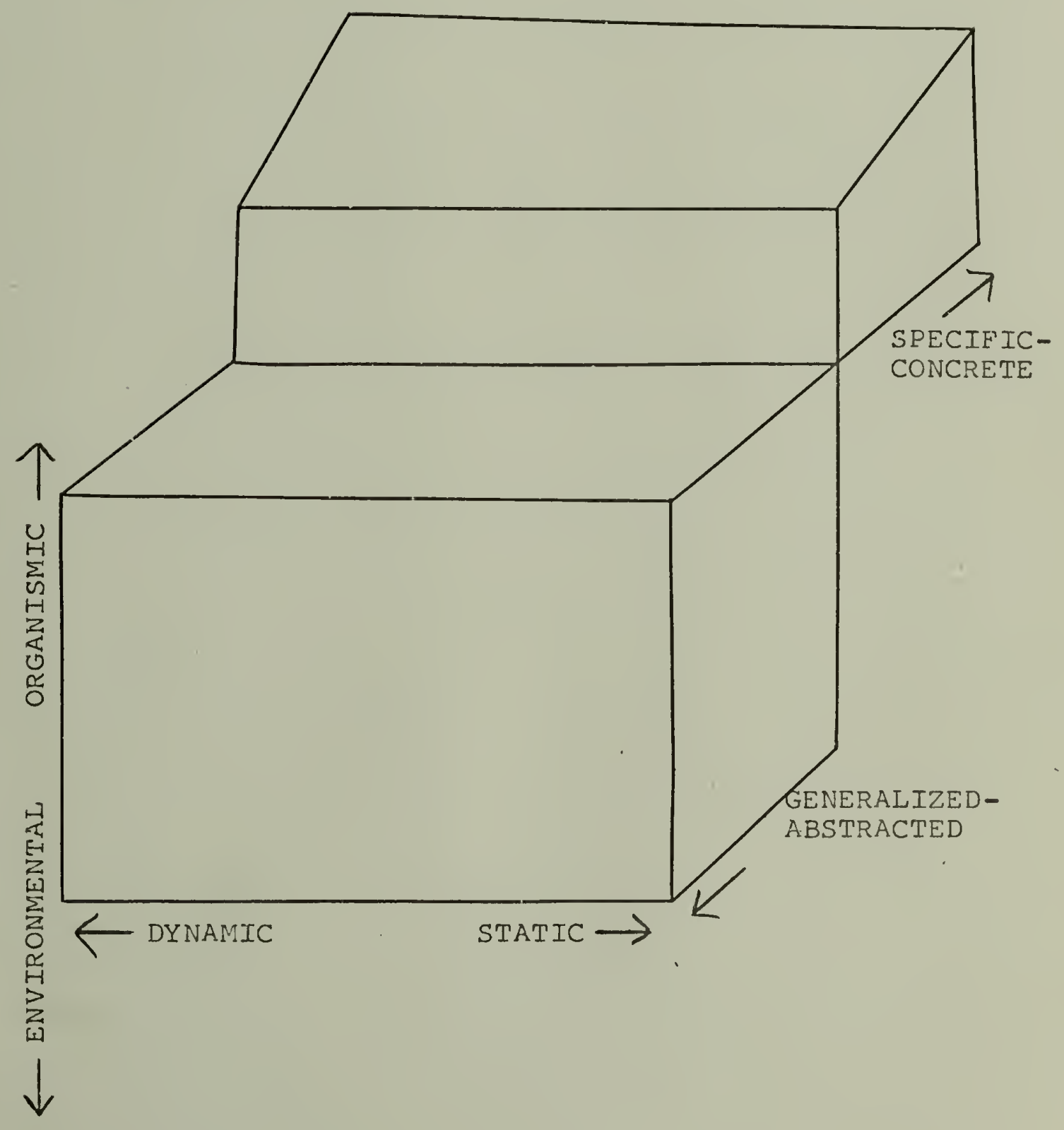


experiences, to general, relating to abstractions from many memory experiences. Specific-concrete metamemory knowledge refers to qualities about memory which a subject may not be aware of, but if queried, can, in the act of remembering, assess. Examples of this type of knowledge include the ability to predict and assess specific storage states, and the ability to differentiate mnemonic functions from other functions. Generalized-abstracted knowledge, on the other hand, is not elicited in the act of remembering, but rather refers to all knowledge about variables that affect memory, such as might be tapped in a questionnaire. This knowledge may be further delineated, however, and as may be seen in Figure 2, another dimension might be in terms of whether it concerns environmental or organismic factors. That is, whether it involves memory abilities and activities, or mnemonics effects of characteristics of materials to be remembered and external memory aids. Obviously, these categories are not so clear cut, and indeed, some very interesting aspects of metamemory probably involve knowledge about the interaction between organismic and environmental variables. Finally, metamemory knowledge may also be distinguished in terms of a continuum with reference to static-dynamic aspects of memory. Examples of each of these types have already been enumerated for specific-concrete metamemory know- 
ledge; the ability to predict and assess storage states pertains to static aspects of memory, and the ability to differentiate mnemonic functions from other nonmnemonic cognitive functions, such as perception, pertains to dynamic aspects of memory. Examples for each specification of generalized-abstracted knowledge may also be enumerated; knowledge about the limits of one's memory capacity refers to static-organismic properties of memory, knowledge about strategies one may employ to facilitate memory concerns dynamic-organismic qualities, knowledge about characteristics that make stimulus materials easy or difficult to remember pertains to staticenvironmental aspects of memory, and knowiedge of external aids that may serve memory reflects dynamic-environmental characteristics of memory

a. Generalized-Abstracted Knowledge. Generalizedabstracted metamemory refers to knowledge about the structure and functioning of memory which is not elicited in the act of remembering, but which is derived from abstractions from many mnemonic experiences. This knowledge encompasses organismic knowledge, i.e., an individual's knowledge about his own memory abilities and activities, as well as environmental knowledge, i.e., his knowledge about mnemonic effects produced by certain characteristics of material to be remembered and external memory aids. Also, organismic and environ- 
mental knowledge both relate to static and dynamic aspects of memory.

1. Organismic Factors

a. Static Aspects: Memory Capacity. Kruetzer, Leonard, \& Flavell (1975) used an interview technique to assess children's knowledge about their own memory capacities. In what will probably be a seminal piece of work, they interviewed twenty children at each of four age levels $(6,7,9$, and 11 years olds), about a variety of questions designed to evaluate a wide range of metamnemonic knowledge. Almost all children recognized that their memories are fallable. When asked, "do you forget", all but a third of the kindergarteners acknowledged that they sometimes do. Additionally, most subjects in each age group knew that information in immediate memory is susceptible to rapid forgetting. When asked if they wanted to phone their friend and someone told them the phone number, would they call right away or get a drink first, the model response for all age groups was to phone first. Thus, this study shows that even very young children have some knowledge about memory capacities.

\section{b. Dynamic Aspects: Memory Strateaies. The} Kruetzer et. al. (1975) study also examined children's knowledge about dynamic aspects of their memories. The majority of children in each age group indicated know- 
ledge about the relationship between study time and probability of recall. When asked, "which child remembered most, the one who studied one minute, or the one who studied five minutes", almost all subjects said that the child who studied five minutes would remember most. Also, a large and equal percentage of subjects in each age group knew that there is savings in memory. When asked whether it would be easier for a boy who was relearning a list of names, or one who hadn't learned it before, to learn the list, most children thought it would be easier for the relearner. Thus, the Kruetzer et. al. (1975) study indicates that even very young children know a considerable amount about the dynamics of their memories. Yet, the authors suggest that older children have a more differentiated concept of self as a mnemonic organism, and are thus more likely to conceptualize memory ability as something that varies over occasions. Indeed, several aspects of the data indicate a more refined metamemory in older subjects. In each case, where follow-up justification questions were asked, older children were able to articulate more, and better, responses. Moreover, several questions directed at irvestigating activities that subjects say they engage in to promote memory, seemed to indicate a developmental increase in metamemory knowledge. For example, more older children 
suggested categorization as an appropriate study plan, and in general, older children gave a greater number of adequate responses to questions concerning plans for storage and retrieval.

Thus, while this study provides evidence that even kindergarten aged children know something about the functioning of their own memories, it seems to suggest a developmental increase in awareness and planfulness in approach to memory problems. Yet, this developmental trend should be interpreted with caution. The interview technique utilized puts excessive verbal demands on subjects, and it may be that this production requirement underestimated younger children's metamemory. In most instances where verbal demands were minimal, developmental differences in memory knowledge were absent. Perhaps a choice task would more adequately assess younger children's metamemory knowledge.

\section{Environmental Factors}

\section{a. Static Aspects: Characteristics of}

Stimulus Materials. Moynaham (1973) investigated the development of awareness of the fact that free recall is facilitated when items to be recalled are from the same conceptual category, rather than from different ones. Children $(7,9$, and 11 year olds) were asked to predict which of two sets of stimuli, one of related and the other of unrelated items, would be easier to 
remember. The results indicated that awareness of the facilitative effect of categorization on recall increases with age. Despite the fact that the facilitative effect was equally strong at all ages, younger children were less likely than older children to predict that the categorized items would be easier to remember. Moynahan suggests that the young children may not have differentiated the act of remembering from the act of naming. The youngest children of ten said that a particular set of items would be easier to remember because they were easier to name.

renney (1975) also investigated children's understanding of the importance of organization for recall, asking whether children realize that organization of some sort, is needed to make a list easy to remember. Additionally, she examined developmental changes in the kinds of relationships children consider useful in remembering. She presented a single word, and had children ( 5,8 , and II year olds) generate short lists, which were either free associates, from the same category, or easy to remember together. When so instructed, all age groups were able to produce words from a taxonomic category, and all age groups took advantage of this type of organizational structure; they clustered in recall. Yet, when the youngest group constructed their own easy-to-remember lists, they rarely selected 
words from the same category. Indeed, for the youngest children, essentially the same kind of lists were generated in response to free association and easy-toremember instructions. In contrast, the older children generally produced items from the same category, regardless of whether asked to compose easy-to-remember or categorized lists.

These two studies thus suggest that young children are facilitated by organized characteristics of stimulus materials to be remembered, at a time prior to when they are cognizant or aware of this fact. Apparently, a categorical quality of memory preceeds metamemory knowledge of it.

The Kruetzer et. al. (1975) study also suggests developmental increases in knowledge about the relative difficulty of remembering various stimulus materials. When asked whether a story or list format would be easier for learning pictures, many children at each age level knew that the story format would be easier. However, there was also an age trend; more older than younger children chose correctly. Likewise, when asked whether a list of opposites, or arbitrarily paired words would be easier to learn, many children in each age group, but more in the older groups, chose the list of opposites.

b. Dynamic Aspects: Use of Externals Aids. 
Memory functioning seems to entail an intimate interplay between the internal and the external. For example, when trying to remember something, which is presumably internally stored in the head, such as the location of a missing object, one thinks of the various likely places it may be, as well as physically explores them; the external environment hopefully cues internal memory. Moreover, many external aids, such as notes, calendars, and even other people, can be used to serve memory. An interesting finding of the kruetzer et. al. (1975) study was that even young children were readily inclined to exploit external resources to serve their own memory processes. Children often replied that they would use other human beings to help them remember, would write notes, use tape recorders, tie strings on their fingers, or would place to-be-remembered objects in places where they would be sure to physically encounter them at retrieval time.

b. Specific-Concrete Knowledge

Specific-concrete metamemory refers to knowledge an individual derives in the act of remembering. This knowledge relates to both static and dynamic aspects of a subject's memory system.

\section{Static Aspects: Memory Monitoring of}

Storage states. Memory monitoring refers to the ability to make specific-concrete metamemory judgments and 
includes predicting and assessing storage states. Although the role of this phenomenon in memory functioning has not yet been determined, it might be hypothesized to be an important component of an executive mechanism; effective deployment of deliberate mnemonic strategies probably depends upon accurate prediction of one's memory capacity, and effective search and retrieval probably depends upon accurate information about the contents of memory.

Flavell, Friedrichs, \& Hoyt (1970) examined developmental changes in children's recall predictions. They assessed subjects' ability to predict the number of items they could retain and recall from their own primary memories. The children $(4,6,8$, and 10 year olds) were presented strings of pictures, and required to predict the longest string they could remember, that is, to predict their immediate memory spans. This thus permitted assessment of children's ability to selfevaluate storage and retrieval capacity. All four age groups over-estimated their actual memory spans. However, accuracy of prediction increased as a function of age; older children remembered more items, as well as predicted they would remember fewer.

In a similar study, Yussen \& Levy (1975) replicated and extended Flavell's et. al. (1970) findings. They demonstrated that children ( 4 and 8 year olds), as 
well as college students (20 year olds), over-estimate their primary memory capacities, but there is a developmental improvement in prediction accuracy across the entire age range studied. Adults remembered more, as well as predicted fewer, than children.

Thus, the results of these two studies, paired with findings of age-related increases in memorization activities, and number of items recalled, suggest a close relationship between the development of the ability to become aware of memory, and actual memory functioning. However, whether there is a causal relation has yet to be determined.

The Flavell et. al. (1970) study also investigated whether children could determine when a set of studied items were sufficiently well memorized to guarantee perfect recall. Over the age range studied $(4,6,8$, and 10 year olds), there was a marked improvement in children's ability to sense when items were sufficiently well memorized to be recalled perfectly. Apparently, the capacity for cognizing one's memory system in this way, also improves during childhood.

Wellman (1975) examined developmental changes in children's recognition predictions. He employed a recall-judgment-recognition paradigm, in which subjects were required to make feeling of knowing judgments, indicating whether they thought they would be 
able to recognize the names of pictures of items they failed to recall. He found evidence for this kind of memory monitoring skill in all age groups $(5,7$, and 9 year olds), however, there was an increase with age, in ability to monitor memory in this way.

Berch \& Evans (1973) examined the development of another metamemory skill, the ability to gauge accuracy of memory responses. They used a confidence rating procedure requiring children to give certainty judgments concerning their recognition of things they had or had not seen before. Both age groups ( 5 and 8 year olds) were able to gauge correctness of their responses to some extent, although judgments of older subjects were somewhat more accurate. Thus, this study demonstrated that children as young as five are capable of this kind of memory monitoring.

\section{Dynamic Asperts: Differentiating Mnemonic}

Functions from Other Functions. Perhaps the first requirement for developing a concept of memory as a dynamic cognitive function, that can to some degree come under one's control, is to discriminate memorization from perception. Appel, Cooper, McCarrell, SimsKnight, Yussen, \& Flavell (1972) investigated children's $(4,7$, and 11 year olds) differentiation of these two processes. They compared subject's recall following instructions to look at, or remember items. They found 
that younger children studied no differently, and subsequently recalled no better, with instructions to memorize items, than with instructions to look at them; under the two instruction conditions they showed the same levels of recall, recall clustering, and various study behaviors. Older children, on the other hand, were more likely to behave differently under the two instruction conditions; they recalled more, clustered more, and showed more study behaviors following remember instructions, than following look instructions. These results thus suggest a developing differentiation between memory and perception, and an increasing ability to become aware of the possibility of evoking certain mnemonic strategies to serve memory. However, the data should be interpreted carefully, since the study did not provide a direct test of behavioral versus conceptual differentiation. It is possible that the younger children's similar performance following instructions to look at or remember items, is attributable to mnemonic processing deficits, not to metamemory deficits. That is, ineffective processing, rather than a lack of conceptual differentiation between memory and perception may have caused this result. The young children may have distinguished these two activities conceptually, but nevertheless, may have been unable to carry out appropriate mnemonic behavior. This study thus points 
out the very real problem encountered when trying to draw inferences about metamemory from behavioral memory data. Indeed, it is this yet unknown relation between metamemory and memory per se which needs to be delineated, but this will require more complex experimental designs.

\section{c. Coordination of Various Metamemory Measures}

One further finding of several metamemory studies should be mentioned. This relates to the hypothesis that various aspects of metamemory might correlate with each other. For example, one could ask whether children who are precocious in their knowledge about some qualities of memory, also tend to be precocious in their knowledge about others. Two studies used multiple metamemory measures, thereby permitting analysis of this question. No evidence supported this kind of relationship, however. Flavell et. al. (1970) found no significant correlation between children's ability to predict their memory spans, and their ability to predict recall readiness. Also, Kruetzer et. al. (1975) found no systematic relationships between all of their metamemory measures. of course, as Kruetzer et. al. (1975) themselves acknowledge, their study was a "descriptive exploration", and was not designed to detect such relationships. Thus, it may be premature to conclude that there are no rela- 
tionships between various measures of metamemory. However, the lack of such relationships does raise the possibility that the assessments have not been entirely reliable. Obviously, this issue needs to be further explored, and better techniques for assessing metamemory need to be developed.

d. Coordination between Metamemory and Memory

If metamemory is indeed a valid notion, and

if it is a distinct component of memory, then questions about the relationship between metamemory and memory per se are of considerable interest. Three possible types of relationships may be hypothesized for the interaction between memory knowledge and memory behavior. Sketches approximating these relationships may be seen in Figure 3. If there is an independent relationship, neither metamemory nor memory affects the other. If there is a unidirectional relationship, it may be of two varieties. In one, memory behavior leads to metamemory awareness, but the reverse does not occur, while in the other metamemory awareness leads to memory behavior, but its reverse does not. Finally, if there is a bidirectional relationship, metamemory and memory are mutually supportive; each tends to elicit the other. It is almost certain that at some point in development each of these types of relationships holds true for some aspect of the relationship between metamemory and 
Figure 3. Memory - Metamemory Relationships

INDEFENDENT
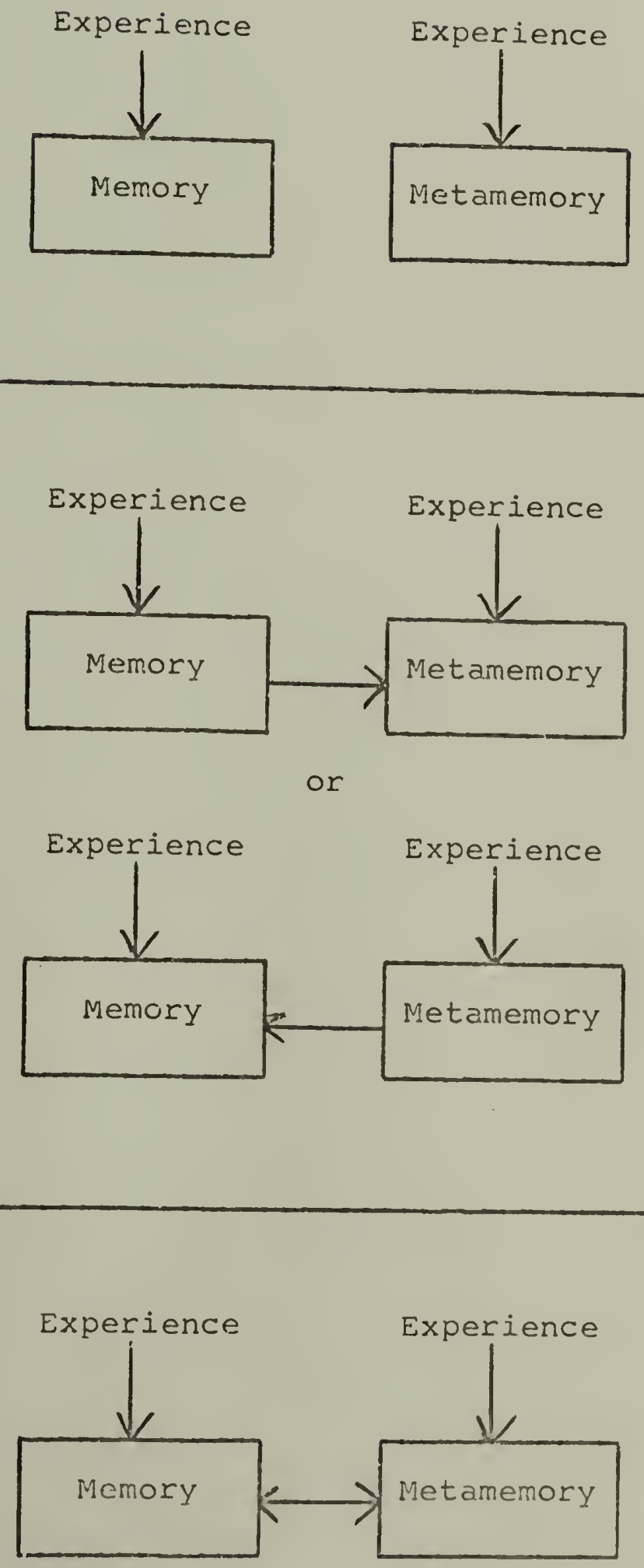
memory. It may be hypothesized, for example, that in childhood, memory development involves an increasing coordination between metamemory and memory, that is, an age-related shift from a predominance of independent relationships to more bidirectional relationships. Yet, through adulthood, as mnemonic activities become increasingly routinized, and perhaps reflexive, this coordination may actually diminish, and the potentially facilitating influence of knowledge about memory may not be actualized. Alternatively, if there is some biological deteriorization of memory in aging, the knowledge about memory that was acquired over the years may no longer be veridical, and this may compound memory problems.

Thus, while questions about the development and coordination of metamemory and memory per se have until now focused on early stages of development, it remains an interesting unexplored problem with respect to normal adult development, and was pursued in this dissertation.

\section{e. Summary}

An appropriate summary of the literature on metamemory might be a quote from a recent paper by Wellman, Drozdol, Flavell, Salatas, \& Ritter (1975).

The study of metamemory has just begun. We know very little about his this knowledge about memory relates to actual 
mnemonic behavior, and about possible developmental changes in this metamemoryto-memory behavior relationship.

(Well, an, Drozal, Flavell, Salatas, \& Ritter, 1975, p. 13)

B. Methodological Issues in studying Memory Aging

1. Pacing Effects

It is well established that older subjects' performance is often affected more by rapid pacing than younger subjects' (see Arenberg, 1973; Davies, 1968). However, since time constraints probably do not largely contribute to the deficits elderly people experience in everyday tasks, research might profitably be directed at assessing aging effects not complicated by timing. That is, one step toward more ecologically valid research would be to eliminate artificial time parameters. Further motivation for such an approach comes from the success various non-cognitive explanations have had in accounting for pacing effects. For example, older subjects' high arousal levels and general cautiousness, have been shown to be detrimental to their performance on speeded tasks.

\section{Cohort Effects}

All of the studies reviewed, used cross-sectional designs. That is, age changes were inferred from aroup differences. Yet, it has been pointed out (see Riegel, 1972; Schaie, 1970, 1973), that these designs inherenty 
confound age of subject, with date of subjects' birth (cohort effects), and date of testing (historical effects). The analysis of development is thus contaminated by generational and cultural variables. Only with the use of appropriate sequential designs, can these effects be truly disentangled. But, this entails considerable additional effort, including testing over a long time span, and is often too difficult and impractical to implement.

It is evident, however, that many of the aging effects described could be attributable to generational effects. For example, in Figure 4, which shows hypothetical developmental curves for three cohort groups, it may be seen that a cross-sectional analysis tends to over estimate age decline. Since the cohort groups did not reach comparable peak performance levels, age differences measured at a single time of testing (cross-sectional analysis) include aging effects as well as this cohort difference. On the other hand, age differences measured over time (longitudinal analysis) include only aging effects. A similar overestimation of aging effects, from cross-sectional analyses, may be seen in Figure 5, which shows hypothetical functions for two cross-sectional and one longitudinal assessment of age change. In either of the two single cross-sectional tests which are depicted, ten year age 
Figure 4. Hypothetical Developmental Functions of Three Cohort Groups

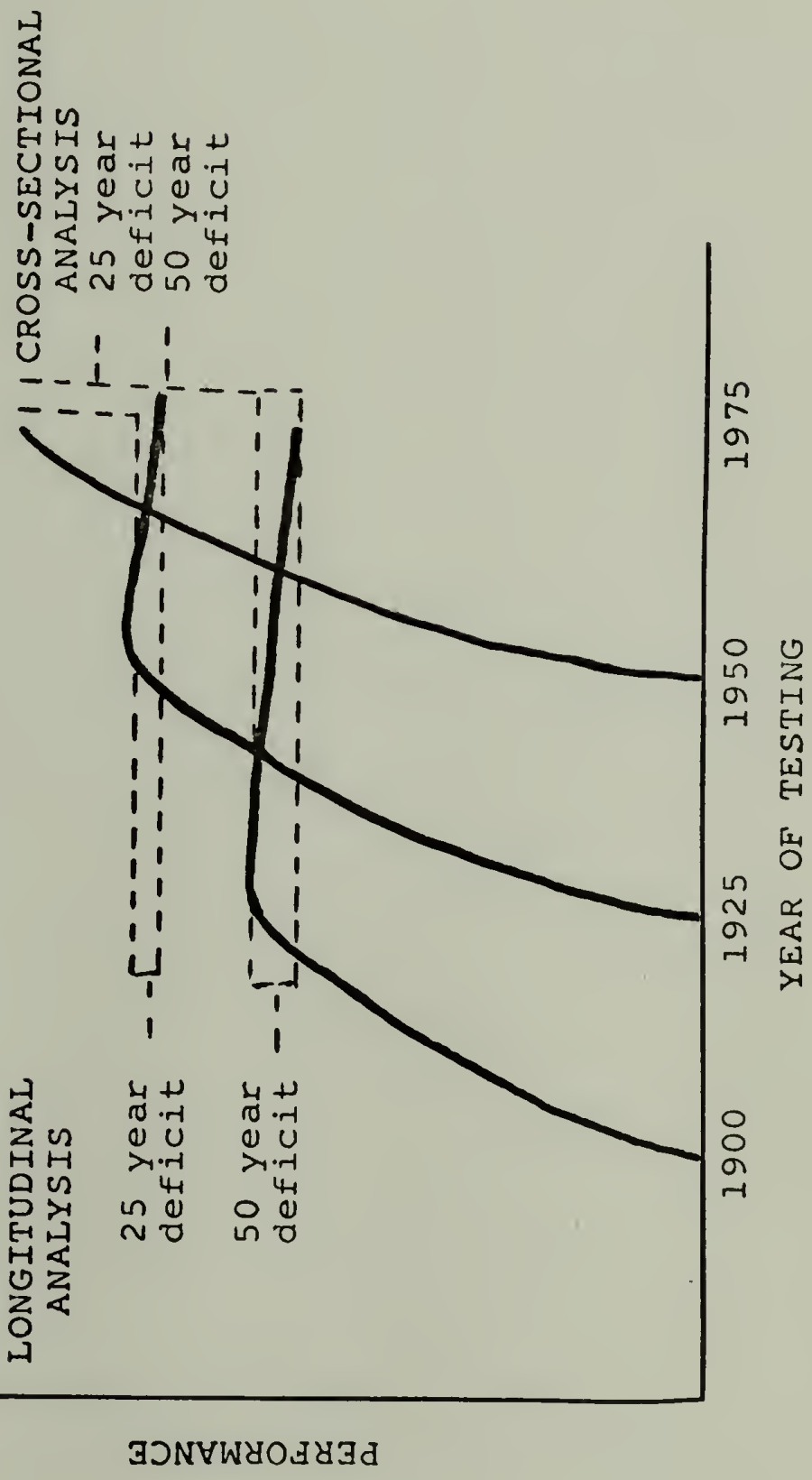


Figure 5. Hypothetical Functions from Two CrossSectional and One Longitudinal Assessment of Age Change

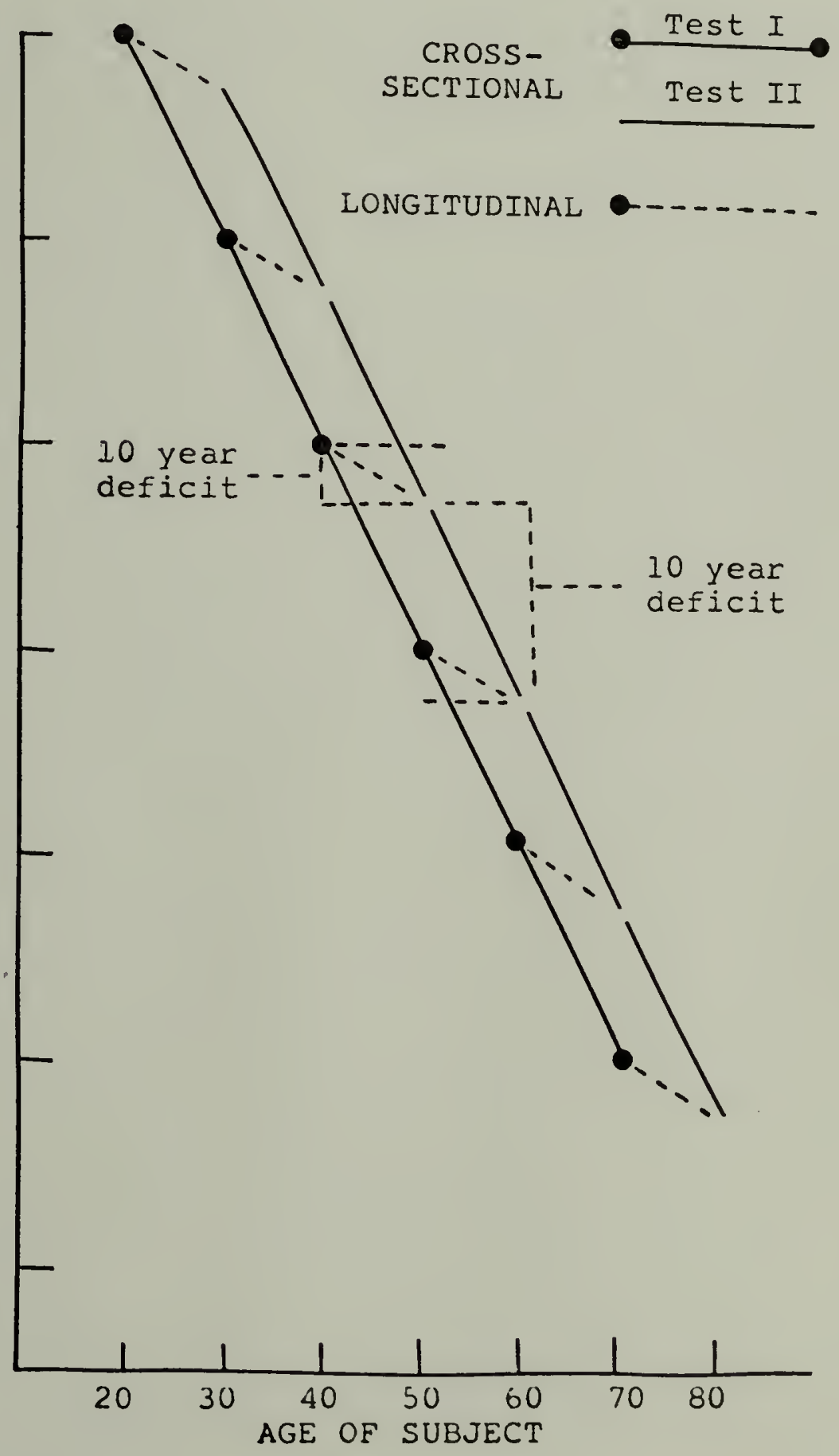


deficits appear quite large. On the other hand, in the longitudinal analysis, ten year age deficits appear much smaller. This apparent discrepancy can be interpreted by comparing performance, at any particular age, of two cohort groups, tested ten years apart. As can be seen, even when tested at comparable agès, considerable performance differences are evident for different cohort groups. Thus, these hypothetical graphs indicate how cohort effects, attributable to generational differences, can easily be confounded, and misinterpreted as aging effects.

This confounding can be hypothesized to have contriouted to many observed memory deficits. For example, if it is assumed that mnemonic production is a skill acquired over the course of extensive educational experiences (see Yendovitskaya, 1971), then production deficiencies would be expected in less educated members of a population. If, as we know to be the case, older members of our society are less likely to have had as many years of formal education as younger people, then observed production deficiencies may be largely generational differences, not developmental impairment associated with aging.

In cross-sectional experiments, one way to assess the contribution of such generational effects is to include design factors that systematically vary level 
of a generational variable hypothesized to be important. of course, this method is not perfect, however, it does permit determination of the course decline, at least at some levels of various generational variables. Thus, for example, many studies have differentiated subjects on the basis of some measure of intelligence, and in general, have found that bright older people show relatively, as well as absolutely, less loss, but in time, even they perform less well (see Botwinick, 1967). II. Statement of Froblem

The purpose of this dissertation was to examine age-related differences in adults' memory. More specifically, it was planned to address three problems. First, it assessed several hypothesized processing differences which may contribute to previously documented memory deficits associated with aging. Second, it provided data on adults' metamemory. And third, it evaluated several hypothesized explanations of aging effects on memory.

A. Processing Differences Contributing to Age Differences in Memory

'1. Episodic Memory

Although several age-related episodic memory performance deficits are now well documented (e.g., Arenberg, 1973; Botwinick, 1973; Craik, 1975; Horn, 1975; Reese, 1975), there is still no consensus about 
what contributes to this decline. The literature on memory changes in normal adult development provides evidence of deficient mnemonic functions at both acquisition and retrieval. Yet, it is probable that similar processing failures can account for memory impairments at both stages of processing. Several hypothesized processing differences can be proposed to account for age-related episodic memory impairment, for example, processing overloads, processing deficits, and strategy deficits. Table 1 lists several processing differences and predicted findings for hypotheses that follow.

a. Processing Overload Hypothesis

1. Mediator Overload. Horn (1974) suggests that "by virtue of having lived longer than younger persons, older persons tend to have been exposed to more opportunities to learn, and therefore, presumably would have learned and stored more than younger persons. Thus, it is not unreasonable to suppose that learning and memory deficits associated with age are, at least in part, a result of interference of one form or another" (p. 67). The notion that interference is a prime contributor of memory deficits in aging has long been in the literature (see Kausler, 1970). It may be suggested, for example, that older people tend to perceive more relationships, and by comprehending 


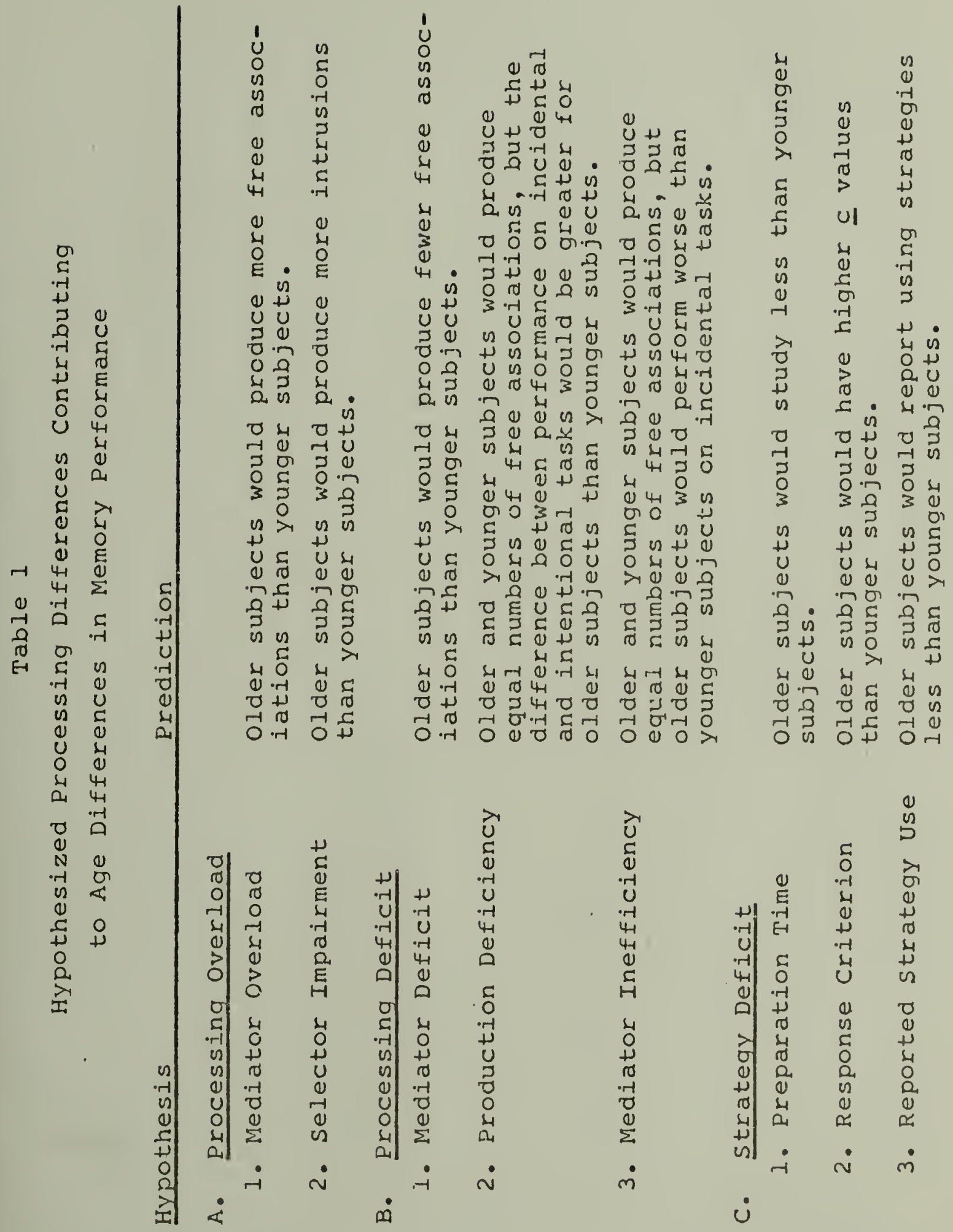


more, they often make tasks more difficult than they would otherwise be. Craik (1975) suggests that older subjects may fail to remember because a greater number of items are associated with each retrieval cue, thereby giving rise to cue overload. One prediction of such a mediator interpretation of an interference hypothesis would be of increased numbers of associations generated for items in a free association task.

\section{Selector Impairment. Even if increased} associations were evident in older subjects, some other mnemonic deficit would need to be hypothesized to account for memory problems. Increased associations could be detrimental if paired with impairment of a selector mechanism; a failure to differentiate between appropriate and inappropriate responses would account for poor memory. Furthermore, even without excessive numbers of associations, selector impairment alone could account for interference effects. The number of intrusions given in recall may be indicative of the effectiveness of a selector mechanism. If older subjects produce more intrusions than younger subjects, it is likely that they operate with an impaired selector mechanism.

Thus, two independent questions, suggested by an interference hypothesis, were asked. First, do older subjects tend to generate more associations to items 
than younger subjects, indicating a possible mediator overload? And second, do older subjects proauce more intrusions, indicating selector impairment?

b. Processing Deficit Hypothesis

Quite an opposite point of view is perhaps presently most prevalent in the literature. Both Craik (1975) and Horn (1975), for example, are most inclined to interpret age-related memory deficits in terms of processing deficits.

1. Mediator Deficiency. Horn (1975) suggests that "adulthood age-decrements in intellectual performances... are due to lack of internally generated associations" (p. 66). Craik (1975) interprets age-related memory decline in terms of older subjects' failure to engage in deep levels of semantic processing, both when presented with materials to be learned, and when given retrieval clies. The observed deficits may also be interpretable in terms of a deficiency of an automatic spread of activation mechanism (Collins \& Loftus, 1974). Older subjects may educe fewer of the possible relevant correlates for any given element. The number of associations elicited in a free association task can be used to evaluate mediator deficits. If there is a deficit in older subjects' ability to generate relevant associated information to stimuli, then they would produce fewer free assciations. 


\section{Production Deficiency. Older subjects may} be capable of producing this kind of elaborative mediating information required for good retention, but they may ordinarily fail to produce it spontaneously. such a production deficiency interpretation can be tested by comparing memory of items subjects expect to remember, with memory of items they do not expect to remember, but to which they produce free associations. If production capacity (i.e., number of associations generated on free association tasks) is equal across age levels, then a production deficiency interpretation would predict age differences on the standard intentional memory task, but not on the incidental task. A production deficiency implies that instructional manipulations are sufficient to increase acquisitional processing, and this improves retention.

3. Mediator Inefficiency. One further interpretation of the processing deficit hypothesis was also evaluated. A mediator inefficiency interpretation predicts memory differences, even if production is equated. That is, if production is equal, regardless of whether it is equalized because of comparable propensities to spontaneously produce, or because of instructions to produce, retention may still be poor, because the mediators that are produced are not utilized effectively. 
Thus, three interpretations of the processing deficit hypothesis were evaluated. Mediator deficiency was assessed from free association data. Production deficiency was assessed by comparing memory on two list types, intentional memorization and incidental association. Finally, mediator inefficiency was assessed by comparing the two age groups' memory levels on lists in which production should have been equated, that is, on the incidental associate list.

c. Strategy Deficit Hypothesis

One additional hypothesis, a strategy deficit hypothesis, was also evaluated. The hypotheses thus far discussed predominately address relatively automatic constructive or elaborative mechanisms. Yet, other less automatic processes may account for age differences. Thus, for example, older subjects may engage in less strategic acquisitional processing. If this were true, the amount of study time used to prepare for intentional memory tasks might differ. Time spent on each task was thus analyzed.

Additionally, older subjects, who are perhaps less self assured, may adapt more conservative, but less effective memory strategies. One way to assess strategic differences associated with aging is to compare levels of errors of commission and omission. If no strategic differences exist, the relative levels of 
each of these error types would be expected to be stable over age. On the other hand, if strategy changes accompany aging, differential omission and commission errors might be expected. Signal detection analysis permits separate evaluation of retentional ( $\left.d^{\prime}\right)$, and response bias (c) factors in recognition performance, and were thus carried out.

\section{Semantic Momory}

Although older subjects' episodic memory performance has consistently been found to be worse than younger subjects', there has been little investigation of age differences in semantic memory. It is possible that memory for more general information (semantic memory) is relatively unimpaired in the elderly. Furthermore, tests of episodic memory generally tap retention of information acquired during an experimental session, but tests of semantic memory involve retention of information acquired at an earlier time. Thus, agerelated differences in retrieval processes probably affect episodic and semantic memory performance equally, but age-related differences in acquisitional processes probably affect episodic memory performance more than semantic memory performance. In order to investigate these hypotheses, the present study included recall and recognition tests of semantic and episodic material. 


\section{B. Metarnemory in Adulthood}

No previous work has apparently assessed adults' metamemory knowledge and possible changes in metamemory associated with aging, although a few single-aged adult studies could be interpreted within this framework (e.g., Blake, 1973; Hart, 1965, 1967). Moreover, research probably still needs to focus on the reliability and validity of present techniques of assessing metamemory, as well as on developing new techniques. For example, previous failures to find correlations between various metamemory measures (Flavell et. al., 1970 ; Kruetzer, et. al., 1975), raises questions about the reliability of the measures employed. In the present work explicit sorts of metamemoty knowledge were assessed with a metamemory questionnaire, and two memory monitoring skills, memory prediction, and confidence ratings, were evaluated on episodic and semantic memory tasks. It was hoped that this variety of metamemory assessments, paired with a variety of memory performance assessments, would be vaiuable in gaining an understanding of the relationship between metamemory and memory capacities, as well as in determining the merits of the various measures employed.

1. Generalized-Abstracted Knowledae: Questionnaire Considerable information about what adults know about memory, can be derived from questionnaires. The 
questionnaire used in this dissertation included explicit questions about memory problems subjects encounter, how subjects conceptualize memory development, particularly the kinds of memory changes they expect to accompany aging, knowledge subjects have about static and dynamic aspects of organismic and environmental factors affecting memory, and memory strategies and aids subjects employ.

\section{Specific-Concrete Knowledge: Memory Monitoring}

Memory monitoring refers to subjects ability to reflect upon, and predict or assess, but not necessarily retrieve, the contents of their memories; it involves judgments about the accessibility of potential, or actual, memory items. Although research has not yet identified the exact function memory monitoring has in ordinary memory behavior, it may be hypothesized to be an important component of an executive mechanism; it probably contributes to efficient instigation, maintainance, and termination of acquisition and retrieval strategies, as well as of memory search. Two memory monitoring skills, memory prediction and memory confidence rating, were examined in this dissertation. prediction skills were assessed with respect to episodic recall and episodic and semantic recognition, and $\mathrm{co}^{\circ}$ idence skills were assessed with respect to episod = and semantic recall and recognition. 


\section{a. Memory Prediction}

Memory prediction studies typically require subjects to predict the number of items they will be able to recall or recognize. Thus, these tasks assess subjects' ability to reflect upon, and self-evaluate their storage and retrieval capacities. Moreover, this ability may well reilect a capacity that is critical for deliberate deployment of effective memory strategies, and essential for competent memory search.

Flavell et. al. (1970) examined recall predictions. They found that children generally overestimate their memory spans, but also, that preciction accuracy improves with age. Yussen \& Levy (1975) extended these findings, showing that college students are more accurate than children, in predicting their memory spans, although they too tend to overestimate their capacities. Although poor ability to predict recall capacity might be expected to impair memory performance, no studies have yet examined this prediction capacity in aging subjects. The present dissertation examined adult age changes in the ability to predict episodic recall and episodic and semantic recognition.

Recognition prediction has also been examined in feeling of knowing experiments, which use recall- 
judgment-recognition paradigms. In these tasks, subjects' feeling of knowing accuracy is examined, that is, when subjects fail to recall items, they are asked to judge whether or not they think they will be able to recognize them. The measure of interest is the probability of correct recognition given a positive feeling of knowing judgment (feeling of knowing hits), relative to the probability of correct recognition given a negative feeling of knowing judgment (feeling of knowing misses).

Hart (1965), who was the first to use this procedure, found that adults could make accurate feeling of knowing judgments about general fact information. Subsequently, both Hart (1967) and Blake (1973), demonstrated adult accuracy in feeling of knowing judgments for recently learned paired associates. Finally, Wellman (1975) showed that even kindergarten aged children are somewhat capable of this memory monitoring skill; they are able to predict at above chance level which pictures, out of those they could not name, they will be able to recognize the names of. Additionally, a developmental improvement in this metamemory ability was observed for children between kindergarten and third grade. No studies have yet examined older adults' ability to monitor memory in this way, although it might be hypothesized that a 
deficit in this metamemory skill would contribute to a more general memory impairment. Thus, this dissertation evaluated older subjects feeling of knowing performance.

\section{b. Confidence Ratings}

Confidence ratings, or certainty judgments, can be used in conjunction with other memory tasks, to assess subjects' ability to gauge the accuracy of their memory performance. With confidence ratings, subjects are not only required to elicit discrete responses, but also to make judgments about how sure they are of their responses. The probability of correct responses given high confidence ratings, and the probability of errors given low confidence ratings, can then be used to determine subjects' accuracy in moritoring their memory performance.

Previous work with adults has demonstrated substantial correlations between confidence ratings and recall (e.g., Murdock, 1966), and recognition accuracy (e.g., Tulving \& Thomson, 1971). Also, Berch \& Evans (1973) showed that kindergarten and third grade children are capable of monitoring their recognition memory states, to some extent, although older children were considerably more accurate than younger children. It is apparent that accurate assessment of the reliability of one's memory is essential to competent performance 
in a wide variety of nonmnemonic tasks. Also, there is a prevalent notion that, to an unfounded degree, older adults lack confidence in their abilities. Thus, it seems important to evaluate aging subjects' memory monitoring capacity for accurately gauging memory performance. In the present dissertation, this was carried out with respect to episodic and semantic recall and recognition.

C. Explanation of Aging Effect

All researchers studying age changes in adulthood apparently conclude that there is at least some memory impairment associated with aging (e.g., Arenberg, 1973; Botwinick, 1973; Craik, 1975; Horn, 1975; Reese, 1975). One objection to this conclusion comes from methodologists, most notably Schaie (1970, 1973) who claims that observed age deficits in cognitive performance can be accounted for by generational differences (cohort effects) (see Figures 4 and 5). It seems likely that cohort effects do account for some of the observed age differences, for example, findings of acquisitional production deficiencies can easily be explained in terms of cohort effects. However, an additional portion of the observed age difference can probably be attributed to other explanations, for example, disuse, self-fulfilling expectation, or biological decay. 


\section{Cohort Effects}

A cohort effects explanation of age differences implies that some factor in the population that happens to be highly correlated with age, is a better explanation of observed age differences than development. For example, if age of subject is a good predictor of number of years of education, and if number of years of education truly affects performance, then random sampling would produce age differences which could more appropriately be attributed to level of education than to development. An experiment which controlled level of education could determine whether additional factors produce age differences. The design of this dissertation therefore, included equal numbers of subjects, in each age group, at each of two educational levels.

At a subtler level, cohort effects could imply that another less quantifiable factor is highly correlated with age, even when educational level is controlled. It is possible that over the years, an equal number of years of education fail to produce equivalent effects. For example, fewer years of schooling in today's television-oriented society, may be sufficient to produce levels of scholarship that are comparable to that which, in the past, required much longer devotion to schooling. Thus, 
possible Age $x$ Education interactions were of interest as well as age and educational main effects.

2. Disuse Hypothesis

One possible explanation of age-related memory decline, suggested by Reese (1975), is that formerly acquired strategies become functionally less available because of disuse. The implication is that because of disuse, mnemonic strategies which were once well established, may be forgotten, or become less well-established, exhibiting deficiencies typical of mnemonic activities not well established. Although the perfect test of such a notion would require manipulations too drastic to contemplate, naturally occurring experiential differences can be analyzed to assess this hypothesis. For example, certain life roles tend to place more or less memory demands on people. Assessing memory demand thus permitted assessment of the disuse hypothesis. If memory demand correlated with memory performance, the hypothesis would be supported, although other self-selection factors could also be contributing to the relationship, and would thus have to be considered.

\section{Expectation Hypothesis}

Another possible explanation of age-related memory deficits, also suggested by Reese (1972), is that expectation of decline tends to produce memory 
impairment. If this hypothesis is correct, a negative correlation between high expectation of memory impairment and memory performance would be expected. Of course, such a correlation would not indicate a causal direction of the relationship, however, the lack of such a finding would invalidate the hypothesis.

4. Biological Hypothesis

Finally, the hypothesis of a biological basis to memory decay has been quite prevalent (e.g., Jarvik \& Cohen, 1973). This notion suggests that physiological wear and tear, and/or biochemical changes actually reduce older people's capacities. If this hypothesis is correct, a positive correlation might be expected between gross measures of good health, and memory performance.

III. Method

A. Design

The design, depicted in Figure 6, was a 2 (Age Level) $\times 2$ (Educational Level) $\times 2$ (Sex) $\times 2$ (Phase Order) multivariate design, in which the following phenomena were examined: episodic and semantic recall performance, and episodic and semantic recognition performance, explicit metamemory knowledge, free associations, episodic recall prediction, episodic and semantic recognition prediction, episodic and semantic recall confidence rating, episodic and 


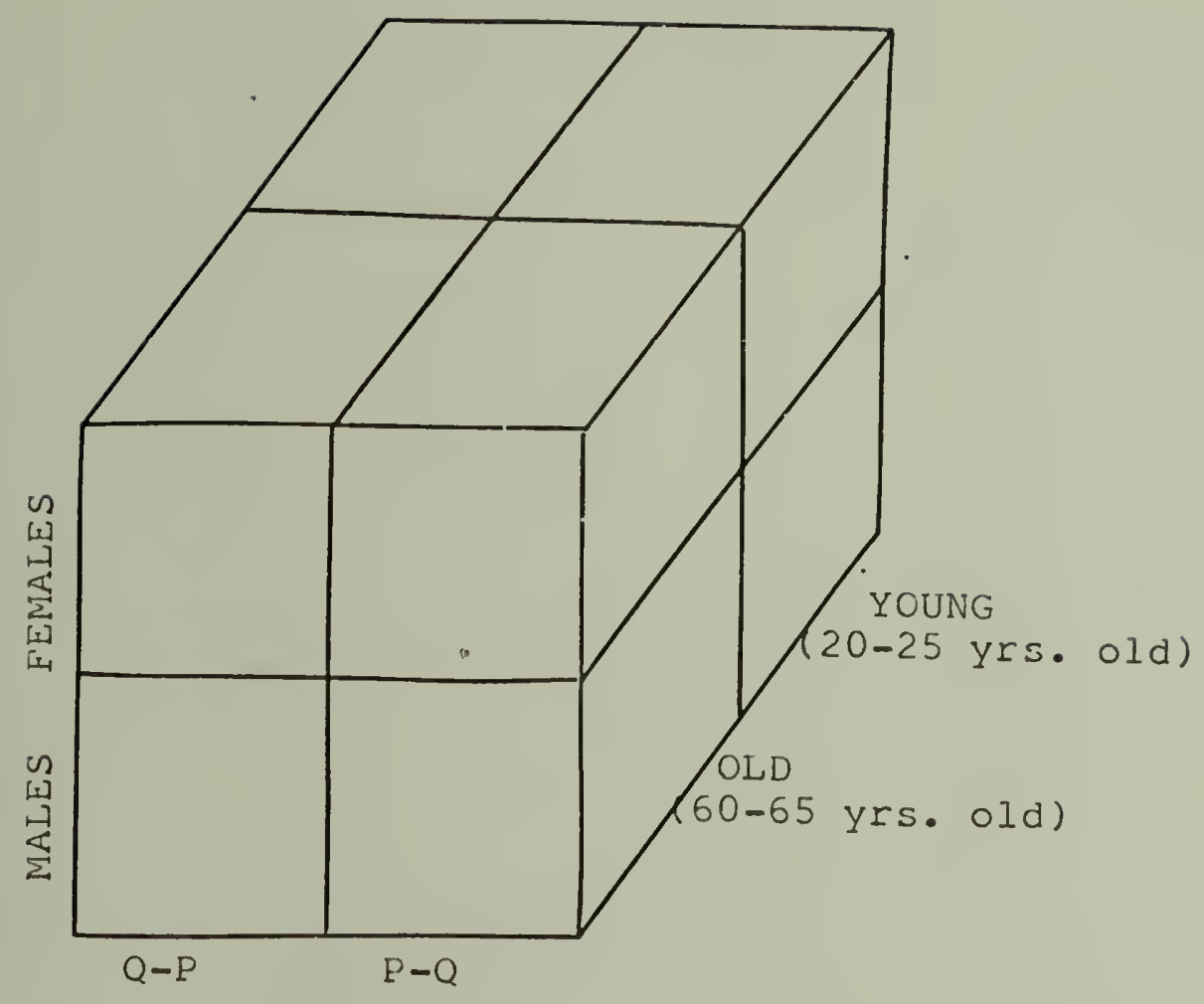

LOW EDUCATION

(High School)

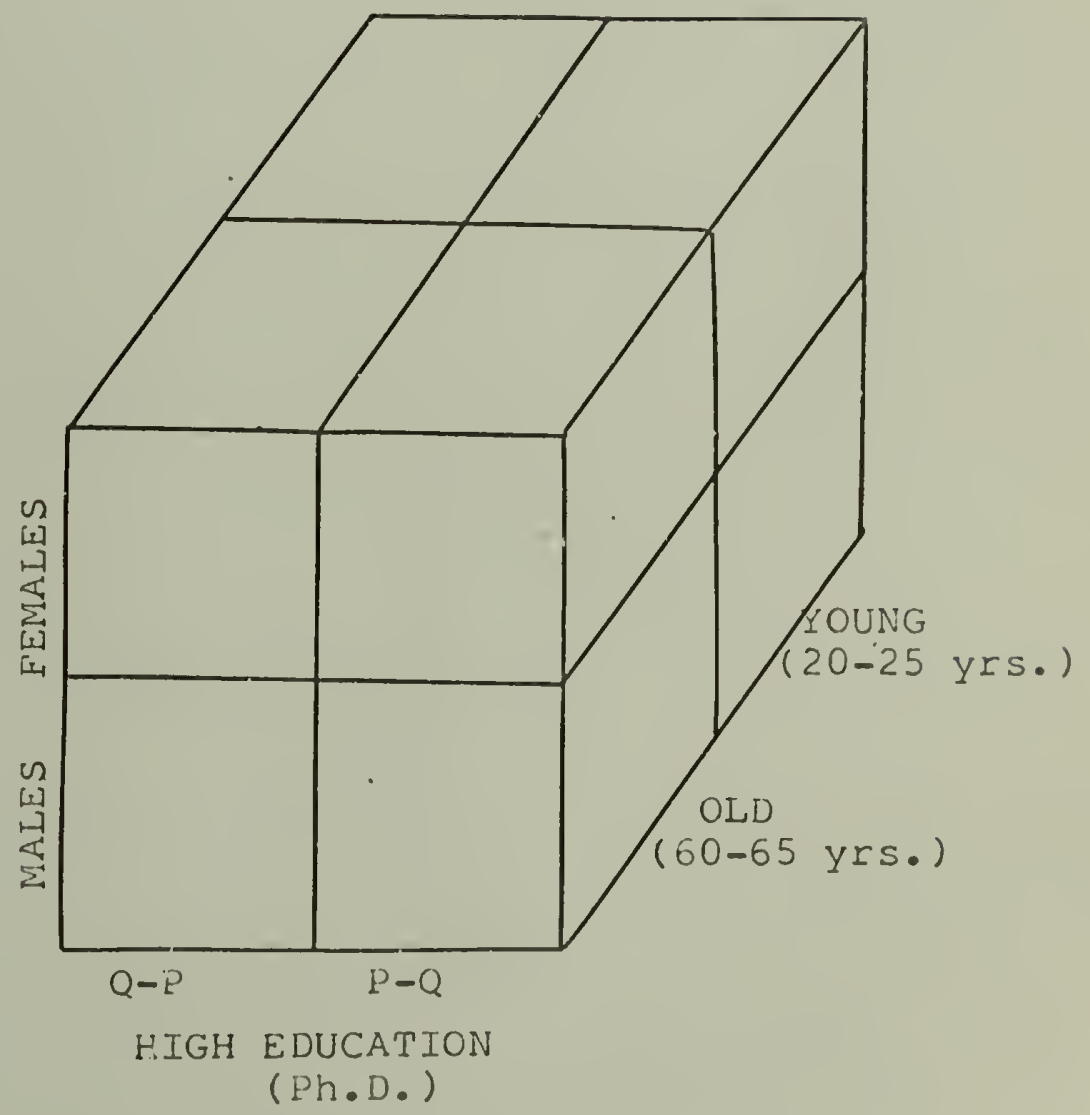

Figure 6. Design Plan 
semantic recognition confidence rating.

B. Subjects

All subjects were drawn from the Amherst, Massachusetts Five-College Academic community. Advertisements in local newspapers were used to locate volunteer subjects, and letters soliciting participants were sent to all University of Massachusetts, Smith College, Mount Holyoke College, Amherst College, and Hampshire college faculty between 60 and 65 , who have Ph.D.s, and to all town of Amherst residents between 60 and 65 , who are listed in the twon registry, and who are not professionals. Those receiving letters were contacted by telephone soon afterwards. Of the 60 to 65 year olds who could be contacted by telephone, $47 \%$ of the Ph.D.S, and $89 \%$ of the non-Ph.D.S, who had completed high school but had no further education, refused to participate. All subjects were offered $\$ 10$ for participating, and all but one female Ph.D. accepted this payment.

There were eight males and eight females in each of four Age $x$ Education groups. Subjects in the low education group completed high school but had no further formal education, and subjects in the high education group had doctoral degrees, or were working towards this degree. Of course, educational level was undoubtedly highly confounded with intelligence, 
and the results were interpreted with this in mind. Subjects in the younger age group were between 20 and 25 years of age (mean age $=23$ years) and subjects in the older age group were between 60 and 65 (mean age = 62 years) except for one female Ph.D. who turned 66 earlier in the week she was tested.

\section{Materials and Procedure}

Subjects worked at their own pace, and recorded the time at the beginning and end of each task. They each completed a background questionnaire, and then participated in two phases of the study, a metamemory questionnaire phase, and a test phase. These were given in a counterbalanced order, so that equal numbers of subjects in each cell of the design received each phase order (i.e., questionnaire first-tests second versus tests first-questionnaire second). All subjects received the same form of questionnaires. For the test phase, each subject had an individualized form, generated by computer, in which the particular items, as well as task orders, were randomized. Table 2 summarizes the procedure, and a sample data sheet may bé seen in Appendix A.

The materials and procedure for each task follow.

1. Background Questionnaire

The background questionnaire (see Appendix A), was designed to ascertain specific physical health 


$$
\begin{gathered}
-67- \\
\text { Table } 2 \\
\text { Summary of Procedure }
\end{gathered}
$$

1. Background Questionnaire

Objective Physical Health Questions

Subjective Physical Health Scale

Subjective Mental Health Scale

Memory Demand Scale

2. Metamemory.Questionnaire (counterbalanced order)

Memory Problems

Expectation of Memory Decay

Memory Knowledge

Memory Strategies

3. Tests (counterbalanced order)

a. Preliminary Tasks

Incidental Association Task

Intentional Episodic Memory Task Random Order

b. Episodic Recall prediction

c. Recall Tasks, Recall Confidence Ratings, and Recognition Predictions

Incidental Association Items (Episodic) Random Intentional Memory Items (Episodic) Order General Information Items (Semantic)

d. Recognition Task and Recognition Confidence Ratings

Incidental Association Items (Episodic) Intentional Memory Items (Episodir) General Information Items (Semantic) 
problems, as well as subjective ratings of physical health, mental health, and memory demands.

\section{Metamemory Questionnaire}

The metamemory questionnaire (see Appendix A) was designed to ascertain memory problems (questions 5-14), expectation of memory change (questions 36-47), memory knowledge (questions 29-60), and memory strateqy use (questions 15-28).

\section{Tests}

\section{a. Preliminary Tasks}

The two preliminary tasks were the first two tasks of the test phase; they were administered in a random order.

\section{Incidental Association Task. A random} sample of 24 unrelated familiar nouns, from a pool of 96 used by Botwinick \& Storandt (1974), were presented to subjects, who were told to generate as many free associations as they could. No time restriction was given, although subjects were told not to dwell on the words, but rather to write down words that immediately came to mind.

\section{Intentional Memory Task. Subjects were} presented a random sample of 24 different nouns, from the pool of 96, and asked to study them, so that they could remember as many of them as possible, for later memory tests. Again, no time limit was set. When 
subjects were finished studying the words, they were told to go on to the next task.

\section{b. Recall prediction}

Subjects were asked to predict how many of the incidental and intentional words they thought they would be able to recall later in the session.

c. Recall Tasks, Recall Confidence Ratings, and Recognition Predictions

The next three tasks of the test phase each entailed recall, recall confidence ratings, and recognition predictions. The incidental association, intentional memory, and general information items were given in a random order.

\section{Incidental Association Items (Episodic}

Memory). Subjects were asked to write down as many of the words they had generated associations to as they could remember. Additionally, they were asked to rate their confidence, on a four-point scale, that each recalled word was indeed on the previous associate list. Then, they were asked to judge how many of the remaining incidental words that they failed to remember, they thought they would be able to recognize.

\section{Intentional Memory Items (Episodic Memory).}

Similarly, subjects were asked to write down as many of the memory words they had been asked to study, as they could remember, to make confidence ratings, and then, 
to judge how many of the remaining memory words that they failed to remember, they thought they would be able to recognize.

\section{General Information Items (Semantic Memory).}

Subjects were presented 24 general information questions used by Botwinick \& Storandt (1974). There were six questions referring to each of the following four time periods; 1890-1909, 1910-1929, 1930-1949, and 1950-1969. Subjects were asked to answer as many questions as they could, and rate their confidence in the accuracy of each answer. Also, for each question, they could not answer they were asked to judge whether they thought they would be able to recognize the correct answer.

\section{d. Recognition Tasks and Confidence Ratings}

The iast three tasks of the test phase entailed recognition and confidence ratings of the incidental association, intentional memory, and general information items. A random order of all of the 24 association words, 24 memory words, 48 remaining words in the pool. 24 correct general information statements and 24 incorrect general information statements, were presented to subjects. They were asked to make old-new or truefalse recognition choices, and four-point scaled confidence ratings. 
IV. Results

\section{A. Associations}

Subjects were asked to generate associations to 24 words. The mean number of associations generated by each Age $x$ Education $x$ Sex group is shown in Table 3. A 2 (Age) $\times 2$ (Education) $\times 2$ (Sex) analysis of variance were performed on these data, and the results are summarized in Table 4. Averaged over subjects the mean number of associations generated was 30 , which is just over one association for each word presented. Younger subjects produced slightly fewer associations than older subjects ( $30 \mathrm{vs}$. 3I), but the Age main effect was nonsignificant. High school educated subjects produced slightly fewer associations than Ph.D. educated subjects ( 30 vs. $3 I$ ), but the Education main effect was also nonsignificant. Males produced fewer associations than females ( 26 vs. 34 ), but the sex main effect was nonsignificant as well. These variables accounted for only $5 \%$ of the variance in number of associations; Age less than $1 \%$, Education less than $1 \%$, and sex $4 \%$.

\section{B. Memory Performance}

1. Episodic Memory

Incidental memory performance was assessed by recall and recognition tests of the 24 association words, and intentional memory performance by recall and recognition tests of: 24 words presented for 
Table 3

Mean Number of Associations Generated by

Each Age $x$ Education $x$ Sex Group

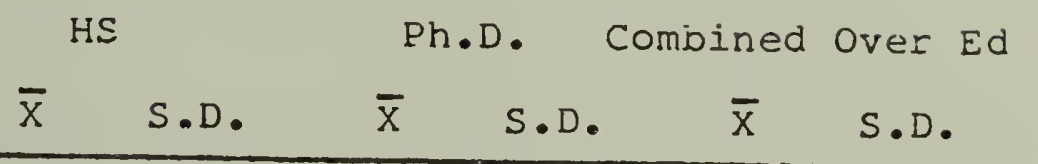

$20-25$

$\begin{array}{lllllll}\text { Males } & 25.88 & 3.7 & 28.50 & 7.1 & 27.19 & 5.6\end{array}$

$\begin{array}{lllllll}\text { Females } & 36.38 & 14.6 & 28.25 & 6.9 & 32.31 & 11.8\end{array}$

$\begin{array}{lllllll}\begin{array}{l}\text { Combined } \\ \text { Over Sex }\end{array} & 31.13 & 11.7 & 28.38 & 6.7 & 29.75 & 9.5\end{array}$

$60-65$

$\begin{array}{lllllll}\text { Males } & 25.63 & 7.5 & 25.88 & 3.8 & 25.75 & 5.7\end{array}$

$\begin{array}{lllllll}\text { Females } & 31.63 & 18.1 & 41.13 & 48.0 & 46.38 & 35.4\end{array}$

$\begin{array}{lllllll}\text { Combined } & 28.63 & 13.7 & 33.50 & 33.9 & 31.06 & 25.5\end{array}$

Over Sex

Combined Over Age

$\begin{array}{lrrrrrr}\text { Males } & 25.75 & 5.7 & 27.19 & 5.7 & 26.47 & 5.6 \\ \text { Females } & 34.00 & 16.1 & 34.69 & 33.8 & 34.34 & 26.0 \\ \begin{array}{l}\text { Combined } \\ \text { Over Sex }\end{array} & 29.88 & 12.6 & 30.94 & 24.2 & 30.41 & 19.1\end{array}$




\section{Table 4}

Summary ANOVA for Associations

$$
\overline{\mathrm{X}}=\text { Associations }
$$

\begin{tabular}{|c|c|c|c|c|}
\hline$d f=1,56$ & 30.41 & $\mathrm{~F}$ & $\mathrm{P}$ & $E t a^{2}$ \\
\hline Age & $\begin{array}{r}-.66 \\
.66\end{array}$ & $<1$ & NS & $<.01$ \\
\hline Education & $\begin{array}{r}-.53 \\
.53\end{array}$ & $<1$ & NS & $<.01$ \\
\hline Sex & $\begin{array}{r}-3.94 \\
3.94\end{array}$ & 2.62 & NS & .04 \\
\hline Age $x \mathrm{Ed}$ & & $<1$ & NS & \\
\hline Age $x$ Sex & & $<1$ & NS & \\
\hline Age $x$ Ed & & $<1$ & NS & \\
\hline Age $x$ Ed $x$ Sex & & 1.06 & NS & \\
\hline Multiple $\mathrm{R}^{2}$ & & & & .05 \\
\hline
\end{tabular}


memorization.

a. Percent Remembered on Recall and Recoanition

Tests

The percentage of items correctly recalled and recognized should indicate retention. Since recognition was tested with a yes-no procedure, however, the percentage of items correctly recognized may reflect guessing as well, as retention. The recognition scores reported here were, therefore, corrected for guessing (Kintsch, 1970). I The mean percentage of the 24 incidental and 24 intentional words correctly recalled and recognized (after correction) by each Age x Education $x$ sex group is shown in Table 5. These recall and recognition scores were submitted to a 2 (Age) $\times 2$ (Education) $\times 2$ (Sex) $\times 2$ (Order) $\times 2$ (Memory (Recall vs. Recognition)) $\times 2$ (Task (Incidental vs. Intentional)) analysis of variance. Older subjects remembered less than younger subjects ( $38 \%$ vs. 51\%), and the Age main effect was statistically significant $(\underline{F}(1,48)=11.34, \mathrm{p}<.001)$. High school educated subjects remembered less than Ph.D. educated subjects $(40 \%$ vs. $50 \%)$, and the Education main effect was statistically significant (F $(1,48$ ) $=6.84, \underline{\mathrm{L}}(.05)$. Males remembered less than females 
Table 5

Mean Percentage of Incidental and Intentional Words Correctly Recalled and Recognized by Each Age $x$ Education $x$ Sex Group

Recall

\section{Incidental Intentional Total}

HS $\bar{x} \quad$ S.D. $\quad \bar{x}$, S.D. $\quad \bar{x}$ S.D.

20 to 25

Males

$\begin{array}{llllll}31.25 & 17.5 & 28.65 & 17.7 & 29.95 & 11.8\end{array}$

Females $\quad 35.94 \quad 24.0 \quad 35.94 \quad 29.4 \quad 35.94 \quad 25.4$

Combined

Over Sex

$$
\begin{array}{llllll}
33.59 & 20.4 & 32.29 & 23.7 & 32.94 & 19.4
\end{array}
$$

60 to 65

Males

$\begin{array}{llll}8.85 & 12.5 & 10.94 & 12.8\end{array}$

$9.90 \quad 11.4$

Females

$35.94 \quad 20.4$

$\begin{array}{rl}45.83 & 17.7\end{array}$

$40.89 \quad 12.2$

Combined

Over Sex

$22.40 \quad 21.5$

28.39

23.4

$25.39 \quad 19.7$

Combined over Age

$\begin{array}{lllllll}\text { Males } & 20.05 & 18.7 & 19.79 & 17.5 & 19.92 & 15.3 \\ \text { Females } & 35.94 & 21.5 & 40.89 & 24.0 & 38.41 & 19.4 \\ \begin{array}{l}\text { Combined } \\ \text { Over Sex }\end{array} & 27.99 & 21.4 & 30.34 & 23.3 & 29.17 & 19.6\end{array}$


Table 5 Continued

Recognition

Incidental Intentional Total

HS

$\overline{\bar{x}} \quad$ S.D. $\quad \overline{\mathrm{x}} \quad$ S.D. $\quad \overline{\mathrm{x}} \quad$ S.D.

20 to 25

$\begin{array}{lllllll}\text { Males } & 59.18 & 27.0 & 52.26 & 23.7 & 55.72 & 25.1\end{array}$

$\begin{array}{lllllll}\text { Females } & 59.80 & 22.1 & 59.29 & 25.3 & 59.54 & 26.6\end{array}$

$\begin{array}{lllllll}\text { Combined } & 59.49 & 26.6 & 55.78 & 23.9 & 57.63 & 25.0\end{array}$

Over Sex

60 to 65

$\begin{array}{lllllll}\text { Males } & 45.04 & 20.3 & 12.97 & 11.1 & 29.01 & 14.2\end{array}$

$\begin{array}{lllllll}\text { Females } & 64.26 & 23.8 & 47.66 & 20.1 & 55.96 & 20.9\end{array}$

$\begin{array}{lllllll}\text { Combined } & 54.65 & 23.6 & 30.32 & 23.8 & 42.48 & 22.2\end{array}$

Over Sex

Combined over Age

Males $\quad \begin{array}{lllllll}52.11 & 24.2 & 32.5 & 27.0 & 42.36 & 24.1\end{array}$

Females $\quad \begin{array}{llllll}62.03 & 25.3 & 53.47 & 22.9 & 57.75 & 23.1\end{array}$

$\begin{array}{lllllll}\text { Combined } & 57.07 & 24.9 & 43.05 & 26.8 & 50.06 & 24.5\end{array}$

Over Sex 
Table 5 continued

Recall

Incidental Intentional Total

Ph.D. $\bar{x} \quad$ S.D. $\quad \bar{x} \quad$ S.D. $\quad \bar{x}$ S.D.

20 to 25

$\begin{array}{lllllll}\text { Males } & 52.08 & 18.5 & 48.96 & 22.50 & 50.52 & 15.4\end{array}$

$\begin{array}{lllllll}\text { Females } & 42.19 & 15.2 & 42.19 & 21.30 & 42.19 & 15.6\end{array}$

$\begin{array}{lllllll}\text { Combined } & 47.14 & 17.1 & 45.57 & 21.4 & 46.35 & 15.6\end{array}$

Over Sex

60 to 65

Males $\quad 22.92 \quad 18.0 \quad 29.17 \quad 20.9 \quad 26.04 \quad 14.0$

$\begin{array}{lllllll}\text { Females } & 25.52 & 14.7 & 26.56 & 33.3 & 26.04 & 21.4\end{array}$

$\begin{array}{lllllll}\text { Combined } & 24.22 & 15.9 & 27.86 & 26.9 & 26.04 & 17.4\end{array}$ Over Sex

Combined over Age

$\begin{array}{lllllll}\text { Males } & 37.50 & 23.2 & 39.06 & 23.3 & 38.28 & 19.0\end{array}$

$\begin{array}{lllllll}\text { Females } & 33.86 & 16.8 & 34.37 & 28.2 & 34.11 & 19.9\end{array}$

$\begin{array}{lllllll}\text { Combined } & 35.68 & 20.0 & 36.72 & 25.6 & 36.20 & 19.3\end{array}$

Over Sex 
Table 5 Continued

Recognition

Incidental Intentional Total

Ph.D. $\quad \bar{x} \quad$ S.D. $\quad \bar{x} \quad$ S.D. $\quad \bar{x} \quad$ S.D.

20 to 25

$\begin{array}{lllllll}\text { Males } & 76.51 & 5.9 & 66.65 & 18.5 & 71.58 & 10.7\end{array}$

Females $\quad 74.80 \quad 14.9 \quad 55.68 \quad 21.3 \quad 65.24 \quad 17.3$

$\begin{array}{lllllll}\text { Combined } & 75.66 & 11.0 & 61.16 & 20.1 & 68.41 & 14.3\end{array}$

Over Sex

60 to 65

$\begin{array}{lllllll}\text { Males } & 65.29 & 12.1 & 48.41 & 24.6 & 56.85 & 16.3\end{array}$

$\begin{array}{lllllll}\text { Females } & 69.89 & 14.3 & 50.91 & 24.1 & 60.40 & 12.6\end{array}$

$\begin{array}{lllllll}\text { Combined } & 67.59 & 13.0 & 49.66 & 23.6 & 58.63 & 16.5\end{array}$

Over Sex

Combined over Age

$\begin{array}{lllllll}\text { Males } & 70.90 & 10.9 & 57.53 & 23.0 & 64.22 & 15.4\end{array}$

$\begin{array}{lllllll}\text { Females } & 72.34 & 14.3 & 53.29 & 22.1 & 62.82 & 17.0\end{array}$

$\begin{array}{lllllll}\text { Combined } & 57.07 & 24.9 & 55.41 & 22.3 & 63.52 & 16.0\end{array}$

Over Sex 
Table 5 Continued

Recall

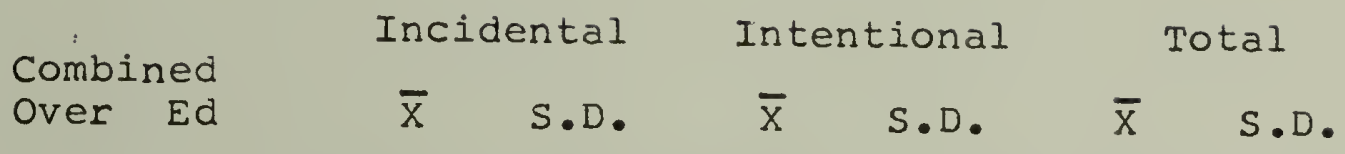

20 to 25

Males $\quad \begin{array}{llllll}41.67 & 20.5 & 38.80 & 22.2 & 40.23 & 17.0\end{array}$

$\begin{array}{lllllll}\text { Females } & 39.06 & 19.7 & 39.06 & 25.0 & 39.06 & 20.6\end{array}$

$\begin{array}{lllllll}\text { Combined } & 40.36 & 19.8 & 38.93 & 23.3 & 39.65 & 18.6\end{array}$

Over Sex

60 to 65

$\begin{array}{lllllll}\text { Males } & 15.89 & 16.6 & 20.05 & 19.2 & 17.97 & 14.9 \\ \text { Females } & 39.73 & 18.0 & 36.20 & 27.6 & 33.46 & 18.5 \\ \begin{array}{l}\text { Combined } \\ \text { Over Sex }\end{array} & 23.31 & 18.6 & 28.12 & 24.8 & 25.72 & 18.3\end{array}$

Combined over Age

$\begin{array}{lllllll}\text { Males } & 28.78 & 22.5 & 29.43 & 22.5 & 29.10 & 19.4 \\ \text { Females } & 34.90 & 19.0 & 37.63 & 26.0 & 36.26 & 19.5 \\ \begin{array}{l}\text { Combined } \\ \text { Over Sex }\end{array} & 31.84 & 20.9 & 33.53 & 24.5 & 32.68 & 19.6\end{array}$


Table 5 Continued

Recognition

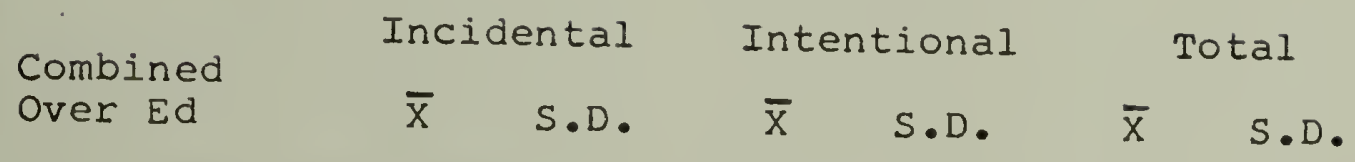

20 to 25

$\begin{array}{lllllll}\text { Males } & 67.84 & 20.9 & 59.46 & 21.8 & 63.65 & 20.4\end{array}$

$\begin{array}{lllllll}\text { Females } & 67.30 & 23.1 & 57.48 & 22.7 & 62.39 & 21.8\end{array}$

$\begin{array}{lllllll}\text { Combined } & 67.57 & 21.7 & 58.47 & 21.9 & 63.02 & 20.8\end{array}$ Over sex

60 to 65

$\begin{array}{lllllll}\text { Males } & 55.16 & 19.2 & 30.69 & 26.0 & 42.93 & 20.6 \\ \text { Females } & 67.08 & 19.2 & 49.29 & 21.5 & 58.18 & 18.8 \\ \begin{array}{l}\text { Combined } \\ \text { Over Sex }\end{array} & 61.12 & 19.8 & 39.99 & 25.3 & 50.55 & 20.9\end{array}$

Combined over Age

$\begin{array}{lllllll}\text { Males } & 61.50 & 20.8 & 45.08 & 27.7 & 53.29 & 22.7 \\ \text { Females } & 67.19 & 20.9 & 53.38 & 22.1 & 60.29 & 20.1 \\ \begin{array}{l}\text { Combined } \\ \text { Over Sex }\end{array} & 64.35 & 20.9 & 49.23 & 25.2 & 56.79 & 21.6\end{array}$


( $41 \%$ vs. $48 \%$ ), and the sex main effect was marginally significant $(\underline{F}(1,48)=3.26, \underline{p}<.08)$. The order main effect was not significant.

The Education $x$ sex interaction was statistically significant $(\underline{F}(1,48)=6.33, \underline{p}<.05$, and may be seen in Figure 7, which shows the mean percentage of items correctiy remembered by males and females in each education group. "HS males remembered significantly less than any of the other sex x Education groups (all ps< $.01)$, and performance of the other three groups was not statistically different. While less education was associated with significantly poorer memory performance in males $(\underline{t}(30)=3.64, p<.001)$, it was not a significant factor in females' performance. Also, while in the HS sample, males remembered significantly less than females $(\underline{t}(30)=2.75, \underline{p}<.01)$, in the Ph.D. sample sex was not a significant factor in performance. The Age $x$ sex interaction was also statistically significant $(\underline{\mathrm{F}}(1,48)=4.48, \underline{\mathrm{p}}<.05$, and may be seen in Figure 8, which shows the mean percentage of items correctly remembered by males and females in each age group. Older males remembered significantly less than any of the other Age $x$ Sex groups (all ps <.01), and performance of the other three groups was not statistically different. While performance decrement associated with aging was statistically significant for males 
Figure 7. Mean Percentage of Items Correctly Remembered by Males and Females in Each Education Group

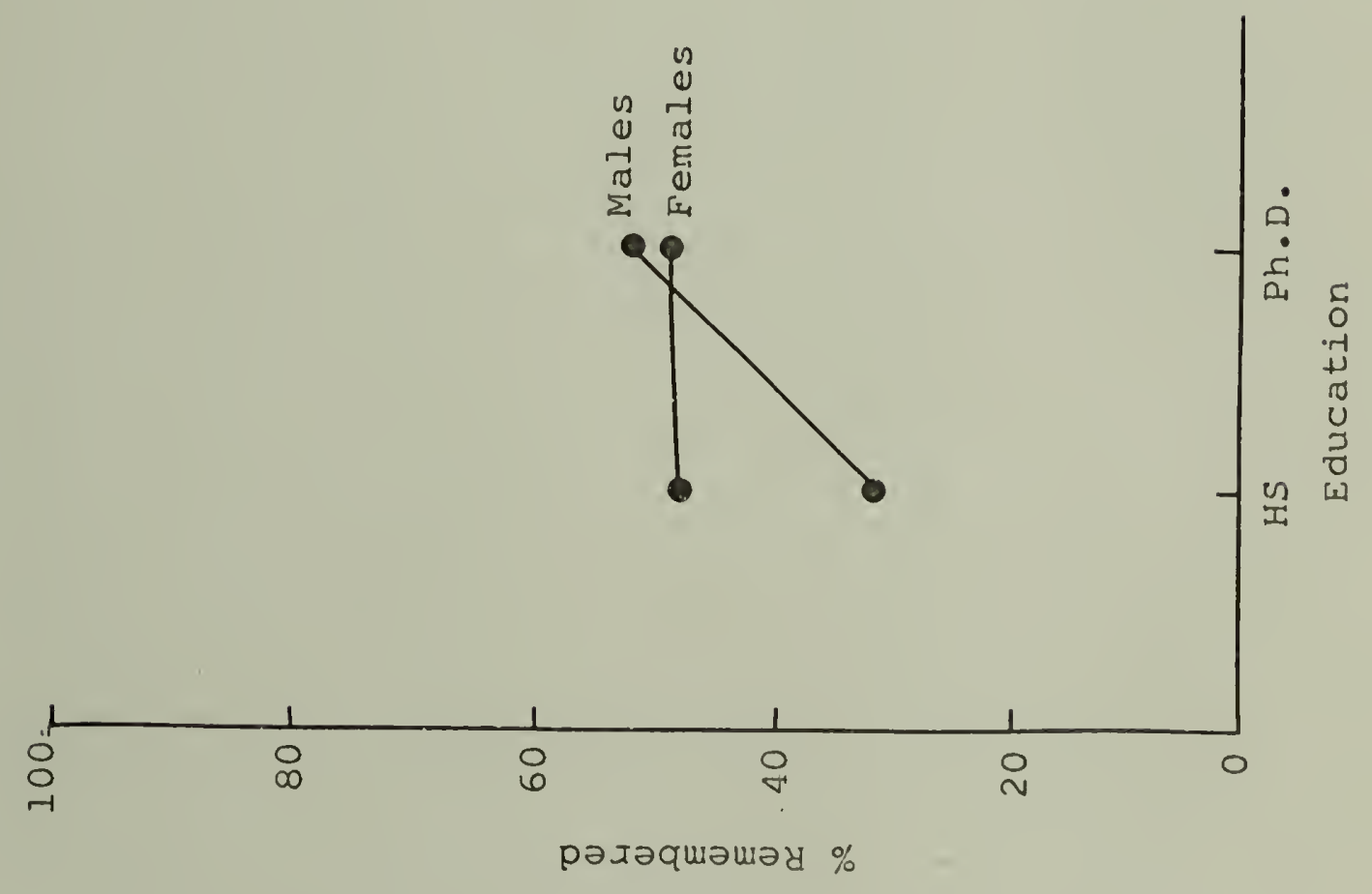


Figure 8. Mean Percentage of Items Correctly Remembered by Males and Females in Each Age Group
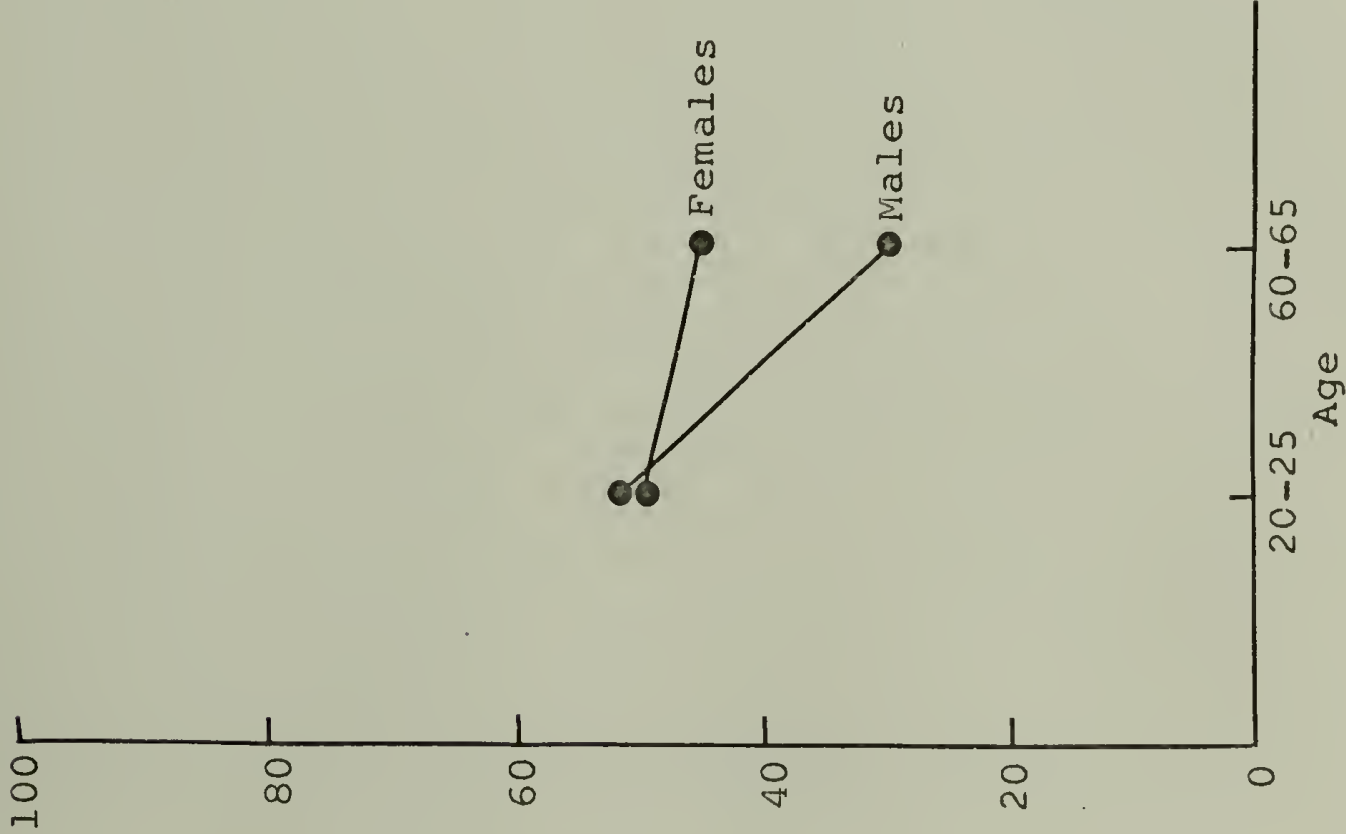

рәләquәшэу \% 
$(\underline{t}(30)=4.02, \underline{D}<.001)$, it failed to reach statistical significance for females. Furthermore, while in the older group males remembered significantly less than females $(\underline{t}(30)=2.74, \underline{p}<.01)$, in the younger group sex was not a significant factor in performance.

Not surprisingly, recognition performance was considerably better than recall. performance $(57 \%$ vs. $33 \%$ ), and the Memory (Recall vs. Recognition) main effect was highly significant $(\underline{E}(1,48)=102.86, \underline{p}$ $<.001)$.

The Task (Incidental vs. Intentional) main effect $(\underline{F}(1,48)=8.89, \underline{p}<.01)$, Memory $x$ Task interaction $(\underline{F}(1,48)=39.52, \underline{\mathrm{p}}<.001)$, and Memory $\times$ Task $\times$ Age interaction were also statistically significant ( $48)=11.68, \underline{p}(.001)$, and may be seen in Figure 9 , which shows the mean percentage of incidental and intentional items correctly recalled and recognized by each age group. Recall of incidental and jntentional items did not differ statistically. for either age group, and while the difference in recognition of incidental and intentional items was greater for older subjects, it was statistically significant for both younger $(\underline{t}(31)=3.94, \underline{p}<.001)$, and older $(\underline{t}(31)=$ $6.67, \mathrm{p}<.001)$ subjects. Furthermore, aging was associated with decreases in recall of incidental 
Figure 9. Nean Percentage of Incidental and Intentional Items Correctly Recalled and Recognized by Each Age Group

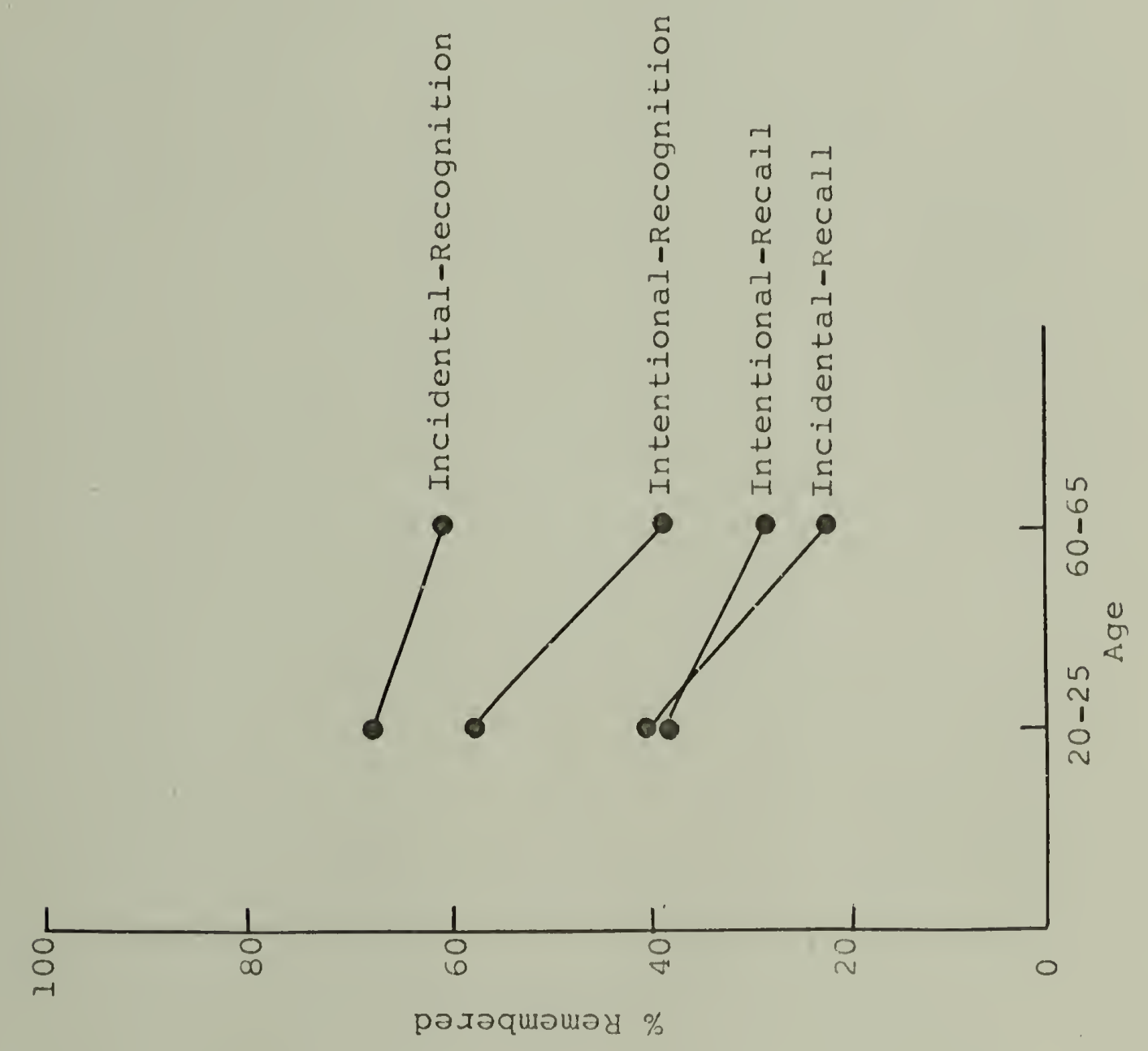


$(\underline{t}(62)=3.55, \underline{p}<.001)$ and intentional $(\underline{t}(62)=$ $1.80, \underline{p}<.08)$ items, and recognition of intentional $(\underline{t}(62)=3.12, \underline{p}<.01)$, but rot incidental items. b. Number of Responses in Recall

The total number of recall responses provides an index of response production. The mean number of correct, incorrect, and total responses on incidental and intentional recall tests for each Age $x$ Education $x$ Sex group is shown in Table 6 . These data were submitted to a 2 (Age) $\times 2$ (Education) $\times 2$ (Sex) $\times 2$ (Order) $\times 2$ (Task (Incidental vs. Intentional)) $\times 2$ (Response (Correct vs. Incorrect)) analysis of variance.

Older subjects produced fewer responses than younger subjects $(7.0$ vs. 10.4) and the Age main effect was statistically significant $(\underline{F}(1,48)=11.98$, $\mathrm{p}(.001)$. High school educated subjects produced fewer responses than Ph.D. educated subjects ( 8.2 vs. 9.2), but the Education main effect was not statistically significant. Males produced fewer responses than females ( 8.0 vs. 9.4$)$, but the sex main effect was also not significant. Likewise, the order main effect was not significant.

The Education $x$ sex interaction was statistically significant $(\underline{E}(1,48)=7.31, \underline{D}<.01)$, and may be seen in Figure 10, which shows the mean number of recall responses by males and females in each education group. 
Table 6

Mean Number of Correct and Incorrect Responses

On Incidental and Intentional Recall Tests For Each Age $x$ Education $x$ Sex Group

Correct Responses

Incidental Intentional Total

$\begin{array}{llllll}\overline{\mathrm{X}} & \mathrm{H} . \mathrm{S} & \overline{\mathrm{X}} & \mathrm{S} . \mathrm{D} . & \overline{\mathrm{X}} & \text { S.D. }\end{array}$

20 to 25

$\begin{array}{lllllll}\text { Males } & 7.50 & 4.21 & 6.88 & 4.26 & 7.19 & 2.84\end{array}$

$\begin{array}{lllllll}\text { Females } & 8.63 & 5.76 & 8.63 & 7.05 & 8.63 & 6.09\end{array}$

$\begin{array}{lllllll}\text { Combined } & 8.06 & 4.91 & 7.75 & 5.70 & 7.91 & 4.65\end{array}$

Over Sex

60 to 65

$\begin{array}{lllllll}\text { Males } & 2.13 & 3.00 & .2 .63 & 3.07 & 2.38 & 2.73\end{array}$

$\begin{array}{lllllll}\text { Females } & 8.63 & 4.90 & 11.00 & 4.24 & 9.81 & 2.94\end{array}$

$\begin{array}{lllllll}\text { Combined } & 5.38 & 5.16 & 6.81 & 5.61 & 6.09 & 4.72\end{array}$

Over Sex

Combined over Age

$\begin{array}{lllllll}\text { Males } & 4.81 & 4.49 & 4.75 & 4.20 & 4.78 & 3.67 \\ \text { Females } & 8.63 & 5.16 & 9.81 & 5.75 & 9.22 & 4.66 \\ \begin{array}{l}\text { Combined } \\ \text { Over Sex }\end{array} & 6.72 & 5.14 & 7.28 & 5.58 & 7.00 & 4.70\end{array}$


Table 6 Continued

Incorrect Responses

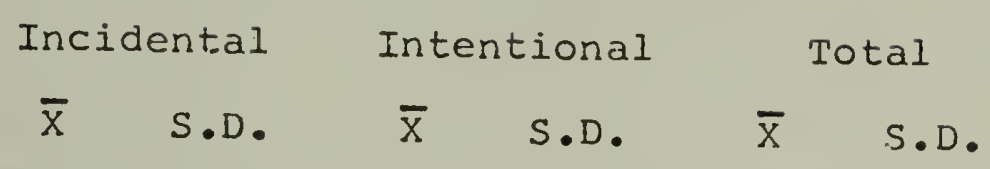

20 to 25

Males $\quad \begin{array}{llllll}1.38 & 1.60 & 1.13 & 1.73 & 1.25 & 1.51\end{array}$

$\begin{array}{lllllll}\text { Females } & 1.75 & 2.61 & .88 & .84 & 1.31 & 1.56\end{array}$

$\begin{array}{lllllll}\text { Combined } & 1.56 & 2.10 & 1.00 & 1.32 & 1.28 & 1.48\end{array}$

Over Sex

60 to 65

$\begin{array}{lrrrrrr}\text { Males } & .63 & 1.19 & 2.13 & 3.04 & 1.38 & 1.66 \\ \text { Females } & .88 & 1.46 & .63 & 1.06 & .75 & .70 \\ \begin{array}{l}\text { Combined } \\ \text { Over Sex }\end{array} & .75 & 1.29 & 1.38 & 2.34 & 1.06 & 1.28\end{array}$

Combined over Age

$\begin{array}{lllllll}\text { Males } & 1.00 & 1.41 & 1.63 & 2.45 & 1.31 & 1.54 \\ \text { Females } & 1.31 & 2.09 & .75 & .93 & 1.03 & 1.20 \\ \begin{array}{l}\text { Combined } \\ \text { Over Sex }\end{array} & 1.16 & 1.76 & 1.19 & 1.87 & 1.17 & .47\end{array}$


Table 6 Continued

Total Responses

$$
\text { Incidental Intentional Total }
$$

HS

$$
\overline{\mathrm{X}} \quad \text { S.D. } \quad \overline{\mathrm{X}} \quad \text { S.D. } \quad \overline{\mathrm{X}} \quad \text { S.D. }
$$

20 to 25

$\begin{array}{lllllll}\text { Males } & 8.88 & 4.55 & 8.00 & 4.18 & 8.44 & 2.74\end{array}$

$\begin{array}{lllllll}\text { Females } & 10.38 & 4.57 & 9.60 & 6.85 & 9.94 & 5.51\end{array}$

$\begin{array}{lllllll}\text { Combined } & 9.63 & 4.47 & 8.75 & 5.53 & 9.19 & 4.27\end{array}$

Over Sex

60 to 65

$\begin{array}{lllllll}\text { Males } & 2.75 & 4.03 & 4.75 & 5.39 & 3.75 & 4.08\end{array}$

$\begin{array}{lllllll}\text { Females } & 9.50 & 4.21 & 11.63 & 3.93 & 10.56 & 2.46\end{array}$

$\begin{array}{lllllll}\text { Combined } & 6.13 & 5.29 & 8.19 & 5.78 & 7.16 & 4.79\end{array}$ Over Sex

Combined over Age

$\begin{array}{lrrrrrr}\text { Males } & 5.81 & 5.22 & 6.38 & 4.95 & 6.09 & 4.14 \\ \text { Females } & 9.94 & 4.27 & 10.56 & 5.50 & 10.25 & 4.13 \\ \begin{array}{l}\text { Combined } \\ \text { Over Sex }\end{array} & 7.88 & 5.14 & 8.47 & 5.57 & 8.17 & 4.58\end{array}$


Table 6 Continued

\begin{tabular}{|c|c|c|c|c|c|c|}
\hline \multirow{3}{*}{ Ph.D. } & \multicolumn{6}{|c|}{ Correct Responses } \\
\hline & \multicolumn{2}{|c|}{ Incidental } & \multicolumn{2}{|c|}{ Intentional } & \multicolumn{2}{|c|}{ Total } \\
\hline & $\bar{x}$ & $S \cdot D$. & $\bar{x}$ & S.D. & $\bar{x}$ & $S . D$. \\
\hline \multicolumn{7}{|l|}{$\begin{array}{c}20 \text { to } 25 \\
\text { Males }\end{array}$} \\
\hline Males & 12.50 & 4.44 & $11.75^{\circ}$ & 5.59 & 12.13 & 3.70 \\
\hline Females & 10.13 & 3.64 & 10.13 & 5.11 & 10.13 & 3.75 \\
\hline $\begin{array}{l}\text { Combined } \\
\text { Over Sex }\end{array}$ & 11.31 & 4.11 & 10.94 & 5.14 & 11.13 & 3.74 \\
\hline \multicolumn{7}{|l|}{60 to 65} \\
\hline Males & 5.50 & 4.31 & 7.00 & 5.01 & 6.25 & 3.35 \\
\hline Females & 6.13 & 3.52 & 6.38 & 8.00 & 6.25 & 5.13 \\
\hline $\begin{array}{l}\text { Combined } \\
\text { Over Sex }\end{array}$ & 5.81 & 3.82 & 6.69 & 6.46 & 6.25 & 4.18 \\
\hline
\end{tabular}

Combined over Age

$\begin{array}{lllllll}\text { Males } & 9.00 & 5.62 & 9.38 & 5.60 & 9.19 & 4.56 \\ \text { Females } & 8.13 & 4.03 & 8.25 & 6.77 & 8.19 & 4.78 \\ \text { Combined } & 8.56 & 4.80 & 8.81 & 6.14 & 8.69 & 4.62 \\ \text { Over Sex } & & & & & & \end{array}$


Table 6 Continued

Incorrect Responses

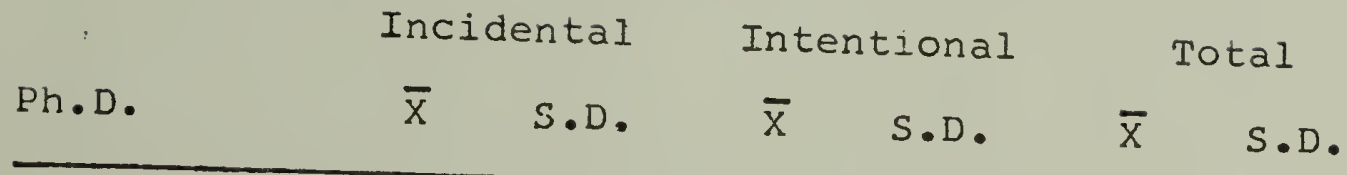

20 to 25

\begin{tabular}{|c|c|c|c|c|c|c|}
\hline Males & .63 & .74 & .50 & .76 & .56 & .63 \\
\hline Females & .38 & .74 & .25 & .46 & .31 & .46 \\
\hline $\begin{array}{l}\text { Combined } \\
\text { Over Sex }\end{array}$ & .50 & .73 & .38 & .19 & .44 & .54 \\
\hline
\end{tabular}

60 to 65

$\begin{array}{lrrrrrr}\text { Males } & .88 & 1.46 & .25 & .46 & .56 & .73 \\ \text { Females } & .75 & .70 & .13 & .35 & .44 & .42 \\ \begin{array}{l}\text { Combined } \\ \text { Over Sex }\end{array} & .81 & 1.11 & .19 & .40 & .50 & .58\end{array}$

Combined over Age

$\begin{array}{lrrrrrr}\text { Males } & .75 & 1.13 & .38 & .62 & .56 & .66 \\ \text { Females } & .56 & .73 & .19 & .40 & .38 & .43 \\ \begin{array}{l}\text { Combined } \\ \text { Over Sex }\end{array} & .66 & .94 & .28 & .52 & .47 & .55\end{array}$


Table 6 Continued

Total Responses

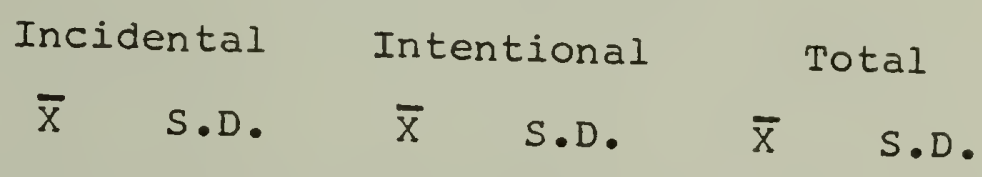

20 to 25

Males

13.134 .45

12.25

5.34

$12.69 \quad 3.71$

Females

$10.50 \quad 4.00$

10.38

5.04

$10.49 \quad 3.73$

Combined

Over Sex

11.814 .31

11.31

5.11

$11.56 \quad 3.78$

60 to 65

$\begin{array}{lllllll}\text { Males } & 6.38 & 3.93 & 7.25 & 5.06 & 6.81 & 3.20 \\ \text { Females } & 6.88 & 3.56 & 6.50 & 7.96 & 6.69 & 5.15 \\ \text { Combined } & 6.63 & 3.63 & 6.88 & 6.46 & 6.75 & 4.14 \\ \text { Over Sex } & & & & & & \end{array}$

Combined over Age

$\begin{array}{lllllll}\text { Males } & 9.75 & 5.35 & 9.75 & 5.65 & 9.75 & 4.52 \\ \text { Females } & 8.69 & 4.11 & 8.44 & 6.74 & 8.56 & 4.76 \\ \begin{array}{l}\text { Combined } \\ \text { Over Sex }\end{array} & 9.22 & 4.72 & 9.09 & 6.16 & 9.16 & 4.60\end{array}$


Table 6 Continued

Correct Responses

\begin{tabular}{lcccccc} 
Combined & \multicolumn{2}{c}{ Incidental } & Intentional & \multicolumn{2}{c}{ Total } \\
Over Ed & $\bar{x}$ & S.D. & $\bar{x}$ & S.D. & $\bar{x}$ & S.D. \\
\hline
\end{tabular}

20 to 25

$\begin{array}{lrrrrrr}\text { Males } & 10.00 & 4.91 & 9.31 & 5.33 & 9.66 & 4.08 \\ \text { Fernales } & 9.38 & 4.72 & 9.38 & 6.00 & 9.38 & 4.95 \\ \begin{array}{l}\text { Combined } \\ \text { Over Sex }\end{array} & 9.69 & 4.75 & 9.34 & 5.58 & 9.52 & 4.46\end{array}$

60 to 65

$\begin{array}{lllllll}\text { Males } & 3.81 & 3.99 & 4.81 & 4.61 & 4.31 & 3.57\end{array}$

$\begin{array}{lllllll}\text { Females } & 7.38 & 4.32 & 8.69 & 6.63 & 8.03 & 4.44\end{array}$

$\begin{array}{lllllll}\text { Combined } & 5.59 & 4.47 & 6.75 & 5.95 & 6.17 & 4.39\end{array}$

Combined over Age

$\begin{array}{lllllll}\text { Males } & 6.91 & 5.41 & 7.06 & 5.41 & 6.98 & 4.65 \\ \text { Females } & 8.38 & 4.56 & 9.03 & 6.23 & 8.70 & 4.67 \\ \begin{array}{l}\text { Combined } \\ \text { Over Sex }\end{array} & 7.64 & 5.02 & 8.05 & 5.87 & 7.84 & 4.70\end{array}$


Table 6 Continued

\section{Incorrect Responses}

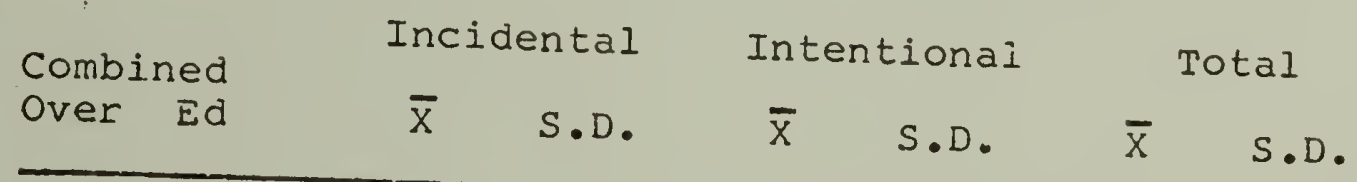

20 to 25

$\begin{array}{lllllll}\text { Males } & 1.00 & 1.27 & .81 & 1.33 & .91 & 1.17 \\ \text { Females } & 1.06 & 1.98 & .56 & .73 & .81 & 1.22 \\ \begin{array}{l}\text { Combined } \\ \text { Over Sex }\end{array} & 1.03 & 1.64 & .69 & 1.06 & .86 & 1.18\end{array}$

60 to 65

Males

$\begin{array}{lll}.75 & 1.29 & 1.19\end{array}$

2.32

$.97 \quad 1.31$

Females

$\begin{array}{lll}.81 & 1.11 \quad .38\end{array}$

.81

.59

.58

Combined

Over Sex

$.78 \quad 1.18$

$.78 \quad 1.76$

$.78 \quad \mathrm{~J} .02$

Combined over Age

$\begin{array}{lrrrrrr}\text { Males } & .88 & 1.26 & 1.00 & 1.87 & .94 & 1.22 \\ \text { Females } & .94 & 1.59 & .47 & .76 & .70 & .95 \\ \begin{array}{l}\text { Comined } \\ \text { Over Sex }\end{array} & .91 & 1.42 & .73 & 1.44 & .82 & 1.09\end{array}$


Table 6 Continued

Total Responses

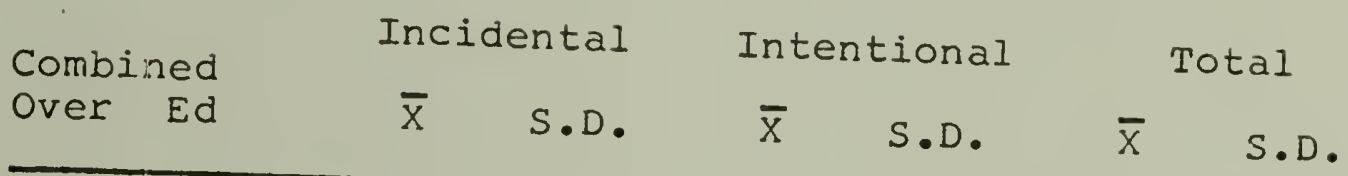

20 to 25

$\begin{array}{lrrrrrr}\text { Males } & 11.00 & 4.87 & 10.13 & 5.12 & 10.56 & 3.84 \\ \text { Females } & 10.44 & 4.15 & 9.94 & 5.83 & 10.19 & 4.55 \\ \begin{array}{l}\text { Combined } \\ \text { Over Sex }\end{array} & 10.72 & 4.46 & 10.03 & 5.40 & 10.38 & 4.15\end{array}$

60 to 65

$\begin{array}{lllllll}\text { Males } & 4.56 & 4.27 & 6.00 & 5.22 & 5.28 & 3.88\end{array}$

$\begin{array}{lllllll}\text { Females } & 8.19 & 4.00 & 9.06 & 6.62 & 8.63 & 4.38\end{array}$

$\begin{array}{lllllll}\text { Combined } & 6.38 & 4.47 & 7.53 & 6.06 & 6.95 & 4.41\end{array}$

Combined over Age

$\begin{array}{lcccccc}\text { Males } & 7.78 & 5.57 & 8.06 & 5.50 & 7.92 & 4.65 \\ \text { Females } & 9.31 & 4.17 & 9.50 & 6.15 & 9.41 & 4.47 \\ \begin{array}{l}\text { Combined } \\ \text { Over Sex }\end{array} & 8.55 & 4.94 & 8.78 & 5.83 & 8.67 & \end{array}$


Figure 10. Mean Number of Recall Responses by Males and Females in each Education Group

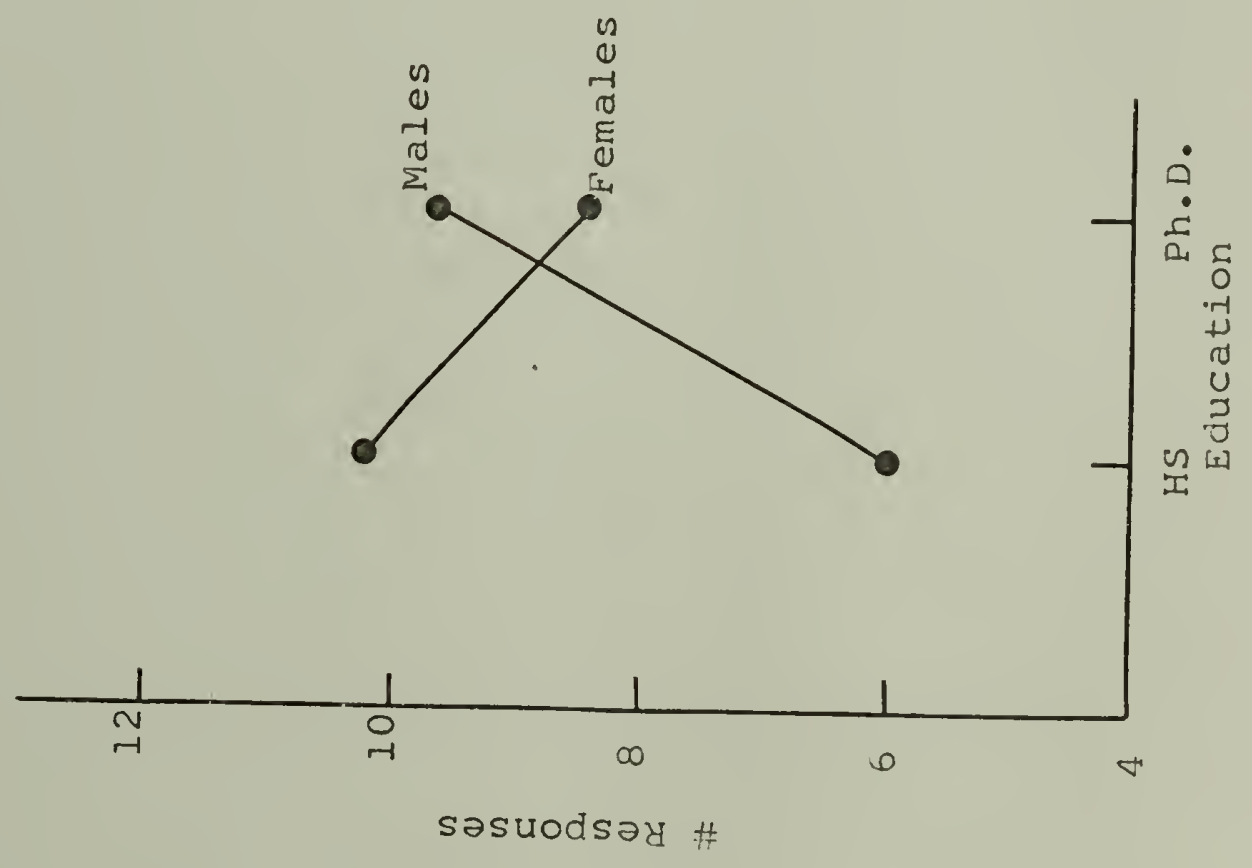


While less education was associated with significantly

fewer recall responses for males $(\underline{t}(30)=2.39, \underline{p}<$ .05), education was not a significant factor in the number of recall responses produced by females. Also, while in the HS sample males produced significantly fewer responses than females ( $\underline{t}\left(30_{-}=2.04, \underline{p}<.01\right.$ ), in the $\mathrm{Ph} . \mathrm{D}$. sample there were no differences in the number of responses produced by males and females.

The number of correct responses was considerably greater then the number of incorrect responses $(7.8$ vs. 0.8), and the Response (Correct vs. Incorrect) main effect was highly significant $(\underline{F}(1,48)=174.84$, $\mathrm{p}(.001)$.

The Age $x$ Response $(\underline{F}(1,48)=9.40, \underline{p}<.01)$ and Age $\mathrm{x}$ Sex $\mathrm{x}$ Response interaction were statistically significant as well $(\underline{F}(1,48)=6.04, \underline{\mathrm{Q}}<.05)$, and may be seen in Figure 11, which shows the mean number of correct and incorrect recall responses by males and females in each Age group. Older males produced significantly fewer correct responses than any of the other Age $x$ Sex groups (all ps $<.05$ ), and the number of correct responses produced by the other three groups was not statistically different. Aging was associated with significantly fewer correct responses for males $(\underline{t}(30)=3.94, \underline{\mathrm{D}}<.001)$, but this tendency failed to reach statistical significance for females. 
Figure 11. Mean Number of Correct and Incorrect Recall Responses by Males and Females in Each Age Group

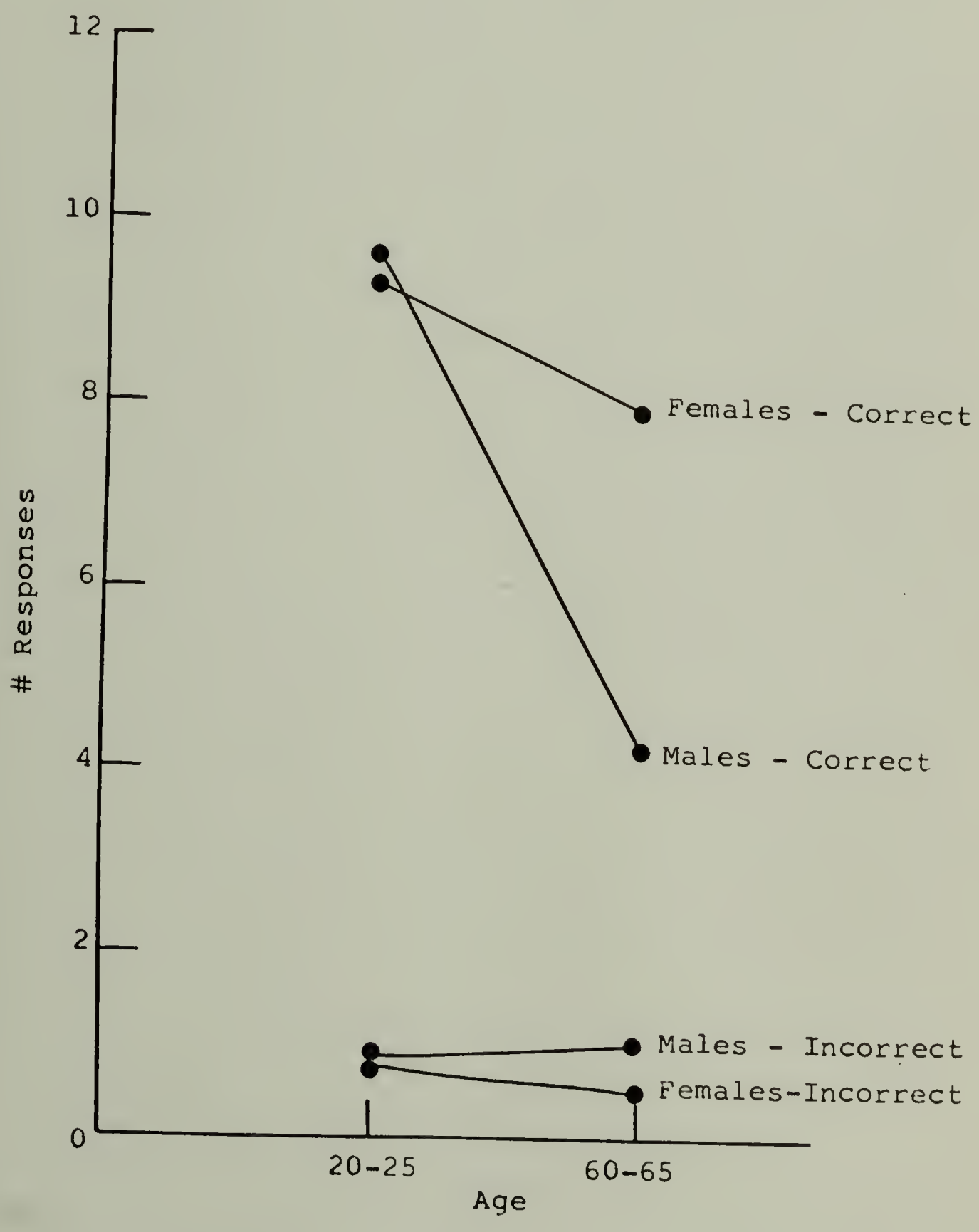


Furthermore, while for older subjects, males produced significantly fewer correct responses than females $(\underline{t}(30)-2.61, \mathrm{p}<.05)$, for younger subjects, sex was not a significant iactor in number of correct responses. None of the Age $x$ Sex groups differed significantly in number of incorrect responses.

The Education $x$ Response $(\underline{F}(1,48)=5.04, \underline{p}<$

.05) and Education $x$ Sex $x$ Response interactions were also statistically significant $(\underline{F}(1,48)=6.74, \underline{p}<$ $.05)$, and may be seen in Figure 12, which shows the mean number of correct and incorrect recall responses by males and females in each Education group. Hs males produced significantly fewer correct responses than any of the other Education $x$ Sex groups (all ps $<.05)$, and the number of correct responses produced by the other three groups was not statistically different. While more education was related to significantly more correct responses for males $\left(\underline{t}\left(30_{-}=3.01\right.\right.$, $\mathrm{p}<.01)$, it was not for females. Furthermore, while for HS subjects, males produced significantly fewer correct responses than females $(\underline{t}(30)=2.99, \underline{p}<.01)$, for Ph.D. subjects, sex was not a significant factor in number of correct responses. More education was related to fewer incorrect responses for both males $(\underline{t}(30)=1.80, \underline{p}<.09)$ and females $(\underline{t}(30)=2.05, \underline{p}<$ 
Figure 12. Mean number of Correct and Incorrect Recall Responses by Males and Females in Each Education Group

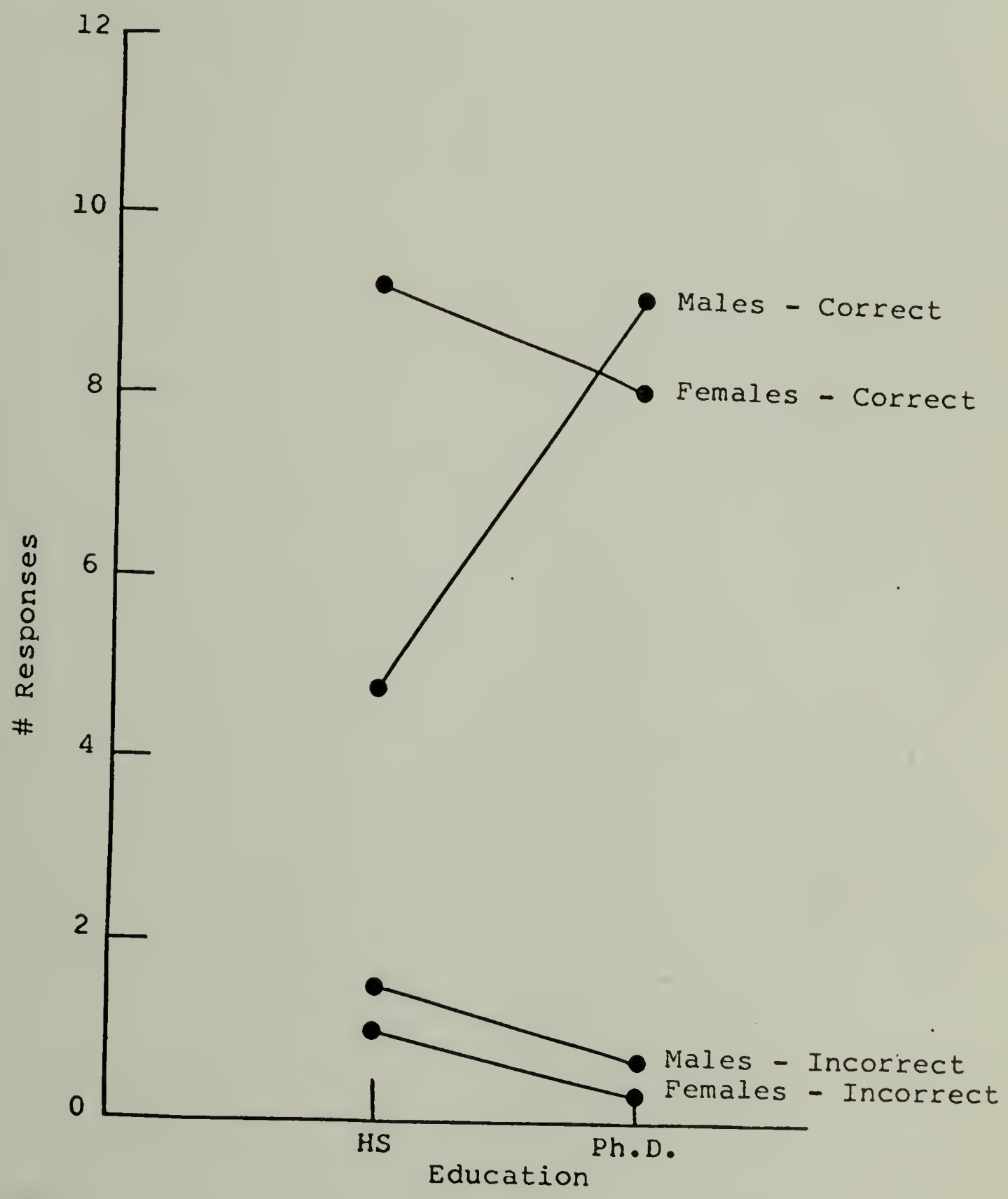


.06). Sex was not a significant factor in the number of incorrect responses produced by either Education group.

\section{c. Intrusions in Recall}

Incorrect responses on incidental and intentional recall tests were categorized according to whether they were from the other list (list intrusions) from the set of associations the subject generated for the association task (associate intrusions), or other. (miscellaneous intrusions). The mean number of list, associate, and miscellaneous intrusions produced by each Age $x$ Education $x$ Sex group is shown in Table 7 . These data were submitted to a 2 (Age) $\times 2$ (Education) $\times 2$ (Sex) $\times 2$ (Order) $\times 2$ (Task (Incidental vs. Intentional)) x 3 (Intrusion (List vs. Associate vs. Miscellaneous)) analysis of variance. The only statistically significant main effect was Education ( $\mathrm{F}(1,48$ ) $=7.00, \mathrm{p}<.05)$; HS subjects produced more intrusions than Ph.D. subjects (.39 vs. 16).

The Task $x$ Intrusion interaction was statistically significant $(\underline{F}(2.96)=7.26, \underline{p}<.01)$, and may be seen in Figure 13, which shows the mean number of list, associate, and miscellaneous intrusions on incidental and intentional recall. More list and associate intrusions were produced on incidental than intentional 


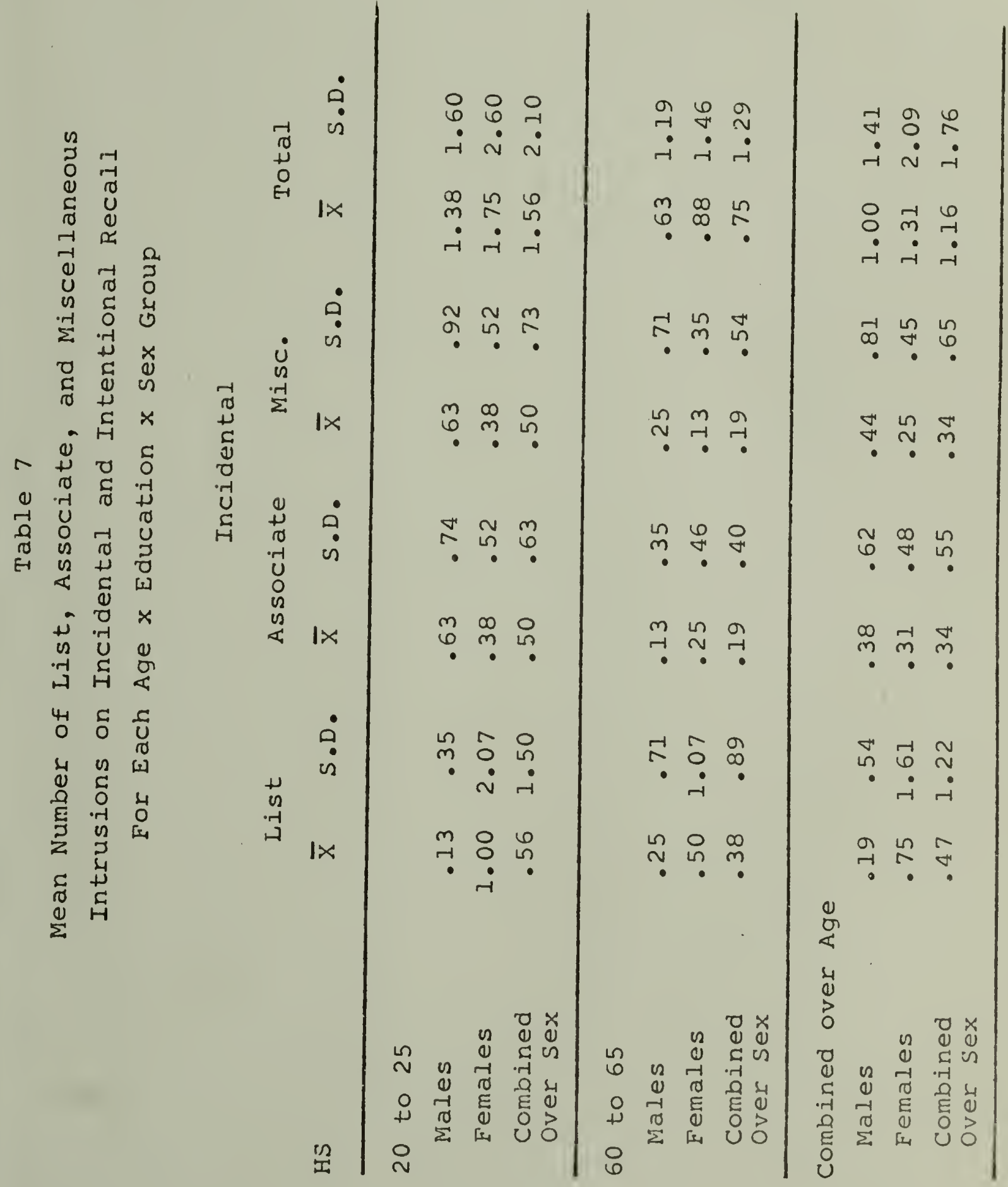


Table 7 Continued

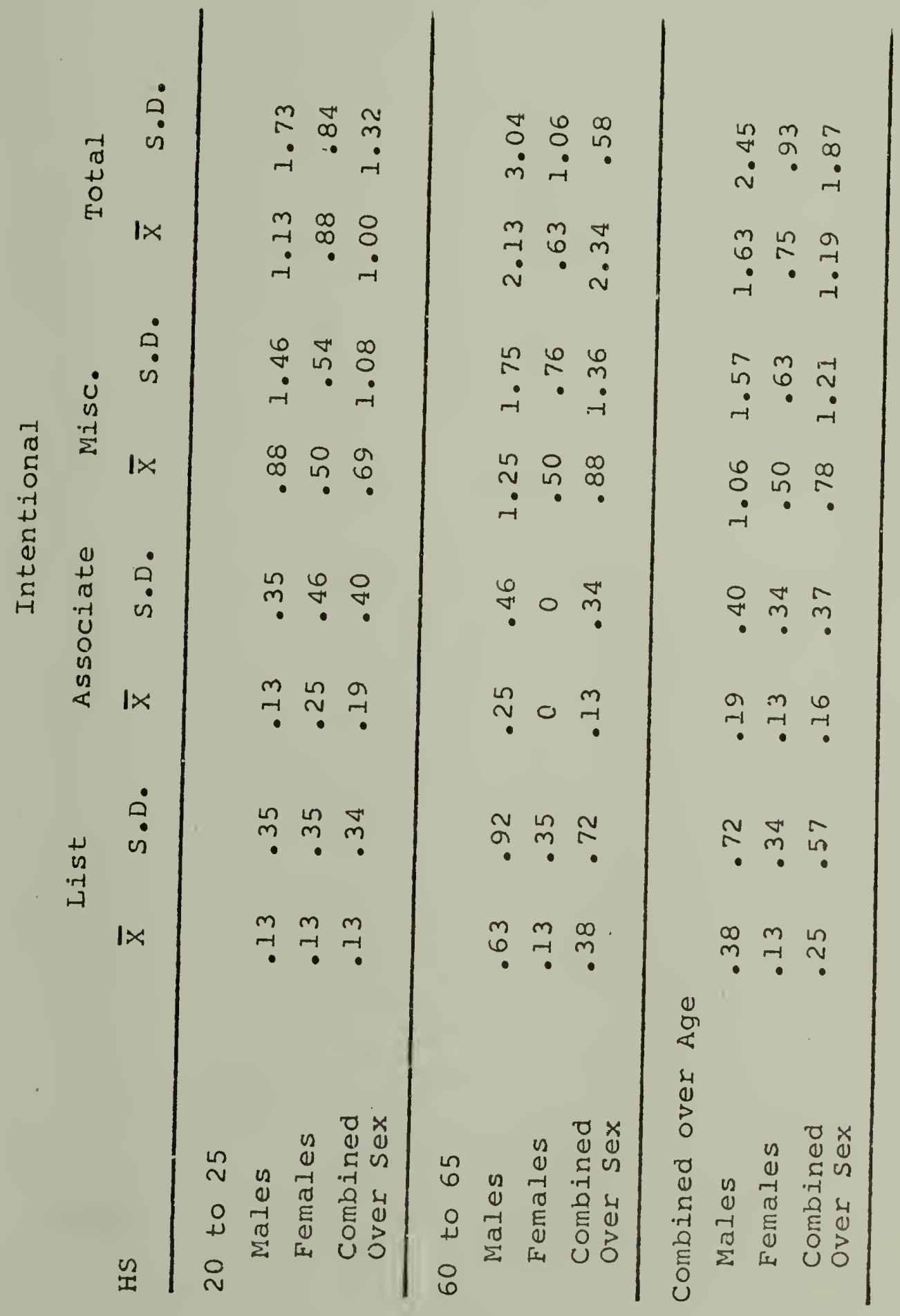


Table 7 Continued

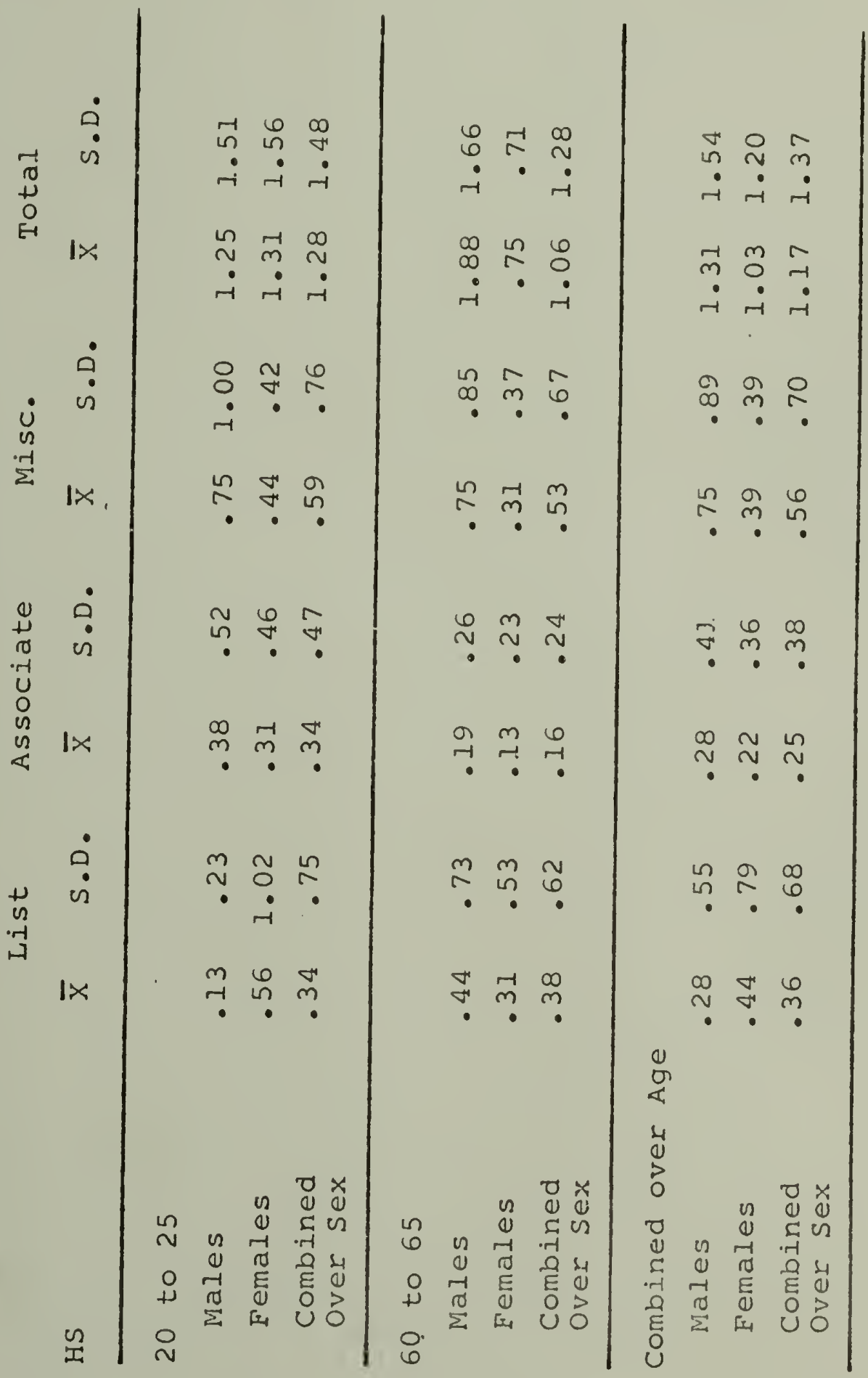


Table 7 Continued

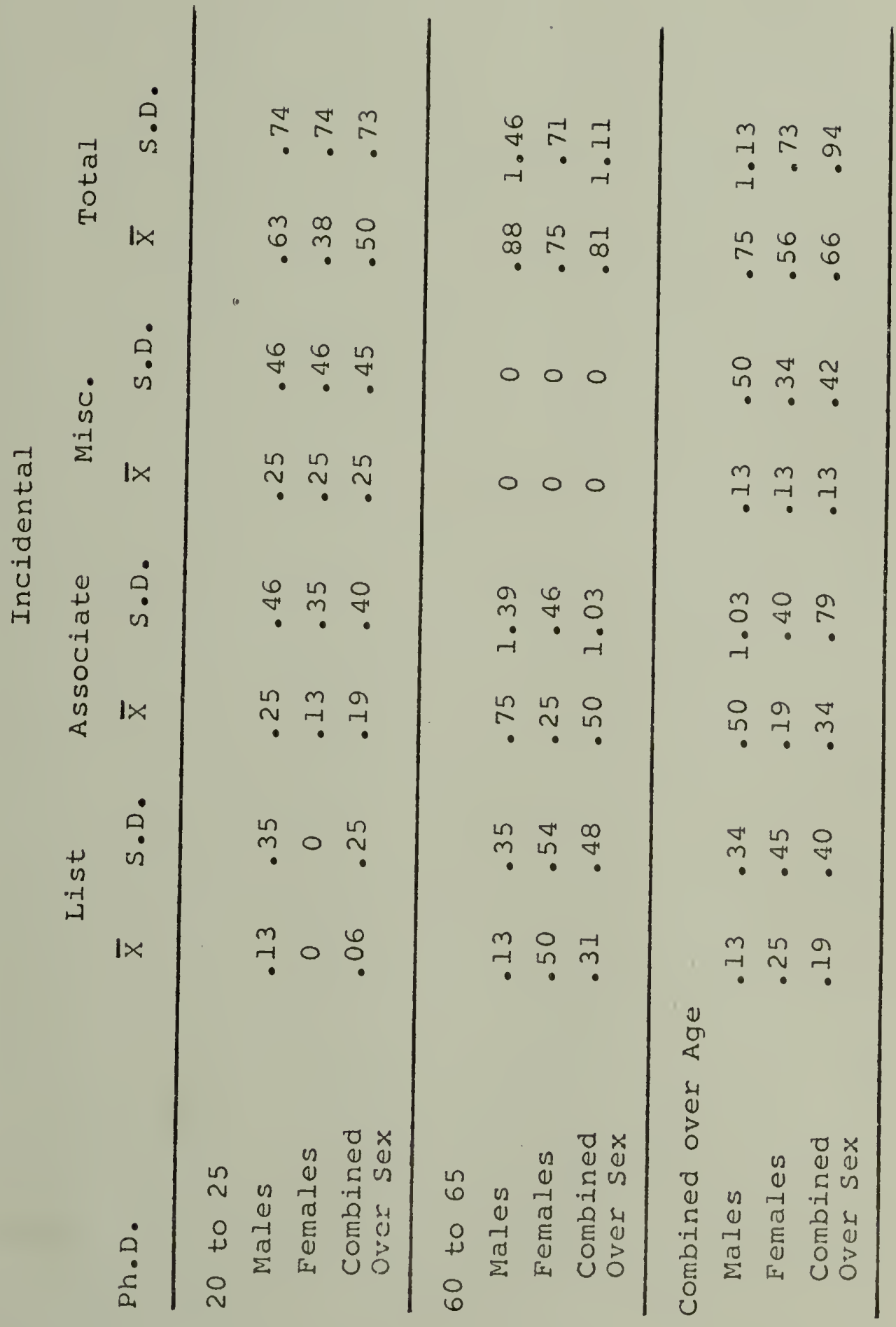


Table 7 Continued

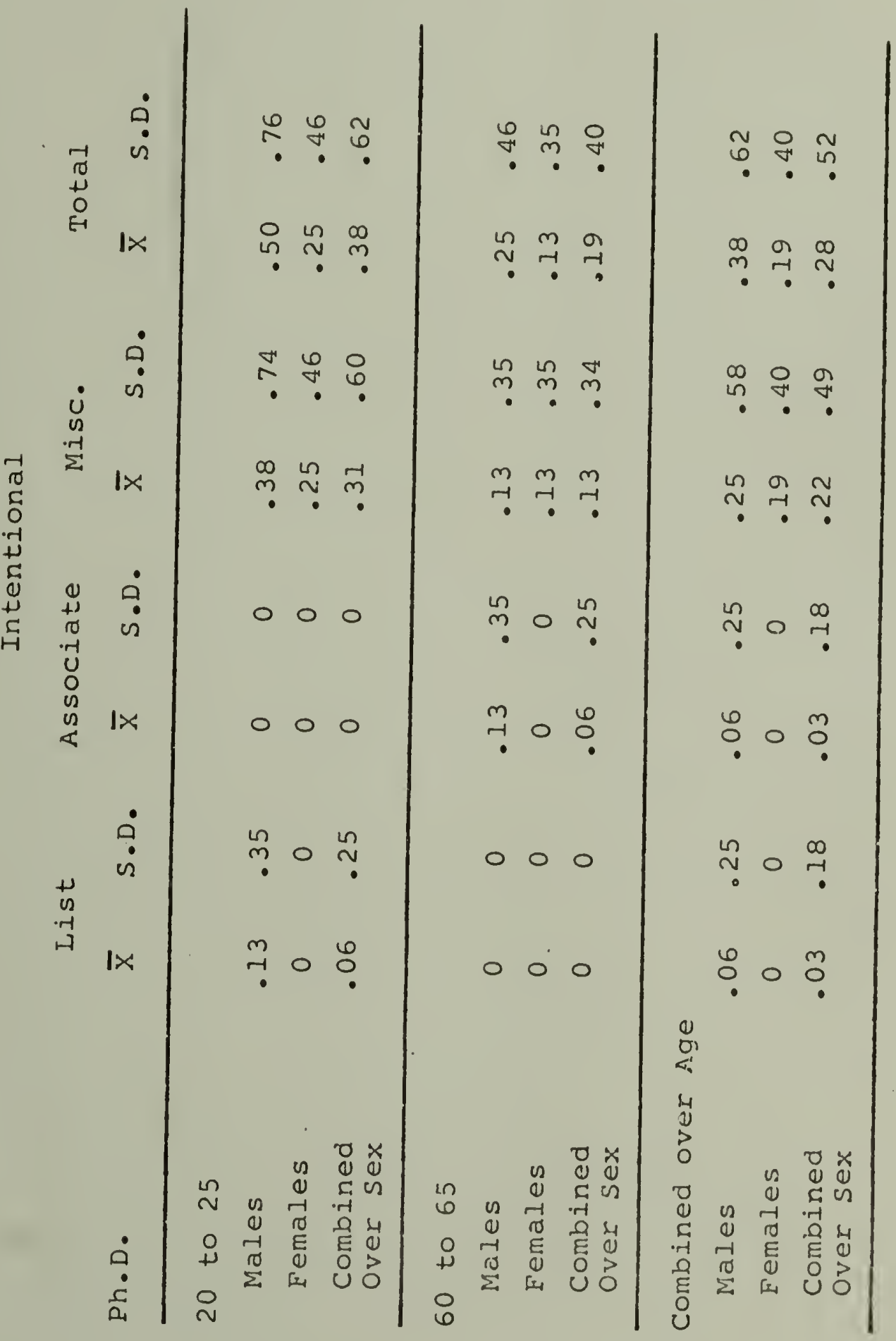


Table 7 Continued

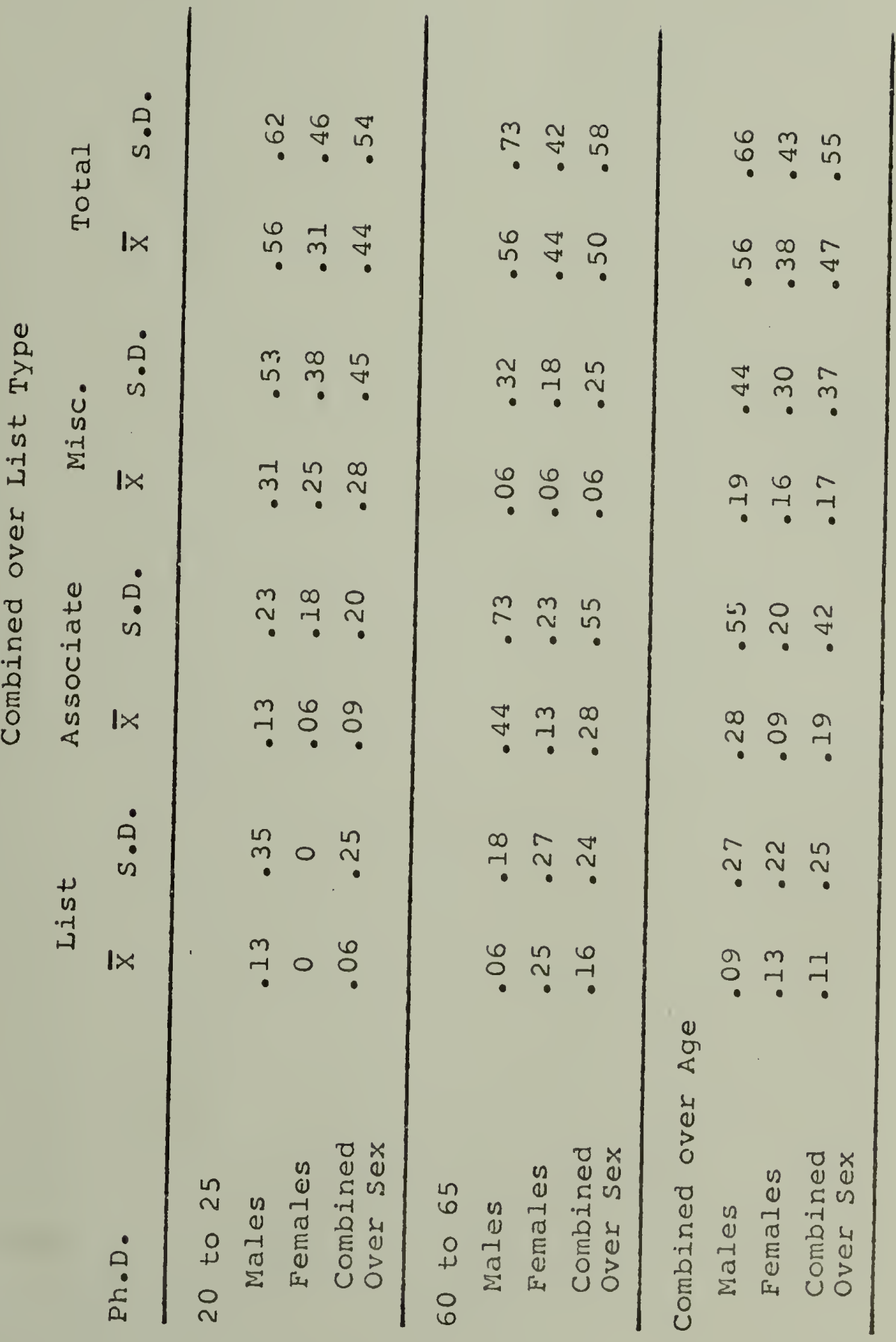


Table 7 Continued

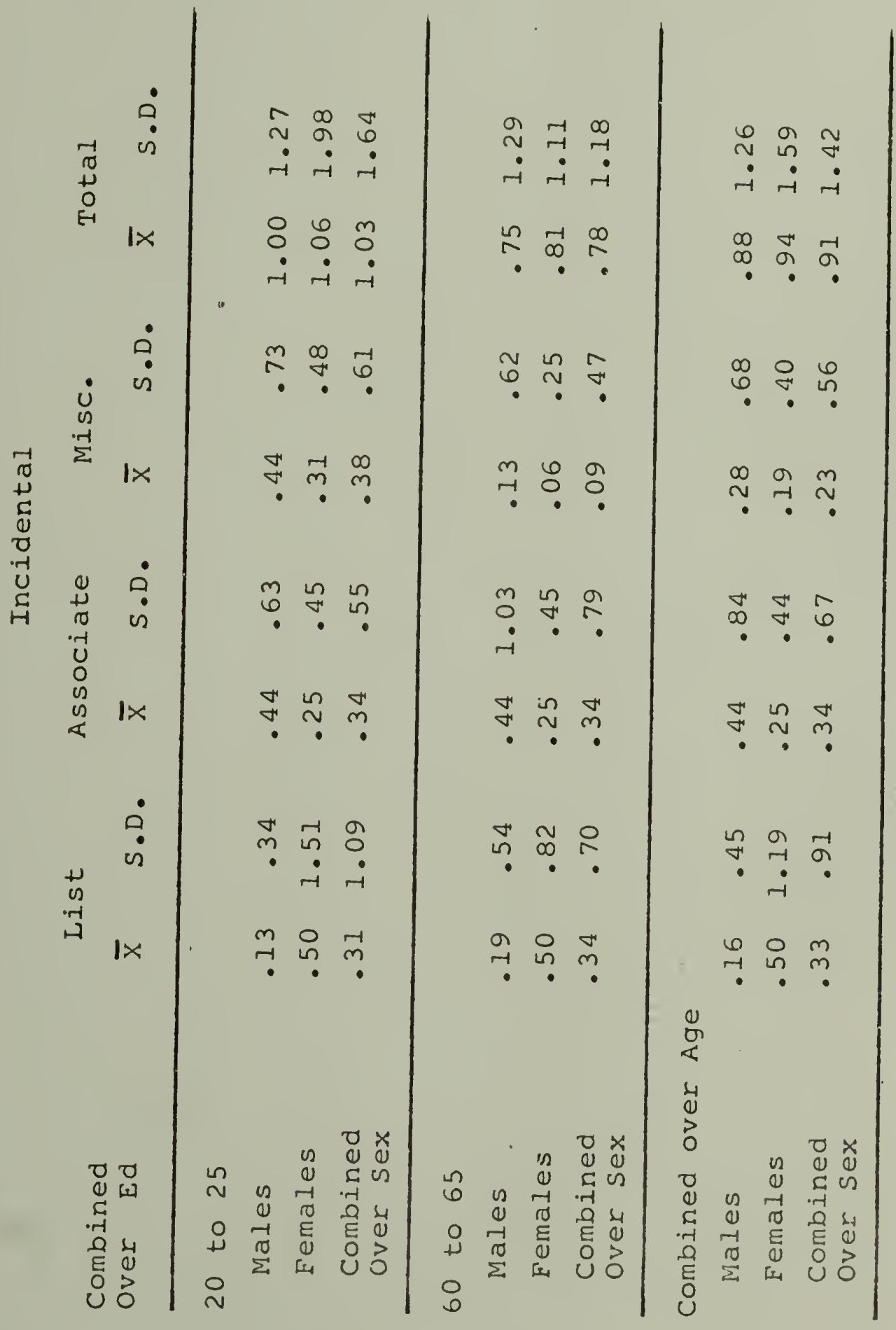


Table 7 Contirued

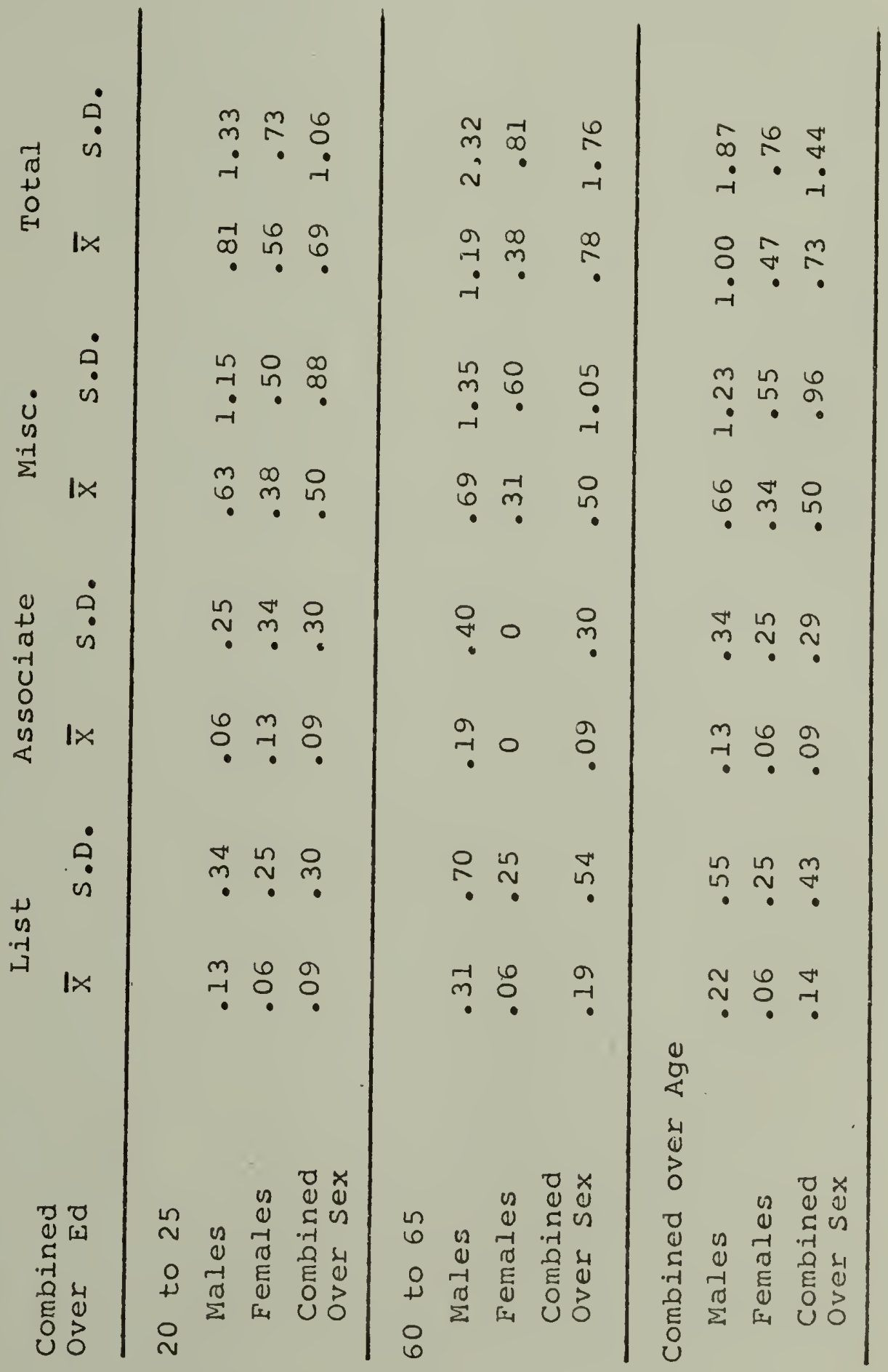


Table 7 Continued

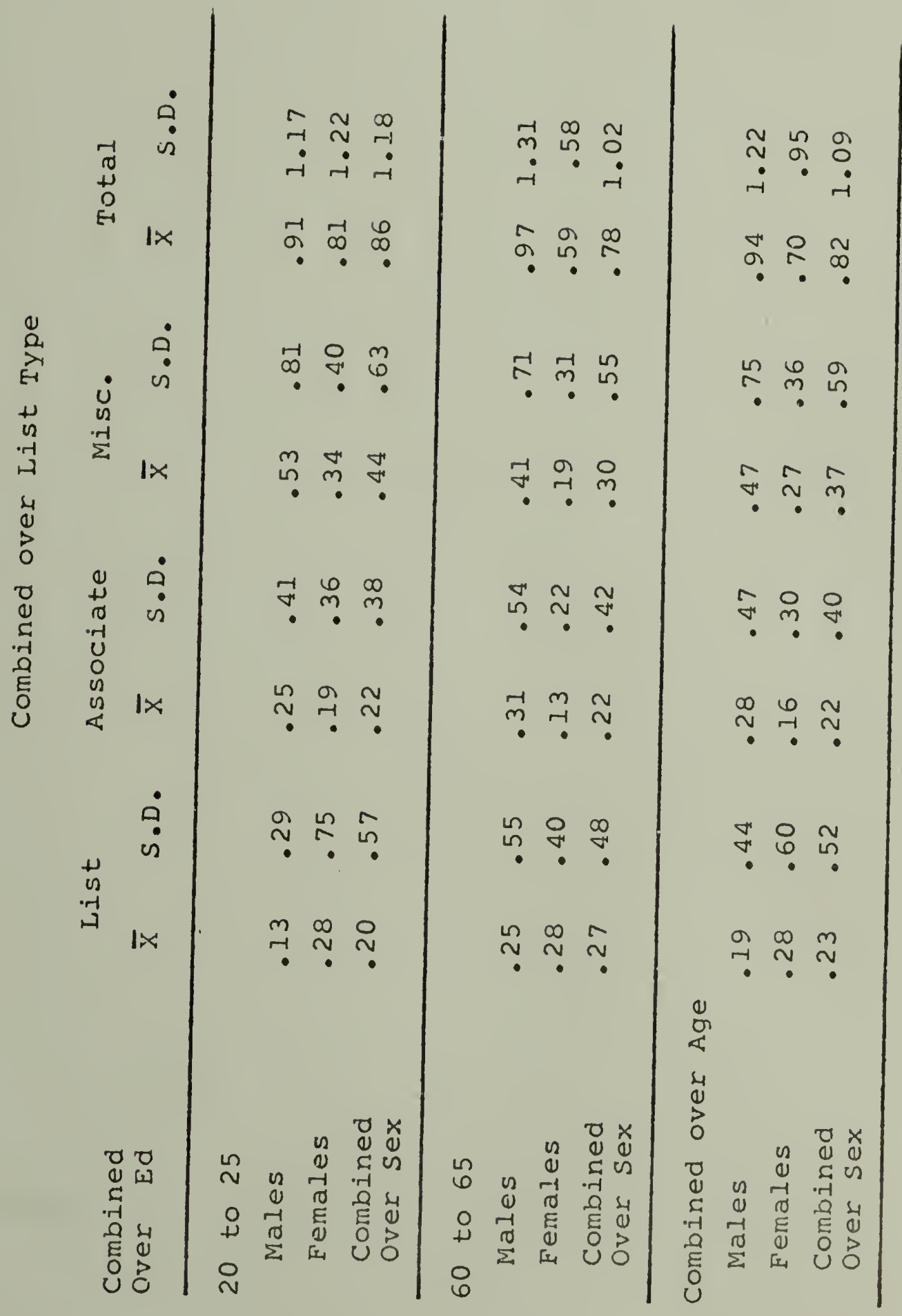


Figure 13. Mean Number of List, Associate, and Miscellaneous Intrusions on Incidental and Intentional Recall

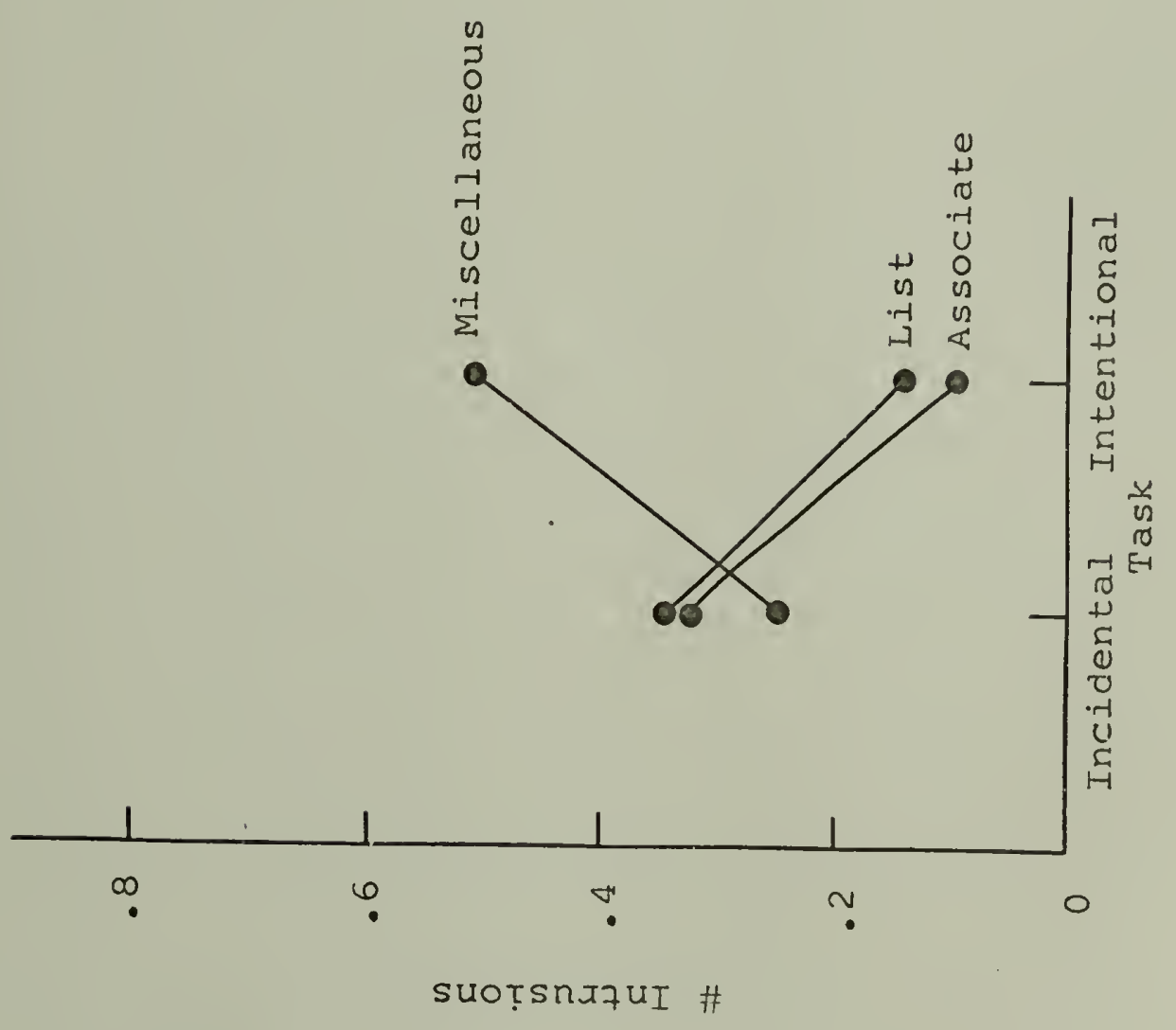


lists, although this difference only reached statistical significance for associate intrusions $(\underline{t}(63)=$ $3.00, \mathrm{p}<.01)$, and more miscellaneous intrusions were produced on intentional than incidental lists $(\underline{t}(63)=$ $1.83, \mathrm{p}<.08)$. On incidental lists there were no statistical differences in the number of intrusions of any type, and on intentional lists no statistical differences in the number of list and associate intrusions, but significantly more miscellaneous intrusions than list $(\underline{t}(63)=3.58, \underline{p}<.001)$, and associate $(\underline{t}(63)=3.83, \underline{p}<.001)$ intrusions.

d. Percent Correct in Recognition

The percentage of incidental, intentional, and new items, correctly recognized by each Age $x$ Education $x$ Sex group is shown in Table 8 . These data were submitted to a 2 (Age) $x 2$ (Education) $x$ 2 (Sex) $x 2$ (Order)x 3 (Item (Incidental vs. Intentional vs. New)) analysis of variance.

Older subjects produced fewer correct recognition responses than younger subjects ( $76 \%$ vs. $82 \%$ ), and the Age main effect was statistically significant $(\underline{F}(I, 48)=6.79, \underline{p}<.05)$. High school educated subjects produced fewer correct recognition responses than Ph.D. educated subjects ( $76 \%$ vs. $82 \%$ ), and the Education main effect was statistically significant 


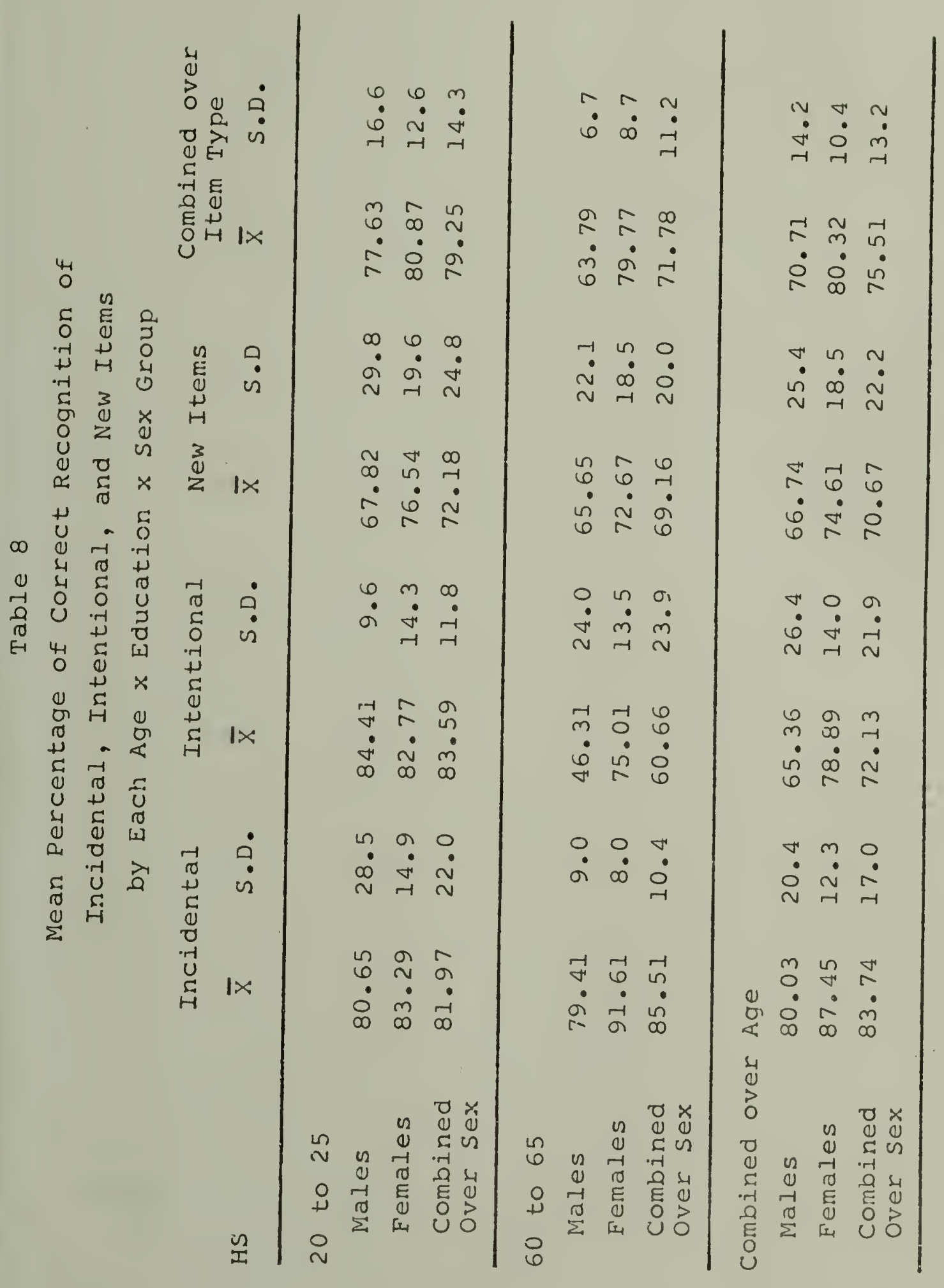


Table 8 Continued

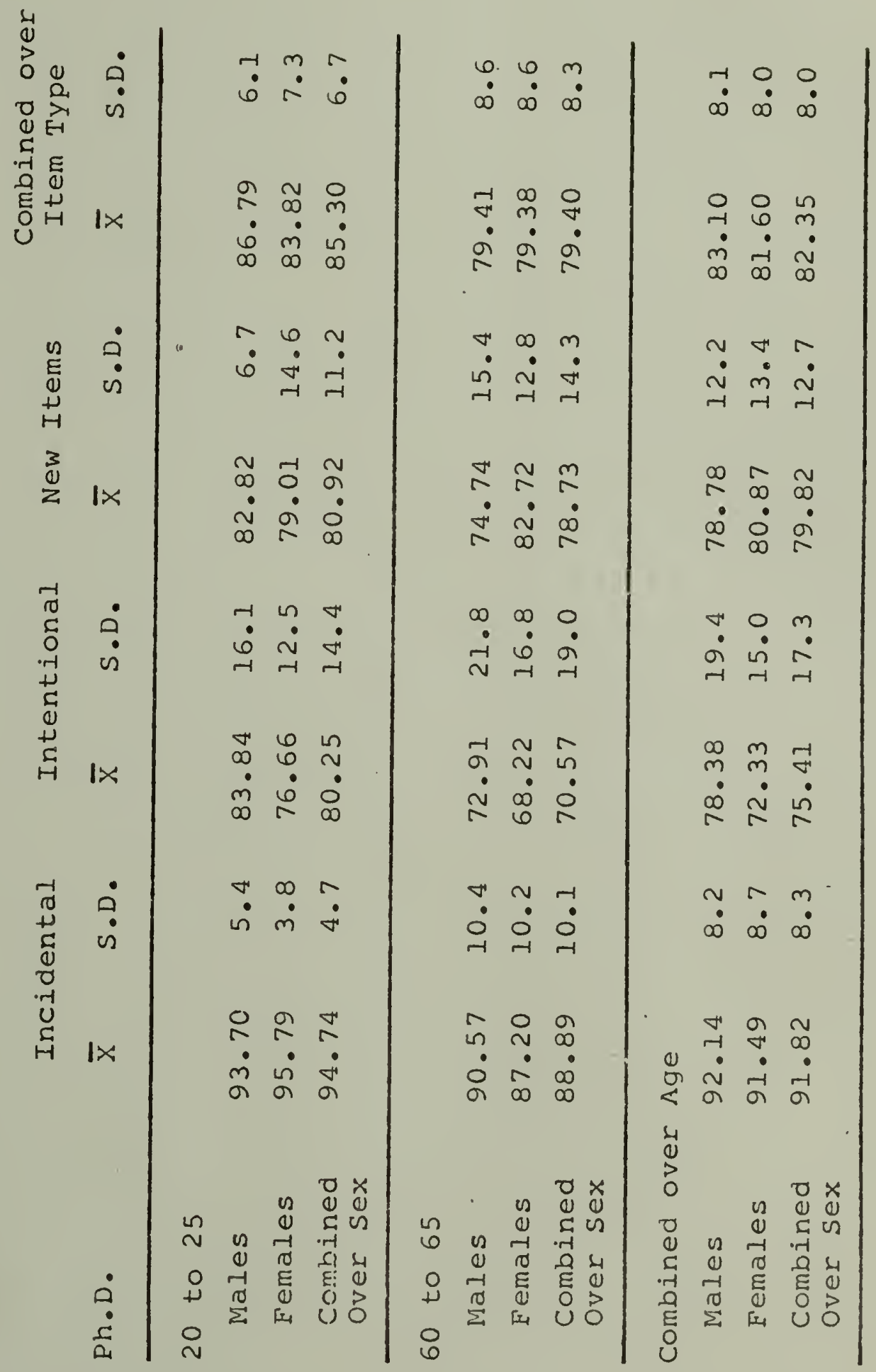


Table 8 Continued

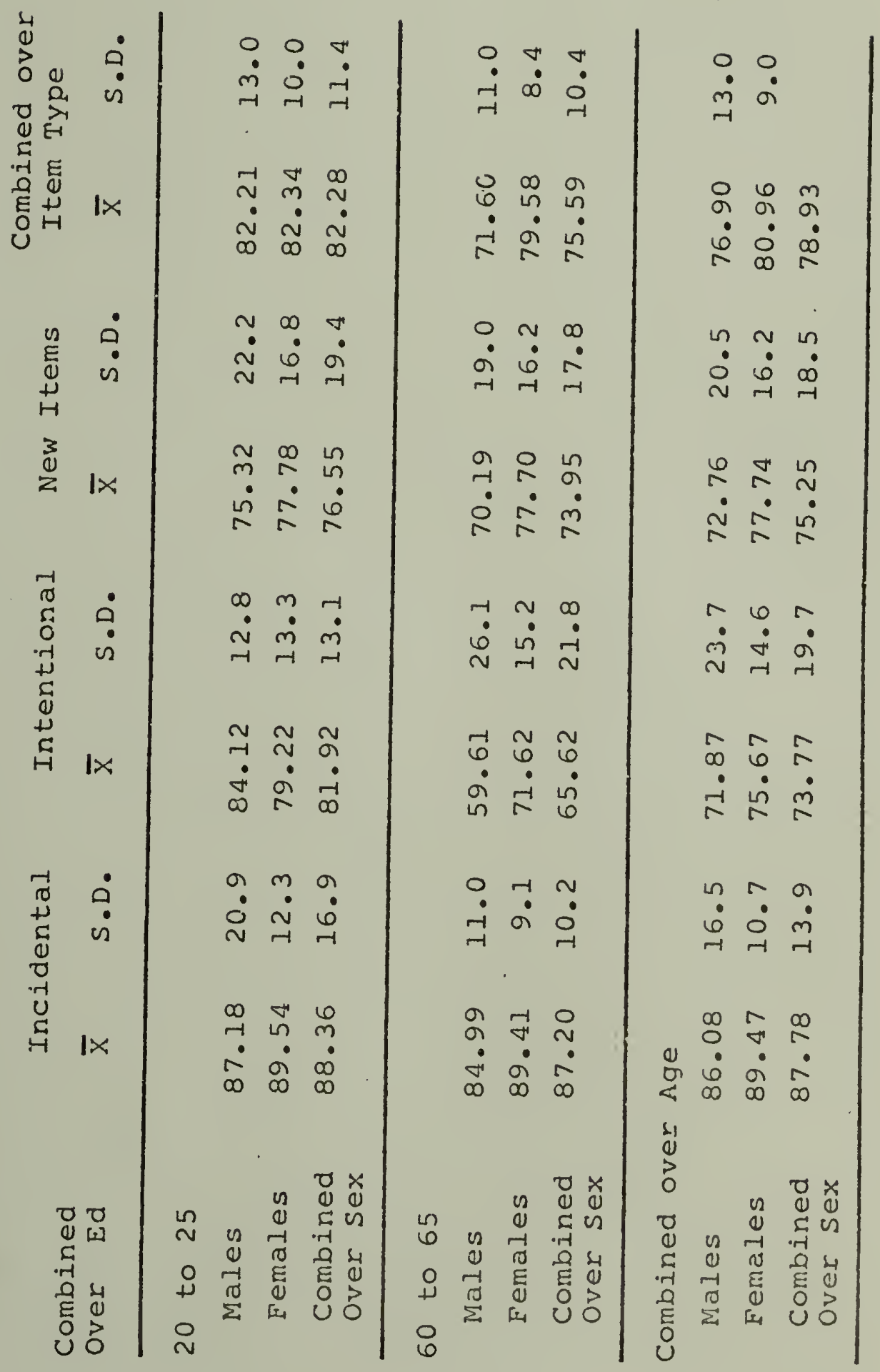


$(\underline{F}(1,48)=7.09, \underline{p}<.05)$. Males produced fewer correct recognition responses than females ( $77 \%$ vs. $81 \%$ ), but the sex main effect was not statistically significant. The order main effect was also not significant. The Education $x$ sex interaction was statistically significant $(\underline{F}(1,48)=4.68, \underline{p}<.05)$, and may be seen in Figure 13, which shows the mean percent correct recognition by males and females in each education group. Hs males correctly recognized significantly fewer items than any of the other sex $x$ Education groups (all ps< .05), and performance of the other three groups was not statistically different. While iess education was associated with poorer recognition performance for males $(\underline{t}(3)=3.06, \underline{p}<.01)$, it was not a significant factor in females recognition performance. Also, while in the HS sample, males correctly recognized significantly fewer items than Remales $(\underline{t}(30)=2.18, \underline{\mathrm{a}}<.05)$, in the Ph.D. sample sex was not a significant factor in recognition performance.

The percentage of correct responses for incidental, intentional, and new items was $88 \%, 74 \%$, and $75 \%$, respectively. The Item main effect was statistically significant $(\underline{E}(2.96)=14.55, \underline{\mathrm{p}}<.001)$, and simple effects tests indicated that there were statistically 
Figure 14. Mean Percent Correct Recognition by Males and Females in Each Education Group
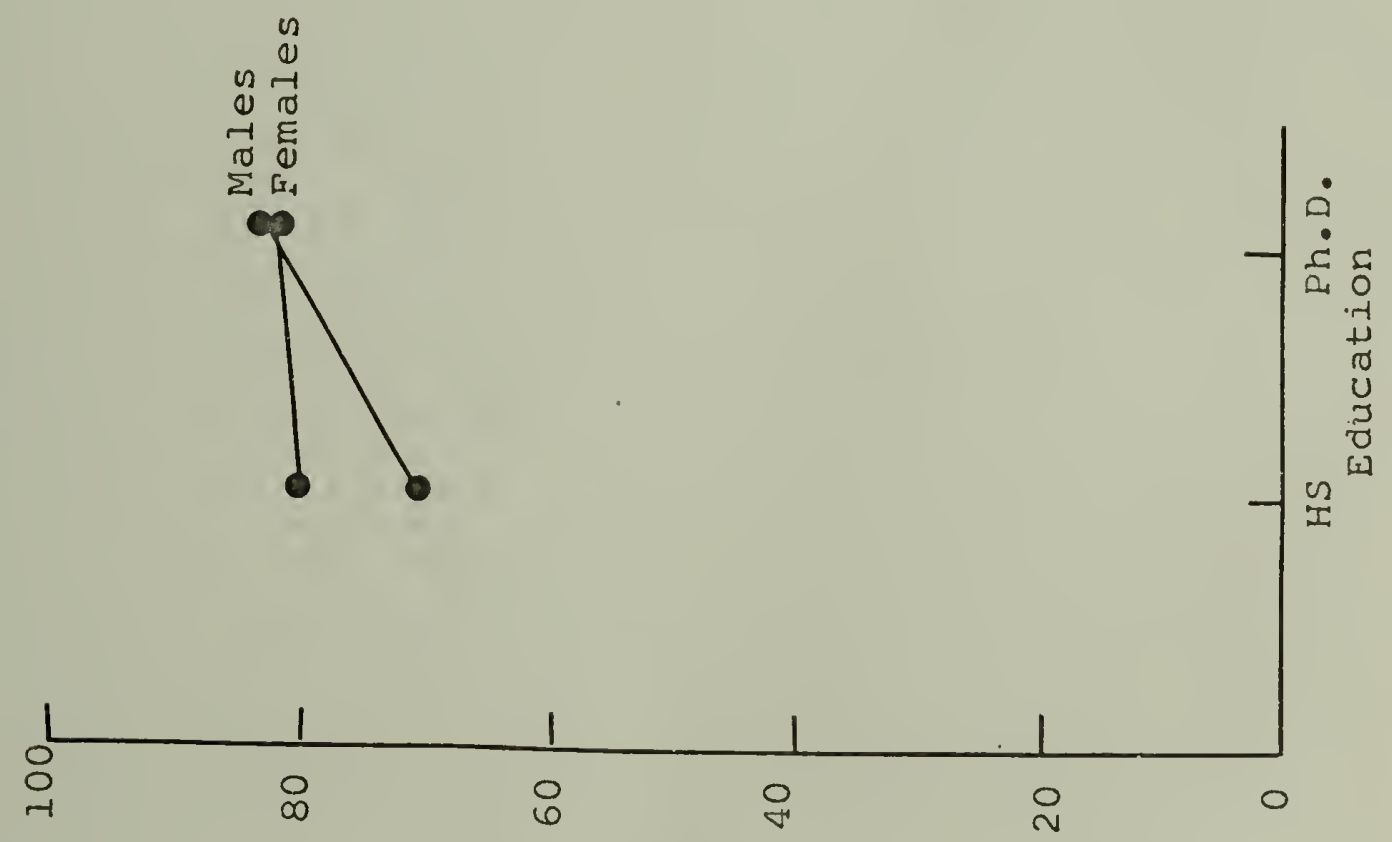

7วәлภоว \% 
more correct responses to incidental items than to intentional $(\underline{t}(63)=5.21, \underline{p}<.001)$, or new $(\underline{t}(63)$ $=5.37, \mathrm{p}<.001)$ items, but statistically comparable percentages of correct responses to intenticnal and new items.

The Age $x$ Item interaction was statistically significant $(\underline{F}(2.96)=4.29, \mathrm{p}<.05)$, and may be seen in Figure 15, which shows the mean percent correct responses for incidental, intentional, and new items by each Age group. Both age groups produced more correct responses to incidental items than to intentional items $(\underline{t s}(3)=1.70$ and $6.42, \mathrm{ps}<.1$ and .001 , for younger and older subjects, respectively. Although this difference was greater for older than younger subjects. Additionally, both age groups produce significantly more correct responses to incidental items than to new items $(\underline{t} s(3)=4.16$ and 3.54 , both ps (.001), but statistically comparable percentages of correct responses to intentional and new items. Furthermore, while older subjects produced significantly fewer correct responses to intentional items than younger subjects $(\underline{t}(62)=3.62, \underline{p}<.001)$, age was not a significant factor in recognition performance on incidental or new items.

e. Signal Detection Analysis of Recognition Signal detection analysis can be used to 
Figure 15. Mean Percent Correct Responses for Incidental, Intentional, and New Items by Each Age Group
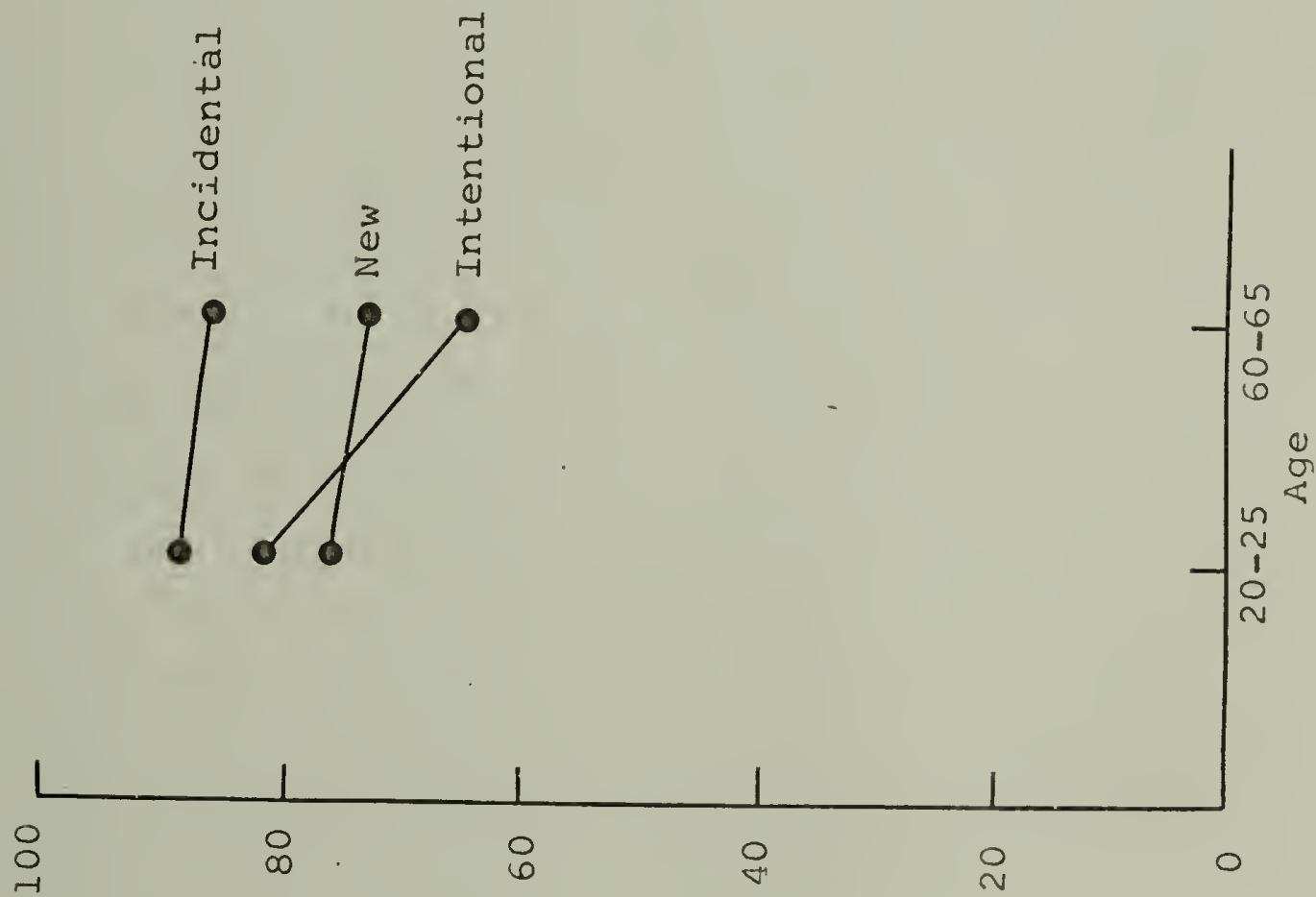

7ววภภดว \# 
separate retention and decision components of recognition (Banks, 1970; Lockhart \& Murdock, 1970). The d' statistic represents retention, and $\underline{C}$, decision components of recognition. Each of these statistics was computed for subject's incidental and intentional recognition performance. Separate 2 (Age) $\times 2$ (Education) $\times 2$ (Sex) $\times 2$ (Order) $\times 2$ (Task (Incidental vs. Intentional)) analyses of variance were performed on the $\underline{d}^{\prime}$ and $\underline{c}$ measures.

The mean ds for younger and older subjects were 2.24 and 1.68 , respectively, and the analysis indicated significantly better retention for younger than older subjects $(\underline{F}(I, 48)=5.60, \underline{p}<.05)$. The mean d's for HS and Ph.D. subjects were 1.67 and 2.24, respectively, and the analysis indicated significantly better retention for $\mathrm{Ph} . \mathrm{D}$. than HS subjects $(\underline{E}(1,48)=$ $5.90, \mathrm{p}<.05)$. The mean $\mathrm{d}$ 's for males and females were 1.70 and 2.21 , respectively, and the analysis indicated significantly better retention for females than males $(\underline{F}(1,48)=4.55, \underline{p}<.05)$. Additionally, d's were higher for incidental than intentional lists (2.30 vs. 1.62), and the Task main effect was statis-. tically significant $(\underline{F}(1,48)=20.98, \underline{p}<.001)$. No other main effects or interactions were statistically significant. 
The mean $\subseteq$ value was .80 , and the analysis of these measures yielded no statistically significant main effects or interactions.

\section{Semantic Memory}

Subjects were asked to answer 24 questions of general information knowledge, in a recall task, and then asked to make true and false responses to 24 true and 24 false general information statements. There were six recall questions and six true and six false recognition statements referring to each of the following four time periods: 1890-1909, 1910-1929, 19301949 , and 1950-1969.

a. Percent Known on Recall and Recognition Tests The mean percentage of items from each time period correctly recalled and recognized (after correction) by each Age $x$ Education $x$ Sex group is shown in Table 9. These recall and recognition scores were submitted to a 2 (Age) $\times 2$ (Education) $\times 2$ (Sex) $\times 2$ (Order) × 2 (Memory (Recall vs. Recognition)) × 4 (Time Period) analysis of variance.

Older subjects made more correct responses than younger subjects ( $50 \%$ vs. $40 \%$ ), and the Age main effect was statistically significant $(\underline{F}(1,48)=5.81$, $\mathrm{p}<.05)$. Ph.D. educated subjects made more correct responses than high school educated subjects (52\% vs. 


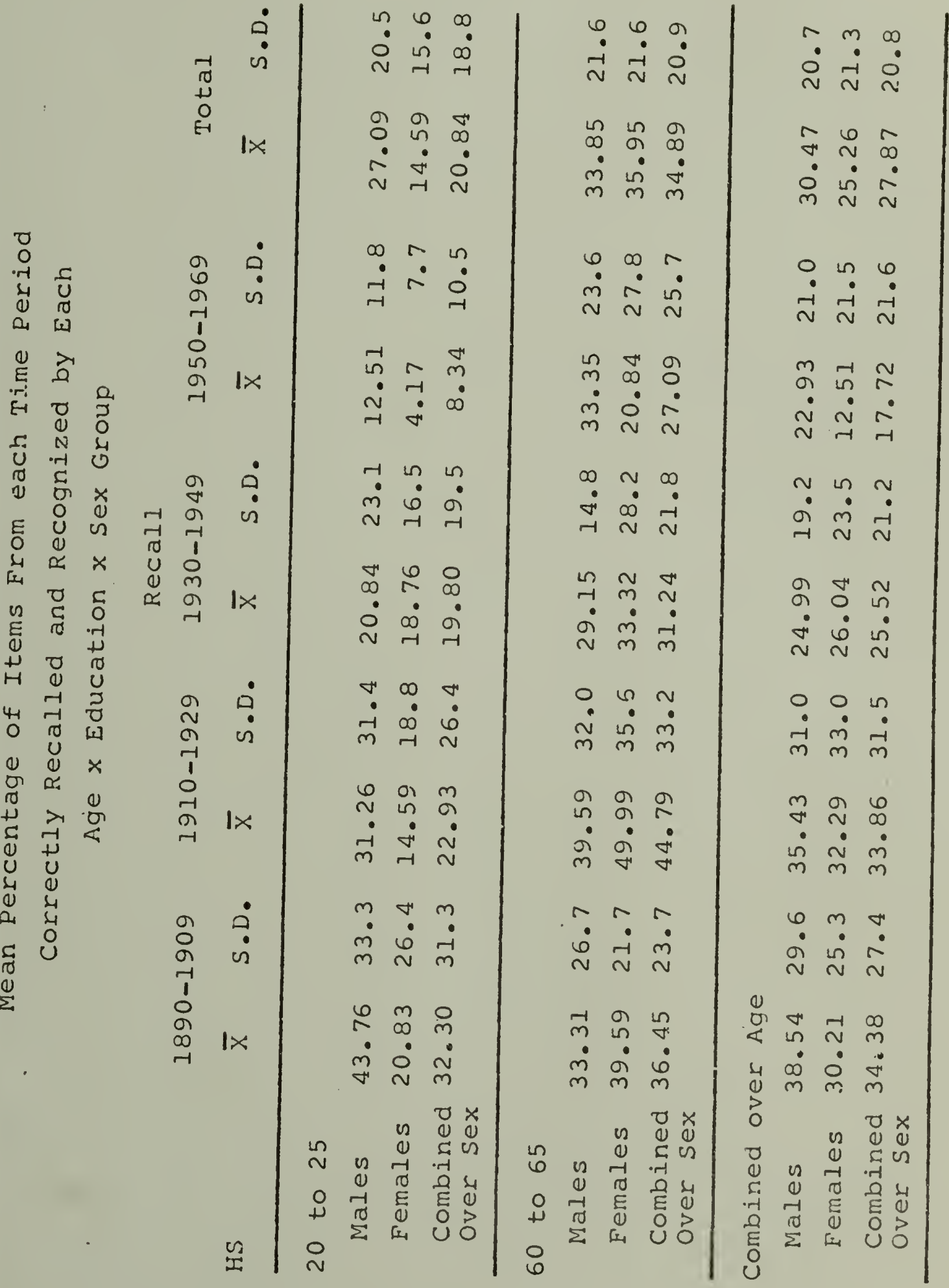


Table 9 Continued

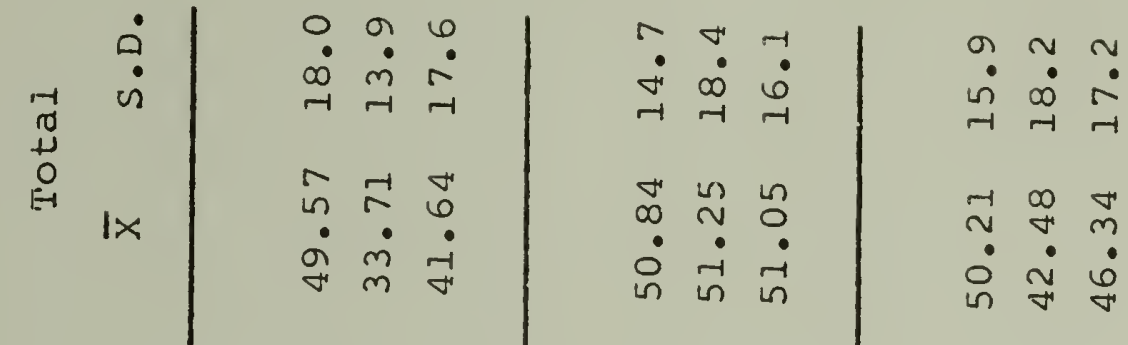

$$
\begin{aligned}
& \begin{array}{lllll}
a & \dot{0} & \infty & n & a \\
\sigma & \dot{1} & \dot{v} & \dot{v} & \dot{v}
\end{array} \\
& \begin{array}{ccc}
\stackrel{-1}{r} & -1 & 0 \\
-1 & \bullet & 0 \\
m & 0
\end{array} \\
& \begin{array}{lll}
0 & m & \infty \\
\infty & \stackrel{m}{v} & \stackrel{n}{v}
\end{array} \\
& \begin{array}{lll}
\because r & a \\
\sim & \dot{m} & m
\end{array} \\
& \begin{array}{lll}
m & 0 & 9 \\
\dot{1} & \ddots & ? \\
\dot{m} & \dot{0} & 0
\end{array} \\
& \stackrel{m}{\sim} \stackrel{\sim}{\sim} \\
& \dot{m} \dot{0} \dot{\circ} \\
& m \rightarrow \underset{\sim}{m}
\end{aligned}
$$

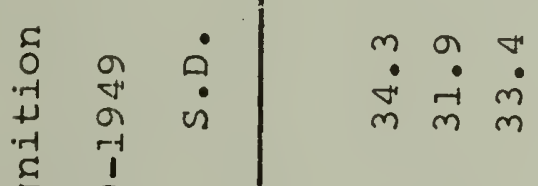

$$
\begin{aligned}
& \begin{array}{ccc}
m & n & \text { n } \\
\stackrel{\sim}{v} & \stackrel{-1}{m} & \stackrel{0}{v}
\end{array}
\end{aligned}
$$

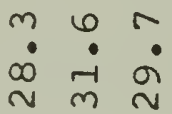

$$
\begin{aligned}
& \stackrel{n}{r} \stackrel{n}{n} \\
& \ddot{n} \dot{\sim} \ddot{\sim}
\end{aligned}
$$

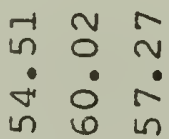

$$
\begin{aligned}
& \text { r-1 } 1009 \\
& \text { - } \\
& \text { a } N \text { in } \\
& \text { a } \\
& \begin{array}{lll}
\because & \infty & \sim \\
\dot{m} & \dot{n} & \dot{n}
\end{array}
\end{aligned}
$$

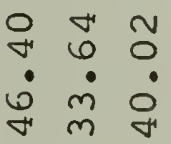

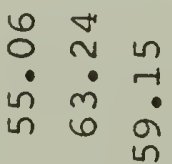

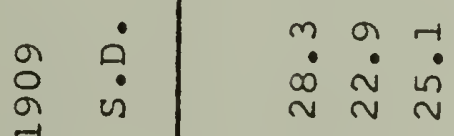

$$
\begin{aligned}
& \begin{array}{lll}
\infty & \leftrightarrow & 0 \\
\text { in } & \stackrel{v}{*} \\
\text { in } & \dot{\sigma} & \dot{n}
\end{array} \\
& \begin{array}{lll}
-1 & \sim \\
\sim & 0 & \dot{4} \\
\sim & \sim &
\end{array} \\
& \begin{array}{lll}
\sim & -1 & \infty \\
\dot{0} & \dot{0} & \dot{\omega} \\
\sim & \sim & \sim
\end{array} \\
& \begin{array}{ll}
r & 1 \\
0 & \\
0 & 1 \\
r & 1 x \\
r-1
\end{array} \\
& \begin{array}{ll}
1 & \\
1 & \\
0 & \\
0 & 1 x
\end{array}
\end{aligned}
$$

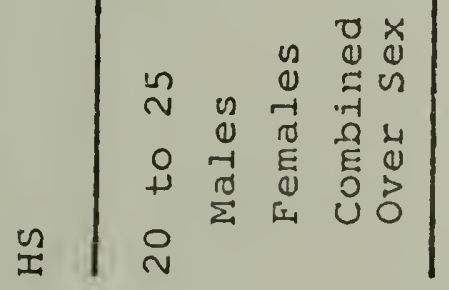

$$
\begin{aligned}
& \begin{array}{ccc}
0 & 0 & \infty \\
m & 0 & 9 \\
\infty & 1 & 0 \\
\text { in } & n & \text { in }
\end{array} \\
& \stackrel{n}{\forall} \infty \\
& \text { 요 } \stackrel{\infty}{\circ} \dot{\circ} \\
& \begin{array}{lll}
\sim & 0 & \sim \\
\sim & \sim & \sim \\
\sim & \sim & \sim
\end{array}
\end{aligned}
$$

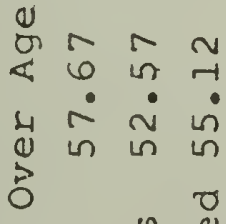

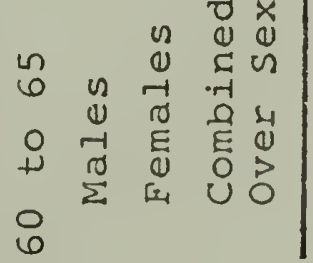


Table 9 Continued

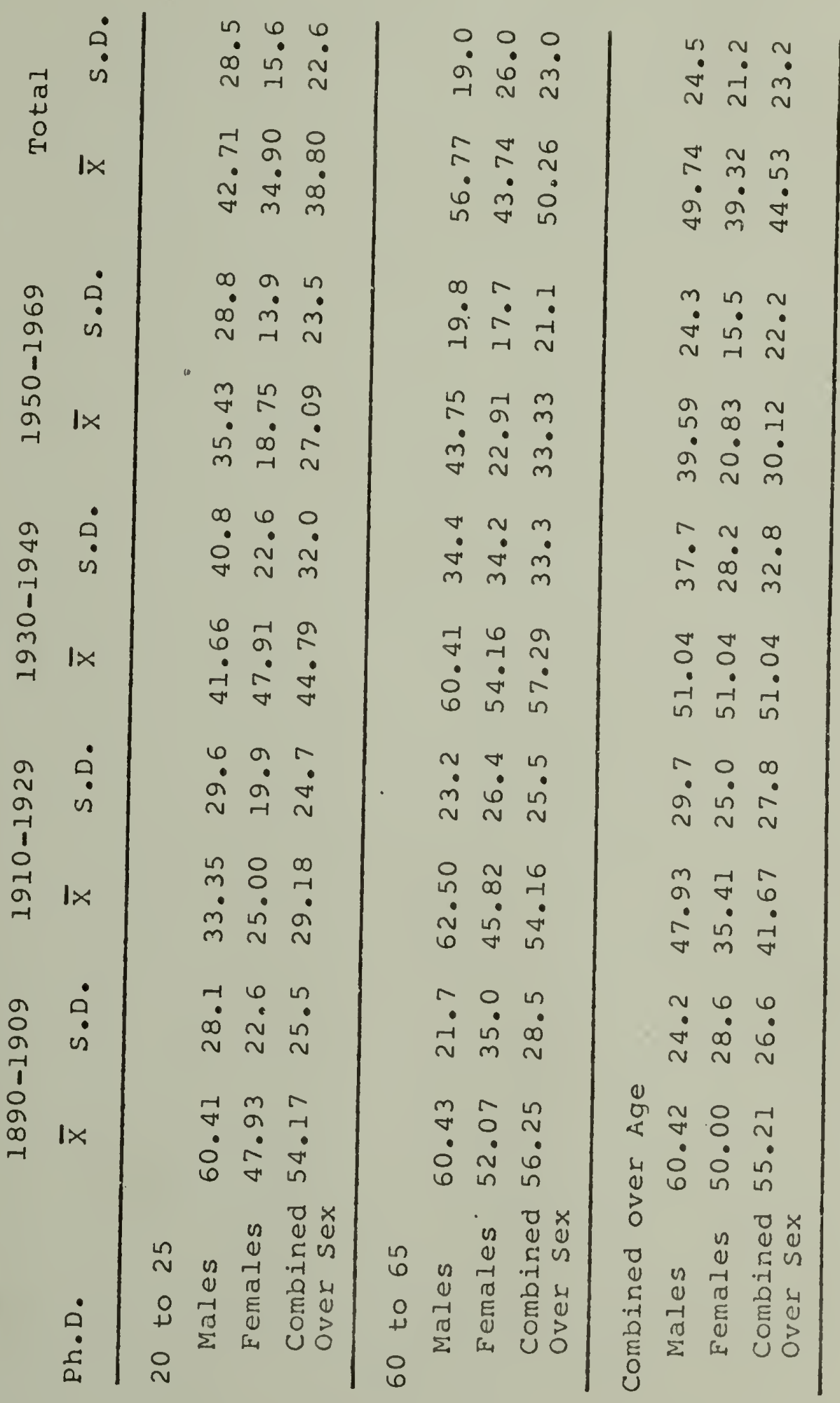


Table 9 Continued

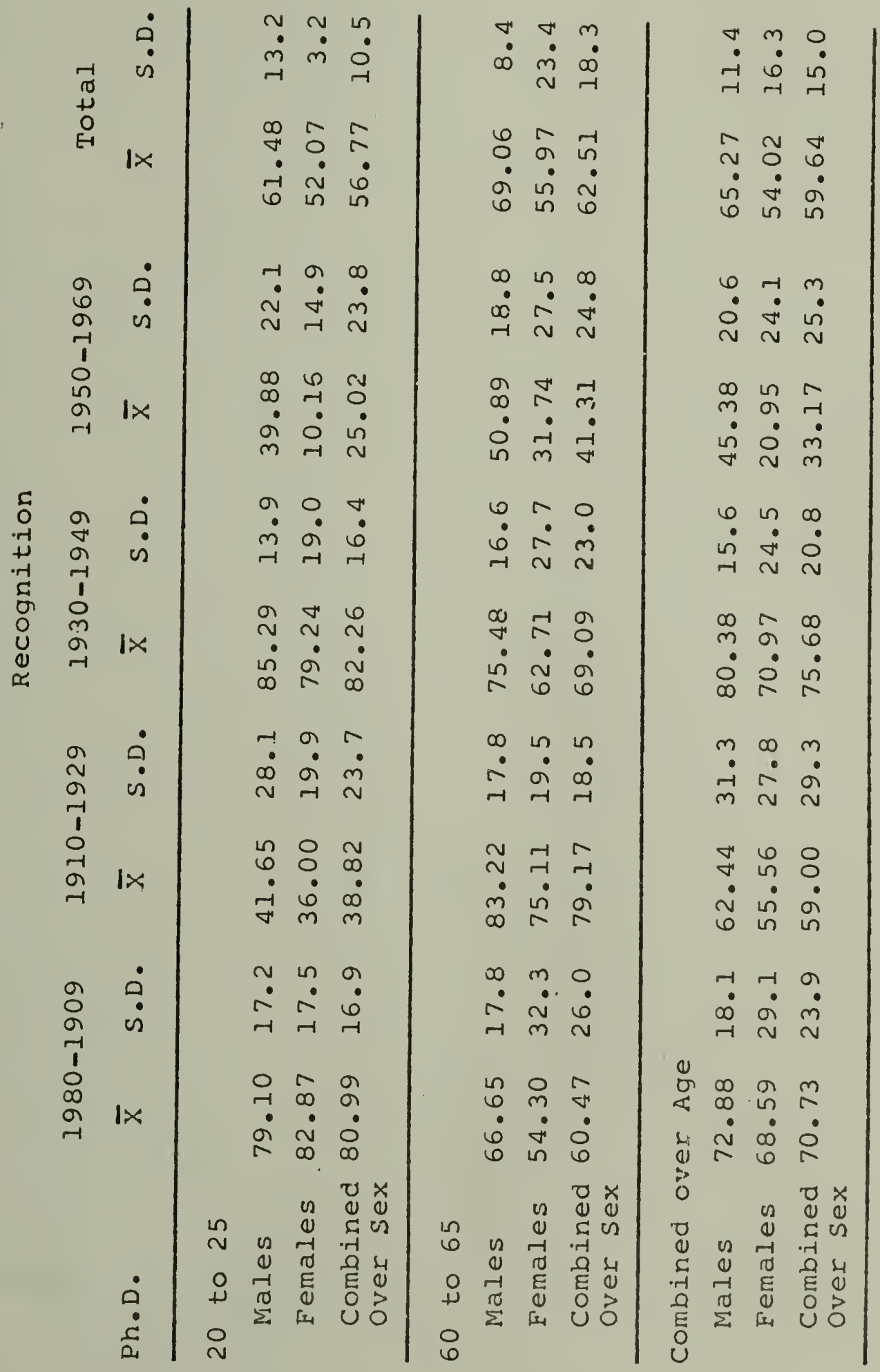


Table 9 Continued

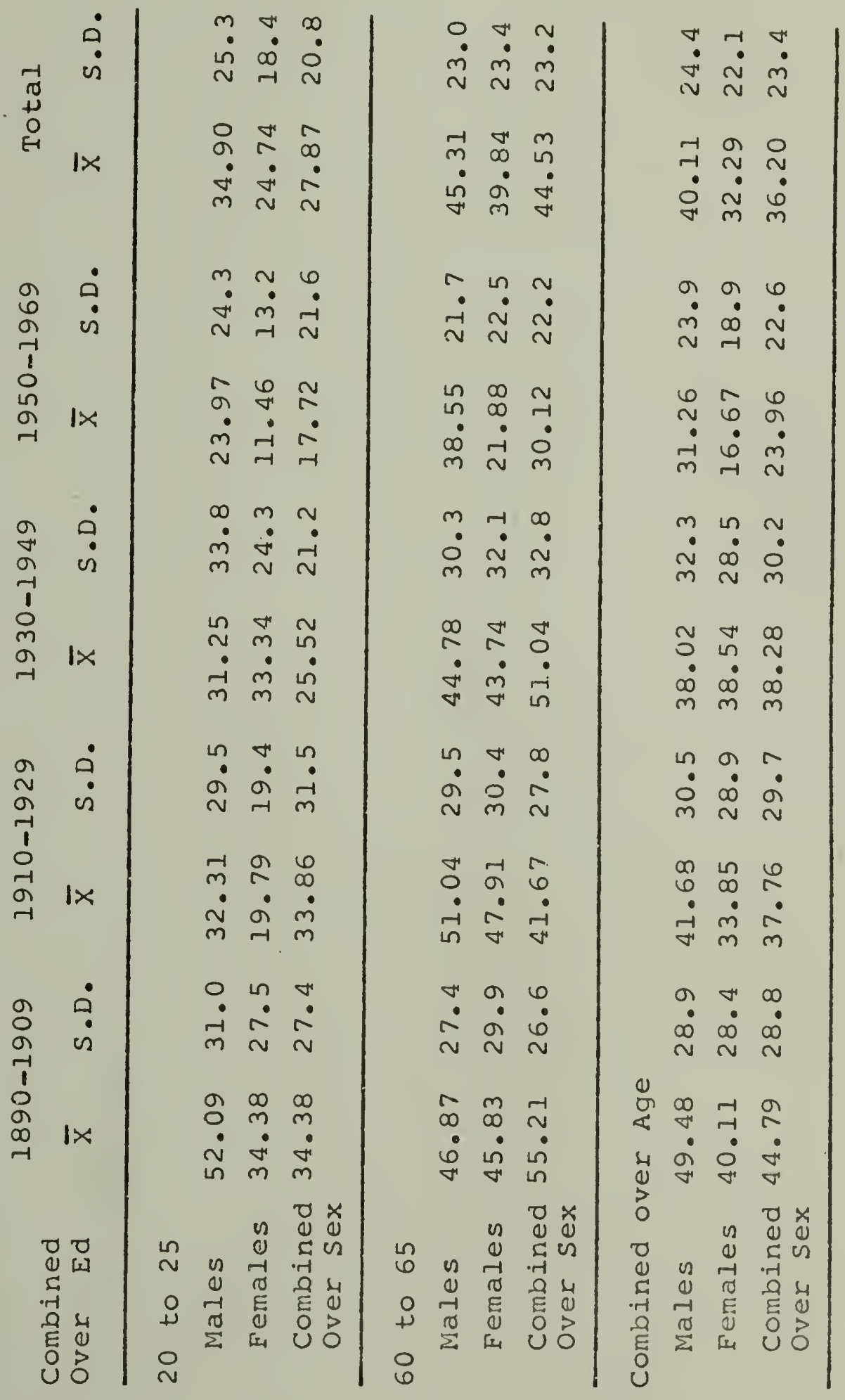


Table 9 Continued

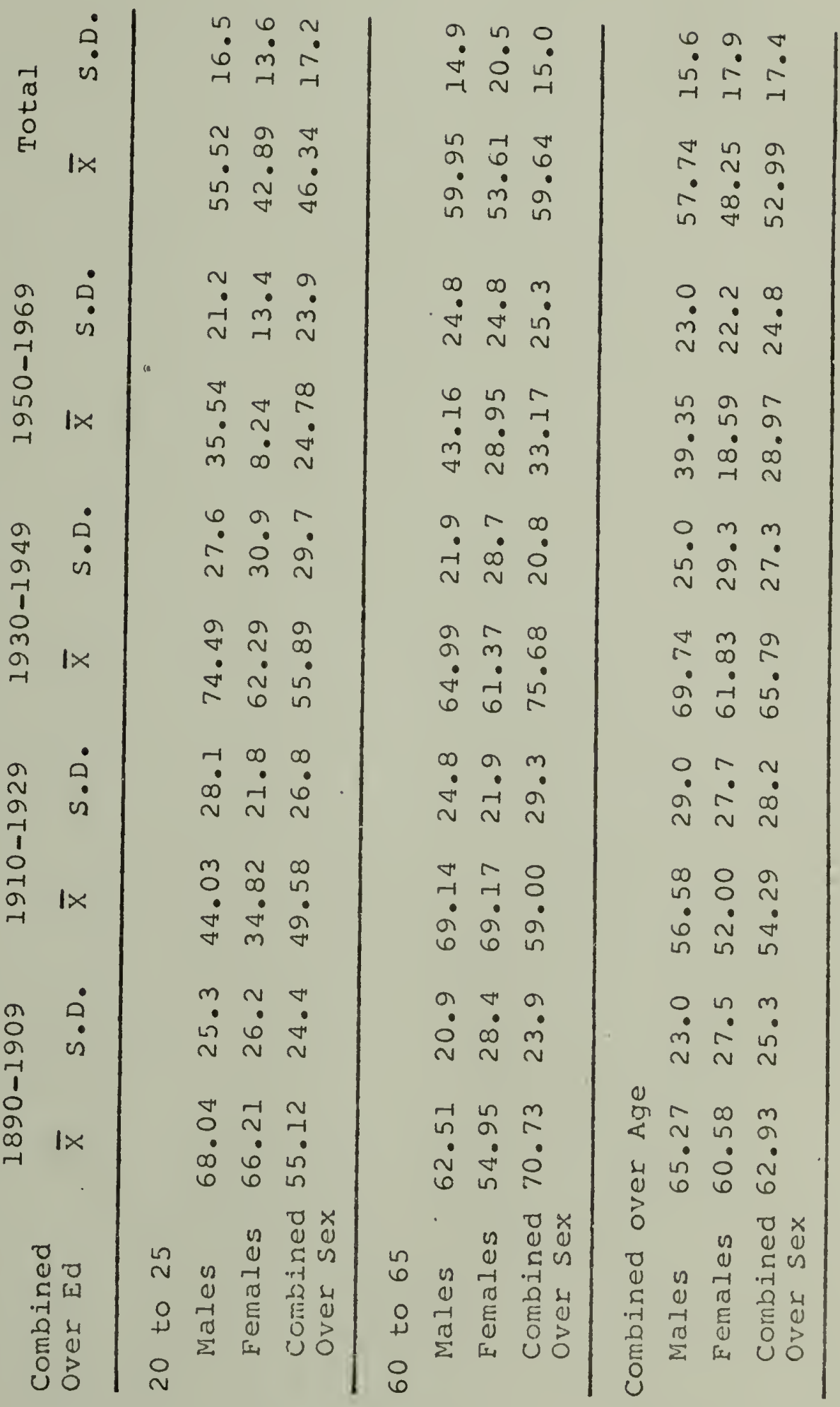


$37 \%)$, and the Education main effect was statistically significant $(\underline{F}(1,48)=12.62, \underline{p}<.001)$. Males made more correct responses than females ( $49 \%$ vs. $40 \%$ ), and the sex main effect was also statistically significant $(\underline{E}(1,48)=4.21, \underline{p}<.05)$. The order main effect, and all interactions of between subject variables were nonsignificant.

Recognition of the facts was considerably better than recall. ( $53 \%$ vs. $36 \%$ ), and the Memory main effect was highly significant $(\underline{F}(1,48)=58.82, \underline{p}<.001)$. The mean percentages of correct responses to questions pertaining to the respective time periods (1890-1909, $1910-1929,1930-1949,1950-1969)$ were $54 \%, 46 \%, 52 \%$, and $26 \%$. The Time Period main effect was statistically significant $(\underline{F}(3,144)=45.85, \underline{p}<.001)$, and simple effects tests indicated that performances on questions from the first and third time periods did not differ, but all other performances did (all ps $<.05)$. Statistically significant interactions were obtained between Time Deriod and Age $(\underline{F}(3,144)=11.89, \mathrm{p}<.01)$, Education $(\underline{F}(3,144)=3.17, \underline{p}<.05)$, sex $(\underline{F}(3,144)=2.78$, $\underline{p}<.05)$, Memory $(\underline{F}(3,144)=8.91, \underline{p}<.001)$, Memory $x$ Age $(\underline{F}(3,144)=3.16, \underline{p}<.05)$, and Memory $x$ Age $x$ Education $(\underline{F}(3,144)=3.01, \underline{D}<.05)$, but since no interesting patterns emerged, they will not be disclissed further. 


\section{b. Number of Responses in Recall}

The mean number of correct, incorrect, and total recall responses produced for questions from each time period by each Age $x$ Education $x$ Sex groups is shown in Table 10. These data were submitted to a 2 (Age) $\times 2$ (Education) $\times 2$ (Sex) $\times 2$ (Order) $\times 2$ (Response (Correct vs. Incorrect)) $\times 4$ (Time Pericd) analysis of variance.

Older subjects produced more responses than younger subjects ( 3.7 vs. 2.5), and the Age main effect was statistically significant $(\underline{F}(1,48)=9.50$, p (.01). Ph.D. educated subjects produced more responses than high school educated subjects $(3.5$ vs. 2.7), and the Education main effect was statistically significant $(\underline{F}(1,48)=4.06, \underline{p}<.05)$. Males produced more responses than females ( $3.3 \mathrm{vs} .2 .8)$, but the sex main effect was not statistically significant. The Order main effect was also not significant, and except for the Sex $x$ Order interaction $(\underline{F}(1,48)=6.20, \underline{p}<$ .05), no interactions of between subject variables were statistically significant.

Considerably more correct than incorrect responses were produced ( 2.2 vs. 0.9), and the Response main effect was highly significant $(\underline{F}(1,48)=59.96, \underline{p}<$ $.001)$. The Response $x$ Education interaction was also statistically significant $(\underline{F}(1,48)=12.33, \underline{p}(.001)$, 
Table 10

Mean Number of Correct and Incorrect Recall Responses For Each Time Period by Each Age $x$ Education $x$ Sex Group

\section{0-1909}

Correct

Incorrect

Total

HS

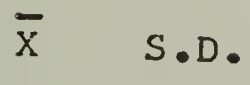

$\bar{X} \quad S . D$.

$\overline{\mathrm{X}} \quad \mathrm{S} \cdot \mathrm{D}$.

20 to 25

Males

Females

Combined

Over Sex

$\begin{array}{ll}2.63 & 2.00 \\ 1.25 & 1.58 \\ 1.94 & 1.88\end{array}$

$.75 \quad .89$

$3.38 \quad 2.62$

$.75 \quad .71$

2.00 1.93

.75 .78

2.692 .33

60 to 65

$\begin{array}{lllllll}\text { Males } & 2.00 & 1.60 & 1.00 & .76 & 3.00 & 1.77 \\ \text { Females } & 2.38 & 1.30 & 1.13 & .64 & 3.50 & 1.51 \\ \text { Combined } & 2.19 & 1.42 & 1.06 & .68 & 3.25 & 1.61 \\ \text { Over Sex } & & & & & \end{array}$

Combined over Age

Males

Females

Combined

Over Sex
2.311 .78

1. 811.52

2.061 .65
.88 .81

$.94 \quad .68$

.91 .73
3. 192.17

2. 751.84

2.971 .99

Ph.D.

20 to 25

Males

Females

Combined

Over Sex

.75

.25 .46

$.50 \quad .52$
4. $38 \quad 1.92$

3.131 .55

3.751 .81 
Table 10 Continued

1890-1909

\begin{tabular}{lrrrrrrr} 
& \multicolumn{2}{c}{ Correct } & \multicolumn{2}{c}{ Incorrect } & \multicolumn{2}{c}{ Total } \\
Ph.D. & $\bar{x}$ & S.D. & $\bar{x}$ & S.D. & $\bar{x}$ & S.D. \\
\hline $\begin{array}{l}60 \text { to } 65 \\
\quad \text { Males }\end{array}$ & 3.63 & 1.30 & 1.00 & 1.07 & 4.63 & .92 \\
$\begin{array}{l}\text { Females } \\
\text { Combined } \\
\text { Over Sex }\end{array}$ & 3.13 & 2.10 & .75 & .71 & 3.88 & 1.96 \\
& 3.38 & 1.71 & .88 & .89 & 4.25 & 1.53
\end{tabular}

Combined over Age

$\begin{array}{lllllll}\text { Males } & 3.63 & 1.46 & .88 & .81 & 4.50 & 1.46 \\ \text { Females } & 3.00 & 1 . \% 1 & .50 & .63 & 3.50 & 1.75 \\ \text { Combined } & 3.31 & 1.60 & .69 & .74 & 4.00 & 1.67 \\ \text { Over Sex } & & & & & & \end{array}$

Combined over Ed 20 to 25

$\begin{array}{lllllll}\text { Males } & 3.13 & 1.86 & .75 & .68 & 3.88 & 2.28 \\ \text { Females } & 2.06 & 1.65 & .50 & .63 & 2.56 & 1.79 \\ \text { Combined } & 2.59 & 1.81 & .63 & .66 & 3.22 & 2.12 \\ \text { Over Sex } & & & & & & \end{array}$

60 to 65

$\begin{array}{lrrrrrr}\text { Males } & 2.81 & 1.64 & 1.00 & .89 & 3.81 & 1.60 \\ \text { Females } & 2.75 & 1.73 & .94 & .68 & 3.69 & 1.70 \\ \text { Combined } & 2.78 & 1.66 & .97 & .78 & 3.75 & 1.63 \\ \text { Over Sex } & & & & & & \end{array}$

Combined over Age

$\begin{array}{lllllll}\text { Males } & 2.97 & 1.73 & .88 & .79 & 3.84 & 1.94 \\ \text { Females } & 2.41 & 1.70 & .72 & .68 & 3.13 & 1.81 \\ \text { Combined } & 2.27 & 1.78 & .80 & .74 & 3.48 & 1.89 \\ \text { Over Sex } & & & & & & \end{array}$


Table 10 Continued

$$
\text { 1910-1929 }
$$

Correct

Incorrect

Total

HS $\bar{X} \quad S . D$.

$\overline{\mathrm{X}} \quad \mathrm{S} . \mathrm{D}$. $\bar{X} \quad$ S.D.

20 to 25

Males

Females

Combined

over Sex

$$
\begin{array}{rr}
1.88 & 1.89 \\
.88 & 1.13 \\
1.38 & 1.59
\end{array}
$$

.631 .41

$2.50 \quad 2.51$

$.50 \quad .76$

$1.38 \quad 1.51$

$.56 \quad 1.09$

$1.94 \quad 2.08$

60 to 65

Males

Females

$2.38 \quad 1.92$

$1.13 \quad 1.64$

$3.50 \quad 1.93$

Combined

Over Sex

2.691 .99

$.75 \quad .46$

$3.75 \quad 2.19$

$.94 \quad 1.18$

3.132 .00

Combined over Age

Males

Females

2.131 .86

$.88 \quad 1.50$

$3.00 \quad 2.22$

Combined

Over Sex

1.941 .98

$.63 \quad .62$

$2.56 \quad 2.19$

$2.03 \quad 1.89$

$.75 \quad 1.14$

$2.78 \quad 2.18$

$\mathrm{Ph}$. D.

20 to 25

Males

Females

$2.00 \quad 1.77$

.50

.93

$2.50 \quad 1.51$

Combined

$1.50 \quad 1.20$

.88

$2.38 \quad 1.60$

Over Sex

$1.75 \quad 1.48$

.69

$2.44 \quad 1.50$

60 to 65

Males

Females

Combined

Over Sex

$\begin{array}{llllll}3.75 & 1.39 & 1.00 & .54 & 4.75 & 1.04 \\ 2.75 & 1.58 & 1.00 & .93 & 3.75 & 1.98 \\ 3.25 & 1.53 & 1.00 & .73 & 4.25 & 1.61\end{array}$


Table 10 Continued

$$
\text { 1910-1929 }
$$

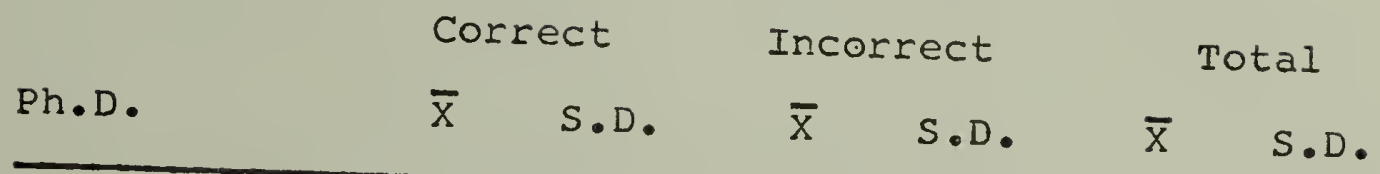

Combined over Age

$\begin{array}{lllllll}\text { Males } & 2.88 & 1.78 & .75 & .78 & 3.63 & 1.71 \\ \text { Females } & 2.13 & 1.50 & .94 & .85 & 3.06 & 1.88 \\ \text { Combined } & 2.50 & 1.67 & .84 & .81 & 2.34 & 1.79 \\ \text { Over Sex } & & & & & & \end{array}$

Combined over Ed 20 to 25

$\begin{array}{lrrrrrr}\text { Males } & 1.94 & 1.77 & .56 & 1.15 & 2.50 & 2.00 \\ \text { Females } & 1.19 & 1.17 & .69 & .79 & 1.88 & 1.59 \\ \text { Combined } & 1.56 & 1.52 & .63 & .98 & 2.19 & 1.80 \\ \text { Over Sex } & & & & & & \end{array}$

60 to 65

$\begin{array}{lrrrrrr}\text { Males } & 3.06 & 1.77 & 1.06 & 1.18 & 4.13 & 1.63 \\ \text { Females } & 2.88 & 1.82 & .88 & .72 & 3.75 & 2.02 \\ \text { Combined } & 2.97 & 1.77 & .97 & .97 & 3.94 & 1.81 \\ \text { Over Sex } & & & & & & \end{array}$

Combined over Age

$\begin{array}{lrrrrrrr}\text { Males } & 2.50 & 1.83 & .81 & 1.3 .8 & 3.31 & 1.98 \\ \text { Females } & 2.03 & 1.73 & .78 & .75 & 2.81 & 2.02 \\ \text { Combined } & 1.89 & 2.69 & .80 & .98 & 3.06 & 2.00 \\ \text { Over Sex } & & & & & & \end{array}$


Table 10 Continued

$$
1930-1949
$$

Correct

$\bar{X} \quad S . D$.
Incorrect

$\bar{X} \quad S . D$.
Total

$\overline{\mathrm{X}} \quad \mathrm{S} . \mathrm{D}$.

20 to 25

Males

Females

1.251 .39

Combined

Over Sex

$1.25 \quad .99$

1.191 .17

$.50 \quad 1.41$

$1.75 \quad 2.19$

$.13 \quad .35$

$1.25 \quad 1.04$

$.31 \quad 1.01$

$1.50 \quad 1.67$

60 to 65

Males

Females

Combined

Over Sex

$\begin{array}{lr}1.75 & .89 \\ 2.00 & 1.69 \\ 1.88 & 1.31\end{array}$

.75

.71

$2.50 \quad 1.41$

$.63 \quad .74$

2.632 .13

$.69 \quad .70$

$2.56 \quad 1.75$

Combined over Age

Males

Females

Combined

Over Sex
1.501 .16

$1.56 \quad 1.41$

$1.53 \quad 1.27$
$.63 \quad 1.09$

.38 .62

.50

.88

$2.13 \quad 1.82$

$1.94 \quad 1.77$

$2.03 \quad 1.77$

Ph.D.

20 to 25

$\begin{array}{lllllll}\text { Males } & 2.50 & 2.45 & .13 & .35 & 2.63 & 2.39 \\ \text { Females } & 2.88 & 1.36 & .13 & .35 & 3.00 & 1.41 \\ \text { Combined } & 2.69 & 1.92 & .13 & .34 & 2.81 & 1.91 \\ \text { Over Sex } & & & & & & \end{array}$

60 to 65

Males

Females

Combined

Over Sex
$3.63 \quad 2.07$

$3.25 \quad 2.05$

$3.44 \quad 2.00$

1.13

.38

.84

$4.75 \quad 1.49$

$.75 \quad .86$

$3.63 \quad 2.00$

4.191 .80 
Table 10 Continued

1930-1949

\begin{tabular}{lccccccc} 
& \multicolumn{3}{c}{ Correct } & \multicolumn{2}{c}{ Incorrect } & \multicolumn{2}{c}{ Total } \\
Ph.D. & $\overline{\mathrm{x}}$ & S.D. & $\overline{\mathrm{x}}$ & S.D. & $\overline{\mathrm{x}}$ & S.D. \\
\hline $\begin{array}{l}\text { Combined over } \\
\text { Males }\end{array}$ & 3.06 & 2.27 & .63 & .81 & 3.69 & 2.21 \\
$\begin{array}{l}\text { Females } \\
\begin{array}{l}\text { Combined } \\
\text { Over Sex }\end{array}\end{array}$ & 3.06 & 1.69 & .25 & .58 & 3.31 & 1.70 \\
\hline
\end{tabular}

Combined over Ed 20 to 25

$\begin{array}{lrrrrrr}\text { Males } & 1.88 & 2.03 & .31 & 1.01 & 2.19 & 2.26 \\ \text { Females } & 2.00 & 1.46 & .13 & .34 & 2.13 & 1.50 \\ \text { Combined } & 1.94 & 1.74 & .22 & .75 & 2.16 & 1.89 \\ \text { Over Sex } & & & & & & \end{array}$

60 to 65

$\begin{array}{lllllll}\text { Males } & 2.69 & 1.82 & .94 & .77 & 3.63 & 1.82 \\ \text { Females } & 2.63 & 1.93 & .50 & .73 & 3.13 & 2.06 \\ \text { Combined } & 2.66 & 1.84 & .72 & .77 & 3.38 & 1.93 \\ \text { Over Sex } & & & & & & \end{array}$

Combined over Age

$\begin{array}{lllllll}\text { Males } & 2.28 & 1.94 & .63 & .94 & 2.91 & 2.15 \\ \text { Females } & 2.31 & 1.71 & .31 & .59 & 2.63 & 1.85 \\ \text { Combined } & 2.30 & 1.81 & .47 & .80 & 2.77 & 2.00 \\ \text { Over Sex } & & & & & & \end{array}$


Table 10 Continued

$$
\text { 1950-1969 }
$$

\begin{tabular}{llllllll} 
& \multicolumn{2}{c}{ Correct } & \multicolumn{2}{c}{ Incorrect } & \multicolumn{2}{c}{ Total } \\
HS & $\bar{x}$ & S.D. & $\bar{x}$ & S.D. & $\bar{x}$ & S.D. \\
\hline $\begin{array}{l}20 \text { to } 25 \\
\text { Males }\end{array}$ & .75 & .71 & 1.63 & 2.00 & 2.38 & 2.56 \\
Females & .25 & .46 & 1.00 & 1.41 & 1.25 & 1.83 \\
$\begin{array}{l}\text { Combined } \\
\text { Over Sex }\end{array}$ & .50 & .63 & 1.31 & 1.70 & 1.81 & 2.23 \\
\hline
\end{tabular}

60 to 65

Males

Females

Combined

Over Sex

$\begin{array}{llllll}2.00 & 1.41 & 1.63 & 1.06 & 3.63 & 1.60 \\ 1.25 & 1.67 & 3.00 & 1.69 & 4.25 & 1.98 \\ 1.63 & 1.54 & 2.31 & 1.54 & 3.94 & 1.77\end{array}$

Combined over Age

$\begin{array}{lrlllll}\text { Males } & 1.38 & 1.26 & 1.63 & 1.54 & 3.00 & 2.16 \\ \text { Females } & .75 & 1.29 & 2.00 & 1.83 & 2.75 & 2.41 \\ \text { Combined } & 1.06 & 1.29 & 1.81 & 1.67 & 2.88 & 2.25 \\ \text { Over Sex } & & & & & & \end{array}$

Ph.D.

20 to 25

Males

Females

$2.13 \quad 1.73$

$.88 \quad .84$

$3.00 \quad 2.07$

Combined

Over Sex

$1.13 \quad .84$

$1.25 \quad 1.17$

$2.38 \quad 1.41$

1.631 .41

1.061 .00

$2.69 \quad 1.74$

60 to 65

$\begin{array}{lrrrrrr}\text { Males } & 2.63 & 1.19 & 1.13 & .35 & 3.75 & 1.28 \\ \text { Females } & 1.38 & 1.06 & 1.50 & 1.31 & 2.88 & 2.17 \\ \text { Combined } & 2.00 & 1.27 & 1.31 & .95 & 3.13 & 1.78 \\ \text { Over Sex } & & & & & & \end{array}$


Table 10 Continued

$1950-1969$

Correct

Incorrect

Total

\begin{tabular}{llllll}
$\bar{x}$ & S.D.D. & $\bar{x}$ & S.D. & $\bar{x}$ & S.D. \\
\hline
\end{tabular}

Combined over Age

Males

Females

2.38

Combined

Over Sex

$1.25 \quad .93$

1.00

.63

$3.38 \quad 1.71$

$1.81 \quad 1.33$

$1.38 \quad 1.20$

$2.63 \quad 1.78$

1.19

.97

3.001 .76

Combined over Ed

20 to 25

Males

Females

$1.44 \quad 1.46$

$1.25 \quad 1.53$

$2 . .9 \quad 2.27$

Combined

Over Sex

$.69 \quad .79$

$1.13 \quad 1.26$

$1.81 \quad 1.68$

$1.06 \quad 1.22$

$1.19 \quad 1.38$

$2.50 \quad 2.02$

60 to 65

Males

Females

Combined

Over Sex

$2.31 \quad 1.30$

$1.38 \quad .81$

$2.69 \quad 1.40$

$1.31 \quad 1.35$

$2.25 \quad 1.65$

$3.56 \quad 2.13$

$1.81 \quad 1.40$

1.811 .36

$3.63 \quad 1.77$

Combined over Age

Males

Females

Combined

Over Sex

\subsection{8}

1.00

1.4
1.43

$1.31 \quad 1.20$

1.691 .55

$1.50 \quad 1.39$

$3.19 \quad 1.93$

$2.69 \quad 2.09$

2.942 .00 
Table 10 Continued

Total

\begin{tabular}{lrrrrrrr} 
& \multicolumn{2}{c}{ Correct } & \multicolumn{2}{c}{ Incorrect } & \multicolumn{2}{c}{ Total } \\
HS & $\bar{x}$ & S.D. & $\bar{x}$ & S.D. & $\bar{x}$ & S.D. \\
\hline $\begin{array}{l}\text { 20 } 25 \\
\text { Males }\end{array}$ & 6.50 & 4.93 & 3.50 & 5.26 & 10.00 & 8.96 \\
Females & 3.50 & 3.74 & 2.38 & 2.50 & 5.88 & 5.79 \\
$\begin{array}{l}\text { Combined } \\
\text { Over Sex }\end{array}$ & 5.00 & 4.50 & 2.94 & 4.02 & 7.94 & 7.59
\end{tabular}

60 to 65

Males

Females

8.135 .19

$4.50 \quad 3.38$

$12.63 \quad 6.05$

Combined

Over Sex

8.635 .18

$5.50 \quad 2.45$

14.136 .66

$8.38 \quad 5.02$

$5.00 \quad 2.90$

13.386 .20

Combined over Age

$\begin{array}{lllllll}\text { Males } & 7.31 & 4.96 & 4.00 & 4.31 & 11.31 & 7.51 \\ \text { Females } & 6.06 & 5.12 & 3.94 & 2.59 & 10.00 & 7.39 \\ \text { Combined } & 6.69 & 4.99 & 3.97 & 3.61 & 10.66 & 7.36 \\ \text { Over Sex } & & & & & & \end{array}$

$\mathrm{Ph} \cdot \mathrm{D}$.

20 to 25

$\begin{array}{lrlllll}\text { Males } & 10.25 & 6.84 & 2.25 & 1.67 & 12.50 & 7.21 \\ \text { Females } & 8.38 & 3.74 & 2.50 & 2.27 & 10.88 & 5.14 \\ \text { Combined } & 9.31 & 5.41 & 2.38 & 1.93 & 11.69 & 6.11 \\ \text { Over Sex } & & & & & & \end{array}$

60 to 65

$\begin{array}{lllllll}\text { Males } & 13.63 & 4.57 & 4.25 & 1.91 & 17.88 & 3.44 \\ \text { Females } & 10.50 & 6.23 & 3.63 & 2.62 & 14.13 & 7.59 \\ \text { Combined } & 12.06 & 5.52 & 3.94 & 2.24 & 16.00 & 6.01 \\ \text { Over Sex } & & & & & & \end{array}$


Table 10 Continued

Total

Correct Incorrect

Total

$\mathrm{Ph} \cdot \mathrm{D}$. $\overline{\mathrm{X}} \quad \mathrm{S} \cdot \mathrm{D}$.

$S \cdot D$. $\overline{\mathrm{X}} \quad \mathrm{S} . \mathrm{D}$.

Combined over Age

$\begin{array}{lrrrrrr}\text { Males } & 11.94 & 5.88 & 3.25 & 2.02 & 15.19 & 6.12 \\ \text { Females } & 9.44 & 5.09 & 3.06 & 2.44 & 12.50 & 6.48 \\ \text { Combined } & 10.69 & 5.56 & 3.16 & 2.20 & 13.84 & 6.35 \\ \text { Over Sex } & & & & & & \end{array}$

Combined over Ed 20 to 25

Males

Females

Combined

Over Sex

$\begin{array}{rrrrrr}8.38 & 6.08 & 2.88 & 3.83 & 11.25 & 7.96 \\ 5.94 & 4.40 & 2.44 & 2.31 & 8.38 & 5.89 \\ 7.16 & 5.37 & 2.66 & 3.12 & 9.81 & 7.04\end{array}$

60 to 65

Males

Females

$10.88 \quad 5.51$

$4.38 \quad 2.66$

$15.25 \quad 5.47$

Combined

Over Sex

$9.56 \quad 5.62$

$4.56 \quad 2.63$

14.136 .90

$10.22 \quad 5.52$

4.47

14.096 .15

Combined over Age

$\begin{array}{lllllll}\text { Males } & 9.63 & 5.85 & 3.63 & 3.33 & 13.25 & 7.02 \\ \text { Females } & 7.50 & 5.30 & 3.50 & 2.66 & 11.25 & 6.95 \\ \text { Combined } & 8.69 & 5.61 & 3.56 & 2.99 & 12.25 & 7.00 \\ \text { Over Sex } & & & & & & \end{array}$


and may be seen in Figure 16, which shows the mean number of correct and incorrect recall responses to general information questions by each Education group. While Ph.D. subjects produced significantly more correct responses than HS subjects $(\underline{t}(62)=3.03, \underline{p}<$ .01), the number of incorrect responses did not vary significantly as a function of Education.

The mean number of responses to questions pertaining to the four time periods were $3.5,3.1,2.8$, and 2.9 , respectively. The Time Period main effect was statistically significant $(\underline{q}(3,144)=6.01, \underline{\mathrm{p}}<$ .001), and simple effects tests indicated that the number of responses produced to questions referring to 1890-1909 was significantly greater than to questions about any other time period (all $\mathrm{p}<.01$ ), but the number of responses to questions about all other time periods was not significantly different. Time Period interacted significantly with Age $(\underline{F}(3,144)=$ $5.40, \mathrm{p}<.001)$, Education $(\underline{\mathrm{F}}(3,144)=4.16, \underline{\mathrm{p}}<.001)$, Age $\times$ Education $(\underline{F}(3,144)=2.73, \underline{p}<.05)$, Response $(\underline{F}(3,144)=22.96, \underline{p}<.001)$, and Response $x$ Sex $(\underline{F}(3,144)=2.91, \underline{p}<.05)$, however, since these were largely uninterpretable, they will not be discussed further.

c. Percent Correct in Recognition 
Figure 16. Mean Number of Correct and Incorrect Resporses to General Information Questions by Each Education Group

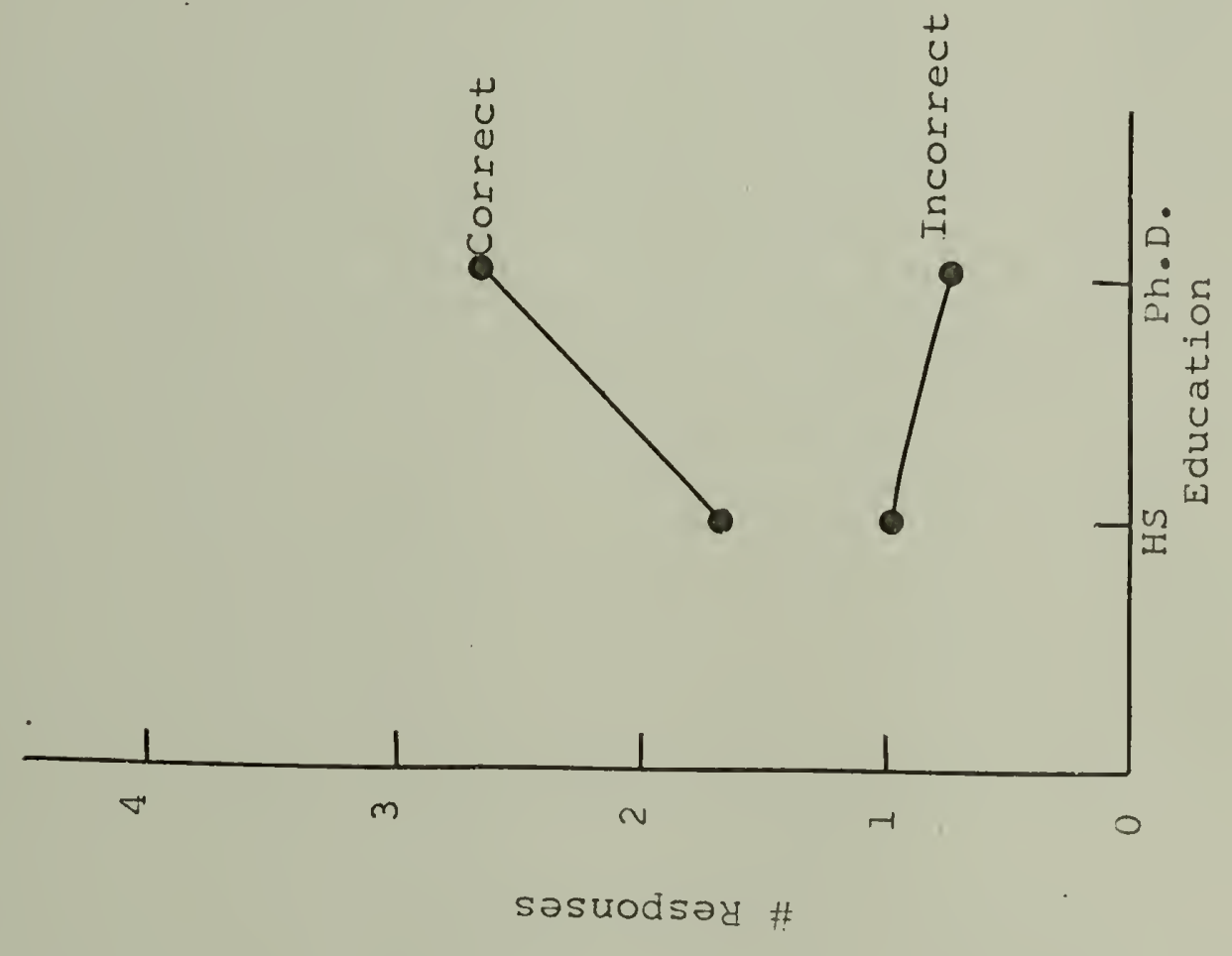


from each time period correctly recognized by each Age $x$ Education $x$ sex group is shown in Table 11 . These data were submitted to a 2 (Age) $\times 2$ (Education) $\times 2$ (Sex) $\times 2$ (Order) $\times 2$ (Item (True vs. False)) $x$ 4 (Time Period) analysis of variance. Older subjects recognized more items correctly than younger subjects ( $78 \%$ vs. $73 \%$ ), and the Age main effect was statistically significant $(\underline{F}(1,48)=9.34$, P (.05). Ph.D. educated subjects recognized more items correctly than HS educated subjects $(79 \%$ vs. $72 \%)$, and the Education main effect was statistically significant $(\underline{F}(1,48)=9.34, \underline{p}<.01)$. Males recognized more items correctly than females (78\% vs. $73 \%$ ), and the sex main effect was statistically significant $(\underline{F}(1,48)=5.30, \underline{p}<.05)$. The order main effect, and all interactions of between subject variables were nonsignificant.

More correct responses were produced to false items than true items ( $78 \%$ vs. $73 \%$ ), and the Item main effect was statistically significant $(\underline{F}(1,48)=4.39$, $\mathrm{p}<.05)$. The mean percentages of correct responses to items from each time period was $81 \%, 76 \%, 83 \%$, and $61 \%$, respectively. The Time Period main effect was statistically significant $(\underline{F}(3,144)=40.23, p<.001)$, and simple effects tests indicated that recognition was different for items from each time period except 


\section{Table 11}

Mean Percentage of True and False Items from Each Time Period Correctly Recognized by Each Age $x$ Education $x$ Sex Group

$$
\text { 1890-1909 }
$$

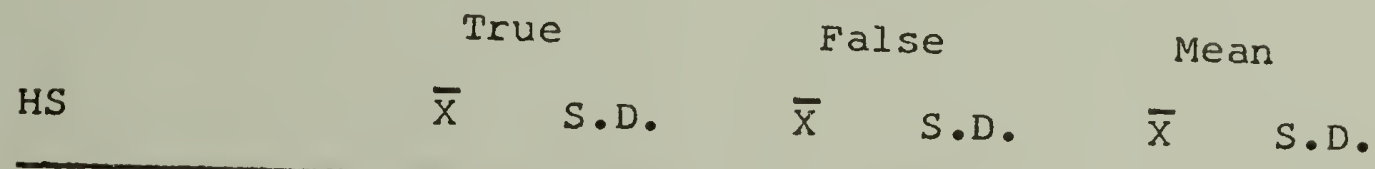

20 to 25

Males

Females

Combined Over Sex

$$
\begin{array}{llllll}
80.81 & 22.5 & 72.00 & 19.3 & 76.41 & 19.2 \\
79.15 & 17.2 & 70.37 & 20.8 & 74.76 & 11.4 \\
79.98 & 19.4 & 71.19 & 19.4 & 75.59 & 15.3
\end{array}
$$

60 to 65

Males

Females

Combined Over Sex

$$
\begin{array}{llllll}
85 . .0 & 16.5 & 72.67 & 15.7 & 79.19 & 12.1 \\
73.06 & 17.8 & 82.55 & 13.4 & 77.81 & 13.1 \\
79.38 & 17.8 & 77.61 & 15.0 & 78.50 & 12.2
\end{array}
$$

Combined over Age

Males

Females

Combined

Over Sex

$$
83,26
$$

19.2

$$
72.34 \quad 17.0
$$$$
77.80
$$

15.6

$$
76.11 \quad 17.2
$$$$
76.46
$$

18.1

$76.08 \quad 12.0$ $79.08 \quad 18.3$ $74.40,17.4$ $77.04 \quad 13.7$

Ph. D.

20 to 25

Males

Females

Combined Over Sex

88. 32.12 .6

95.82

7.7

89.58

8.6 88.31

9.7

94.6

10.6

91.48

8.8

$85.82 \quad 11.2$

95.23

9.090 .53

8.5 
Table 11 Continued

$$
\text { 1890-1909 }
$$

True

False

Mean

Ph.D.

$\overline{\mathrm{X}} \quad$ S.D. $\quad \overline{\mathrm{X}} \quad$ S.D. $\quad \overline{\mathrm{X}} \quad$ S.D.

60 to 65

Males

Females

$85.39 \quad 13.9$

$81.24 \quad 10.7$

83.31

8.9

Combined

Over Sex

$\begin{array}{lllll}79.80 & 20.8 & 72.37 & 21.9\end{array}$

$76.09 \quad 18.2$

$\begin{array}{llllll}82.59 & 17.3 & 76.81 & 17.3 & 79.70 & 14.4\end{array}$

Combined over Age

$\begin{array}{lrllllr}\text { Males } & 84.36 & 12.8 & 88.53 & 11.7 & 86.44 & 9.1 \\ \text { Females } & 84.06 & 16.3 & 83.51 & 20.2 & 83.78 & 16.0 \\ \text { Combined } & 84.21 & 14.4 & 86.02 & 16.5 & 85.11 & 12.8 \\ \text { Over Sex } & & & & & & \end{array}$

Combined over Ed

20 to 25

$\begin{array}{lllllll}\text { Males } & 82.07 & 17.7 & 83.92 & 18.8 & 82.99 & 15.9 \\ \text { Females } & 83.73 & 14.3 & 82.51 & 20.3 & 83.12 & 13.1 \\ \text { Combined } & 82.90 & 15.8 & 83.21 & 19.2 & 83.06 & 14.3 \\ \text { Over Sex } & & & & & & \end{array}$

60 to 65

$\begin{array}{lllllll}\text { Males } & 85.54 & 14.7 & 76.96 & 13.7 & 81.25 & 10.5 \\ \text { Females } & 76.43 & 19.0 & 77.46 & 18.3 & 76.95 & 15.4 \\ \begin{array}{l}\text { Combined } \\ \text { Over Sex }\end{array} & 80.99 & 17.3 & 77.21 & 15.9 & 79.10 & 13.1\end{array}$

Combined over Age

$\begin{array}{lllllll}\text { Males } & 83.81 & 16.1 & 80.44 & 16.5 & 82.12 & 13.3 \\ \text { Females } & 80.08 & 17.0 & 79.98 & 19.2 & 80.03 & 14.4 \\ \begin{array}{l}\text { Combined } \\ \text { Over Sex }\end{array} & 81.94 & 16.5 & 80.21 & 17.8 & 81.09 & 13.8\end{array}$


Table 11 Continued

$$
1910-1929
$$

True

False

Mean

HS

$\bar{X} \quad S \cdot D$.

$\bar{X} \quad$ S.D.

$\bar{x} \quad S . D$.

20 to 25

Males

Females

Combined

Over Sex

$\begin{array}{llllll}65.18 & 22.8 & 77.09 & 17.7 & 71.13 & 19.2 \\ 50.02 & 17.8 & 83.62 & 12.6 & 66.83 & 12.5 \\ 57.60 & 21.2 & 80.36 & 15.2 & 68.98 & 15.8\end{array}$

60 to 65

Males

Females

$71.74 \quad 20.5$

$83.32 \quad 12.6$

$77.53 \quad 11.7$

Combined

Over Sex

$79.32 \quad 19.5$

83.9121 .9

$81.62 \quad 11.9$

75.5319 .7

$83.62 \quad 17.2$

$79.57 \quad 11.6$

Combined over Age

Males

Females

Combined

Over Sex

\subsection{6} 64.68

66.51
21.2 23.5 .

80.21

83.77

15.2

74.33

74.22

81.9916 .1

74.38

22.1

15.7

Ph.D.

20 to 25

Males

Females

68.7

58.31

20.8

70.84

24.8

$69.79 \quad 16.0$

Combined

Over Sex

$63.52 \quad 20.4$

77.66

14.9

67.99

9.9

74.25

20.1

$68.99 \quad 12.9$

60 to 65

Males

Females

Combined Over sex

87.49

89.84

11.7

95.84

11.8

91.66

8.9

88.16

12.2

85.29

16.6

87.56

9.8

$88.16 \quad 11.6$ 14.9 89.619 .3 
Table 11 Continued

$$
\text { 1910-1929 }
$$

True

False

Mean

Ph.D. $\quad \bar{x} \quad$ S.D. $\quad \bar{X} \quad$ S.D. $\quad \bar{x} \quad$ S.D.

Combined over Age

$\begin{array}{lllllll}\text { Males } & 78.11 & 18.9 & 83.34 & 22.8 & 80.73 & 16.9 \\ \text { Females } & 74.08 & 22.8 & 81.48 & 15.7 & 77.78 & 13.9 \\ \text { Combined } & 76.09 & 20.7 & 82.41 & 13.3 & 79.25 & 15.3 \\ \text { Over Sex } & & & & & & \end{array}$

Combined over Ed 20 to 25

$\begin{array}{lllllll}\text { Males } & 66.96 & 21.1 & 73.96 & 21.0 & 70.46 & 17.1 \\ \text { Females } & 54.17 & 18.7 & 80.64 & 13.7 & 67.41 & 10.9 \\ \text { Combined } & 60.56 & 20.7 & 77.30 & 17.8 & 68.93 & 14.2 \\ \text { Over Sex } & & & & & & \end{array}$

60 to 65

Males

Females

Combined Over Sex

$\begin{array}{llllll}79.61 & 18.1 & 89.58 & 13.4 & 84.60 & 12.4 \\ 84.58 & 16.6 & 84.60 & 18.8 & 84.59 & 1.1 .0 \\ 82.10 & 17.3 & 87.09 & 16.3 & 84.59 & 11.5\end{array}$

Combined over Age

$\begin{array}{lllllll}\text { Males } & 73.28 & 20.4 & 81.77 & 19.1 & 77.53 & 16.3 \\ \text { Females } & 69.38 & 23.3 & 82.62 & 16.3 & 76.00 & 13.9 \\ \text { Combined } & 71.33 & 21.8 & 82.20 & 17.6 & 76.76 & 15.0 \\ \text { Over Sex } & & & & & & \end{array}$


Table Il Continued

$1930-1949$

\begin{tabular}{lccccccc} 
& \multicolumn{2}{c}{ True } & \multicolumn{2}{c}{ False } & \multicolumn{2}{c}{ Mean } \\
HS & $\overline{\mathrm{x}}$ & S.D. & $\overline{\mathrm{x}}$ & S.D. & $\overline{\mathrm{x}}$ & S.D. \\
\hline $\begin{array}{l}20 \text { to } 25 \\
\text { Males }\end{array}$ & 83.30 & 21.8 & 80.41 & 15.0 & 81.86 & 17.1 \\
$\begin{array}{l}\text { Females } \\
\text { Combined }\end{array}$ & 64.57 & 27.3 & 74.60 & 28.0 & 69.69 & 22.3 \\
Over Sex & 73.94 & 25.7 & 77.51 & 21.9 & 75.72 & 20.2 \\
\hline
\end{tabular}

60 to 65

Males

Females

Combined Over Sex

$\begin{array}{llllll}71.62 & 14.3 & 82.91 & 17.9 & 77.27 & 11.1 \\ 75.83 & 21.9 & 84.31 & 17.3 & 80.02 & 15.8 \\ 73.72 & 18.0 & 83.56 & 17.0 & 78.64 & 13.3\end{array}$

Combined over Age

$\begin{array}{lllllll}\text { Males } & 77.46 & 18.8 & 81.66 & 16.0 & 79.56 & 14.2 \\ \text { Females } & 70.20 & 24.6 & 79.41 & 23.0 & 74.80 & 19.4 \\ \text { Combined } & 73.83 & 21.9 & 80.53 & 19.5 & 77.48 & 16.9 \\ \text { Over Sex } & & & & & & \end{array}$

$\mathrm{Ph} \cdot \mathrm{D}$.

20 to 25

Males

Females $89.51 \quad 15.2$

95.82

7. 7

$92.67 \quad 6.9$

Combined Over Sex

92.2111 .2

$87.09 \quad 14.9$

89.65

9.5

$90.86 \quad 13.0$ $91.46 \quad 12.3$

91.16

8.2

60 to 65

Males

Females

$83.67 \quad 13.3$

91.84

8.9

87.76

8.3

Combined Over Sex $80.27 \quad 22.4$ $82.50 \quad 25.9$ $81.39 \quad 13.9$ $81.98 \quad 17.9$ $87.17 \quad 19.3$ 84.5111 .5 
Table 11 Continued

$1930-1949$

True

False

Mean

$\mathrm{Ph} \cdot \mathrm{D}$.

$\overline{\mathrm{X}} \quad \mathrm{S} \cdot \mathrm{D}$.

$\bar{X} \quad S . D$.

$\overline{\mathrm{X}} \quad$ S.D.

Combined over Age

Males

Females

86.59

86.24

Combined

Over Sex

86.42

14.2

93.84

8.3

90.21

7.8

18.2

84.79

20.6

$85.52 \quad 12.3$

89.3116 .1

$87.87 \quad 10.4$

Combined over Ed

20 to 25

Males

Females

86.41

18.4

$88.12 \quad 14.0$

$87.26 \quad 13.8$

Combined

Over Sex

$78.39 \quad 24.7$

80.84

22.6

$79.62 \quad 19.5$

82.40

21.8

84.48

18.9

$83.49 \quad 17.1$

60 to 65

Males

Females

$77.65 \quad 14.7$

$87.38 \quad 14.4$

$82.51 \quad 10.9$

Combined

Over Sex

$78.05 \quad 21.5$

$83.36 \quad 21.3$

$80.70 \quad 14.4$

$77.85 \quad 18.1$

$85.37 \quad 18.0$

$81.61 \quad 12.6$

Combined over Age

Males

82.03

Females $78.22 \quad 22.8$

87.75

14.0

$84.89 \quad 12.5$

Combined Over Sex $80.13 \quad 20.0$ $82.10 \quad 21.7$ $20.16 \quad 16.9$ $\begin{array}{llll}84.92 & 18.3 & 82.52 & 14.9\end{array}$ 
Table 11 Continued

$$
\text { 1950-1969 }
$$

True

False

Miean

HS.

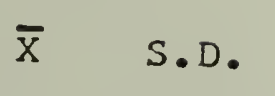

$\overline{\mathrm{X}} \quad \mathrm{S} \cdot \mathrm{D}$.

$$
\overline{\mathrm{X}} \quad \mathrm{S} \cdot \mathrm{D} \text {. }
$$

20 to 25

Males

Females

Combined

Over Sex

$$
\begin{array}{ll}
64.56 & 20.7
\end{array}
$$

62.50

17.3

$63.53 \quad 14.7$

$55.06 \quad 21.3$

41.71

14.9

48.39

9.5

$59.81 \quad 20.9$

52.11

18.9

55.96

14.3

60 to 65

Males

Females

Combined

Over Sex

58.
53.2
55.
Age

Combined over Age

Males

Females

Combined

Over Sex

$61.44 \quad 24.1 \quad 64.59 \quad 19.1$

$\begin{array}{llll}54.16 & 20.8 & 55.64 & 27.2\end{array}$

$63.02 \quad 19.0$

$57.80 \quad 22.5$

$60.12 \quad 23.6$

$54.90 \quad 13.6$

$58.96 \quad 16.8$

$\mathrm{Ph} \cdot \mathrm{D}$.

20 to 25

Males

Females

Combined

Over Sex

$60.42 \quad 17.7 \quad 75.29^{\prime} 20.1$

$67.86 \quad 15.9$

$\begin{array}{lllllll}48.81 & 27.0 & 43.75 & 16.2 & 46.28 & 16.9\end{array}$

$\begin{array}{lllllll}54.62 & 22.9 & 59.52 & 24.0 & 57.07 & 19.4\end{array}$

60 to 65

Males

Females

$\begin{array}{llllll}71.27 & 13.7 & 79.64 & 16.9 & 75.46 & 9.4\end{array}$

Combined

Over Sex

$59.25 \quad 17.5 \quad 66.25 \quad 30.6$

$62.75 \quad 19.5$

$65.26 \quad 16.4$

$72.94 \quad 24.9$

$69.10 \quad 16.2$ 
Table 11 Continued

1950-1969

\begin{tabular}{lcccccc} 
& \multicolumn{2}{c}{ True } & \multicolumn{2}{c}{ False } & \multicolumn{2}{c}{ Mean } \\
Ph.D. & $\bar{x}$ & S.D. & $\bar{x}$ & S.D. & $\bar{x}$ & S.D. \\
\hline $\begin{array}{llllll}\text { Combined over Age } \\
\text { Males }\end{array}$ & 65.85 & 16.3 & 77.46 & 18.1 & 71.66 & 13.2 \\
Females & 54.03 & 22.7 & 55.00 & 26.4 & 54.52 & 19.6 \\
$\begin{array}{l}\text { Combined } \\
\text { Over Sex }\end{array}$ & 59.94 & 20.3 & 66.23 & 25.0 & 63.09 & 18.6
\end{tabular}

Combined over Ed

20 to 25

Males

Females

Combined

Over Sex

$\begin{array}{llllll}62.49 & 18.7 & 68.89 & 19.3 & 65.69 & 15.0 \\ 51.94 & 23.7 & 42.73 & 15.0 & 47.33 & 13.3 \\ 57.22 & 21.7 & 55.81 & 21.6 & 56.51 & 16.7\end{array}$

60 to 65

Males

Females

Combined Over Sex

$$
\begin{aligned}
& 54.80 \\
& 56.2 \\
& 60.5 \\
& \text { Age }
\end{aligned}
$$

Males

Females

Combined Over Sex

63.65

54.10

$58.87 \quad 21.3$

$63.17 \quad 24.3$

$67.34 \quad 16.7$

$54.71 \quad 16.6$

$61.02 \quad 17.7$ 
Table II Continued

Total

True

False

Mean

HS

$$
\begin{array}{llllll}
\bar{x} & S \cdot D \cdot & \bar{x} & S . D . & \bar{x} & S . D .
\end{array}
$$

20 to 25

Males

Females

Combined

Over Sex

$$
\begin{array}{rrrrrr}
73.46 & 14.5 & 73.00 & 9.9 & 73.23 & 11.6 \\
62.20 & 11.3 & 67.56 & 12.8 & 64.69 & 7.4 \\
67.85 & 13.9 & 70.29 & 11.4 & 69.06 & 10.3
\end{array}
$$

60 to 65

$\begin{array}{lllllll}\text { Males } & 71.85 & 13.3 & 76.40 & 11.4 & 74.12 & 9.6 \\ \text { Females } & 70.37 & 13.2 & 80.06 & 17.2 & 75.22 & 9.6 \\ \text { Combined } & 71.11 & 12.8 & 78.73 & 14.2 & 74.67 & 9.3 \\ \text { Over Sex } & & & & & & \end{array}$

Combined over Age

Males

Females

Combined Over Sex

$$
72.65
$$

66.29

69.47
$5 \quad 13.5$

12.6

13.2
$74.70 \quad 10.5$

16.0

13.3
$73.68 \quad 10.3$

$70.05 \quad 9.8$

$71.87 \quad 10.0$

$\mathrm{Ph} \cdot \mathrm{D}$.

20 to 25

Males

Females 75.50 71.91

10.8

84.44

9.9

79.97

8.0

Combined

Over Sex

73.71

$9.8 \quad 75.78$

7.3

73.85

3.0

10.0

80.11

9.5

76.91

6.7

60 to 65

Males

Females

Combined Over Sex
$81.96 \quad 7.3$

87.14

6.6

84.55

77.29 13.2
$76.80 \quad 21.7$ $\begin{array}{llllll}79.62 & 10.0 & 81.87 & 16.4 & 80.75 & 10.4\end{array}$

$76.95 \quad 13.5$ 
Table 11 Continued

Total

True

False

Mean

$\mathrm{Ph} \cdot \mathrm{D}$.

$\bar{x} \quad S \cdot D$.

$\overline{\mathrm{X}} \quad \mathrm{S} \cdot \mathrm{D}$.

$\overline{\mathrm{X}} \quad \mathrm{S} . \mathrm{D}$.

Combined over Age

Males

Females

78.73

$9.5 \quad 85.79$

8.3

82.26

6.6

Combined

Over Sex

$\begin{array}{llll}74.60 & 11.6 \quad 76.19 & 15.6\end{array}$

75.40

9.6 $76.66 \quad 10.6$ 80.99 13.2 78.83

8.8

Combined over Ed

20 to 25

$\begin{array}{lrrrrrr}\text { Males } & 74.48 & 12.4 & 78.72 & 11.3 & 76.60 & 10.3 \\ \text { Females } & 67.06 & 11.4 & 71.68 & 10.9 & 69.37 & 7.2 \\ \text { Combined } & 70.77 & 12.3 & 75.20 & 11.5 & 72.99 & 9.4 \\ \text { Over Sex } & & & & & & \end{array}$

60 to 65

$\begin{array}{lrrrrrr}\text { Males } & 76.90 & 11.6 & 81.77 & 10.6 & 79.33 & 9.0 \\ \text { Females } & 73.83 & 13.2 & 78.33 & 19.0 & 76.08 & 11.3 \\ \text { Combined } & 75.37 & 12.3 & 80.05 & 15.2 & 77.10 & 10.2 \\ \text { Over Sex } & & & & & & \end{array}$

Combined over Age

$\begin{array}{lrrrrrr}\text { Males } & 75.69 & 11.9 & 80.25 & 10.8 & 77.97 & 9.6 \\ \text { Females } & 70.44 & 12.6 & 25.01 & 15.6 & 72.73 & 9.9 \\ \text { Combined } & 73.07 & 12.4 & 77.63 & 13.6 & 75.35 & 10.0 \\ \text { Over Sex } & & & & & & \end{array}$


the first and third (all ps<.08). Time Period interacted significantly with Age $(\underline{F}(3,144)=8.85, p<$ $.001), \operatorname{Sex}(\underline{E}(3,144)=2.71, \underline{D}<.05)$, Age $x$ Education $(\underline{E}(3,144)=3.50, \underline{p}<.05)$, Item $(\underline{F}(3,144)=4.01$, $\underline{\mathrm{p}}(.01)$, Item $\times$ Age $(\underline{\mathrm{F}}(3,144)=3.96, \underline{\mathrm{p}}<.01)$, and Item $\times$ Age $\times \operatorname{Sex}(\underline{F}(3,144)=4.44, \mathrm{D}<.01)$, however, since these were largely uninterpretable, tney will not be discussed further.

\section{d. Signal Detection Analysis of Recoanition}

Separate 2 (Age) $\times 2$ (Education) $\times 2$ (Sex) $x$ 2 (Order) x 4 (Time Period) analyses of variance were performed on $\underline{d}$ ' and $\underline{c}$ statistics. The mean $\mathrm{d}^{\prime} \mathrm{s}$ for younger and older subjects were 1.74 and 1.97 , respectively, but the analysis indicated no significant age difference in retention of general information knowledge. The mean d's for $\mathrm{HS}$ and $\mathrm{Ph} . \mathrm{D}$. subjects were 1.65 and 2.07 , respectively, and the analysis indicated a marginally significant superiority in retention of $\mathrm{Ph} . \mathrm{D}$. subjects $(\underline{\mathrm{F}}(1,48)=3.85, \underline{\mathrm{D}}<.01)$. The mean d's for males and females were 1.60 and 2.12 , respectively, and the analysis indicated significantly better retention for females than males $(\underline{E}(1,48)=$ $5.95,25.05)$.

The mean $\subseteq$ values for younger and older subjects were . 86 and 1.03, respectively, but the Age main effect was nonsignificant. The mean $\subseteq$ values for $H S$ and 
Ph.D. subjects were .76 and 1.13 , respectively, and the analysis indicated that HS subjects were significantly less conservative than Ph.D. subjects $(\underline{F}(1,48)$ $=6.50, \mathrm{p}(.05)$. The mean $\subseteq$ values for males and females were .75 and 1.14 , respectively, and the analysis indicated that males were significantly less conservative than females $(\underline{E}(1,48)=7.90, \underline{p}<.01)$.

\section{Summary "}

To summarize the results for memory performance, 2 (Age) $\times 2$ (Education) $\times 2$ (Sex) analyses of variance on the mean number of episodic and semantic items correctly recalled and recognized, as well as average episodic and semantic memory performance are summarized in Table 12.

\section{a. Episodic Memory Performance}

Younger subjects remembered more than older subjects and Age accounted for $13 \%$ of the variance in recall performance and $11 \%$ in recognition performance. Ph.D. subjects remembered more than HS subjects, and Education accounted for $3 \%$ of the variance in recall performance and $5 \%$ in recognition performance. Females remembered more than males and sex accounted for $3 \%$ of the variance recall performance and $2 \%$ in recognition performance. These variables accounted for $20 \%$ of the variance in episodic recall performance, and $18 \%$ in 


\section{Table 12}

Summary ANOVAs for Episodic and Semantic Memory Performance

\begin{tabular}{|c|c|c|c|c|}
\hline \multirow[b]{2}{*}{$d f=1,56$} & \multicolumn{4}{|c|}{$\begin{array}{c}\text { Episodic } \\
\overline{\mathrm{x}}=\text { Recall }\end{array}$} \\
\hline & 7.84 & $\mathrm{~F}$ & $\mathrm{P}$ & $E t a^{2}$ \\
\hline Age & $\begin{array}{r}1.67 \\
-1.67\end{array}$ & 11.31 & $<.01$ & .13 \\
\hline Education & $\begin{array}{r}-.84 \\
.84\end{array}$ & 2.88 & $<.1$ & .03 \\
\hline Sex & $\begin{array}{r}-.86 \\
.86\end{array}$ & 2.99 & $<.09$ & .03 \\
\hline Age $\times E d$ & & 2.37 & NS & \\
\hline Age $x$ Sex & & 4.05 & $<.05$ & \\
\hline Ed $x$ Sex & & 7.48 & 5.01 & \\
\hline Age $x$ Ed $x$ Sex & & 1.01 & NS & \\
\hline Multiple $R^{2}$ & & & & .20 \\
\hline 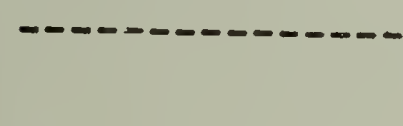 & 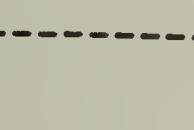 & Sema & ic & $-\ldots$ \\
\hline & & $\bar{x}=R$ & $=211$ & \\
\hline $\mathrm{d} f=$ & 8.70 & F & $\mathrm{P}$ & $E t a^{2}$ \\
\hline Age & $\begin{array}{r}-1.52 \\
1.52\end{array}$ & 5.58 & $<.05$ & .07 \\
\hline Education & $\begin{array}{r}-2.02 \\
2.02\end{array}$ & 9.86 & $c .01$ & .13 \\
\hline Sex & $\begin{array}{r}.95 \\
-.95\end{array}$ & 2.21 & NS & .03 \\
\hline Age $\times E d$ & & $<1$ & NS & \\
\hline Age $x$ Sex & & $<1$ & NS & \\
\hline Ed $x \operatorname{Sex}$ & & $<1$ & NS & \\
\hline Age $x$ Ed $x$ Sex & & $<1$ & NS & \\
\hline Multiple $\mathrm{R}^{2}$ & & & & .24 \\
\hline
\end{tabular}


Table 12 Continued

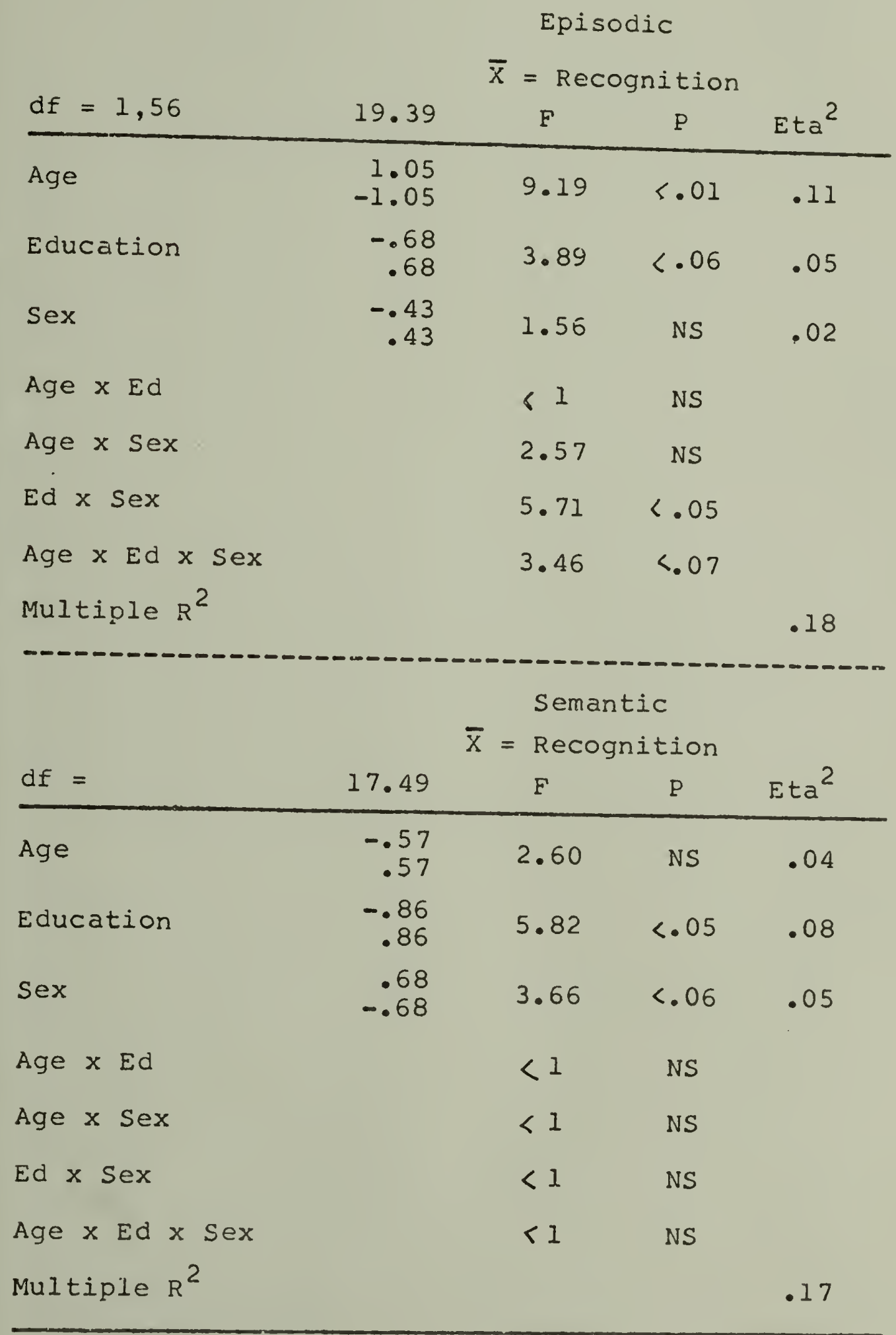


Table 12 continued

Episodic

$$
\overline{\mathrm{X}}=\text { Total }
$$

\begin{tabular}{lrccc} 
df $=1,56$ & 13.61 & $\mathrm{~F}$ & $\mathrm{P}$ & $\mathrm{Eta}^{2}$ \\
\hline Age & 1.36 & 14.04 & $<.001$ & .15 \\
& -1.36 & & & \\
Education & -.76 & 4.42 & $<.05$ & .05 \\
& .76 &. & & \\
Sex & -.65 & 3.16 & $<.08$ & .03
\end{tabular}

Age $x E d$ $<1 \quad$ NS

Age $x$ sex $4.58<.05$

Ed $x$ Sex $9.06<.01$

Age $x$ Ed $x$ Sex $2.48 \quad$ NS

Multiple $R^{2}$

Semantic

$\overline{\mathrm{X}}=\operatorname{Total}$

$\mathrm{df}=\quad \begin{array}{llll}13.10 & \mathrm{~F} & \mathrm{P} & \mathrm{Eta}^{2}\end{array}$

Age

$-1.04$

1.04

6.09

$<.05 \quad .08$

Education

$-1.44$

11.52

$<.01$

.14

Sex

.82
-.82

3.72

$<.06$

.05

Age $x E d$

$<1$ NS

Age $x$ Sex

< 1 NS

Ed $x$ Sex

51

NS

Age $x$ Ed $x$ Sex

1.15

NS

Multiple $R^{2}$ 
episodic recognition performance. Furthermore, summed over recall and recognition, $23 \%$ of the variance could be accounted for: $15 \%$ by Age, $5 \%$ by Education, and $3 \%$ by sex.

\section{b. Semantic Memory Performance}

Older subjects knew more general information facts than younger subjects, and Age accourted for $7 \%$ of the variance in recall performance and $3 \%$ in recognition performance. Ph.D. subjects knew more general information facts than HS subjects, and Education accounted for $13 \%$ of the variance in recall performance and $8 \%$ in recognition performance. Males responded correctly to more items than females, and sex accounted for $3 \%$ of the variance in recall performance and $5 \%$ in recognition performance. These variables accounted for $24 \%$ of the variance in semantic recall performance and $17 \%$ in semantic recognition performance. Furthermore, summed over recall and recognition $27 \%$ of the variance could be accounted for: $8 \%$ by Age, $14 \%$ by Education, and $5 \%$ by sex.

\section{Memory Information}

1. Metamemory Questionnaire

The mean percentage of subjects in each Age, Education, and sex group giving each response on the questionnaire is shown in Appendix B. Chi square values are also reported for the 17 questions on which 
subjects in the two Age groups responded differently, the 13 questions on which subjects in the two Education groups responded differently, and the 9 questions on which subjects in the two sex groups responded differently. There were virtually no differences in questionnaire responses of subjects in the two Order groups.

\section{Memory Demands}

Subjects were asked to assess the memory demand generally on them, on an 11 point scale $(0=$ minimal thru 10 = excessive). These responses were submitted to a 2 (Age) $\times 2$ (Education) $\times 2$ (Sex) analysis of variance, the results of which are summarized in Table 13. Overall the mean response was 7.09 . Younger subjects reported greater memory demand than older subjects ( 7.43 vs. 6.75$)$, but the Age main effect was nonsignificant. HS subjects reported less memory demand than Ph.D. subjects (6.34 vs. 7.84), and the Education main effect was statistically significant $(\underline{F}(1,56)=9.86$, $\mathrm{p}(.01)$. Males reported less memory demand than females (6.62 vs. 7.56), and the sex main effect was marginally significant $(\underline{F}(1,56)=3.85, \underline{p}<.06)$. These variables accounted for $21 \%$ of the variance in memory demand: Age 3\%, Education $14 \%$, and Sex $5 \%$.

3. Memory Problems

A composite score of memory problems was computed 
Table 13

Summary ANOVAS for Memory Information Measures

$$
\overline{\mathrm{X}}=\text { Memory Demands }
$$

\begin{tabular}{|c|c|c|c|c|}
\hline$d f=1,56$ & 7.09 & $F$ & $\mathrm{P}$ & $E t a^{2}$ \\
\hline Age & $\begin{array}{r}.34 \\
-.34\end{array}$ & 2.07 & NS & .03 \\
\hline Education & $\begin{array}{r}-.75 \\
.75\end{array}$ & 9.86 & $<.01$ & .14 \\
\hline Sex & $\begin{array}{r}-.47 \\
.47\end{array}$ & 3.85 & $<.06$ & .05 \\
\hline Age $x E d$ & & $<1$ & NS & \\
\hline Age $x$ Sex & & 1.39 & NS & \\
\hline Ed $x$ Sex & & $<1$ & NS & \\
\hline $\begin{array}{l}\text { Age } x \text { Ed } x \text { Sex } \\
\text { Multiple } \mathrm{R}^{2}\end{array}$ & & 51 & NS & .21 \\
\hline
\end{tabular}

$$
\overline{\mathrm{X}}=\text { Memory Problems }
$$

\begin{tabular}{lrrrr} 
df $=1,56$ & 17.73 & $F$ & $P$ & $E^{2}$ \\
\hline Age & $\begin{array}{r}-1.80 \\
1.80\end{array}$ & 7.77 & $<.01$ & .11
\end{tabular}

$\begin{array}{lrlll}\text { Education } & .80 & & & \\ & -.80 & 1.53 & \text { NS } & .02 \\ \text { Sex } & .95 & & & \\ & -.95 & 2.19 & \text { NS } & .03\end{array}$

Age $x \mathrm{Ed}$

$<1 \quad$ NS

Age $x$ Sex

$<1 \quad$ NS

Ed $x$ Sex

$3.67 \quad .06$

Age $x$ Ed $x$ Sex

$<1$

NS

Multiple $\mathrm{R}^{2}$ 
Table 13 Continued

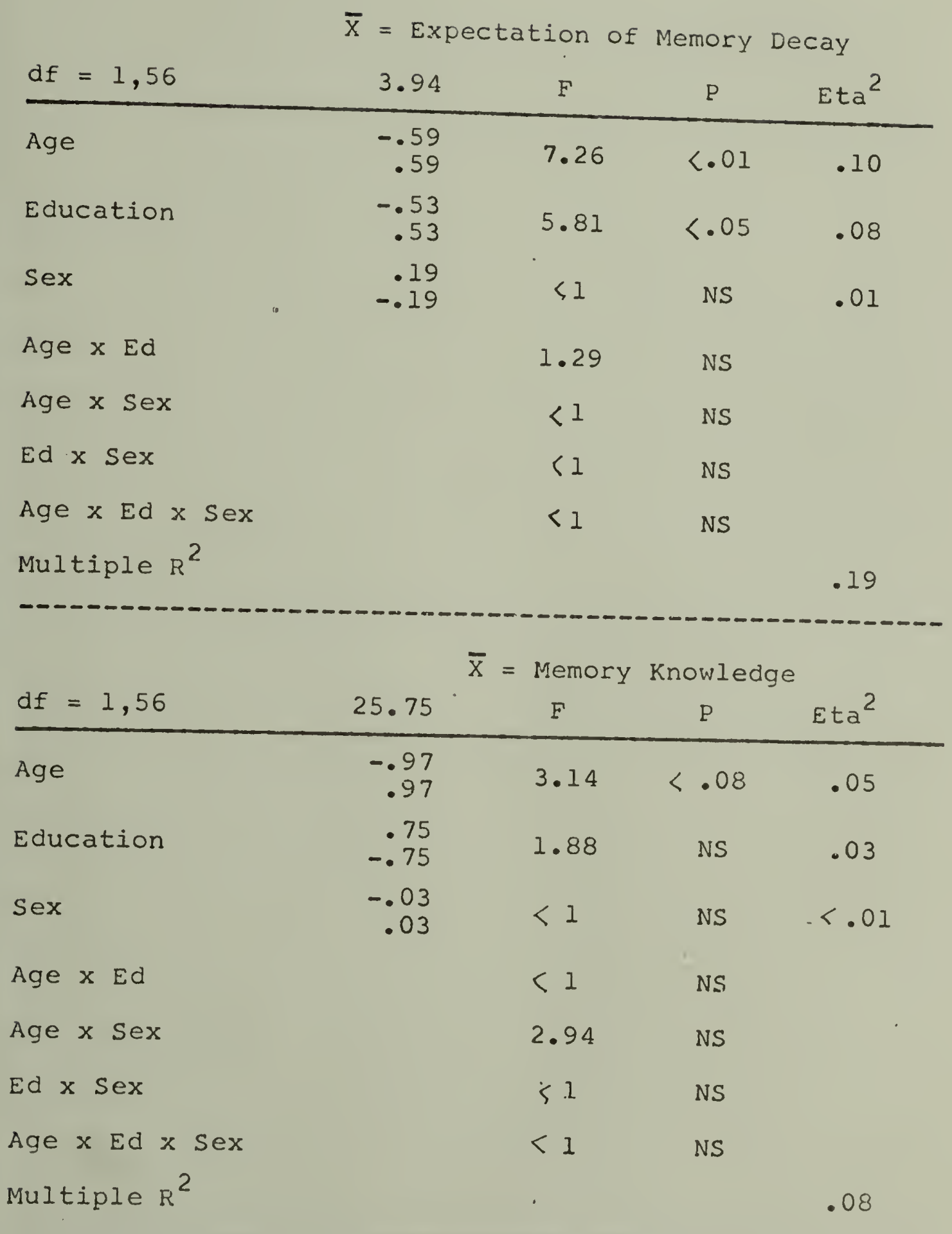


Table 13 Continued

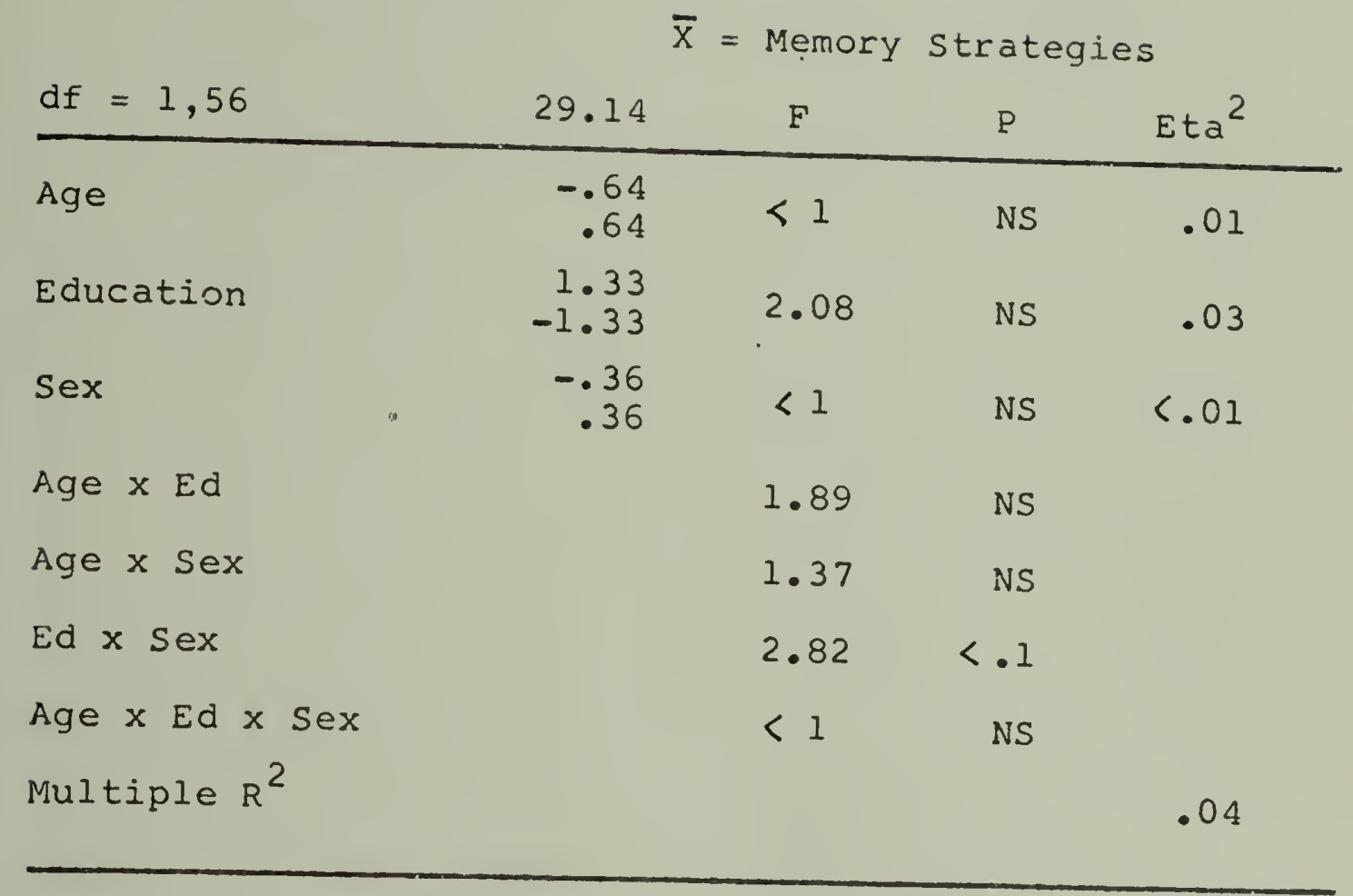


by summing responses on questions 5 thru 14 on the metamemory questionnaire (see Appendix B). These composite scores could range from 0 to 40 , with higher score indicative of more memory problems. These scores were submitted to a 2 (Age) $\times 2$ (Education) $x$ 2 (Sex) analysis of variance, the results of which are summarized in Table 13. Overall the mean score was 17.73. Younger subjects reported tewer memory problems than older subjects (15.93 vs. 19.53), and the Age main effect was statistically significant $(\underline{F}(1,56)$ $=7.77, \mathrm{p}(.01)$. HS subjects reported more memory problems than Ph.D.subjects (18.53 vs. 16.93), but the Education main effect was nonsignificant. Males reported more memory problems than females (18.68 vs. 16.78), but the sex main effect was also nonsignificant. These variables accounted for $15 \%$ of the variance in memory problems: Age $11 \%$, Education $2 \%$, and Sex $3 \%$.

\section{Expectation of Memory Decay}

A composite score of expectation of memory decay was computed by summing responses on questions 41,43 , and 45 on the metamemory questionnaire (see Appendix B). These composite scores could range from 0 to 12 , with higher scores indicative of greater expectation of memory decay. These scores were submitted to a 2 (Age) $x 2$ (Education) $\times 2$ ( $\operatorname{sex}$ ) analysis of variance, the 
results of which are summarized in Table 13. Overall the mean score was 3.94. Younger subjects expected memory decay less than older subjects (3.35 vs. 4.53), and the Age main effect was statistically significant $(\underline{F}(1,56)=7.28, \underline{\mathrm{p}}<.01)$. HS subjects expected memory decay more than Ph.D. subjects (5.03 vs. 4.47$)$, and the Education main effect was statistically significant $(\underline{F}(1,56)=5.81, \underline{p}<.05)$. Males expected memory decay more than females ( 4.13 vs. 3.75 ), but the sex main effect was also nonsignificant. These variables accounted for 19\% of the variance in expectation of memory decay: Age 10\%, Education $8 \%$, and Sex $1 \%$.

\section{Memory Knowledge}

A composite score of memory knowledge was computed by summing the number of unsure responses on questions 29 thru 60 on the metamemory questionnaire (see Appendix B). These composite scores could range from 0 to 31 , with higher scores indicative of less memory knowledge. These scores were submitted to a 2 (Age) $\times 2$ (Education) $x 2$ (Sex) analysis of variance, the results of which are summarized in Table 13. Overall the mean score was 25.75. Younger subjects demonstrated more memory knowledge than older subjects (24.78 vs. 26.72), and the Age main effect was marginally significant $(\underline{F}(1,56)=$ $3.14, \mathrm{p}<.08)$. HS subjects demonstrated less memory knowledge than Ph.D. subjects (26.50 vs. 25.00), but 
the Education main effect was nonsignificant. Males and females demonstrate about the same amount of memory knowledge, $(25.72$ vs. 25.78) and the sex main effect was nonsignificant. These variables accounted for only $8 \%$ of the variance in memory knowledge: Age 5\%, Education $3 \%$, and sex less than $1 \%$.

6. Memory Strategies and Aids

A composite score of use of memory strategies and aids was computed by summing responses on questions 15 thru 28 on the metamemory questionnaire (see Appendix B). Composite scores could range from 0 to 52 , with higher scores indicating more use of memory strategies and aids. These scores were submitted to a 2 (Age) $\times 2$ (Education) $\times 2$ (Sex) analysis of variance, the results of which are summarized in Table 13. Overall the mean score was 29.14. Younger subjects said they used memory strategies and aids less than older subjects (28.50 vs. 29.14), but the Age main effect was nonsignificant. HS subjects said they used memory strategies and aids more than Ph.D. subjects (30.47 vs. 27.81, but the Education main effect was also nonsignificant. Males said they used memory strategies and aids more than females (28.78 vs. 28.06), but the sex main effect was nonsignificant as well. These variables accounted for only $4 \%$ of the variance in memory strategy and aids use: Age $1 \%$, Education $3 \%$, and sex $1 \%$. 


\section{Memory Predictions}

Subjects were asked to make predictions concerning how many items they thought they would correctly recall and recognize. These predictions, as well as difference scores computed by subtracting performance scores from predictions, and absolute values of the difference scores are reported below.

\section{Episodic Memory}

\section{a. Recall and Recognition Predictions}

Mean predictions of the number of incidental and intentional items to be correctly recalled and recognized are shown in Table 14. These data were submitted to a 2 (Age) $\times 2$ (Education) $\times 2$ (sex) $\times 2$ (order) $\times 2$ (Memory (Recall vs. Recognition)) $\times 2$ (Task (Incidental vs. Intentional)) aralysis of variance.

Predictions of younger subjects were larger than those of older subjects (15.64 vs. 13.29, and the Age main effect was statistically significant $(E(1,48)=$ $5,69, \underline{\mathrm{p}}(.05)$. Predictions of Ph.D. educated subjects were larger than those of HS educated subjects (14.63 vs. 14.30), but the Education main effect was nonsignificant. Predictions of females were larger than those of males (15.25 vs. 13.69), but the sex main effect was also nonsignificant. Likewise, the order main effect was nonsignificant. 
Table 14

Mean Predictions of the Number of Incidental and Intentional Items correctly Recalled and Recognized by Each Age $x$ Education $x$ Sex Group

Recall

Incidental Intentional Mean

\begin{tabular}{|c|c|c|c|c|c|c|}
\hline HS & $\bar{x}$ & $S \cdot D$. & $\bar{x}$ & S.D. & $\bar{x}$ & S.D. \\
\hline \multicolumn{7}{|l|}{20 to 25} \\
\hline Males & 12.25 & 5.37 & 11.63 & 5.90 & 11.94 & 5.22 \\
\hline Females & 11.63 & 6.76 & $13 . .13$ & 5.06 & 12.38 & 4.14 \\
\hline $\begin{array}{l}\text { Combined. } \\
\text { Over Sex }\end{array}$ & 11.94 & 5.90 & 12.38 & 5.37 & 12.16 & 4.68 \\
\hline \multicolumn{7}{|l|}{60 to 65} \\
\hline Males & 10.75 & 5.92 & 8.13 & 3.94 & 9.44 & 4.76 \\
\hline Females & 12.25 & 6.14 & 12.13 & 5.33 & $12.19^{\circ}$ & 5.59 \\
\hline $\begin{array}{l}\text { Combined } \\
\text { Over Sex }\end{array}$ & 11.50 & 5.88 & 10.13 & 4.98 & 10.81 & 5.22 \\
\hline
\end{tabular}

Combined over Age

$\begin{array}{lllllll}\text { Males } & 11.50 & 5.51 & 9.88 & 5.18 & 10.69 & 5.00 \\ \text { Females } & 11.94 & 6.25 & 12.63 & 5.05 & 12.28 & 4.87 \\ \text { Combined } & 11.72 & 5.80 & 11.25 & 5.22 & 11.48 & 4.92 \\ \text { Over Sex } & & & & & & \end{array}$

$\mathrm{Ph}$. D.

20 to 25

Males

$10.88 \quad 5.64$

$10.88 \quad 2.64$

$10.88 \quad 3.64$

Females

12.006 .63

$13.63 \quad 4.66$

12.815 .12

Combined

Over Sex

$\begin{array}{llllll}11.44 & 5.98 & 12.50 & 3.92 & 11.84 & 4.41\end{array}$


Table 14 Continued

Recall

\begin{tabular}{lccccccc} 
& \multicolumn{2}{c}{ Incidental } & \multicolumn{2}{c}{ Intentional } & \multicolumn{2}{c}{ Mean } \\
Ph.D. & $\overline{\mathrm{x}}$ & S.D. & $\overline{\mathrm{x}}$ & S.D. & $\overline{\mathrm{x}}$ & S.D. \\
\hline 60 to 65 & & & & & & \\
$\quad$ Males & 8.63 & 4.90 & 11.25 & 6.43 & 9.94 & 4.91 \\
$\quad$ Females & 7.25 & 6.23 & 11.00 & 6.50 & 9.13 & 5.64 \\
$\quad \begin{array}{l}\text { Combined } \\
\text { Over Sex }\end{array}$ & 7.94 & 5.46 & 11.13 & 6.25 & 9.53 & 5.12
\end{tabular}

Combined over Age

$\begin{array}{lllllll}\text { Males } & 9.75 & 5.24 & 11.06 & 4.75 & 10.41 & 4.20 \\ \text { Females } & 9.63 & 6.68 & 12.31 & 5.63 & 10.97 & 5.54 \\ \text { Combined } & 9.69 & 5.91 & 11.69 & 5.17 & 10.69 & 4.85 \\ \text { Over Sex } & & & & & \end{array}$

Combined over Ed

20 to 25

$\begin{array}{lllllll}\text { Males } & 1.1 .56 & 5.37 & 11.25 & 4.44 & 11.41 & 4.38 \\ \text { Females } & 11.81 & 6.47 & 13.38 & 4.70 & 12.59 & 4.62 \\ \text { Combined } & 11.69 & 5.85 & 12.31 & 4.62 & 12.00 & 4.47 \\ \text { Over Sex } & & & & & & \end{array}$

60 to 65

$\begin{array}{lrrrrrr}\text { Males } & 9.69 & 5.36 & 9.69 & 5.40 & 9.69 & 4.68 \\ \text { Females } & 9.75 & 6.51 & 11.56 & 5.77 & 10.66 & 5.65 \\ \text { Combined } & 9.72 & 5.87 & 10.63 & 5.58 & 10.17 & 5.13 \\ \text { Over Sex } & & & & & & \end{array}$

Combined over Age

$\begin{array}{lllllll}\text { Males } & 10.63 & 5.36 & 10.47 & 4.93 & 10.55 & 4.54 \\ \text { Females } & 10.78 & 6.47 & 12.47 & 5.26 & 11.63 & 5.17 \\ \text { Combined } & 10.70 & 5.90 & 11.47 & 5.16 & 11.09 & 4.86 \\ \text { Over Sex } & & & & & & \end{array}$


Table 14 Continued

Recognition

Incidental Intentional

HS, $\overline{\mathrm{X}}$ S.D. $\overline{\mathrm{x}}$ S.D. $\overline{\mathrm{x}}$ S.D.

20 to 25

Males

Females

Combined

over Sex

$\begin{array}{llllll}19.88 & 5.49 & 19.00 & 6.02 & 19.44 & 5.47 \\ 19.38 & 5.48 & 18.75 & 4.17 & 19.06 & 4.47 \\ 19.63 & 5.30 & 18.88 & 5.01 & 19.25 & 4.83\end{array}$

60 to 65

Males

Females

Combined

Over sex

$\begin{array}{rrrrrr}9.63 & 8.33 & 9.63 & 7.52 & 9.63 & 6.24 \\ 21.63 & 3.85 & 19.13 & 5.06 & 20.38 & 4.27 \\ 15.63 & 8.82 & 14.38 & 7.90 & 15.00 & 7.58\end{array}$

Combined over Age

Males

Females

Combined

Over Sex
$14.75 \quad 8.63$

$20.50 \quad 4.72$

$.27 .63 \quad 7.44$
$14.31 \quad 8.17$

$18.94 \quad 4.48$

16.636 .89
$14.53 \quad 7.60$

$19.72 \quad 4.27$

$17.13 \quad 6.61$

Ph.D.

20 to 25

Males

$19.75 \quad 2.05$

$19.88 \quad 3.48$

$19.81 \quad 2.10$

Females

$19.13 \quad 4.02$

$18.50 \quad 3.42$

18.812 .96

Combined

Over Sex

$19.44 \quad 3.10$

19.19

3.41

$19.31 \quad 2.54$

$60^{\circ}$ to 65

Males

$17.75 \quad 5.15$

19.13

2.53

$18.44 \quad 3.44$

Females

$16.88 \quad 4.97$

17.50

4.47

$17.19 \quad 3.89$

Combined

Over Sex

17.314 .91

18.31

3.61

$17.81 \quad 3.61$ 
Table 14 Continued

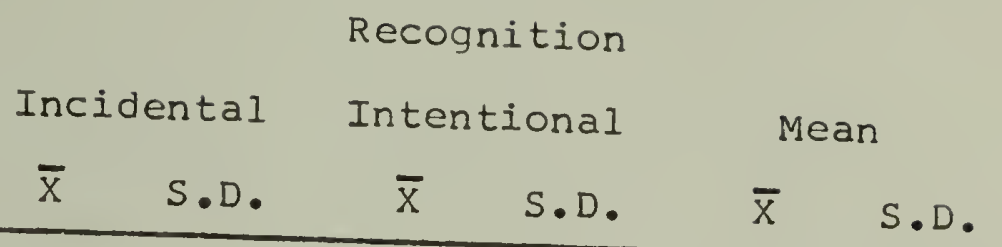

Combined over Age

$\begin{array}{lllllll}\text { Males } & 18.75 & 3.92 & 19.50 & 2.97 & 19.13 & 2.84 \\ \text { Females } & 18.00 & 4.52 & 18.00 & 3.88 & 18.00 & 3.45 \\ \text { Combined } & 18.38 & 4.18 & 18.75 & 3.48 & 18.56 & 3.16 \\ \text { Over Sex } & & & & & & \end{array}$

Combined over Ed

20 to 25

$\begin{array}{lllllll}\text { Males } & 19.81 & 4.00 & 19.44 & 4.77 & 19.63 & 4.01 \\ \text { Females } & 19.25 & 4.64 & 18.63 & 3.69 & 18.94 & 3.67 \\ \text { Combined } & 19.53 & 4.27 & 19.03 & 4.22 & 19.28 & 3.79 \\ \text { Over Sex } & & & & & & \end{array}$

60 to 65

$\begin{array}{lllllll}\text { Males } & 13.69 & 7.90 & 14.38 & 7.31 & 14.03 & 6.66 \\ \text { Females } & 19.25 & 4.95 & 18.31 & 4.69 & 18.78 & 4.27 \\ \text { Combined } & 16.47 & 7.07 & 16.34 & 6.36 & 16.41 & 6.01 \\ \text { Over Sex } & & & & & & \end{array}$

Combined over Age

$\begin{array}{lllllll}\text { Males } & 16.75 & 6.90 & 16.91 & 6.60 & 16.83 & 6.11 \\ \text { Females } & 19.25 & 4.72 & 18.47 & 4.15 & 18.86 & 3.92 \\ \text { Combined } & 18.00 & 6.00 & 17.69 & 5.52 & 17.84 & 5.19 \\ \text { Over Sex } & & & & & & \end{array}$


The Age $x$ Education $x$ Sex interaction was statistically significant $(\underline{F}(1,48)=4.34, \underline{\mathrm{p}}<.05)$, and may be seen in Figure 17, which shows the mean predictions of memory performance by males and females in each Age $x$ Education group. Memory predictions of older HS males were lower than any of the other groups, and were statistically different from all groups but older female Ph.D.s (all ps $<.05)$. Predictions of the other groups were not statistically different.

Predictions were very similar for memory of incidental and intentional items (14.35 vs. 14.58), and the Task main effect was nonsigrificant. The Task x Education interaction was statistically significant $(\underline{F}(1,48)$ $=4.72, \mathrm{p}(.05)$, however, and may be seen in Figure 18, which shows the mean predictions for incidental and intentional memory performance by each Education group. High school educated subjects predicted they would remember more incidental items than intentional items, while Ph.D. educated subjects predicted they would remember more intentional items than incidental items. None of these differences reached statistical significance, however.

Subjects predicted they would correctly recognize considerably more items than they predicted they would correctly recall (17.34 vs. 11.09), and the Memory (Recall vs. Recognition) main effect was highly signif- 
Figure 17. Mean Predictions of Memory Performance by Males and Females in Each Age $x$ Education Group

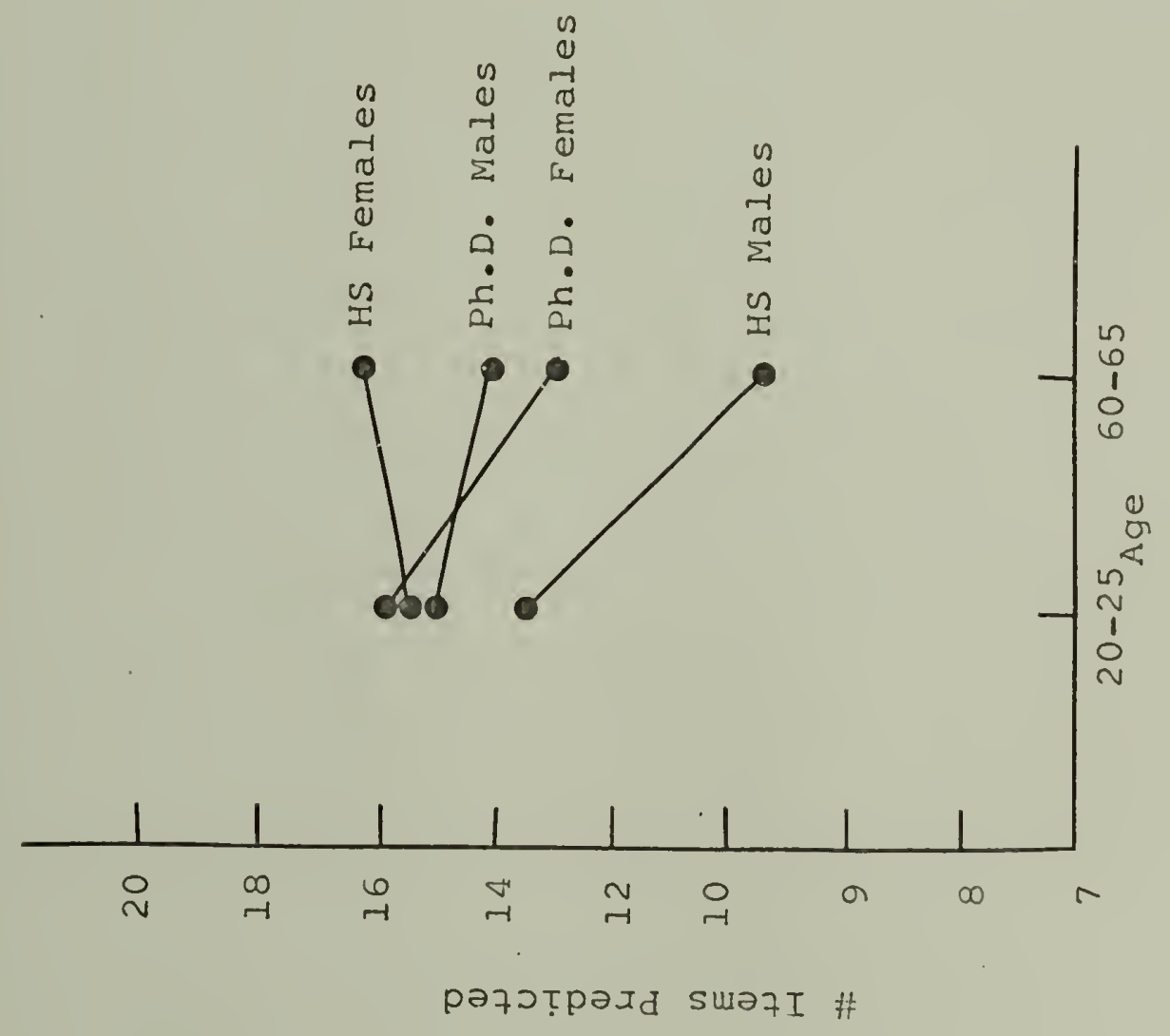


Figure 18. Mean Predictions for Incidental and Intentional Memory Performance by Each Education Group
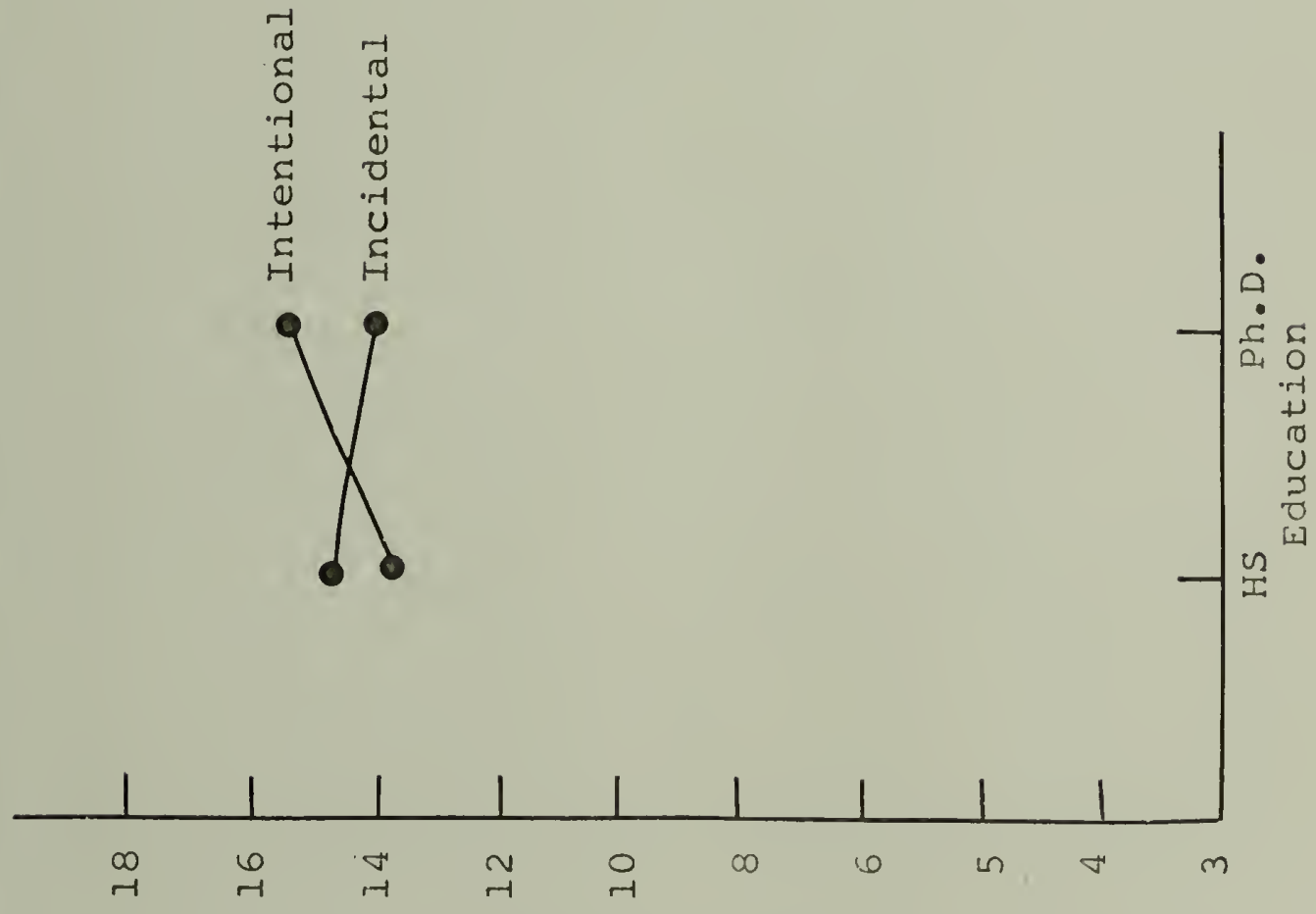

$$
\text { рә7วтฺрәда suə7I \# }
$$


icant $(\underline{F}(1,48)=105.50, \underline{p}<.001)$. The Memory $x$ Age $x$ Sex interaction was also statistically significant $(\underline{F}(1,48)=4.03, \underline{p}<.05)$, and may be seen in Figure 19, which shows the mean predictions of recall and recognition memory performance by males and females in each Age group. For recall, there were no significant differences in predictions, and for recognition, only older males predicted significantly lower performance than the other groups (all $\mathrm{p}<.05)$.

The Memory $x$ Education $x$ Sex interaction was also statistically significant $(\underline{F}(1,48)=4.62, \underline{p}<.05)$, and may be seen in Figure 20, which shows the mean predictions of recall and recognition memory performance by males and females in each Education group. For recall, there were no significant differences in prediction, and for recognition, only HS males predicted significantly lower performance than the other groups (all ps $<.05)$.

b. Differences Between Prediction and Performance Difference scores of prediction and performance were calculated to evaluate over- and under-prediction. The mean difference scores on recall and recognition of incidental and intentional items by each Age $x$ Education $x$ sex group are shown in Table 15. These data were submitted to a 2 (A.ge) $x 2$ (Education) $\times 2$ (Sex) x 2 (Order) $\times 2$ (Memory (Recall vs. Recognition)) $x$ 
Figure 19. Mean Predictions of Recall and Recognition Memory Performance by Males and Females in Each Age Group

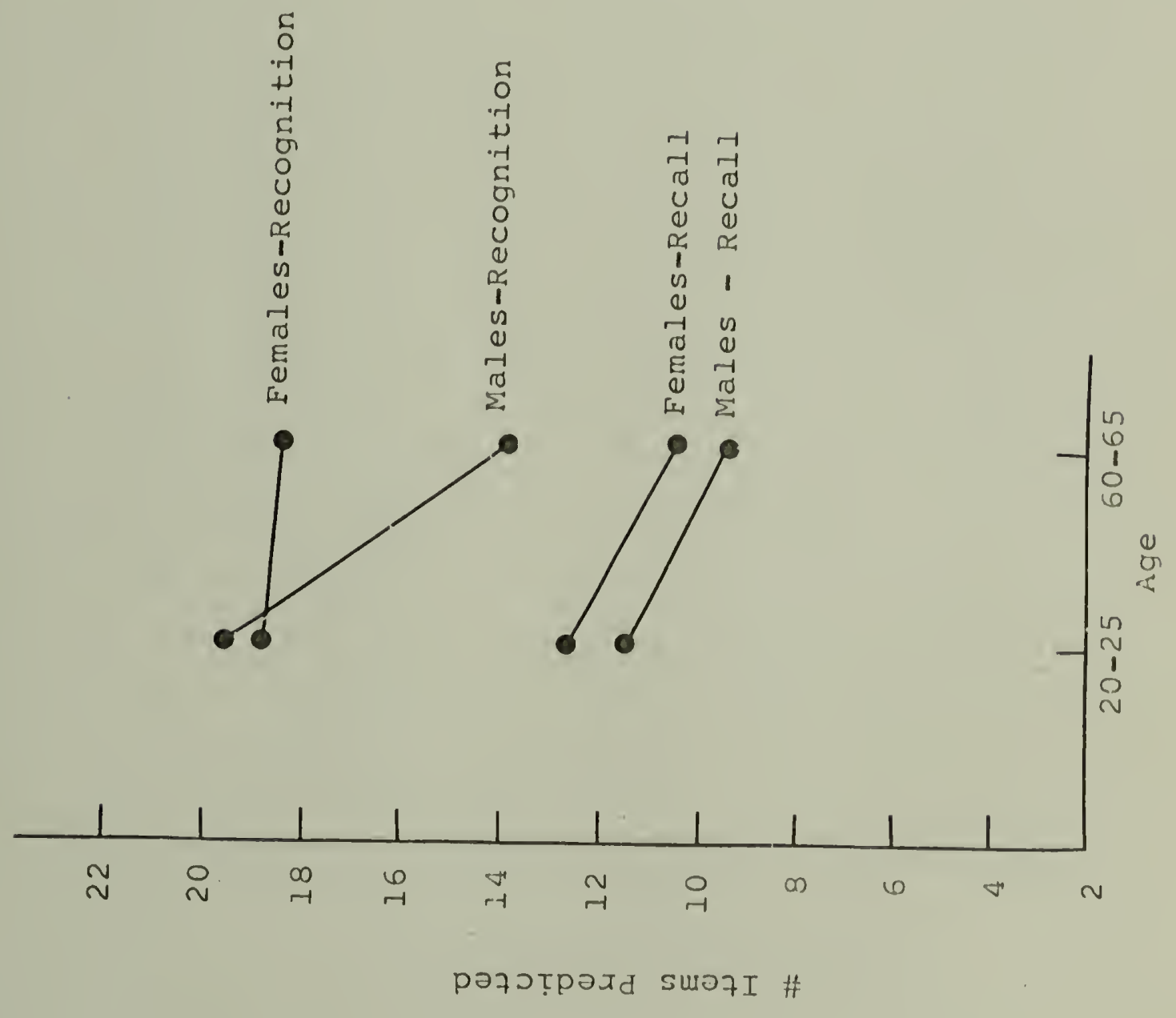


Figure 20. Mean Fredictions of Recall and Recognition Memory Performance by Males and Females in Each Education Group

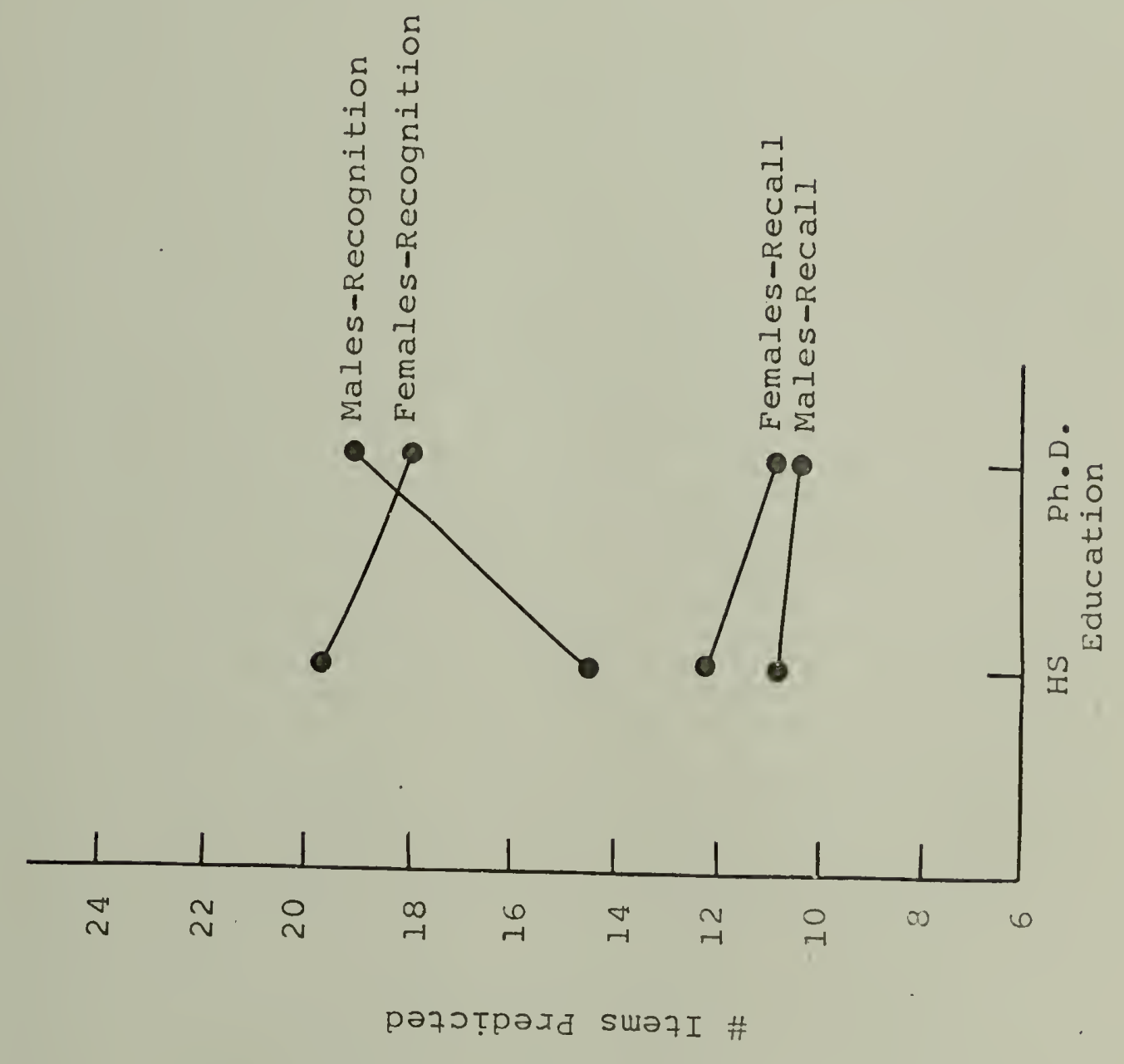


Table 15

Mean Difference Scores of Prediction and Performance on Recall and Recognition of Incidental and Intenticnal Items by Each Age $x$ Education $x$ Sex Group

\begin{tabular}{|c|c|c|c|c|c|c|}
\hline \multirow[b]{2}{*}{ HS } & \multicolumn{5}{|c|}{ Recall } & \\
\hline & $\bar{x}$ & $S \cdot D$ & $\bar{x}$ & $S \cdot D$. & $\bar{x}$ & S.D. \\
\hline \multicolumn{7}{|l|}{20 to 25} \\
\hline Males & 4.75 & 6.25 & 4.75 & 4.89 & 4.75 & 3.51 \\
\hline Females & 3.00 & 7.43 & 4.50 & 4.96 & 3.75 & 4.82 \\
\hline $\begin{array}{l}\text { Combined } \\
\text { Over Sex }\end{array}$ & 3.88 & 6.69 & 4.63 & 4.76 & 4.25 & 4.10 \\
\hline \multicolumn{7}{|l|}{60 to 65} \\
\hline Males & 8.63 & 6.00 & 5.50 & 5.26 & 7.06 & $5 \cdot 36$ \\
\hline Females & 3.63 & 5.07 & 1.13 & 7.24 & 2.38 & 5.24 \\
\hline $\begin{array}{l}\text { Combined } \\
\text { Over Sex } \\
\end{array}$ & 6.13 & 5.95 & 3.31 & 6.52 & 4.72 & 5.66 \\
\hline \multicolumn{7}{|c|}{ Combined over Age } \\
\hline Males & 6.69 & 6.25 & 5.13 & 4.92 & $5 \cdot 91$ & 4.53 \\
\hline Females & 3.31 & 6.15 & 2.81 & 6.24 & 3.05 & 4.91 \\
\hline $\begin{array}{l}\text { Combined } \\
\text { Over Sex }\end{array}$ & 5.00 & 6.34 & 3.97 & 5.65 & 4.48 & 4.87 \\
\hline
\end{tabular}

$\mathrm{Ph} . \mathrm{D}$.

20 to 25

Males $-1.63$

9.24

$-.88$

5.30

$-1.25$

5.73

Females 1.88

5.22

3.50

1.51

2.69

2.40

Combined

$\begin{array}{lll}.13 & 7.47 & 1.31\end{array}$

4.39

.72

4.70 
Table 15 Continued

Recall

Incidental Intentional Mean

\begin{tabular}{lllllll} 
Ph.D. & $\bar{x}$ & S.D. & $\bar{x}$ & S.D. & $\bar{x}$ & S.D. \\
\hline 60 to 65 & & & & & & \\
$\quad$ Males & 3.13 & 3.94 & 4.25 & 4.40 & 3.69 & 2.99 \\
$\quad \begin{array}{l}\text { Females } \\
\text { Combined }\end{array}$ & 1.13 & 7.42 & 4.63 & 6.84 & 2.88 & 5.61 \\
Over Sex & 2.13 & 5.83 & 4.44 & 5.56 & 3.28 & 4.36
\end{tabular}

Combined over Age

$\begin{array}{lllllll}\text { Males } & .75 & 7.29 & 1.69 & 5.40 & 1.22 & 5.10 \\ \text { Females } & 1.50 & 6.21 & 4.06 & 4.82 & 2.78 & 4.17 \\ \text { Combined } & 1.13 & 6.67 & 2.88 & 5.18 & 2.00 & 4.65 \\ \text { Over Sex } & & & & & & \end{array}$

Combined over Ed

20 to 25

$\begin{array}{lllllll}\text { Males } & 1.56 & 8.30 & 1.93 & 5.72 & 1.75 & 5.54 \\ \text { Females } & 2.44 & 6.23 & 4.00 & 3.58 & 3.22 & 3.72 \\ \text { Combined } & 2.00 & 7.23 & 2.97 & 4.81 & 2.48 & 4.70 \\ \text { Over Sex } & & & & & & \end{array}$

60 to 65

Males

Females

4.88

4.73

5.38

4.54

Combined

2.38

6.27

2.88

7.04

2.63

5.25

Over Sex

4.13

6.14

3.88

5.99

4.00

5.02

Combined over Age

$\begin{array}{lllllll}\text { Males } & 3.72 & \% .33 & 3.41 & 5.38 & 3.56 & 5.31 \\ \text { Fernales } & 2.41 & 6.15 & 3.44 & 5.52 & 2.92 & 4.49 \\ \text { Combined } & 3.06 & 6.74 & 3.42 & 5.41 & 3.24 & 4.89 \\ \text { Over Sex } & & & & & & \end{array}$


Table 15 Continued

Recognition

\begin{tabular}{|c|c|c|c|c|c|c|}
\hline \multirow[b]{2}{*}{ HS } & \multicolumn{2}{|c|}{ Incidental } & \multicolumn{2}{|c|}{ Intentional } & \multicolumn{2}{|c|}{ Mean } \\
\hline & $\bar{x}$ & S.D. & $\bar{x}$ & S.D. & $\bar{x}$ & S.D. \\
\hline \multicolumn{7}{|l|}{20 to 25} \\
\hline Males & .52 & 9.75 & -1.26 & 6.28 & -.37 & 6.86 \\
\hline Females & -.61 & 4.06 & -1.12 & 3.27 & -.87 & 2.98 \\
\hline $\begin{array}{l}\text { Combined } \\
\text { Over Sex }\end{array}$ & -.05 & 7.24 & -1.19 & 4.83 & -.62 & 5.12 \\
\hline \multicolumn{7}{|l|}{60 to 65} \\
\hline Males & -9.43 & 8.67 & -1.49 & 7.64 & -5.46 & 6.56 \\
\hline Females & -.36 & 4.89 & 1.12 & 5.57 & .38 & 4.76 \\
\hline $\begin{array}{l}\text { Combined } \\
\text { Over Sex }\end{array}$ & -4.90 & 8.26 & -.18 & 6.60 & -2.54 & 6.30 \\
\hline
\end{tabular}

Combined over Age

$\begin{array}{lrrrrrr}\text { Males } & -4.46 & 10.29 & -1.37 & 6.76 & -2.92 & 7.00 \\ \text { Females } & -.49 & 4.35 & .00 & 4.56 & .24 & 3.89 \\ \text { Combined } & -2.47 & 8.03 & -.69 & 5.71 & -1.58 & 5.73 \\ \text { Over Sex } & & & & & & \end{array}$

Ph.D.

20 to 25

$\begin{array}{lrrrrrr}\text { Males } & -2.74 & 2.12 & -.25 & 1.27 & -1.49 & 1.13 \\ \text { Females } & -3.86 & 3.99 & .10 & 3.64 & -1.88 & 3.25 \\ \text { Combined } & -3.30 & 3.14 & -.07 & 2.64 & -1.69 & 2.36 \\ \text { Over sex } & & & & & & \end{array}$

60 to 65

$\begin{array}{lrrrrrr}\text { Males } & -3.99 & 5.50 & 1.63 & 5.78 & -1.18 & 3.97 \\ \text { Females } & -4.05 & 3.89 & 1.13 & 1.73 & 1.46 & 2.40 \\ \text { Combined } & -4.02 & 4.60 & 1.38 & 4.13 & -1.32 & 3.18 \\ \text { Over Sex } & & & & & & \end{array}$


Table 15 Continued

Recognition

\section{Incidental Intentional Mean}

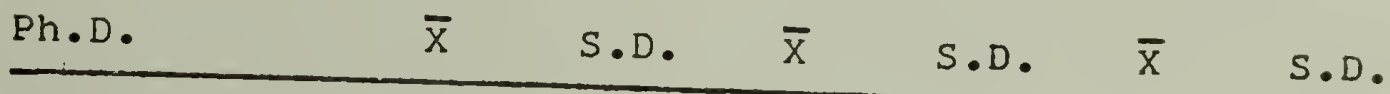

Combined over Age

$\begin{array}{lllllll}\text { Males } & -3.36 & 4.08 & .69 & 4.16 & -1.34 & 2.83 \\ \text { Females } & -3.96 & 3.81 & .61 & 2.80 & -1.67 & 2.77 \\ \text { Combined } & -3.66 & 3.89 & .65 & 3.49 & -1.50 & 2.76 \\ \text { Over Sex } & & & & & & \end{array}$

Combined over Ed

20 to 25

Males

Females

$$
-1.11
$$

Combined Over sex

$$
\begin{array}{r}
2.24 \\
-1.67
\end{array}
$$

$$
\begin{array}{ll}
7.02 & -.75 \\
4.24 & -.51
\end{array}
$$$$
-.75
$$$$
4.41
$$$$
-.93
$$$$
4.79
$$$$
3.40
$$$$
-1.38
$$$$
3.06
$$$$
5.73
$$$$
-.63
$$

3.87

$-1.15$

3.96

60 to 65

$\begin{array}{lrrrrrr}\text { Males } & -6.71 & 7.56 & .07 & 6.74 & -3.32 & 5.69 \\ \text { Females } & -2.21 & 4.68 & 1.12 & 3.99 & -.54 & 3.76 \\ \text { Combined } & -4.46 & 6.59 & .60 & 5.47 & -1.93 & 4.95 \\ \text { Over Sex } & & & & & & \end{array}$

Combined over Age

$\begin{array}{lrrrrrr}\text { Males } & -3.91 & 7.72 & -.34 & 5.62 & -2.13 & 5.31 \\ \text { Females } & -2.22 & 4.39 & .31 & 3.74 & -.96 & 3.40 \\ \text { Combined } & -3.07 & 6.29 & -.02 & 4.74 & -1.54 & 4.46 \\ \text { Over Sex } & & & & & & \end{array}$


2 (Task (Incidental vs. Intentional)) analysis of variance.

Summed over subjects, memory (recall vs. recognition), and task (incidental vs. intentional), there was a small tendency towards over-prediction (.85). Older subjects over-predicted more than younger subjects ( 1.03 vs. .67 ), but the Age main effect was nonsignificant. High school educated subjects overpredicted more than Ph.D. educated subjects ( 1.45 Vs. .25), but the Education main effect was also nonsignificant. Females over-predicted more than males (.98 vs. .72), but the Sex main effect was nonsignificant, as well. Likewise, the order main effect, and all interactions of between subject variables, were nonsignificant.

There was a slight mean tendency to under-predict memory for incidental items $(-.002)$, but a mean tendency to over-predict memory for intentional items $(+1.70)$, and the Task (Incidental vs. Intentional) main effect was statistically significant $(\underline{F}(1,48)=$ 7.74, $\mathrm{p}(.01)$. Additionally, the Task $x$ Education interaction was statistically significant $(\underline{F}(1,48)=$ $4.69, \mathrm{p}<.05)$, and may be seen in Figure 21 , which shows the mean difference in prediction and performance on incidental and intentional memory tests, by each Education group. High school educated subjects made 
Figure 21. Mean Differences in Prediction and Performance on Incidental and Intentional Memory Tests by Each Education Group

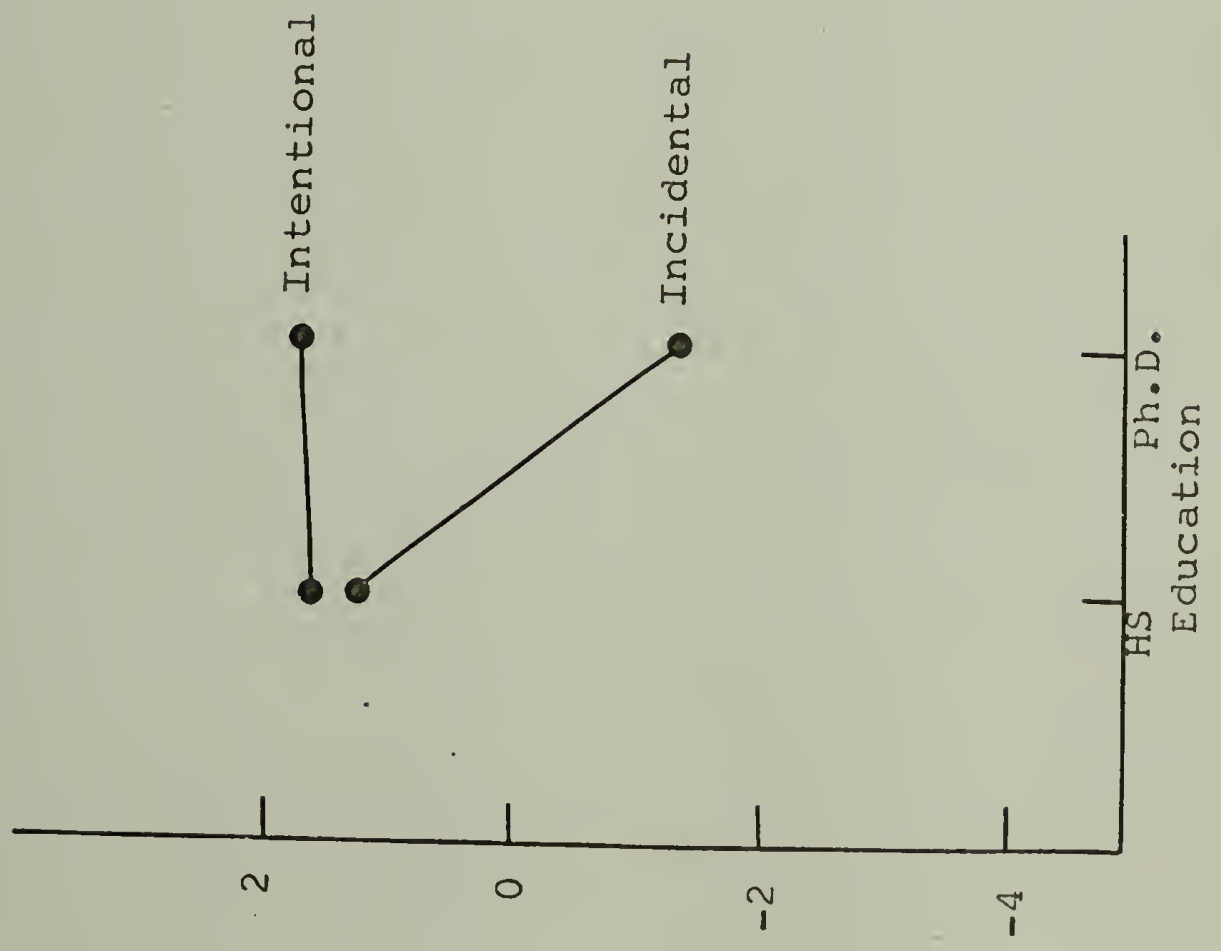

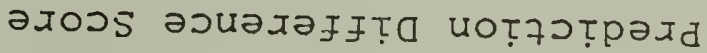


comparable over-predictions of performance on incidental and intentional memory tests, while $\mathrm{Ph} . \mathrm{D}$. educated subjects over-predicted memory of intentional items, but under-predicted memory of incidental items.

There was a mean tendency to over-predict recall performance $(+3.24$, but a mean tendency to under-predict recognition performance $(-1.54)$, and the Memory (Recall vs. Recognition) main effect was statistically significant $(\underline{F}(1,48)=42.24, \underline{p}(.001)$.

The Memory $x$ Task interaction was also statistically significant $(\underline{F}(1,48)=4.62, \underline{p}<.05)$, and may be seen in Figure 22, which shows the mean difference in prediction and performance on incidental and intentional recall and recognition memory test. For recall, comparable over-predictions were observed for incidental and intentional items. For recognition, however, significantly greater under-predictions were observed for incidental than intentional items $(\underline{t}(63)=3.66, \underline{\mathrm{p}}<$ $.001)$.

c. Absolute Differences Between Prediction and Performance

Mean absolute values of differences in prediction and performance were calculated to provide an estimate of prediction accuracy. The means of these prediction accuracy scores on recall and recognition of incidental and intentional items are shown in Table 16. These data 
Figure 22. Mean Differences in Prediction and Performance on Incidental and Intentional Recall and Recognition Tests

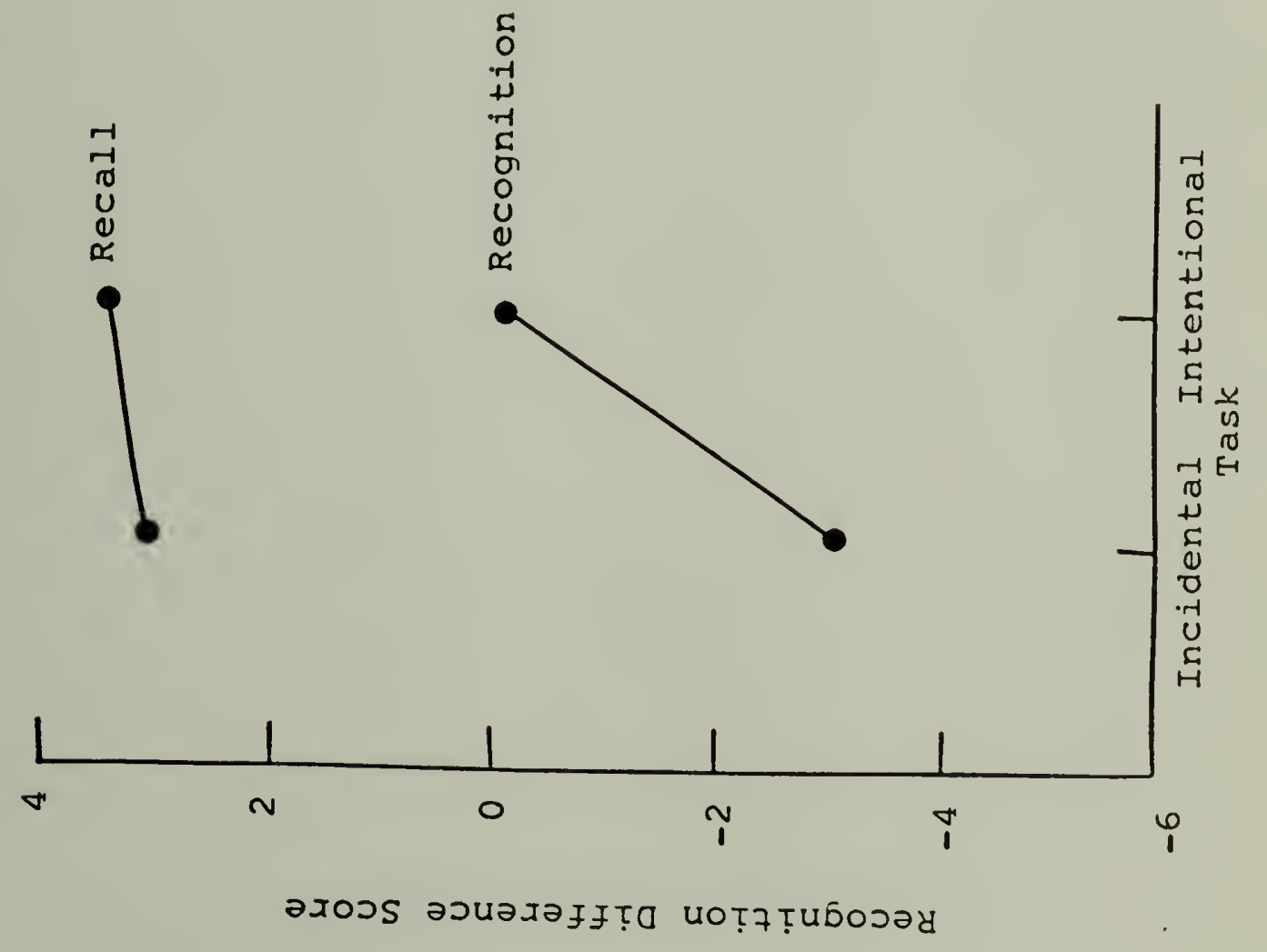


Table 16

Mean Prediction Accuracy Scores on Recill and Recognition of Incidental and Intentional Items by Each Age $x$ Education $x$ Sex Group

\begin{tabular}{|c|c|c|c|c|c|c|}
\hline \multirow[b]{3}{*}{ HS } & \multicolumn{6}{|c|}{ Recall } \\
\hline & \multicolumn{2}{|c|}{ Incidental } & \multicolumn{2}{|c|}{ Intentional } & \multicolumn{2}{|c|}{ Mean } \\
\hline & $\bar{x}$ & $S . D$. & $\bar{x}$ & $S \cdot D$ & $\bar{x}$ & S.D. \\
\hline \multicolumn{7}{|l|}{20 to 25} \\
\hline Males & 6.25 & 4.50 & 4.75 & 4.89 & 5.50 & 3.94 \\
\hline Females & 6.25 & 4.56 & 5.00 & 4.38 & 5.63 & 3.62 \\
\hline $\begin{array}{l}\text { Combined } \\
\text { Over Sex } \\
\end{array}$ & 6.25 & 4.37 & 4.88 & 4.49 & 4.25 & 4.10 \\
\hline \multicolumn{7}{|l|}{60 to 65} \\
\hline Males & 8.63 & 6.00 & 6.00 & 4.60 & 7.31 & 4.96 \\
\hline Females & 3.88 & 4.85 & 5.63 & 4.21 & 4.75 & 3.13 \\
\hline $\begin{array}{l}\text { Combined } \\
\text { Over Sex }\end{array}$ & 6.25 & 5.81 & 5.81 & 4.26 & 4.72 & 5.66 \\
\hline
\end{tabular}

Combined over Age

Males

Females

Combined Over Sex
7.34

5.06

6.25
5.27

4.71

5.06
5.38

5.31

5.34
4.63

4.16

4.33
6.41

5.19

5.80
4.42

3.30

3.89

$\mathrm{Ph} \cdot \mathrm{D}$.

20 to 25

Males

Females

Combined Over Sex

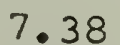

5.13

4.38

2.67

3.50

1.51

5.88

2.50

4.63

2.62

3.94

2.1

4.06

1. 32

6.00

4.18

4.97

2.15 
Table 16 Continued

\section{Recall}

\begin{tabular}{lllllll} 
& \multicolumn{2}{c}{ Incidental } & \multicolumn{2}{c}{ Intentional } & \multicolumn{2}{c}{ Mean } \\
Ph.D. & $\bar{x}$ & S.D. & $\bar{x}$ & S.D. & $\bar{x}$ & S.D. \\
\hline 60 to 65 & & & & & & \\
Males & 3.63 & 3.42 & 4.25 & 4.40 & 3.94 & 2.60 \\
Females & 5.38 & 4.84 & 6.38 & 4.98 & 5.88 & 4.32 \\
$\quad \begin{array}{l}\text { Combined } \\
\text { Over Sex }\end{array}$ & 4.50 & 4.15 & 5.31 & 4.67 & 4.91 & 3.58
\end{tabular}

Combined over Age

$\begin{array}{lllllll}\text { Males } & 5.50 & 4.63 & 4.31 & 3.52 & 4.91 & 2.66 \\ \text { Females } & 5.00 & 3.78 & 4.94 & 3.86 & 4.97 & 3.22 \\ \text { Combined } & 5.25 & 4.17 & 4.63 & 3.64 & 4.94 & 2.91 \\ \text { Over Sex } & & & & & & \end{array}$

Combined over Ed 20 to 25

$\begin{array}{lllllll}\text { Males } & 6.81 & 4.69 & 4.56 & 3.81 & 5.69 & 3.19 \\ \text { Females } & 5.44 & 3.69 & 4.25 & 3.26 & 4.84 & 2.76 \\ \text { Combined } & 6.13 & 4.21 & 4.41 & 3.49 & 5.27 & 2.97 \\ \text { Over Sex } & & & & & & \end{array}$

60 to 65

Males

Females $6.13 \quad 5.38$ $5 \cdot 13$ 4.44 5.63 4.20

Combined Over Sex

4.63

4.75

6.00

4.47

$5.31 \quad 3.69$

$5.38 \quad 5.05$

5.56

4.41

$5.47 \quad 3.89$

Combined over Age

$\begin{array}{lllllll}\text { Males } & 6.47 & 4.98 & 4.84 & 4.08 & 5.66 & 3.67 \\ \text { Females } & 5.03 & 4.20 & 5.13 & 3.95 & 5.08 & 3.21 \\ \text { Combined } & 5.75 & 4.63 & 4.98 & 3.99 & 5.37 & 3.43 \\ \text { Over Sex } & & & & & & \end{array}$


Table 16 Continued

Recognition

\begin{tabular}{llllllll} 
& \multicolumn{2}{c}{ Incidental } & \multicolumn{2}{c}{ Intentional } & \multicolumn{2}{c}{ Mean } \\
& $\bar{x}$ & S.D. & $\bar{x}$ & S.D. & $\bar{x}$ & S.D. \\
\hline $\begin{array}{l}\text { to } 25 \\
\quad \text { Males }\end{array}$ & 6.01 & 7.36 & 5.26 & 3.09 & 5.64 & 3.87 \\
$\quad \begin{array}{l}\text { Females } \\
\text { Combined } \\
\text { Over Sex }\end{array}$ & 3.12 & 2.41 & 2.13 & 2.63 & 2.63 & 1.38 \\
& 4.56 & 5.50 & 3.70 & 3.21 & -.62 & 5.12
\end{tabular}

60 to 65

$\begin{array}{lrrrrrr}\text { Males } & 11.44 & 5.24 & 6.38 & 3.80 & 8.91 & 3.58 \\ \text { Females } & 3.37 & 3.33 & 4.38 & 3.25 & 3.88 & 3.10 \\ \text { Combined } & 7.41 & 5.94 & 5.38 & 3.57 & -2.54 & 6.30 \\ \text { Over sex } & & & & & & \end{array}$

Combined over Age

Males

Females

Combined Over Sex
8.72

3.25

5.98
6.78

2.81

5.81
5.82

3.25

5.54
3.39

3.08

3.45
7.27

3.25

5.26
3.90

2.41

3.78

Ph. D.

20 to 25

$\begin{array}{lllllll}\text { Males } & 2.74 & 2.11 & .74 & 1.03 & 1.74 & 1.22 \\ \text { Females } & 4.37 & 3.34 & 2.39 & 2.59 & 3.38 & 2.44 \\ \text { Combined } & 3.56 & 2.82 & 1.57 & 2.08 & 2.56 & 2.05 \\ \text { Over Sex } & & & & & & \end{array}$

60 to 65

Males

Females

Combined Over Sex

$\begin{array}{llllll}4.75 & 4.76 & 4.63 & 3.46 & 4.69 & 3.58 \\ 4.06 & 3.88 & 1.63 & 1.19 & 2.85 & 2.07 \\ 4.41 & 4.21 & 3.13 & 2.94 & 3.77 & 2.98\end{array}$


Table 16 Continued

Recognition

\begin{tabular}{llllllll}
\multicolumn{1}{c}{} & \multicolumn{2}{c}{ Incidental } & \multicolumn{2}{c}{ Intentional } & \multicolumn{2}{c}{ Mean } \\
Ph.D. & $\overline{\mathrm{x}}$ & S.D. & $\overline{\mathrm{x}}$ & S.D. & $\overline{\mathrm{x}}$ & S.D. \\
\hline Combined over & Age & & & & & \\
$\quad$ Males & 3.75 & 3.71 & 2.69 & 3.18 & 3.22 & 3.00 \\
Females & 4.22 & 3.50 & 2.01 & 1.98 & 3.11 & 2.20 \\
$\begin{array}{l}\text { Combined } \\
\text { Over Sex }\end{array}$ & 3.98 & 3.55 & 2.35 & 2.63 & 3.16 & 2.59
\end{tabular}

Combined over Ed

20 to 25

$\begin{array}{lllllll}\text { Males } & 4.38 & 5.50 & 3.00 & 3.22 & 3.69 & 3.33 \\ \text { Females } & 3.75 & 2.88 & 2.26 & 2.52 & 3.00 & 1.95 \\ \text { Combined } & 4.06 & 4.33 & 2.63 & 2.87 & 3.35 & 2.71 \\ \text { Over Sex } & & & & & & \end{array}$

60 to 65

$\begin{array}{lllllll}\text { Males } & 8.09 & 5.94 & 5.50 & 3.63 & 6.80 & 4.09 \\ \text { Females } & 3.72 & 3.51 & 3.01 & 2.76 & 3.36 & 2.60 \\ \text { Combined } & 5.91 & 5.29 & 4.25 & 3.41 & 5.08 & 3.80 \\ \text { Over Sex } & & & & & & \end{array}$

Combined over Age

$\begin{array}{lllllll}\text { Males } & 6.23 & 5.94 & 4.25 & 3.61 & 5.24 & 3.99 \\ \text { Females } & 3.73 & 3.16 & 2.63 & 2.63 & 3.18 & 2.27 \\ \text { Combined } & 4.98 & 4.88 & 3.44 & 3.23 & 4.21 & 3.39 \\ \text { Over Sex } & & & & & & \end{array}$


were submitted to a 2 (Age) $\times 2$ (Education) $\times 2$ (Sex) x 2 (Order) x 2 (Memory (Recall vs. Recognition)) x 2 (Task (Incidental vs. Intentional)) analysis of variance.

Younger subjects were more accurate in predicting memory performance than older subjects (4.31 vs. 5.27), and the Age main effect was marginally significant $(\underline{F}(I, 48)=3.24, \underline{p}<.08)$. Ph.D. educated subjects were more accurate than HS educated subjects (4.05 vs. 5.53), and the Education main effect was statistically significant $(\underline{F}(1,48)=7.55, \underline{D}<.01)$. Females were more accurate than males ( 4.13 vs. 5.45), and the sex main effect was also statistically significant $(E(1,48)$ $=6.03, \mathrm{p}(.05)$. The order main effect was nonsignificant.

The Education $x$ Sex interaction was statistically significant $(\underline{F}(1,48)=5.84, \underline{p}<.05)$, and may be seen in Figure 23, which shows the mean prediction accuracy scores on episodic memory tasks by males and females in each Education group. High school educated males were significantly less accurate in predicting memory performarice than any other Education $x$ sex group (all ps (.01). While for HS subjects, males predicted significantiy more poorly than females $(\underline{t}(30)=2.82, \mathrm{p}<$ .001), for Ph.D. subjects there was no sex difference in accuracy of predicting memory performance. Further- 
Figure 23. Mean Prediction Accuracy Scores on Episodic Memory Tasks for Males and Females in Each Education Group

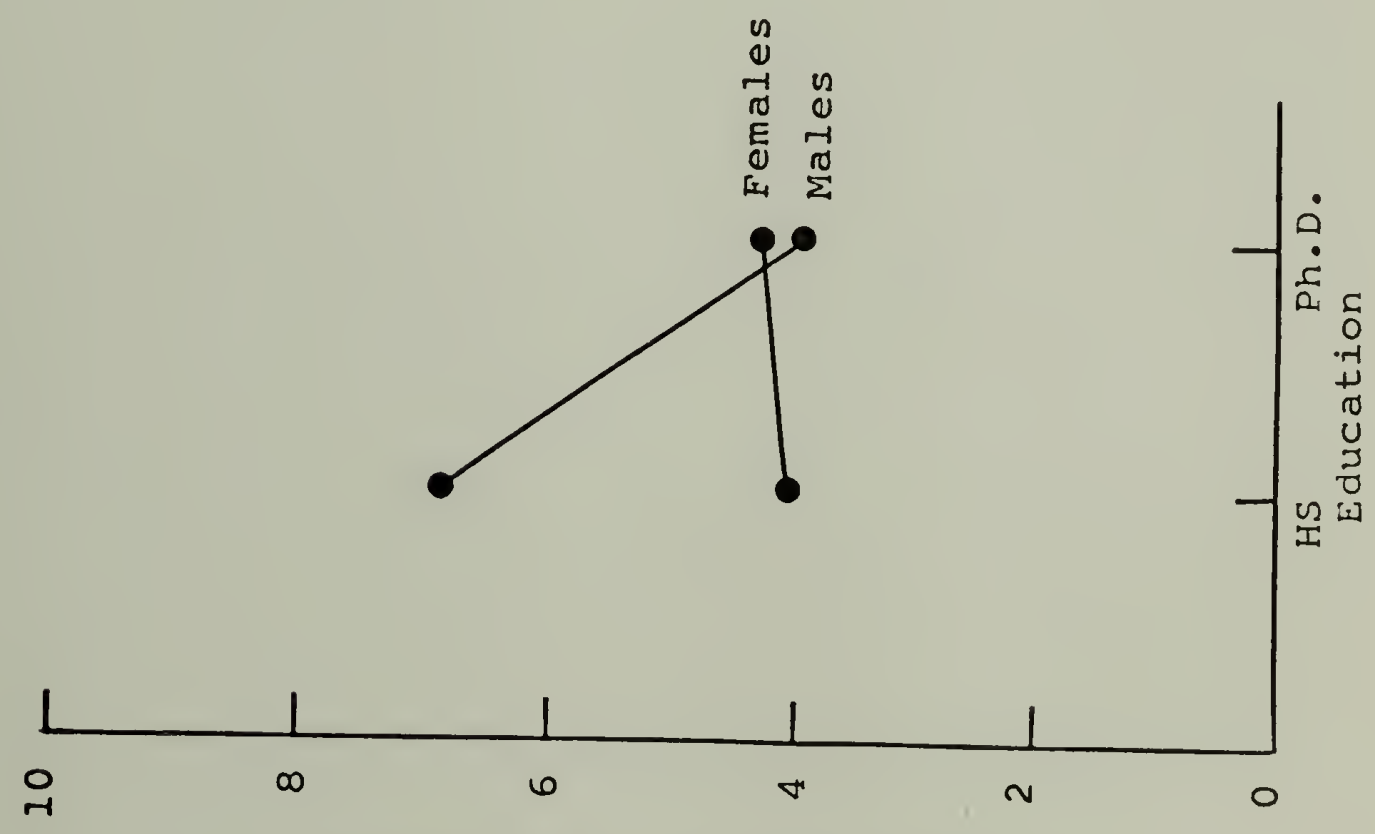

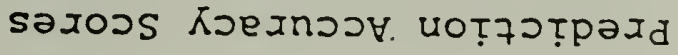


more, while for males less education was associated with poorer prediction $(\underline{t}(30)=3.10, \underline{\mathrm{p}}<.01)$, for females education did not have an effect on prediction accuracy .

Prediction of intentional items was more accurate than prediction of incidental items (4.21 vs. 5.37), and the Task (Incidental vs. Intentional) main effect was statistically significant $(\underline{\mathrm{F}}(1,48)=5.63, \mathrm{p}<.05)$. Prediction of recognition was more accurate than prediction of recall ( 4.21 vs. 5.37), and the Memory main effect was marginally significant $(\underline{F}(1,48)=3.98, \underline{p}<$ $.06)$.

2. Semantic Memory

a. Recognition Predictions

Mean predictions of the number of general information knowledge facts to be correctly recognized by each Age $x$ Education $x$ sex group are shown in Table 17. These data were submitted to a 2 (Age) $\times 2$ (Education) $\times 2$ (Sex) $\times 2$ (Order) analysis of variance. Predictions of older subjects were higher than those of younger subjects (19.38 vs. 17.03), and the Age main effect was statistically significant $(E(1,48)$ $=5.09, \underline{p}(.05)$. Predictions of $\mathrm{HS}$ and $\mathrm{Ph} . \mathrm{D}$. educated subjects were almost identical (18.25 vs. 18.16), and the Education main effect was nonsignificant. Predictions of males were higher than females (19.69 vs. 


\section{Table 17}

Mean Predictions of the Number of General Information Knowledge Facts to be correctly Recognized by Each Age $x$ Education $x$ Sex Group

\begin{tabular}{|c|c|c|c|c|}
\hline \multicolumn{2}{|c|}{ HS } & \multicolumn{2}{|c|}{$\mathrm{Ph} \cdot \mathrm{D}$. } & $\begin{array}{l}\text { Combined Ove } \\
\text { Education }\end{array}$ \\
\hline & $S . D$. & $\bar{x}$ & $S . D$. & $S \cdot D$. \\
\hline
\end{tabular}

\section{0 to 25}

Males

Females

Combined

Over Sex

$\begin{array}{llllll}21.88 & 2.10 & 17.63 & 3.16 & 19.75 & 3.40 \\ 11.75 & 6.75 & 16.88 & 3.80 & 14.31 & 5.92 \\ 16.81 & 7.12 & 17.25 & 3.40 & 17.03 & 5.49\end{array}$

60 to 65

Males

Females

$19.13 \quad 3.94$

$20.13 \quad 3.04$

$19.63 \quad 3.44$

Combined

Over Sex

$$
\begin{aligned}
& 20.25 \\
& 19.69 \\
& \text { Age }
\end{aligned}
$$

Males

$$
20.0
$$

3.37

$18.88 \quad 3.26$

19.69

3.36

Females

16.00

6.43

$1.7 .44 \quad 5.24$

$16.72 \quad 5.93$

Combined

Over sex

$18.25 \quad 5.66$

$18.16 \quad 4.36$

$18.20 \quad 5.01$ 
16.72), and the sex main effect was statistically significant $(\underline{E}(1,48)=8.17, \underline{p}<.01)$. The order main effect was nonsignificant.

The Age $x$ sex interaction was statistically significant $(\underline{F}(1,48)=5.65, \underline{p}<.05)$, and may be seen in Figure 24, which shows the mean predictions for general information recognition by males and females in each Age group. Young females predicted they would correctly recognize fewer general information facts than any of the other Age $x$ sex groups (all ps $<.05)$. While for younger subjects, females made significantly lower predictions than males $(\underline{t}(30)=$ $3.19, \mathrm{p}(.01)$, for older subjects there were no sex differences in predictions. Furthermore, while for females, greater age was associated with significantly higher predictions $(\underline{t}(30)=2.48, \underline{p}<.05)$, age was not a significant factor in males' predictions. The Education $x$ Age $x$ Sex interaction was also statistically significant $(\mathrm{F}(1,48)=9.23, \mathrm{p}<.01)$, and may be seen in Figure 25, which shows the mean predictions of general information recognition by males and females in each Age $x$ Education group. The predictions of younger HS males were significantly higher than those of any of the other young groups (all ps $<.01)$, while the predictions of females were lower than those of any other group (all ps $<.09)$. 
Figure 24. Mean Predictions for General Information Recognition by Males and Females in Each Age Group

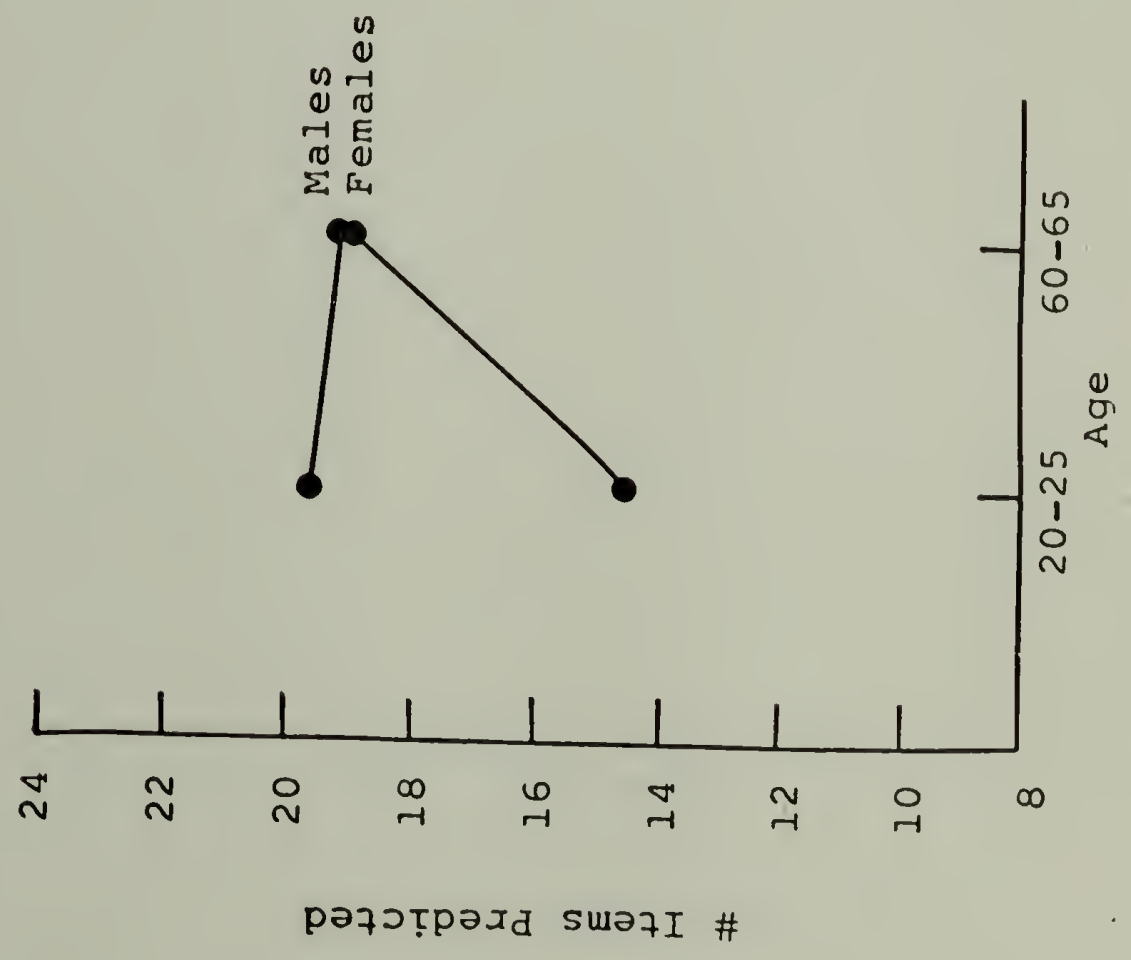


Figure 25. Mean Predictions of General Information Recognition by Males and Females in Each Age $x$ Education Group

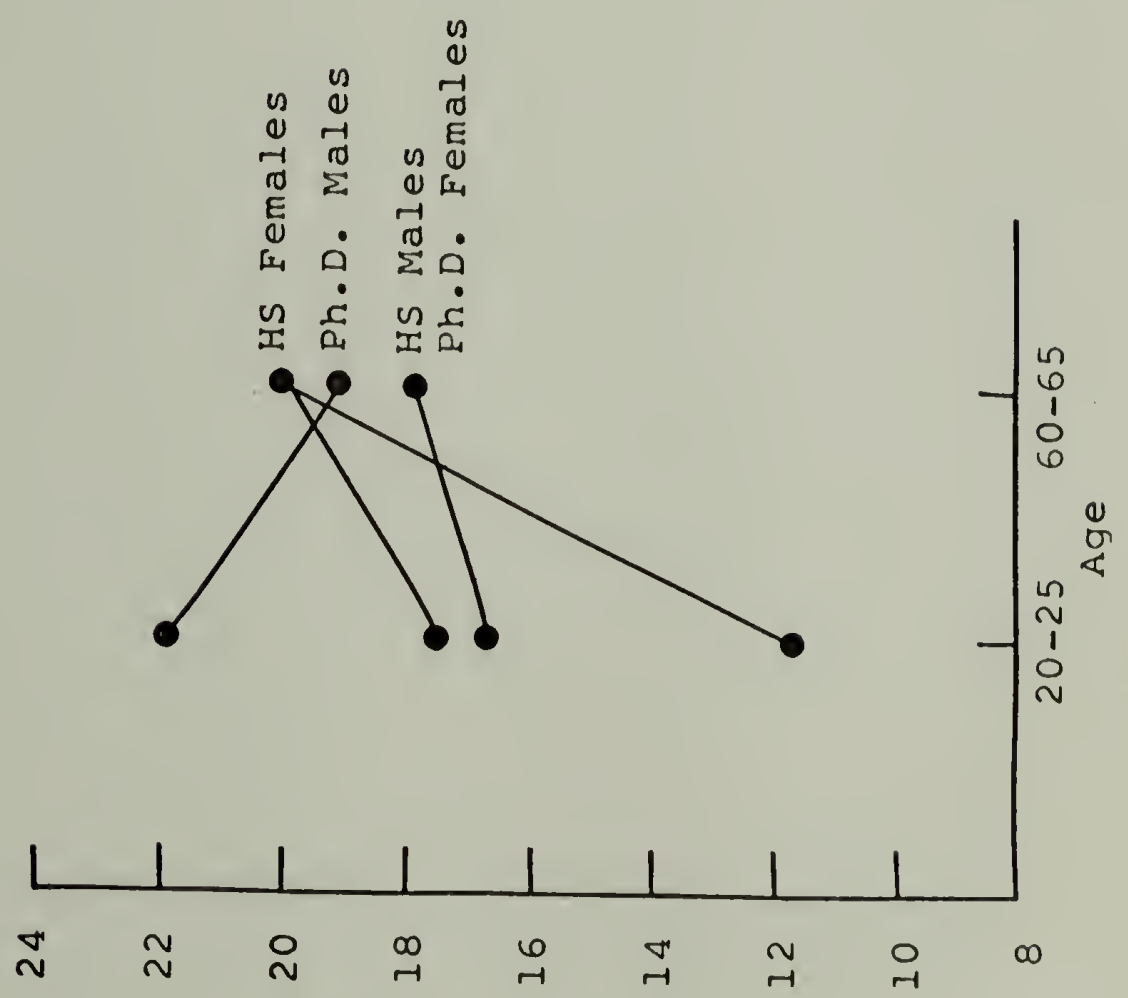

рә7วтุрวлd sแว7I \# 
b. Differences between Prediction and Performance Mean difference scores of prediction and performance were calculated to evaluate over- and underprediction. The mean difference scores on general information knowledge recognition by each Age $x$ Education $x$ sex group are shown in Table 18. These data were submitted to a 2 (Age) $\times 2$ (Education) $\times 2$ (Sex) $x 2$ (order) analysis of variance.

Summed over subjects, there was a small tendency towards over-prediction of recognition performance (.71). Older subjects over-predicted more than younger subjects ( 1.31 vs..II), but the Age main effect was nonsignificant. While HS educated subjects overpredicted recognition performance $(+1.62)$, Ph.D. educated subjects under-predicted it (-.19), and the Education main effect was marginally significant $(\underline{F}(1,48)=3.10, \underline{\mathrm{p}}<.09)$. While males over-predicted recognition performance $(+1.15)$, females under-predicted it (-.09), but the sex main effect was not significant. Subjects tested after being given the memory questionnaire under-predicted performance (-.55) while subjects tested before being given the memory questionnaire over-predicted performance (1.97); the Order main effect was statistically significant $(\underline{F}(1,48)$ $=5.99, \mathrm{p}(.05)$. All interactions were nonsignificant. 


\section{Table 18}

Mean Difference scores of Prediction and Performance on General Information Knowledge Recognized by Each Age x Education $x$ Sex Group

\begin{tabular}{llll} 
HS & Ph.D. & $\begin{array}{c}\text { Combined Over } \\
\text { Education }\end{array}$ \\
\hline
\end{tabular}

20 to 25

Males

Females

$\begin{array}{rrrrrr}4.24 & 2.91 & -.50 & 3.33 & 1.87 & 3.89 \\ -3.12 & 6.20 & -.17 & 5.51 & -1.64 & 5.87 \\ .56 & 6.03 & -.33 & 4.40 & .11 & 5.22\end{array}$

Combined

Over Sex

60 to 65

$\begin{array}{lllrlll}\text { Males } & 1.95 & 4.23 & .37 & 2.68 & 1.16 & 3.52 \\ \text { Females } & 3.93 & 2.87 & -.48 & 5.39 & 1.46 & 4.63 \\ \text { Combined } & 2.67 & 3.57 & -.06 & 4.13 & 1.31 & 4.04 \\ \text { Over Sex } & & & & \end{array}$

Combined over Age

$\begin{array}{lrrrrrr}\text { Males } & 3.10 & 3.70 & -.07 & 2.96 & 1.51 & 3.67 \\ \text { Females } & .13 & 5.76 & -.32 & 5.27 & -.09 & 5.43 \\ \text { Combined } & 1.62 & 4.99 & -.19 & 4.20 & .71 & 4.67 \\ \text { Over Sex } & & & & & \end{array}$




\section{c. Absolute Difference Between Predictjon and}

\section{Performance}

Mean absolute values of differences in prediction and performance were calculated to provide an estimate of prediction accuracy. The means of these prediction accuracy scores on general information recognition by each Age $x$ Education $x$ Sex group are shown in Table 19. These data were submitted to a 2 (Age) $\times 2$ (Education) $\times 2$ (Sex) $\times 2$ (Order) analysis of variance.

Older subjects predicted more accurately than younger subjects ( 3.27 vs. 4.00$)$, but the Age main effect was nonsignificant. Ph.D. educated subjects predicted more accurately than HS educated subjects ( 3.05 vs. 4.22), but the Education main effect was nonsignificant. Males predicted more accurately than females ( 3.17 vs. 4.10 ) but the sex main effect was also nonsignificant. Likewise, the order main effect, and all interactions were nonsignificant.

\section{d. probability Correct Responding Given Positive} and Negative Feeling of Knowing Judgments

The mean probability of a correct response on true and false items, given positive and negative feeling of knowing judgments, for each Age $x$ Education $x$ Sex group is shown in Table 20. These data were submitted to a 2 (Age) $\times 2$ (Education) $\times 2$ (Sex) 
Table 19

Mean Prediction Accuracy Scores on General

Information Knowledge Recognition by

Each Age $x$ Education $x$ Sex Group

\begin{tabular}{lllllll} 
& \multicolumn{2}{c}{ HS } & \multicolumn{2}{c}{ Ph.D. } & \multicolumn{2}{c}{$\begin{array}{c}\text { Combined Ove } \\
\text { Education }\end{array}$} \\
& $\bar{x}$ & S.D. & $\bar{x}$ & S.D. & $\bar{x}$ & S.D. \\
\hline $\begin{array}{l}\text { to } 25 \\
\text { Males }\end{array}$ & 1.30 & 2.81 & 2.74 & 1.67 & 3.52 & 2.38 \\
Females & 5.37 & 4.08 & 3.56 & 3.99 & 4.47 & 4.01 \\
Combined & 4.84 & 3.43 & 3.15 & 2.98 & 4.00 & 3.28 \\
Over Sex & & & & & &
\end{tabular}

60 to 65

Males

Females

$3.52 \quad 2.84$

2.10

1.52

2.81

2.32

$3.68 \quad 2.43$

$3.78 \quad 3.60$

$3.73 \quad 2.97$

Combined

Over Sex

$3.60 \quad 2.55$

2.94

2.81

$3.27 \quad 2.66$

Combined over Age

$\begin{array}{lllllll}\text { Males } & 3.91 & 2.76 & 2.42 & 1.58 & 3.17 & 2.34 \\ \text { Females } & 4.53 & 3.36 & 3.67 & 3.67 & 4.10 & 3.49 \\ \text { Combined } & 4.22 & 3.04 & 3.05 & 2.85 & 3.63 & 2.98 \\ \text { Over Sex } & & & & & & \end{array}$


Table 20

Mean Probability of a Correct Response on True and False Items Given Positive and Negative Feeling of knowing Judgments for Each Age $x$ Education $x$ Sex Group

Positive

\begin{tabular}{lllllll} 
HS & \multicolumn{3}{c}{ True } & \multicolumn{2}{c}{ False } & \multicolumn{2}{c}{ Total } \\
\hline 20 to 25 & & S.D. & $\bar{x}$ & S.D. & $\bar{x}^{2}$ & S.D \\
Males & .75 & .13 & .74 & .07 & .74 & .09 \\
Females & .74 & .19 & .72 & .12 & .73 & .11 \\
$\begin{array}{l}\text { Combined } \\
\text { Over Sex }\end{array}$ & .74 & .16 & .73 & .10 & .74 & .10
\end{tabular}

60 to 65

$\begin{array}{lllllll}\text { Males } & .76 & .14 & .81 & .14 & .78 & .11 \\ \text { Females } & .75 & .13 & .83 & .18 & .79 & .11 \\ \text { Combined } & .75 & .13 & .82 & .16 & .79 & .11\end{array}$

Combined over Age

$\begin{array}{lllllll}\text { Males } & .75 & .13 & .78 & .11 & .76 & .10 \\ \text { Females } & .74 & .16 & .78 & .16 & .76 & .11 \\ \text { Combined } & .75 & .14 & .78 & .14 & .76 & .10 \\ \text { Over Sex } & & & & & & \end{array}$

Ph.D.

20 to 25

$\begin{array}{lllllll}\text { Males } & .80 & .11 & .91 & .06 & .86 & .07 \\ \text { Females } & .81 & .10 & .80 & .10 & .81 & .70 \\ \text { Combined } & .81 & .11 & .86 & .10 & .83 & .07 \\ \text { Over Sex } & & & & & & \end{array}$


Table 20 Continued

Positive

\begin{tabular}{lllllll}
\multicolumn{1}{c}{ Ph.D. } & $\overline{\mathrm{x}}$ & S.D. & $\overline{\mathrm{x}}$ & S.D. & $\overline{\mathrm{x}}$ & S.D. \\
\hline 60 to 65 & & & & & & \\
$\quad$ Males & .85 & .06 & .90 & .06 & .87 & .02 \\
Females & .81 & .13 & .82 & .12 & .82 & .11 \\
$\begin{array}{l}\text { Combined } \\
\text { Over Sex }\end{array}$ & .83 & .10 & .86 & .10 & .85 & .08
\end{tabular}

Combined over Age

$\begin{array}{lllllll}\text { Males } & .83 & .09 & .90 & .06 & .86 & .05 \\ \text { Females } & .81 & .11 & .81 & .11 & .81 & .09 \\ \text { Combined } & .82 & .10 & .86 & .10 & .84 & .08 \\ \text { Over Sex } & & & & & & \end{array}$

Combined over Ed 20 to 25

$\begin{array}{lllllll}\text { Males } & .77 & .12 & .83 & .11 & .80 & .10 \\ \text { Females } & .77 & .15 & .76 & .12 & .77 & .10 \\ \text { Combined } & .77 & .14 & .80 & .11 & .78 & .10 \\ \text { Over Sex } & & & & & & \end{array}$

60 to 65

$\begin{array}{lllllll}\text { Males } & .80 & .11 & .85 & .11 & .83 & .09 \\ \text { Females } & .78 & .13 & .83 & .15 & .80 & .11 \\ \text { Combined } & .79 & .12 & .84 & .13 & .82 & .10 \\ \text { Over Sex } & & & & & & \end{array}$

Combined over Age

$\begin{array}{lllllll}\text { Males } & .79 & .12 & .84 & .11 & .81 & .09 \\ \text { Females } & .78 & .14 & .79 & .14 & .79 & .10 \\ \text { Combined } & .78 & .13 & .82 & .13 & .80 & .10 \\ \text { Over Sex } & & & & & & \end{array}$


Table 20 Continued

Negative

\begin{tabular}{lllllll}
\multicolumn{1}{c}{ HS } & $\bar{x}$ & \multicolumn{2}{c}{ True } & \multicolumn{2}{c}{ Total } \\
20 to 25 & & & & & & \\
$\quad$ Males & .46 & .36 & .46 & .42 & .46 & .39 \\
Females & .45 & .21 & .65 & .21 & .55 & .09 \\
Combined & .45 & .28 & .55 & .34 & .50 & .28 \\
Over Sex & .45 & & & & & S.D.
\end{tabular}

60 to 65

Males

Females

Combined

Over Sex

$\begin{array}{llllll}.43 & .33 & .55 & .29 & .49 & .25 \\ .36 & .33 & .56 & .34 & .46 & .24 \\ .39 & .32 & .55 & .31 & .47 & .24\end{array}$

Combined over Age

$\begin{array}{lllllll}\text { Males } & .45 & .34 & .50 & .36 & .47 & .31 \\ \text { Females } & .40 & .27 & .60 & .28 & .50 & .18 \\ \text { Combined } & .42 & .30 & .55 & .32 & .49 & .25 \\ \text { Over Sex } & & & & & & \end{array}$

$\mathrm{Ph} \cdot \mathrm{D}$.

20 to 25

$\begin{array}{lllllll}\text { Males } & .63 & .17 & .66 & .20 & .65 & .13 \\ \text { Females } & .45 & .32 & .66 & .22 & .56 & .10 \\ \text { Combined } & .54 & .27 & .66 & .20 & .60 & .12 \\ \text { Over Sex } & & & & & & \end{array}$

60 to 65

$\begin{array}{lllllll}\text { Males } & .68 & .34 & .71 & .35 & .69 & .17 \\ \text { Females } & .30 & .40 & .54 & .38 & .42 & .25 \\ \text { Combined } & .49 & .41 & .62 & .36 & .56 & .25 \\ \text { Over Sex } & & & & & & \end{array}$


Table 20 Continued

Negative

\begin{tabular}{|c|c|c|c|c|c|c|}
\hline \multirow{2}{*}{$\mathrm{Ph} \cdot \mathrm{D}$. } & \multicolumn{2}{|c|}{ True } & \multicolumn{2}{|c|}{ False } & \multicolumn{2}{|c|}{ Total } \\
\hline & $\bar{x}$ & S.D. & $\bar{x}$ & $S \cdot D$. & $\bar{x}$ & S.D \\
\hline \multicolumn{7}{|c|}{ Combined over Age } \\
\hline Males & .66 & .26 & .68 & .27 & .67 & .15 \\
\hline Females & .38 & .36 & .60 & .30 & .49 & .20 \\
\hline $\begin{array}{l}\text { Combined } \\
\text { Over Sex }\end{array}$ & .52 & .34 & .64 & .29 & .58 & .20 \\
\hline
\end{tabular}

Combined over Ed 20 to 25

$\begin{array}{lllllll}\text { Males } & .55 & .29 & .56 & .34 & .55 & .30 \\ \text { Females } & .45 & .26 & .66 & .21 & .55 & .09 \\ \text { Combined } & .50 & .27 & .61 & .28 & .55 & .22\end{array}$

60 to 65

$\begin{array}{lllllll}\text { Males } & .56 & .35 & .63 & .32 & .59 & .23 \\ \text { Females } & .33 & .36 & .55 & .35 & .44 & .24 \\ \begin{array}{l}\text { Combined } \\ \text { Over Sex }\end{array} & .44 & .37 & .59 & .33 & .52 & .24\end{array}$

Combined over Age

$\begin{array}{lllllll}\text { Males } & .55 & .32 & .59 & .33 & .57 & .26 \\ \text { Females } & .39 & .31 & .60 & .29 & .50 & .19 \\ \text { Combined } & .47 & .32 & .60 & .30 & .53 & .23 \\ \text { Over Sex } & & & & & & \end{array}$


x 2 (Order) $\times 2$ (Item (True vs. False)) $\times 2$ (Prediction (Positive vs. Negative)) analysis of variance. While the mean probability of making a correct response given a positive feeling of knowing juagment was .80 , it was only .53 given a negative feeling of knowing judgment, and the Prediction main effect was highly significant $(\underline{F}(1,48)=77.90, \underline{P}<.001)$. Prediction did not interact significantly with any other variables, however,

e. Differences in Probability correct Responding Given Positive and Neqative Feeling of Knowing Judgments

The mean differences in probability correct responding given positive or negative feeling of knowing judgments were calculated to evaluate feeling of knowing accuracy. These difference scores for true and false items are shown in Table 21. These data were submitted to a 2 (Age) $\times 2$ (Education) $\times 2$ (Sex) $\times 2$ (Order) x 2 (Item (True vs. False)) analysis of variance.

The difference in probability correct responding given positive and negative feeling of knowing judgments was greater for older than younger subjects (.30 vs. .23) but the Age main effect was nonsignificant. The difference in probability correct responding given positive and negative feeling of knowing judgments was very similar for HS and Ph.D. subjects (.28 vs. .26), and the Education main effect was nonsignificant. The difference in probability correct responding given positive 
Table 21

Mean Difference of Probability Correct Responding On True and False Items Given Positive or Negative Feeling of Knowing Judgments

\begin{tabular}{|c|c|c|c|c|c|c|}
\hline \multirow{2}{*}{ HS } & \multicolumn{2}{|c|}{ True } & \multicolumn{2}{|c|}{ False } & \multicolumn{2}{|c|}{ Total } \\
\hline & $\bar{x}$ & $S \cdot D$. & $\bar{x}$ & S.D. & $\bar{x}$ & $S . D$ \\
\hline 0 to 25 & & & & & & \\
\hline Males & .28 & .38 & .29 & .42 & .29 & .39 \\
\hline Females & .29 & .22 & .08 & .25 & .18 & .15 \\
\hline $\begin{array}{l}\text { Combined } \\
\text { Over Sex }\end{array}$ & .29 & .30 & .18 & .35 & .23 & .29 \\
\hline
\end{tabular}

60 to 65

$\begin{array}{lllllll}\text { Males } & .33 & .30 & .26 & .40 & .30 & .26 \\ \text { Females } & .39 & .39 & .28 & .34 & .33 & .30 \\ \text { Combined } & .36 & .34 & .27 & .36 & .31 & .27\end{array}$

Combined over Age

$\begin{array}{lllllll}\text { Males } & .30 & .33 & .28 & .40 & .29 & .32 \\ \text { Females } & .34 & .31 & .18 & .31 & .26 & .24 \\ \text { Combined } & .32 & .32 & .23 & .35 & .27 & .28 \\ \text { Over Sex } & & & & & & \end{array}$

Ph.D.

20 to 25

$\begin{array}{lllllll}\text { Males } & .17 & .19 & .25 & .18 & .21 & .12 \\ \text { Females } & .35 & .31 & .14 & .24 & .25 & .15 \\ \text { Combined } & .26 & .27 & .19 & .21 & .23 & .13 \\ \text { Cver Sex } & & & & & & \end{array}$


Table 21 Continued

\begin{tabular}{lllllll}
\multicolumn{1}{c}{ Ph.D. } & $\bar{x}$ & S.D. & $\bar{x}$ & S.D. & $\bar{x}^{\text {Total }}$ & S.D. \\
\hline $\begin{array}{l}60 \text { to } 65 \\
\text { Males }\end{array}$ & .16 & .32 & .19 & .33 & .18 & .16 \\
$\begin{array}{l}\text { Females } \\
\text { Combined }\end{array}$ & .51 & .37 & .28 & .40 & .40 & .28 \\
$\begin{array}{l}\text { Over Sex } \\
\text { Combined over }\end{array}$ & Age & .38 & .24 & .36 & .29 & .25 \\
$\begin{array}{l}\text { Males } \\
\text { Females }\end{array}$ & .17 & .25 & .22 & .26 & .19 & .14 \\
$\begin{array}{l}\text { Combined } \\
\text { Over Sex }\end{array}$ & .30 & .34 & .21 & .33 & .32 & .23 \\
\hline
\end{tabular}

Combined over Ed 20 to 25

$\begin{array}{lllllll}\text { Males } & .23 & .30 & .27 & .31 & .25 & .28 \\ \text { Females } & .32 & .26 & .11 & .24 & .21 & .15 \\ \text { Combined } & .27 & .28 & .19 & .29 & .23 & .22 \\ \text { Over Sex } & & & & & & \end{array}$

60 to 65

$\begin{array}{lllllll}\text { Males } & .24 & .31 & .23 & .36 & .24 & .22 \\ \text { Females } & .45 & .37 & .28 & .36 & .36 & .28 \\ \text { Combined } & .35 & .35 & .25 & .35 & .30 & .26 \\ \text { Over Sex } & & & & & & \end{array}$

Combined over Age

$\begin{array}{lllllll}\text { Males } & .24 & .30 & .25 & .33 & .24 & .25 \\ \text { Females } & .39 & .32 & .19 & .31 & .29 & .23 \\ \text { Combined } & .31 & .32 & .22 & .32 & .27 & .24\end{array}$


and negative feeling of knowing judgments was somewhat greater for females than males (.29 vs. .24) but the Sex main effect was also nonsignificant. Likewise, the Order and Item main effects, and all interactions were nonsignificant.

\section{Summary}

To summarize the results for memory prediction accuracy, 2 (Age) $\times 2$ (Education) $\times 2$ (Sex) analyses of variance on mean prediction accuracy on episodic recall, recognition, average episodic prediction, and semantic recognition are summarized in Table 22.

\section{a. Episodic Memory Predictions}

Younger subjects were more accurate than older subjects, but Age accounted for less than 3.\% of the variance in recall prediction accuracy and $7 \%$ in recognition prediction accuracy. Ph.D. subjects were more accurate than HS subjects, but Education accounted for $2 \%$ of the variance in recall prediction accuracy and $10 \%$ in recognition prediction accuracy. Females were more accurate than males, but sex accounted for only $1 \%$ of the variance in recall prediction accuracy and $10 \%$ in recognition prediction accuracy. These variables accounted for $2 \%$ of the variance in episodic recall prediction accuracy, but $26 \%$ in episodic recognition prediction accuracy. Furthermore, summed over recall and recognition $21 \%$ of the variance could be accounted 


\section{Table 22}

Summary ANOVAs for Episodic and Semantic Prediction Accuracy

Episodic

$d f=1,56$

Age

Education

sex

Age $x$ Ed

Age $x$ Sex

Ed $x$ Sex

Age $x$ Ed $x$ Sex

Multiple $\mathrm{R}^{2}$

$$
\bar{x}=\operatorname{Recall}
$$

$\begin{array}{rccc}5.37 & \mathrm{~F} & \mathrm{P} & \operatorname{Eta}^{2} \\ -.10 & <.1 & \text { NS } & <.01 \\ .10 & & & \\ .43 & <.1 & \text { NS } & .02 \\ .43 & & & \\ .29 & <.1 & \text { NS } & .01\end{array}$

$<\cdot 1 \quad$ NS

<. $\quad$ NS

3.44

$<.07$

Multiple $\mathrm{R}^{2} \mathrm{O}$

$\overline{\mathrm{x}}=$ Recognition

\begin{tabular}{lrrcc} 
df $=1,56$ & 4.21 & $F$ & $P$ & $E^{2}$ \\
\hline Age & -.87 & 6.16 & $<.05$ & .07 \\
& .87 & & & \\
Education & 1.05 & 9.01 & $<.01$ &. .10 \\
& -1.05 & 10 & & .10 \\
Sex & -1.03 & 8.72 & $<.01$ & .10
\end{tabular}

Age $\times$ Ed <. $\quad$ NS

Age $x$ Sex

$3.88<.06$

Ed $x$ Sex

$7.87<.01$

Age $x E d x$ Sex

$<1$

NS

Multiple $R^{2}$ 
Table 22 Continued

Episodic

$$
\bar{x}=\text { Total }
$$

\begin{tabular}{|c|c|c|c|c|}
\hline $\mathrm{df}=1,56$ & 4.79 & $F$ & $\mathrm{P}$ & $E t a^{2}$ \\
\hline Age & $\begin{array}{r}-.48 \\
.48\end{array}$ & 3.21 & $<.08$ & .04 \\
\hline Education & $\begin{array}{r}.74 \\
-.74\end{array}$ & 7.48 & $<.01$ & .09 \\
\hline Sex & $\begin{array}{r}.66 \\
-.66\end{array}$ & 5.97 & $<.05$ & .07 \\
\hline Age $x$ Ed & & $<1$ & NS & \\
\hline Age $x$ Sex & & 1.05 & NS & \\
\hline Ed $x$ Sex & & 5.79 & $<.05$ & \\
\hline $\begin{array}{l}\text { Age } x \text { Ed } x \text { Sex } \\
\text { Multiple } \mathrm{R}^{2}\end{array}$ & & 1.33 & NS & .21 \\
\hline
\end{tabular}

Semantic

$d f=1,56$

Age

Education

Sex

Age $x$ Ed

Age $x$ Sex

Ed $x$ Sex

Age $x$ Ed $x$ Sex

Multiple $\mathrm{R}^{2}$

\section{$\overline{\mathrm{X}}=$ Recognition}

3.63 F P Eta ${ }^{2}$

.36

$-.36$

$<1$

NS

.01

.59

$-.59$

2.43

NS

.04

$-.47$

1.54

NS

.03

$<1$

NS

$<1 \quad$ NS

$<1 \quad$ NS

rI NS 
for: $4 \%$ by Age, $9 \%$ by Education, and $7 \%$ by Sex.

b. Semantic Memory Predictions

Only recognition predictions were assessed for semantic memory. Older subjects were more accurate than younger subjects, but Age accounted for only 1\% of the variance. Ph.D. subjects were more accurate than HS subjects, but Education accounted for only $4 \%$ of the variance. Males were more accurate than females, but sex accounted for only $3 \%$ of the variance. These variables accounted for $8 \%$ of the variance in semantic recognition prediction.

\section{E. Memory Confidence}

Subjects were asked to make confidence judgments for each recall and recognition response, on a four point scale, with higher ratings indicating more confidence. For earh subject mean confidence ratings were computed for correct and incorrect responses on episodic and semantic recall and recognition tests. Additionally, when both correct and incorrect responses were produced, difference scores of mean confidence ratings on correct and incorrect responses, were computed.

1. Episodic Memory.

a. Confidence Ratings on Hits and False Alarms on

Recall and Recoanition Tests

Mean confidence ratings for hits (correct responses) on old items) and false alarms (incorrect 
responses on old items) on recall and recognition memory tests for each Age $x$ Education $x$ Sex groups are shown in Table 23. These data were submitted to a 2 (Age) $\times 2$ (Education) $\times 2$ (Sex) $\times 2$ (order) $\times 2$ (Memory (Recall vs. Recognition)) x 2 (Response (Correct vs. Incorrect)) analysis of variance.

The mean confidence rating of younger subjects was higher than those of older subjects (3.31 vs. 2.91), but the Age main effect was nonsignificant. The mean confidence rating of $\mathrm{HS}$ and $\mathrm{Ph} . \mathrm{D}$. subjects was almost identical ( 3.13 vs. 3.11 ), and the Education main effect was also nonsignificant. The mean confidence rating of males and females was also almost identical (3.11 and 3.13), and the sex main effect was nonsignificant as well. The order main effect was also nonsignificant.

The Age $x$ Education interaction was statistically significant, $(\underline{F}(1,26)=8.15, \underline{p}<.01)$, and may be seen in Figure 26, which shows the mean confidence ratings on memory tests for each Age $x$ Education group. Younger HS subjects produced the highest confidence ratings, and older HS subjects the lowest. There was a significant decrease with age in confidence ratings for HS subjects $(\underline{t}(22)=3.85, \underline{\mathrm{P}}<.001)$, but not for Ph.D. subjects.

The mean confidence rating for recall was higher 


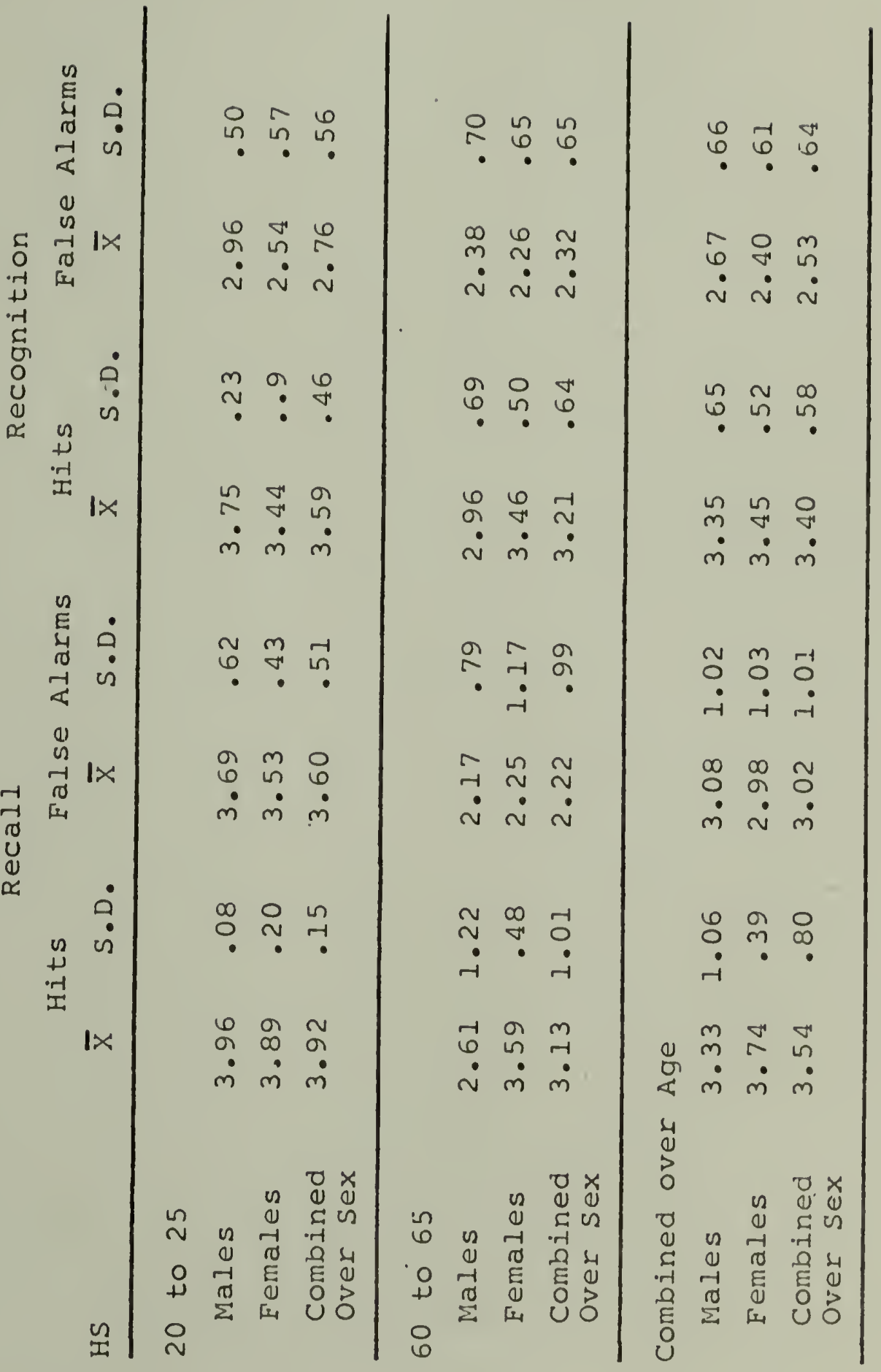


Table 23 Continued

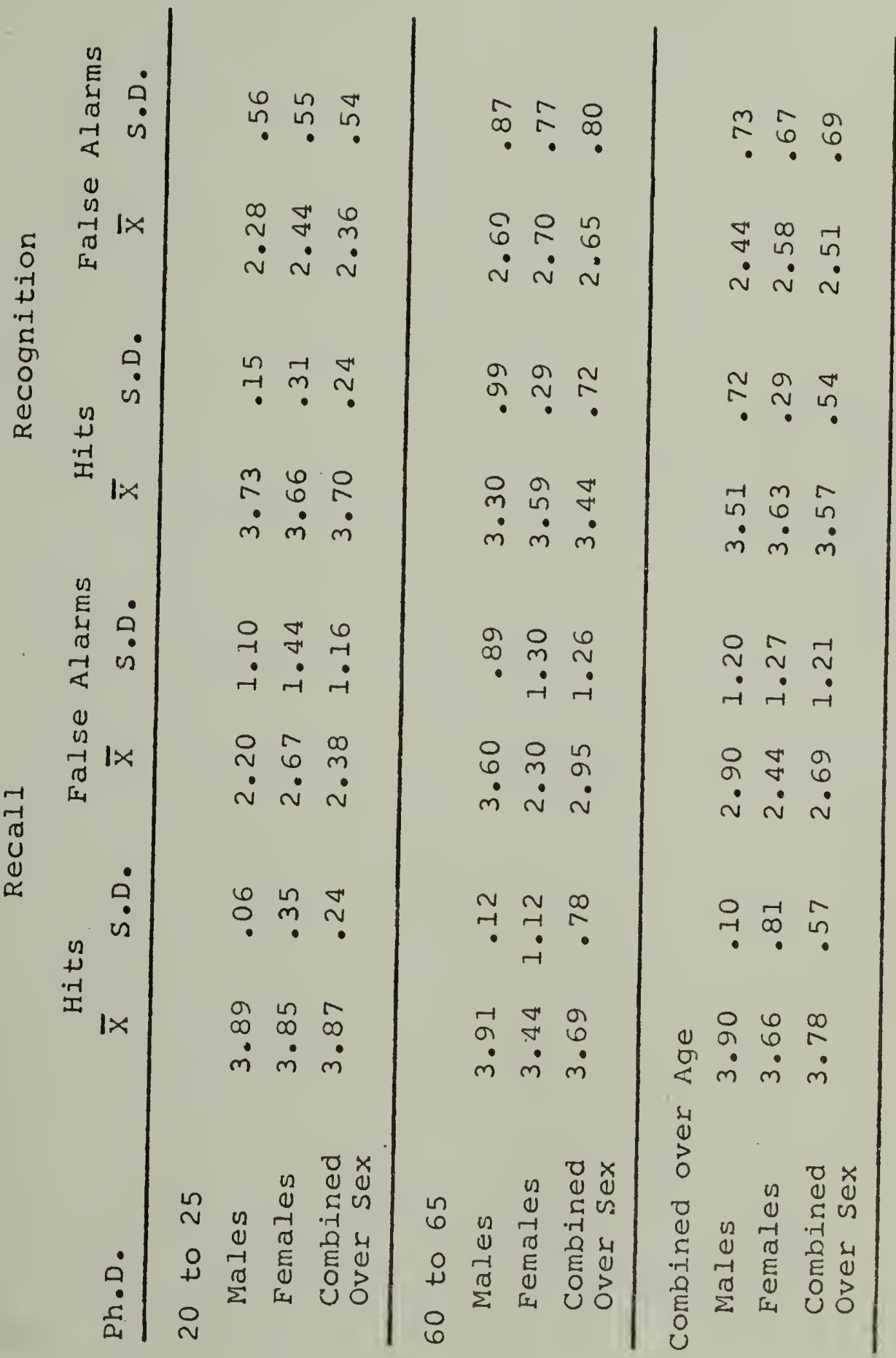


Table 23 Continued

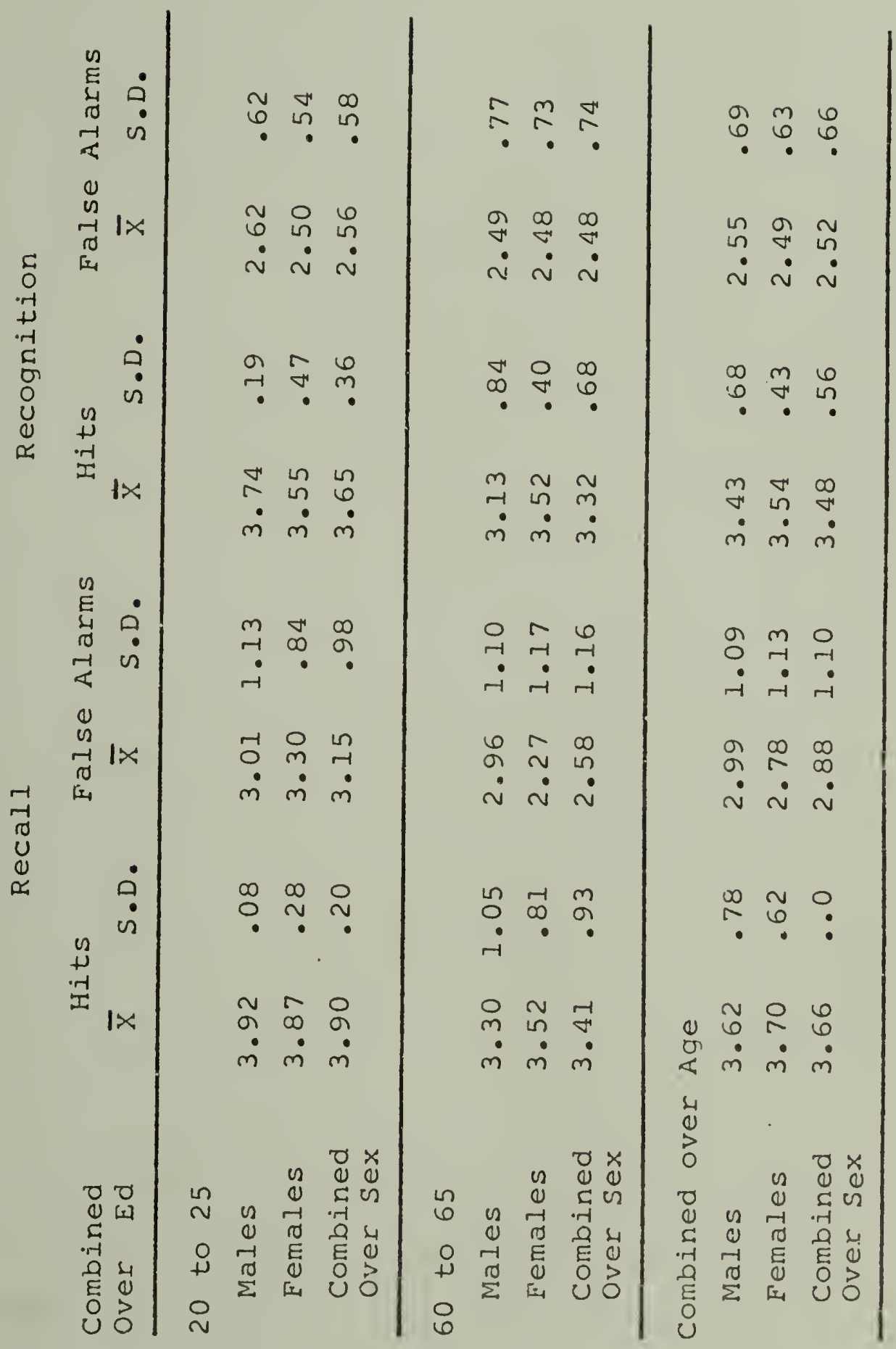


Figure 26. Mean Confidence Ratings on Episodic Memory Tests for Each Age $x$ Education Group

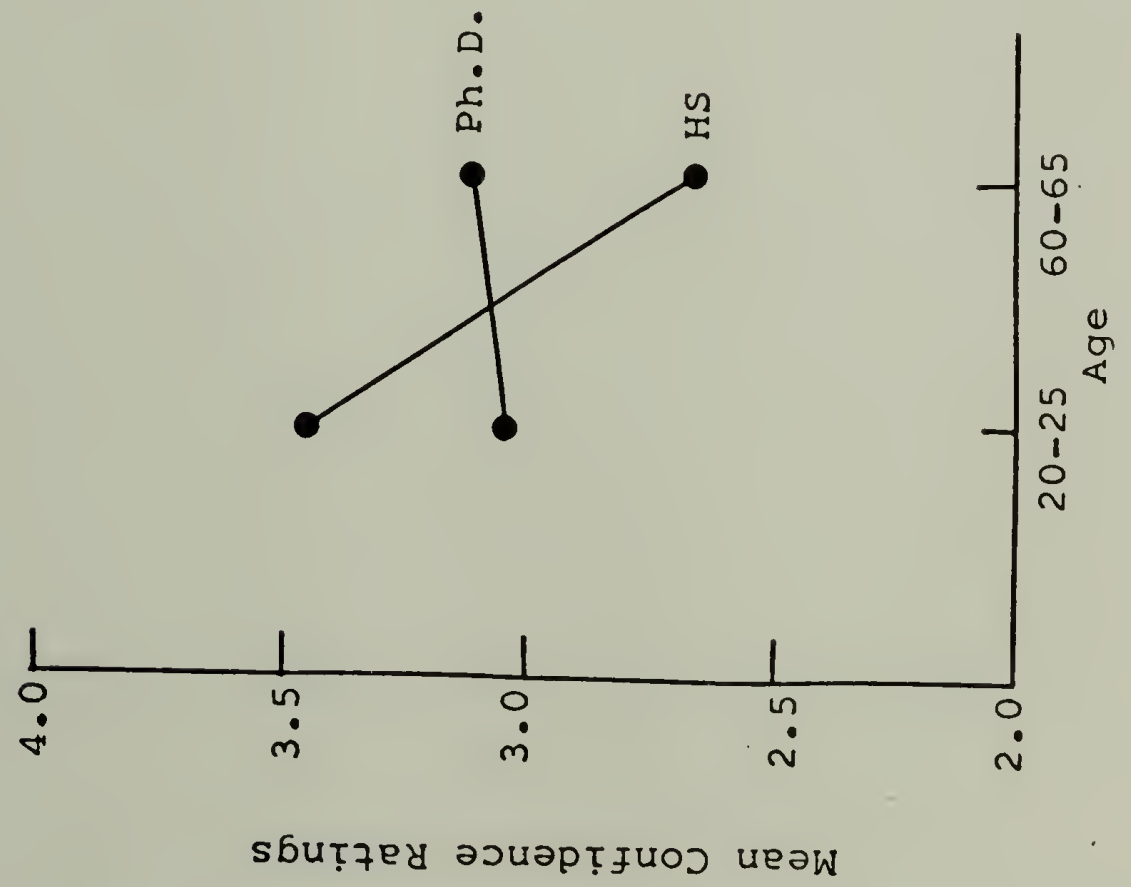


than for recognition ( 3.26 vs. 3.01 ), but the Memory main effect was nonsignificant. The mean confidence rating for correct responses was higher than for incorrect responses (3.57 vs. 2.71), and the Response main effect was highly significant $(\underline{F}(1,26)=72.89$, p (.001), and interacted significantly with Age $x$ Education $(\underline{F}(1,26)=8.08, \underline{p}<.01)$, and Age $x$ sex $(\underline{F}(1,26)=4.22, \underline{P}<.05)$.

b. Difference Scores for Confidence Ratings on Hits and False Alarms on Recall and Recognition Tests

The mean difference scores for confidence ratings of hits and false aiarms were calculated to evaluate confidence accuracy. These difference scores on recall and recognition tests for each Age $x$ Education $x$ sex group are shown in Table 24. These scores were submitted to a 2 (Age) $\times 2$ (Education) $\times 2$ (Sex) x 2 (Order) x 2 (Memory (Recall vs. Recognition)) analysis of variance. The mean difference score was greater for younger than older subjects (.89 vs. .78) but the Age main effect was nonsignificant. The mean difference score was lower for HS than Ph.D. subjects (.76 vs. .94), but the Education main effect was also nonsignificant. The mean difference score was lower for males than females (.74 and .92), and the sex main effect was marginally significant $(\underline{E}(1,26)=3.46, \underline{\mathrm{p}}$ .08). The order main effect was nonsignificant. 
Table 24

Mean Difference Scores for Confidence Ratings On Hits and False Alarms on Recall and Recognition Memory Tests for Each Age x Education $x$ Sex Group

\begin{tabular}{llllllll} 
& \multicolumn{2}{c}{ Recall } & \multicolumn{2}{c}{ Recognition } & \multicolumn{2}{c}{ Total } \\
& $\bar{x}$ & S.D. & $\bar{x}$ & S.D. & $\bar{x}$ & S.D. \\
\hline to 25 & & & & & & \\
$\quad$ Males & .52 & .74 & .91 & .33 & .55 & .25 \\
$\quad \begin{array}{l}\text { Females } \\
\text { Combined }\end{array}$ & .56 & .36 & .90 & .43 & .62 & .37 \\
Over Sex & & .48 & .90 & .38 & .60 & .32
\end{tabular}

60 to 65

$\begin{array}{lrrrrrr}\text { Males } & .14 & .47 & .58 & .44 & .44 & .45 \\ \text { Females } & 1.54 & .99 & 1.20 & .64 & 1.34 & .56 \\ \text { Combined } & .98 & 1.07 & .89 & .62 & .98 & .68 \\ \text { Over Sex } & & & & & & \end{array}$

Combined over Age

$\begin{array}{lrrrrrr}\text { Males } & .31 & .58 & .73 & .42 & .50 & .34 \\ \text { Females } & 1.09 & .89 & 1.05 & .55 & .93 & .57 \\ \text { Combined } & .79 & .86 & .89 & .51 & .76 & .53 \\ \text { Over Sex } & & & & & & \end{array}$

Ph.D.

20 to 25

$\begin{array}{lrrrrrr}\text { Males } & 1.67 & 1.10 & 1.45 & .58 & 1.55 & .80 \\ \text { Females } & 1.00 & .87 & 1.17 & .38 & 1.08 & .45 \\ \text { Combined } & 1.42 & 1.01 & 1.32 & .50 & 1.37 & .70 \\ \text { Over Sex } & & & & & & \end{array}$


Table 24 Continued

\begin{tabular}{|c|c|c|c|c|c|c|}
\hline \multirow[b]{2}{*}{$\mathrm{Ph} . \mathrm{D}$} & \multicolumn{2}{|c|}{ Recall } & \multicolumn{2}{|c|}{ Recognition } & \multicolumn{2}{|c|}{ Total } \\
\hline & $\bar{x}$ & S.D. & $\bar{x}$ & S.D. & $\overline{\mathrm{x}}$ & S.D. \\
\hline \multicolumn{7}{|l|}{60 to 65} \\
\hline Males & .72 & 1.37 & .70 & .46 & .36 & .51 \\
\hline Females & $1 \cdot 52$ & .59 & .89 & .76 & .81 & .81 \\
\hline $\begin{array}{l}\text { Combined } \\
\text { Over Sex }\end{array}$ & 1.20 & .92 & .79 & .61 & .59 & .68 \\
\hline \multicolumn{7}{|c|}{ Combined over Age } \\
\hline Males & 1.40 & 1.16 & 1.07 & .64 & .96 & .89 \\
\hline Females & 1.26 & .72 & 1.02 & .61 & .91 & .67 \\
\hline $\begin{array}{l}\text { Combined } \\
\text { Over Sex }\end{array}$ & 1.33 & .94 & 1.05 & .61 & .94 & .78 \\
\hline
\end{tabular}

Combined over Ed 20 to 25

$\begin{array}{lrrrrrr}\text { Males } & 1.24 & 1.10 & 1.20 & .54 & 1.05 & .77 \\ \text { Females } & .73 & .58 & 1.02 & .42 & .75 & .42 \\ \text { Combined } & .98 & .89 & 1.11 & .49 & .89 & .62 \\ \text { Over Sex } & & & & & \end{array}$

60 to 65

$\begin{array}{lrrrrrr}\text { Males } & .34 & .77 & .64 & .44 & .40 & .46 \\ \text { Females } & 1.53 & .84 & 1.04 & .70 & 1.10 & .70 \\ \text { Combinea } & 1.05 & .99 & .84 & .61 & .78 & .69 \\ \text { Over Sex } & & & & & \end{array}$

Combined over Age

$\begin{array}{lrrrrrr}\text { Males } & .85 & 1.04 & .91 & .56 & .74 & .71 \\ \text { Females } & 1.15 & .82 & 1.03 & .57 & .92 & .60 \\ \text { Combined } & 1.02 & .92 & .97 & .56 & .84 & .65 \\ \text { Over Sex } & & & & & & \end{array}$


The Age $x$ Education interaction was statistically significant $(\underline{F}(1,26)=8.08, \underline{p}(.01)$, and may be seen in Figure 27, which shows the mean difference scores for confidence ratings on hits and false alarms on memory tests for each Age $x$ Education group. For Ph.D. subjects, there was a statistically significant decrease with age in the difference between confidence ratings on correct and incorrect responses $(\underline{t}(16)=$ 2.41, p (.05), while for HS subjects there was a nonsignificant increase with age in this difference. Additionally, education was associated with significantly greater difference scores for younger subjects $(\underline{t}(20)=3.46, \underline{p}(.01)$, but nonsignificantly smaller difference scores for older subjects.

The Age $x$ sex interaction was statistically significant $(\underline{F}(1,26)=4.22, \underline{p}<.05)$, and may be seen in Figure 28, which shows the mean difference scores for confidence ratings on hits and false alarms on episodic memory tests for each Age $x$ Sex group. Increasing age was associated with a significant decrease in difference scores for males $(\underline{t}(17)=2.21, \underline{p}<.05)$, and a nonsignificant increase for females. Additionally, for older subjects, females' difference scores were significantly higher than males $(\underline{t}(18)=2.58$, $\underline{\mathrm{p}}<.05)$, but for younger subjects they were nonsignificantly lower. The mean difference score was higher for recall than 
Figure 2.7. Mean Difference Scores Between Confidence Ratings on Hits and False Alarms on Memory Tests for Each Age x Education Group

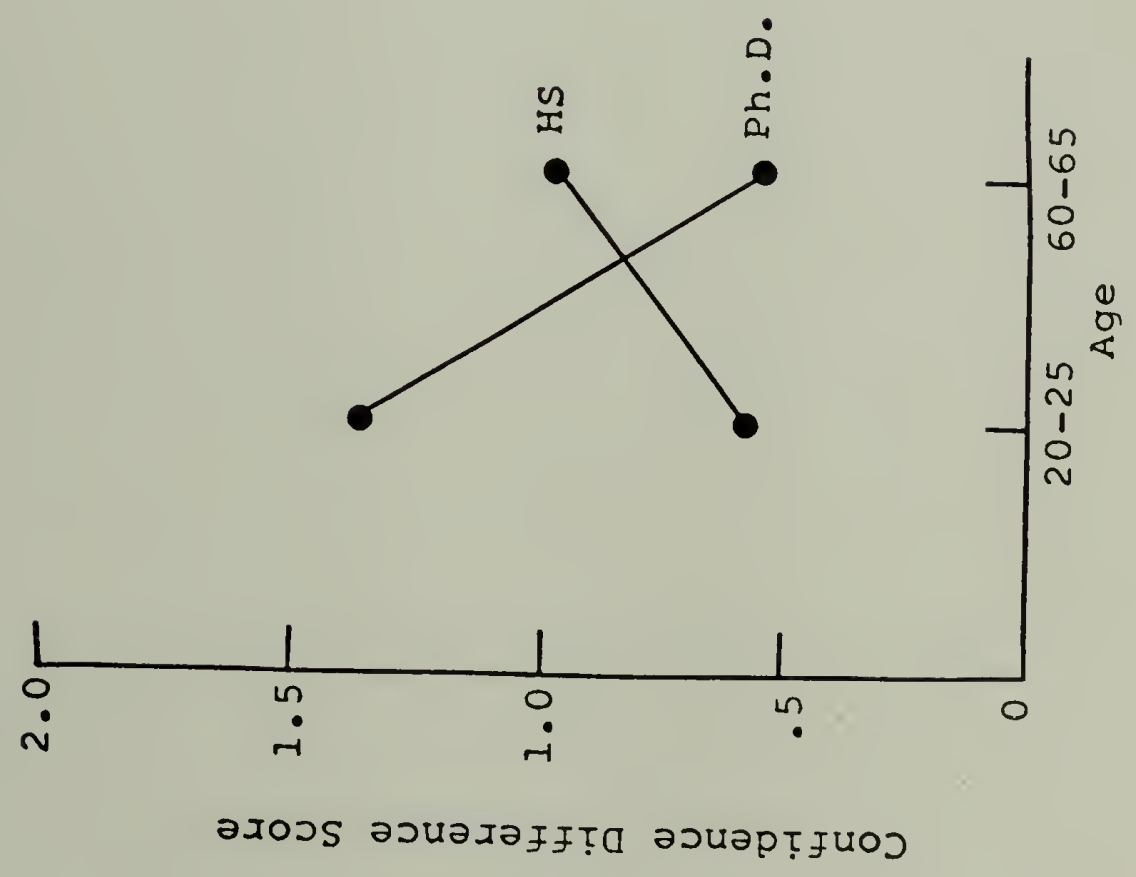


Figure 28. Mean Difference Scores for Confidence Ratings on Hits and False Alarms on Episodic Memory Tests for Each Age $x$ Sex Group

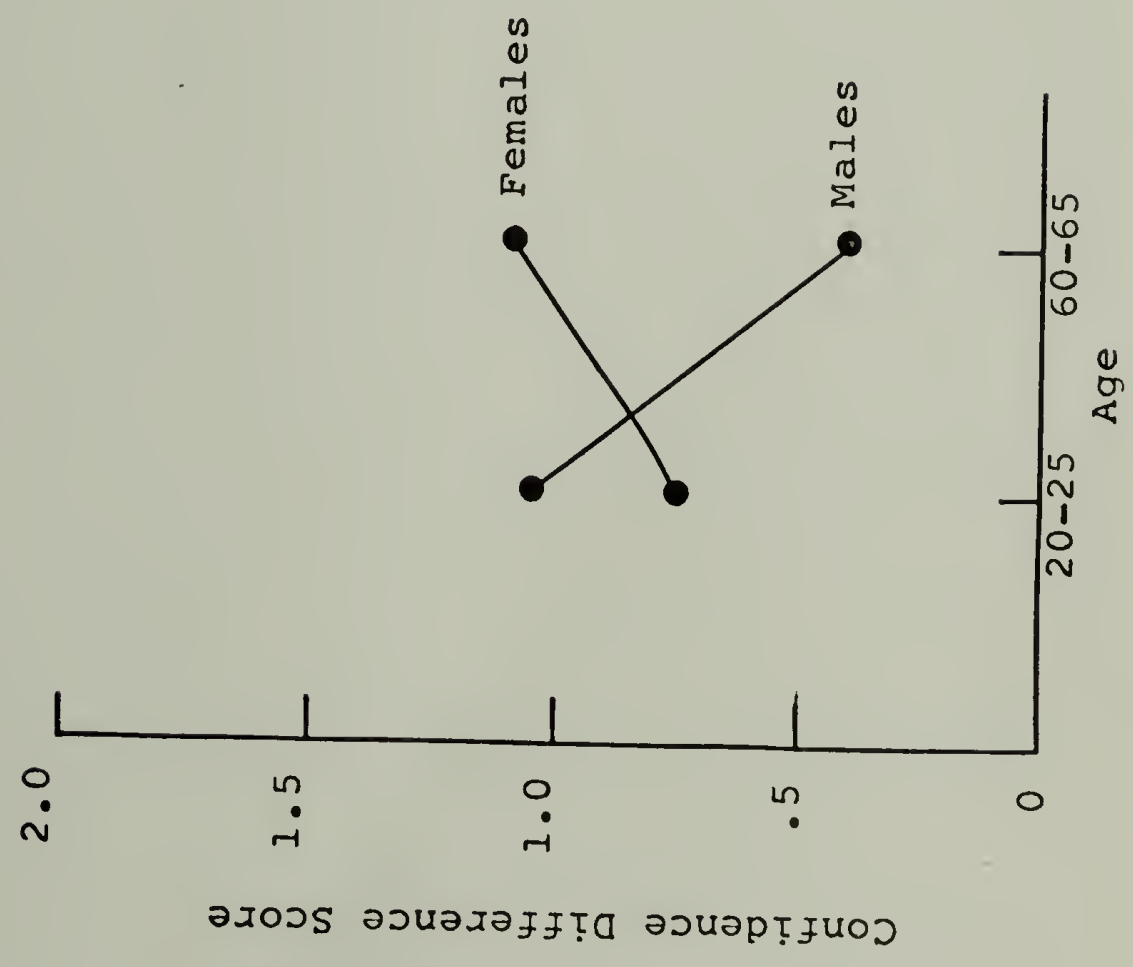


recognition tests (1.02 vs. .97), but the Memory main effect was nonsigrificant.

c. Confidence Ratings on Old and New Recognition Items

The mean confidence ratings on correct and incorrect old and new recognition items for each fige $x$ Education $x$ Sex group is shown in Table 25. These data were submitted to a 2 (Age) $\times 2$ (Education) $\times 2$ (Sex) $x$ 2 (Order) $\times 2$ (Item (Old vs. New)) x 2 (Response. (Correct vs. Incorrect)) analysis of variance. The additional information gained from this analysis concerns confidence ratings on old vs. new items. The mean confidence ratings on old items was higher than on new items $(3.04$ vs. 2.67), and the Item main effect was statistically significant $(\underline{F}(1,46)=63.26, \underline{p}(.001)$.

The Item $x$ Response interaction was also statistically significant $(\underline{F}(1,46)=19.49, \underline{\mathrm{p}}<.001)$, and may be seen in Figure 29, which shows the mean confidence ratings on correct and incorrect old and new recognition items. While for correct items significantly higher confidence ratings were obtained for old than new items $(\underline{t}(63)=8.13, \underline{p}(.001)$, for incorrect items statistically comparable confidence ratings were obtained for old and new items.

d. Difference Scores for Confidence Ratings on old and New Recoanition Items 
Table 25

Mean Confidence Ratings on Correct and Incorrect old and New Items on Recognition Memory Test For Each Age $x$ Education $x$ Sex Group

Correct

\begin{tabular}{|c|c|c|c|c|c|c|}
\hline \multirow[b]{2}{*}{ HS } & \multicolumn{2}{|l|}{ Old } & \multicolumn{2}{|c|}{ New (CR) } & \multicolumn{2}{|c|}{ Total } \\
\hline & $\bar{x}$ & S.D. & $\bar{x}$ & S.D. & $\overline{\mathrm{x}}$ & S.D. \\
\hline \multicolumn{7}{|l|}{20 to 25} \\
\hline Males & 3.75 & .23 & 3.45 & .43 & 3.60 & .28 \\
\hline Females & 3.44 & .59 & 2.95 & .71 & 3.19 & .56 \\
\hline $\begin{array}{l}\text { Combined } \\
\text { Over Sex }\end{array}$ & 3.59 & .46 & 3.20 & .62 & 3.40 & .47 \\
\hline
\end{tabular}

60 to 65

$\begin{array}{lllllll}\text { Males } & 2.96 & .69 & 2.26 & .54 & 2.61 & .54 \\ \text { Females } & 3.46 & .50 & 2.56 & .99 & 3.01 & .71 \\ \text { Combined } & 3.21 & .64 & 2.41 & .79 & 2.81 & .64 \\ \text { Over Sex } & & & & & & \end{array}$

Combined over Age

$\begin{array}{lllllll}\text { Males } & 3.35 & .65 & 2.85 & .77 & 3.10 & .66 \\ \text { Females } & 3.45 & .52 & 2.76 & .86 & 3.10 & .62 \\ \text { Combined } & 3.40 & .58 & 2.81 & .80 & 3.10 & .63 \\ \text { Over Sex } & & .56 & & & & \end{array}$

Ph.D.

20 to 25

Males

Females

$3.73-15$

2.84

.32

3.29

.19

Combined

3.60 .31

2.43 .91

3.05

.51

Over Sex

$3.70 \quad .24$

$2.64 \quad .69$

3.17 
Table 25 Continued

\section{Correct}

\begin{tabular}{|c|c|c|c|c|c|c|}
\hline \multirow{2}{*}{$\mathrm{Ph} \cdot \mathrm{D}$. } & \multirow{2}{*}{$\begin{array}{l}\text { Old } \\
\bar{x}\end{array}$} & \multirow{2}{*}{$\begin{array}{c}\text { Hits) } \\
\text { S.D. }\end{array}$} & \multicolumn{2}{|c|}{ New (CR) } & \multicolumn{2}{|c|}{ Total } \\
\hline & & & $\bar{x}$ & S.D. & $\bar{x}$ & S.D. \\
\hline \multicolumn{7}{|l|}{60 to 65} \\
\hline Males & 3.30 & .99 & 2.82 & .69 & 3.06 & .73 \\
\hline Females & 3.59 & .29 & 2.95 & .71 & 3.27 & .44 \\
\hline $\begin{array}{l}\text { Combined } \\
\text { Over Sex }\end{array}$ & 3.44 & .72 & $2: 88$ & .68 & 3.16 & .59 \\
\hline
\end{tabular}

Combined over Age

$\begin{array}{lllllll}\text { Males } & 3.51 & .72 & 2.83 & .52 & 3.17 & .53 \\ \text { Females } & 3.63 & .29 & 2.69 & .83 & 3.16 & .47 \\ \text { Combined } & 3.57 & .54 & 2.76 & .69 & 3.16 & .49 \\ \text { Over Sex } & & & & & & \end{array}$

Combined over Ed 20 to 25

$\begin{array}{lllllll}\text { Males } & 3.74 & .19 & 3.14 & .48 & 3.44 & .28 \\ \text { Females } & 3.55 & .47 & 2.69 & .83 & 3.12 & .52 \\ \begin{array}{l}\text { Combined } \\ \text { Over Sex }\end{array} & 3.65 & .36 & 2.92 & .71 & 3.28 & .44\end{array}$

60 to 65

$\begin{array}{lllllll}\text { Males } & 3.13 & .84 & 2.54 & .66 & 2.83 & .66 \\ \text { Females } & 3.52 & .40 & 2.75 & .86 & 3.14 & .58 \\ \text { Combined } & 3.32 & .68 & 2.65 & .76 & 2.99 & .63 \\ \text { Over Sex } & & & & & . & \end{array}$

Combined over Age

$\begin{array}{lllllll}\text { Males } & 3.43 & .68 & 2.84 & .65 & 3.14 & .59 \\ \text { Females } & 3.54 & .43 & 2.72 & .83 & 3.13 & .54 \\ \text { Combined } & 3.48 & .56 & 2.78 & .74 & 3.13 & .56 \\ \text { Over Sex } & & & & & & \end{array}$


Table 25 Continued

Incorrect

\begin{tabular}{lcccccc} 
& Old & (Misses) & \multicolumn{2}{c}{ New (FA) } & \multicolumn{2}{c}{ Total } \\
HS, & $\bar{x}$ & S.D. & $\bar{x}$ & S.D. & $\bar{x}$ & S.D \\
\hline 20 to 25 & & & & & & \\
$\quad$ Males & 3.31 & .41 & 2.96 & .50 & 3.14 & .43 \\
$\quad$ Females & 2.68 & .68 & 2.54 & .57 & 2.61 & .56 \\
$\quad \begin{array}{l}\text { Combined } \\
\text { Over Sex }\end{array}$ & 3.02 & .62 & 2.75 & .56 & 2.89 & .55
\end{tabular}

60 to 65

Males

Females

$\begin{array}{ll}2.41 & .43 \\ 2.20 & .95\end{array}$

$2.38 \quad .70$

$2.39 \quad .50$

Combined

Over Sex

$2.30 \quad .72$

$2.26 \quad .65$

2.23 .73

$\begin{array}{lllll}2.32 & .65 & 2.31 & .61\end{array}$

Combined over Age

Males

Females

2.8

Combined

Over Sex

$\begin{array}{ll}2.86 & .62 \\ 2.42 & .84 \\ 2.65 & .76\end{array}$

$2.67 \quad .66$

$2.76 \quad .60$

$2.40 \quad .61$

$2.40 \quad .66$

$\mathrm{Ph} \cdot \mathrm{D}$.

20 to 25

Males

Females

Combined

Over Sex

$\begin{array}{ll}2.79 & .43 \\ 2.12 & .56 \\ 2.45 & .60\end{array}$

2.28

.253

.35

$.244 \quad .55$

2.29

.43

2.36

.54

2.42

.40

60 to 65

$\begin{array}{lllllll}\text { Males } & 2.61 & .93 & 2.60 & .87 & 2.61 & .69 \\ \text { Females } & 2.70 & .63 & 2.70 & .77 & 2.70 & .66 \\ \text { Combined } & 2.66 & .77 & 2.65 & .80 & 2.65 & .65 \\ \text { Over Sex } & & & & & & \end{array}$


Table 25 Continued

Incorrect

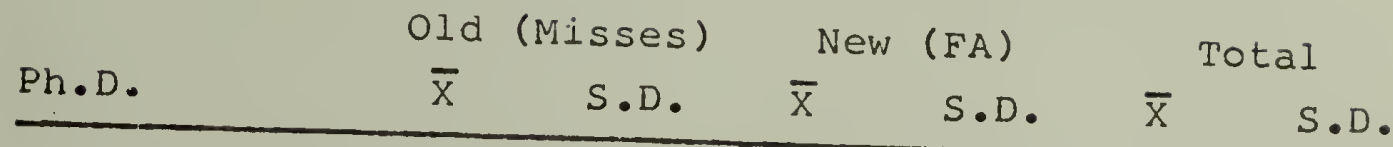

Combined over Age

$\begin{array}{lllllll}\text { Males } & 2.70 & .71 & 2.44 & .73 & 2.57 & .53 \\ \text { Females } & 2.41 & .66 & 2.58 & .67 & 2.51 & .59 \\ \text { Combined } & 2.56 & .69 & 2.51 & .69 & 2.54 & .55 \\ \text { Over Sex } & & & & & & \end{array}$

Combined over Ed 20 to 25

$\begin{array}{lllllll}\text { Males } & 3.05 & .49 & 2.62 & .62 & 2.83 & .49 \\ \text { Females } & 2.38 & .67 & 2.50 & .54 & 2.45 & .51 \\ \text { Combined } & 2.72 & .67 & 2.56 & .58 & 2.65 & .53 \\ \text { Over Sex } & & & & & & \end{array}$

60 to 65

$\begin{array}{lllllll}\text { Males } & 2.51 & .71 & 2.49 & .77 & 2.50 & .59 \\ \text { Females } & 2.45 & .82 & 2.48 & .73 & 2.46 & .72 \\ \text { Combined } & 2.48 & .75 & 2.48 & .74 & 2.49 & .65 \\ \text { Over Sex } & & & & & & \end{array}$

Combined over Age

$\begin{array}{lllllll}\text { Males } & 2.78 & .66 & 2.55 & .69 & 2.67 & .56 \\ \text { Females } & 2.42 & .74 & 2.49 & .63 & 2.46 & .62 \\ \text { Combined } & 2.60 & .72 & 2.52 & .66 & 3.50 & .54 \\ \text { Over Sex } & & & & & & \end{array}$


Figure 29. Mean Confidence Ratings on Correct and Incorrect Old and New Recognition Items

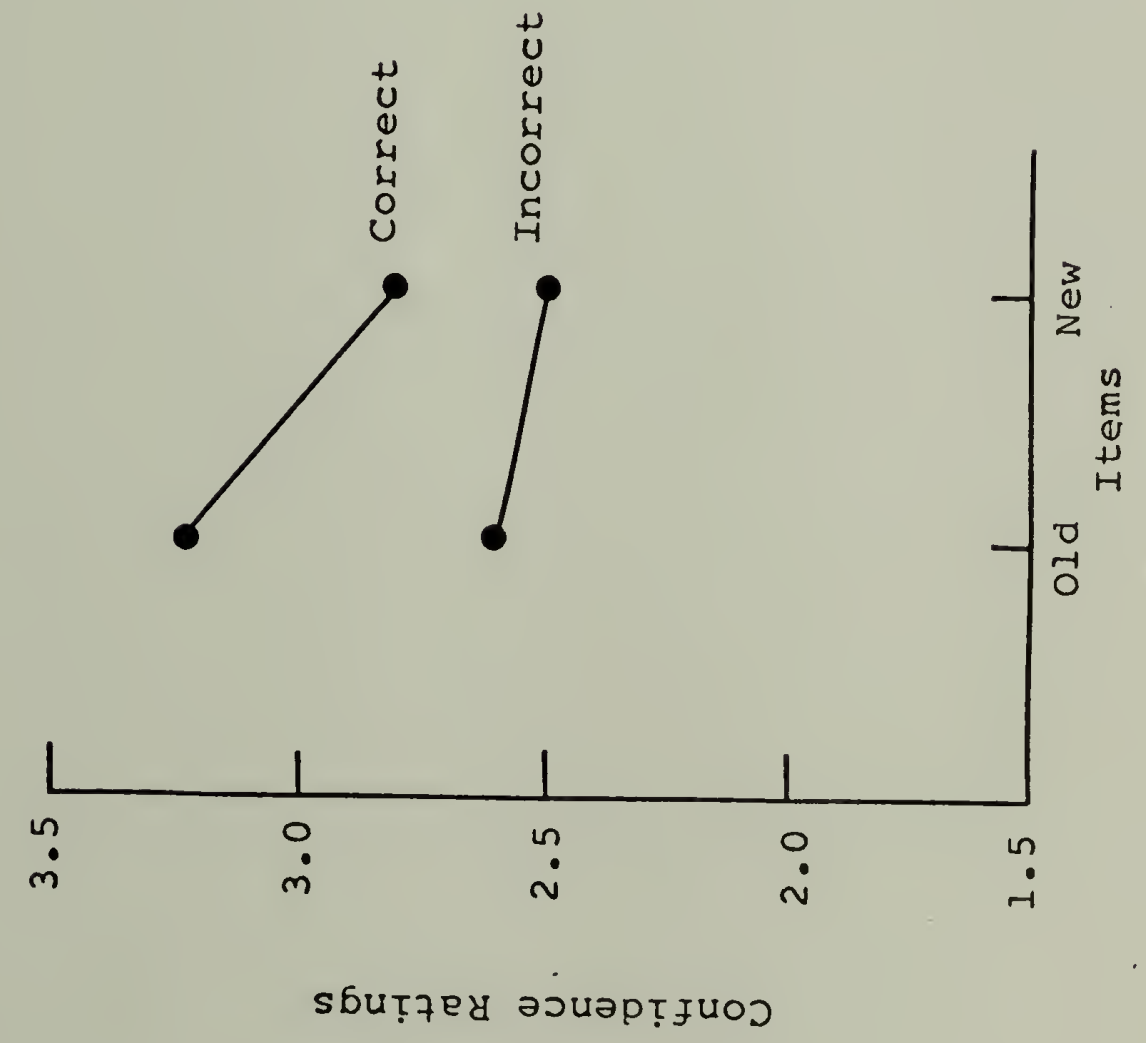


on correct and incorrect old and new recognition items for each Age $x$ Education $x$ Sex group are shown in Table 26. The mean difference scores for old items was higher than for new items $(.89 \mathrm{vs} . .31)$, and the Item main effect was statistically significant (F $(1,46)=19.49, \underline{\mathrm{p}}(.001)$.

2. Sernantic Memory

a. Confidence Ratings on Hits and False Alarms on Recall and Recoanition Tests

The mean confidence ratings for hits and false alarms on general information recall and recognitjon tests for each Age $x$ Education $x$ sex group are shown in Table 27. These data were submitted to a 2 (Age) $\times 2$ (Education) $\times 2$ (Sex) $\times 2$ (Order) $\times 2$ (Memory (Recall vs. Recognition)) × 2 (Response (Correct vs. Incorrect)) analysis of variance.

The mean confidence rating for younger subjects was lower than for older subjects (2.77 vs. 2.97), but the Age main effect was nonsignificant. The mean confidence rating for HS subjects was lower than for Ph.D. subjects (2.82 vs. 2.94), but the Education main effect was also nonsignificant. The mean confidence rating for males was greater than for females (2.94 vs. 2.83), but the sex main effect was nonsignificant as well. The order mair effect, as well as all interactions of between subjects variables, were also nonsignificant. 
Table 26

Mean Difference Scores for Confidence Ratings on Correct and Incorrect old and New Items Recognition Memory Tests for Each

Age $x$ Education $x$ Sex Group

\begin{tabular}{|c|c|c|c|c|c|c|}
\hline \multirow[b]{2}{*}{ HS } & \multicolumn{2}{|c|}{ Old } & \multicolumn{2}{|c|}{ New } & \multicolumn{2}{|c|}{ Total } \\
\hline & $\bar{x}$ & $S \cdot D$. & $\bar{x}$ & S.D. & $\bar{x}$ & S.D. \\
\hline \multicolumn{7}{|l|}{20 to 25} \\
\hline Males & .50 & .26 & .56 & .27 & .53 & .27 \\
\hline Females & .71 & .55 & .51 & .57 & .41 & .57 \\
\hline $\begin{array}{l}\text { Combined } \\
\text { Over Sex }\end{array}$ & .60 & .55 & .48 & .44 & .57 & .20 \\
\hline \multicolumn{7}{|l|}{60 to 65} \\
\hline Males & .55 & .62 & -.12 & .38 & .22 & .19 \\
\hline Females & 1.26 & .75 & .31 & .75 & .78 & .44 \\
\hline $\begin{array}{l}\text { Combined } \\
\text { Over Sex }\end{array}$ & .90 & .76 & .10 & .62 & .50 & .44 \\
\hline
\end{tabular}

Combined over Age

$\begin{array}{lrrrrrr}\text { Males } & .52 & .47 & .20 & .47 & .36 & .23 \\ \text { Females } & 1.00 & .70 & .36 & .65 & .70 & .36 \\ \text { Combined } & .76 & .63 & .28 & .57 & .53 & .34 \\ \text { Over Sex } & & & & & & \end{array}$

$\mathrm{Ph} . \mathrm{D}$.

20 to 25

$\begin{array}{lllllll}\text { Males } & .95 & .44 & .56 & .41 & .75 & .23 \\ \text { Females } & 1.54 & .51 & .19 & .90 & .83 & .28 \\ \text { Combined } & 1.24 & .55 & .39 & .68 & .79 & .25 \\ \text { Over Sex } & & & & & & \end{array}$


Table 26 Continued

\begin{tabular}{|c|c|c|c|c|c|c|}
\hline \multirow[b]{2}{*}{$\mathrm{Ph} . \mathrm{D}$. } & \multicolumn{2}{|c|}{ Old } & \multicolumn{2}{|c|}{ New } & \multicolumn{2}{|c|}{ Total } \\
\hline & $\bar{x}$ & $S . D$. & $\overline{\mathrm{x}}$ & S.D. & $\overline{\mathrm{x}}$ & S.D. \\
\hline 60 to 65 & & & & & & \\
\hline Males & .78 & 1.45 & .22 & .71 & .45 & .48 \\
\hline Females & .89 & .55 & .25 & .48 & .57 & .36 \\
\hline $\begin{array}{l}\text { Combined } \\
\text { Over Sex }\end{array}$ & .84 & 1.03 & .24 & .59 & .51 & .42 \\
\hline
\end{tabular}

Combined over Age

$\begin{array}{lrrrrrr}\text { Males } & .87 & 1.01 & .39 & .59 & .50 & .40 \\ \text { Females } & 1.21 & .61 & .22 & .68 & .69 & .34 \\ \text { Combined } & 1.05 & .83 & .31 & .63 & .65 & .37 \\ \text { Over Sex } & & & & & & \end{array}$

Combined over Ed 20 to 25

$\begin{array}{lrrrrrr}\text { Males } & .74 & .42 & .56 & .34 & .65 & .22 \\ \text { Females } & 1.15 & .67 & .31 & .72 & .72 & .28 \\ \text { Combined } & .94 & .59 & .43 & .57 & .68 & .25 \\ \text { Over Sex } & & & & & & \end{array}$

60 to 65

$\begin{array}{lrrrrrr}\text { Males } & .66 & 1.05 & .05 & .58 & .33 & .38 \\ \text { Females } & 1.07 & .66 & .28 & .61 & .68 & .40 \\ \text { Combined } & .87 & .89 & .17 & .60 & .50 & .42 \\ \text { Over Sex } & & & & & & \end{array}$

Combined over Age

$\begin{array}{lrrrrrr}\text { Males } & .70 & .79 & .30 & .54 & .49 & .34 \\ \text { Females } & 1.11 & .66 & .29 & .66 & .70 & .35 \\ \text { Combined } & .89 & .74 & .31 & .59 & .59 & .36 \\ \text { Over Sex } & & & & & & \end{array}$




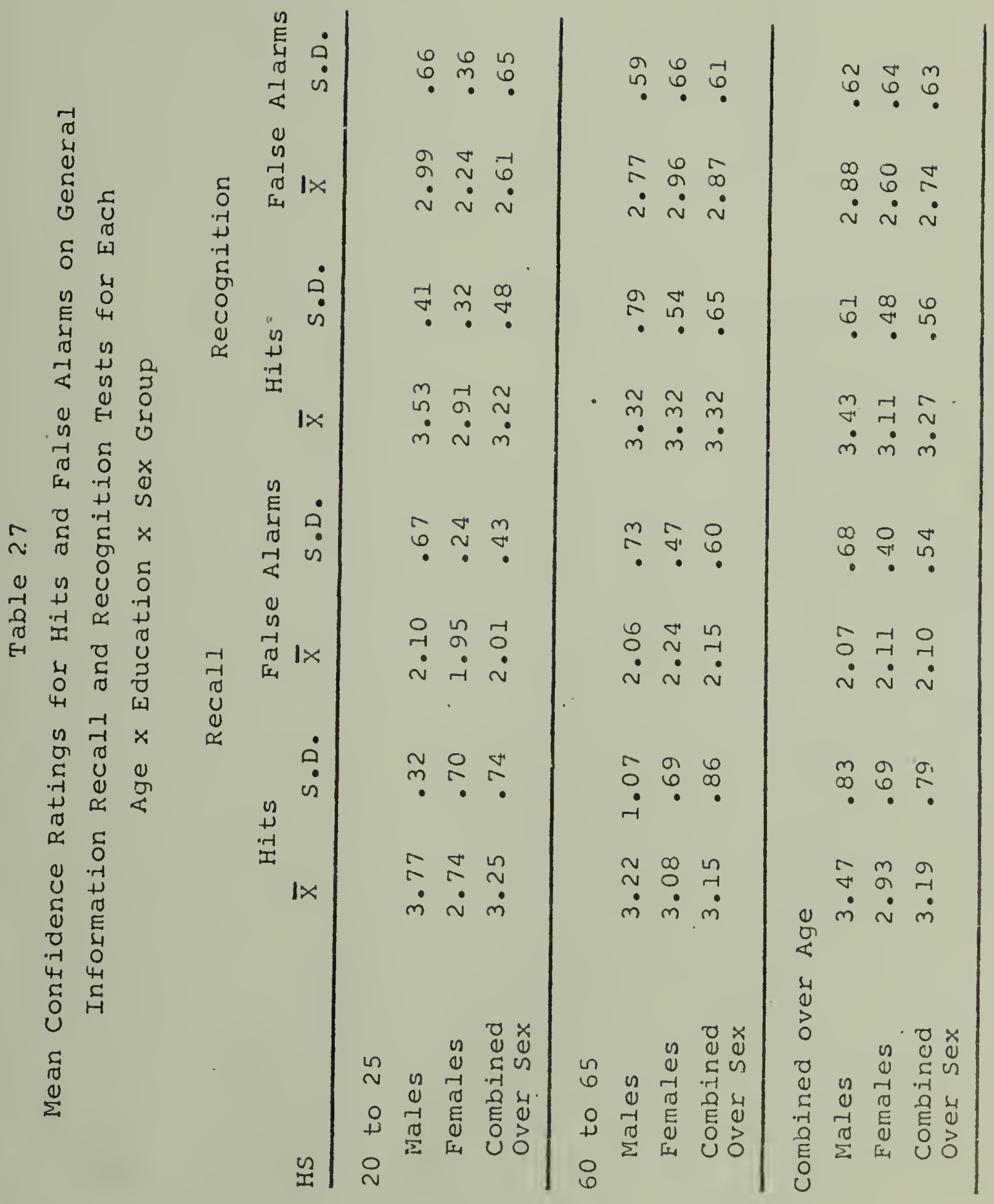


Table 27 Continued

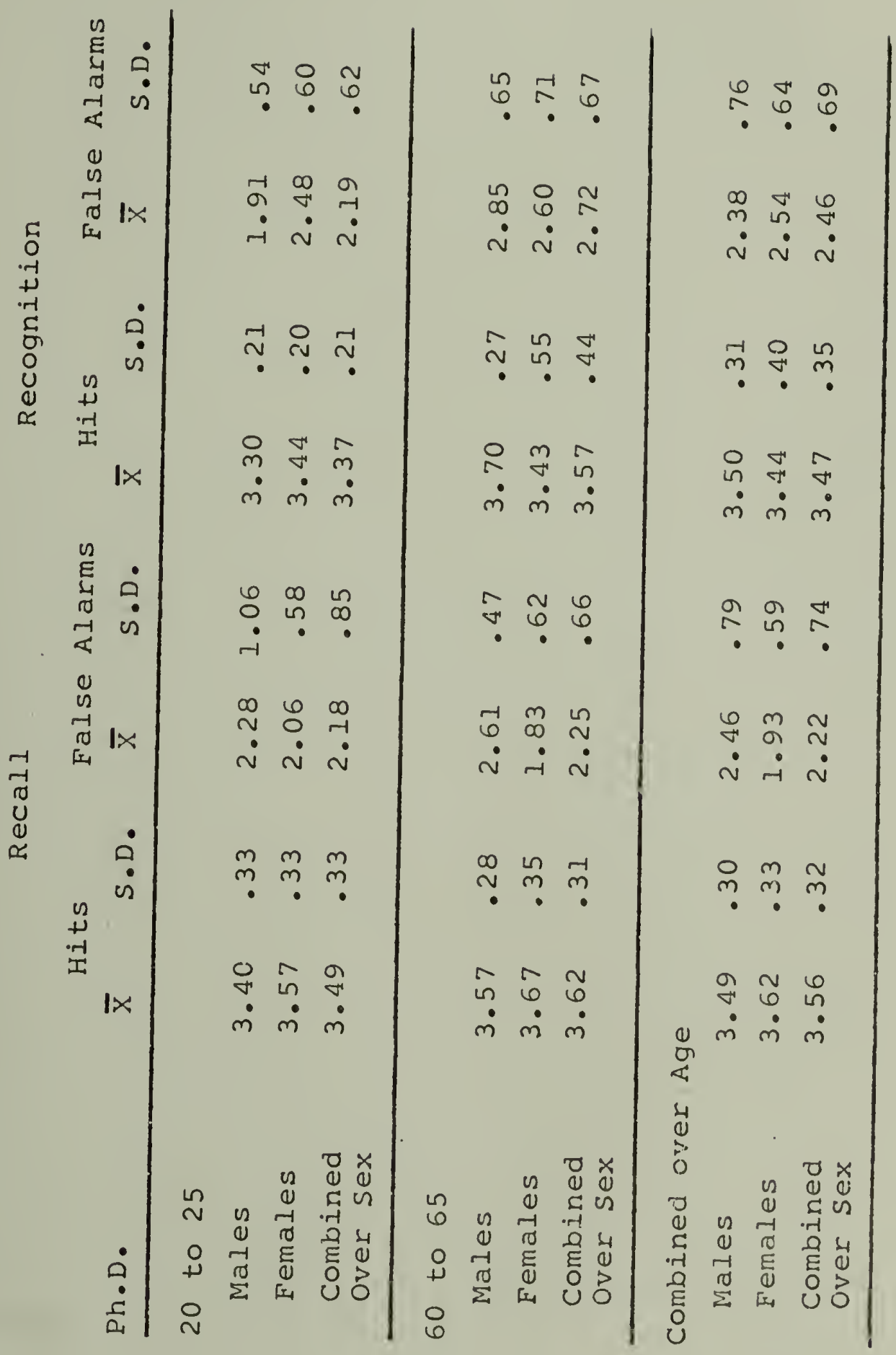


Table 27 Continued

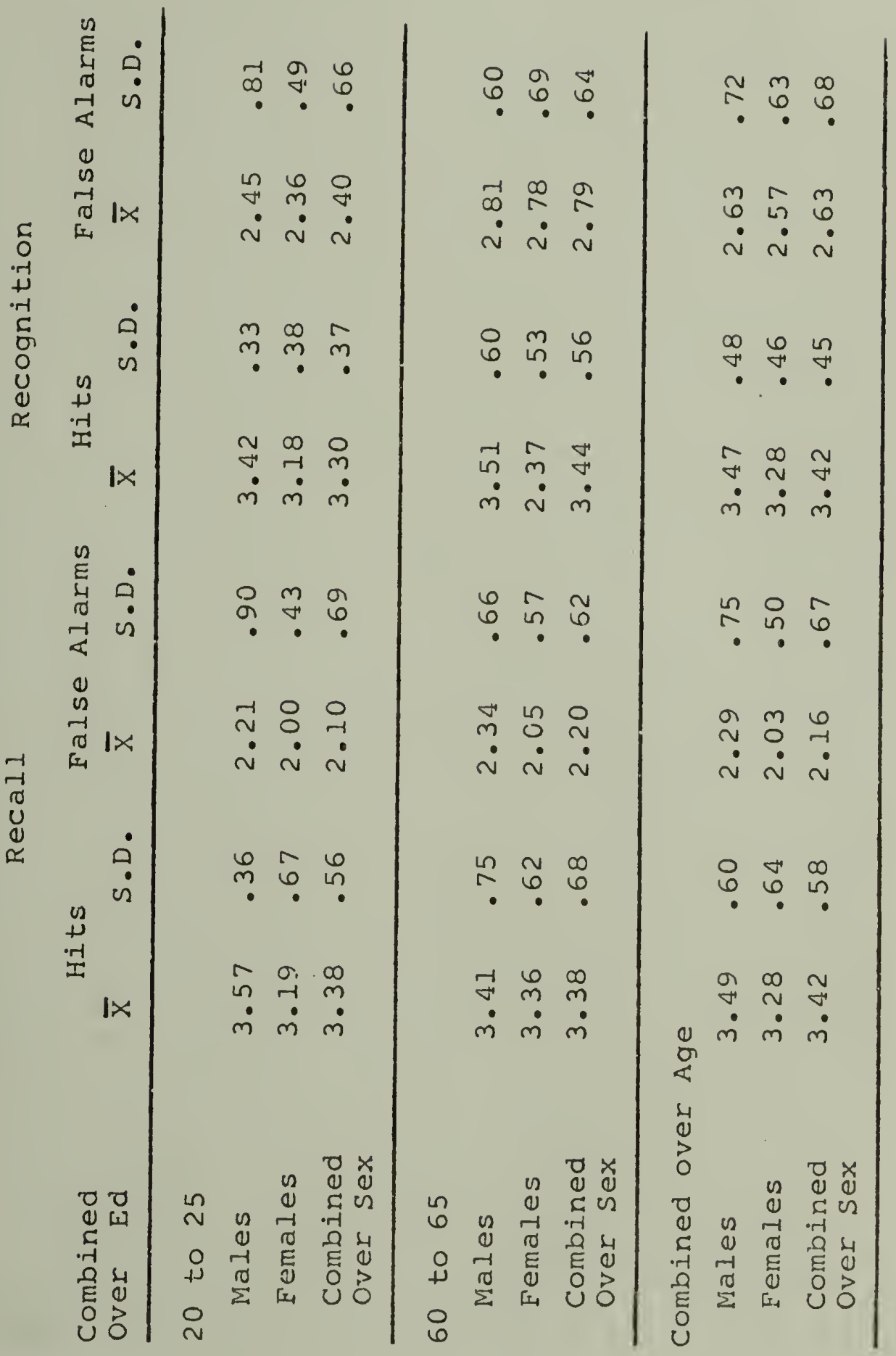


The mean confidence rating on recognition tests was higher than on recall tests (3.00 vs. 2.77), and the Memory main effect was statistically significant $(\underline{F}(1,36)=6.22, \underline{p}<.05)$. The mean confidence rating for correct responses was higher than for incorrect responses ( $3.39 \mathrm{vs} .2 .38$ ), and the Response main effect was highly significant $(\underline{F}(1,36)=49.05, \underline{p}<.001)$.

The Response $x$ Education interaction was also statistically significant $(\underline{F}(1,36)=7.68, \underline{p}<.01)$, and may be seen in Figure 30 , which shows the mean confidence ratings for hits and false alarms on general information knowledge tests for each Education group. Confidence ratings on correct responses were higher for Ph.D. than HS subjects $(\underline{t}(54)=2.33, \underline{p}<.05)$, but on incorrect responses they were no different for the two education groups.

The Response $x$ Memory (Recall vs. Recognition) interaction was also statistically significant $(\underline{E}(1,36)$ $=9.40, \mathrm{p}(.01)$, and may be seen in Figure 31 , which shows the mean confidence ratings for hits and false alarms on general information recall and recognition tests. Confidence ratings for incorrect responses were higher on recognition than recall tests $(\underline{t}(53)=3.84$, $\mathrm{p}(.001)$, but for correct responses they were the same on recall and recognition tests. 
Figure 30. Mean Confidence Ratings for Hits and False Alarms on General Information knowledge Tests for Each Education Group

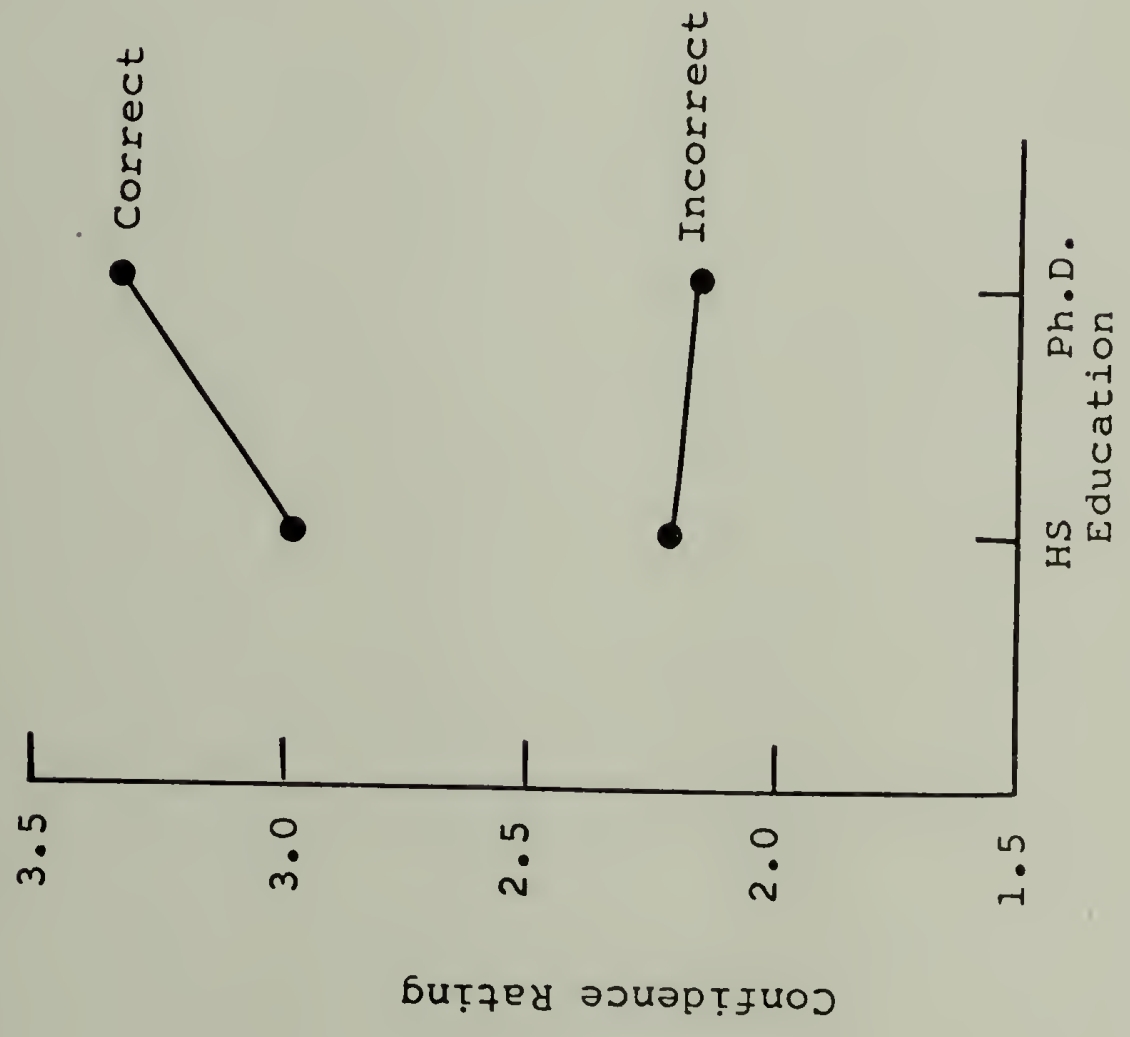


Figure 31. Mean Confidence Ratings for Hits and False Alarms on General Information Recall and Recognition Tests

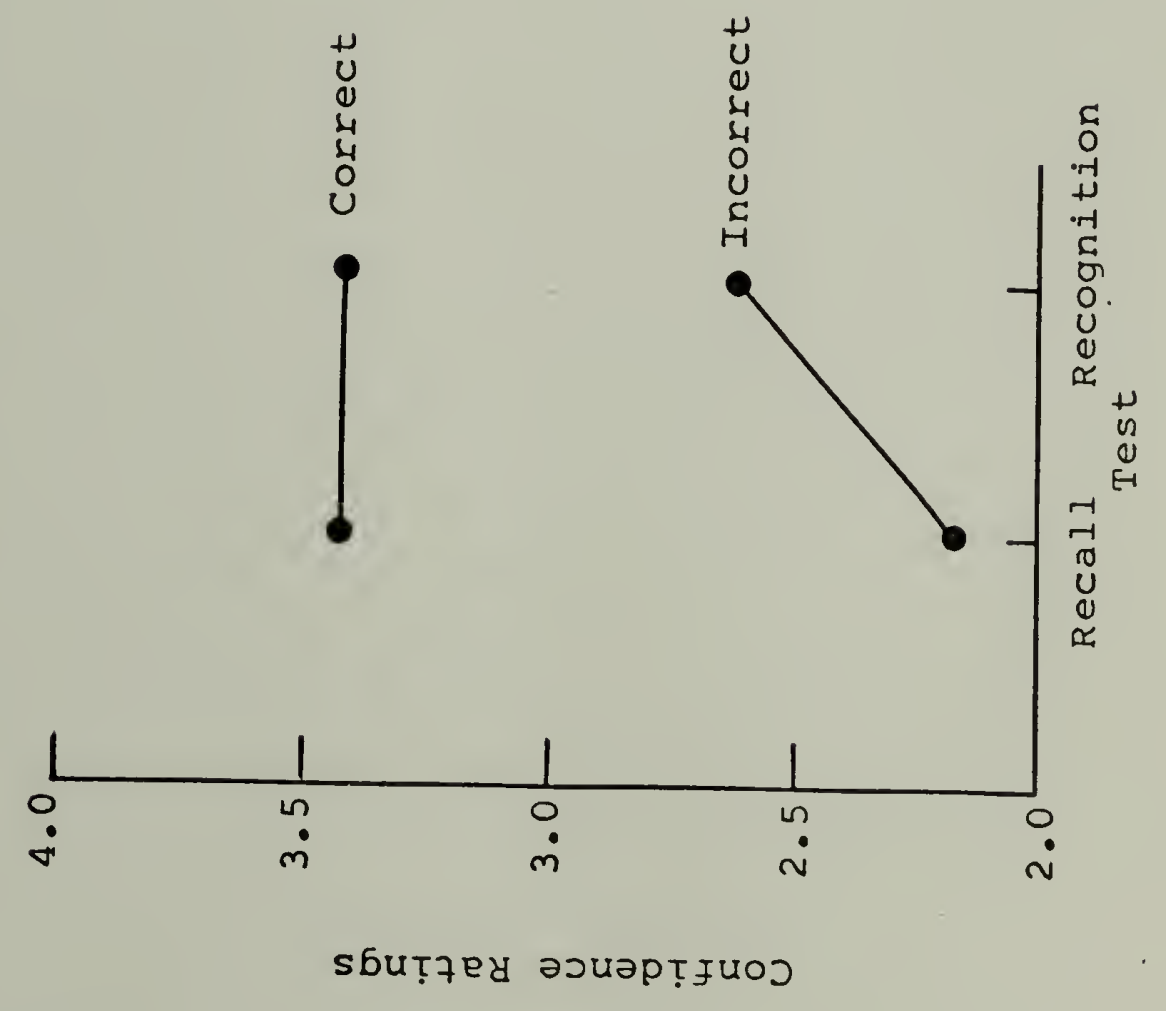


b. Difference Scores for Confidence Ratings on Hits and False Alarms on Recall and Recognition Tests The mean difference scores for confidence ratings on hits and false alarms were calculated to evaluate confidence accuracy. These difference scores on general information recall and recognition tests for each Age $x$ Education $x$ Sex group are shown in Table 28. These scores were submitted to a 2 (Age) $\times 2$ (Education) $\times 2$ (Sex) $\times 2$ (Order) $\times 2$ (Memory (Recall vs. Recognition)) analysis of variance.

The mean difference score for younger subjects was greater than for older subjects (1.14 vs. .91), but the Age main effect was nonsignificant. The mean difference score for HS subjects was lower than for Ph.D. subjects (.90 vs. 1.18), and the Education main effect was statistically significant $(\underline{F}(1,36)=7.68, \underline{p}<.01)$. The mean difference scores for males and females were almost identical ( 1.01 vs. 1.00), and the sex main effect was nonsignificant. The order main effect was also nonsignificant.

The mean difference score for recall tests was higher than for recognition tests (1.28 vs. .81), and the Memory main effect was statistically significant $(\underline{F}(1,36)=9.40, \underline{\mathrm{p}}(.01)$.

c. Confidence Ratings on True and False Recognition Items 
Table 28

Mean Difference Scores for Confidence Ratings on Hits and False Alarms on General Information Recall and Recognition Tests for Each Age $x$ Education $x$ Sex Group

\begin{tabular}{lrrrrrr} 
& \multicolumn{2}{c}{ Recall } & \multicolumn{2}{c}{ Recognition } & \multicolumn{2}{c}{ Total } \\
HS & $\bar{x}$ & S.D. & $\bar{x}$ & S.D. & $\bar{x}$ & S.D. \\
\hline $\begin{array}{l}\text { to } 25 \\
\text { Males }\end{array}$ & 1.56 & .37 & .62 & .24 & 1.12 & .20 \\
$\begin{array}{l}\text { Females } \\
\text { Combined } \\
\text { Over Sex }\end{array}$ & 1.95 & .45 & .67 & .25 & .83 & .36 \\
\hline
\end{tabular}

60 to 65

$\begin{array}{lrrrrrr}\text { Males } & 1.35 & .32 & .56 & .65 & .84 & .62 \\ \text { Females } & .96 & .94 & .36 & .64 & .60 & .59 \\ \text { Combined } & 1.14 & .73 & .56 & .63 & .71 & .59 \\ \text { Over Sex } & & & & & \end{array}$

Combined over Age

$\begin{array}{lrrrrrr}\text { Males } & 1.43 & .34 & .59 & .49 & .94 & .51 \\ \text { Females } & .95 & . .75 & .51 & .50 & .69 & .51 \\ \text { Combined } & 1.17 & .63 & .55 & .49 & .80 & .52 \\ \text { Over Sex } & & & & & & \end{array}$

Ph. D.

20 to 25

$\begin{array}{lrrrrrr}\text { Males } & 1.12 & .93 & 1.39 & .65 & 1.24 & .58 \\ \text { Females } & 1.49 & .69 & .97 & .46 & 1.29 & .29 \\ \text { Combined } & 1.29 & .82 & 1.18 & .58 & 1.26 & .45 \\ \text { Over Sex } & & & & & & \end{array}$


Table 28 continued

\begin{tabular}{llllllll} 
& \multicolumn{2}{c}{ Recall } & \multicolumn{2}{c}{ Recognition } & \multicolumn{2}{c}{ Total } \\
Ph.D. & $\bar{x}$ & S.D. & $\bar{x}$ & S.D. & $\bar{x}$ & S.D \\
\hline $\begin{array}{l}\text { to } 65 \\
\quad \text { Males }\end{array}$ & .96 & .55 & .85 & .62 & .91 & .46 \\
Females & 1.84 & .72 & .83 & .48 & 1.35 & .34 \\
$\quad \begin{array}{l}\text { Combined } \\
\text { Over Sex }\end{array}$ & 1.37 & .76 & .84 & .54 & 1.11 & .45
\end{tabular}

Combined over Age

$\begin{array}{lrrrrrr}\text { Males } & 1.04 & .73 & 1.12 & .67 & 1.06 & .54 \\ \text { Females } & 1.68 & .70 & .90 & .46 & 1.32 & .30 \\ \text { Combined } & 1.33 & .77 & 1.01 & .58 & 1.18 & .45 \\ \text { Over Sex } & & & & & & \end{array}$

Combined over Ed 20 to 25

$\begin{array}{lrrrrrr}\text { Males } & 1.28 & .78 & 1.03 & .63 & 1.20 & .47 \\ \text { Females } & 1.24 & .63 & .82 & .39 & 1.08 & .39 \\ \text { Combined } & 1.26 & .69 & .92 & .52 & 1.14 & .42 \\ \text { Over Sex } & & & & & & \end{array}$

60 to 65

$\begin{array}{lllllll}\text { Males } & 1.13 & .49 & .71 & .63 & .87 & .52 \\ \text { Females } & 1.40 & .93 & .59 & .60 & .95 & .61 \\ \text { Combined } & 1.26 & .74 & .65 & .61 & .91 & .56 \\ \text { Over Sex } & & & & & & \end{array}$

Combined over Age

$\begin{array}{lllllll}\text { Males } & 1.19 & .62 & .86 & .64 & 1.01 & .51 \\ \text { Females } & 1.33 & .80 & .71 & .51 & 1.00 & .52 \\ \text { Combined } & 1.28 & .72 & .81 & .61 & 1.04 & .50 \\ \text { Over Sex } & & & & & & \end{array}$


incorrect true and false items on general information recognition tests for each Age $x$ Education $x$ Sex group is shown in Table 29. These data were submitted to a 2 (Age) $\times 2$ (Education) $\times 2$ (Sex) $\times 2$ (Order) $\times 2$ (Item (True vs. False)) x 2 (Response (Correct vs. Incorrect)) analysis of variance. The additional information gained in this analysis concerns confidence ratings on true vs. False items. The mean confidence rating on true items was lower than on false items (2.86 vs. 2.93), but the Item main effect was nonsignificant.

The Item $x$ Response interaction was statistically significant $(\underline{F}(1,48)=9.22, \underline{p}<.01)$, however, and may be seen in Figure 32, which shows the mean confidence ratings for correct and incorrect true and false items on general information knowledge recognition tests. For correct responses, significantly higher confidence ratings were observed for true than false items $(\underline{t}(63)=2.21$, $\underline{p}(.05)$, while for incorrect responses significantly higher confidence ratings were observed for false than true items $(t(63)=11.53$, $\mathrm{p}<.001)$.

\section{d. Difference Scores for Confidence Ratinas on}

True and False Recoanition Items

The mean difference scores for confidence ratings on correct and incorrect true and false items 
Table 29

Mean Confidence Ratings on Correct and Incorrect True and False Items on General Information Recognition Tests for Each Age $x$ Education $x$ Sex Group

Correct

\begin{tabular}{|c|c|c|c|c|c|c|}
\hline \multirow[b]{2}{*}{ HS } & \multicolumn{2}{|c|}{ True } & \multicolumn{2}{|c|}{ False } & \multicolumn{2}{|c|}{ Mean } \\
\hline & $\bar{x}$ & S.D. & $\bar{x}$ & S.D. & $\bar{x}$ & S.D \\
\hline \multicolumn{7}{|l|}{20 to 25} \\
\hline Males & 3.53 & .41 & 3.58 & .35 & 3.56 & .38 \\
\hline Females & 2.91 & .32 & 2.72 & .50 & 2.82 & .37 \\
\hline $\begin{array}{l}\text { Combined } \\
\text { Over Sex }\end{array}$ & 3.22 & .48 & 3.15 & .61 & 3.19 & .53 \\
\hline \multicolumn{7}{|l|}{60 to 65} \\
\hline Males & 3.32 & .79 & 3.24 & .60 & 3.28 & .68 \\
\hline Females & 3.32 & .54 & 2.86 & .55 & 3.09 & .49 \\
\hline $\begin{array}{l}\text { Combined } \\
\text { Over Sex }\end{array}$ & 3.22 & .65 & 3.05 & .59 & 3.19 & .58 \\
\hline
\end{tabular}

Combined over Age

$\begin{array}{lllllll}\text { Males } & 3.43 & .61 & 3.41 & .50 & 3.42 & .55 \\ \text { Females } & 3.12 & .48 & 2.79 & .51 & 2.95 & .44 \\ \text { Combined } & 3.27 & .56 & 3.10 & .59 & 3.19 & .54 \\ \text { Over Sex } & & & & & & \end{array}$

Ph.D.

20 to 25

Males

Females

$3.30 \quad .21$

$3.24 \quad .37$

$3.27 \quad .2$ ?

Combined

Over Sex

$3.37 \quad .21$

$3.34 \quad .28$

$3.39 \quad .19$

3.29 .32

3.33 .23 
Table 29 Continued

Correct

\begin{tabular}{|c|c|c|c|c|c|c|}
\hline \multirow[b]{2}{*}{$\mathrm{Ph} \cdot \mathrm{D}$. } & \multicolumn{2}{|c|}{ True } & \multicolumn{2}{|c|}{ False } & \multicolumn{2}{|c|}{ Mean } \\
\hline & $\bar{x}$ & S.D. & $\bar{x}$ & S.D. & $\bar{x}$ & S.D. \\
\hline \multicolumn{7}{|l|}{50 to 65} \\
\hline Males & 3.70 & .27 & 3.59 & .23 & 3.65 & .22 \\
\hline Females & 3.43 & .55 & 3.60 & .29 & 3.51 & .35 \\
\hline $\begin{array}{l}\text { Combined } \\
\text { Over Sex } \\
\end{array}$ & $\begin{array}{c}3.57 \\
. \\
\end{array}$ & .44 & 3.60 & .25 & 3.58 & .29 \\
\hline
\end{tabular}

Combined over Age

$\begin{array}{lllllll}\text { Males } & 3.50 & .31 & 3.41 & .35 & 3.46 & .31 \\ \text { Females } & 3.44 & .40 & 3.47 & .30 & 3.45 & .28 \\ \begin{array}{l}\text { Combined } \\ \text { Over Sex }\end{array} & 3.47 & .31 & 3.44 & .32 & 3.46 & .29\end{array}$

Combined over Ed 20 to 25

$\begin{array}{lllllll}\text { Males } & 3.42 & .33 & 3.41 & .39 & 3.41 & .35 \\ \text { Females } & 3.18 & .38 & 3.03 & .50 & 3.10 & .41 \\ \text { Combined } & 3.30 & .37 & 3.22 & .48 & 3.26 & .41 \\ \text { Over Sex } & & & & & & \end{array}$

60 to 65

$\begin{array}{lllllll}\text { Males } & 3.51 & .60 & 3.42 & .47 & 3.47 & .52 \\ \text { Females } & 3.37 & .53 & 3.23 & .57 & 3.30 & .47 \\ \text { Combined } & 3.44 & .56 & 3.32 & .52 & 3.38 & .50 \\ \text { Over Sex } & & & & & & \end{array}$

Combined over Age

$\begin{array}{lllllll}\text { Males } & 3.47 & .48 & 3.41 & .43 & 3.44 & .44 \\ \text { Females } & 3.28 & .46 & 3.13 & .54 & 3.20 & .44 \\ \begin{array}{l}\text { Combined } \\ \text { Over Sex }\end{array} & 3.37 & .48 & 3.27 & .50 & 3.32 & .45\end{array}$


Table 29 Continued

Incorrect

\begin{tabular}{llllllll}
\multicolumn{1}{c}{ HS } & $\bar{x}$ & True & \multicolumn{2}{c}{ False } & \multicolumn{2}{c}{ Mean } \\
\hline 20 to 25 & & & & & & & \\
$\quad$ Males & 2.93 & .66 & 2.99 & .66 & 2.96 & .62 \\
$\quad \begin{array}{l}\text { Females } \\
\text { Combined }\end{array}$ & 1.99 & .44 & 2.34 & .36 & 2.11 & .28 \\
Over Sex & 2.46 & .73 & 2.61 & .65 & 2.54 & .64
\end{tabular}

60 to 65

Males

Females

Combined

Over Sex

$\begin{array}{ll}2.45 & .89 \\ 1.77 & .61 \\ 2.11 & .81\end{array}$

2.77

.59

2.61

.72

2.96

.66

2.36

.44

2.87

.61

$2.49 \quad .59$

Combined over Age

$\begin{array}{lllllll}\text { Males } & 2.69 & .80 & 2.88 & .62 & 2.79 & .67 \\ \text { Females } & 1.88 & .52 & 2.60 & .64 & 2.24 & .38 \\ \text { Combined } & 2.28 & .78 & 2.74 & .63 & 2.51 & .60 \\ \text { Over Sex } & & & & & & \end{array}$

$\mathrm{Ph} \cdot \mathrm{D}$.

20 to 25

Males

Females

2.10 .51

1.91 .54

2.01

.40

Combined

Over Sex

$2.21 \quad .85$

2.48 .60

2.34

.67

$2.16 \quad .68$

2. 19

.62

2.18

.56

60. to 65

$\begin{array}{lllllll}\text { Males } & 2.78 & .59 & 2.85 & .65 & 2.81 & .59 \\ \text { Females } & 2.56 & .39 & 2.60 & .71 & 2.58 & .21 \\ \text { Combined } & 2.67 & .50 & 2.72 & .67 & 2.70 & .45 \\ \text { Over Sex } & & & & & & \end{array}$


Table 29 Continued

Incorrect

\begin{tabular}{|c|c|c|c|c|c|c|}
\hline \multirow{2}{*}{$\mathrm{Ph} \cdot \mathrm{D}$. } & \multicolumn{2}{|c|}{ True } & \multicolumn{2}{|c|}{ False } & \multicolumn{2}{|c|}{ Mean } \\
\hline & $\bar{x}$ & $S \cdot D \cdot$ & $\overline{\mathrm{x}}$ & $S \cdot D \cdot$ & $\overline{\mathrm{x}}$ & S.D \\
\hline Combined over & Age & & & & & \\
\hline Males & 2.44 & .64 & 2.38 & .76 & 2.41 & .64 \\
\hline Females & 2.39 & .66 & 2.54 & .64 & 2.46 & .49 \\
\hline $\begin{array}{l}\text { Combined } \\
\text { Over Sex }\end{array}$ & 2.42 & .64 & 2.46 & .69 & 2.44 & .56 \\
\hline
\end{tabular}

Combined over Ed 20 to 25

$\begin{array}{lllllll}\text { Males } & 2.52 & .72 & 2.45 & .81 & 2.49 & .70 \\ \text { Females } & 2.10 & .66 & 2.36 & .49 & 2.23 & .51 \\ \text { Combined } & 2.31 & .71 & 2.40 & .66 & 2.36 & .62 \\ \text { Over Sex } & & & & & & \end{array}$

60 to 65

$\begin{array}{lllllll}\text { Males } & 2.61 & .75 & 2.81 & .60 & 2.71 & .64 \\ \text { Females } & 2.16 & .64 & 2.78 & .69 & 2.47 & .32 \\ \text { Combined } & 2.39 & .72 & 2.79 & .64 & 2.59 & .52 \\ \text { Over Sex } & & & & & & \end{array}$

Combined over Age

$\begin{array}{lllllll}\text { Males } & 2.57 & .72 & 2.63 & .72 & 2.60 & .67 \\ \text { Females } & 2.13 & .64 & 2.57 & .63 & 2.35 & .45 \\ \text { Combined } & 2.35 & .71 & 2.60 & .67 & 2.47 & .58 \\ \text { Over Sex } & & & & & & \end{array}$


Figure 32. Mean Confidence Ratings for Correct and Incorrect True and False Items on General Information Recognition Tests

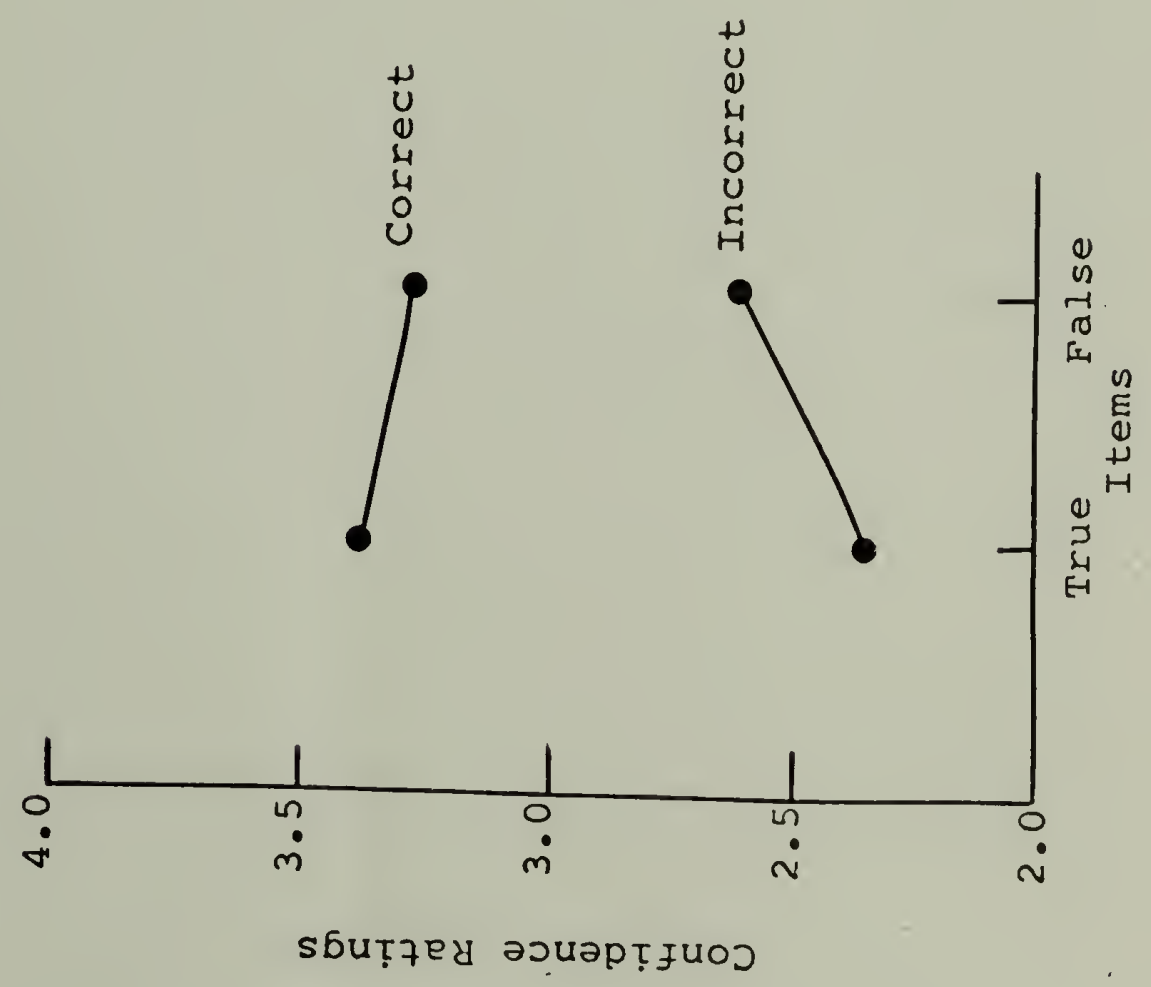


on general information recognition tests for each Age $x$ Education $x$ Sex group is shown in Table 30. The mean difference score was higher for true than false items ( $1.05 \mathrm{vs} . .70)$, and the Item main effect was statistically significant $(\underline{F}(1,48)=9.22, \underline{.}<.01)$.

\section{Summary}

To summarize the results for memory confidence accuracy, 2 (Age) $\times 2$ (Education) $\times 2$ (Sex) analyses of variance on the mean differences between confidence ratings on correct and incorrect episodic and semantic recall and recognition responses, as well as on average episodic and semantic confidence are summarized in Table 31.

\section{a. Episodic Memory Confidence}

Younger subjects' recall confidence ratings were more accurate than those of older subjects, while older subjects' recognition confidence ratings were more accurate than those of younger subjects, however, Age accounted for only $1 \%$ of the variance in recall confidence accuracy and $2 \%$ in recognition confidence accuracy. Ph.D. subjects were more accurate than HS subjects, and Education accounted for $9 \%$ of the variance in recall confidence accuracy and $4 \%$ in recognition confidence accuracy. Females were more accurate than males and sex accounted for $3 \%$ of the variance in recall confidence accuracy and $3 \%$ in recognition confi- 


\section{Table 30}

Mean Difference Scores for Confidence Ratings On Correct and Incorrect True and False Items on General Information Recognition For Each Age $x$ Education $x$ Sex Group

\begin{tabular}{|c|c|c|c|c|c|c|}
\hline \multirow[b]{2}{*}{ HS } & \multicolumn{2}{|c|}{ True } & \multicolumn{2}{|c|}{ False } & \multicolumn{2}{|c|}{ Total } \\
\hline & $\bar{x}$ & $S . D$. & $\bar{x}$ & S.D. & $\bar{x}$ & S.D. \\
\hline \multicolumn{7}{|l|}{20 to 25} \\
\hline Males & .69 & .50 & .67 & .25 & .68 & .28 \\
\hline Females & .92 & .44 & .49 & .56 & .70 & .32 \\
\hline $\begin{array}{l}\text { Combined } \\
\text { Over Sex }\end{array}$ & .81 & .47 & .57 & .44 & .69 & .29 \\
\hline \multicolumn{7}{|l|}{60 to 65} \\
\hline Males & .88 & .94 & .54 & .61 & .67 & .73 \\
\hline Females & 1.56 & .78 & -.11 & .82 & .72 & .52 \\
\hline $\begin{array}{l}\text { Combined } \\
\text { Over Sex }\end{array}$ & 1.22 & .91 & .19 & .73 & .70 & .61 \\
\hline
\end{tabular}

Combined over Age

\begin{tabular}{lrrrrrr} 
Males & .79 & .75 & .61 & .45 & .68 & .55 \\
Females & 1.24 & .70 & .19 & .74 & .71 & .41 \\
$\begin{array}{l}\text { Combined } \\
\text { Over Sex }\end{array}$ & 1.02 & .74 & .38 & .65 & .70 & .48 \\
\hline
\end{tabular}

Ph.D.

20 to 25

\begin{tabular}{lrrrrrr} 
Males & 1.20 & .48 & 1.32 & .81 & 1.26 & .53 \\
Females & 1.41 & .69 & .86 & .52 & 1.05 & .51 \\
Combined & 1.29 & .57 & 1.09 & .70 & 1.15 & .51 \\
Over Sex & & & & & & \\
\hline
\end{tabular}


Table 30 Continued

\begin{tabular}{|c|c|c|c|c|c|c|}
\hline \multirow[b]{2}{*}{$\mathrm{Ph} \cdot \mathrm{D}$. } & \multicolumn{2}{|c|}{ True } & \multicolumn{2}{|c|}{ False } & \multicolumn{2}{|c|}{ Total } \\
\hline & $\bar{x}$ & S.D. & $\bar{x}$ & S.D. & $\bar{x}$ & S.D. \\
\hline \multicolumn{7}{|l|}{60 to 65} \\
\hline Males & .92 & .58 & .85 & .51 & .83 & .53 \\
\hline Females & .87 & .82 & 1.00 & .71 & .93 & .21 \\
\hline $\begin{array}{l}\text { Combined } \\
\text { Over Sex }\end{array}$ & .90 & .68 & .93 & .61 & .88 & .40 \\
\hline \multicolumn{7}{|c|}{ Combined over Age } \\
\hline Males & 1.06 & .53 & 1.10 & .71 & 1.05 & .56 \\
\hline Females & 1.12 & .78 & .93 & .60 & .99 & .38 \\
\hline $\begin{array}{l}\text { Combined } \\
\text { over Sex }\end{array}$ & 1.09 & .65 & 1.01 & .65 & 1.02 & .47 \\
\hline
\end{tabular}

Combined over Ed 20 to 25

$\begin{array}{lrrrrrr}\text { Males } & .96 & .54 & 1.02 & .69 & .99 & .51 \\ \text { Females } & 1.15 & .60 & .67 & .56 & .87 & .45 \\ \text { Combined } & 1.05 & .57 & .84 & .64 & .93 & .48 \\ \text { Over Sex } & & & & & & \end{array}$

60 to 65

$\begin{array}{lllllll}\text { Males } & .90 & .76 & .69 & .56 & .75 & .62 \\ \text { Females } & 1.21 & .85 & .45 & .93 & .83 & .40 \\ \text { Combined } & 1.06 & .81 & .56 & .78 & .79 & .52 \\ \text { Over Sex } & & & & & & \end{array}$

Combined over Age

$\begin{array}{lllllll}\text { Males } & .93 & .65 & .86 & .64 & .87 & .58 \\ \text { Females } & 1.1 .8 & .73 & .56 & .77 & .85 & .42 \\ \text { Combined } & 1.05 & .70 & .70 & .72 & .86 & .50 \\ \text { Over Sex } & & & & & & \end{array}$


Table 31

Summary ANOVAs for Episodic and Semantic Confidence Accuracy

\begin{tabular}{|c|c|c|c|c|}
\hline $\begin{array}{l}\text { Episodic } \\
d f=1.56\end{array}$ & 1.02 & $\begin{array}{l}\bar{X}= \\
F\end{array}$ & pli & $E t a^{2}$ \\
\hline Age & $\begin{array}{r}-.03 \\
.04\end{array}$ & $<1$ & NS & $<.01$ \\
\hline Education & $\begin{array}{r}-.23 \\
.32\end{array}$ & 4.04 & .06 & .09 \\
\hline $\operatorname{sex}$ & $\begin{array}{r}-.17 \\
.14\end{array}$ & 1.54 & NS & .03 \\
\hline Age $x$ Ed & & $<1$ & NS & \\
\hline Age $x$ Sex & & 4.99 & .05 & \\
\hline Ed $x$ Sex & & 1.07 & NS & \\
\hline $\begin{array}{l}\text { Age } x \text { Ed } x \text { Sex } \\
\text { Multiple } R^{2}\end{array}$ & & $<1$ & NS & .14 \\
\hline Semantic & & $\bar{x}=$ & 11 & 2 \\
\hline $\mathrm{df}=1,56$ & 1.26 & F & $\mathrm{P}$ & $E t a{ }^{2}$ \\
\hline Age & $\begin{array}{r}.00 \\
.00\end{array}$ & $<1$ & NS & $<.01$ \\
\hline Education & $\begin{array}{r}-.09 \\
.07\end{array}$ & $<1$ & NS & .01 \\
\hline $\operatorname{sex}$ & $\begin{array}{r}-.07 \\
.07\end{array}$ & $<1$ & NS & .01 \\
\hline Age $x$ Ed & & $<1$ & NS & \\
\hline Age $x$ Sex & & $<1$ & NS & \\
\hline Ed $x$ Sex & & 8.40 & $<.01$ & \\
\hline $\begin{array}{l}\text { Age } x \text { Ed } x \text { Sex } \\
\text { Multiple } R^{2}\end{array}$ & & $<1$ & NS & .03 \\
\hline
\end{tabular}


Table 31 Continued

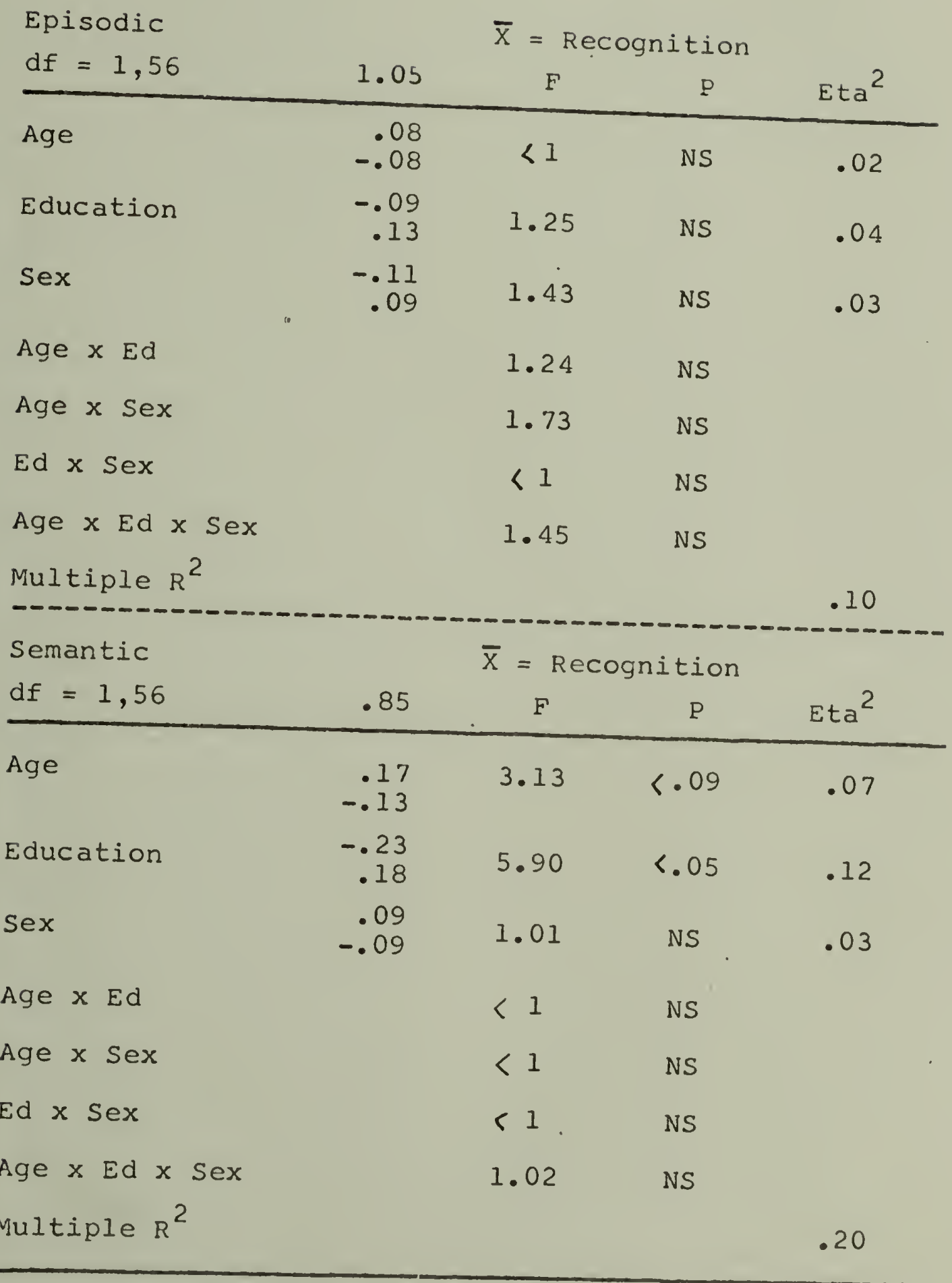


Table 31 Continued

\begin{tabular}{|c|c|c|c|c|}
\hline $\begin{array}{l}\text { Episodic } \\
\mathrm{d} f=1,56\end{array}$ & 1.03 & $\begin{array}{l}\bar{X}= \\
F\end{array}$ & $\stackrel{t a l}{t}$ & $E t a^{2}$ \\
\hline Age & $\begin{array}{r}.02 \\
-.02\end{array}$ & $<1$ & NS & $<.01$ \\
\hline Education & $\begin{array}{r}-.16 \\
.22\end{array}$ & 4.43 & $<.05$ & .10 \\
\hline sex & $\begin{array}{r}-.14 \\
.11\end{array}$ & 2.42 & NS & .04 \\
\hline Age $x E d$ & & 1.39 & NS & \\
\hline Age $x$ Sex & & 5.65 & $<.05$ & \\
\hline Ed $x$ Sex & & $<1$ & NS & \\
\hline $\begin{array}{l}\text { Age } x \text { Ed } x \text { Sex } \\
\text { Multiple } R^{2}\end{array}$ & & $<1$ & NS & .16 \\
\hline Semantic & & $\bar{x}=$ & tal & \\
\hline $\begin{array}{l}\text { Semantic } \\
\mathrm{d} f=1,56 \\
\end{array}$ & 1.06 & $\mathrm{~F}$ & $P$ & $E t a^{2}$ \\
\hline Age & $\begin{array}{r}.08 \\
-.06\end{array}$ & 1.17 & NS & .03 \\
\hline Education & $\begin{array}{r}-.16 \\
.13\end{array}$ & 5.22 & .05 & .10 \\
\hline Sex & $\begin{array}{r}.01 \\
-.01\end{array}$ & $<1$ & NS & $<.01$ \\
\hline Age $x$ Ed & & r & NS & \\
\hline Age $x$ Sex & & $<1$ & NS & \\
\hline$E d^{\prime} x \operatorname{Sex}$ & & 5.99 & $<.05$ & \\
\hline $\begin{array}{l}\text { Age } x \text { Ed } \times \text { Sex } \\
\text { Multiple } R^{2}\end{array}$ & & $<1$ & NS & .12 \\
\hline
\end{tabular}


dence accuracy. These variables accounted for $14 \%$ of the variance in episodic recall confidence accuracy and $10 \%$ in episodic recognition confidence accuracy. Furthermore, summed over recall and recognition, 16\% of the variance could be accounted for: $1 \%$ by Age, 10\% by Education, and $4 \%$ by Sex.

b. Semantic Memory Confidence

There were no differences in the accuracy of recall confidence ratings of younger and older subjects but younger subjects' recognition confidence ratings were more accurate than those of older subjects, and Age accounted for $7 \%$ of the variance in recognition confidence accuracy. Ph.D. subjects were more accurate than HS subjects, and Education accounted for $10 \%$ of the variance in recall confidence accuracy and $12 \%$ in recognition confidence accuracy. Females' recall confidence ratings were more accurate than males', while males' recognition confidence ratings were more accurate than females, however, sex accounted for only $1 \%$ of the variance in recall confidence accuracy and $3 \%$ in recognition confidence accuracy. These variables accounted for $3 \%$ of the variance in semantic recall confidence accuracy, and $20 \%$ in semantic recognition confidence accuracy. Furthermore, summed over recall and recognition $12 \%$ of the variance could be accounted for: $3 \%$ by Age, $10 \%$ by Education, and less than $1 \%$ by Sex. 


\section{F. Times}

Subjects worked on tasks at their own pace, but they were asked to record the time at the beginning and end of each task. The mean time spent on each task by each Age $x$ Education $x$ sex group is shown in Table 32. These times were submitted to a 2 (Age) $x$ 2 (Education) $\times 2$ (Sex) $\times 2$ (Order) $\times 9$ (Task (Background Questionnaire vs. Metamemory Questionnaire vs. Association vs. Memory Study vs. Recall Prediction vs. Incidental Recall vs. Intentional Recall vs. General Information Recall vs. Recognition)) analysis of variance. The average time spent on all tasks was 79 minutes. Younger subjects spent less time than older subjects ( 68 vs. 89), and the Age main effect was statistically significant $(\underline{E}(1,48)=14.45, \underline{\mathrm{p}}<.001)$. HS subjects spent more time than Ph.D. subjects ( 86 vs. 71), and the Education main effect was also statistically significant $(\underline{F}(1,48)=7.31, \underline{p}<.01)$. Males spent less time than females ( $77 \mathrm{vs}$. 80 ), but the sex main effect was nonsignificant. The order main effect was also nonsignificant.

The mean time spent on the background questionnaire was 5 minutes, on the metamemory questionnaire $16 \mathrm{~min}-$ utes, generating associations 4 minutes, studying intentional memory items 3 minutes, predicting intentional and incidental recall 2 minutes, recalling incidental 


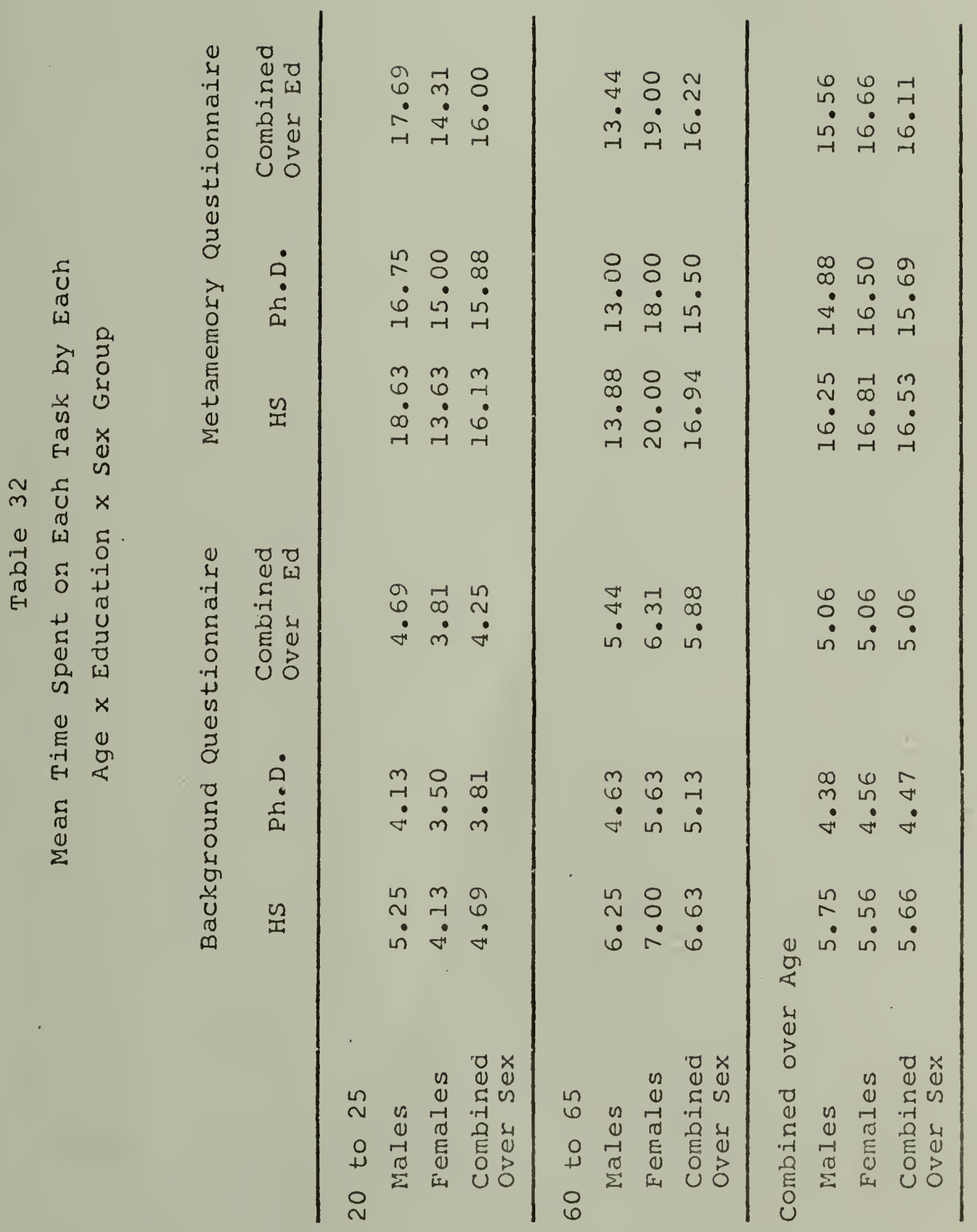


Table 32 Continued

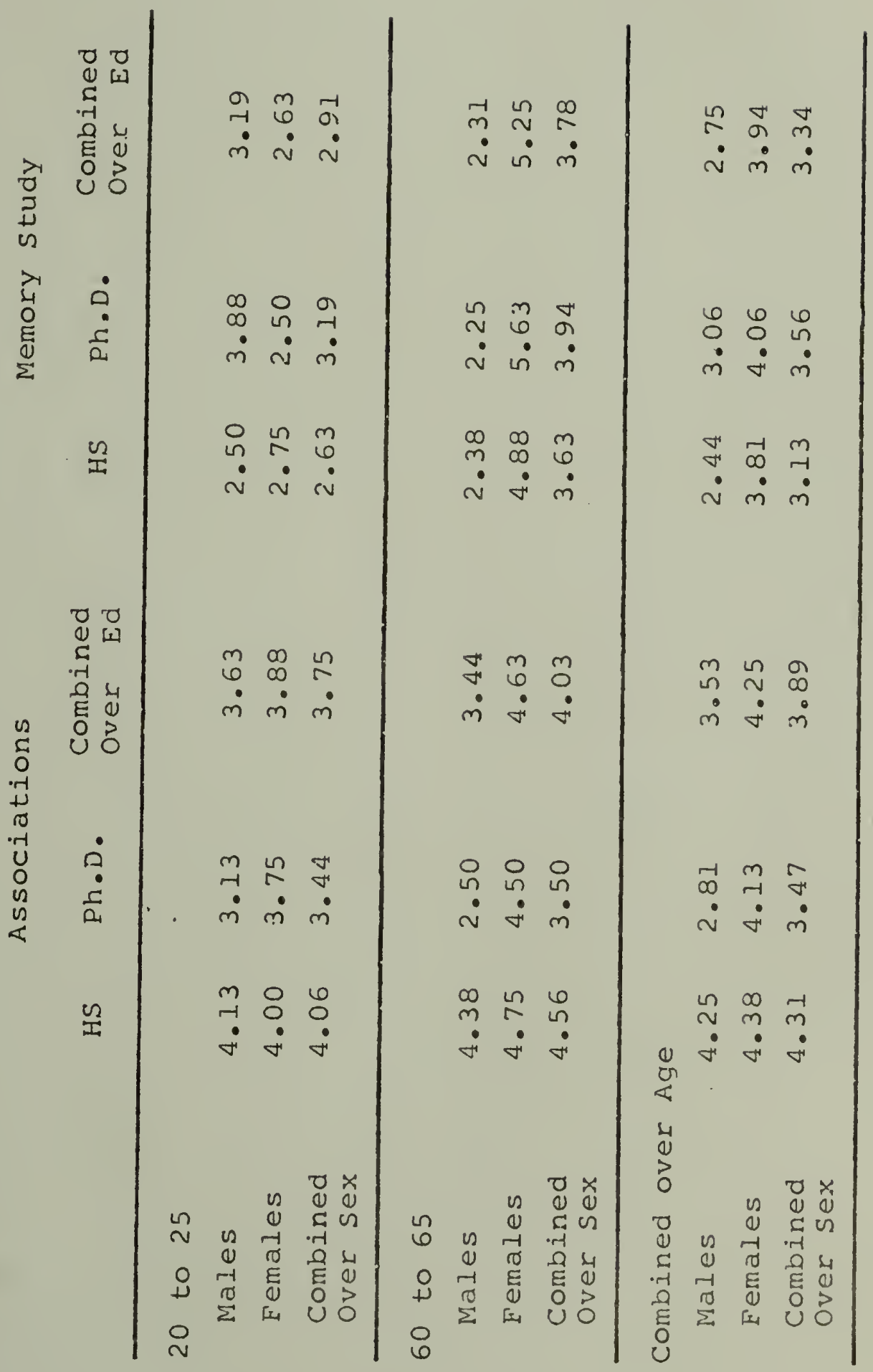


Table 32 Continued

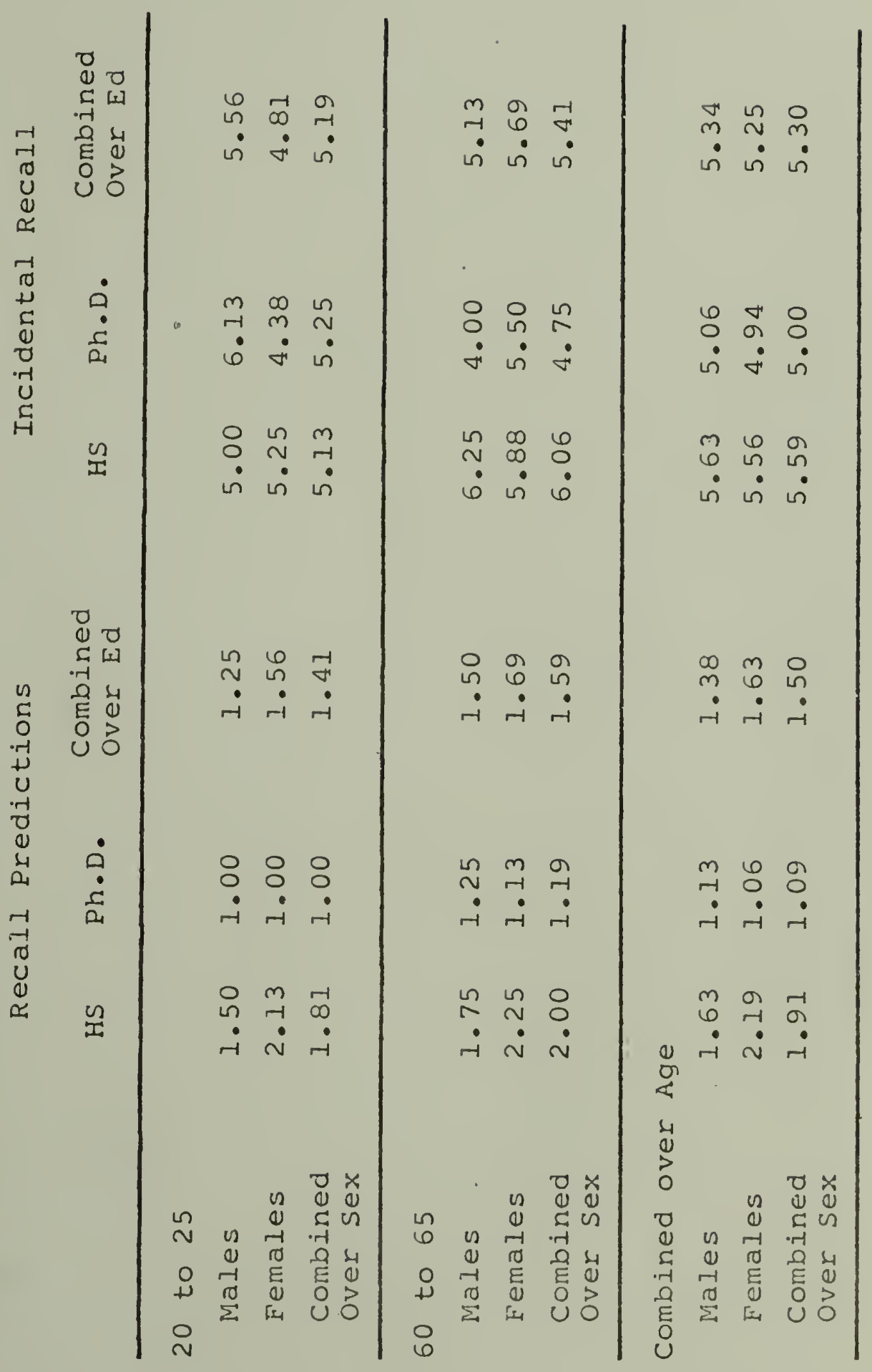


Table 32 continued

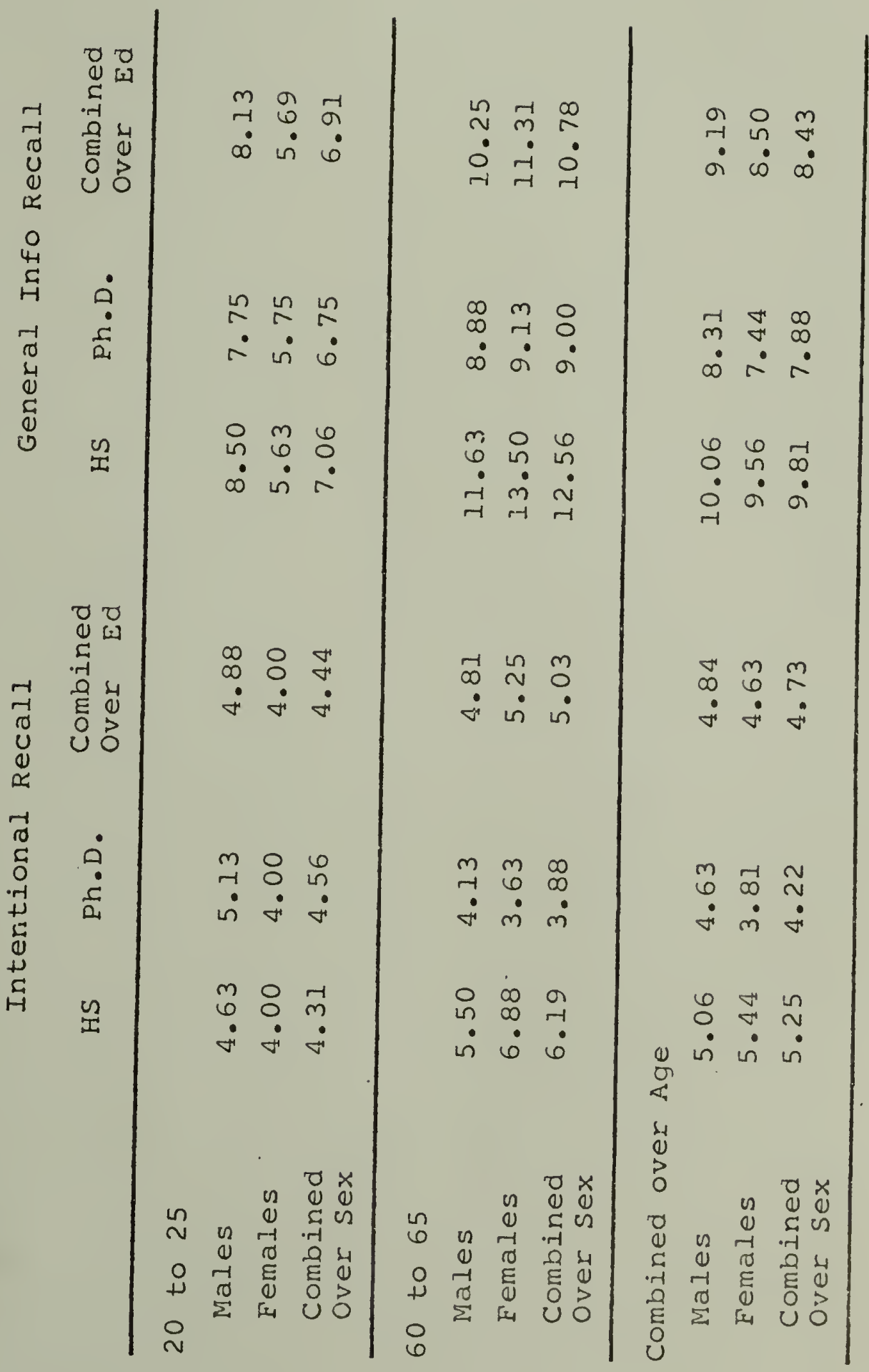


Table 32 Continued

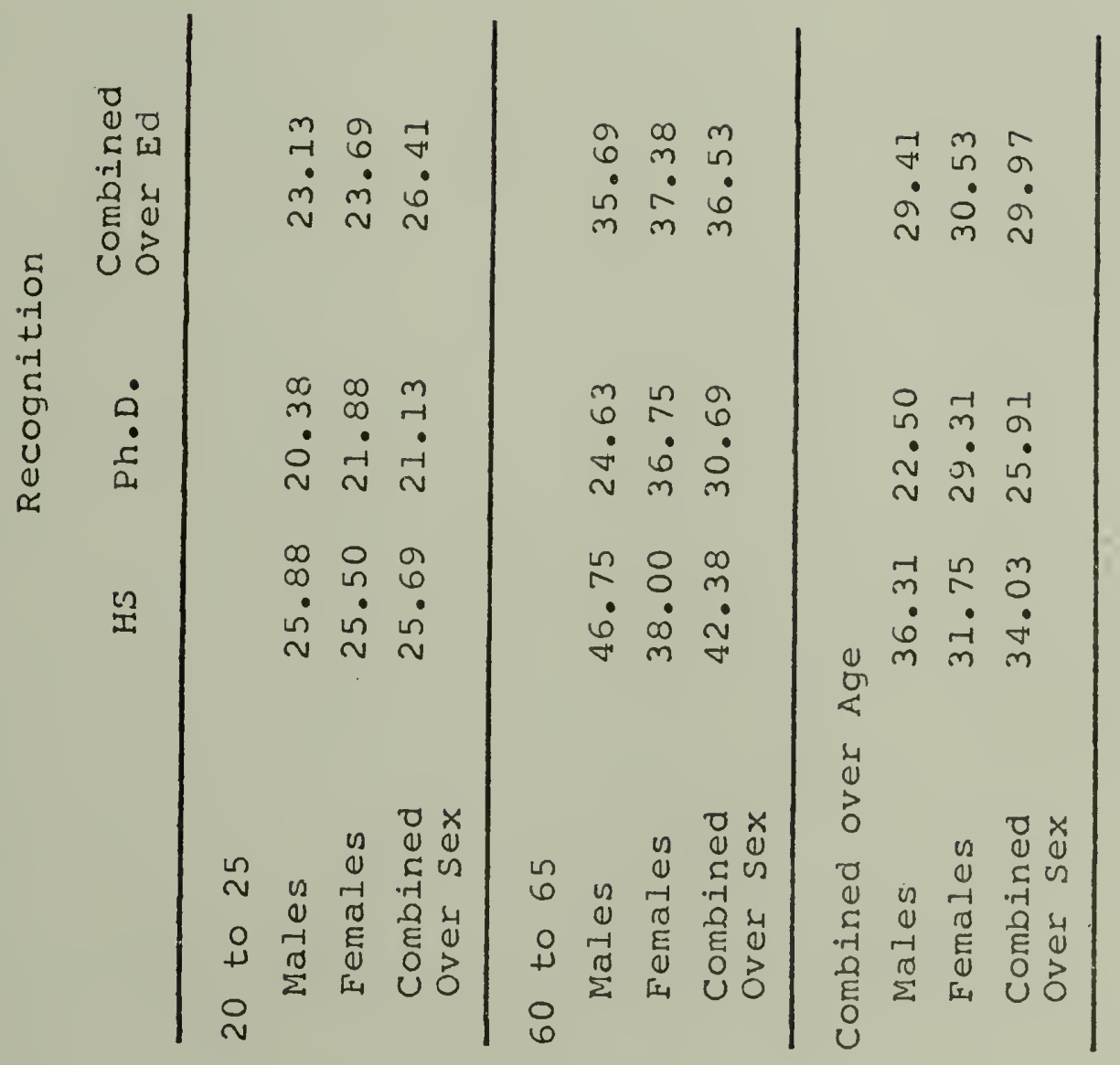


items 5 minutes, recalling intentional items 5 minutes, recalling general information facts 9 minutes, and responding to the recognition task 20 minutes. The Task main effect was highly significant $(\underline{F}(8,384)=$ 266.43, $\mathrm{p}(.001)$. Moreover, Task interacted significantiy with Age $(\underline{F}(8,384)=14.54, \underline{\mathrm{p}}<.001)$, and $\mathrm{Ed}-$ ucation $(\underline{F}(8,384)=5.12, \mathrm{p}<.05)$.

Mean times spent on each task by the two Age groups may be seen in Figure 33. Older subjects spent significantly more time than younger subjects on the background questionnaire $(\underline{t}(48)=2.63, \underline{p}<.05)$, general information recall $(\underline{f}(48)=3.69, \mathrm{p}<.001)$, and the recognition task $(\underline{t}(48)=5.64, \mathrm{p}<.001)$. Age did not have a significant effect on the amount of time spent on any of the other tasks.

Mean times spent on each task by the two Education groups may be seen in Figure 34. HS subjects spent significantly more time than $\mathrm{Ph} . \mathrm{D}$. subjects on the background questionnaire $(\underline{t}(48)=3.61, \underline{\mathrm{p}}(.001)$, predicting recall $(\underline{t}(48)=2.13, \underline{p}<.05)$, and the recognition task $(\underline{t}(48)=3.49, \underline{p}<.001)$. Education did not have a significant effect on the amount of time spent on any of the other tasks.

Time spent preparing for memory tasks, that is generating associations to incidental items, and studying intentional items, was of particular interest. The 
Figure 33. Mean Times Spent on Each Task by the Two Age Groups

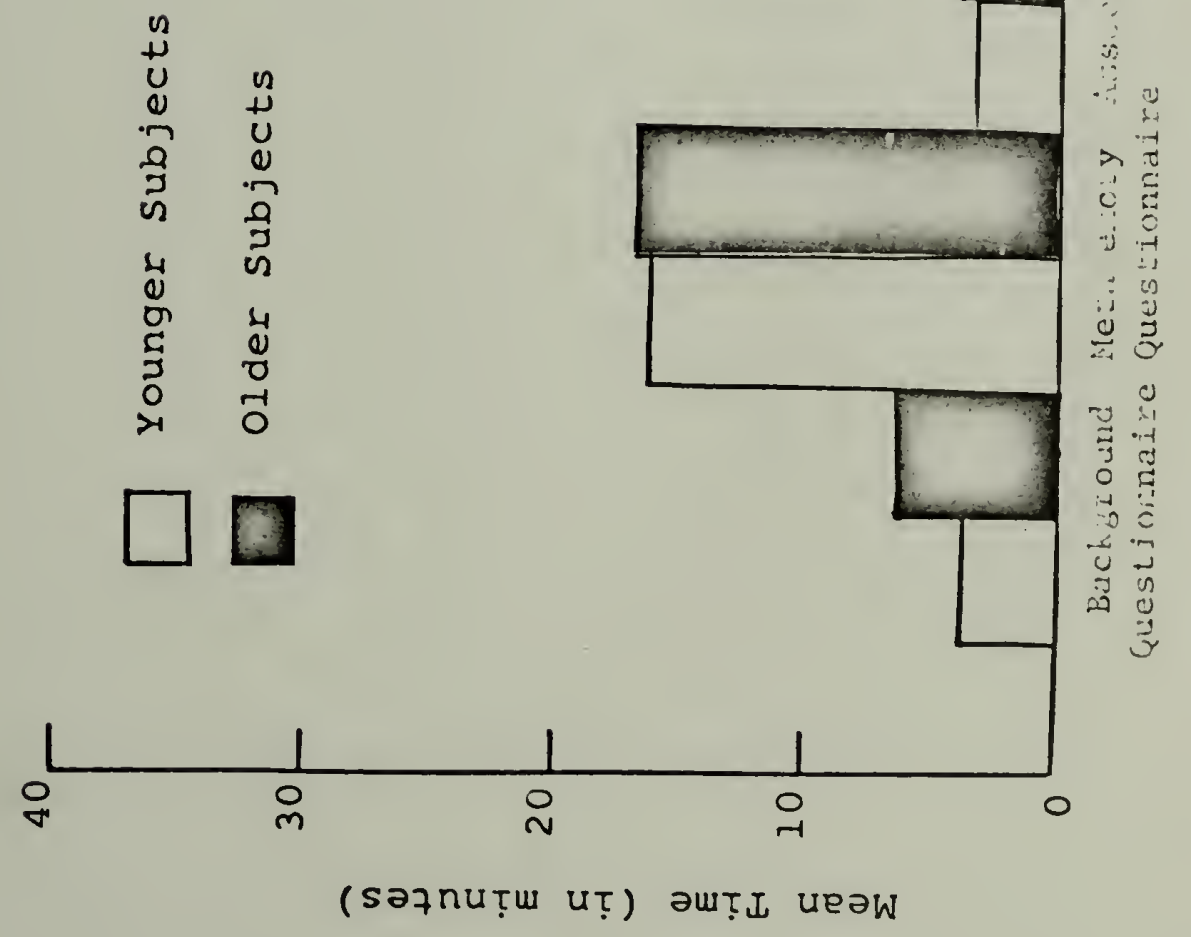


Figure 33 Cont.

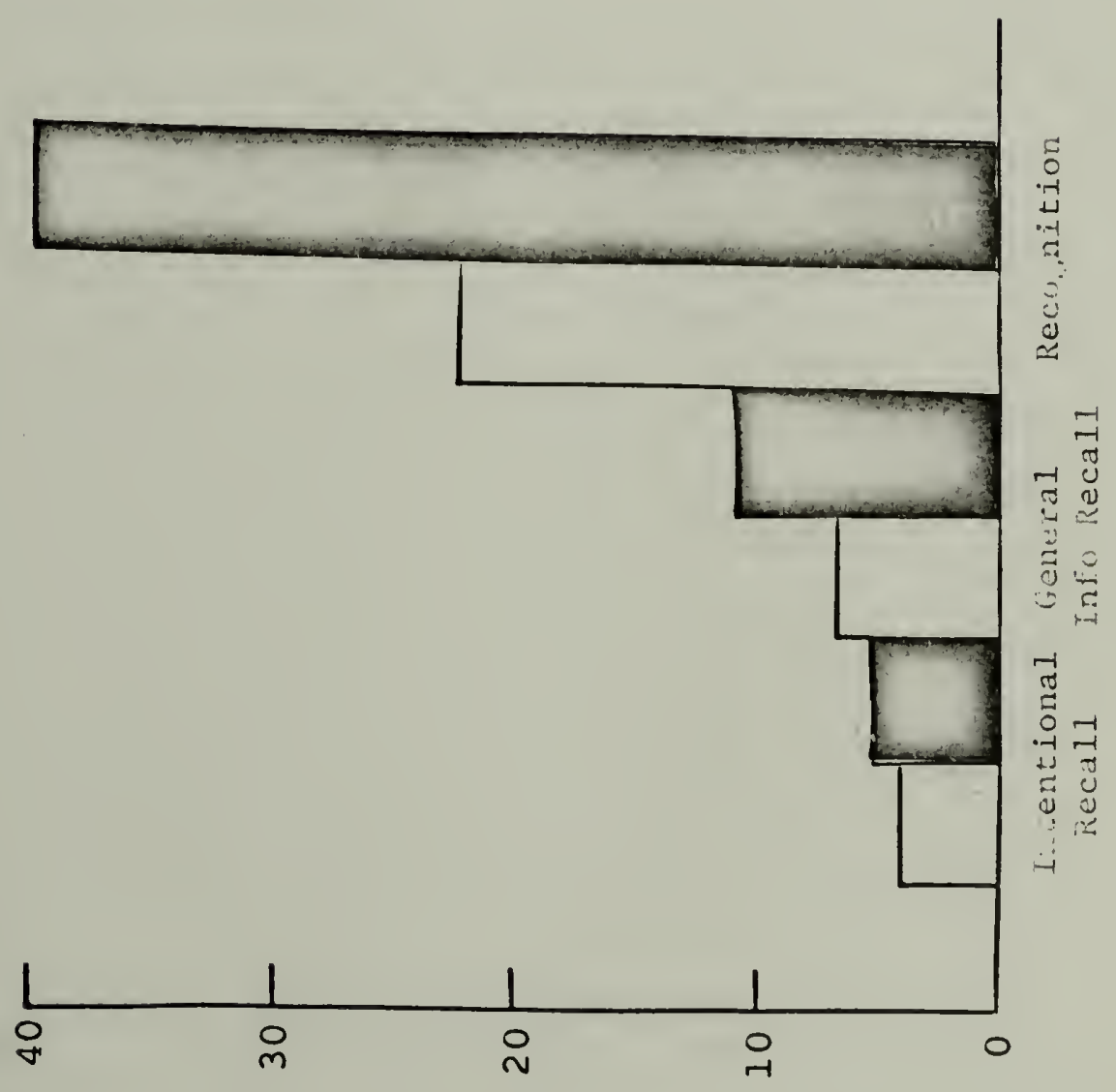


Figure 34. Mean Times Spent on Each Task by the Two Education Groups

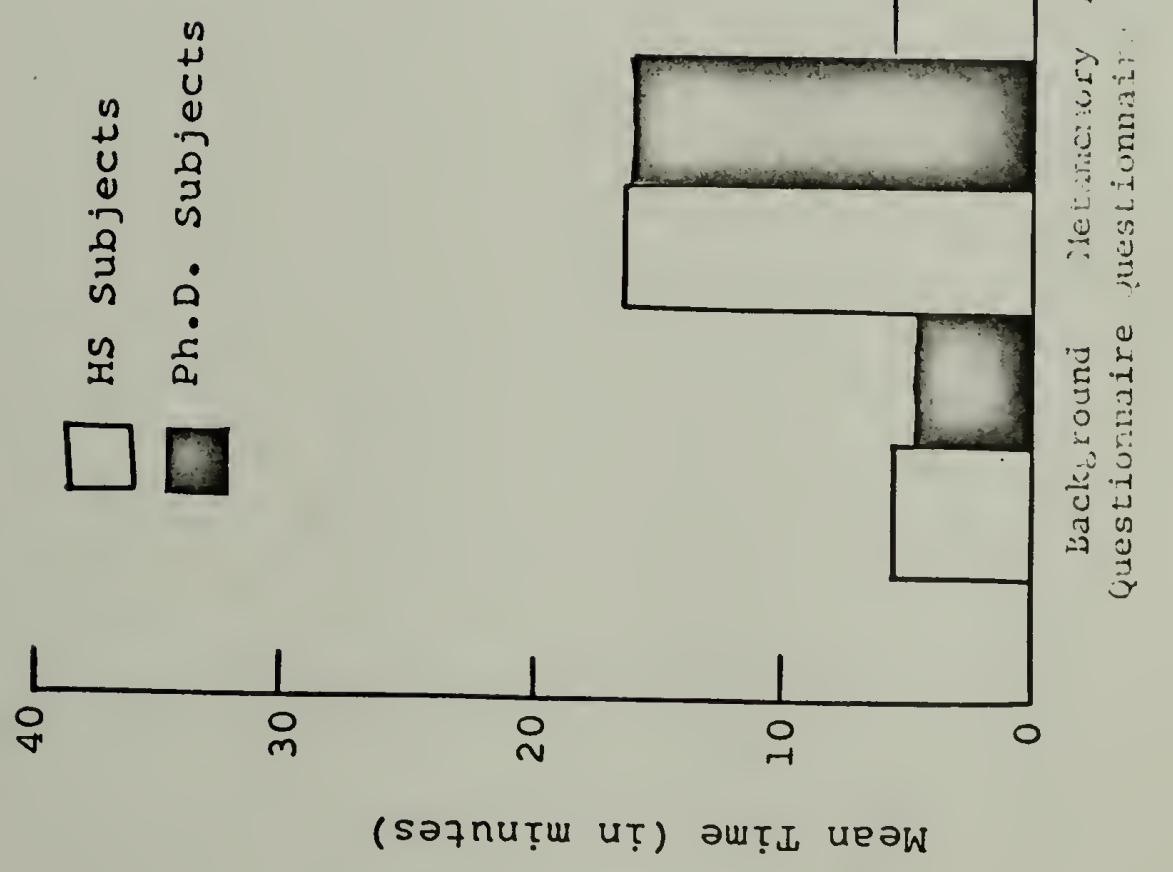


Figure 34 Cont.

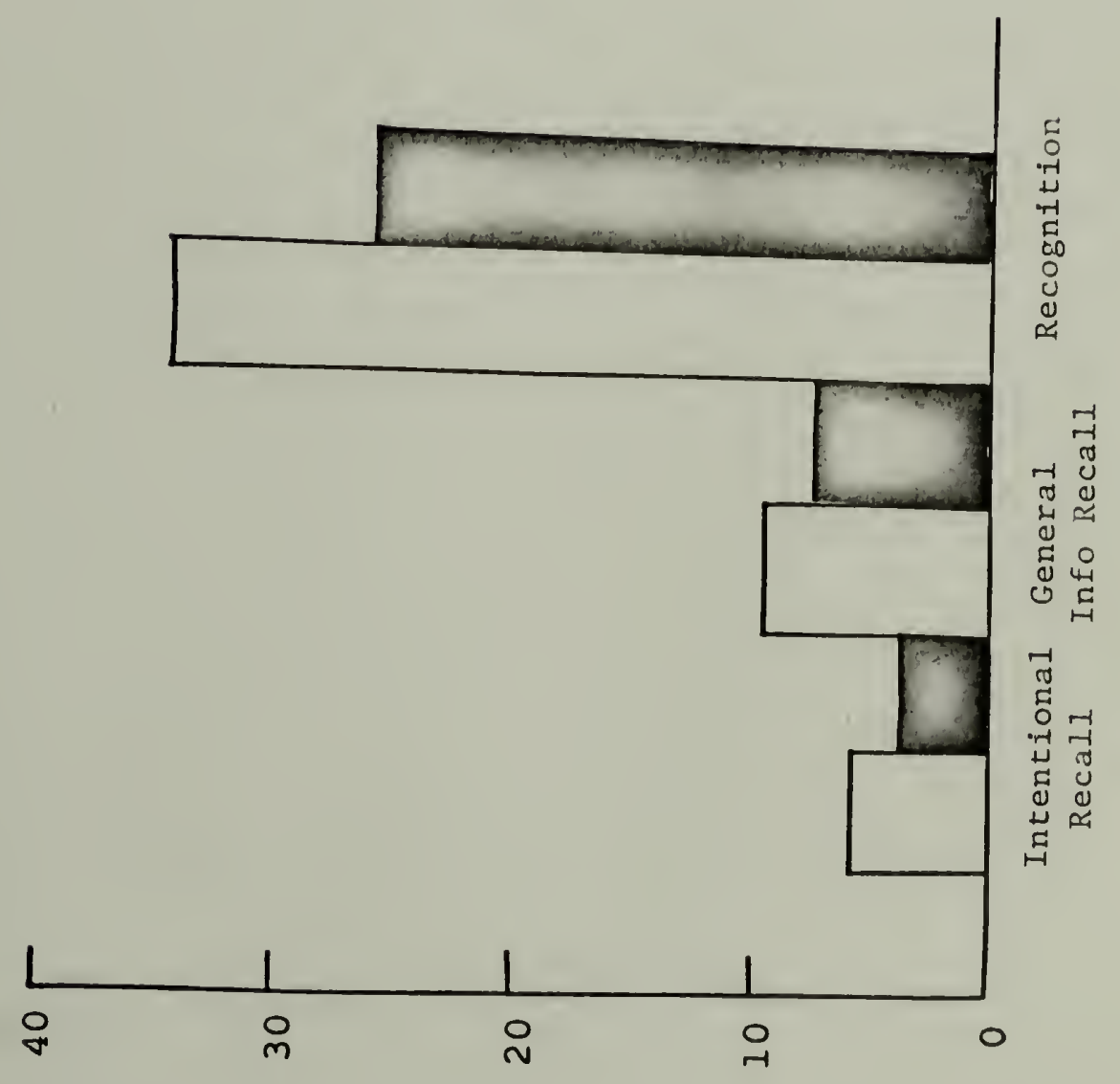


mean time spent generating associations was $3.89 \mathrm{~min}$ utes, and the mean time studying was 3.34 minutes. These times were not statistically different.

A composite preparation time score was computed by summing the time spent generating associations to incidental items and studying intentional items. These measures were submitted to a 2 (Age) $\times 2$ (Education) $x 2$ (Sex) analysis of variance, the results of which are summarized in Table 33. Overall the mean preparation time was 7.23 minutes. Younger subjects spent less time preparing for memory tasks than older subjects (6.65 vs. 7.81), but the Age main effect was nonsignificant. HS subjects spent more time preparing for memory tasks than Ph.D. subjects (7.03 vs. 7.43), but the Education main effect was also nonsignificant. Males spent less time preparing for menory tasks than females ( 6.28 vs. 8.18), but the sex main effect was nonsignificant as well. These variables accounted for only $4 \%$ of the variance in preparation time: Age 1\%, Education less than $1 \%$, and Sex $3 \%$.

G. Health Information

a. Objective Physical Health

An objective physical health score was computed by summing health problems reported on the background questionnaire. Larger scores indicated poorer health. These scores were submitted to a 2 (Age) $x$ 
Table 33

Summary ANOVA for Preparation Time

$$
\overline{\mathrm{X}}=\text { Preparation Time }
$$

\begin{tabular}{lrccc} 
df $=1,56$ & 7.23 & $F$ & P & $E^{2}$ \\
\hline Age & -.58 & $<1$ & NS & .01 \\
Education & .58 & & & \\
Sex & .20 & $<1$ & NS & $<.01$ \\
Age x Ed & -.95 & 1.62 & NS & .03 \\
Age x Sex & .95 & $<1$ & NS & \\
Ed $x$ Sex & & 2.19 & NS & \\
Age $x$ Ed $x$ Sex & & $<1$ & NS & \\
Multiple ${ }^{2}$ & $<1$ & NS & \\
\end{tabular}


2 (Education) $\times 2$ (Sex) analysis of variance, the results of which are summarized in Table 34. Overall the mean score was 6.70. Younger subjects had fewer health problems than older subjects (4.52 vs. 8.78), and the Age main effect was statistically significant $(\underline{F}(1,56)=24.09, \underline{p}<.001)$. HS subjects had fewer health problems than Ph.D. subjects (6.28 vs. 7.12), but the Education main effect was nonsignificant. Males had fewer health problems than females (5.40 vs. 8.00), and the sex main effect was statistically significant $(\underline{F}(1,56)=9.38, \underline{p}<.01)$. These variables accounted for $34 \%$ of the variance in objective physical health: Age $24 \%$, Education $1 \%$, and Sex $10 \%$.

b. Subjective Physical Health

Subjects were asked to make subjective ratings of their physical health on an 11 point scale $10=$ poor thru 10 - excellent). These responses were submitted to a 2 (Age) $\times 2$ (Education) $\times 2$ (Sex) analysis of variance, the results of which are summarized in Table 34. Overall the mean score was 7.55. Younger subjects gave lower ratings of physical health than older subjects (7.44 vs. 7.66 ), but the Age main effect was nonsignificant. HS subjects gave lower ratings of physical health than Ph.D. subjects $(7.53$ vs. 7.57), but the Education main effect was also nonsignificant. Males gave lower ratings of physical health 


\section{Table 34}

Summary ANOVAs for Health Information Measures

Objective $\overline{\mathrm{X}}=$ Physical Health

\begin{tabular}{|c|c|c|c|c|}
\hline$d f_{1}=1,56$ & 6.70 & F & $\mathrm{P}$ & $E t a^{2}$ \\
\hline Age & $\begin{array}{r}-2.08 \\
2.08\end{array}$ & 24.09 & $<.001$ & .24 \\
\hline Education & $\begin{array}{r}-.42 \\
.42\end{array}$ & $<I$ & NS & .01 \\
\hline Sex & $\begin{array}{r}-1 \cdot 30 \\
1.30\end{array}$ & 9.38 & $<.01$ & .10 \\
\hline
\end{tabular}

Age $\times E d$

SI NS

Age $x$ Sex

$6.86<.05$

Ed $x$ Sex

< I NS

Age $x E d \times$ Sex

$<1$

NS

Multiple $R^{2}$

Subjective

$\overline{\mathrm{x}}=$ Physical Health

\begin{tabular}{|c|c|c|c|c|}
\hline$d f=1,56$ & 7.55 & $F$ & $\mathrm{P}$ & Eta ${ }^{2}$ \\
\hline Age & $\begin{array}{r}-.11 \\
.11\end{array}$ & $<1$ & NS & $<.01$ \\
\hline Education & $\begin{array}{r}-.02 \\
.02\end{array}$ & $<1$ & NS & $<.01$ \\
\hline Sex & $\begin{array}{r}-.39 \\
.39\end{array}$ & 3.00 & $<.09$ & .04 \\
\hline Age $\times \mathrm{Ed}$ & & $<1$ & NS & \\
\hline Age $x$ Sex & & 5.22 & $<.05$ & \\
\hline Ed $x$ sex & & 5.22 & 5.05 & \\
\hline Age $x$ Ed $x$ Sex & & $<1$ & NS & \\
\hline Multiple $\mathrm{R}^{2}$ & & & & .05 \\
\hline
\end{tabular}


Table 34 Continued

Subjective

$\mathrm{df}=1,56$

Age

Education

Sex

Age $x$ Ed

Age $x$ Sex

Ed $x \operatorname{Sex}$

Age $x$ Ed $x$ Sex

Multiple $\mathrm{R}^{2}$

$$
\bar{x}=\text { Mental Health }
$$

$\begin{array}{rlll}7.50 & F & P & E t a^{2} \\ -.03 & <1 & \text { NS } & <.01 \\ .03 & & \end{array}$

$-.53$

.53

4.57

$<.05$

.06

$-.50$

.50

4.05

$<.05$

.05

$<1$

NS

$<1$ NS

9.89

$<.01$

1.01

NS 
than females (7.16 vs. 7.94), and the sex main effect was marginally significant $(\underline{F}(1,56)=3.00, \underline{p}<.09)$. These variables accounted for only $5 \%$ of the variance in subjective physical health: Age 1\%, Education 1\%, and $\operatorname{sex} 4 \%$

\section{c. Subjective Mental Health}

Subjects were asked to make subjective ratings of their mental health on an 11 point scale $(0=$ poor thru 10 = excellent). These responses were subritted to a 2 (Age) $\times 2$ (Education) $\times 2$ (Sex) analysis of variance, the results of which are summarized in Table 34. Overall the mean score was 7.50 . Younger subjects gave lower ratings of mental health than older subjects ( 7.47 vs. 7.53), but the Age main effect was nonsignificant. HS subjects gave lower ratings of mental health than Ph.D. subjects (.697 vs. 8.03), and the Education main effect was statistically significant $(\underline{F}(1,56)=$ $4.57, \underline{p}(.05)$. Males gave lower ratings of mental health than females ( 7.00 vs. 8.00$)$, and the sex main effect was statistically significant $(\underline{F}(1,56)=4.05$, $\underline{\mathrm{p}}(.05)$. These variables accounted for $11 \%$ of the variance in subjective mental health: Age less than $1 \%$, Education $6 \%$, and sex $5 \%$.

H. Relationshios Between Variables

1. Health and Memory Information

Correlations between health and memory information 
measures for the entire sample are shown in Table 35 . Somewhat surprisingly, objective and subjective physical health measures did not correlate significantly, although subjective physical and mental health ratings did $(\underline{r}=.56, \underline{p}<.001)$. More health problems were associated with more memory problems $(\underline{\underline{r}}=.19, \underline{p}<.07)$, and more memory problems were associated with poorer subjective mental health ratings $(\underline{r}=.17, \underline{p}<.1)$. poor subjective mental health ratings were related to greater expectation of memory decay $(\underline{r}=-.23, \underline{p}<.05)$, as were more memory problems $(\underline{r}=.19, \underline{\mathrm{p}}<.07)$, and less memory knowledge $(\underline{r}=.19, \underline{p}<.08)$. Greater memory demands were related to fewer health problems ( $\underline{r}=$ $.17, \underline{p}<.09)$, and better subjective physical $(\underline{r}=.18$, $\mathrm{p}<.05)$ and mental $(\underline{\mathrm{r}}=.44, \mathrm{p}<.001$ ) health ratings. Greater memory demands were also related to fewer memory problems $(\underline{r}=.38, \underline{p}<.001)$, and more memory knowledge $(\underline{\underline{F}}=-.17, \underline{\mathrm{p}}<.09)$. More use of memory strategies was related to poorer subjective physical ( $\underline{r}=-.17$, $\underline{\mathrm{p}}<$ $.09)$ and mental health ratings ( $\underline{r}=-.22, \underline{p}<.05)$, and also to more memory problems $(\underline{r}=.32, \underline{p}<.01)$, but less memory knowledge $(\underline{r}=.23, \underline{p}<.05)$.

2. Memory Prediction. Confidence, and Performance Correlations for average episodic and semantic memory performance, prediction accuracy, and confidence accuracy, for the entire sample are shown in Table 36 , 
Table 35

Correlations Between Health and Memory

Measures for Entire Sample

All

\begin{tabular}{|c|c|c|c|c|c|c|c|}
\hline $\begin{array}{c}\text { All } \\
\text { Subjects }\end{array}$ & SPH & SMH & MP & EMD & MD & MK & MS \\
\hline \multirow{2}{*}{$\mathrm{SPH}$} & -.0383 & & & & & & \\
\hline & .382 & & & & & & \\
\hline \multirow{2}{*}{ SMH } & .0094 & .5617 & & & & & \\
\hline & .471 & .001 & & & & & \\
\hline \multirow{2}{*}{ MP } & .1947 & -.0447 & -.1680 & & & & \\
\hline & .062 & .363 & .002 & & & & \\
\hline \multirow{2}{*}{ EMD } & .1377 & -.2275 & -.1114 & .1882 & & & \\
\hline & .139 & .035 & .190 & .068 & & & \\
\hline \multirow{2}{*}{ MD } & -.1737 & .1837 & .4397 & -.3801 & -.1444 & & \\
\hline & .085 & .073 & .001 & .001 & .127 & & \\
\hline \multirow{2}{*}{ MK } & .0662 & .0219 & -.0619 & .1389 & .1859 & -.1745 & \\
\hline & .301 & .432 & .314 & .137 & .071 & .084 & \\
\hline \multirow[b]{2}{*}{ MS } & .1286 & -.1744 & -.2155 & .3243 & .1487 & -.0984 & .2250 \\
\hline & .156 & .084 & .044 & .004 & .121 & .220 & .037 \\
\hline
\end{tabular}


Table 36

Correlations for Average Episodic and Semantic Memory Performance, Prediction, and Confidence for the Entire Sample

Al1

\begin{tabular}{|c|c|c|c|c|c|}
\hline subjects & EPRED & ECON & SMEM & SPRED & SCON \\
\hline EPRED & $\begin{array}{c}-.6147 \\
.001\end{array}$ & & & & \\
\hline ECON & $\begin{array}{l}.3857 \\
.001\end{array}$ & $\begin{array}{c}-.4037 \\
.001\end{array}$ & & & \\
\hline SMEM & $\begin{array}{l}.1635 \\
.098\end{array}$ & $\begin{array}{r}-.470 \\
.123\end{array}$ & $\begin{array}{l}.2697 \\
.017\end{array}$ & & \\
\hline SPRED & $\begin{array}{c}-.1237 \\
.165\end{array}$ & $\begin{array}{l}.0688 \\
.295\end{array}$ & $\begin{array}{c}-.1380 \\
.145\end{array}$ & $\begin{array}{c}-.4418 \\
.001\end{array}$ & \\
\hline SCON & $\begin{array}{l}.0526 \\
.341\end{array}$ & $\begin{array}{l}.0467 \\
.358\end{array}$ & $\begin{array}{l}.1983 \\
.061\end{array}$ & $\begin{array}{l}.3579 \\
.002\end{array}$ & $\begin{array}{c}-.0616 \\
.316\end{array}$ \\
\hline
\end{tabular}


and for each Age, Education, and Sex group, in Appendix C. Separate correlations for episodic and semantic recall and recognition performance, prediction accuracy, and confidence accuracy are also shown in Appendix C.

More accurate episodic memory prediction was associated with better episodic memory performance $(\underline{r}=.61$, $\mathrm{p}$ (.001), and more accurate episodic confidence rating was also associated with better episodic memory performance $(\underline{r}=.39, \underline{p}<.001)$. Likewise, more accurate semantic memory prediction was associated with better semantic memory performance $(\underline{r}=-.44, \underline{p}<.001)$ and more accurate semantic memory confidence rating was associated with better semantic memory performance ( $\underline{r}=$ $.36, \mathrm{p}<.01)$.

Accuracy of episodic prediction and confidence rating was related $(\underline{\underline{x}}=-.40, \underline{\mathrm{p}}<.001$ ), but accuracy of semantic prediction and confidence rating was not. Episodic and semantic memory performance was marginally related $(\underline{\underline{r}}=.16, \underline{\mathrm{p}}<.1)$, as was episodic and semantic confidence rating accuracy $(\underline{r}=.20, \underline{p}<.07)$, but episodic and semantic prediction accuracy was not related. Similar patterns of results were obtained for each Age, Education, and Sex group.

\section{Predictors of Memory Performance}

Correlations of episodic and semantic memory performance with health and memory information measures, 
preparation time, and number of associations, for the entire sample are shown in Table 37. More preparation time was associated with better episodic memory performance $(\underline{r}=.42, \underline{p}<.001)$, as was the production of more associations $(\underline{r}=.36, \underline{p}(.01)$. Also, high subjective health ratings $(\underline{r}=.18, \underline{p}<.08)$, and more memory knowledge $(\underline{r}=-.27, \underline{p}<.05)$ were related to good episodic memory performance, but only high expectation of memory decay was related to good semantic memory performance $(\underline{\underline{r}}=.27, \underline{\mathrm{p}}<.05)$.

4. Regression Analyses

a. Episodic Memory

Stepwise multiple regression analyses were carried out to ascertain the best predictors of episodic memory performance. Summaries of these analyses are shown in Table 38. For average episodic memory performance, the first variable to enter the prediction equation was preparation time, which accounted for $18 \%$ of the variance. The second variable added was Age, which accounted for an additional 19\% of the variance. Subjective physical health accounted for an additional. $7 \%$ of the variance, and Education 5\%. Summed together, these variables accounted for $47 \%$ of the variance in episodic memory performance.

b. Semantic Memorv

Stepwise multiple regression analyses were 


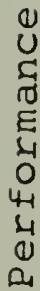

บ
0
บ
บ

क

$\begin{array}{llllllllllll}0 & 0 & \pi & 0 & m & 0 & 0 & \pi & 0 & 0 & \pi & N\end{array}$

$4 ?$ ? $\because$ ?

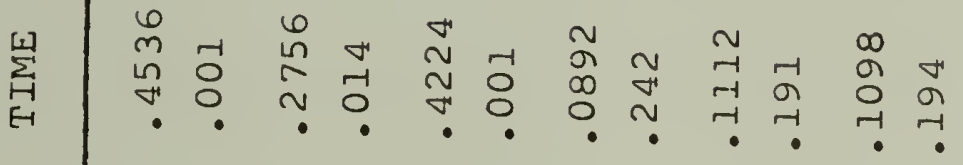

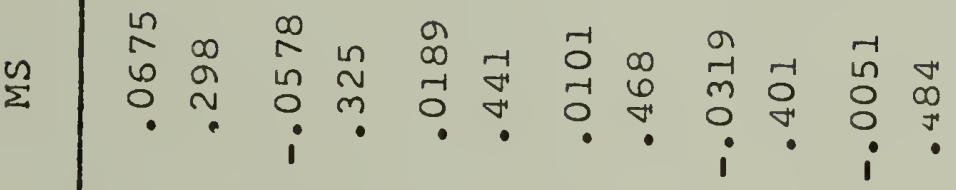

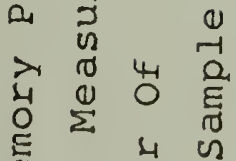
40

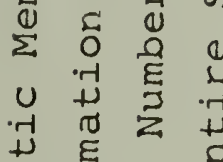

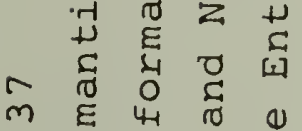

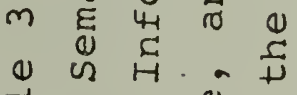

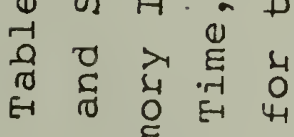

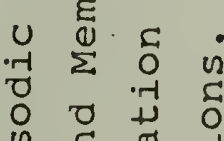

结

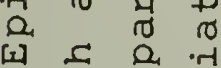

[य] 탐

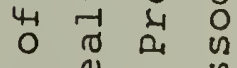

द 尘 先

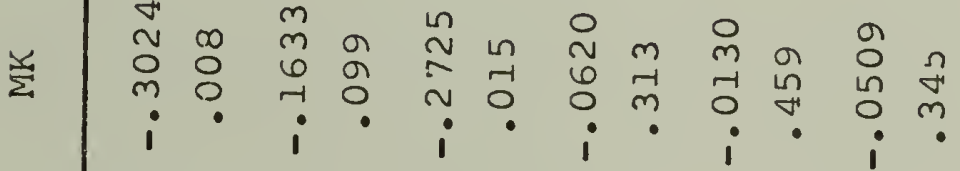

$\stackrel{0}{\Sigma}$

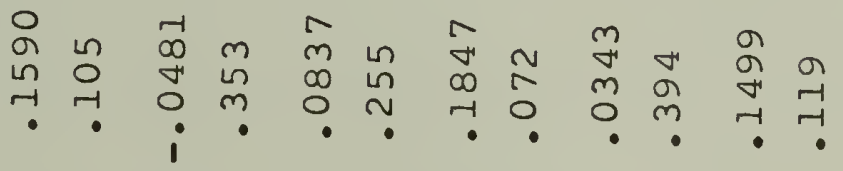

$\sum_{\text {ind }}^{\circ}$

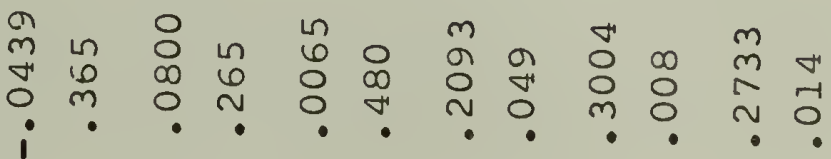

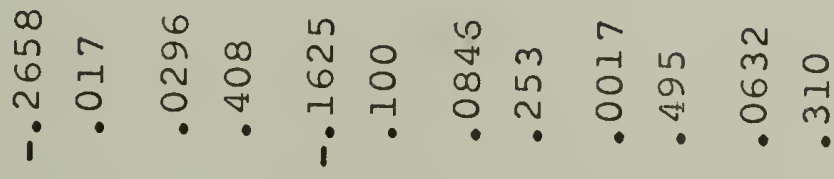

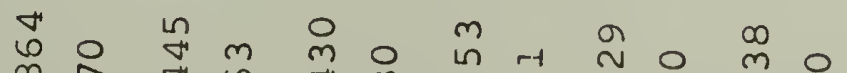

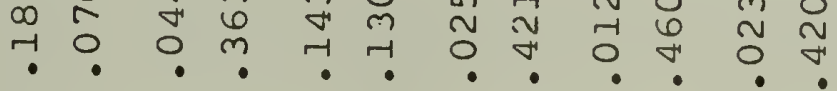

若

$-13$

$\stackrel{\infty}{\Sigma}$

蛋

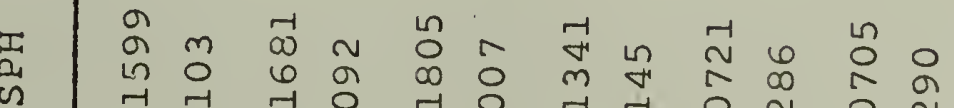

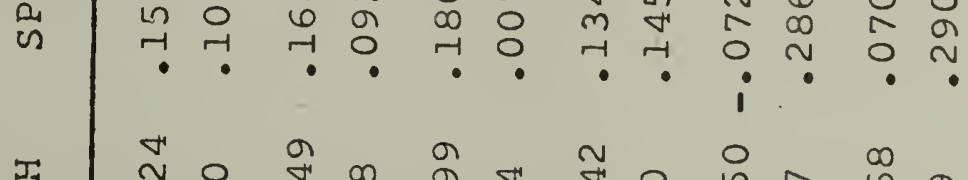

4

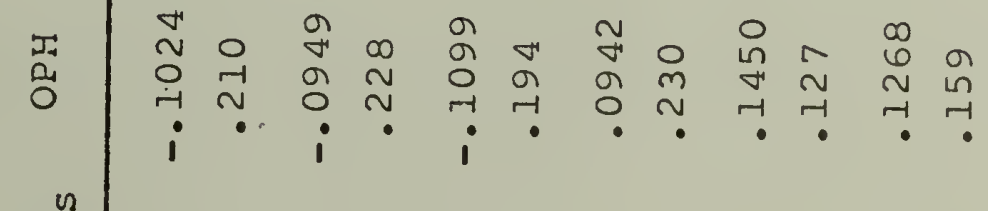

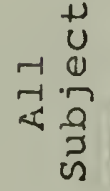

突

$\sum_{\text {: }}^{\circlearrowright}$

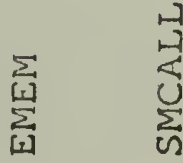

Uั

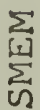




\section{Table 38}

Summary of Multiple Regression for Episodic And Semantic Memory Performance

\begin{tabular}{|c|c|c|c|c|}
\hline \multirow[b]{2}{*}{$\begin{array}{l}\text { Episodic } \\
\text { Memory }\end{array}$} & \multirow[b]{2}{*}{ Variable } & \multicolumn{2}{|c|}{ Recall } & \multirow[b]{2}{*}{$\mathrm{R}^{2}$} \\
\hline & & $F$ & $\mathrm{P}$ & \\
\hline \multirow{14}{*}{$----1--$} & TIME & 16.03 & $<.001$ & .21 \\
\hline & AGE & 15.90 & $<.001$ & .37 \\
\hline & MP & 5.75 & $<.05$ & .43 \\
\hline & $\mathrm{SPH}$ & 4.65 & $<.05$ & .47 \\
\hline & MK & 3.16 & $<.09$ & .49 \\
\hline & MS & 3.54 & $<.07$ & .52 \\
\hline & $E D$ & 3.16 & $<.09$ & .55 \\
\hline & \multicolumn{4}{|c|}{ Recognition } \\
\hline & AGE & 7.77 & $<.01$ & .11 \\
\hline & TIME & 7.40 & $<.01$ & .21 \\
\hline & ED & 4.21 & $<.05$ & .26 \\
\hline & $\mathrm{SPH}$ & 3.73 & $<.06$ & .30 \\
\hline & $M D$ & 3.25 & $<.08$ & .34 \\
\hline & ASSOC & 2.87 & <.I & .37 \\
\hline & \multicolumn{4}{|c|}{ Total } \\
\hline & TIME & 13.47 & $<.001$ & .18 \\
\hline & AGE & 17.63 & $<.001$ & .36 \\
\hline & SPH & 5.85 & $<.05$ & .42 \\
\hline & $E D$ & 5.97 & $<.05$ & .47 \\
\hline
\end{tabular}


Table 38 continued

Sernantic Memory
Recall

Variable

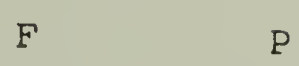

$R^{2}$

\begin{tabular}{cccc} 
ED & 9.39 & $<.01$ & .13 \\
AGE & 5.71 & $<.05$ & .21 \\
EMD & Recognition & .09 \\
ED & 6.15 & $<.05$ & .14 \\
SEX & 3.17 & $<.08$ & .18 \\
ED & 3.11 & $<.09$ & .15 \\
AGE & 10.58 & $<.01$ & .26 \\
SEX & 6.05 & $<.05$ & $<.06$ \\
\hline
\end{tabular}


carried out to ascertain the best predictors of semantic memory performance. Summaries of these analyses are shown in Table 38. For average semantic memory performance the first variable to enter the prediction equation was Education, which accounted for $15 \%$ of the variance. The second variable added was Age, which accounted for an additional $8 \%$ of the variance, and sex accounted for an another $5 \%$. summed together, these variables accounted for $27 \%$ of the variance in semantic memory performance.

V. Discussion

A. Processing Differences Contributing to Age Differences in Memory

1. Episodic Memory

In this study, as in previous studies, (e.g•, Botwinick \% Storandt, 1974; Bromery, 1958; Buschke, 1974; Craik, 1968; Craik, \& Mason, 1969; Denny, 1974; Erber, 1974; Eysenck, 1974; Hultsch, 1969, 1971, 1975; Laurence, $1967 \mathrm{a}, \mathrm{b})$ 60-year olds were found to perform significantly worse than 20-year olds on episodic memory tasks. Unlike previous findings (e.g., Botwinick \& Storandt, 1974; Craik, 1971; Erber, 1974; Gordon \& Clark, 1974) however, comparable aging effects were observed for recall and recognition. This difference may be explained by the fact that recognition tests in the present study always followed a number of other, 
possibly interfering tasks, and were probably thus more difficult.

Three hypothesized processing differences contributing to age-related episodic memory impairment were hypothesized, and Table 39 summarizes the data most pertinent to each of these hypotheses which will be discussed in the following sections.

\section{a. Processing Overload Hypothesis}

It is possible that because older people have lived longer and had more experiences, they have fuller, and perhaps overloaded memories (e.g., Craik, 1975; Horn, 1975; Kausler, 1970). If this is true, retrieval cues may trigger many associations, and this may interfere with task specific retention.

1. Mediator Overload. One purpose of including the association task in the present study was to assess the hypothesis that older subjects produce an overload of mediators. Since there were no differences in the number of free associations older and younger subjects generated this hypothesis was not supported. Of course, the number of associations generated in this overt association task may not reflect covert associative processes adequately, and it may thus be useful to examine age differences on other measures of associative activity.

2. Selector Impairment. Regardless of whether or not older subjects experience increased associative 
Table 39

Summary of Data Most Relevant to Hypothesized Processing Differences Contributing To

Age Difference in Episodic Memory Performance

\begin{tabular}{|c|c|c|c|}
\hline VAR IABLE & $20-25$ & $60-65$ & $\mathrm{p}$ \\
\hline \# Associations & 30 & 31 & NS \\
\hline \# Intrusions " & .86 & .78 & NS \\
\hline Incidental Recall & $40 \%$ & $23 \%$ & $<.001$ \\
\hline Incidental Recognition & $68 \%$ & $61 \%$ & NS \\
\hline $\begin{array}{l}\text { Recall Difference } \\
\text { (Incidental > Intentional) }\end{array}$ & $1 \%$ & $-5 \%$ & NS \\
\hline $\begin{array}{l}\text { Recognition Difference } \\
\text { (Incidental > Intentional) }\end{array}$ & $9 \%$ & $21 \%$ & $<.05$ \\
\hline Preparation Time & 3 & 4 & NS \\
\hline Response Criterion & .85 & .75 & NS \\
\hline Reported Strategy Use & 29 & 30 & NS \\
\hline
\end{tabular}


activity, an impaired selector mechanism could produce interference effects. That is, a failure to differentiate between appropriate and inappropriate responses could account for poor memory performance. Since large numbers of recail intrusions might indicate selector impairment, number of intrusions were examined. Both younger and older subjects produced very few intrusions, and the level of intrusions was almost identical for the two age groups. Thus, there was no evidence of selector impairment.

\section{b. Processing Deficit Hypothesis}

Many investigators have suggested that processing deficits can account for older subject's poorer memory performance (e.g., Canestrari, 1968; Craik, 1975; Denney, 1974; Eysenck, 1974; Horn, 1975; Hulicka \& Grossman, 1967; Hulicka \& Weiss, 1965; Hultsch, 1969, 1971; Moenster, 1972; Wimer \& Wigdor, 1958). For example, in direct contrast to the processing overload hypothesis, a processing deficit hypothesis suggests that older subjects internally generate fewer associations than younger subjects, and thus have fewer retrieval cues available.

\section{Mediator Deficiency. In so far as the} number of associations generated in the association task was indicative of internal associative processing, the association data is again relevant. As already 
indicated, however, the number of associacions produced by each age group was comparable; therefore, the capacity to produce mediators probably does not contribute to memory performance deficits. Again, this conclusion rests upon the assumption that the overt free association task reflected covert associative processing capacity.

\section{Production Deficiency. Both age groups} recognized more incidental than intentional items. It may thus be concluded that, when faced with the intentional memory task, they did not engage in the type of associative activity involved in the free association tasks, yet this type of processing facilitated recognition. It may be concluded that both age groups suffered from a production deficiency with respect to the associative activity involved in generating free associations; the association instructions improved retention. However, there was a significant Age $x$ Item Type interaction on recognition performance. Older subjects apparently suffered from a more severe production deficiency than younger subjects.

3. Mediator Inefficiency. This associative processing production deficiency may thus be added to the growing list of production deficiencies contributing to older subjects' poorer memory performance (e.g•, Canestrari, 1968; Eysenck, 1974; Hulicka \& Grossman, 
1967; Hultsch, 1969, 1971). Age differénces were observed on incidental recall, even though acquisitional processing was presumably equated, that is, older subjects produced as many associations as younger subjects. It seems likely then, that the associations generated by older subjects were not utilized as efficiently as by younger subjects. Thus, as in previous studies which have manipulated semantic mediational processing (e.g., Eysenck, 1974; Hultsch, 1969, 1971), some of the agerelated performance decrement in the present study was probably attributable to mediational inefficiency. Of course, the nature of this inefficiency is not clear, and further work will surely need to address this problem. Finally, since the mediational inefficiency was observed on recall but not recognition, it might be hypothesized that primarily retrieval deficits contributed. to the inefficiency. On the other hand, a production deficiency was observed for intentional recognition, where retrieval demands were minimal. Thus, age decrement in memory performance were probably attributable to processing deficits at both acquisition and retrieval.

\section{c. Strategy Deficit Hypothesis}

Less automatic, more subject controlied strategic processing deficits could also contribute to age differences in memory. One gross indication of acquisitional strategy use might be the amount of time subjects 
take in preparing for memory tasks. Since there were no age differences in the amount of study time used in preparing for intentional memory tasks, however, support for this kind of a strategy deficit by older subjects was not obtained.

In recognition tasks it is possible to ascertain decision strategy differences. For example, if subjects adopt a conservative criterion for judging old items old, they would produce more errors of ommission, but not commission. Signal detection analyses were carried out to separately assess retentional and decision components of recognition. While older subjects' retention was poorer than younger subjects, no age differences in response criterion was found. Finally, subjects responses to questions concerning memory strategy use also failed to support the strategy deficit hypothesis, for a few memory strategies, such as concentrating harder and asking other people's help, older subjects actually reported greater strategy use than younger subjects.

\section{Semantic Memory}

While older subjects performed worse than younger subjects on episodic memory tasks, they performed better than younger subjects on semantic memory tasks. Using the same materials, Botwinick \& Storandt (1974) found a similar, though nonsignificant trend. Retention of information presupposes prior exposure to it, 
and it is quite possible that older subjects' superior semantic memory performance merely reflected age differences in exposure to the particular facts tested. Additionally, the observed age differences may have reflected age differences in exposure to other information, relevant to the particular facts that were tested. Alternatively, an age advantage in skills needed for good performance on retention tests of more general, ecologically valid material may be suggested. It seems most probably however, that the age reversals on semantic memory tasks were attributable to differential knowledge concerning the specific facts tested, and related information, not to differential memory processing. Examination of age effects on semantic memory tasks that utilize material equally well known by all age groups are still needed.

B. Metamemory in Adulthood

Metamemory is a newly conceptualized cognitive component of memory (e.g., Flavell \& Wellman, 1976; Kreutzer, Leonard \& Flavell, 1976), thought to play an important role in control of other mnemonic processes. Although no developmental studies of metamemory in adults have yet been reported, the present study assessed adults explicit metamemory knowledge and competence in two monitoring skills, memory prediction and confidence rating. 
1. Generalized-Abstracted Knowledae: Questionnaire

Generalized-Abstracted metamemory knowledge is not elicited in the act of remembering, but rather refers to more general knowjedge about the structure and functioning of memory. Although considerably more work will be needed to thoroughly assess adults' explicit knowledge about memory, and to relate this knowledge to memory performance, the present study provides preliminary data about adults' reflections on memory, and the information about memory that they have abstracted from life-long experiences.

Subjects' responses to explicit questions indicated how they assessed the memory demands they encounter, memory strategies and aids they use, memory changes they expect, and knowledge they have about the way memory functions.

Most subjects thought they encountered more memory demands than average, though these were not rated as excessive. As might be expected, graduate students and academicians thought they encountered greater memory demands than nonacademics. It was interesting too, that females thought they encountered greater memory demands than males, and younger subjects somewhat greater memory demands than older subjects.

On several questions assessing memory problems, and use of memory strategies and aids, subjects indi- 
cated experiencing some, but not excessive, memory difficulties, and making some use of memory strategies and aids. Older and less educated subjects indicated experiencing more memory problems than younger and more educated subjects, and also indicated they made more use of at least a few memory strategies and aids. Another aspect of the metanemory questionnaire assessed expectation of memory decay. Very few subjects thought their memories would improve with age, but less than half were certain that their memories would get worse with age. Older and less educated subjects tended to expect aging to be associated with memory decay more than younger and more educated subjects.

It is also interesting, and perhaps gratifying, that many subjects' inferences about the functioning of memory were quite consonant with findings of cognitive psychologists. For example, most subjects thought it easiest to remember related, organized, interesting, understandable, and visual materials.

\section{Specific-Concrete Knowledge: Memory Monitoring}

specific-concrete metamemory knowledge is derived in the act of remembering and memory monitoring which involves specific-concrete metamemory knowledge refers to subjects' ability to reflect upon, and predict or assess, but not necessarily retrieve, the contents of 
their memories; it involves judgments about the accessibility of potential, or actual, memory items. Although the exact function of memory monitoring is not yet known, it has been hypothesized to be an important component of an executive mechanism; it probably contributes to efficient instigation, maintainence, and termination of acquisition, search and retrieval. Two memory monitoring skills, memory prediction and memory confidence rating were examined.

\section{a. Memory Prediction}

1. Episodic Memory. After generating associations to 24 incidental items, and studying 24 intentional items, subjects were asked to predict how many of these items they would be able to recall correctly, and after recalling as many items as they could, they were asked to predict how many they would be able to recognize correctly.

In general, subjects' predictions were within about a standard deviation of performance, and the mean absolute deviation score was under 5 items. Subjects predicted they would correctly recognize more items than they predicted they would recall, and indeed, they did recognize more items than they recalled. Furthermore, for recognition they tended to under-predict performance, but for recall they tended to over-predict performance. Additionally, 
recognition predictions were more accurate than recall predictions. It is possible, then, that adults are more competent in monitoring recognition than recall. Alternatively, however, it is possible that the recall. attempts, which preceded recognition predictions, provided information which facilitated recognition prediction. It might be interesting then, to more systematically examine training effects on memory prediction.

Subjects predicted they would remember comparable numbers of incidental and intentional items. Apparently, they believed that generating associations to words was as effective in enhancing retention as the activities they engaged in during deliberate memorization study time. Indeed, this was true; recall of incidental and intentional items was comparable, and recognition of incidental items was actually better than recognition of intentional items.

Group differences in prediction accuracy were also of interest. In general, there was some tendency for females to predict more accurately than males, Ph.D.s more accurately than HS subjects, and younger subjects. more accurately than older subjects. While the direction of these results were fairly consistent for all retention tasks (i.e., incidental and intentional recall and recognition), and paralleled those found for memory performance, they were only statistically 
significant for recognition prediction. It is possible then, that these group differences reflect differences in a facilitating effect of recall, on recognition prediction, rather than differences in a more generalized prediction skill.

\section{Semantic Memory. After subjects tried to} recall each general information question, they were asked to predict whether or not they would be able to recognize the correct answer. Thus, overall predictions, as well as item-by-item feeling of knowing judgments, were available for analysis of semantic recognition prediction.

In general, subjects tended to over-predict recognition performance, but their predictions were within about one standard deviation of performance, and the mean absolute devaition score was under 4 items. Additionally, subjects were much more likely to correctly recognize items to which they had given positive rather than negative, feeling-of-knowing judgments; the mean probability of a correct response given a positive feeling-of-knowing judgment was .80 , while given a negative feeling-of-knowing judgment, it was .53 . This then indicates that subjects were able to discriminate potentially rememberable items from those they would not be able to remember. There were no significant group differences in accuracy of either 
of these forms of semantic recognition prediction. b. Memory Confidence

1. Episodic Memory. Subjects were asked to make confidence ratings for each recall and recognition response. There were no significant Age, Education, or sex main effects in overall level of confidence, although young HS subjects tended to be more confident than other subjects, and old HS subjects less confident. All subjects were considerably more confident when they made correct responses than when they made incorrect responses. This thus provides evidence of competent memory monitoring of episodic material. The difference between confidence ratings on correct and incorrect responses was used as an index of confidence accuracy. At least in the younger age group, more education was associated with more accurate confidence ratings. However, neither Age nor sex were predictive of confidence accuracy.

\section{Semantic Memory. Subjects also made} confidence ratings for recall and recognition responses to general information knowledge questions. There were no Age, Education, or sex main effects in overall level of confidence. All subjects were considerably more confident when they made correct responses then when they made incorrect responses, thus providing evidence of competence in memory monitoring 
of semantic material. Furthermore, more educated subjects again gave more accurate confidence ratings, and neither Age nor sex predicted confidence accuracy.

\section{c. Coordination of Various Metamemory Measures}

Although the aspects of metamemory that have been examined could involve very different processes, it seems more likely that commor monitoring skills were assessed. For example, if memory prediction and memory confidence rating are both components of a memory executor, accuracy in these two skills might be expected to correlate. Prediction and confidence accuracy of episodic memory correlated significantly, thus supporting the notion that, in a laboratory, task, prediction and confidence are related monitoring skills. No reliable correlations were obtained for prediction and confidence accuracy of semantic memory, however. It is perhaps not too surprising that prediction and confidence skills were less related for semantic tasks; since prediction precedes responding, but confidence rating follow it, the additional information brought to bear in responding in semantic tasks could account for this differential relationship.

d. Coordination Between Metamemory and Memory If metamemory is an important component of memory, then memory monitoring accuracy should corre* late with memory performance. The demonstration of 
statistically significant relationships of this sort were important findings of the present work. For episodic memory the correlation between prediction accuracy and performance was approximately +.60 , and between confidence accuracy and performance +.40 . For semantic memory these correlations were about +.45 and +.35 , respectively. This then supports the notion that prediction and confidence skills are related to mechanisms involved in effective memory performance.

Additionally, similar correlations were obtained for each Age, Education, and Sex group. The coordination between metamemory and memory thus appears comparable for these groups, and apparently remains stable over the age range studied.

Another finding, albeit negative, speaks to the issue of the coordination between metamemory and memory. There were essentially no order effects: whether memory tests followed or preceded the metamemory questionnaire memory and metamemory assessments were the same. It may be concluded then, that in a short run situation, such as the single session of laboratory tasks administered in this study, the cognizing, or reflecting about memory, that is required for filling out a metamemory questionnaire, does not significantly influence memory performance. Additionally, engaging in a series 
of memory tasks does not significantly influence the way subjects monitor memory or respond to a questionnaire about memory. An interesting, though seemingly contradictory side note, is that $64 \%$ of the subjects indicated on a post experimental questionnaire that they thought participating in the study taught them something about memory.

\section{e. Age Differences in Metamemory}

An issue of particular interest in this dissertation was possible adult age differences in metamemory. If generalized-abstracted metamemory knowledge, or specific-concrete metamemory skills, are acquired through memory experiences, then clder, more experienced adults might be expected to demonstrate more metamemory sophistication. On the other hand, if aging produces memory deterioration, then older adults' prior knowledge about memory may become inaccurate and their metamemory might appear inferior. Little evidence of systematic age differences in explicit metamemory knowleage, memory monitoring skills, or the coordination between metamemory and memory were observed. It may thus be tentatively concluded that metamemory does not develop or deteriorate between 20 and 65 years of age.

C. Explanation of Aqina Effects

The observed age deficits were probably not attri- 
butable to methodological considerations, such as age differences in the effects of pacing or demand characteristics. Subjects worked at their own pice, and

a post-experimental questionnaire failed to reveal group differences in anxiety associated with participating in the experiment. Additionally, since comparable aging deficits were observed for academicians and nonacademicians, it seems unlikely that the unfamiliar, and perhaps threatening, university environment accounted for age differences; older academicians certainly should have felt at home.

Several possible explanations of aging effects were hypothesized, and data relevant to each will be discussed.

\section{a. Cohort Effects}

In cross-sectional studies development is inferred from group differences, however, cohort effects, rather than age change, may contribute to age differences. That is, it has been suggested (e.g., Riegel, 1972; Shaie, 1970, 1973) that older people are actually less competent than younger people, but this is the result of group differences, not age chance. It may be hypothesized, for example, that in our society age is confounded with education, and ecucational differences, rather than developmental chinge, can account for age effects on episodic memor\%. 
In the present study, two educational groups were included and the portion of variance accounted for by Age and Education were evaluated separately. Although Education accounted for approximately $5 \%$ of the variance in episodic memory performance, Age was apparently more important, and it accounted for about $15 \%$ of the variance. Furthermore, when multiple regression analyses were carried out, Age was found to be a better predictor of episodic memory performance than education. Also, Age $x$ Education interactions were generally not obtained, Age appeared to have comparable effects on HS and Ph.D. subjects. Thus a cohort explanation of aging, at least in its more obvious interpretation, received little support.

There were, however, consistent findings that are relevant to cohort effects, in so far as group differences were probably related to cultural and generational factors. Education $\mathrm{x}$ Sex interactions were obtained on several dependent measures, indicating significant education effects for males, but not females. This is not too surprising, since, as a result of cultural practices, education is probably more confounded with intelligence for males than females. Moreover, the fact that education is probably more confounded with intelligence for younger females than older females, probably contributed to the Age $:$ sex interactions. 
Finally, one further comment concerning cohort effects seems in order. While the observed age differences on episodic memory tasks were probably not attributable to gross age-related group differences in education (i.e., cohort effects), this investigator could not help but be struck by the seemingly important possibly generationally related group differences in attitude and perspective that were apparent in talking with subjects. Furthermore, it subjectively appeared that the different historical societies in which subjects developed had a tremendous influence on the way they behave every decade of their lives. These dimensions of aging seem too substantive to leave uninvestigated.

\section{b. Disuse Hypothesis}

The disuse hypothesis (e.g•, Reese, 1975), predicts that memory grows worse from lack of functioning; an atrophy metaphor is surely implied. In the present study subjects made subjective ratings of memory demands, and these ratings were correlated with memory performance, and ratings of memory problems. If disuse contributes to memory decay, memory demand ratings would be expected to correlate positively with memory performance, and negatively with memory problems. Of course, these correlations would in no way justify the causative relationship implied by the hypothesis; 
people who experience memory impairment probably adjust their lives to minimize memory demands.

There was no evidence of a relationship between rated memory demands and episodic memory performance. Yet, a significant negative correlation was obtained between subjects' ratings of memory demands and memory problems. It is possible then, that when subjects find themselves experiencing memory problems, they try to minimize memory demands. Alternatively, the negative correlation between memory demands and memory problems may indicate that limited exercise of memory processes leads to memory impairment. Furthermore, the lack of correlation between memory demands and episodic memory performance, coupled with the significant relationship between memory demands and memory problems, may suggest that the relationship is restricted to those memory problems which subjects see as related to the memory demands they rated.

\section{c. Expectation Hypothesis}

The expectation hypothesis (e.g., Reese, $1972)$, predicts that expectation of memory decay contributes to memory decay. An expectation of memory decay score was computed from subjects responses to several questions evaluating expectation of memory change, and these scores were correlated with memory performance, and ratings of memory problems. If ex- 
pectation of memory decay contributes to memory decay, a negative correlation would be expected between expectation of memory decay and memory performance, and a positive correlation would be expected between expectation of memory decay and memory problems. Again, these correlations would not justify the causative relationship implied by the hypothesis; memory impairment probably leads to expectation of further memory impairment. There was no evidence of a relationship between expectation of memory decay and episodic memory performance for either age group, however, a marginally significant correlation between expectation of memory decay and memory problems was obtained. It is possible then, that encountering memory problems leads to expectation of memory decay. Alternatively, while the expectation of memory decay hypothesis was not strongly supported, expectation of memory decay may contribute to the type of memory problems encountered in daily routines.

\section{d. Biological Hypothesis}

The biological hypothesis (e.g., Jarvik \& Cohen, 1973) predicts that factors contributing to poor health also contribute to poor memory. Subjects were asked to list physical health problems, as well as to make subjective ratings of their physical and mental health. If the biological hypothesis is valid, the number of health problems reported by subjects would 
be expected to correlate negatively with memory performance, and health ratings would be expected to correlate positively.

All of these correlations were in the predicted direction, although they were small, and failed to reach statistical significance. Yet, when subjective physical health ratings were entered into the regression equation, prediction of episodic memory performance was improved. Thus, there was some support for the biological hypothesis. It is probable, however, that more medicaliy precise health measures would support the biological hypothesis more strongly; more medically sophisticated investigations could probably also isolate particular health problems associated with memory decrements.

VI. Conclusions

This dissertation was concerned with memory and aging. The study had three major focusses: 1) evaluation of processing differences contributing to age differences in adults' memory performance, 2) examination of metamemory in adults, and 3) explanations of memory aging. In general, the following summary statements can be made. Processing Differences Contributina to Aae Differences in Memory

1. There are sizable age decrements in episodic recall 
and recognition of high school and Ph.D. educated subjects.

2. Older subjects' poor memory performance is probably not attributable to mediator overload, selector impairment, mediator capacity deficit, or strategy use.

3. All subjects suffer from an associative processing production deficiency, but this is more severe for older subjects.

4. Older subjects' poor memory performance is also attributable to mediator inefficiency:

5. Retrieval deficits probably contribute to age-related memory performance decrements, and acquisitional deficits are surely an important factor.

\section{Metamemory in Adults}

6. Adults have a wide range of generalized-abstracted knowledge about memory, as well as considerable competence in two memory monitoring skills, memory prediction and memory confidence rating.

7. At least in laboratory controlled episodic memory tasks, competence at memory prediction and memory confidence rating are related, and perhaps more importantly, competence in each is predictive of proficient memory performance.

8. Metamemory probably remains stable between 20 and 65 . 


\section{Explanations of Memory Aging}

9. It is unlikely that cohort effects, at least in their most obvious interpretation, account for observed age differences.

10. Memory demands and expectations of memory decay correlate positively with ratings of memory problems, but there is no such relationship between these variables and memory performance; the notion of socially sanctioned "aging" roles contributing to aging incompetencies therefore, remains tenable, but somewhat restricted.

11. A biological explanation of aging is supported by some tendency for poor health to be related to poor memory• 
References

Anders, T. R., Fozard, J. L., \& Lillyquist, T. D. The effects of age upon retrieval from short-term memory. Developmental Psychology, 1972, 6, 214217.

Anderson, J. R., \& Bower, G. H. Human Associate Memory. New York: Wiley, 1973.

Appel, L. F., Cöoper, R. G., McCarrel, N., Sims-Knight, J., Yussen, S. R., \& Flavell, J. H. The development of the distinction between perceiving and memorizing. Child Development, 1972, 43, 1351-1381. Arenberg, D. Cognition and aging: Verbal learning, memory, problem solving, and aging. In Eisdorfer \& Lawton (Eds.), The Psychology of Adult Development and Aqing. Washington, D. C.: American Psychological Association, 1973.

Atkinson, R. C., \& Shiffrin, R. M. Human memory: A proposed system and its control processes. In Spence \& Spence (Eds.), Advances in the Psychology of Learning and Motivation Research and Theory. Volumne II. New York: Academic Press, 1968. Bahrick, H. P., Bahrick, P. O. Wittlinger, R。P. Fifty years of memory for names and faces: A cross-sectional approach. Journal of Experimental Psychology: Generil, 1975, 104, 54-75. 
Banks, W. P. Signal detection theory and human memory. Psychological Bulletin, 1970, 74, 55-62.

Berch, D. B., \& Evans, R. C. Decision processes in children's recognition memory. Journal of Experimental Child Psychology, 1973, 16, 148-164. Blacke, M. Prediction of recognition when recall fails: Exploring the feeling of knowing phenomenon. Journal of Verbal Learning and Verbal Behavior, 1973, 12, 311-319.

Botwinick, J. Cognitive Processes in Maturity and old Age. New York: Springer, 1967.

Botwinick, J. Aging and Behavior. New York: Springer, 19.73 .

Botwinick, J., \& Storandt, M. Memory, Related Functions and Age. Springfield, Illinois: Thomas, 1974. Bromely, D. B. Some effects of age on short-term learning and memory. Journal of Gerontology, 1958, 13 , 398-406.

Buschke, H. Two stages in learring by children and adults. Bulletin of Psychonomic society, 1974, 2 , 392-394.

Canestrai, R. E. Age changes in acquisition. In

Talland (Ed.), Human Aging and Behavior. New York: Academic Press, 1968.

Collins, A. M., \& Loftus, E. F. A spreading activation theory of semantic processing. Bolt, Beranek, and 
Newman, Inc., Report No. 2711, 1974.

Collins, A. M., \& Quillian, M. R. Retrieval time for

semantic memory. Journal of Verbal Learning and

Verbal Behavior, 1969, 8, 240-247.

Craik, F. I. M. Short-term memory and the aging process.

In Talland (Ed.), Human Aging and Behavior. New

York: Academic Press, 1968.

Craik, F. I. M. Age differences in recognition memory.

Quarterly Journal of Experimental Psychology, 1971 , $23,316-323$

Craik, F. I. M. Signal detection analysis of age dif-

ferences in divided attention. Paper presented at the meetings of the American Psychological Association, Montreal, 1973.

Craik, F. I. M. Age difference in human memory. In

Birren \& Schaie (Eds.), The Handbook of the Psy-

chology of Aging. New York: Uan Nostrand Rein-

hold, (In press).

Craik, F. I. M., \& Lockhart, R. S. Levels of processing:

A framework for memory research. Journal of Verbal

Learning and Verbal Behavior, 1972, 11, 671-684.

Craik, F. I. M., \& Masani, P. A. Age and intelligence

differences in coding and retrieval of word lists.

British Journal of Psychology, 1969, 60, 315-319.

Davies, D. R. Age differences in paced inspection tasks.

In Talland (Ed.), Humar Aqing and Behavior. New 
York: Academic Press, 1968.

Denney, N. W. Clustering in middle and old age. Developmental Psychology, 1974, 10, 471-475.

Erber, J. T. Age differences in recognition memory. Journal of Gerontology, 1974, 29, 177-181.

Eysenck, M. W. Age differences in incidental learning. Developmental Psychology, 1974, 10, 936-941.

Flavell, J. H. Developmental studies of mediated memory. In Reese \& Lipsitt (Eds.), Advances in Child Development and Behavior. Volumne 5. New York: Academic Press, 1970. Flavell, J. H., Friedricks, A. G., \& Hoyt, J. D. Developmental changes in memorization processes. Cognitive Psychology, 1970, I, 324-340. Gordon, S. K., \& Clark, W. C. Application of signal detection theory to prose recall and recognition in elderly and young adults. Journal of Gerontoloqy, $1974,29,64-72$.

Hart, J. T. Memory and the feeling of knowing experience. Journal of Educational Psychology, 1965, 56, 205216.

Hart, J. T. Memory and the memory monitoring process. Journal of Verbal Learning and Verbal Behavior, $1967, \underline{6}, 685-691$.

Horn, J. L. Human abilicies. A review of research and theory in the early 1970's. Unpublished manuscript, 
University of Denver, 1975.

Hulicka, I. M•, \& Grossman, J. L. Age group comparisons

for the use of mediators in paired-associate learning. Journal of Gerontology, 1967, 22, 46-51. Hulicka, I. M., \& Weiss, R. Age differences in retention as a function of learning. Journal of ConSulting Psycholoay, 1965, 29, 125-219.

Hultsch, D. F. Adult age difference in the organization of free recall. Developmental Psychology, 1969, I, $673-678$ 。

Hultsch, D. F. Adult age differences in free classification and free recall. Developmental Psychology, $1971, \underline{4}, 338-342$.

Hultsch, D. F. Adult age differences in retrieval:

Trace-dependent and cue-dependent forgetting. Developmental Psychology, 1975, 11, 197-201. Jarvik, J.F., \& Cohen, D. A biobehavioral approach to intellectual changes with aging. In Eisdorfer \& Lawton (Eds.), The Psycholody of Adult Development and Aging. Washington, D.C.: American Psychological Association, 1973.

Kausler, D. H. Retention-forgetting as a nomological network for developmental research. In Goulet \& Baltes (Eds.), Life-Span Developmental Psycholoay: Research and Theory. New York: Academic Press, 1970. 
Kay, H. Theories of learning and aging. In Birren (Ed.), Handbook of Aging and the Individual.

Chicago: University of Chicago Press, 1959. Kimmel, D. C. Adulthood and Aging. New York: Wiley, 1974.

Kintsch, w. Learning, Memory, and Conceptual Processes. New York: Wiley, 1970.

Kruetzer, M. A., Leonard, C., \& Flavell, J. H. An interview study of children's knowledge about memory. Monographs of the Society for Research in Child Development, 1975, 40, No. 159.

Laurence, M. W. A developmental look at the usefulness of list categorization as an aid to free recall. Canadian Journal of Psychology, 1967, 21, 153165.

Laurence, M. W. Memory loss with age: A test of two strategies for its retardation. Psychonomic Science, 1967, 9, 209-210.

Lockhart, R. S., \& Murdock, B. B. Memory and the theory of signal detection. Psychological Bulletin, 1970, 74, 100-109.

Mandler, G. Organization and memory. In Spence \& Spence (Eds.), The Psychology of Learning and Motivation: Advances in Research and Theory. Volume 1, New York: Academic Press, 1967. 
Miller, G. A. The magical number seven, plus or minus two: Some limits on our capacity for processing information. The Psychological Review, 1956, 63, $81-97$.

Moenster, P. A. Learning and memory in relation to age. Journal of Gerontology, 1972, 27, 361-363. Moynahan, E. D. The development of knowleage concerning the effect of categorization upon free recall. Child Development, 1973, 4ㅗ, 238-246. Murdock, B. B. The criterion problem in short-term memory. Journal of Experimental Psychology, 1966, 72, $317-324$.

Paivio, A. Imagery and Verbal Processes. New York: Holt, Rinehart, and Winston, 1971. Piaget, J., \& Inhelder, B. Memory and Intelligence. New York: Basic Books, 1973. Reese, H. W. Life-span models of memory. Paper presented at the meetings of the Gerontological Society at San Juan, 1972.

Reese, H. W. Information processing and the development of memory. Unpublished manuscript, University of West Virginia, 1975. Riegel, K. F. Time and change in the development of the individual and \$ociety. Advances in Child Development and Behavior: Volume 7, 1972. Rumelhardt, D. E., Lindisay, P.H•, \& Norman, D. A. A 
process model for long-term memory. In Tulving \& Donaldson (Eds.), Organization of Memory. New York: Academic Press, 1972.

Schaie, K. W. A reinterpretation of age related changes in cognitive structure and functioning. In Goulet \& Baltes (Eds.), Life-Span Developmental Psychology: Research and Theory. New York: Academic Press, 1970 .

Schaie, K. W. Methodological problems in descriptive developmental research on adulthood and aging. In Nesselroade \& Reese (Eds.), Life-Span Developmental Psychology: Methodoloaical Issues. New York: Academic Press, 1973.

Sternberg, S. High-speed scanning in human memory. Science, 1966, 153, 652-654.

Taub, H. H. Memory span, practice and aging. Journal of Gerontology, $1973,28,335-338$.

Tenney, Y. J. The child's conception of organization and recall. Journal of Experimental Child Psychology, $1975,19,100-114$.

Tulving, E. Episodic and semantic memory. In Tulving \& Donaldson (Eds.), Organization of Memory. New York: Academic Press, 1972. Tulving, E., \& Madigan, S. A. Memory and verbal learning. In Mussen \& Rosenzweig (Eds.), Annual Review of Psychology. Volume 21. Palo Alto, CA: George Banta Company, 1970. 
Tulving, E., \& Thomson, D. M. Retrieval processes in recognition memory: Effects of associative context. Journal of Experimental Psychology, 1971 , 87, $116-124$.

Watkins, M. J. Concept and measurement of primary memory• Psychological Bulletin, 1974, 81, 695711.

Watkins, M. J., \& Tulving, E. Episodic memory: When recognition fails. Journal of Experimental:

General, 1975, 104, 5-29.

Waugh, N. C., \& Norman, D. A. Primary memory. Psychological Review, 1965, 72, 89-104.

Wellman, H. M. The development of memory monitoring: The feeling of knowing experience. Unpublished doctoral dissertation, University of Minnesota, 1975.

Wellman, H. A., Drozal, J. G., Flavell, J.H., Salatas, H., \& Ritter, K. Metamemory development and its possible role in the selection of behavior. Paper presented at the Society for Research in Child Development meetings at Denver, Colorado, 1975. Wickelgren, W. A. Age and storage dynamics in continuous recognition memory. Developmental Psycholoay, 1975, 11, 165-169.

Wimer, R. E., \& Wigdor, B. T. Age differences in retention of learning. Journal of Gerontology, 1958, 13, 291-295. 
Yendovitskaya, T. V. Development of memory. In Zaporozhets \& Elkonin (Eds.), The Psychology of Preschool Children. Cambridge, MA: MIT Press, 1971.

Yussen, S. R., \& Levy, V. M. Developmental changes in predicting one's own span of short-term memory. Journal of Experimental Child Psychology, 1975 , 19, 502-508. 
$-313-$

Appendix A

Sample Data Sheet 
DLEASE DECORT TISE TTME

- NAME

ADORESS

CAIE

GIDIN TATE

- TELFPHONE ADE

HOH MANY PEOPLE nO YOU LIVE WTHH

c. ARE YOU MEFPIEN

- HOH MAIYY CHILUEEN TO YOU HAYE

$\therefore$ - WHAT IS YOLIO DRESENT OCCUFATION

(. WHAT RIO YOU LIO PEFOE E THAT

_ OIO YOU CLAOLEIE UI FH SCHCJL

(.. HOW MANY YEAFS OF ECHOOI.IN:S HOVE YRU HAO PAST HIGH SCHOOL

6 WHAT IS THE HTEHEST REGIEL YOU HQVË इAFNEO

- ARE YOII PEESËHYY A SIIIRENT

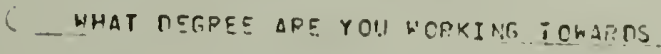
$W H E N$

, HAVE YOIJ EVEF HAO G COUFSE OH, KE:10PY

_ CO YOU PRACTICE TOANCCENTEPITL MECITATION

DO YOU SYCKE

OO YOU ORITK AI C, GHOL HOW MUCH

COO YOU NEEC RLLASSES

$\therefore$ nO YOU HAVE a MEAPIPIG PFIOELEM

CO YOII HAVE FPEOMIIENT HEAOACHES

_CC YOU OFTEN_HAVE STOMACH FPOBL

DO YOU HAVE HIP.H PLOJO PRESSUFE

DO YOII HAVR IILCERS

-. DO YOII HOVE LHY C'MF ONIS HELLIH PROCLEMS

PLEASE LIST THEH

HAVE YOII EVTP REEN HOCDITA. IZEE

HOW OFTEN

FOF WHAT

DLEASE CIETLE A NUMB:D PATING, YOHD MENIAL PEALTH OR ARJUSTMENT

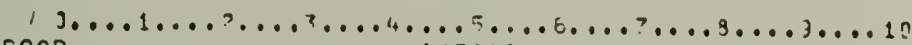
FONP 
DLEAS? CIPRLE A NIMGTO PQIING YOJP PHYCICAL HEALTH

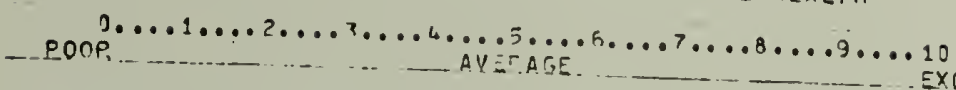

PLEASE CIITLE A IUU:BED PATING TH: MEYORY AND COGHITIV

OF YOUR WCPK

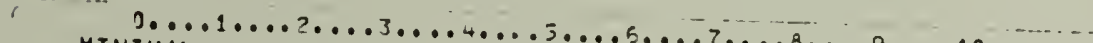
MINIMAL

C

\section{PLEASE FECOF THE TIME}

(

C 
$-316-$

PLEACF RTCOPO THE TIN:

AS YOII ALI EATY KNOH, WE ATE STLOYING MEMOFY, AND THE DUESTICPNAIPE THET FCLLCWE HLLL GEUE US SCI E IHPORTANT INFOPMATIC AR OUT WHAT PCOELE KNOH ARCUT MLMOPY. WE REALIZE

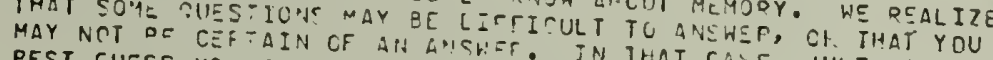

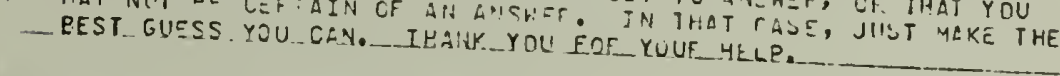

- ONE KINO OF TACK HE IISE TC STUTY MEMOFY INUOLVES R.SCALL, EOP EXAMPL E, AFTER SHOWTAG FCOPL Z ? P CTUUES, WE ASK THEM TO NAME AS YANY OF THEN $\triangle S$ THEY CAH JCMEM?ZD. OL DASE ILL IH THE PEDPLE AI - ACH OF THE AGES LISTER WOULC OSC NIAMES YOU THINK

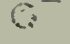

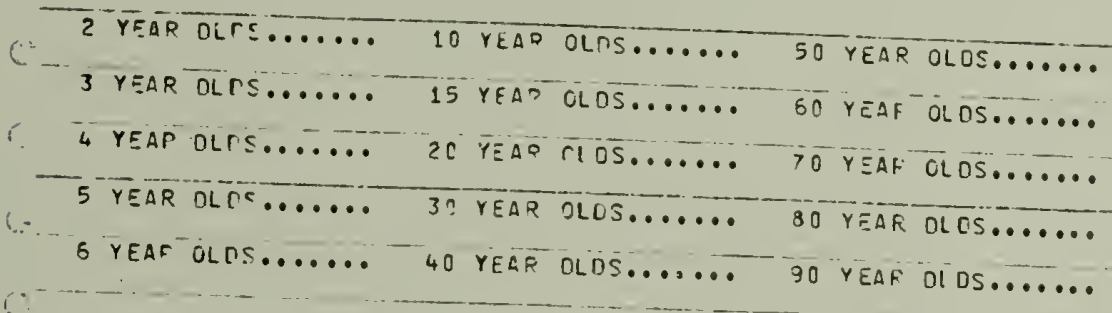

(1. ANOTHEF KIN.O OF TASK USEO TO STUDY 11 EMORY INVOLVLS RECOFNITION FOF FXAYPLE, AFTEE SHOWING P=OFL ? 4 FICTUIES, WE SHOH THEM 48

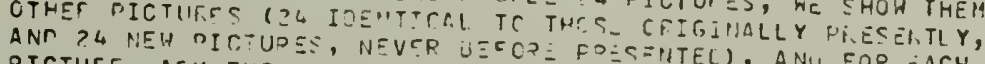

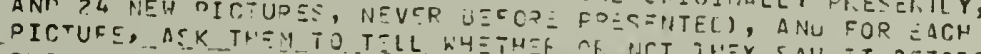
PLEASE FILI IN THE TLOLE INOICATIPG HOW II'EY SAH IT.BEFNGE. YOU THIVK FEOPIE AT EACH OF THE AGTS LOW YAIIYOF THE 4 R PICTURES

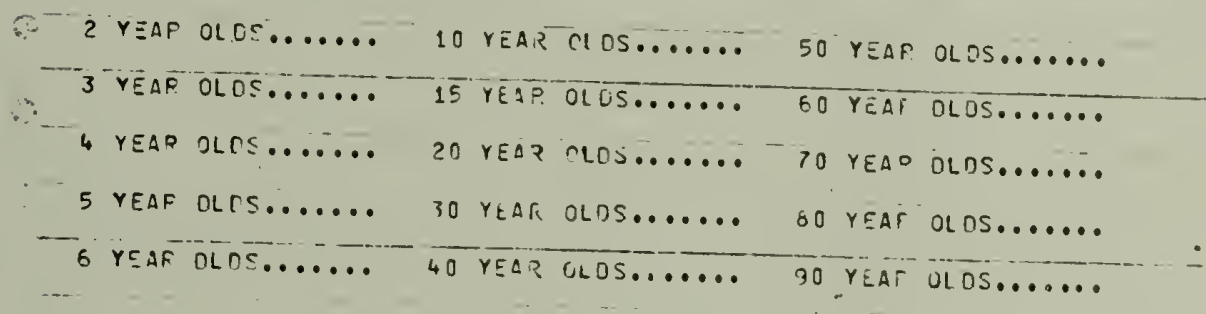


PLEASE AMSWEP THE FOLLOHING ZUESTIZNS AN2 IHEN CIRCLE IME

NUMAEF ABCVE THE RESFONSE THAT IS CLOSEST IO YOUK THOICE

1 HTK OFTEN HAS THER EE SN SOMCONE IN YOINO EAMILY HHO HAS HAO CIFFICULTY -... H.R.ME

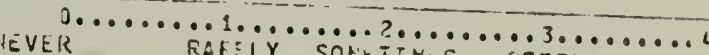

2. MOH OFTEN HAS THEOE BE EN SOMEONE IN YCUR FAMIIY WHO HAS EXOEPTIONALLY ACTIVE. ANO

(

\section{ALEFT IN OLO AGE}

(

\section{.} NEV $=R^{2}$ RARELY SOMETIMES

OFTEN

ALWAYS

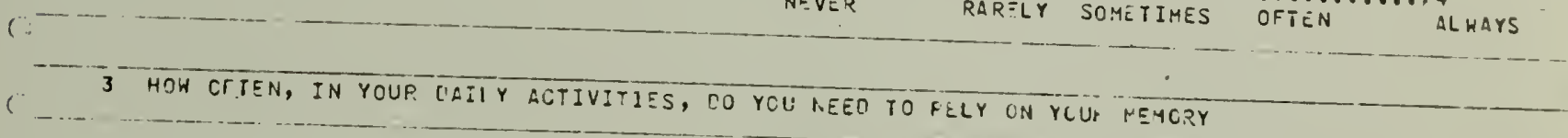

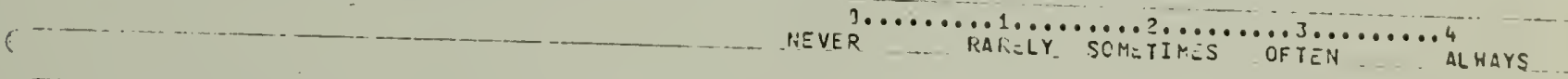

4 HOH CFTEN: DO YOU PARTICULARLY NOTICE YOUK MEHORY

N

5 HOK OFTEN DO YOU EXPEPIEAICE OIFFICULTY REMEMEERING THINAS$$
\text { r. }
$$

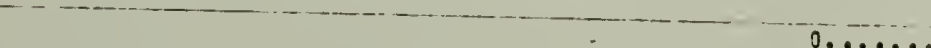

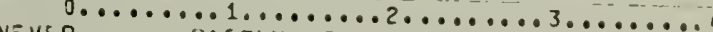

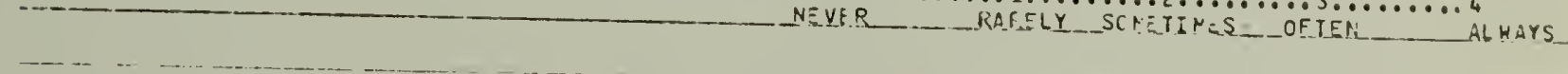

E HEH CFTEN DO YOU HAVE CIFFICULTY FEME:HSFING IHINGS THAT HAPPENEU A FEH RINUTES

7 HOW RFTEN DO YOU HAVE OIFFICILTY FEMEIBEKILG IHIIGJ THAT MAPFNEO A FEH DAYS OR NEVLR

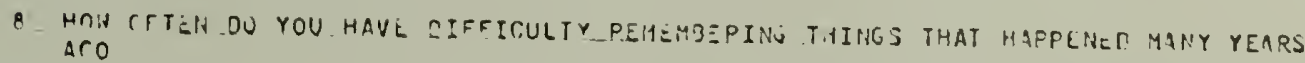

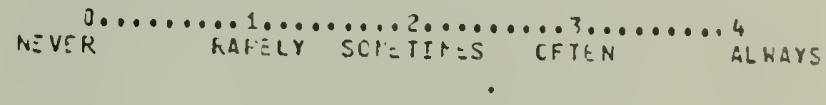




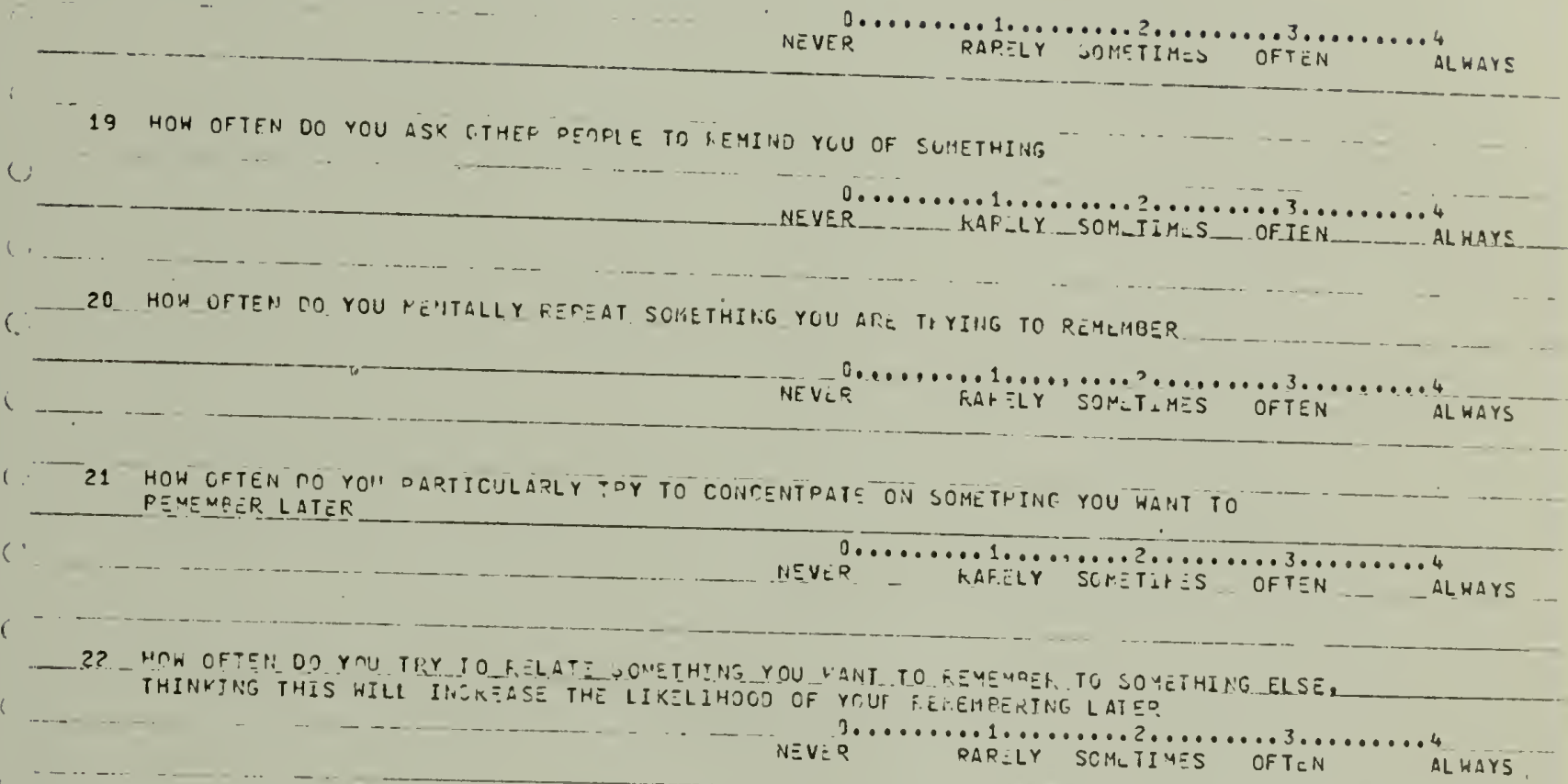

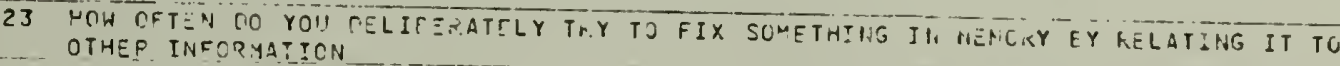

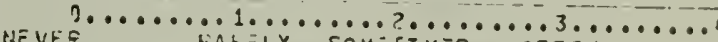
NEVER _RARELY SOMEIIMES OFTEN

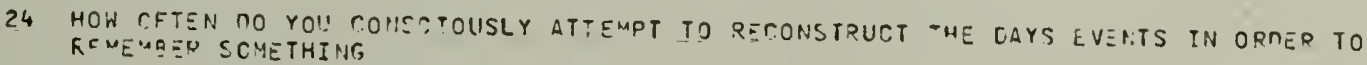

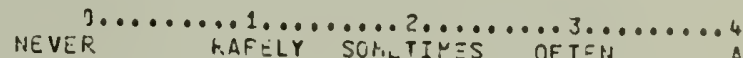
hafely somitires often alhays

25 HCH CFTEN DO YOU TPY TO TWINK OF THINGS THAI FELATE TO SOMETHING YOJ FOKGOT, HOPIIF. IT HALL FKIHG IT TO MINO

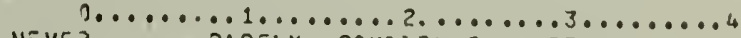

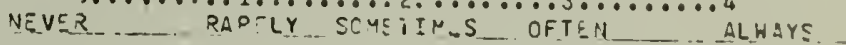

26 HOH CTTEN OQ YOU FIGGGE TN OEI IEEFATE ATTEHPTS TO REIEHEER INFOH IATICN NEV VR

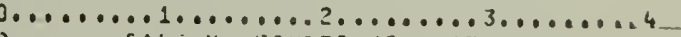
HE VFR RATELY SOMLTIMES OFTEN AL WAYS

27 MRW GETEN CO YOU LDOK ITS THINCS YOU CAN NOT REMEMHER 
a HOW OFTEN DO YOIJ FOHG: T THINGS

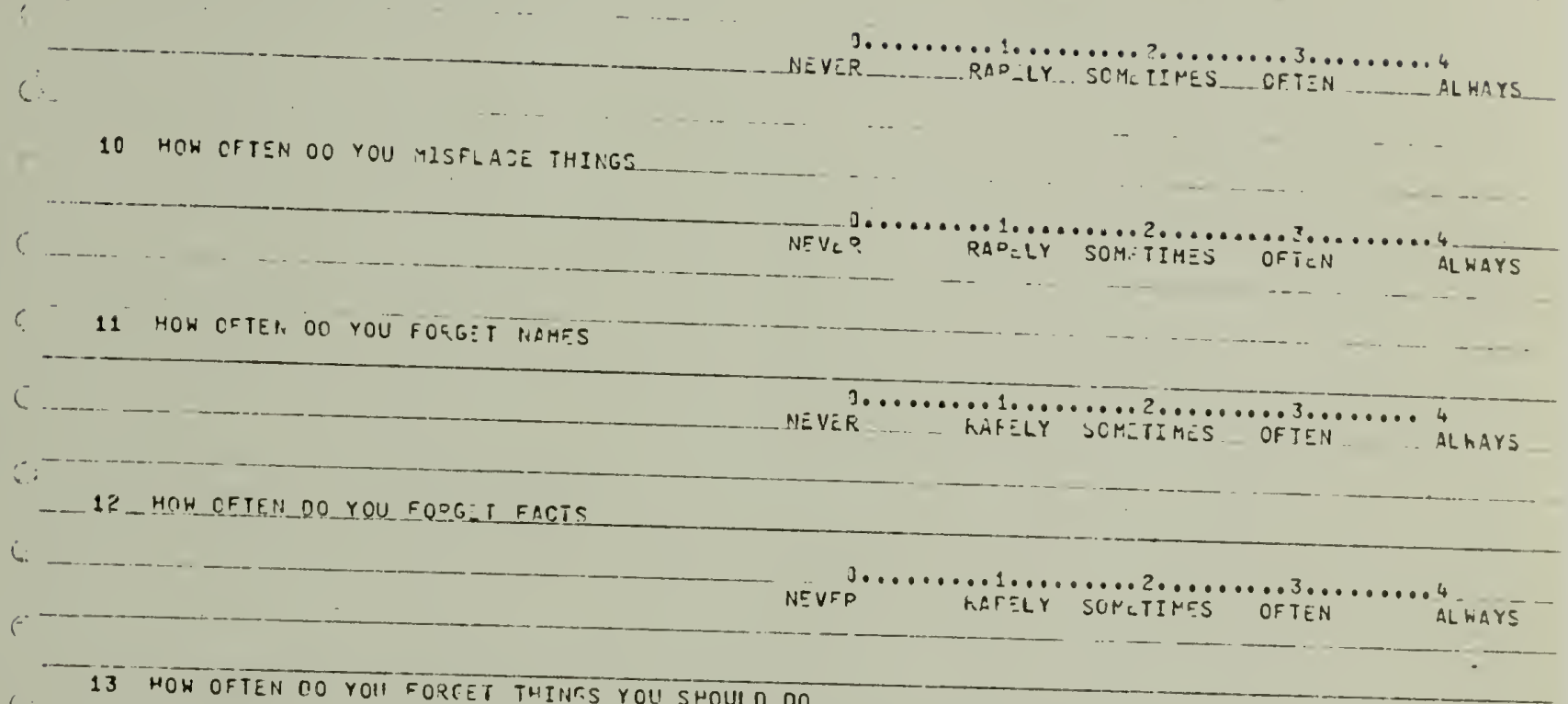

13 HOW OFTEN DO YOII FOREET THINTS YOU SHOULD DO

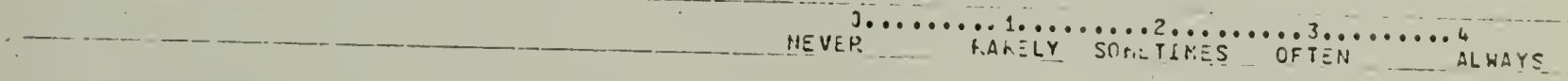

14 HOW OFTEN DO YOU FUFEET APPOI ITMENTS

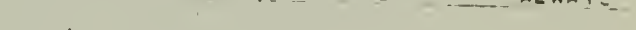

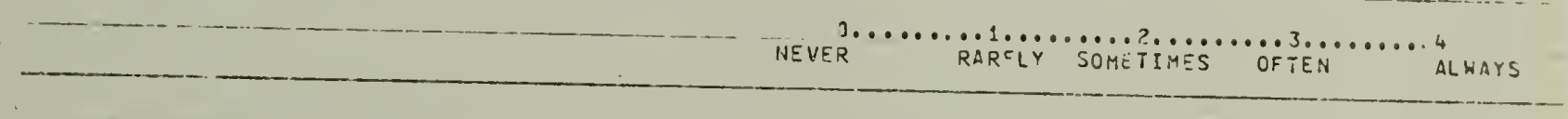

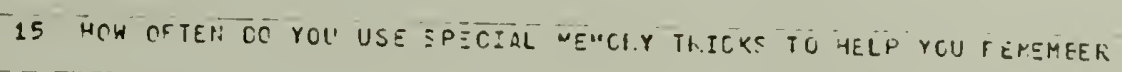

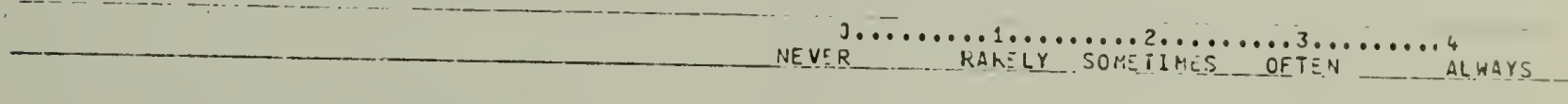

16 HOW CFTEN DO YOU HFITE A SHUPPII.G LIST

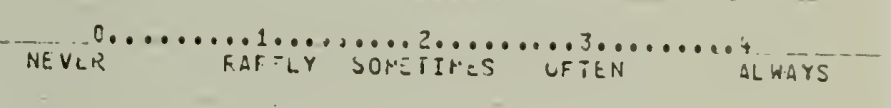

17 HOW OETEN OO YOU HPITE APPOINTMENTS PN A CALEHOAO TO HELP YOU REMCMBEP THEM

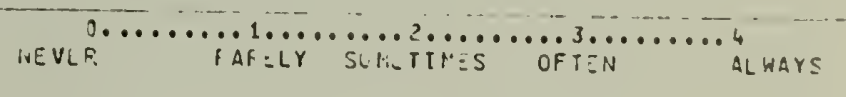




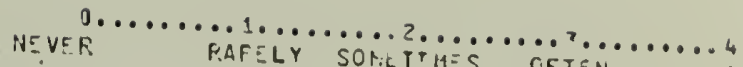
R.AFELY SORILTIHES OFTEN WLWAYS

28 HOH OFTEN DO YOLI ASK OTUEK DEOPLE THIIIGS YOU CAN NOT PEREMBEP

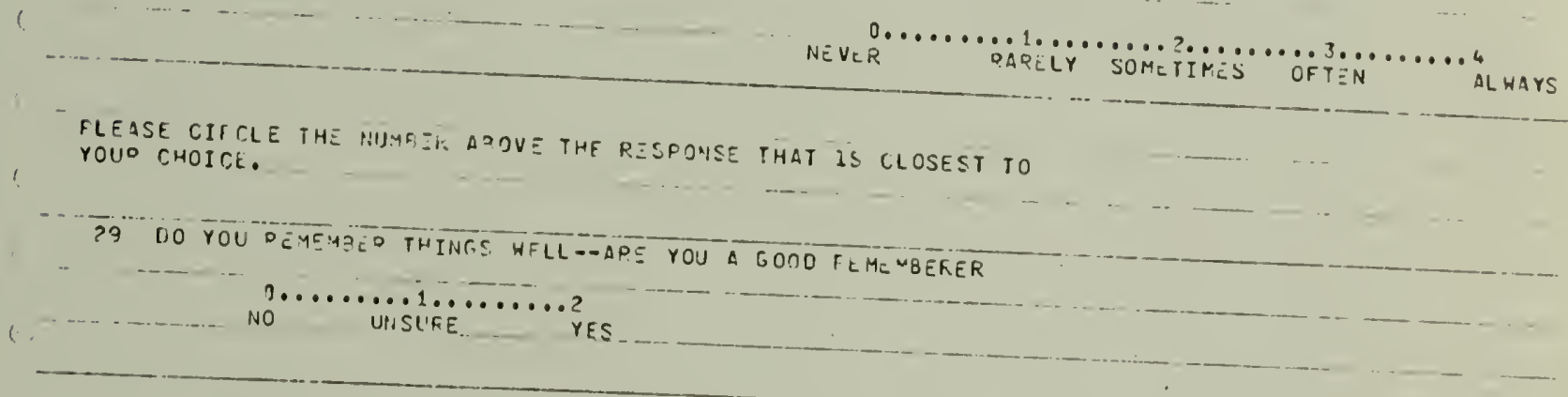

30 CAN YOU REMEMPE? EETTEP, THAN YOUR, FOIENOS

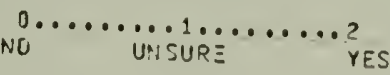

31 WHEN YOU CAN NOT FEMEYJEK SOMETHIMG OC YGU FINO IT UPSETTINF

no $\ldots \ldots \ldots$............

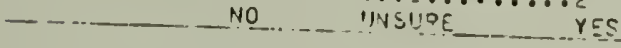

32 DC YOUI HAVE MCFE OIFFICILTY H.EMET,DERTNG THINES WHEN YOU AFE TIRED

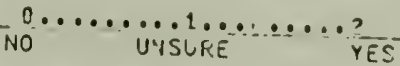

33 DO YOU HAVE YODE NIFFICILTY REMEIIEE?ING THINGS WHEN YOU ARE PRESSURED

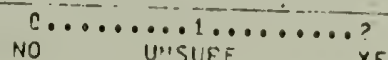

U'SUFE YES

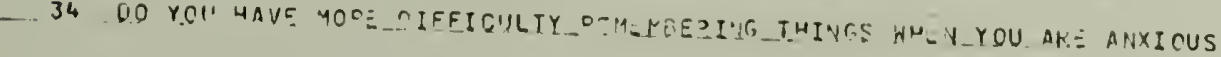

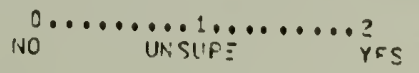

35 APE YOIS MORE IIKEIY TO FOKR:T THINGS HHEN YOUT: MIHO IS PFEOCCUPIEO

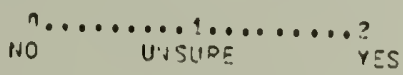


उE OVER THE YEAPS HAVE YOU FECOME MOFE AWAFIC OF YUUT. MEMCRY

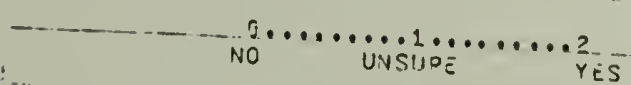

$$
(\because \ldots \text { NO UNSUDE YES }
$$

37 OVER THE YEARE HAVL YCU NUTICF O AIIY CHANGES IA YOUE HEHOFY C............... NO $\cdots$ UISURE $\cdots \cdots$ ?

$6-$

3A DO YOU USE MEMOOY AICS MOPE OFTEN THAN YOIIFRFVIOUISLY OID (

r.

C.

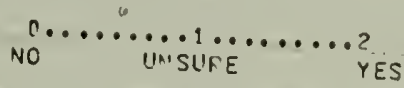

39 OVEP THE VEARS "AS YOI!P MEIORY IMPROVEO

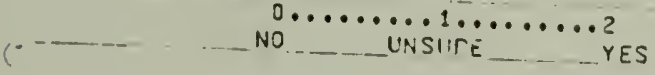

40 NUEF. THE YEAFS HAS YOUQ MEYOFY BECOME HOPSE

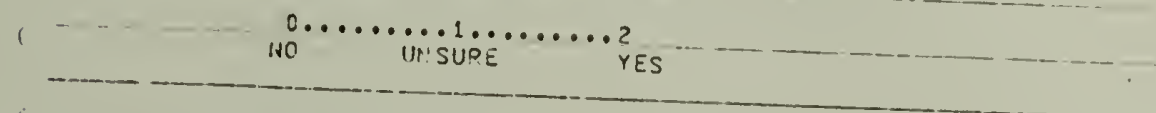

41 DO YOU THINK YOUD MEMORY WILL CHANGE AS YOU GET OLDER

-. 42 DO YOII THTNK YOUF, MEROQY WILL GFT BETTEK WHEN YCU TLT OLOER

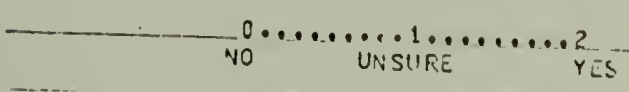

43 DO YCU THINK YOUR VIEPO?Y WILL GET WOS SE WHEN YOU GET OLOER

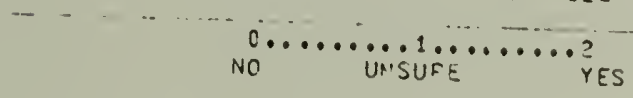

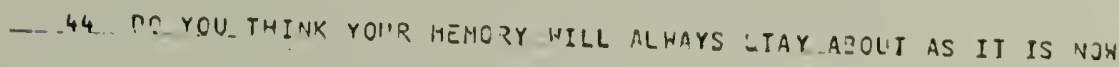

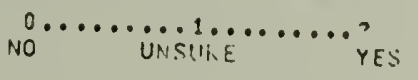


45 OC YOU THINK YOU HILL FOHCET THINCS MOSE EASILY WHEN YOU GCI OLEEK

(

6

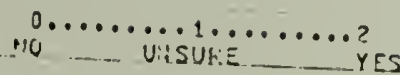

DO YOU THIHK YOII WILL.F ME UYEI, YOF Ë OETAILS AE YOU GCT OLDEP NO $\ldots \ldots \ldots 1 \ldots \ldots \ldots, \ldots$

NO $\cdots \cdots \cdots$ in: SUP

47 OO YCll CONSCICUSLY TFY TO MEMINZZE THINGS MOTE THAN YOU USEO TO

( NO

4R DO YOU FEME" "OCE SOME KINNS OF THINISS PETTER THAIT OTHEFS

c

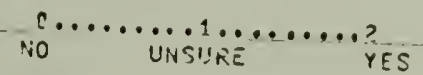

$\epsilon$

40 DO YOU FIND TT EACIER TO PEYL:IRER ORGANIZEU THI:GS THAN UNORGANIZEO THINGS

!

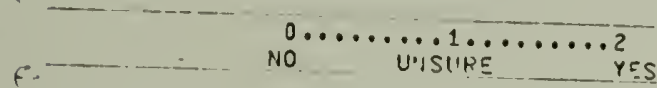

SO. IS IT EASTED TO DEMEME E? VISUAL THINGS THAN VEREAL THINGS

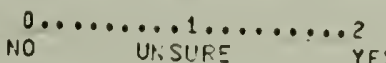

51 TO YCU FINO IT EASIEF TO CEYEMDEF GIZZAFE THINGS THAN USUAL THINGS

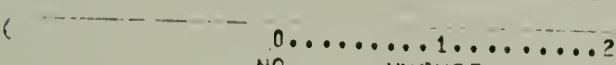
nO $\ldots \ldots$ U.

52 OO YCU FINO IT EASIEY TO FERE"DEF THIHGS YOU ARE ROST INTERESTEO IN

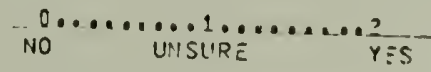

53 DC YOU FIND IT EASIEF TO REREMEF CUNCRETE THTHT.S THAT, R.BSTHACT THINGS

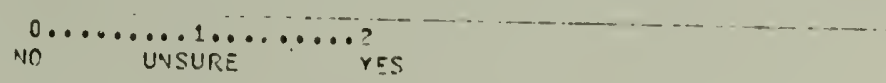


54 DO YOIH FIHO IT EATIEP TO REYEMTID

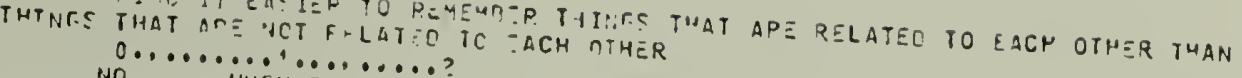

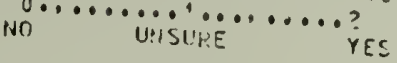

$5 c_{1}$

AFE THERE SOYE KINICS OF THINGS THAT AF FEALLY HAFO TO FEMEMEER

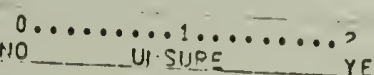

56 AFS YOU ETPECEALLY LIKELY TO FONGET UH.PLEASAH'T THINGS

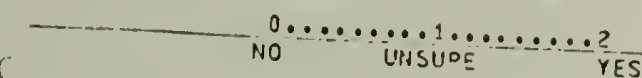

(

57 DO YCU hAVE MOFE LIFFICULTY FFIENBE

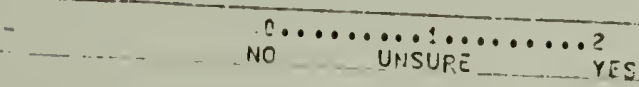

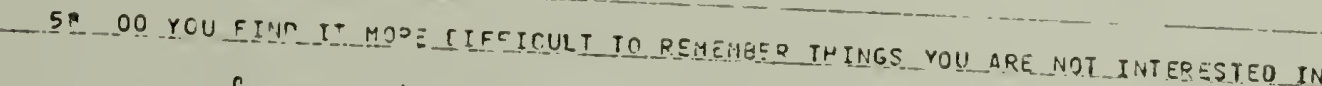

(

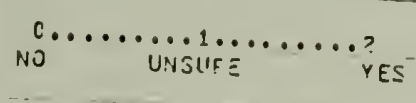

59 กO YOU FIND IT MODE OIFFICULT TO PEMEH3ER UNFAMILIAT. THAN FAMILIAF THINGS

$0 \ldots \ldots \ldots 1 \ldots \ldots \ldots$ ? NO $\ldots$ UNSIIEZ $\cdots$ UES

60 DO YOU FIND IT MOEE UIFFISULT TO KE"'EMEER THINGS YOI DO NOT FEALLY UNDEFSTAND ND $\ldots \ldots \ldots 1 \ldots \ldots$ ? 


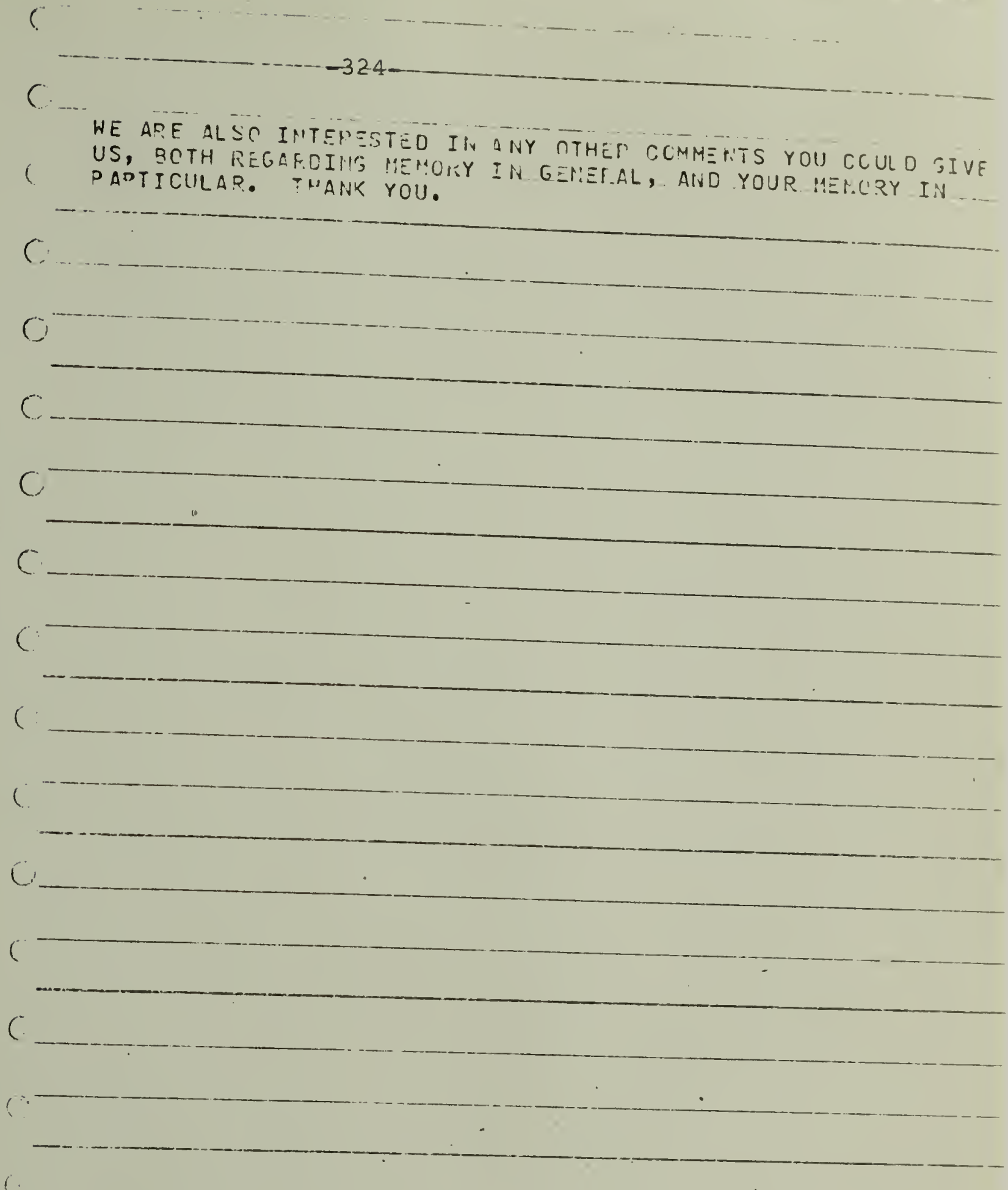

FLEASE RECCRO THE TIME 
PLEASÉ RECORO THE IIYE

1

- THIS IS AR ASSTCITATON TASK. PLALE WOITE DOHN ANYTHIMG THAT

AS MANY ASSORIATIOIS AS YOU WOS BOIIISS TO MIND. YUU MAY KKITE

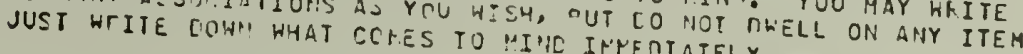

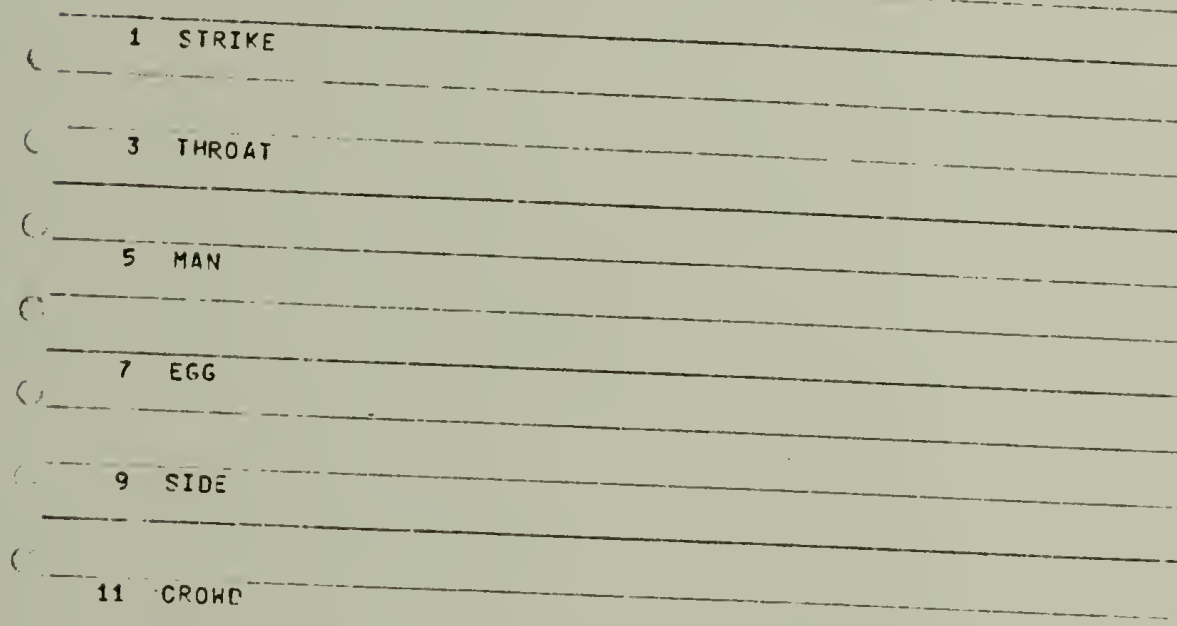

\section{FRONT}

4 HEIGHT

6 DECK

8 DERT

10 HAT

$12 S E A T$

\section{MATTER}

14 NET

15 MILK

16 HHEAT

17 GUEST

$\because$

19108

18

OAY

$-$

$20 \quad 80 \mathrm{AT}$

21 AIR

22 IIME

23 PAP

24 SOUND

PLFASF RECURO THE TIIE 
PLEASE RECOPT THE TI'AE

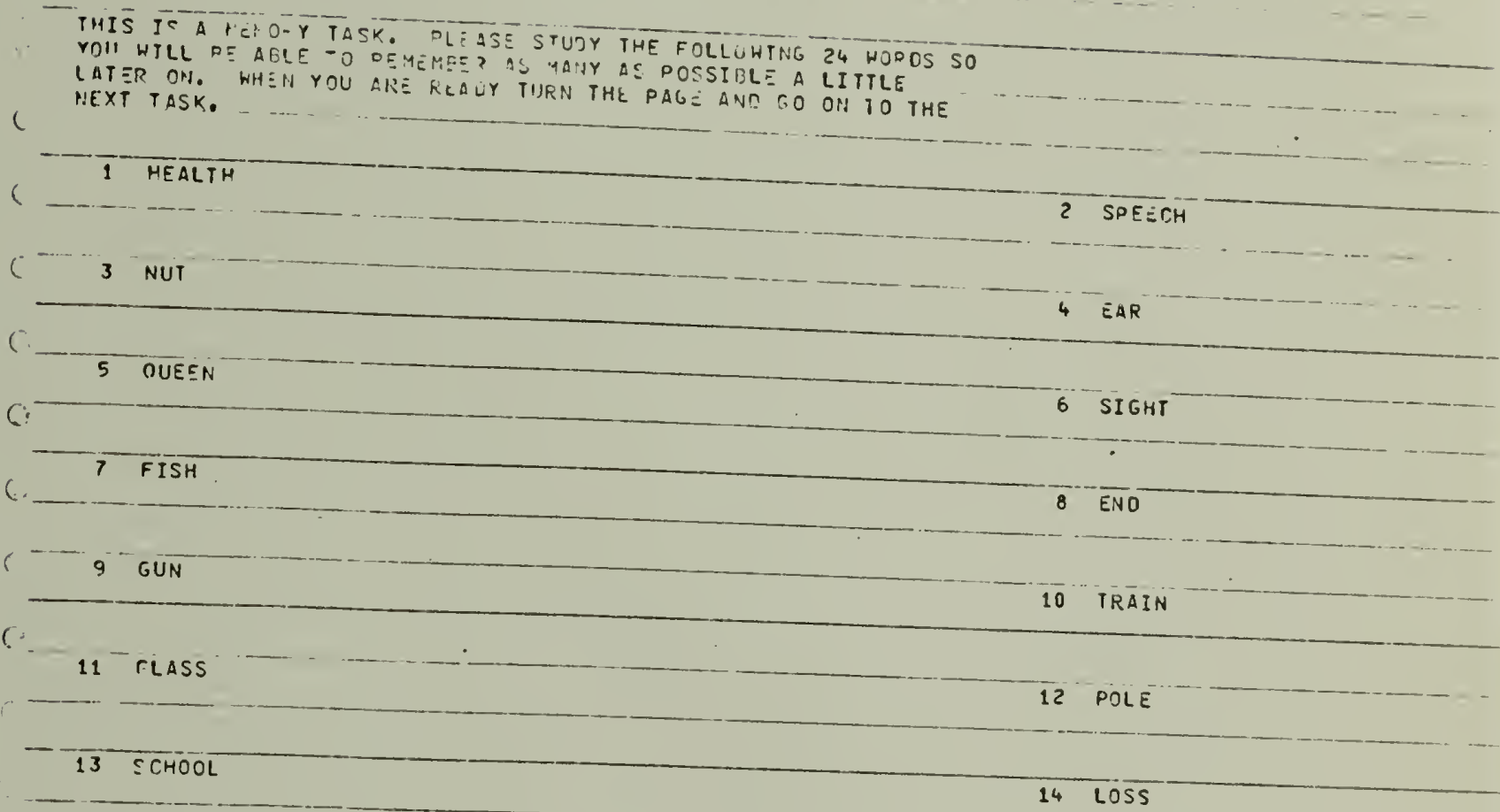

16 TASK

$17 \mathrm{LAH}$

19 HILL

21 NoIse

23 PF $\perp$ NCE

oLEASE RECAKO THE TIME
$18 \quad 36000$

20 IHING

$22^{\circ}$ CHILO

26 B4r 
PLEASE PECORN THE TIME

- (

HOW MANY OF THE 24 WORNS WILL THE TEMORY TASK JU YJU THINK YOU

?

( $\therefore$

HOW MANY OF 1 HE 24 WOFCS ON THE ASSOCIATICI TASK DO YOU THINK
YOIS WILL OF AELE TO_KECALL COFE CTLY.

()

PLEASE RECOPO THE TIME

C i.

6

(C: 
PLEASE RECOFO IHE TIHE

YOU WETE FI EVIRUSLY R.IVEM 24 WCH DS IN STUDY III A MEMOEY TASK.

PLEASF WRITE TOWN ALL OF THE WOROS YOU CIN REMEIREO FRO' THE

MEMOSY TASK, AND FOR. SACH hOTR, YOU THIMK YOU FE YFMECR, CIFCLE

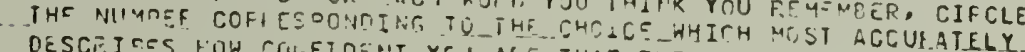

DESCEI MES HOW CGIOFIOENT YCJ AFE THAT THE WLKE WAS ON THE

$(\ldots$

(

C.

(

1

,

VERY UNSUFE

?

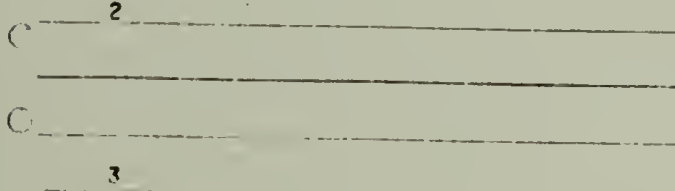

VIPY UNSUE

$1, \ldots \ldots \ldots . \ldots \ldots \ldots+\ldots \ldots \ldots$

YEKY SURE

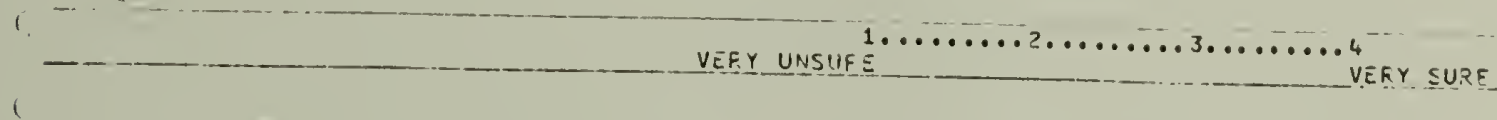

4

VEFY UNSUCE

1........................

VERY SURE

i.

\&.

6

KENY UNSUEE

$1 \ldots \ldots \ldots . \ldots \ldots \ldots, \ldots \ldots, \ldots, \ldots$

VEFY SURE

7

- - - - - -

VFfY UNSURE $1 \ldots \ldots \ldots 2 \ldots \ldots \ldots$ VEFY SURE

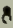

$1, \ldots \ldots \ldots, \ldots \ldots \ldots+\ldots \ldots, \ldots$ 


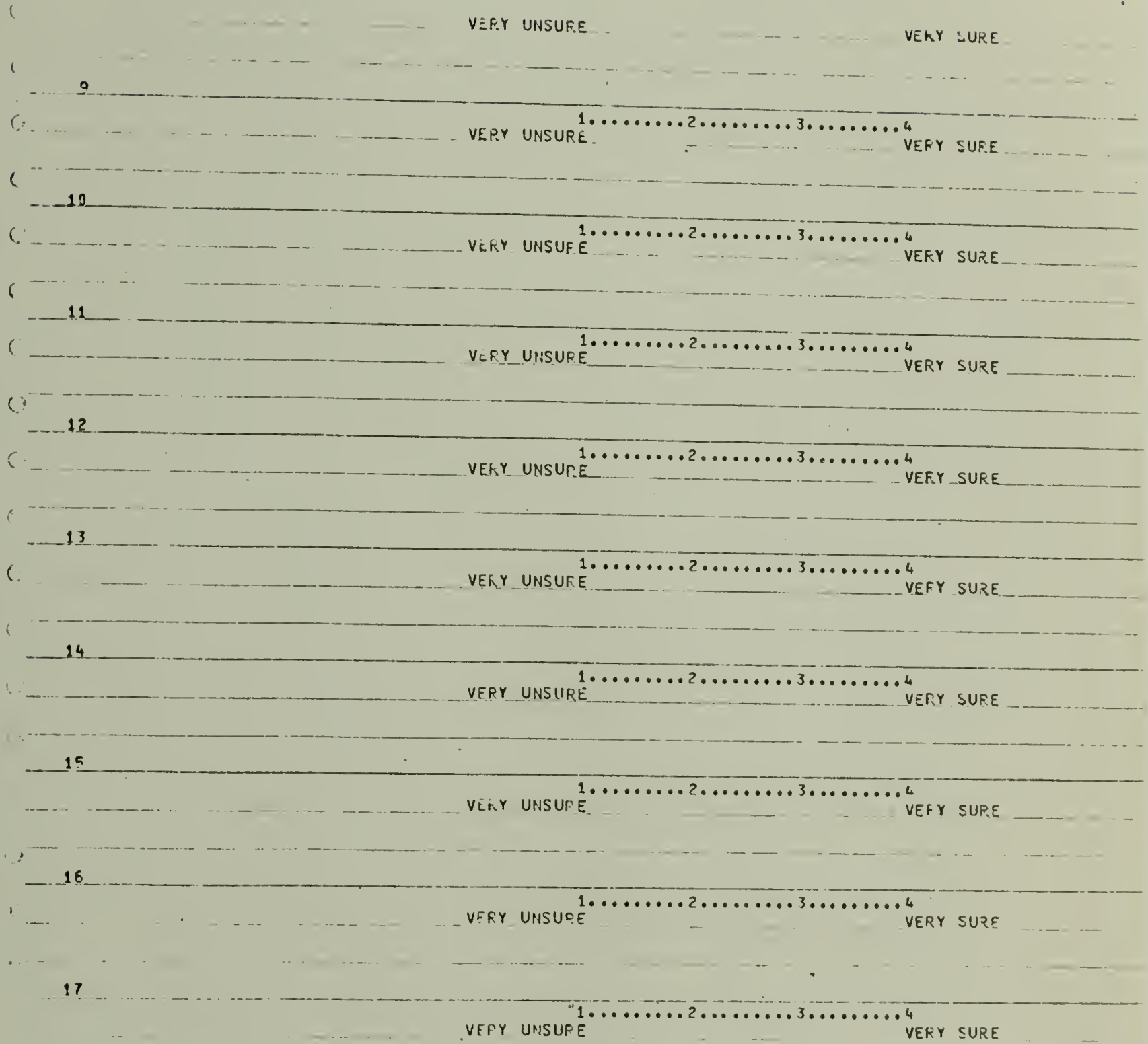

VEPY UNSURE

VERY SURE

IA

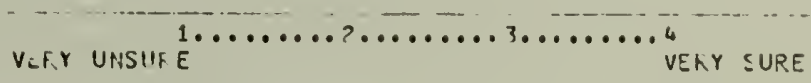




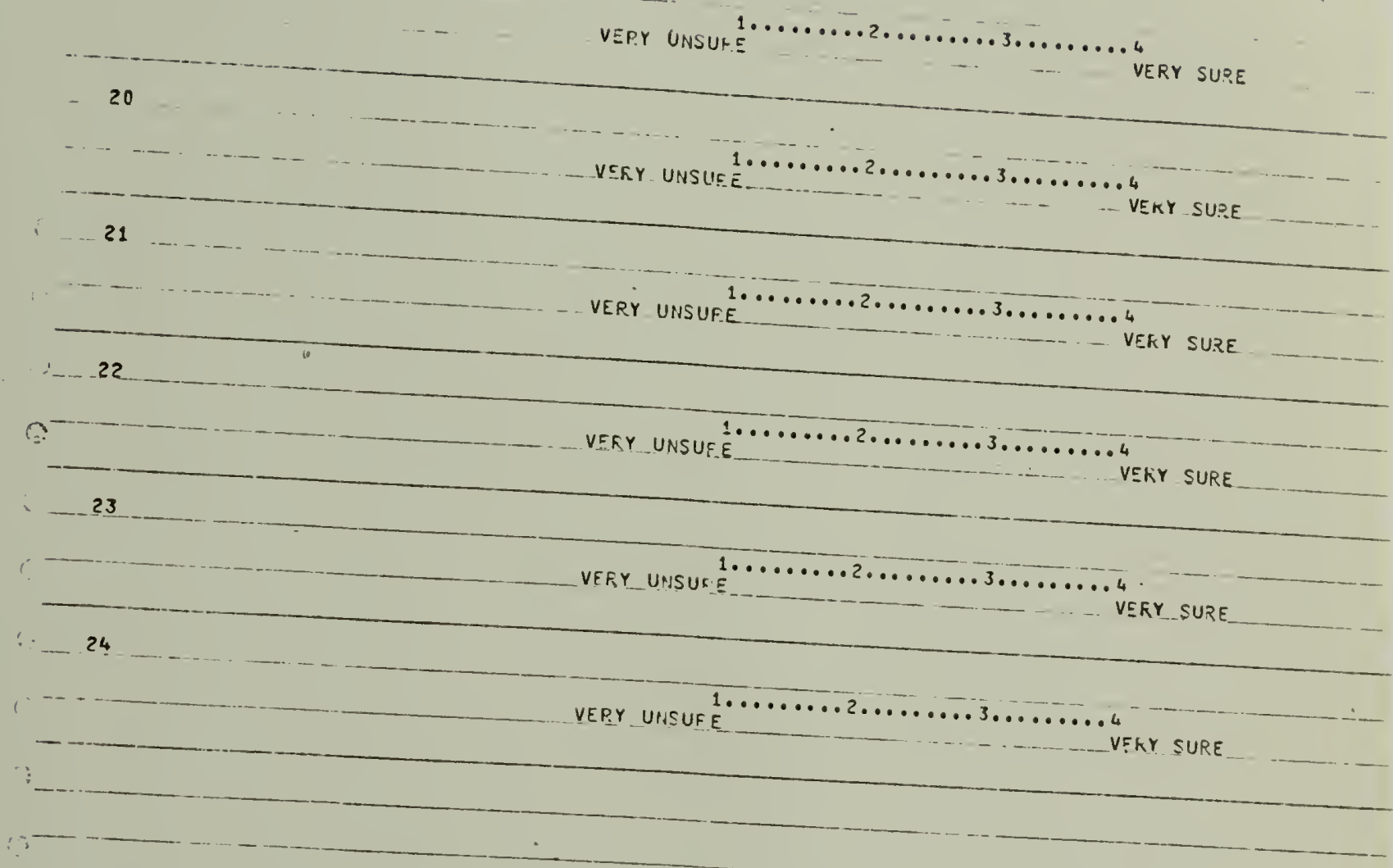

IF YOU WEKE GIVEN A REROSHTTION TEST HHICH SONIAINEO ALL OF

YOU OIO OI THIS RECALL TEST.

HOW MANY OF THE METOFY WOGLS THAT YOU FAILLO TO KECALL JU YOU
THINK YOS KILL B: AELE TO OECCGNIZ TODRECTLY.

PLEASF RECRRT THE TIHE 
PLEASE FECOZN THE TIME

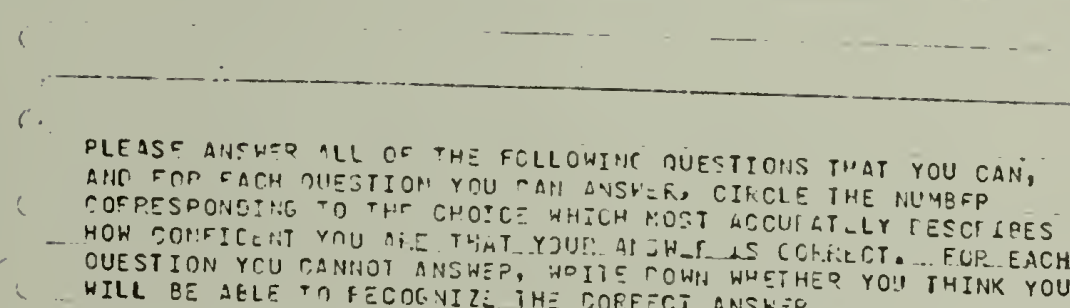

WILL BE LELE TO FECOGNIZE. IHE CORFECT ANSWER.

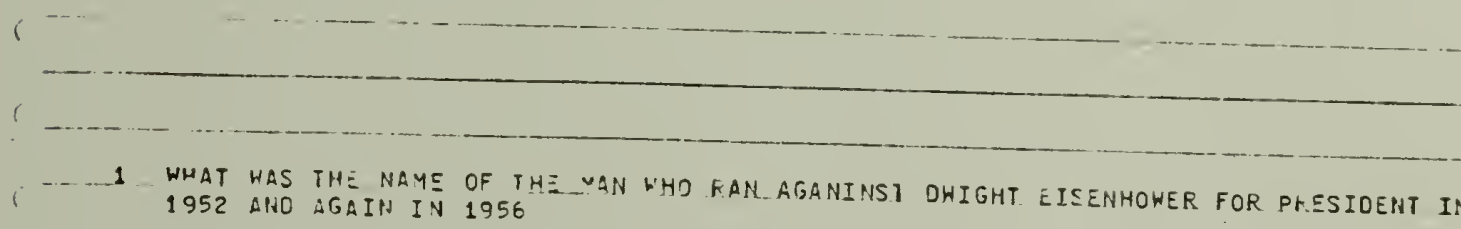

DE YOU THIIK YOU WOULO PECOGHIZE THE CORRECT ANSHER UERY UNSURE

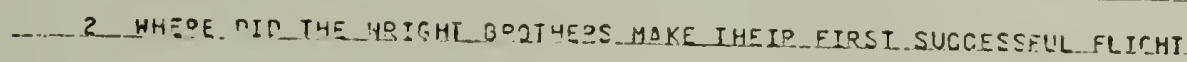

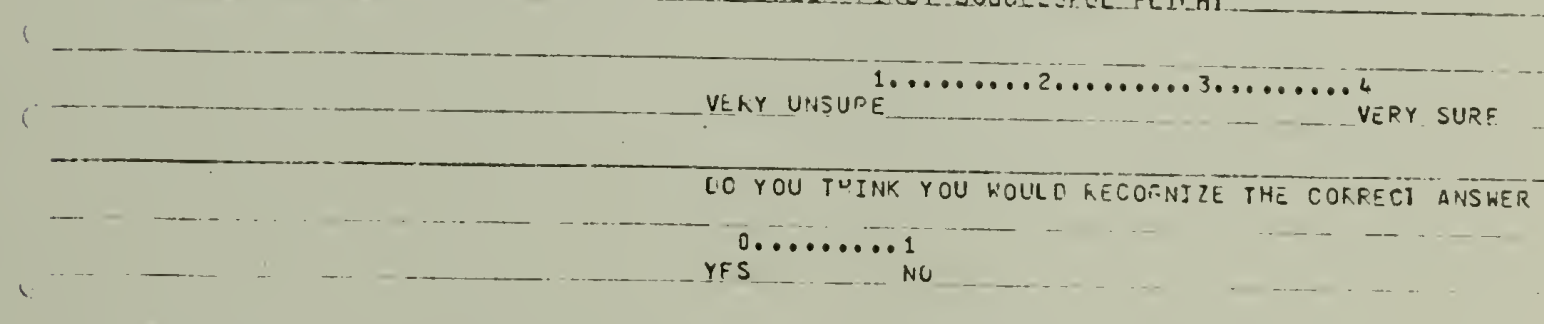
3 HHAT WAS THE NANE OF THE MAN KHO BECOME PKESIOENT HHEN PFESICENI RCKINLEY WAS
ASSASSINATEO IN 1900

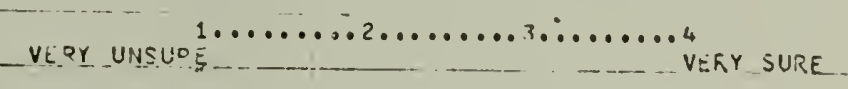

in YOU THIHK YOU WOULL F. ECORMIZL THE COFHECT ANSHCR

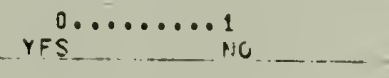

4 WHERT (IN WHAT STATE) DIO THE GEMMAN UIKITIFLE, TH_ VUN HINOLP AEOG, BURN ANO 


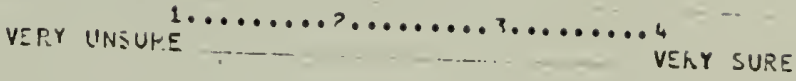

CO YOU THINK YOU WOULO RECOTNIZE THE CORFECT ANSWER

6 $0 . \ldots \ldots 1$

YES

No

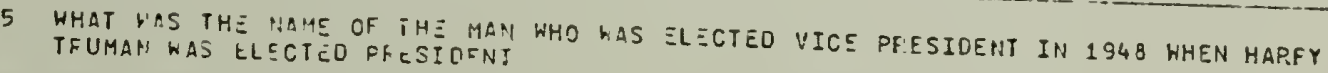

6 HHAT WAS THE HAME CF THE CUXEK WHO WAS NICKHAY EO GETTLEMAN JIM

('.

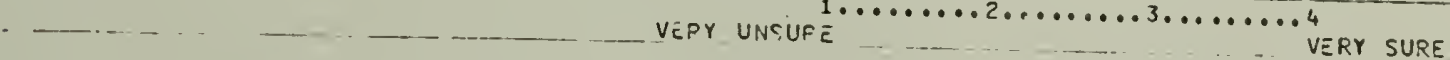

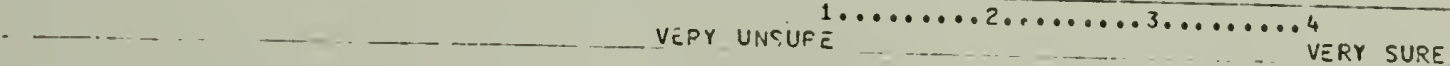

CO YOU THINK YOU WOILL RECOCHIZE THE CORPECT ANSHER

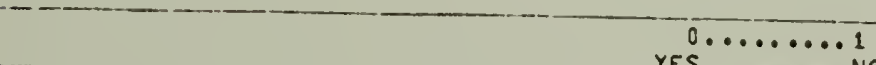

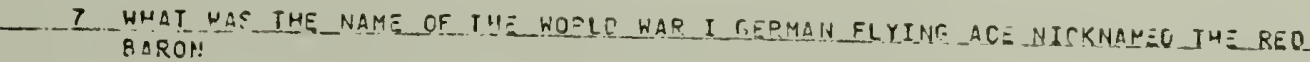

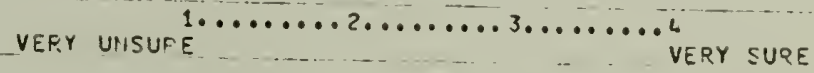

OO YOU THINK YOU WOLLE KECOTNIZE THE COKFECT ANSWER O..........

YES

NO

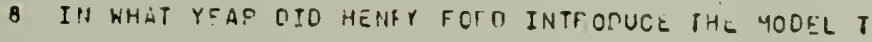

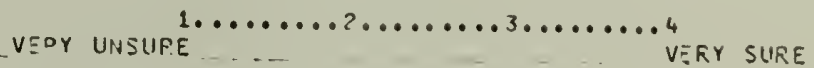

LC YCII THINK YUU WOULO FECOGNIZE THE COKAFT ANSHEQ э....... 1 

- IH WHAT STATE DIO THE EIF.I LEGAL ELECTROCUTION FOO MURCEO IN THE UNITEU STATES
OCCUR

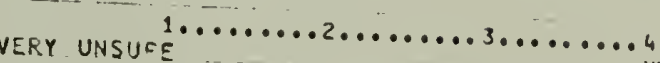

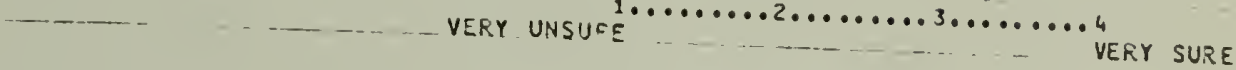

CO YOU THINK YOU WOULO RECOGNIZ THE COKKECT ANSHER $0 \ldots \ldots \ldots 1$ YES NO

10. ON WHAT OATE (CAY, MONTH,YEAR) OIO THE JAPANESC EOMB. FEATL HAFEOF

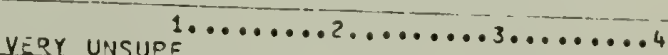

LO YOU THINK YOU WOULO FECOGNIZE IHE CORFECT ANSHER $0 . \ldots \ldots \ldots 1$ YES NL

11 WHAT WAS THE HARE OF THE HAH WHOSE DEATH SET UFF WORLO HAR I

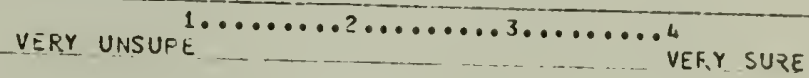

DO YOU THINK YOU WOULL RECORNIZE THE CORFECT ANSHER

$\ldots Y_{E S}^{0} \cdots \cdots \cdots 1$

- 12 WHAT CO TUE INITIALS WCTU STAHO FOR

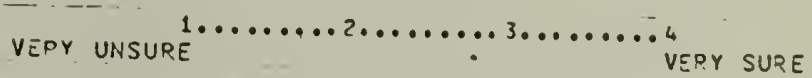

CO YOU THINK YOU WOUIO TECOGN TE THE CURSECT AHSWER YES

13 WHAT WAS THE NAME OF THE MAN NHO TISCOVEFEN THE NORTH POLE 
VEKY IINSHRE

(

i

(

GO YCII THIHK YOU WOULO KECOGIIZE THE COHFECT ANSWE? YES ..... 1

6

(

DO YOU THINK YOU WOULO RECOGNLZE THE CORFECT ANSHER

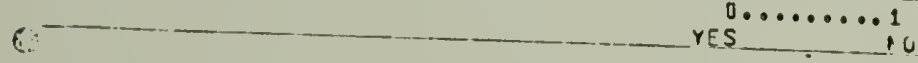

C 15 WHAT WAS THE MAME CF THE TMMMANDER OF THE FAMCUS FLYING IITERS OF HORLO HAR II

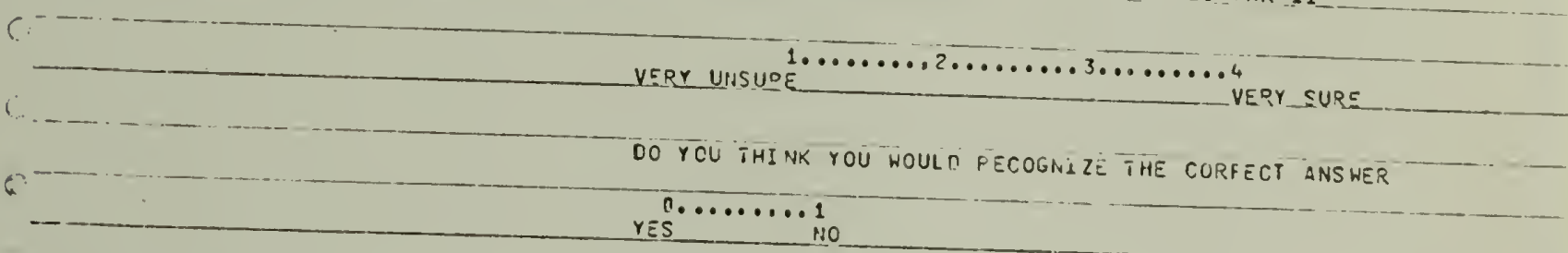
16 WHAT WAS THE NAMS OF THE SHIP WHICH HIT AH IC? BERE AND SAHK OH I IS MAIDER VOYAGE
JH 191 ?

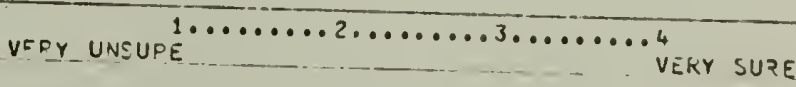
DO YOII THINK YOU WOULO RECOGNIZE IHE CORPFCT ANSHER

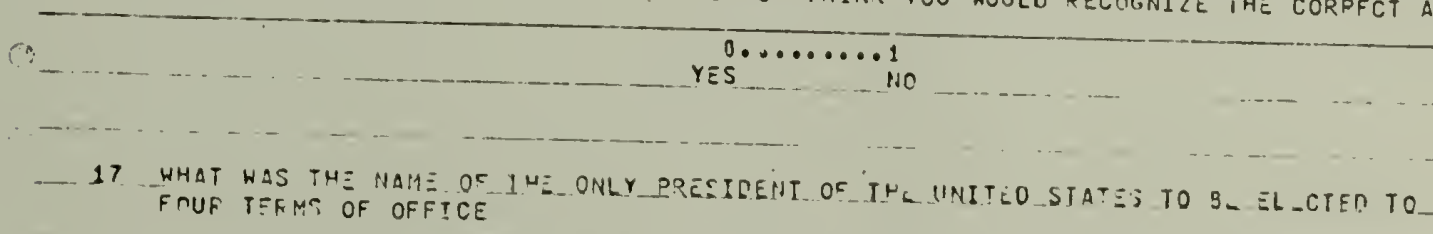

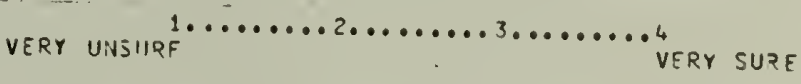

CO YOU THIHK YOU WOLLO FECOGMIZ THE COKPECT ANSWER YES 


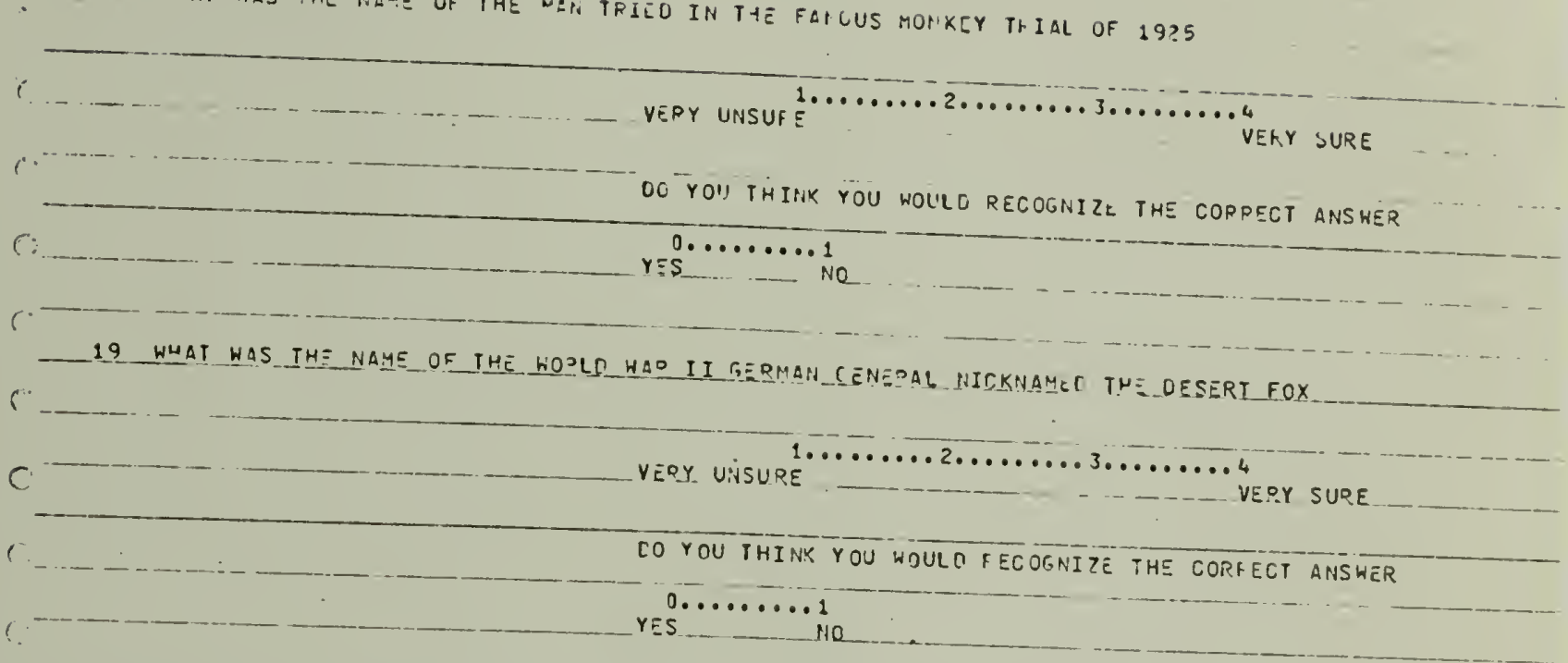

20 WHAT WAS THE NAME OF IHE MA! WHO ASSASSINATEO DF. YAKTIN LUTHEK KING

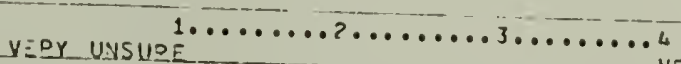

(3)

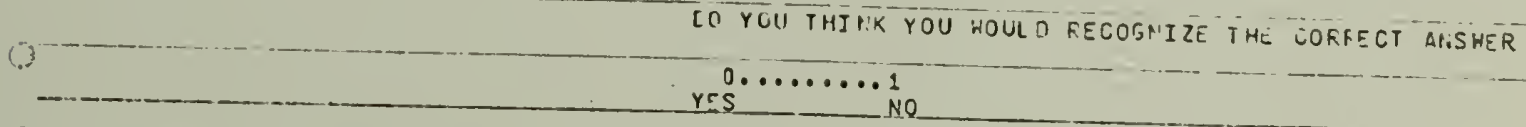

i)

21 TN WHAT CTTY WAS ORESIOENT JOHN F. KEIMIEOY ASSASSINATED

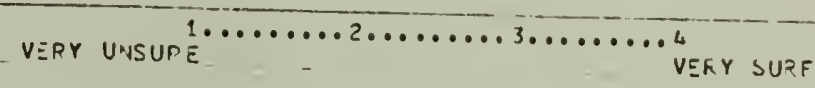

CO YUII THINK YOU HOULC OFCORINIZ THE CORRECT ANSWER YES $\cdots \cdots \cdots+$ NO

- 22. WUAT WAS THE NAY OF IUE FLALEIN HUICU LINRRERT FLEH_THE ATIANTIC

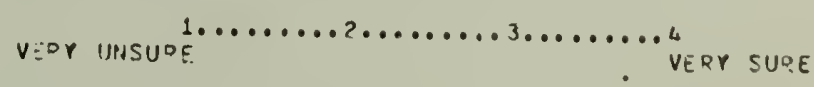


DO YOU THINK YOLI WOULU PECORINIZE THE CORRECT ANSHER

()$^{--\cdots}$ $0 .$. YES

(j) CONGTESSICNAL INVESIIEAIICNS OF COMMUNISM IN IHE EATLY 1950 SS ASSOCIATED HITH

(

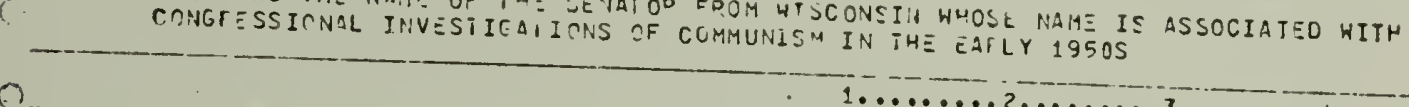

VERY URSIIRE

VERY SURE

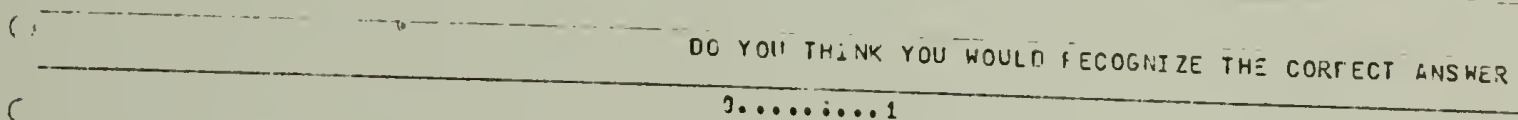

C YES - 24

24 WHAT HAS THE HAME OF IHE FIFST MON TO SET FOCT ON THE MOCN

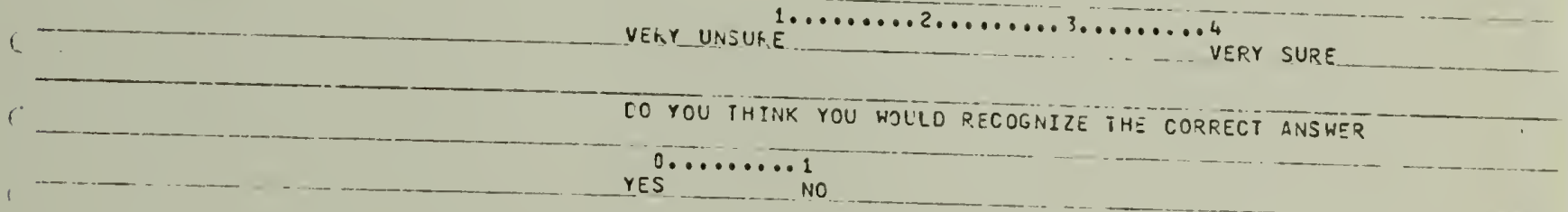

DLEASE RECORE THE TINE 
PLEASE RETORO TH= TIME

YOII WEDE PEEVTMUSLY GIVEV ? 4 ITEMT TO CEVERAT HOFLIS TO IN AN

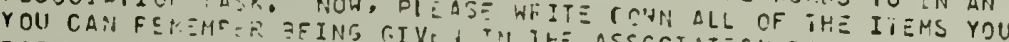

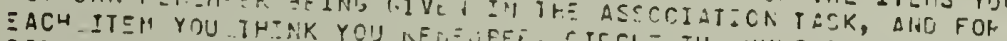

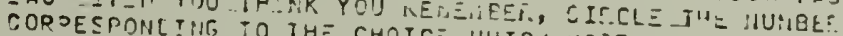

HOW CONFIL NT YOU APE THAT HHICH MOST ACCUDLTELY RESCOISES

ASSOCIATIOH: TASK.

(

(

?

1

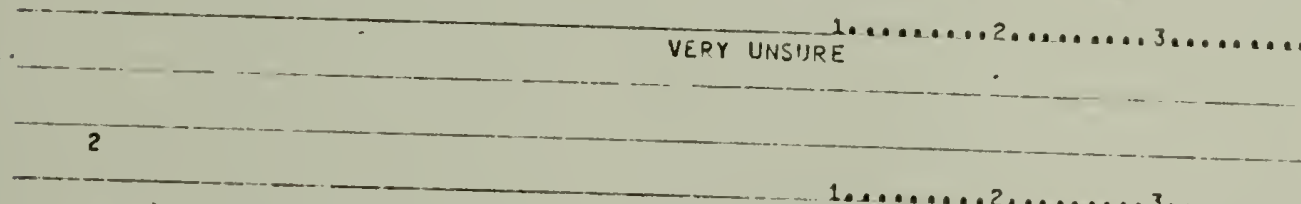

VERY UNSTIRE $1 \ldots \ldots \ldots \ldots 2 \ldots \ldots \ldots \ldots 3 \ldots \ldots \ldots \ldots$

VERY SURE

VERT UNSURE

VERY SURE

3

VERY UNSIRE

VERY SURE

VERY UNSIIOE

VERY SURE

5

VERY UNSURE

VERY SURE

$\epsilon$

VERT UINSIIOE

VERY SURE

7

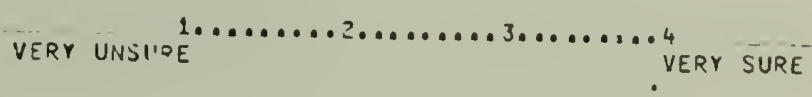


VERY UNSUOE $1 \ldots \ldots \ldots . \ldots \ldots \ldots . \ldots \ldots$

9

VEPT UNSUDE $1 \ldots \ldots \ldots .2 \ldots \ldots \ldots 3 \ldots \ldots \ldots 4$ VEFY SURE -

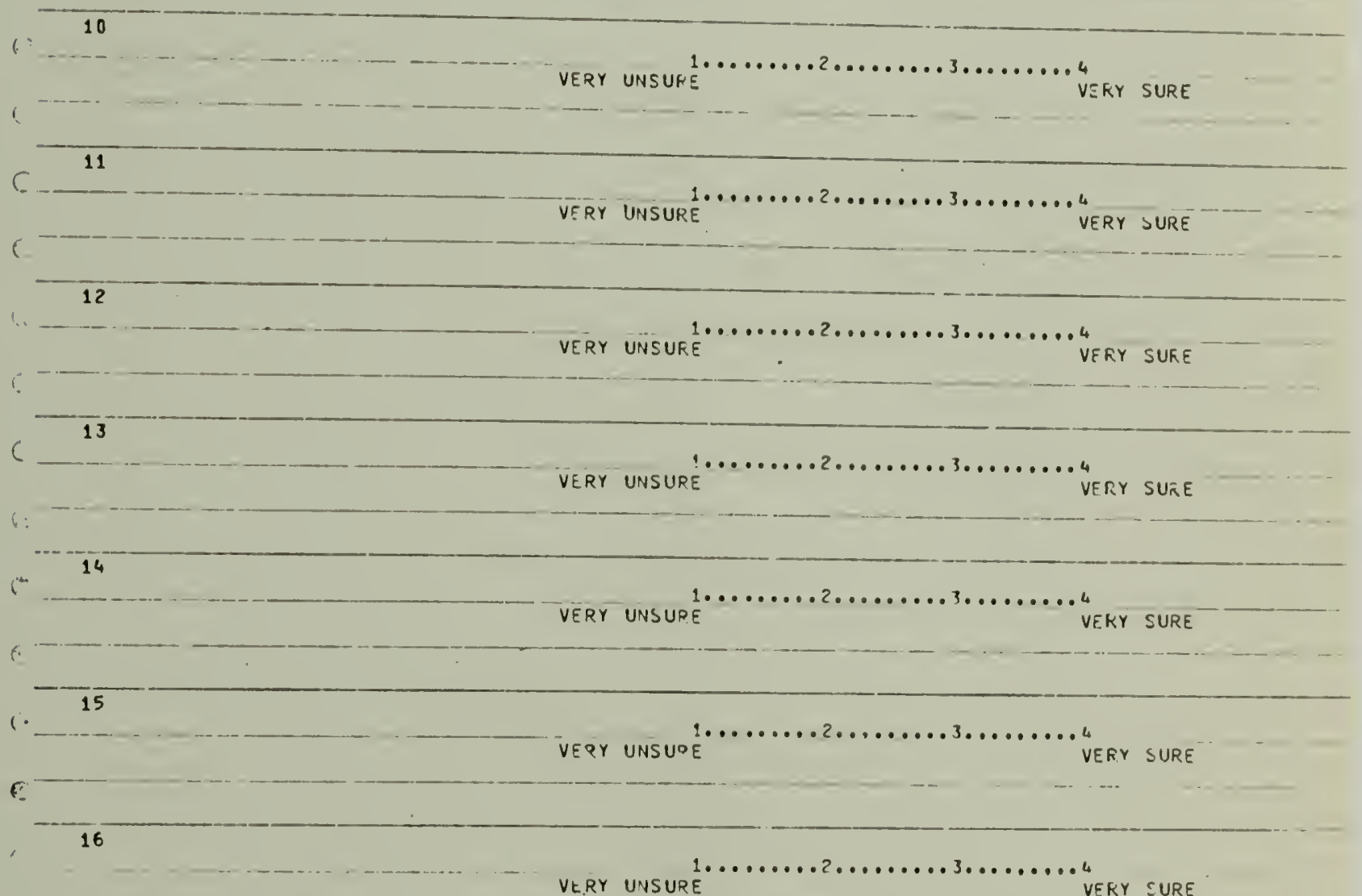
VERY UNSURE VERY SURE

17

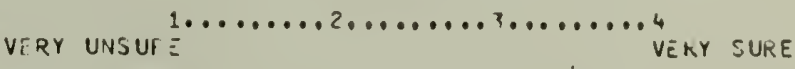


$-339-$

19

$$
\text { VERY UNSURE } 1 \ldots \ldots \ldots 2 \ldots \ldots \ldots, \ldots \ldots, 4 \text { VERY SURE }
$$$$
\text { (.) } 20
$$

VERY UASUKE

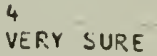

$$
\text { C. } 21
$$

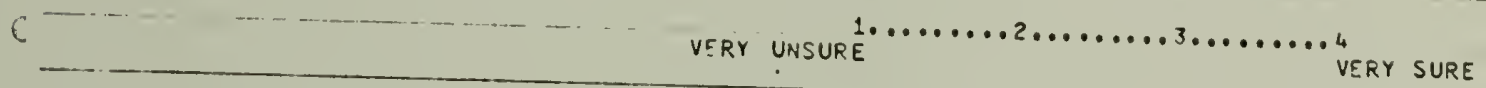

$$
\text { VERY UISURE } 1 \ldots \ldots \ldots 2 \ldots \ldots \ldots \ldots \ldots \ldots \ldots 4 \ldots \text { VEY SURE }
$$

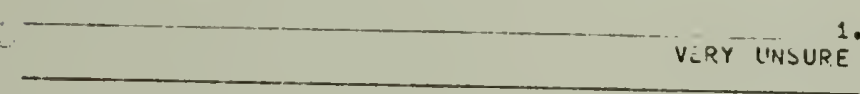

. 6

IF YOII WEPE GTVEN A PECOINITION TESI WUICH CONTAINES ALL OE

THE ASSOCTATION ITEMS, CO YOU TUIHK YOU WOULO GET HOFE COKKECT
THAN YOU MTO ON THIS FECAL. IEST.

HOW MANY OF THE ASSOCIATION HATOS THAT YOU FAILEO TO KECALL DO THINK YOU UILI AE ASIE TO FECORIIIE CCIFECTLY.

1 piease peccho the time 
PLEASE FECOFC THE TIKE

THIS IS A FERCGRITTOIA TASK. THEP. ARE THO KINCS CE ITEMS

OF EACH OF THE WOPCS PLEAS INT CATE WHETHEP THE HOTO IS OLO

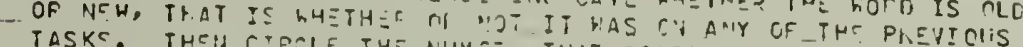

THAT ONST ACCHIATE THE NUWSE TH:T CCIRESFCHOS TL THE CHCTC

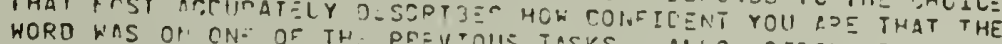

II. E. ASSCRIAT OOA TF Y PFEV YOLIS TASKS. ALSO, CIRCLE IHE TASK

MAKE A COPFIE

STATEMENTS PL ISE TAITICAT FL THAT JURGNEITT. FOF ELCH UF THE

STATEMENTS PLEISE THIITATE HAETHEO YCU THINK II IS TRUZ OR

1.

FALSE, ANC YOU', CONFTDENCE IN THAT JUCCYENT.

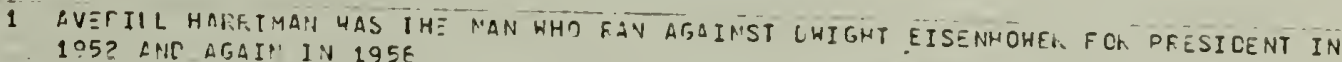

TRUE $\ldots \ldots \cdots+1$ FALSE

2 CHILO

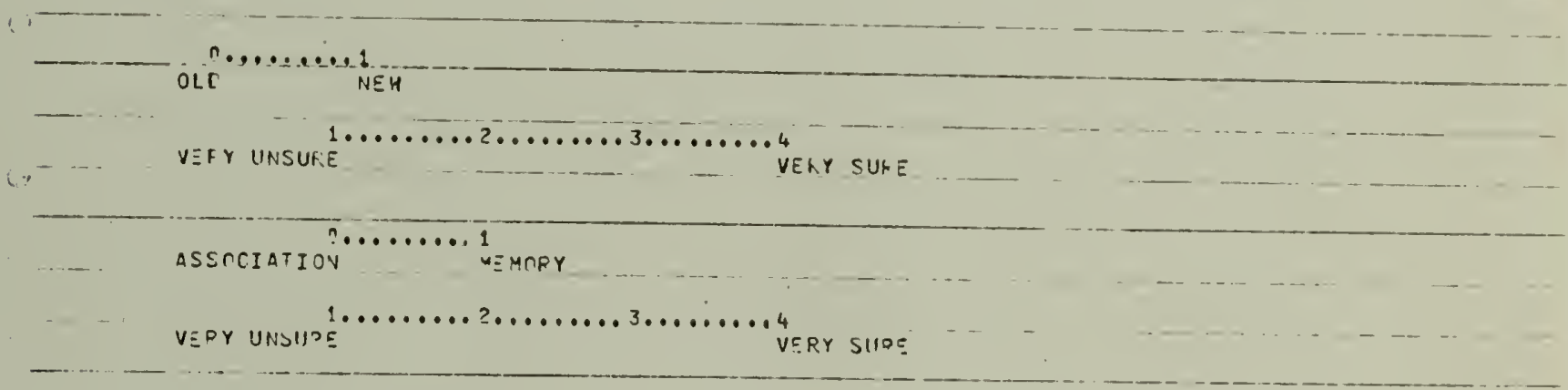

3 JOHN RELHH: HAS THE FIISST TAN TO SET FCOT ON THE MOON

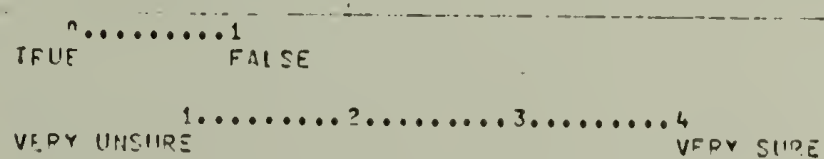



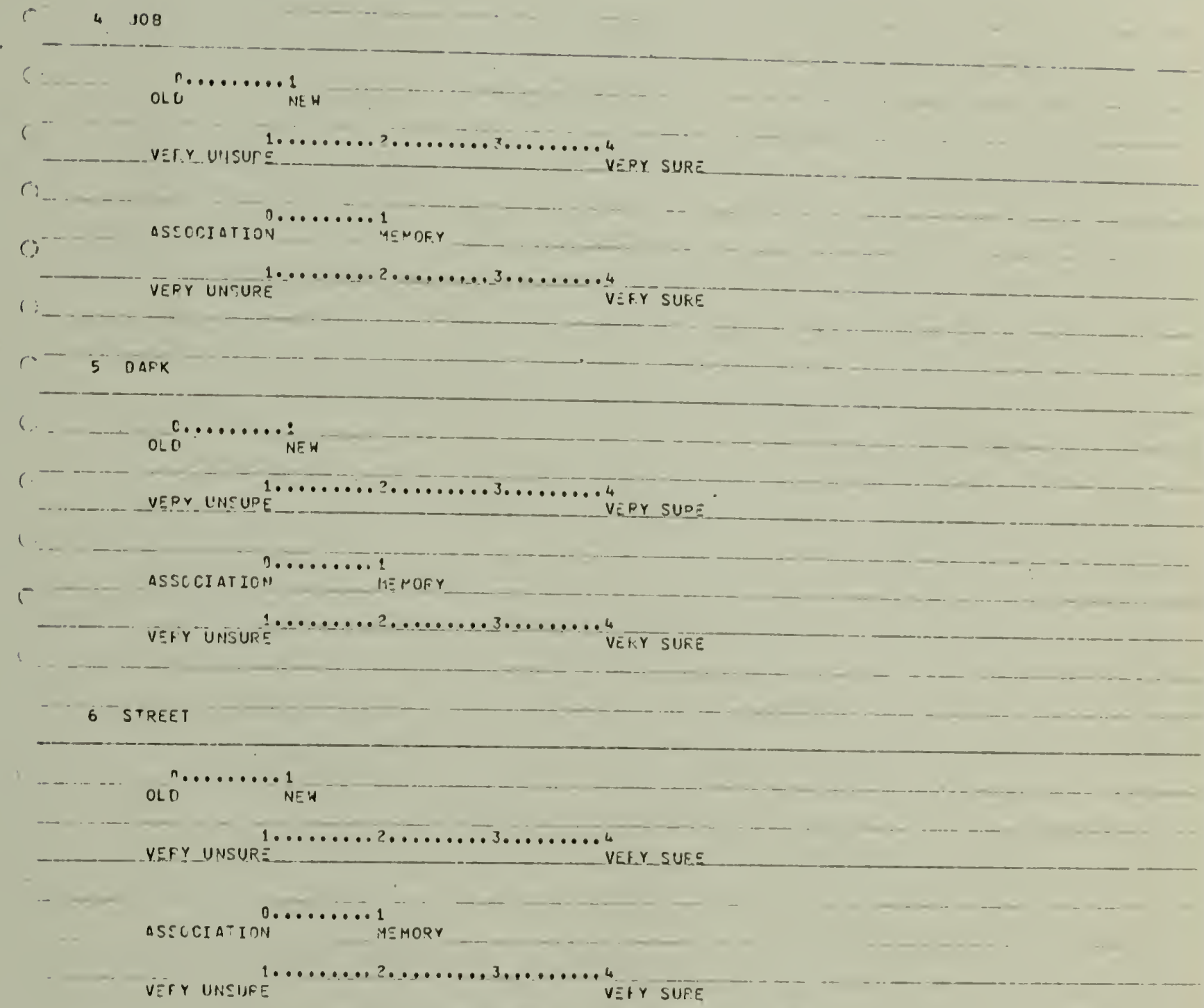

7 C. OUr

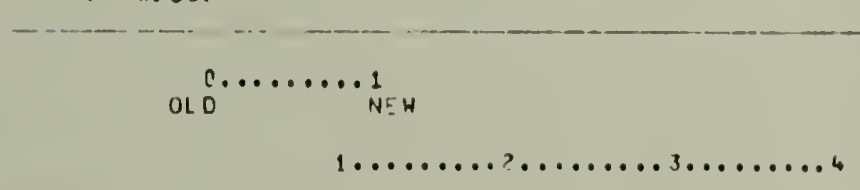


VEFY UISTIIT.

VERY SUFE

ASSOCIAIICN $\ldots \ldots \ldots 1$

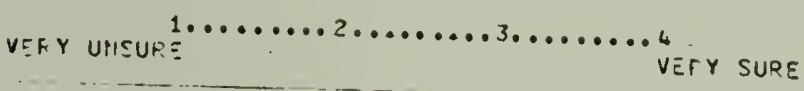

8 ITE

t.

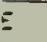

10 GEOFGF WASHTNGTON WLS THE UALY PRESICSNT OF THE UHITEO STATES TS EE ELECTEO TO
( TCUD TERMS OF OFEICE

11 HEPBERT HOOVED WAS THE HAN WHO FETAME PRESIOENT WHEN OPESIOENT MCKINLEY NAS ASSASEINATEO IN 1703 .

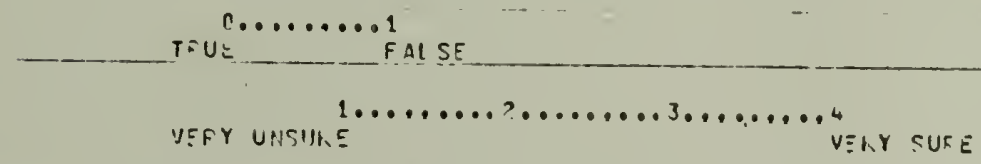


12 POBEDT E. FEADY HAS THE TAN HHJ OISCOVEDLO THE NORTM POLE

_.......................... THE NORTH POLE

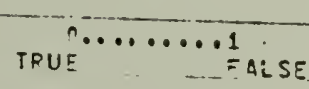

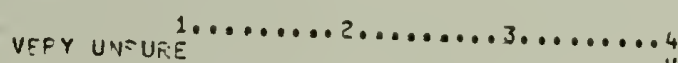

VEIIY SURE

13 EPHIN ROMMEL HAS THE WCPLO HAD II GERMAN CENERAL NICKNAMED THE LESEKT FOX

VEKY UHSUFE $1 \ldots \ldots \ldots \ldots ? \ldots \ldots \ldots 3 \ldots \ldots \ldots$ VALSE

15 WORTH

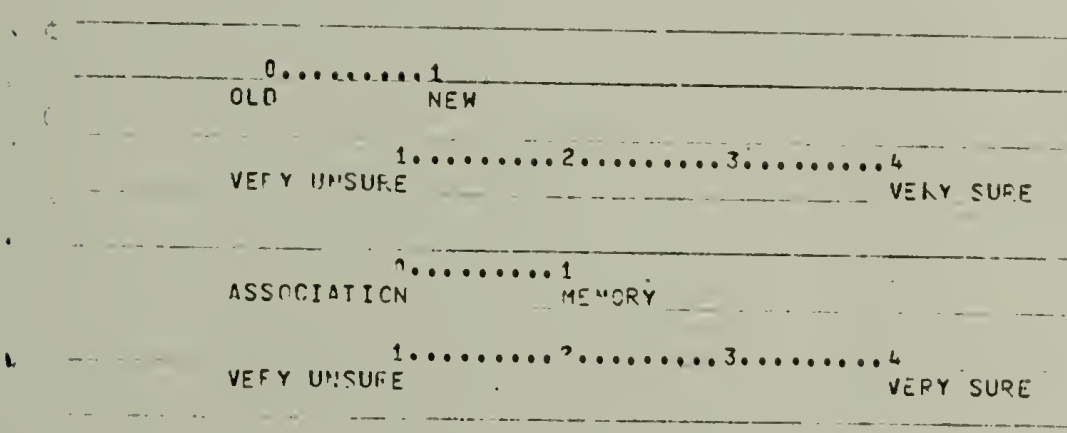

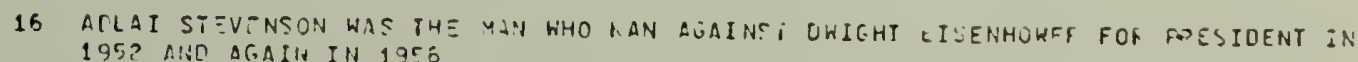


$-344-$

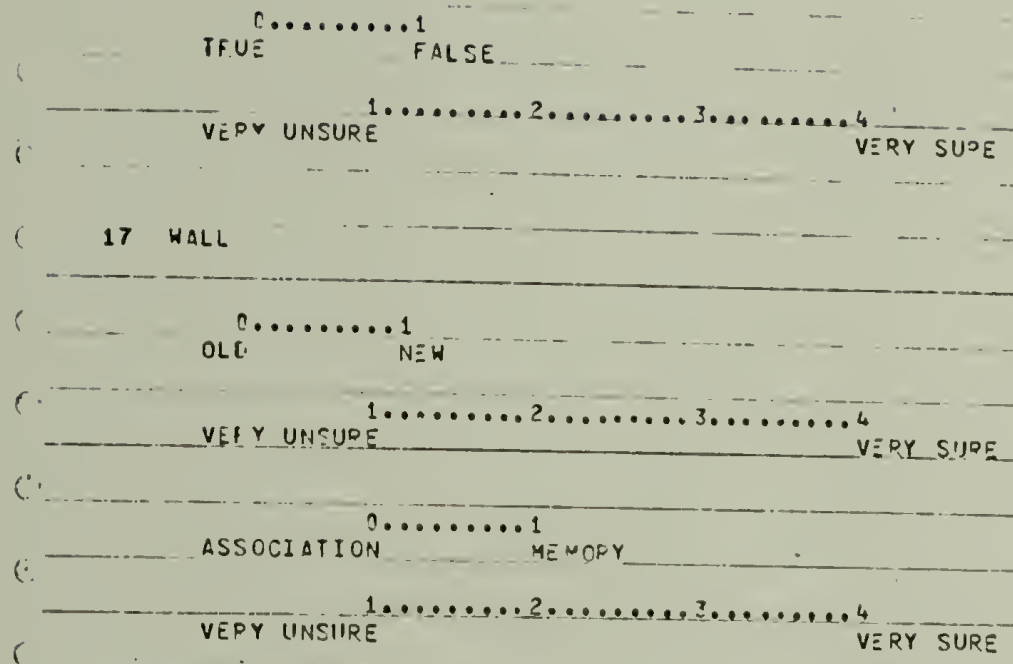

18 RIDGE

C$-{ }_{O L C} \cdots \cdots \cdots$ NEH $^{1}$ VERY UNSURE $1 \ldots \ldots \ldots 2 \ldots \ldots \ldots 3 \ldots \ldots \cdots 4$ VERY SHOOS ASSOCIATION $3 \ldots \ldots \ldots$ MEMORY VERY UNSURE VERY SURE

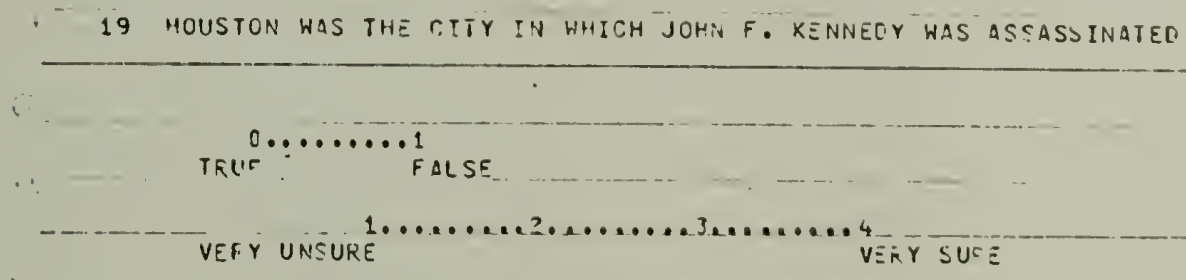

20 POLE

$$
\text { QL } \stackrel{n}{n} \cdots \cdots \cdot \frac{1}{N-W}
$$


-

VEPY UHSURE

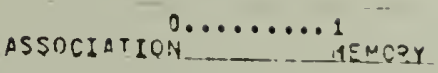

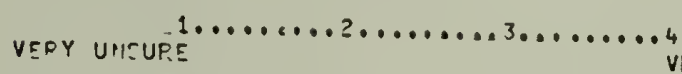

$Y \equiv$ F.Y SUR:

VEFY SUKE

21 TIME

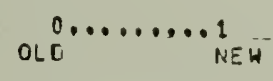

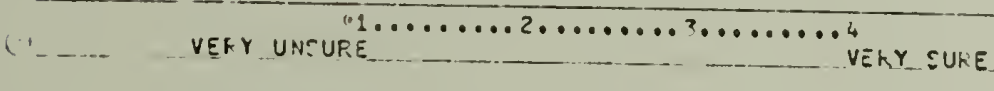

VSSCCIAIION

i.

22 BF.ANCH

$0 \ldots \ldots \ldots 1$

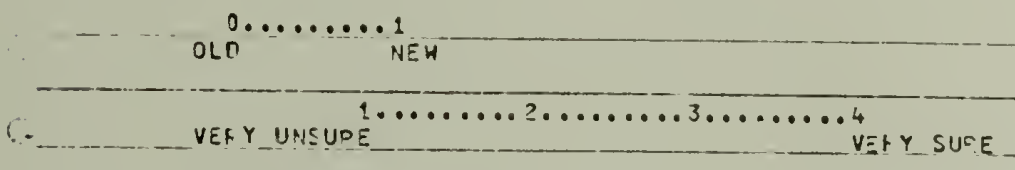

DSSOCIATION

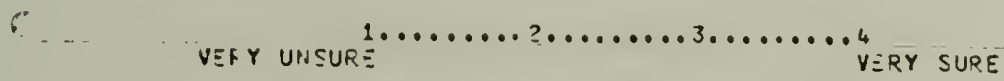

$G$

23 JACK FUPY WAS THE MAN WHO ISSASSINATCO OF. MAFTIY LUTHEK KING

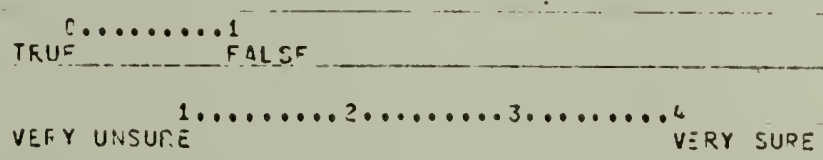

$24 \quad M A P$

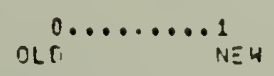




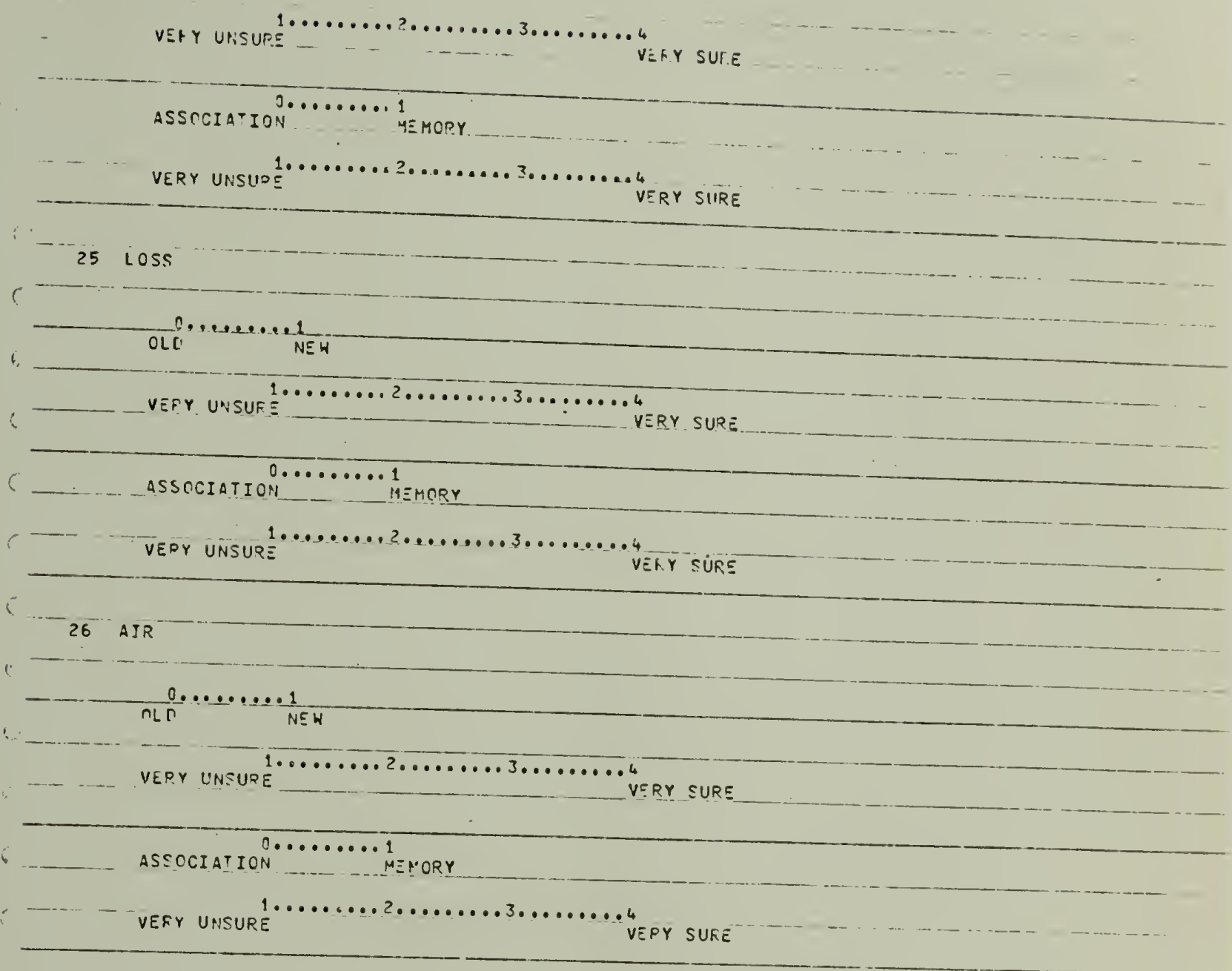

27 KNIFE
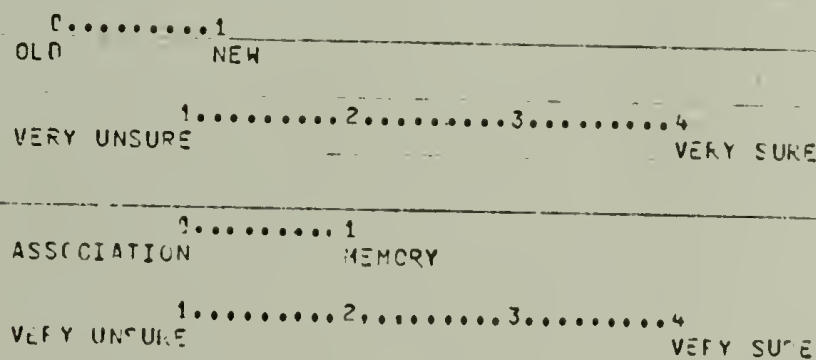
?8 HHEEL

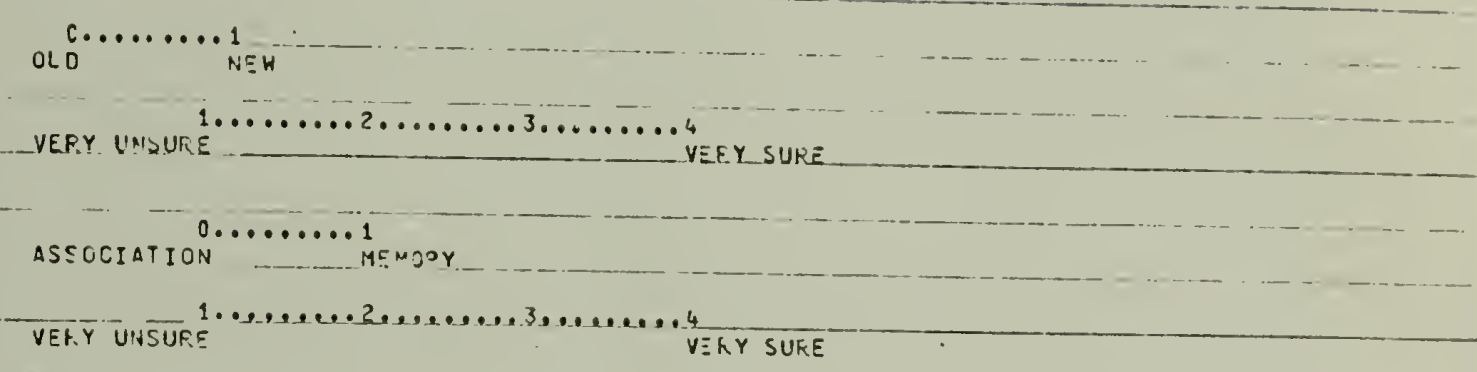

$C^{\prime}$ VEF.Y UHSURE

VEKY SURE

C 29 OALLAS WAS THE CITY IN WHECH FFESIOENT JOHN F. KEMNELY WAJ ASSASSINATSD C.............

TPUE

FALSE

C. VEFFY UNSUPE

VEAY SURE

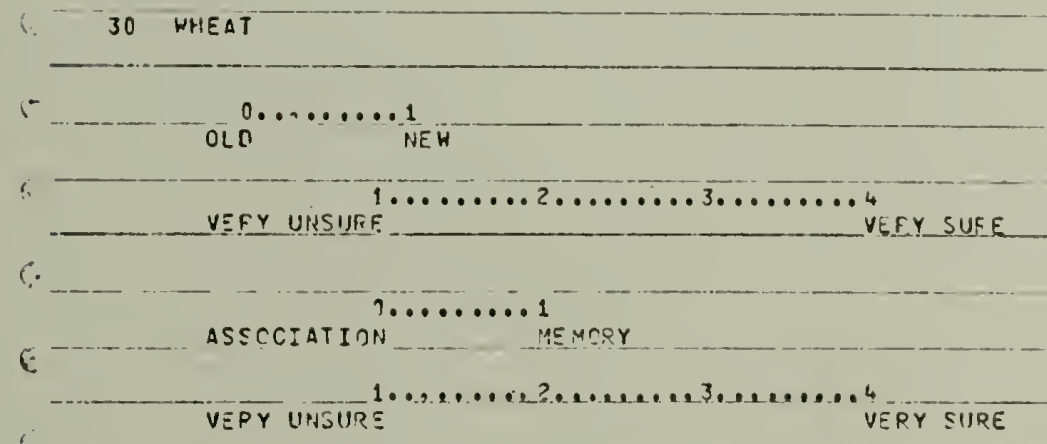

31 TRAIN

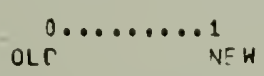

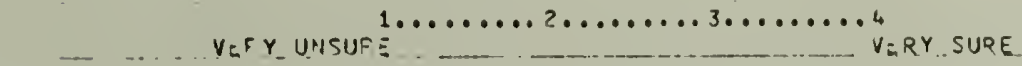

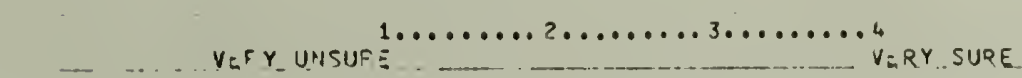

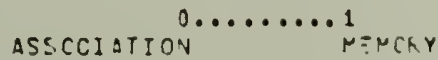




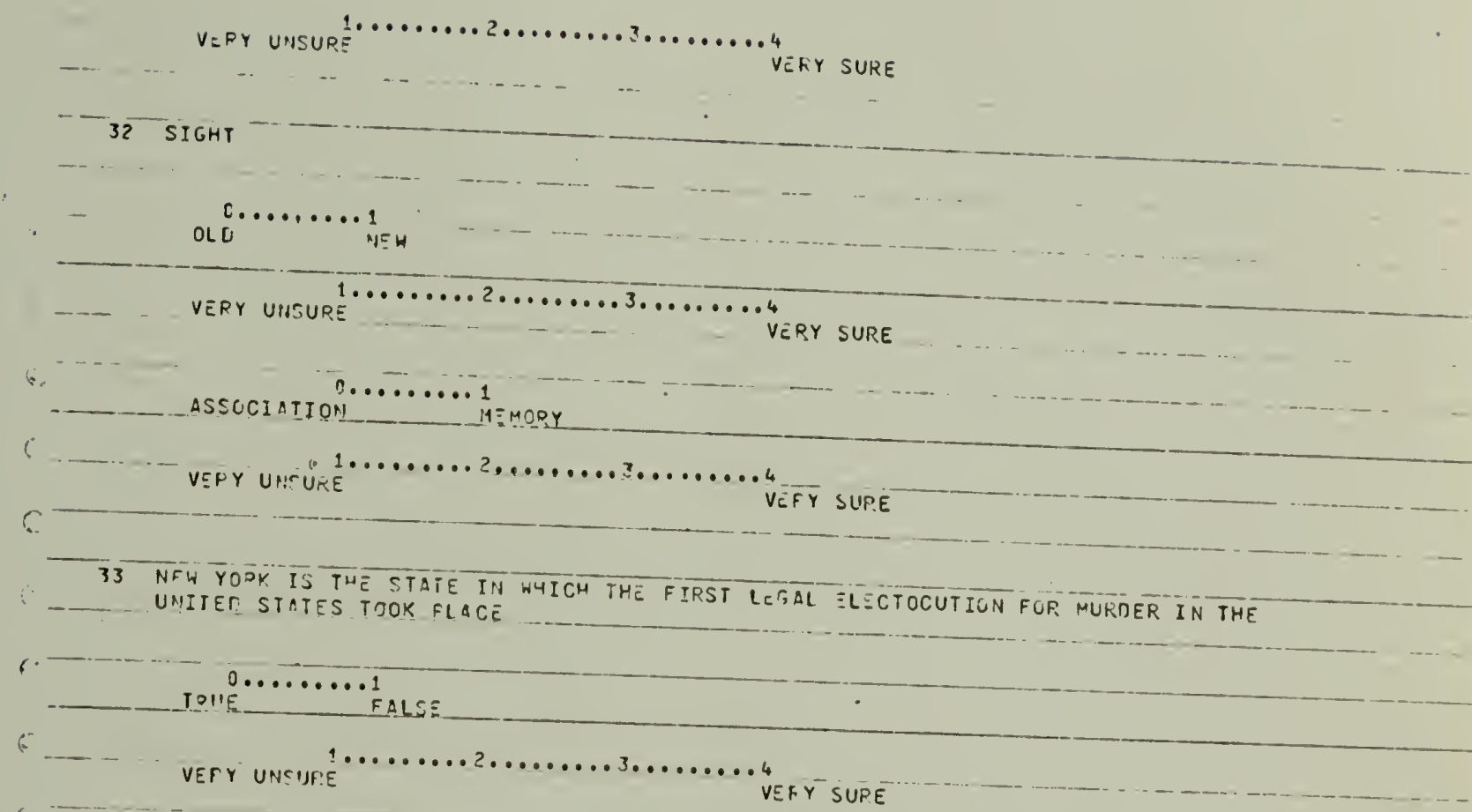


ASSTCEATION MEMOEY

VEPV URSURE 1 .........................

$V \equiv R Y$ SUDE

36 CHESAFEAKT WAS THE SHIP WHICH HIT AN ICEBCFG ANO SAT. OH, ITS HAIDLN VUYAGF IN 1912

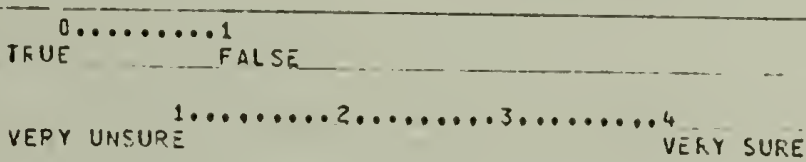

VERY SURE

37 MONTH

$$
\text { OL }-\cdots \cdots 1 \text { NEH }
$$

VERY L'NSURE $\ldots \ldots \ldots 2, \ldots \ldots \ldots 3, \ldots \ldots \ldots$ VERY SURE

$0 . \ldots \ldots \ldots 1$

ASSUCIAIICN MEROF

VEFY UnEUPE $1 \ldots \ldots \ldots 2 \ldots \ldots \ldots, \ldots \ldots$ VEFY SUFE

38 FQONT

$0 \ldots \ldots \ldots 1$

OLO

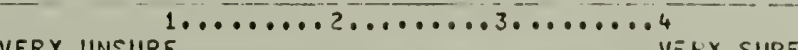

VERY UNEURE

n.........

ASSRCIATION MERCRY

VEF Y UNSUEE

$1 \ldots \ldots \ldots . \ldots . \ldots \ldots 3_{0} \ldots \ldots \ldots$

VEFY SURE

39 F.VEOETT TTOKCEN WAS THE SENATOE FOOY HISCONSIN WHOSE NALE TS ASSOCIATEO HITH CONGF ESSIONAL IUVESTICATIONS CF COMYUNISY IN THE EATLY 1950S

1.

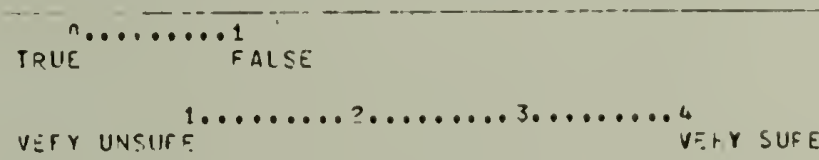


$40 \mathrm{rOOAL}$

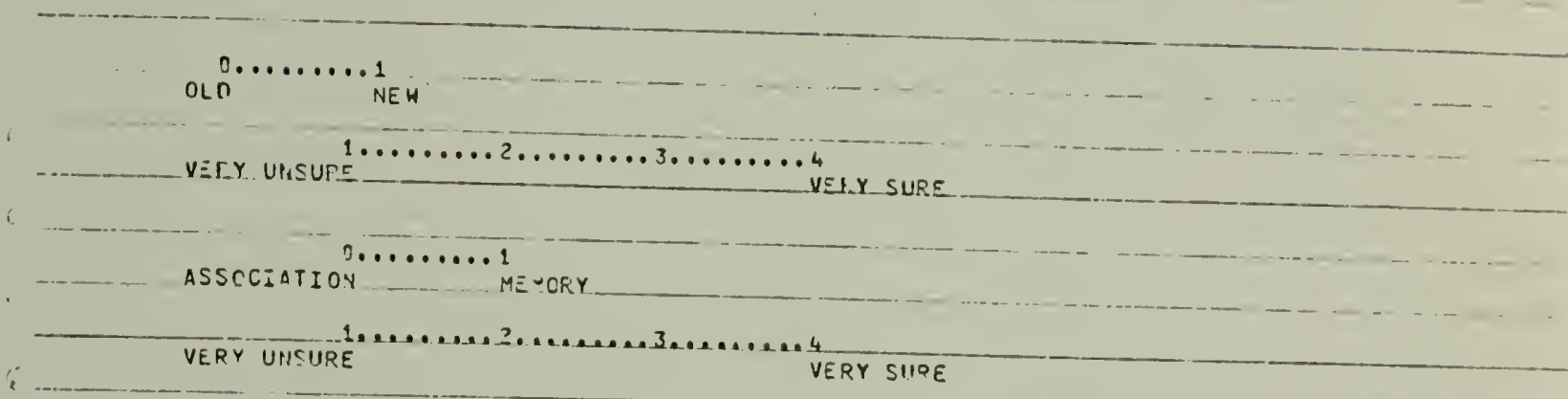

41 DECEMEEF 7,1941 WIS IHE TATE THE JAFANESE EOTBEO PEAKL HABOR

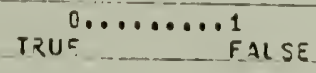

VERY INSURE

42 NEIL ARPSTRONG HAS THE FIFST MAN TO SET FOOT ON TME R CON

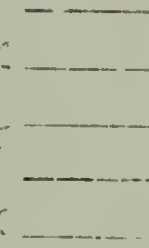

C.........

TRUF FALSE

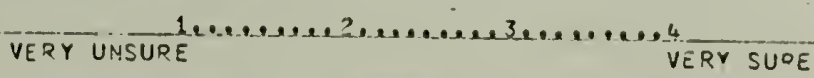
3 NEH JERSFY IS WHEFE THE PEKMAN JIRISIFLE, THE VOH HUNOENBEKE, EURNEO ANO
CFASHED

TEUF $0 \ldots$ PALSE

VEPY UNSIIRE $1 \ldots \ldots \ldots 2, \ldots \ldots \ldots 3 \ldots \ldots \ldots$ VERY SURE

44 MATTEF

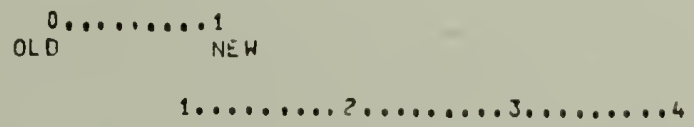


VER.Y UNSYYRE

VEKY SUY

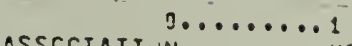

ASSCCIAIIUN._..... 1 EMORY

VEKY UNSURE

VEFY SURE

45 KITTY WAHK IS WHEOE THE WRIGHT BDOT HERS MADE THEIR FIPST SUCCCSSFIL FLIGHT

$-0 . \cdots \cdots \cdot 1$

IRUE

FALSE

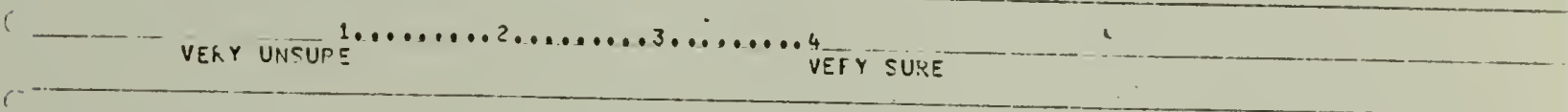

46 PORCH VERY UNSURE

I

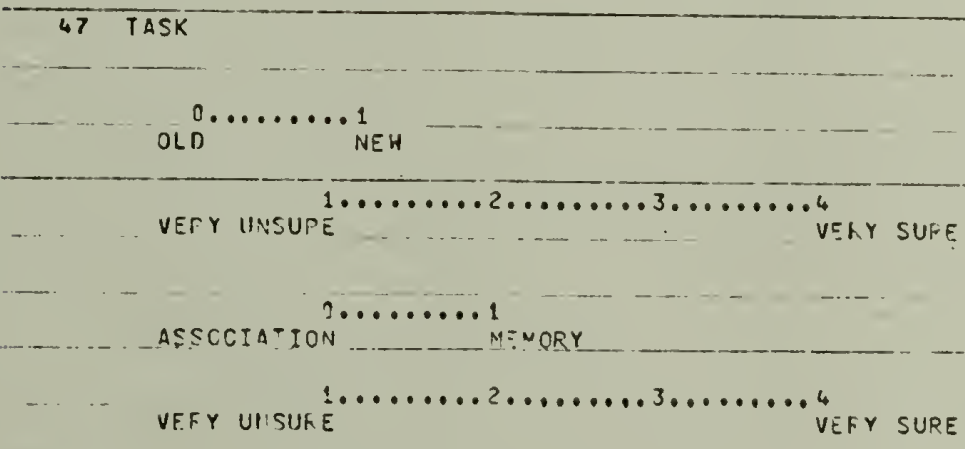

48 NEH YAYPSHIKE IS HHEFE THE GEFYAN ODALISULE, THE VUN HINÜ NAEHG, EURNEU AND CFASHFO

\section{$\ldots \ldots \ldots$....}




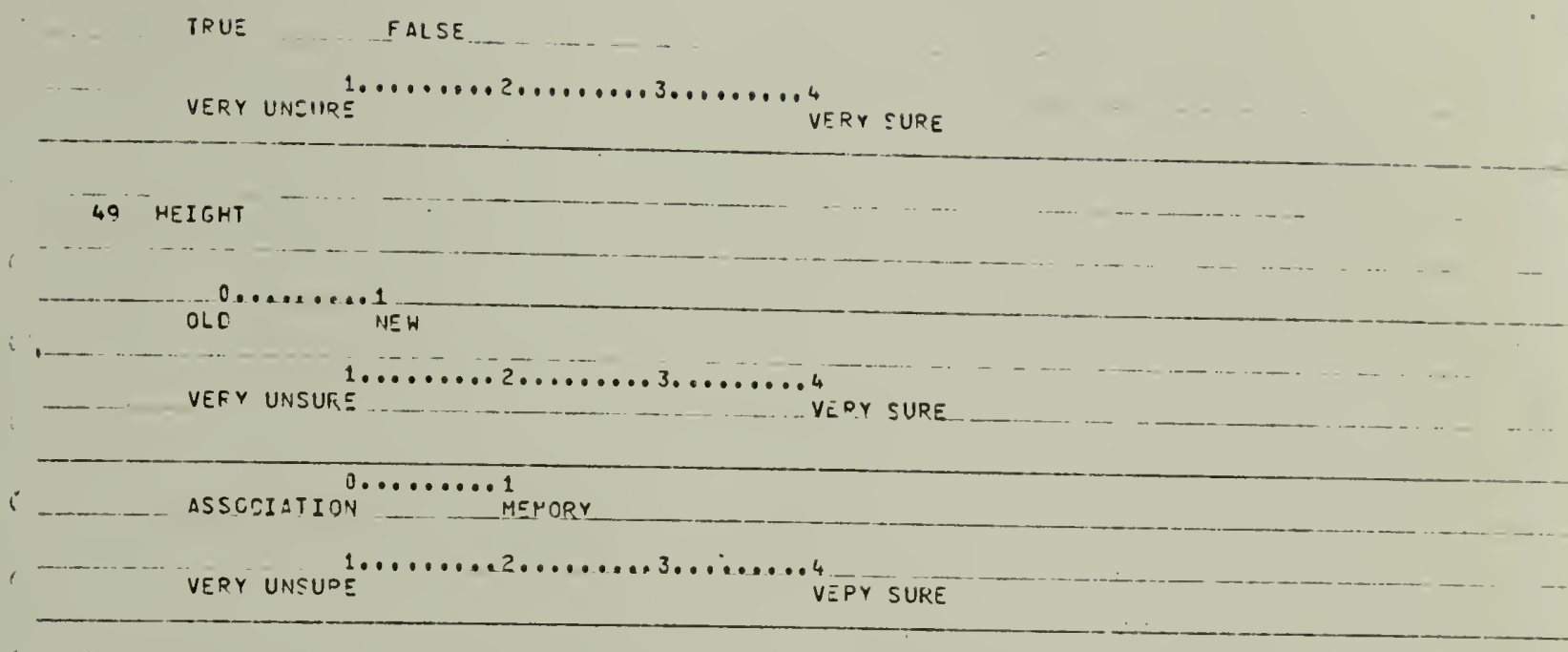

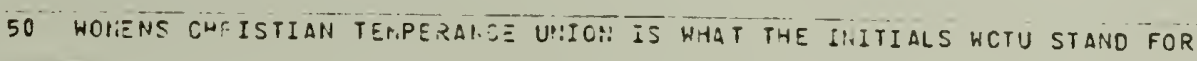

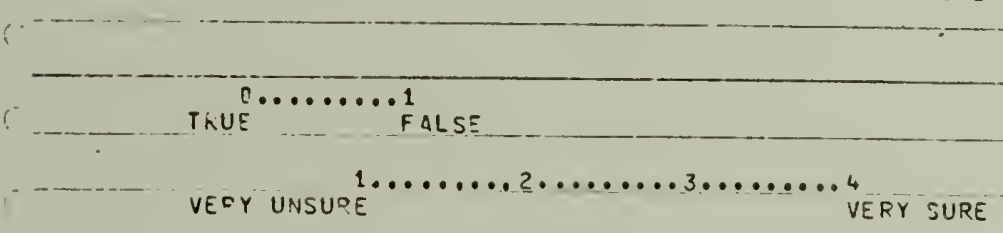

51 AFCHOUJKE FEKJ:NATHO WAS THE MAN HHOSE OEATH SET OFF HORLO HAR I

-

VERE UNSURE

52 FFIFNO

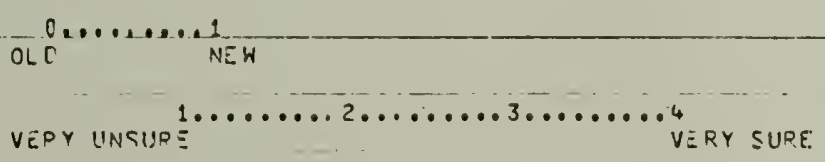

1

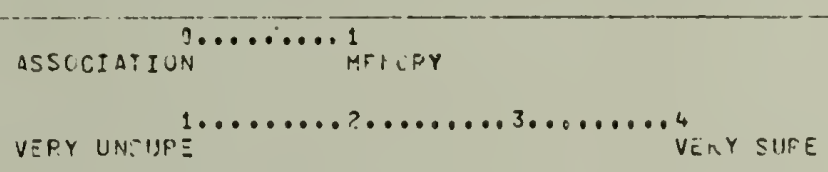


53 NETK

OL $0 \cdots \cdots \cdots$ NEH

VEF_Y_UPISUF.E

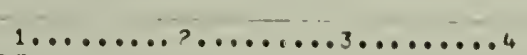

VEF.Y SUEE

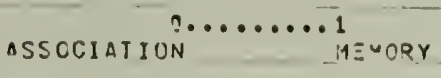

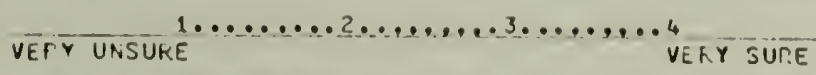

$54^{-} \mathrm{HILL}$

OL ${ }_{n} \cdots \cdots+1$

1........................

VEFY UMTISRE.

VEF Y SUPE

ASSOCIATION $2 \cdots \cdots \cdots$ M

VEF Y UINSUP:

VGFY SURE

55 PLYTH SFIFIT HAS THE PLANE IN WHICH LINOECRGH FLEH THE ATLAHIIC

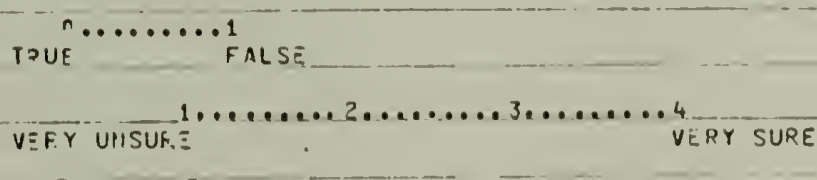

56 MILK

OLO $D \ldots \ldots+1$ NEH

1

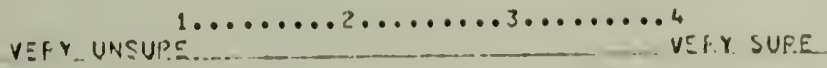

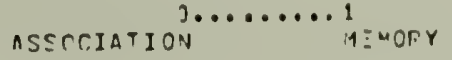




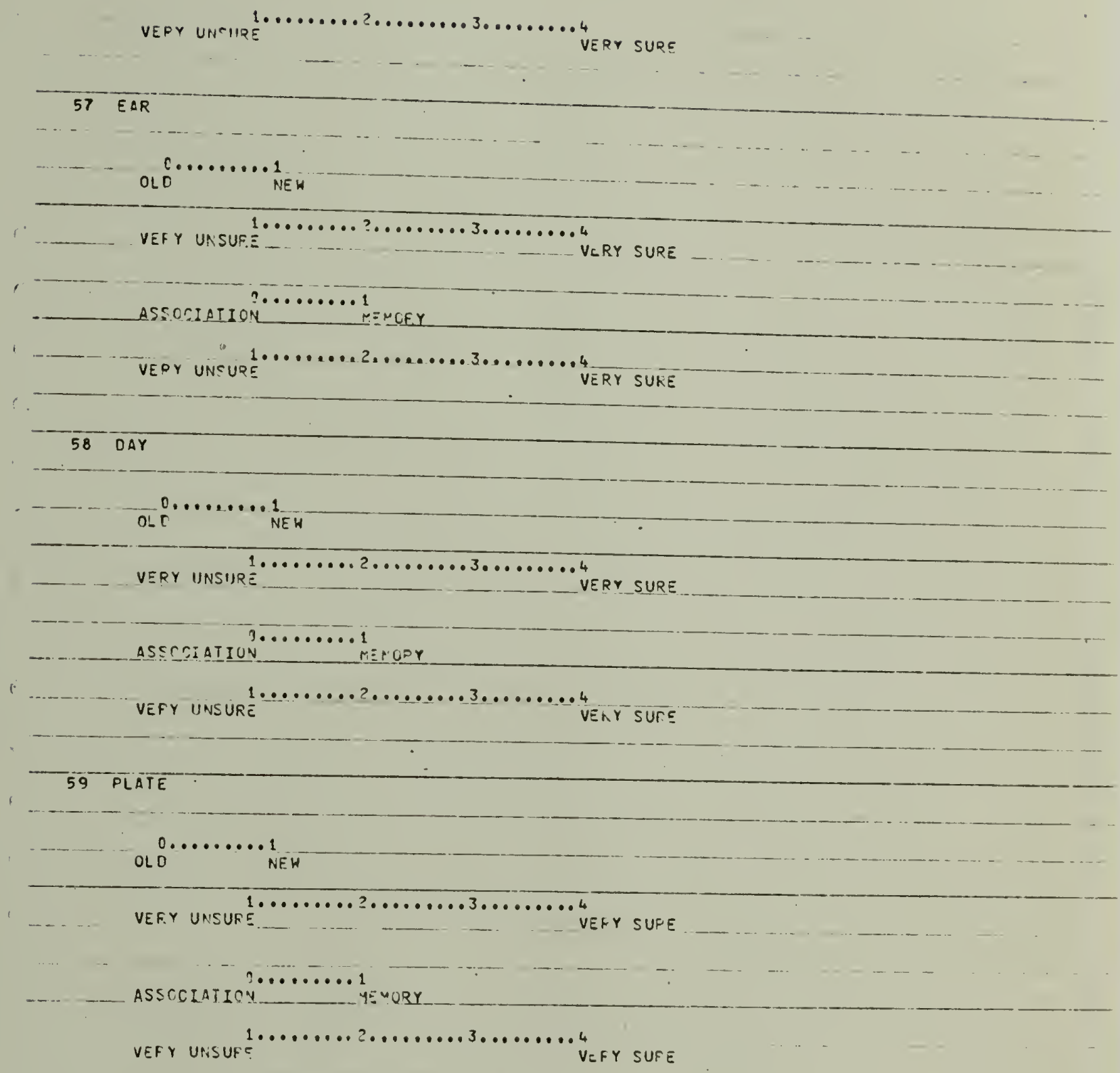

1 GC CLAITE CHTNAULT WAS THE COMMANOER DF TRE FAMOLS FLYING TIGERS OF WORLD HAS II

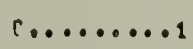




$$
-355-
$$

TF.UE FALSE

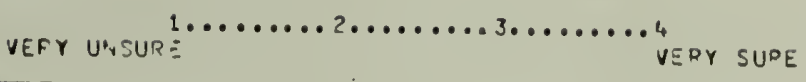

61 FACE

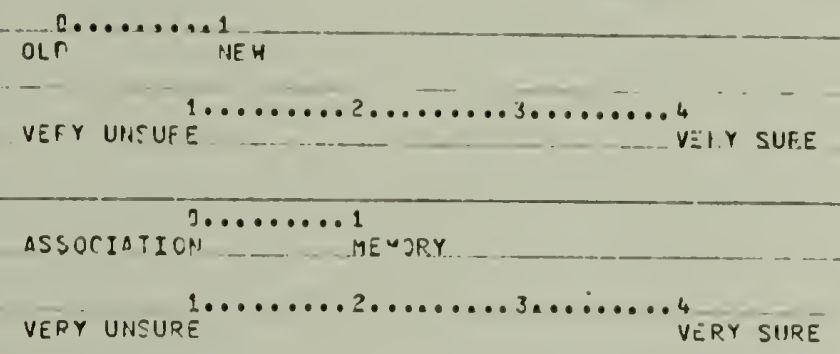

62 CIKTIS LEHAY WAS THE COHMENOSF OF THE FAHCUS FLYIHG TIGEFS OF HOFLO WAR II

TRUE

VERY LINSURL

VERY SURE

63 CAR

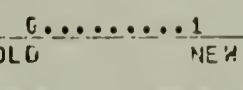

VEFY UISUPE $V \equiv R Y$ SURE

ASSCRIATION - veliftir

VEPY UNSURE

VEPY SURE

64 SHAPE

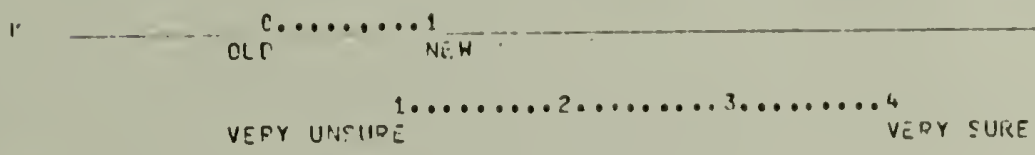




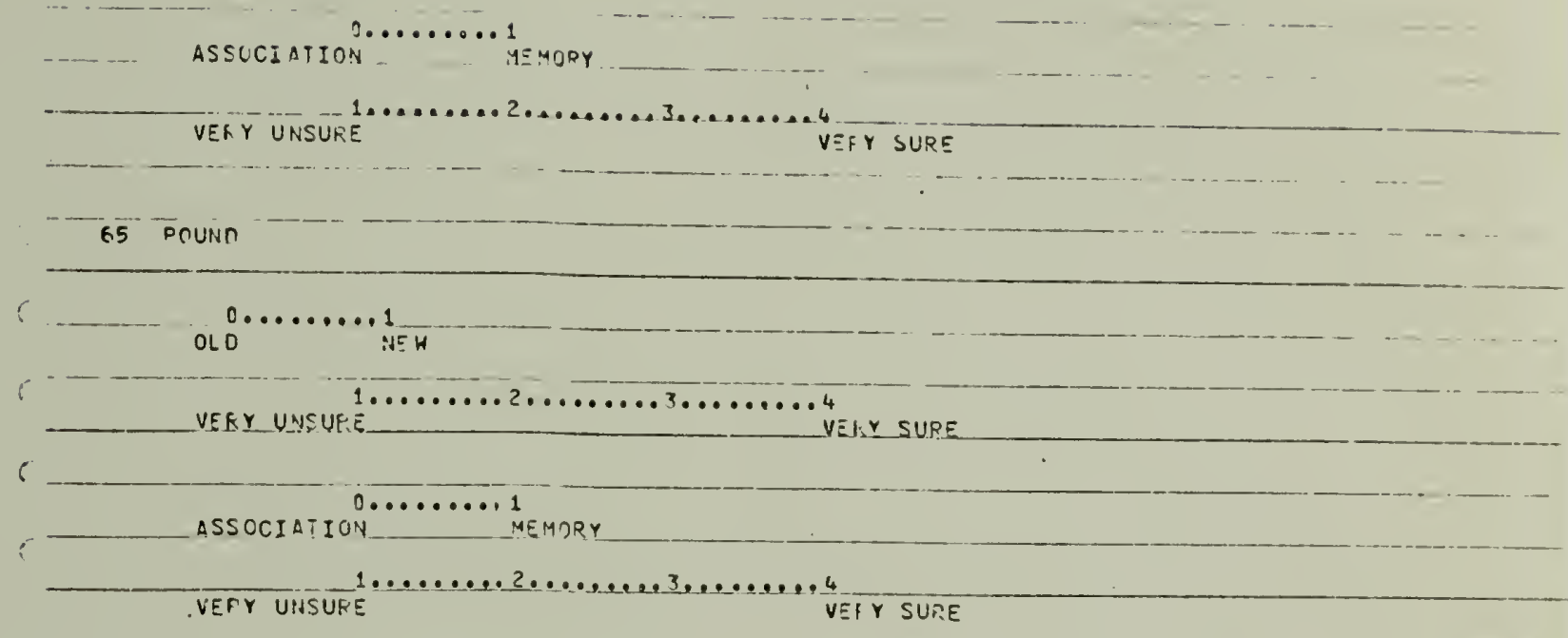

66 JACK SCURVY WAS THE MAN TH.IED IN THE FAMOUS MONKEY IH IAL OF 1925

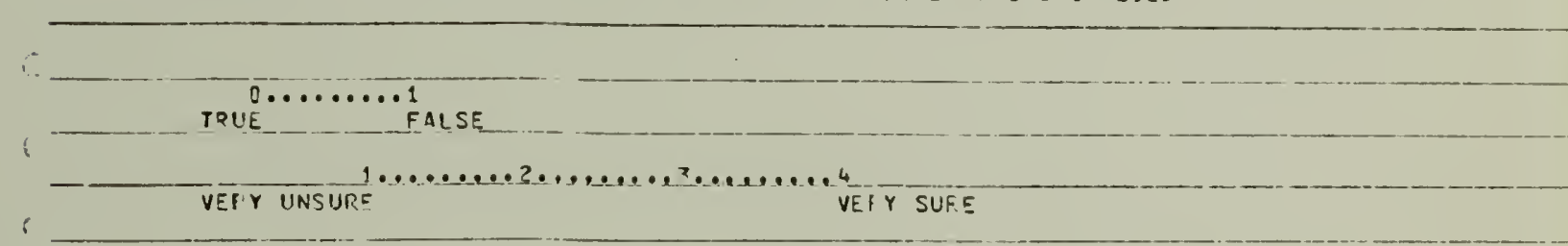

67 ALFKET LANDON WAS THE YAN WHC HAS CLECTEO VICE-FELSECENT IN 1948 WHEN HAR.PY TFUMAN HAS ELECIEO PRESINENT

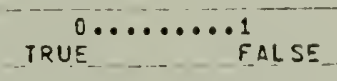

VEPY UNSUFE

68 eor

OL $[\cdots \ldots \ldots$ NEH

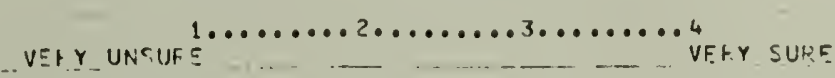

assoctation 


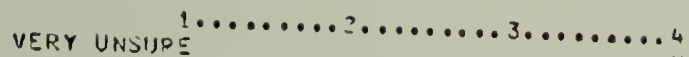

VERY SUPE

69 CALIFCRIIA TS THE STATE IN WHICH THE FIPST LEGAL ELECTKOCUTICH FOI IAUKOEF. IN THE
UHITEN STATLS TOOK PLACE TRU VERY UNSURS

6<smiles>CCCCCC</smiles>

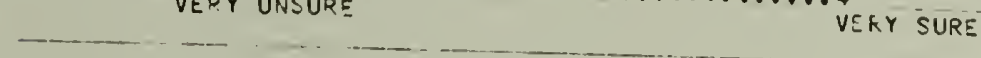

70 JAMES DEFFEEY HAS THE BOXER. HHO HAS MICKIVAMED GENTLEMAN JIM

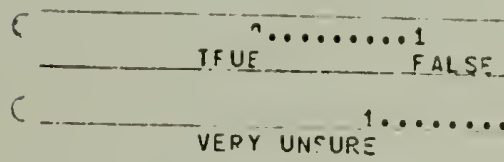

\section{$71 \mu I L$}

$0 \ldots \ldots \ldots 1$

VERY UNTURE $1, \ldots \ldots \ldots 2 \ldots \ldots \ldots 3 \ldots \ldots \ldots 4$

VERY SURE

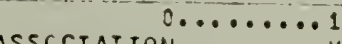

ASECCIAIION_ MEMOPY

VEF Y UNSUPE $1 \ldots \ldots \ldots \ldots \ldots \ldots \ldots \ldots$ VEKY SURE

72 HIRE

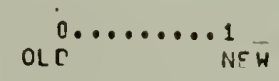

vety unSURE $1 \ldots \ldots \ldots . \ldots \ldots \ldots, \ldots \ldots \ldots 4$

VerY SUPE

ASECCIAIION

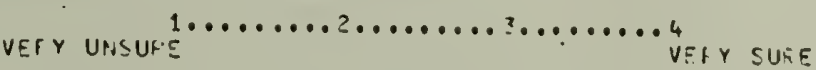


73 OECK

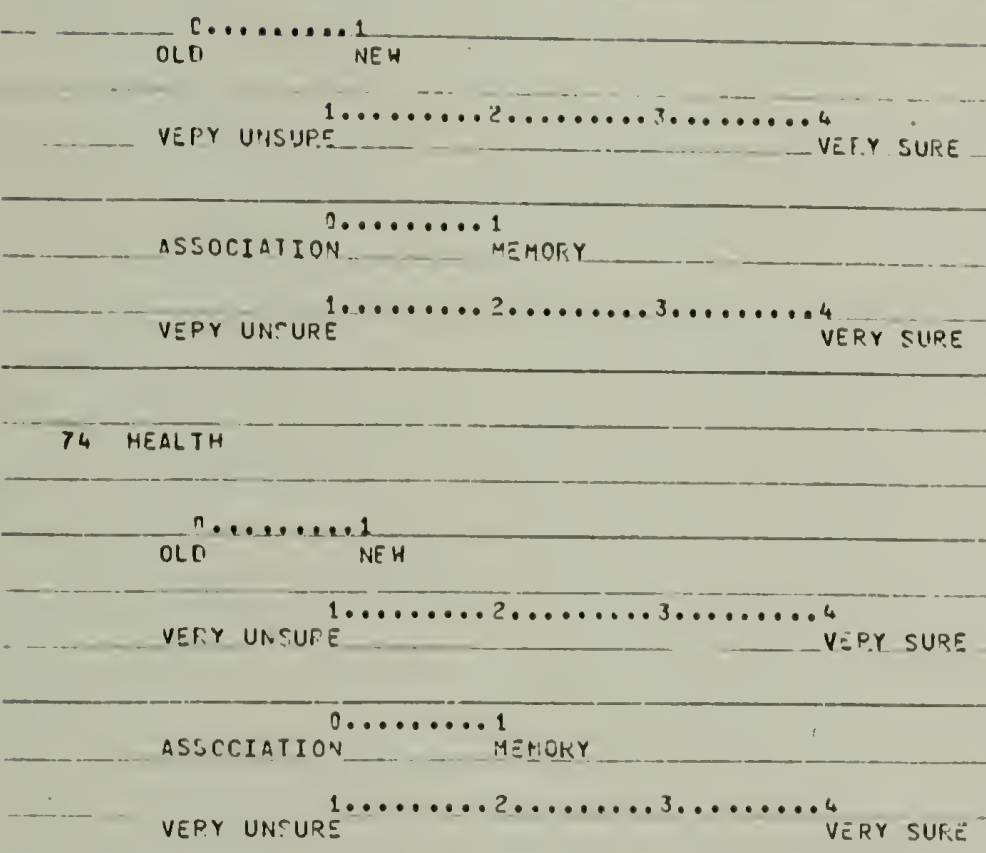

75 SPIRIT OF ST. LOUIS WAS THE PLANE IN WHICH LIM.ORERGH FLEH THE ATLANTIC

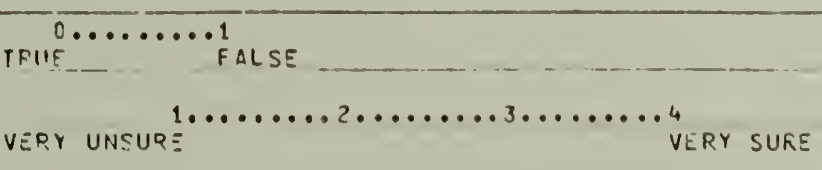

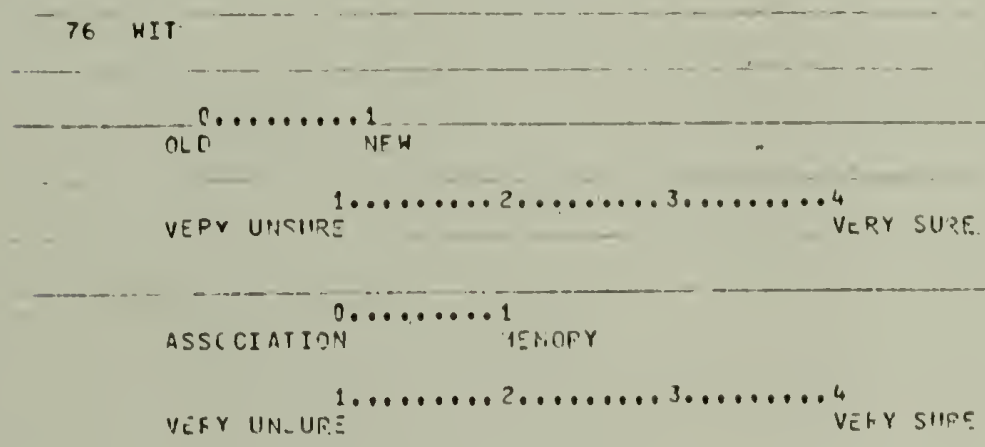


77 SPEECH

DSECCIATION VEFY UNSURE

78 JOSEPH MCRARTHY HAS ITE SEMATOP FKOY WISCONSIM WHOS NAME IS ASSOCTATEO WITH CONGF FSIIONAL INVESTIGAIICNS LF COMNUPISM IN THE EAFIY $1950 S$

TRUE

79 NOISE

$$
\text { OLD }
$$

- $\ldots \ldots \ldots \ldots 2 \ldots \ldots \ldots \ldots 3 \ldots \ldots \ldots \ldots 4$ $Y E T Y$ Y IINSUOE

80 EGG

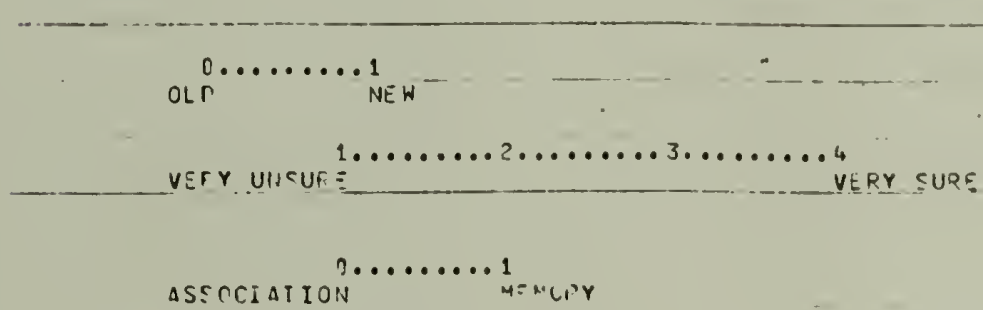


$-360-$

VI FY SURE

81 PATH

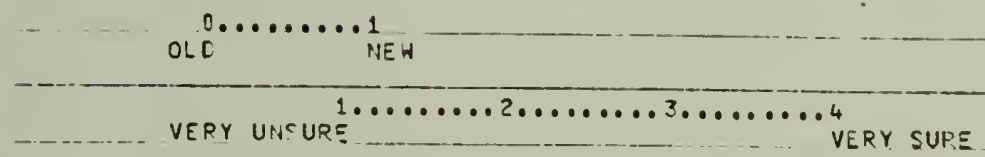

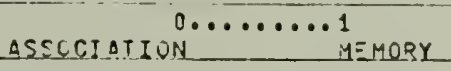

$C_{0}$

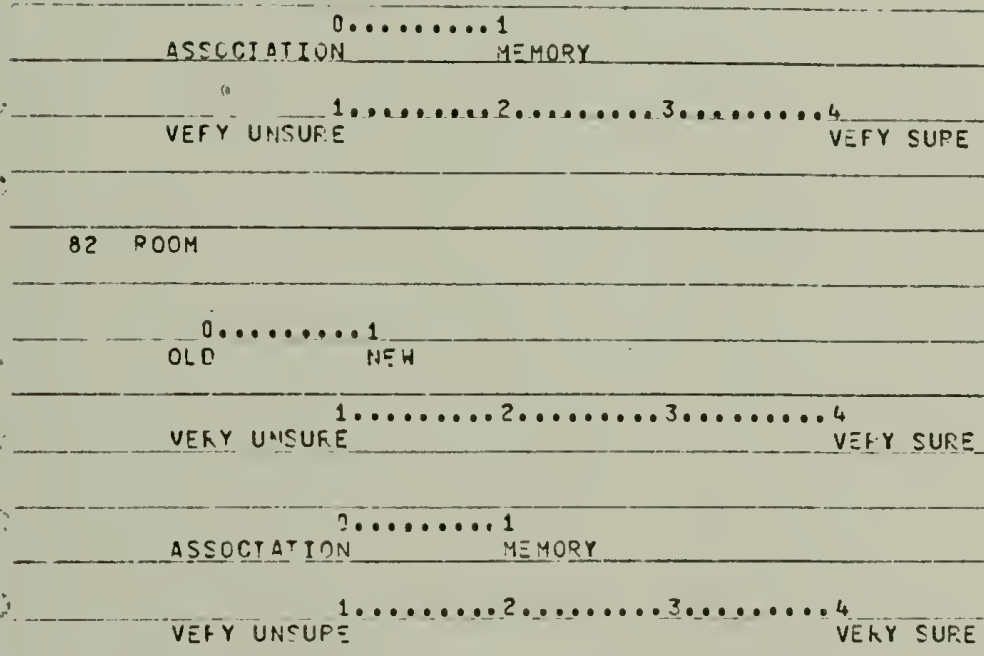

$\Rightarrow 831908$ WAS THE YEAR HENRY FORD INTKOUUCEO THE MODEL T
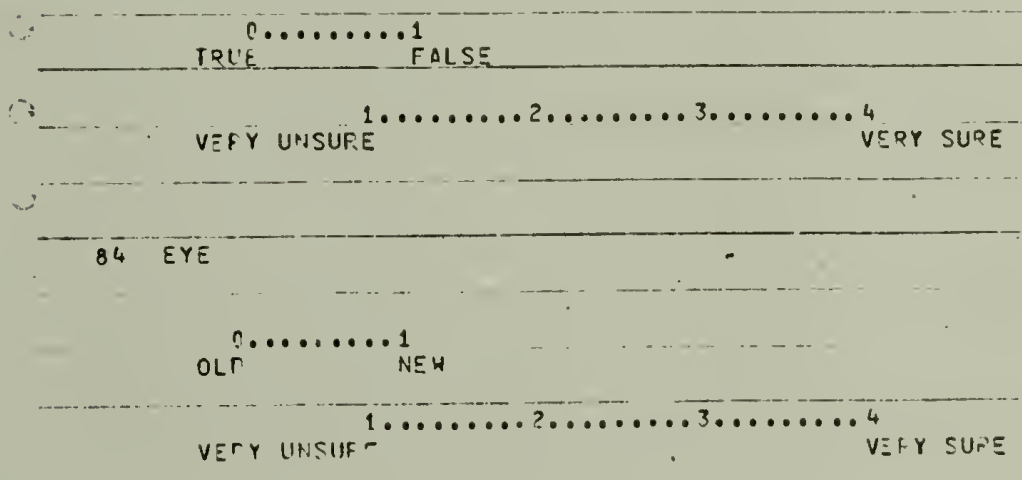
ASSCCIATION

ME MOFY

VEPY UNSURE

VER SURE

1912.

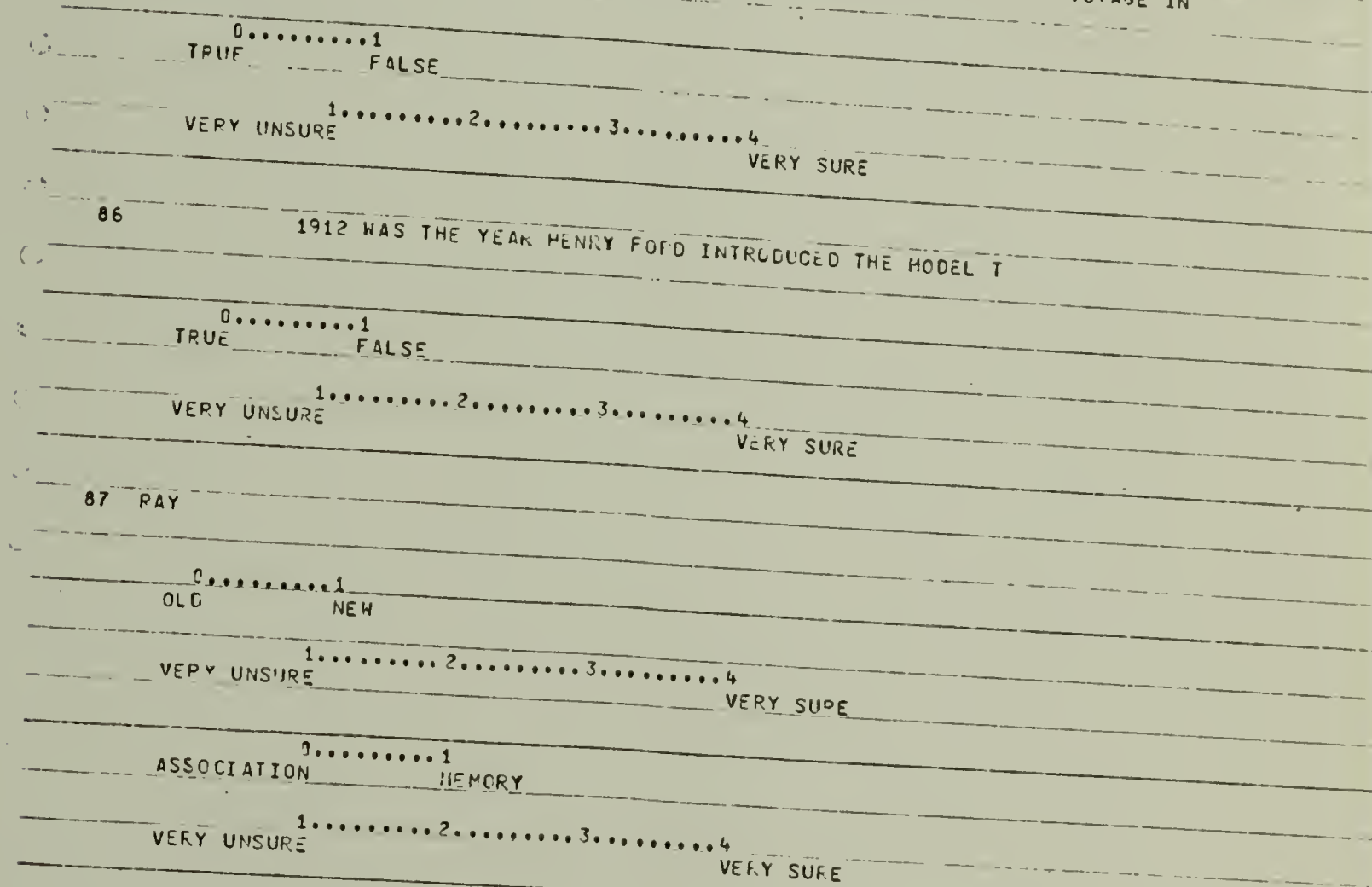

OA 1059 WAS THE YEAP THE RUSSIANS OPBITTEU THE FIRST SATELLITE

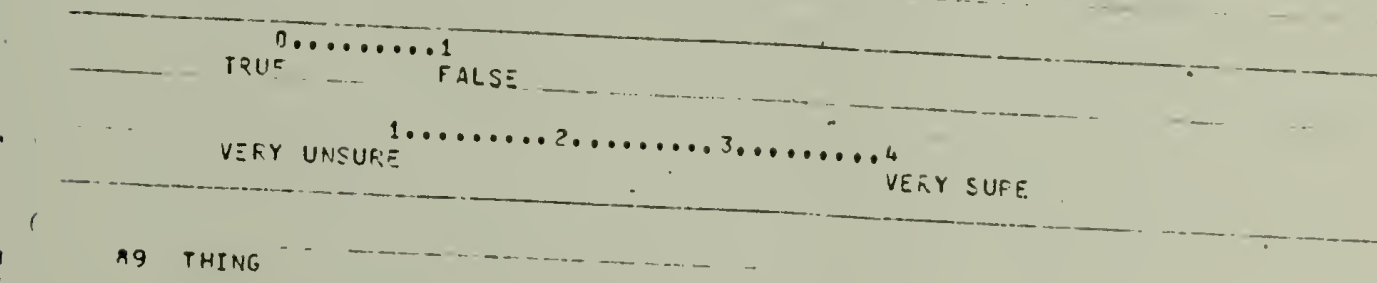




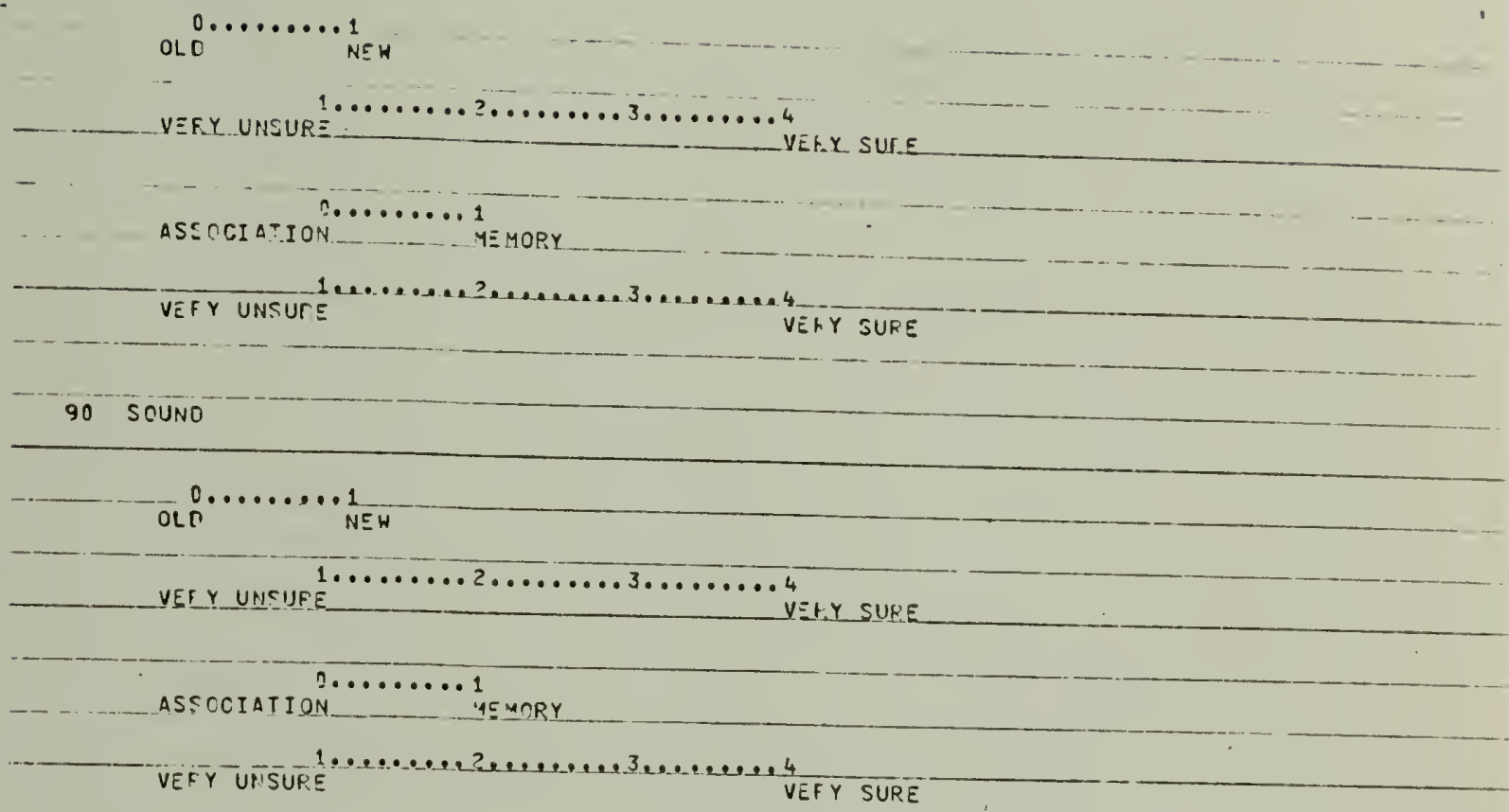

91 GUN

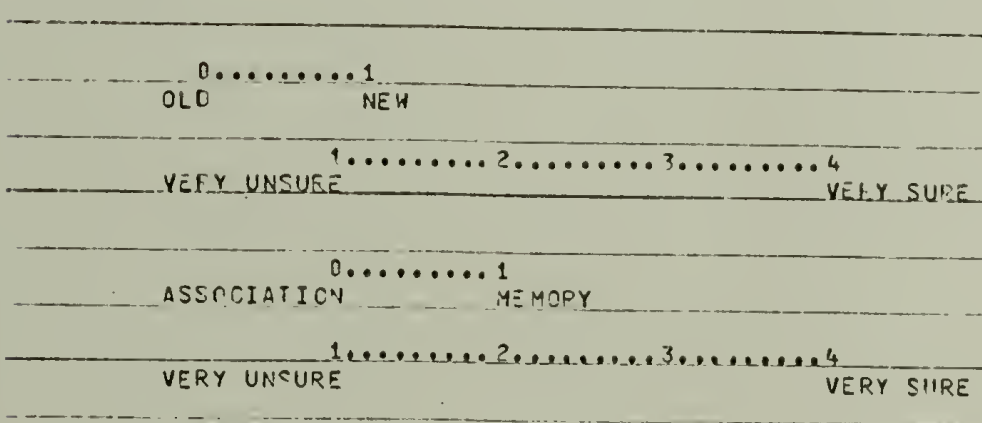

92 NIIT

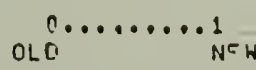

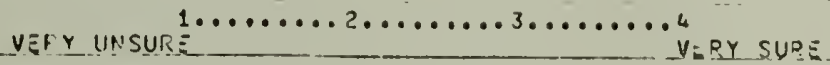

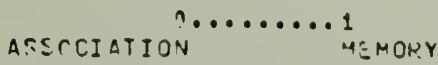


$-363-$

VERY UNSUOE

Vefr sUfE

93 HASHITGTOI. CIVILIAH TAX URIIT IS WHAT THE INITIALS WTTU STANO FOR

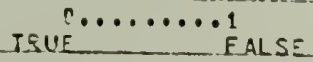

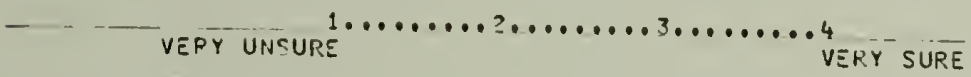

O4 BAROH HILBUR VOP! WPIGHT WAS THE WORLO HAR I GEOMAN FLYING ACE NICKNAMED THE REO

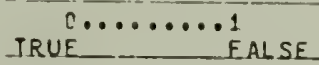

VEFY UHSURE VEKY SURE

95 PRINCE

$O L C \cdots \cdots+1 \overline{N E H}$

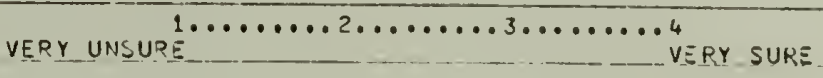

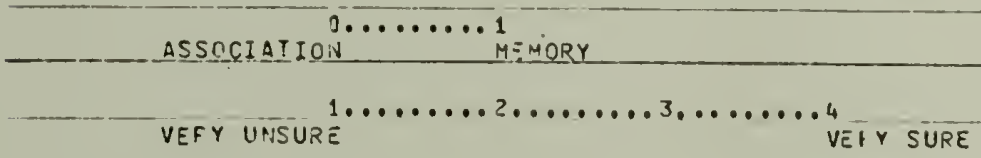

96 HIRHLANE PARK IS HHERL THE WRIGHT BROTHEPS YALE THEIR FIRST SUCEESSFIL FLIGHT

TPUE

VEFY UNSUR

vent sune

97 PLONO

OL $\ldots \ldots \ldots$ NEW $_{\text {N }}$ 


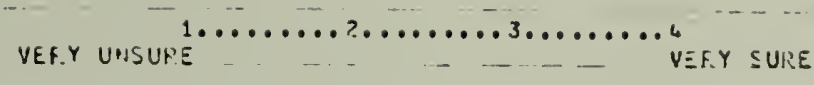
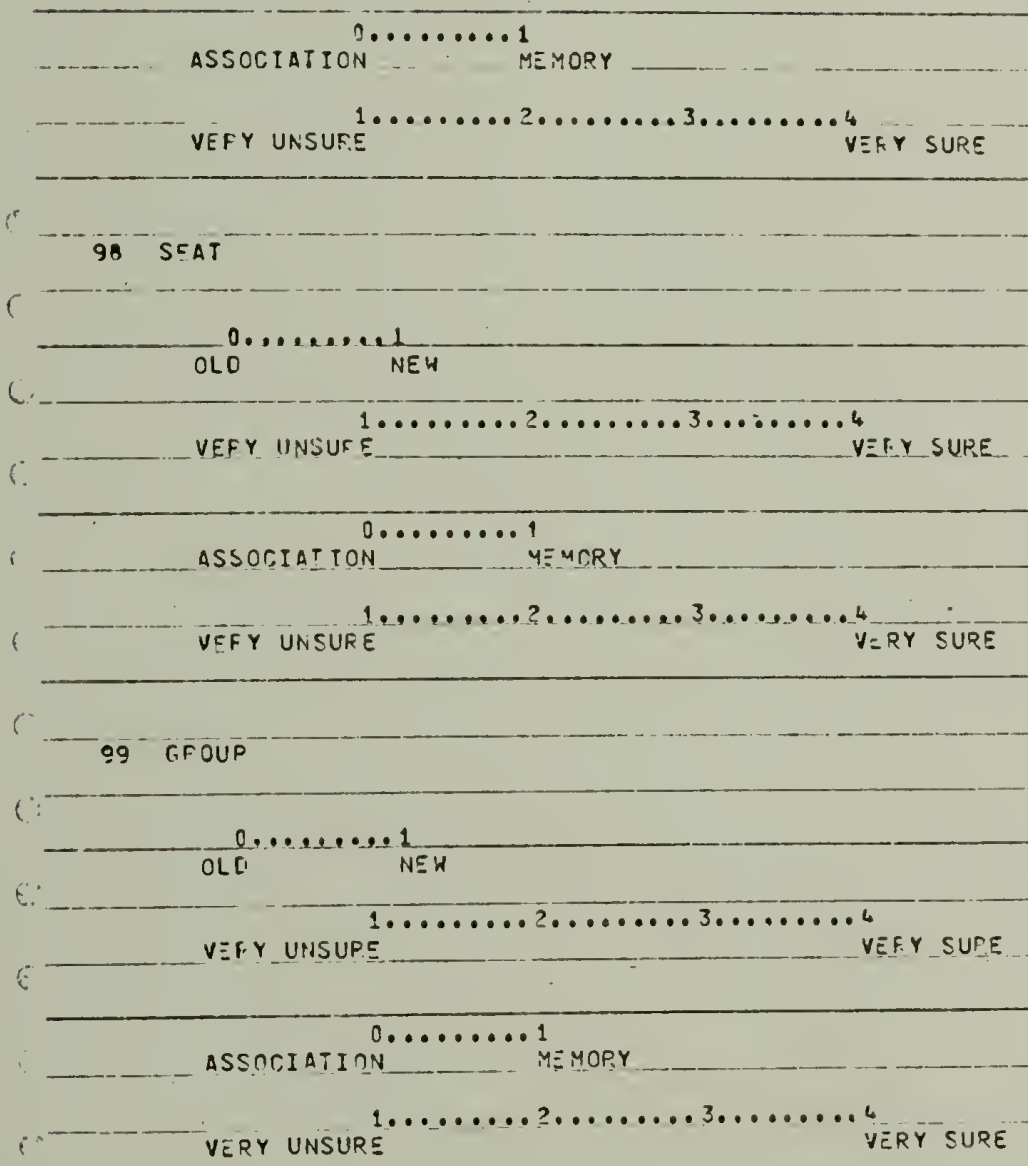

\section{TEATH}

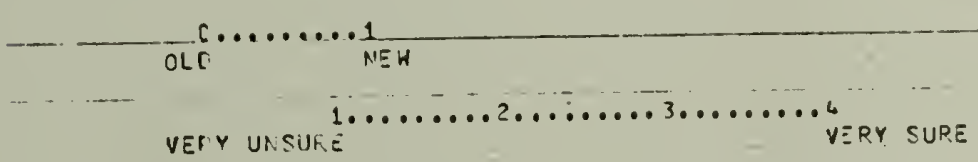

1

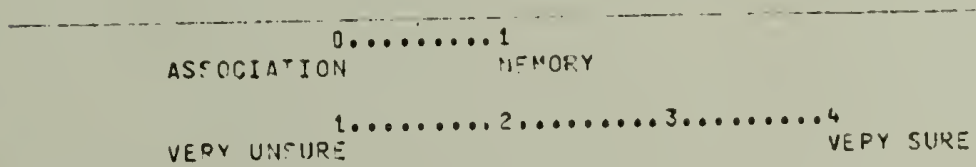



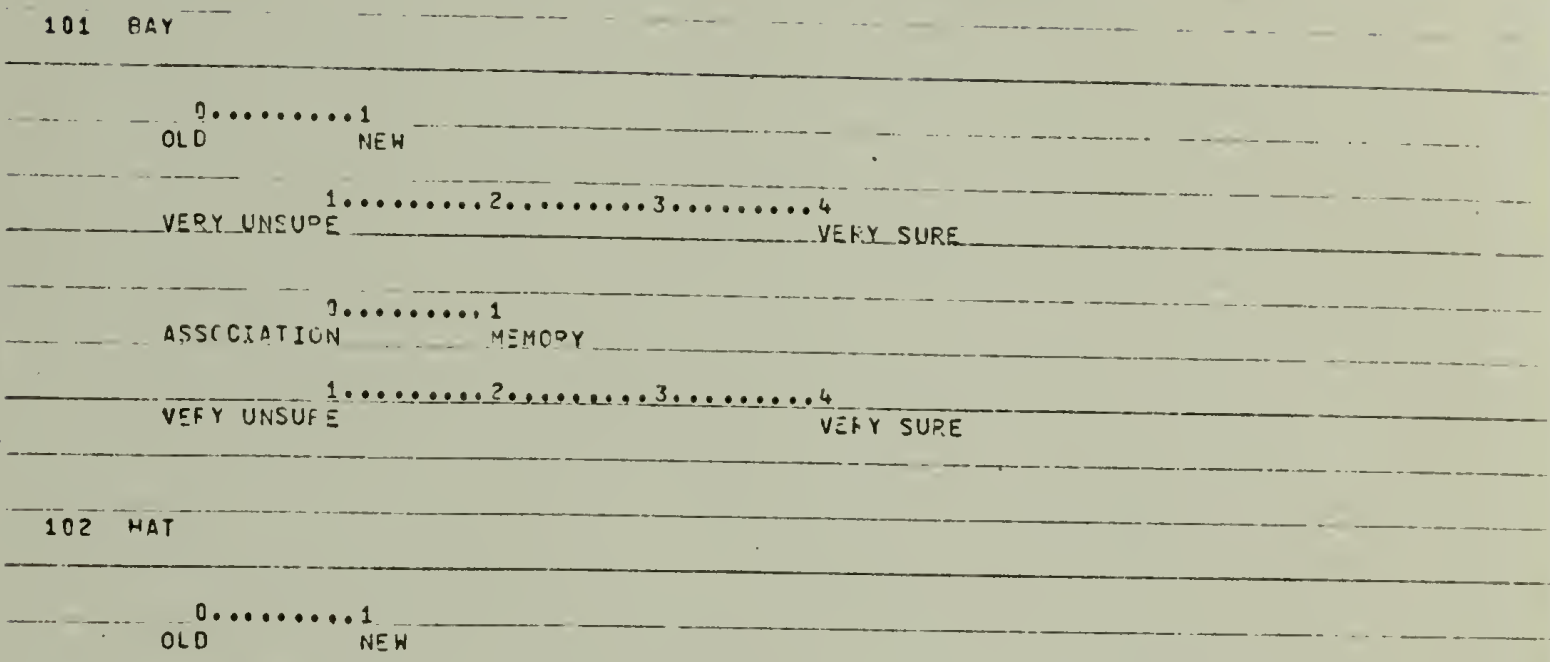

YEKY UNSURE

$1 \ldots \ldots \ldots \ldots \ldots \ldots \ldots \ldots \ldots$

YEF.Y SURE

ASSCETATION

VEKY UNSUIS

103 JAMES COPEETT WAS THE BOXER WHO HAS NICKNAMEO GENTLEMAN JIY

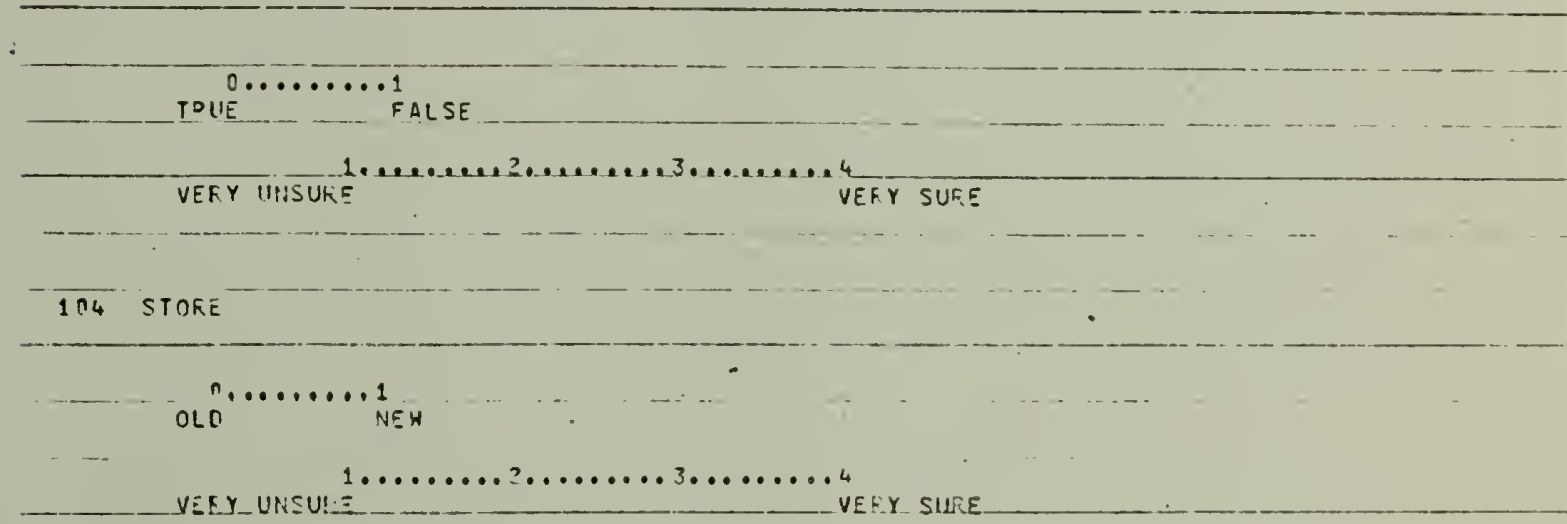

1

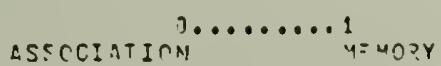




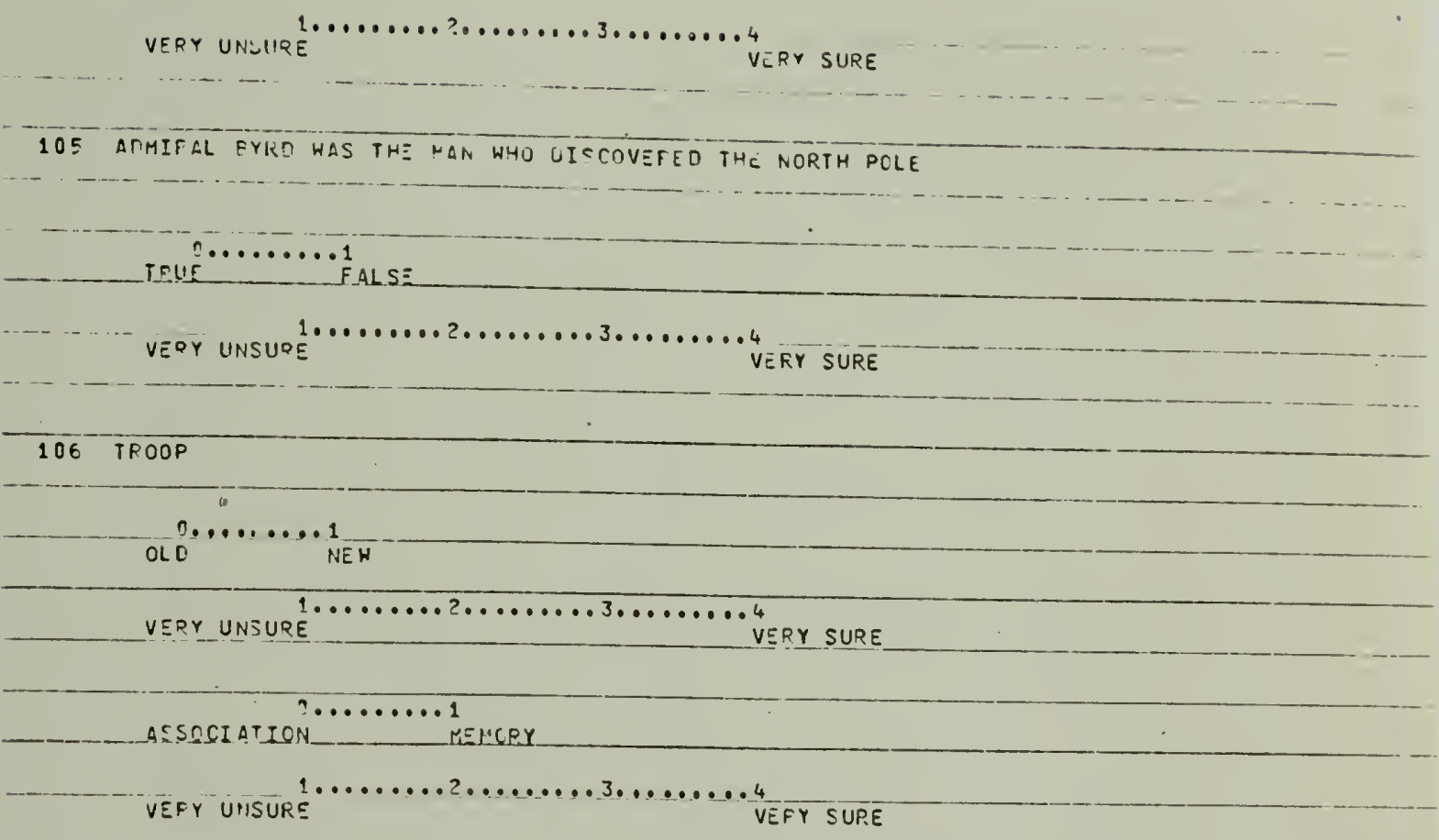

$107 \quad L A 4$

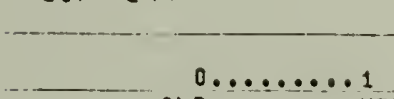

OLD NEY

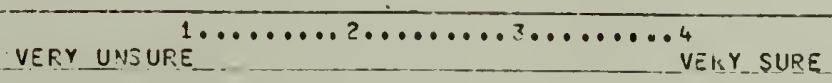

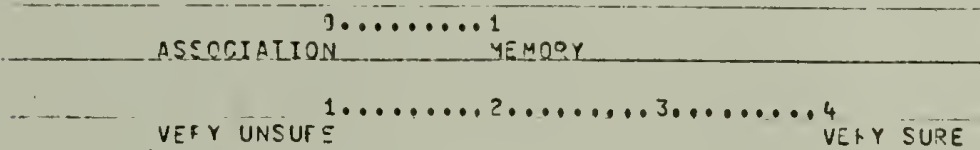

10 A MAT
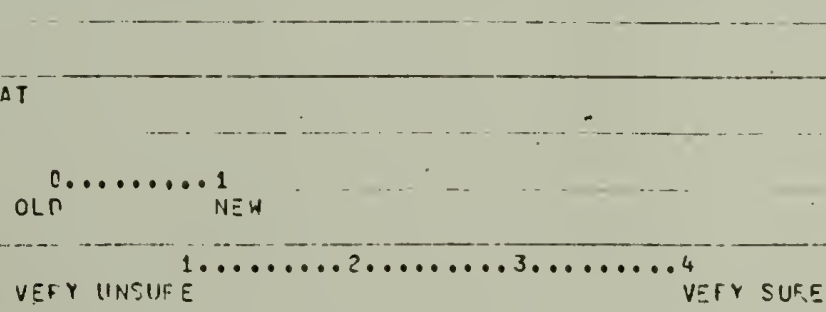
ASECCIATION MEMORY

vef.Y URSUFE $1 \ldots \ldots \ldots 2 \ldots \ldots \ldots \ldots \ldots 4_{\text {VERY SURE }}^{4}$

109 ALVIH BAFKLEY WAS THE HAN WHO WAS ELECTED VICF PRESITENT IN 1948 WHEN HAFFY
TFUMAI, WAE ELECTEI PFESEDEHT

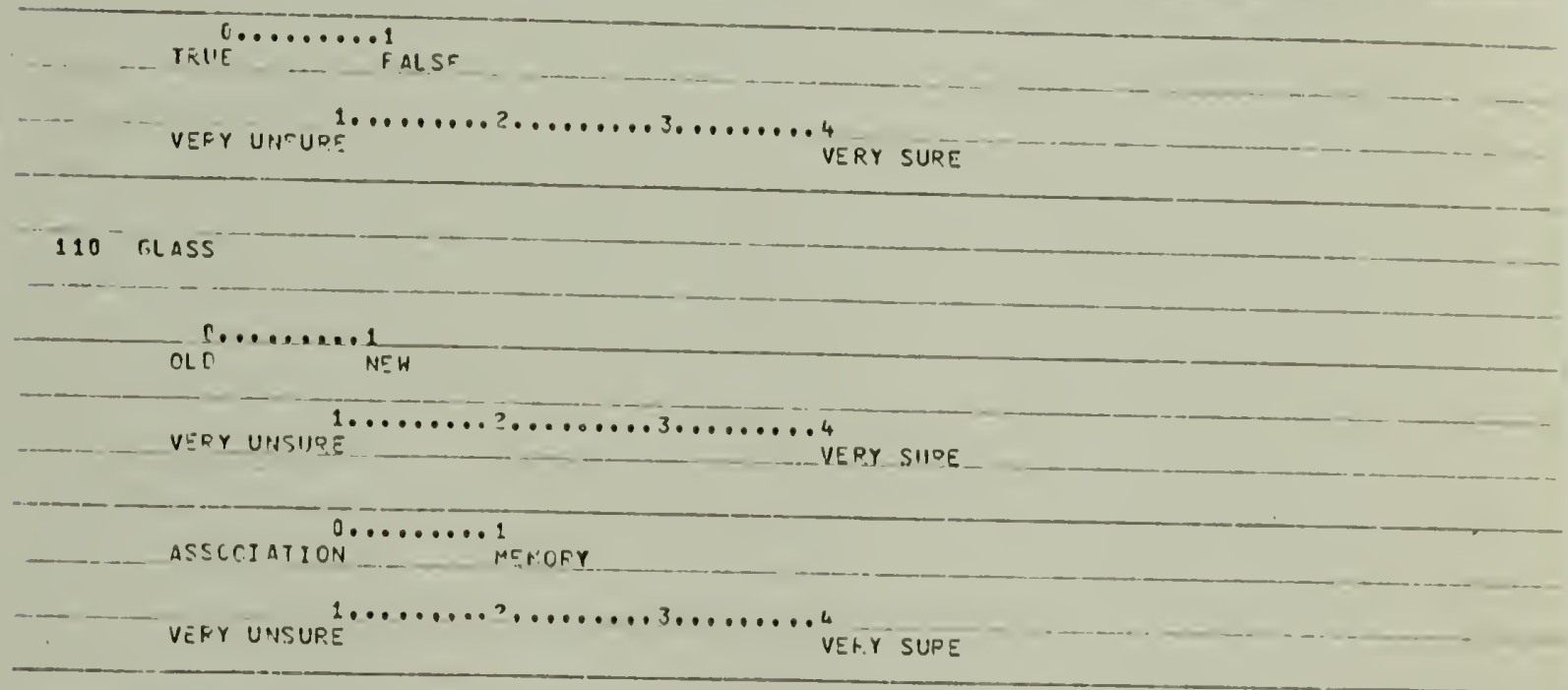

111 QUEEN
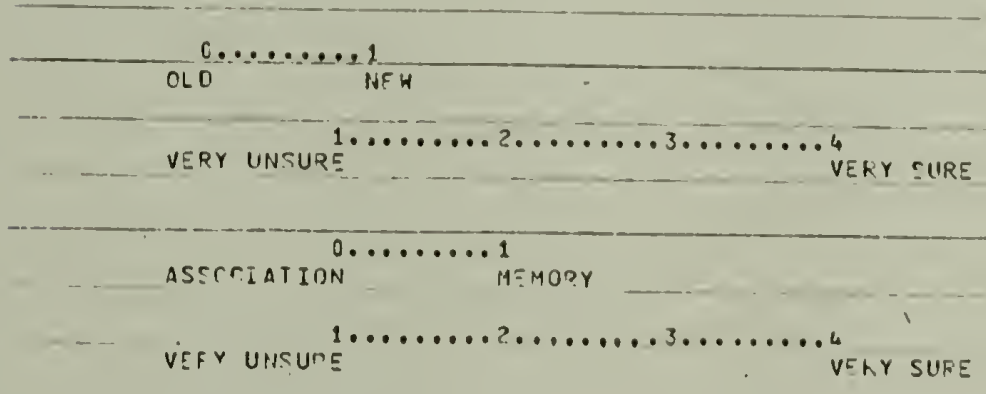


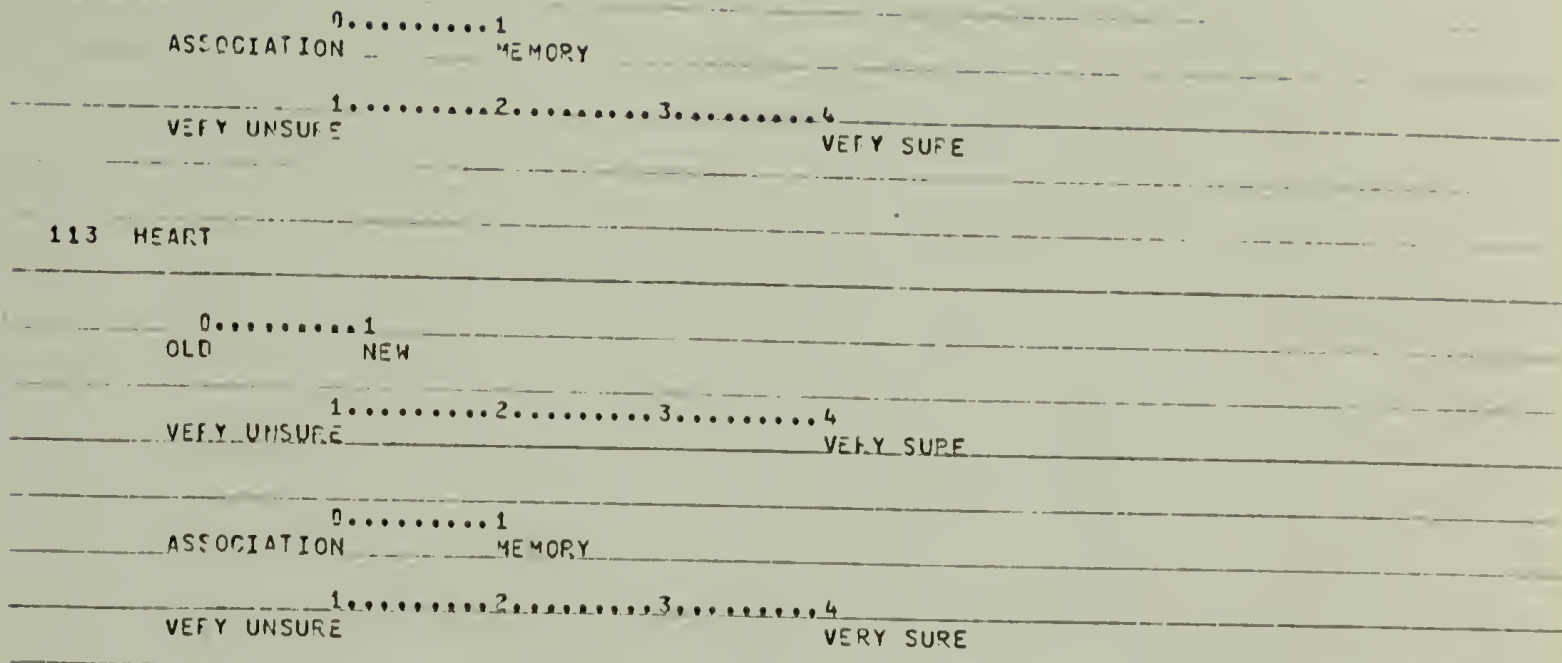

114 HEL $\bar{P}$

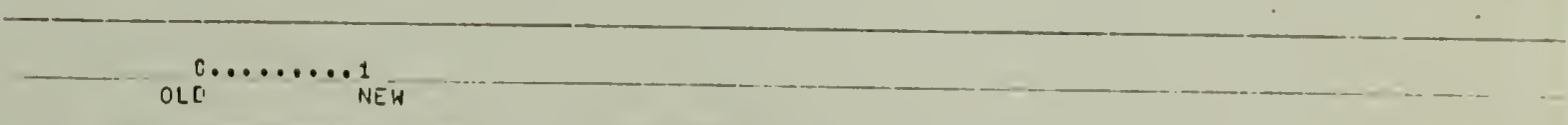

$1, \ldots \ldots \ldots 2, \ldots \ldots \ldots, \ldots \ldots \ldots$

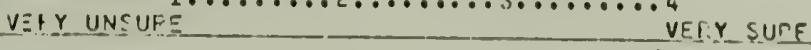

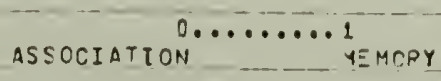

VEPY UNGURE $1, \ldots, \ldots, \ldots, 2, \ldots, \ldots, 1, \ldots \ldots, \ldots 4$

VERY SUPE

115 LOG

$O L C \cdots \cdots+$ NEH

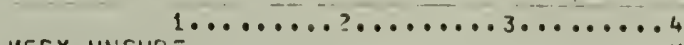

VERY SURE

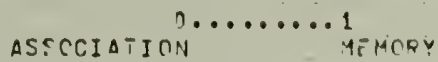

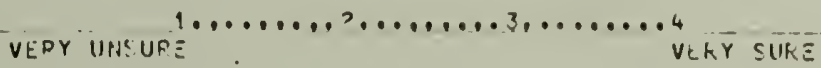

11E PFO 


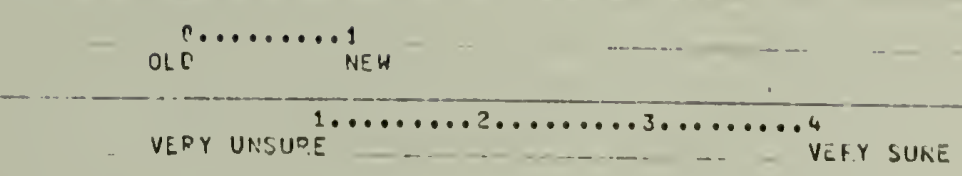

ASECEIAIION

VEFY LINGURE $1 \ldots \ldots \ldots \ldots 2 \ldots \ldots \ldots \ldots, \ldots \ldots \ldots$

\section{VEFY SUP.E}

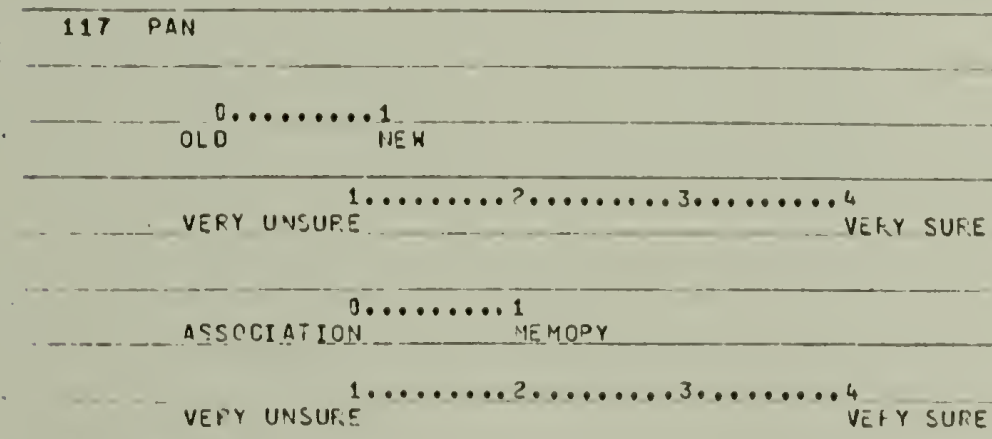

118 JOHN T. SCOPES WAS THE MAN TIIEO IN THE FAMOUS MOHKEY TKIAL OF 1925

TRUE

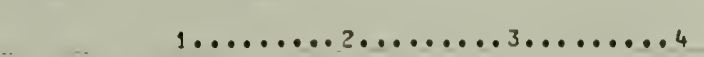

VERY Y UNCUTE

VER Y SURE

\section{$119 \cos T$}
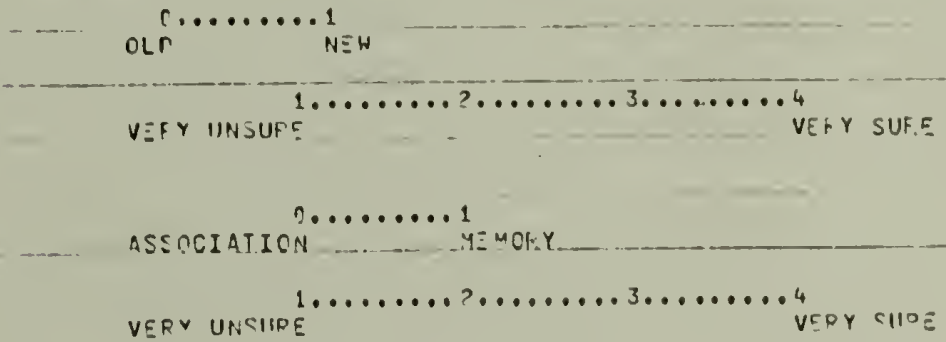
$1205 \mathrm{CHOOL}$

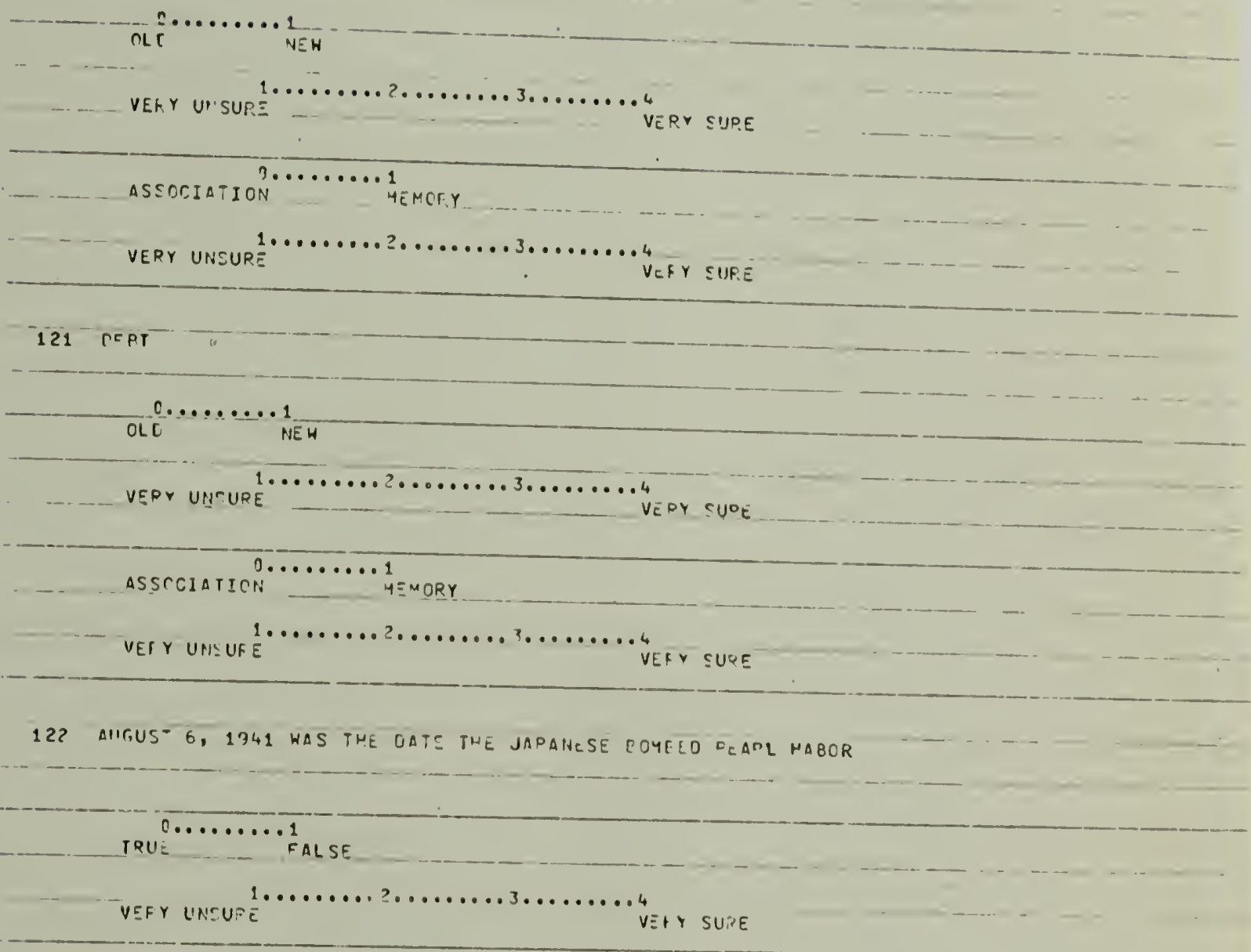

1231912 WAS THE YEAQ HENPY FORU INTOOOUCED THE MCDEL I

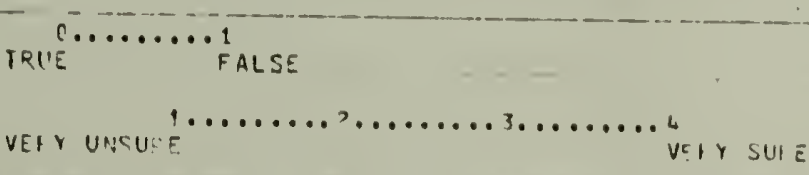

124 FIELI 


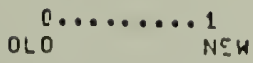

VEr.y_UHSUR $1 \ldots \ldots \ldots 2 \ldots \ldots \ldots 3 \ldots \ldots \ldots$

ASSORIATION $\ldots \ldots \ldots 1$

VETY UTSUREE $1 \ldots \ldots \ldots 2 \ldots \ldots \ldots . \ldots \ldots \ldots 4$ VERY SURE

125 NET

$O L n \ldots \ldots$ n

$1, \ldots \ldots \ldots 2 \ldots \ldots \ldots 3 \ldots \ldots \ldots, \ldots$

VECY SUEE

ASSOCTATION $0 \cdots \cdots \cdots 1$ ME MORY

VERY UNSUIDE $\ldots \ldots \ldots 2 \ldots \ldots \ldots 3 \ldots \ldots \ldots 4$ VFRY SURE

126 FISH

OLC $\cdots \cdots \cdots$ NEH

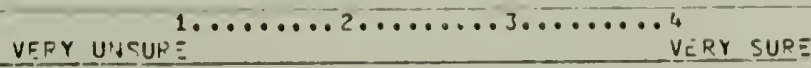

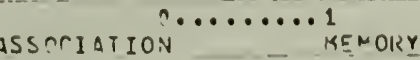

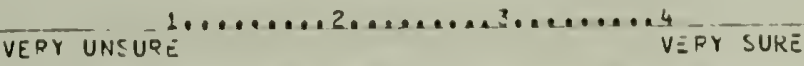

127 NORTH

OL $[\cdots \cdots \cdots$ NEW

VERV INSTIRE $1, \ldots \ldots \ldots 2 \ldots \ldots \ldots 3 \ldots \ldots \ldots$ V VERY SHOE

$\operatorname{ascciatir.4}$ 


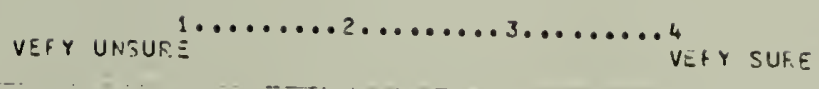

128 KAISED WILHETM HAS THE MAN WHOSE TEATH SET OFF WOPLE WAF I

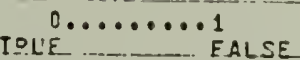

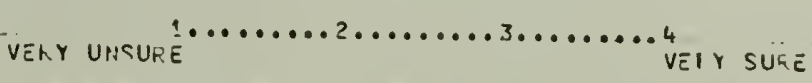

129 JAMES EADL RAY WAS THE MAN WHS ASSASSTNATLO [F. MARTIN LUTHEF KING

IRUE
VETY UNSUFE

130 RUEST

$-0 \ldots \ldots \ldots 1$

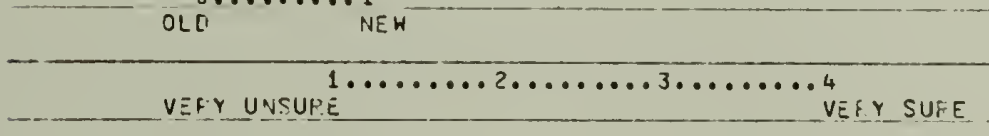

ASSCCIATION

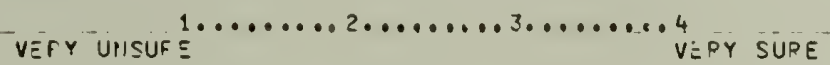

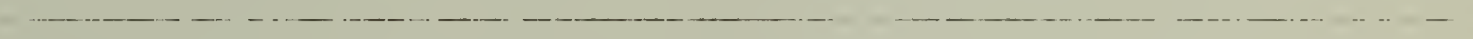

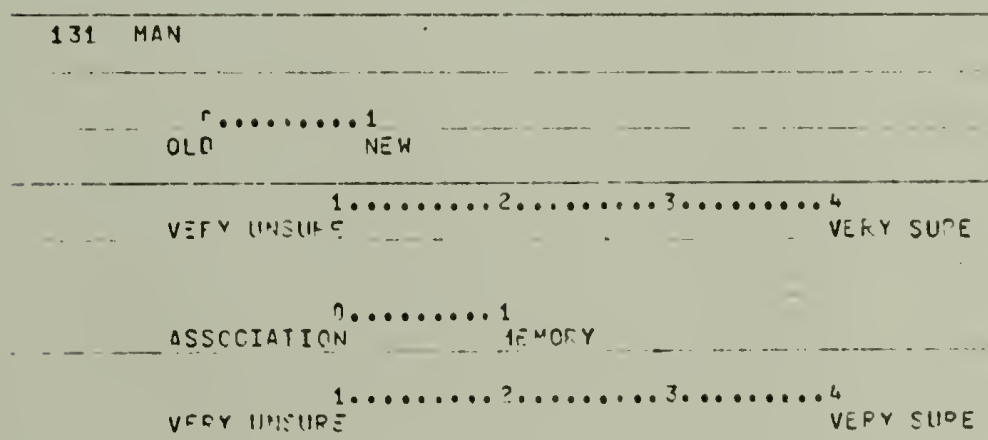




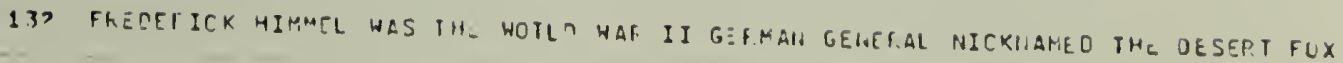

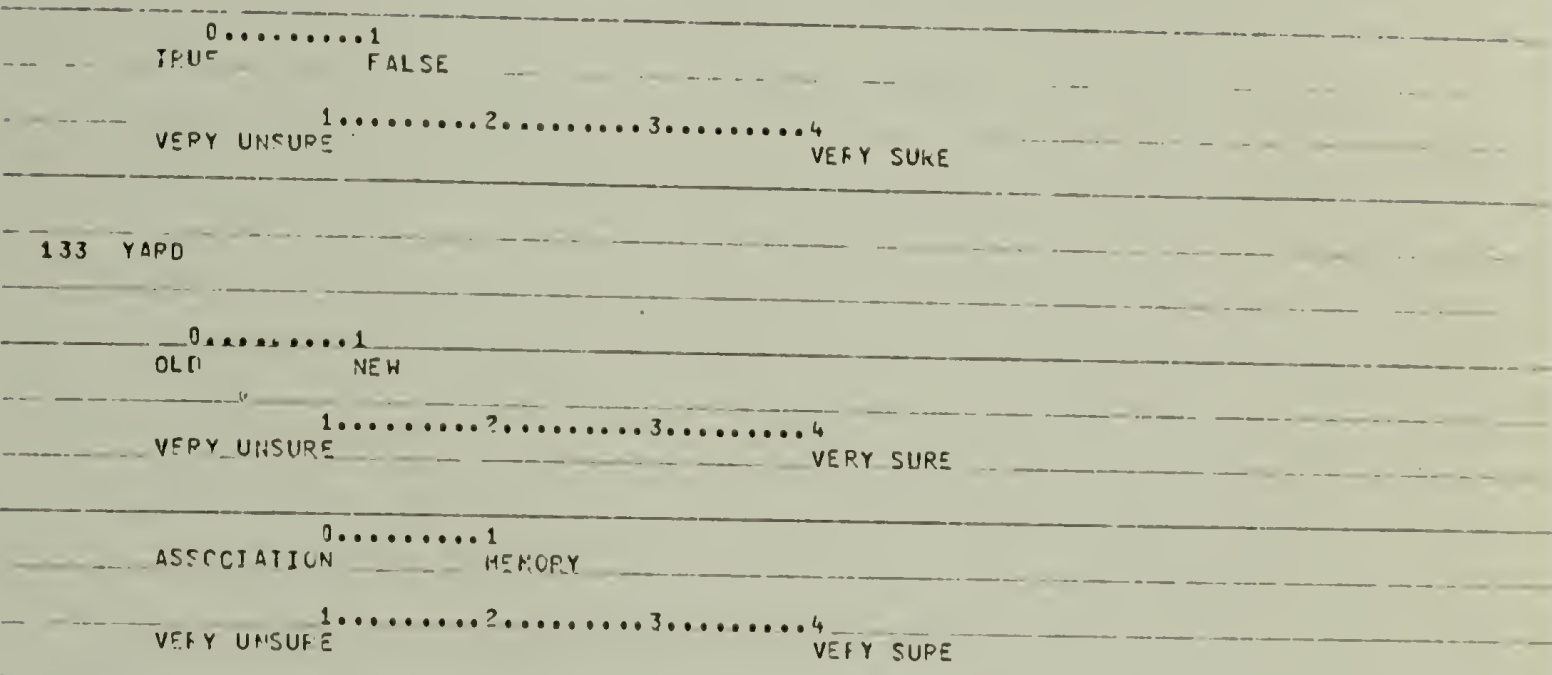

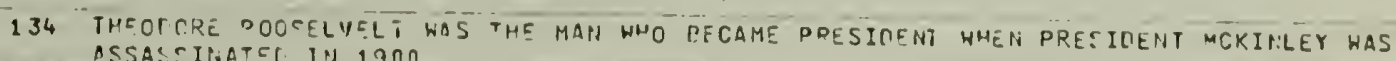
ASSASEIMATEL IN 1300

c........

TFUL FALSE.

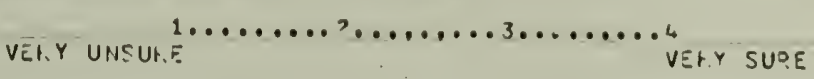

$135^{\circ}$ r.DOHTM

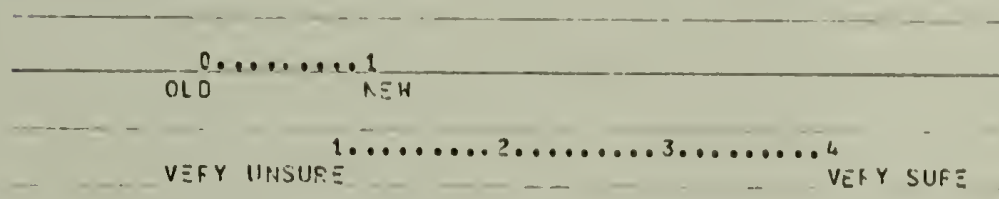

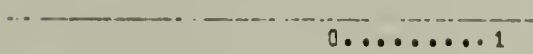

asscritation

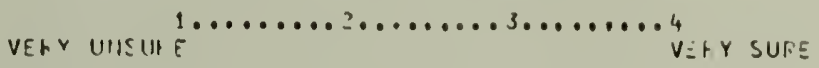

$136 \sin i$ 
$-374-$

OLC $\cdots \cdots \cdots$ NEH

VEI Y UHSUEE YEFY SUFE

ASSOCIATION_..... I

VER UHSURE

VERY SIIOE

1371957 WAS THE YEAF THE RUSSIANS ORPITTEO THE F,FIST SATELLITE

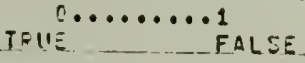

VEOR UHSUR.

VEPY SURE

138 VOICE

OL $[\cdots \cdots \cdots+\cdots$

VERY UISSIIOE

$V=0 Y$ SIIRE

ASSOCIAIICN $\cdots \cdots \cdots 1$ MEMCF

VERYY UNSUFE

VEKY SUF.E

$13 \overline{9}$ FND

OLC ${ }^{0} \ldots \ldots$ NEH

YEOY UNCUOE

YEFY IUEE

ASSCCIATISN J..... 1

VEFr UM!SUT Ë

VER Y SUF

140 STOIK 


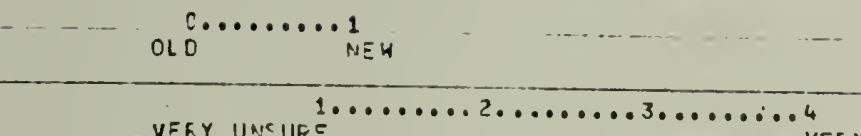

VEKY UNSURE

$0 . \ldots \ldots \ldots 1$

ASSOCIAIION

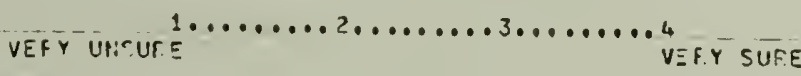

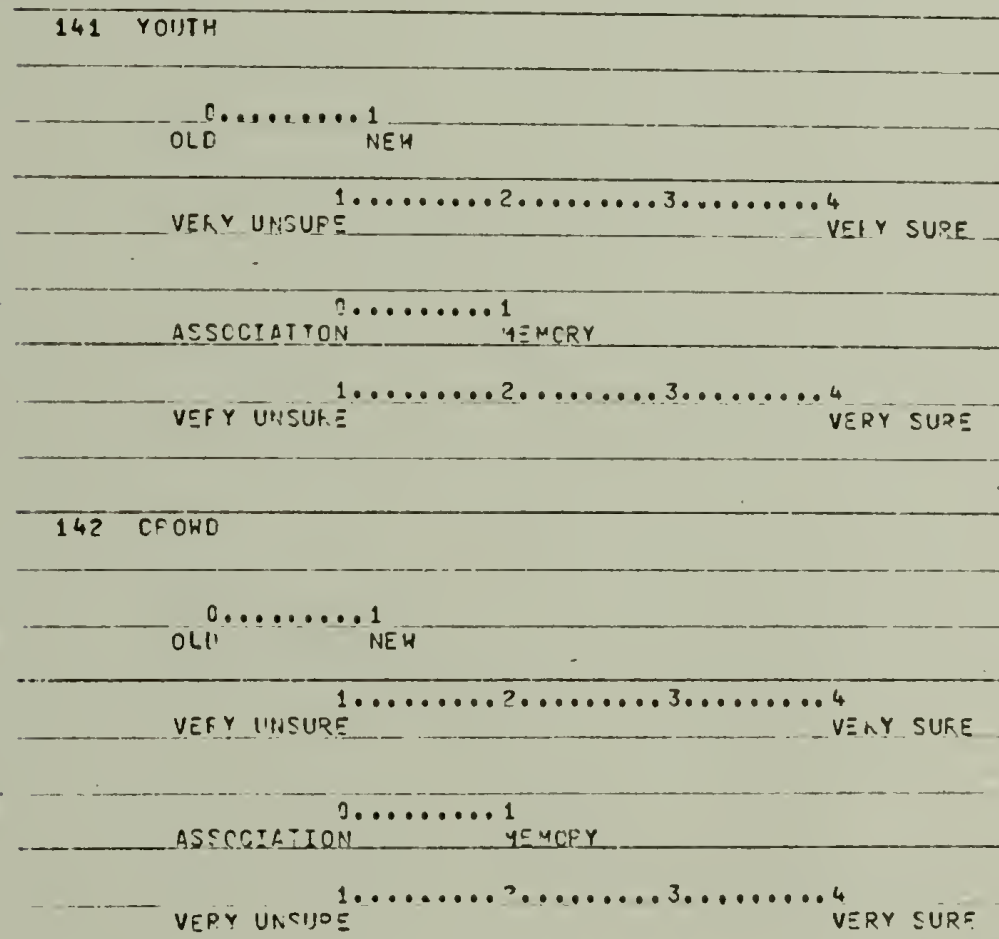

143 TIARKI IA TUCSEVIT WLS THE OMLY FFESICENT UF THE UNETED STATES TO BE ELLCTED TO Fulln TEF."S OF CFFICE

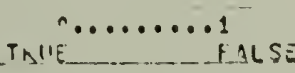

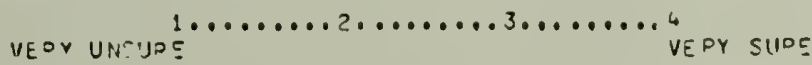


$-376-$

144 WFALTH

$-\ldots$ OLC $\ldots \ldots \ldots 1$

VEP.Y UIHIITEE VEPY SURE

ASSOCI ATION

VEFY UNIURE 4 VEFY SURE

PLEASE PECORO THE TIME 


$$
-377-
$$

Appendix B

Metamemory Questionnaire Responses 
Percentage of Subjects in Each Age, Education, and Sex Group Giving Each Response

On the Questionniare

Please answer the following questions and then circle the number above the response that is closest to your choice.

1. How often has their beer someone in your family who has had difficulty remembering things in old age?

$0 \ldots \ldots \ldots . \ldots \ldots . \ldots . \ldots . . . . . . . .4$ Never Rarely Sometimes Often Always

\begin{tabular}{cccccc} 
& 6.5 & 22.6 & 48.4 & 19.4 & 3.2 \\
Age & 0 & 28.1 & 34.4 & 31.3 & 6.3 \\
& 3.2 & 25.4 & 41.3 & 25.4 & 4.8 \\
\multirow{4}{*}{ Ed } & 3.1 & 21.9 & 40.6 & 25.0 & 9.4 \\
& 3.2 & 29.0 & 41.9 & 25.8 & 0 \\
& 3.2 & 25.4 & 41.3 & 25.4 & 4.8 \\
\multirow{2}{*}{ Sex } & 3.1 & 18.8 & 53.1 & 18.8 & 6.3 \\
& 3.2 & 32.3 & 29.0 & 32.3 & 3.2 \\
& 3.2 & 25.4 & 41.3 & 25.4 & 4.8
\end{tabular}

2. How often has there been someone in your family who was exceptionally active and alert in old age?

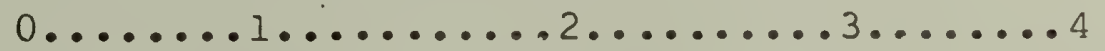

$\begin{array}{crrrr}\text { Never } & \text { Rarely } & \text { Sometimes } & \text { Often } & \text { Always } \\ 3.2 & 16.1 & 25.8 & 45.2 & 9.7 \\ 3.1 & 12.5 & 21.9 & 46.9 & 15.9 \\ 3.2 & 14.3 & 23.8 & 46.0 & 12.7 \\ 6.3 & 18.8 & 12.5 & 50.0 & 12.5 \\ 0 & 9.7 & 35.5 & 41.9 & 12.9 \\ 3.2 & 14.3 & 23.8 & 46.0 & 12.7 \\ 3.1 & 9.4 & 25.0 & 50.0 & 12.5 \\ 3.2 & 19.4 & 22.6 & 41.9 & 12.9 \\ 3.2 & 14.3 & 23.8 & 46.0 & 12.7\end{array}$


3. How often in your daily activities, do you need to rely on your memory?

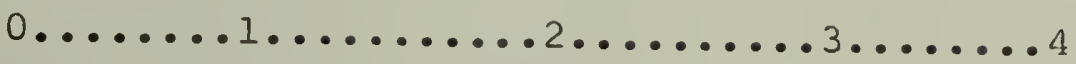

Never Rarely Sometimes Often Always

\begin{tabular}{|c|c|c|c|c|c|}
\hline Age & $\begin{array}{l}0 \\
0 \\
0\end{array}$ & $\begin{array}{l}0 \\
0 \\
0\end{array}$ & $\begin{array}{l}9.7 \\
6.3 \\
7.9\end{array}$ & $\begin{array}{l}64.5 \\
71.9 \\
68.3\end{array}$ & $\begin{array}{l}25.8 \\
21.9 \\
23.8\end{array}$ \\
\hline$E$ & $\begin{array}{l}0 \\
0 \\
0\end{array}$ & $\begin{array}{l}0 \\
0 \\
0\end{array}$ & $\begin{array}{r}12.5 \\
3.2 \\
7.9\end{array}$ & $\begin{array}{l}62.5 \\
74.2 \\
68.3\end{array}$ & $\begin{array}{l}25.0 \\
22.6 \\
23.8\end{array}$ \\
\hline $2 x$ & $\begin{array}{l}0 \\
0 \\
0\end{array}$ & " $\quad \begin{array}{l}0 \\
0 \\
0\end{array}$ & $\begin{array}{l}9.4 \\
6.5 \\
7.9\end{array}$ & $\begin{array}{l}65.6 \\
71.0 \\
68.3\end{array}$ & $\begin{array}{l}25.0 \\
22.6\end{array}$ \\
\hline
\end{tabular}

4. How often do you particularly notice your memory?

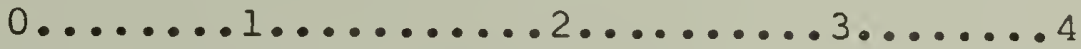

$\begin{array}{cccrr}\text { Never } & \text { Rarely } & \text { Sometimes } & \text { Often } & \text { Always } \\ 0 & 6.5 & 16.1 & 67.7 & 9.7 \\ 0 & 6.3 & 37.5 & 46.9 & 9.4 \\ 0 & 6.3 & 27.0 & 57.1 & 9.5 \\ 0 & 9.4 & 21.9 & 56.3 & 12.5 \\ 0 & 3.2 & 32.3 & 58.1 & 6.5 \\ 0 & 6.3 & 27.0 & 57.1 & 9.5 \\ 0 & 12.5 & 28.1 & 53.1 & 6.3 \\ 0 & 0 & 25.8 & 61.3 & 12.9 \\ 0 & 6.3 & 27.0 & 57.1 & 9.5\end{array}$

5. How often do you experience difficulty remembering things? Age $-x^{2}(4)=11.08, p<.05$

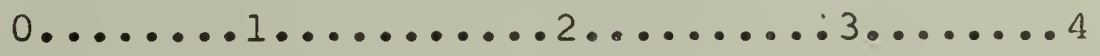

Never Rarely Sometimes Often Always

\begin{tabular}{cccccc} 
& 3.2 & 38.7 & 41.9 & 12.9 & 3.2 \\
Age & 0 & 9.4 & 59.4 & 31.3 & 0 \\
& 1.6 & 23.8 & 50.8 & 22.2 & 1.6 \\
& 3.1 & 21.9 & 40.6 & 31.3 & 3.1 \\
\multirow{3}{*}{ Ed } & 0 & 25.8 & 61.3 & 12.9 & 0 \\
& 1.6 & 23.8 & 50.8 & 22.2 & 1.6 \\
& 3.1 & 21.9 & 59.4 & 15.6 & 0 \\
Sex & 0 & 25.8 & 41.9 & 29.0 & 3.2 \\
& 1.6 & 23.8 & 50.8 & 22.2 & 1.6
\end{tabular}


6. How often do you have difficulty remembering things that happened a few minutes ago? Age $-x^{2}(3)=8.05$, $\mathrm{p} .05, \mathrm{Ed}-\mathrm{x}^{2}(3)=8.87, \mathrm{p}<.05$.

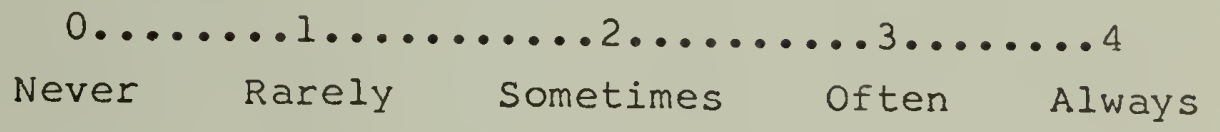

\begin{tabular}{rrrrrr} 
& 12.9 & 51.6 & 35.5 & 0 & 0 \\
Age & 9.4 & 46.9 & 21.9 & 21.9 & 0 \\
& 11.1 & 49.2 & 28.6 & 11.1 & 0 \\
\multirow{3}{*}{ Ed } & 12.5 & 31.3 & 40.6 & 15.6 & 0 \\
& 9.7 & 67.7 & 16.1 & 6.5 & 0 \\
\multirow{3}{*}{ Sex } & 11.1 & 49.2 & 28.6 & 1.11 & 0 \\
& 6.3 & 56.3 & 28.1 & 9.4 & 0 \\
& 16.1 & 41.9 & 29.0 & 12.9 & 0 \\
& 11.1 & 49.2 & 28.6 & 11.1 & 0
\end{tabular}

7. How often do you have difficulty remembering things that happened a few days or weeks ago?

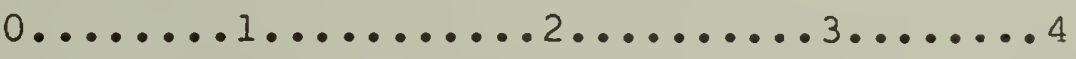

$\begin{array}{ccccc}\text { Never } & \text { Rarely } & \text { Sometimes } & \text { Often } & \text { A.Iways } \\ 6.5 & 41.9 & 35.5 & 16.1 & 0 \\ 3.1 & 18.8 & 46.9 & 31.3 & 0 \\ 4.8 & 30.2 & 41.3 & 23.8 & 0 \\ 3.1 & 28.1 & 40.6 & 28.1 & 0 \\ 6.5 & 32.3 & 41.9 & 19.4 & 0 \\ 4.8 & 30.2 & 41.3 & 23.8 & 0 \\ 3.1 & 21.9 & 50.0 & 25.0 & 0 \\ 6.5 & 38.7 & 32.3 & 22.6 & 0 \\ 4.8 & 30.2 & 41.3 & 23.8 & 0\end{array}$

8. How often do you have difficulty remembering things that happened many years ago? $\mathrm{Ed}-\mathrm{x}^{2}(4)=17.53$, pK.01.

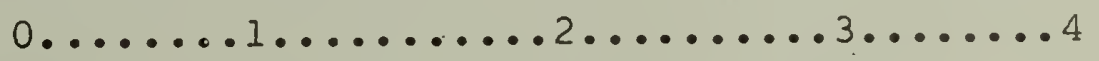

Never Rarely Sometimes often Always

\begin{tabular}{cccccc} 
& 3.2 & 48.4 & 29.0 & 12.9 & 6.5 \\
Age & 0 & 28.1 & 43.8 & 21.9 & 6.3 \\
& 1.6 & 38.1 & 36.5 & 17.5 & 6.3 \\
\multirow{3}{*}{ Ed } & 3.1 & 46.9 & 37.5 & 0 & 12.5 \\
& 0 & 29.0 & 35.5 & 35.5 & 0 \\
& 1.6 & 38.1 & 36.5 & 17.5 & 6.3 \\
Sex & 3.1 & 34.4 & 31.3 & 25.0 & 6.3 \\
& 0 & 41.9 & 41.9 & 9.7 & 6.5 \\
& 1.6 & 36.5 & 36.5 & 17.5 & 6.3
\end{tabular}


9. How often do you forget things. Age $-x^{2}(3)=6.96$, $\mathrm{p}<.08$.

\begin{tabular}{|c|c|c|c|c|c|}
\hline \multicolumn{6}{|c|}{ 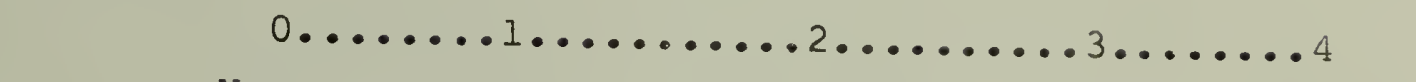 } \\
\hline & Never & Rarely & Sometimes & Often & Always \\
\hline Age & $\begin{array}{l}3.1 \\
0 \\
1.6\end{array}$ & $\begin{array}{l}34.4 \\
12.5 \\
23.4\end{array}$ & $\begin{array}{l}50.0 \\
56.3 \\
53.1\end{array}$ & $\begin{array}{l}12.5 \\
31.3 \\
21.9\end{array}$ & $\begin{array}{l}0 \\
0 \\
0\end{array}$ \\
\hline$E \mathrm{~d}$ & $\begin{array}{l}3.1 \\
0 \\
1.6\end{array}$ & $\begin{array}{l}21.9 \\
25.0 \\
23.4\end{array}$ & $\begin{array}{l}50.0 \\
56.3 \\
53.1\end{array}$ & $\begin{array}{l}25.0 \\
18.8 \\
21.0\end{array}$ & $\begin{array}{l}0 \\
0 \\
0\end{array}$ \\
\hline Sex & $\begin{array}{l}2.1 \\
0 \\
0\end{array}$ & $\begin{array}{l}15 \cdot 6 \\
31 \cdot 3 \\
23 \cdot 4\end{array}$ & $\begin{array}{l}56.3 \\
50.0 \\
53.1\end{array}$ & $\begin{array}{l}25.0 \\
18.8 \\
21.9\end{array}$ & $\begin{array}{l}0 \\
0 \\
0\end{array}$ \\
\hline \multicolumn{4}{|c|}{$\begin{array}{l}\text { 10. How often do you misplace things? } \\
8.58 \text {, p }<.08 \text {. }\end{array}$} & \multicolumn{2}{|c|}{ Age $-x^{2}(4)=$} \\
\hline \multicolumn{6}{|c|}{$0 \ldots \ldots \ldots . \ldots \ldots \ldots . \ldots \ldots \ldots$} \\
\hline & Never & Rarely & Sometimes & Often & Always \\
\hline Age & $\begin{array}{l}3.1 \\
0 \\
1.6\end{array}$ & $\begin{array}{l}62 \cdot 5 \\
31 \cdot 3 \\
46 \cdot 9\end{array}$ & $\begin{array}{l}18 \cdot 8 \\
31 \cdot 3 \\
25 \cdot 0\end{array}$ & $\begin{array}{l}15.6 \\
34.4 \\
25.0\end{array}$ & $\begin{array}{c}0 \\
3.1 \\
1.6\end{array}$ \\
\hline$E \mathrm{~d}$ & $\begin{array}{c}3.1 \\
0 \\
1.6\end{array}$ & $\begin{array}{l}43.8 \\
50.0 \\
46.9\end{array}$ & $\begin{array}{l}18.8 \\
31.3 \\
25.0\end{array}$ & $\begin{array}{l}34.4 \\
15.0 \\
25.0\end{array}$ & $\begin{array}{c}0 \\
3.1 \\
1.6\end{array}$ \\
\hline Sex & $\begin{array}{c}3.1 \\
0 \\
1.6\end{array}$ & $\begin{array}{l}37.5 \\
56.3 \\
46.9\end{array}$ & $\begin{array}{l}28 \cdot 1 \\
21.9 \\
25.0\end{array}$ & $\begin{array}{l}28.1 \\
21.9 \\
25.0\end{array}$ & $\begin{array}{c}3.1 \\
0 \\
1.6\end{array}$ \\
\hline
\end{tabular}

11. How often do you forget names?

\begin{tabular}{|c|c|c|c|c|}
\hline Never & Rarely & sometimes & often & Always \\
\hline $\begin{array}{c}6.3 \\
0 \\
3.1\end{array}$ & $\begin{array}{r}21.9 \\
6.3 \\
14.1\end{array}$ & $\begin{array}{l}31 \cdot 3 \\
31 \cdot 3 \\
31 \cdot 3\end{array}$ & $\begin{array}{l}31.3 \\
56.3 \\
43.8\end{array}$ & $\begin{array}{l}9.4 \\
6.3 \\
7.8\end{array}$ \\
\hline $\begin{array}{c}6.3 \\
0 \\
3.1\end{array}$ & $\begin{array}{r}9.4 \\
18.8 \\
14.1\end{array}$ & $\begin{array}{l}34.4 \\
28.1 \\
31.3\end{array}$ & $\begin{array}{l}37.5 \\
50.0 \\
43.8\end{array}$ & $\begin{array}{r}12.5 \\
3.1 \\
7.8\end{array}$ \\
\hline $\begin{array}{l}3.1 \\
3.1 \\
3.1\end{array}$ & $\begin{array}{r}9.4 \\
18.8 \\
14.1\end{array}$ & $\begin{array}{l}31 \cdot 3 \\
31 \cdot 3 \\
31 \cdot 3\end{array}$ & $\begin{array}{l}46.9 \\
40.6 \\
43.8\end{array}$ & $\begin{array}{l}9.4 \\
6.3 \\
7.8\end{array}$ \\
\hline
\end{tabular}


12. How often do you forget facts? Sex $-x^{2}(3)=9.95$, $\mathrm{p}<.05$.

\begin{tabular}{|c|c|c|c|c|c|}
\hline & Never & Rarely & Sometimes & Often & Always \\
\hline Age & $\begin{array}{c}3.1 \\
0 \\
1.6\end{array}$ & $\begin{array}{l}21.9 \\
21.9 \\
21.9\end{array}$ & $\begin{array}{l}46.9 \\
50.0 \\
48.4\end{array}$ & $\begin{array}{l}28.1 \\
28.1 \\
28.1\end{array}$ & $\begin{array}{l}0 \\
0 \\
0\end{array}$ \\
\hline $\mathrm{Ed}$ & $\begin{array}{l}0 \\
0 \\
0\end{array}$ & $\begin{array}{c}3.1 \\
0 \\
1.6\end{array}$ & $\begin{array}{l}18.8 \\
25.0 \\
21.9\end{array}$ & $\begin{array}{l}46.9 \\
50.0 \\
48.4\end{array}$ & $\begin{array}{l}31.2 \\
25.0 \\
28.1\end{array}$ \\
\hline Sex & $\begin{array}{c}3.1 \\
0 \\
1.6\end{array}$ & $\begin{array}{r}6.3 \\
37.5 \\
21.9\end{array}$ & $\begin{array}{l}59.4 \\
37.5 \\
48.4\end{array}$ & $\begin{array}{l}21.3 \\
25.0 \\
28.1\end{array}$ & $\begin{array}{l}0 \\
0 \\
0\end{array}$ \\
\hline
\end{tabular}

13. How often do you forget things you should do? Ed $\mathrm{x}^{2}(4)=8.59, \mathrm{p}<.08$.

$0 \ldots \ldots \ldots 1 \ldots \ldots \ldots . \ldots . \ldots . \ldots . \ldots 4$

$\begin{array}{rccrc}\text { Never } & \text { Rarely } & \text { Sometimes } & \text { Often } & \text { Always } \\ 9.4 & 43.8 & 24.4 & 12.5 & 0 \\ 3.1 & 43.8 & 37.5 & 12.5 & 3.1 \\ 6.3 & 43.8 & 35.9 & 12.5 & 1.6 \\ 6.3 & 28.1 & 50.0 & 15.6 & 0 \\ 6.3 & 59.4 & 21.9 & 9.4 & 3.1 \\ 6.3 & 43.8 & 35.9 & 12.5 & 1.6 \\ 6.3 & 34.4 & 40.6 & 15.6 & 3.1 \\ 6.3 & 53.1 & 31.3 & 9.4 & 0 \\ 6.3 & 43.8 & 35.9 & 12.5 & 1.6\end{array}$

14. How often do you forget appointments?

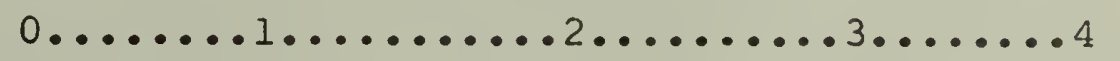

Never Rarely Sometimes Often Always

\begin{tabular}{cccccc} 
& 25.0 & 53.1 & 18.8 & 3.1 & 0 \\
Age & 21.9 & 59.4 & 18.8 & 0 & 0 \\
& 23.4 & 56.3 & 18.8 & 1.6 & 0 \\
\multirow{3}{*}{ Ed } & 15.6 & 56.3 & 25.0 & 3.1 & 0 \\
& 31.3 & 56.3 & 12.5 & 0 & 0 \\
& 23.4 & 56.3 & 18.8 & 1.6 & 0 \\
\multirow{3}{*}{ Sex } & 18.8 & 53.4 & 28.1 & 0 & 0 \\
& 28.1 & 59.4 & 9.4 & 3.1 & 0 \\
& 23.4 & 56.3 & 18.8 & 1.6 & 0
\end{tabular}


15. How often do you use special memory tricks to help you remember? Ed $-x^{2}(4)=9.07, p<.06$.

\begin{tabular}{|c|c|c|c|c|}
\hline Never & Rarely & Sometimes & Often & Always \\
\hline $\begin{array}{l}25.0 \\
18.8 \\
21.9\end{array}$ & $\begin{array}{l}25.0 \\
31.3 \\
28.1\end{array}$ & $\begin{array}{l}31.3 \\
28.1 \\
29.7\end{array}$ & $\begin{array}{l}18.8 \\
15.6 \\
17.2\end{array}$ & $\begin{array}{c}0 \\
6.3 \\
3.1\end{array}$ \\
\hline $\begin{array}{l}28.1 \\
15.6 \\
21.9\end{array}$ & $\begin{array}{l}21.9 \\
34.4 \\
28.1\end{array}$ & $\begin{array}{l}18.8 \\
40.6 \\
29.7\end{array}$ & $\begin{array}{r}28.1 \\
6.3 \\
17.2\end{array}$ & $\begin{array}{l}3.1 \\
3.1 \\
3.1\end{array}$ \\
\hline $\begin{array}{l}28.1 \\
15.6 \\
21.9\end{array}$ & $\begin{array}{l}28.1 \\
28.1 \\
28.1\end{array}$ & $\begin{array}{l}21.9 \\
37.5 \\
29.7\end{array}$ & $\begin{array}{l}15.6 \\
18.8 \\
17.2\end{array}$ & $\begin{array}{c}6.3 \\
0 \\
3.1\end{array}$ \\
\hline
\end{tabular}

16. How often do you write a shopping list?

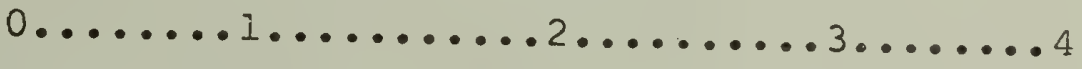

$\begin{array}{rcccc}\text { Never } & \text { Rarely } & \text { Sometimes } & \text { Often } & \text { Always } \\ 12.5 & 31.3 & 25.0 & 15.6 & 15.6 \\ 9.4 & 18.8 & 12.5 & 28.1 & 31.3 \\ 10.9 & 25.0 & 18.8 & 21.9 & 23.4 \\ 6.3 & 25.0 & 18.8 & 21.9 & 28.1 \\ 15.6 & 25.0 & 18.8 & 21.9 & 18.8 \\ 10.9 & 25.0 & 18.8 & 21.9 & 23.4 \\ 15.6 & 28.1 & 15.6 & 25.0 & 15.6 \\ 6.3 & 21.9 & 21.9 & 18.8 & 31.3 \\ 10.9 & 25.0 & 18.8 & 21.9 & 23.4\end{array}$

17. How often do you write appointments in a calendar to help you remember them? $E d-x^{2}(4)=8.38, p<.08$; Sex $-x^{2}(4)=10.65, p<.05$.

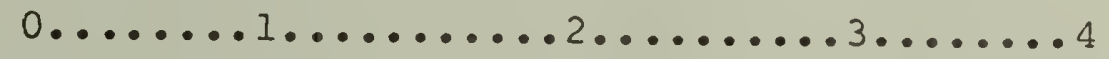

\begin{tabular}{rrrrrr} 
& Never & Rarely & Sometimes & Often & Always \\
Age & 12.5 & 28.1 & 25.0 & 1.8 .8 & 15.6 \\
& 6.3 & 15.6 & 12.5 & 37.5 & 28.1 \\
\multirow{2}{*}{ Ed } & 9.4 & 21.9 & 18.8 & 28.1 & 21.9 \\
& 12.5 & 15.6 & 18.8 & 18.8 & 34.4 \\
\multirow{2}{*}{ Sex } & 6.3 & 28.1 & 18.8 & 37.5 & 9.4 \\
& 9.4 & 21.9 & 18.8 & 28.1 & 21.9 \\
& 15.6 & 25.0 & 10.9 & 31.3 & 6.3 \\
& 3.1 & 18.8 & 7.8 & 25.0 & 37.5 \\
& 9.4 & 21.9 & 18.8 & 28.1 & 21.9
\end{tabular}


18. How often do you write yourself reminder notes? Ed $-x^{2}(4)=10.04, p<.05$

\begin{tabular}{|c|c|c|c|c|}
\hline Never & Rarely & Sometimes & Often & A.lways \\
\hline $\begin{array}{r}9.7 \\
13.8 \\
11.7\end{array}$ & $\begin{array}{l}41.9 \\
20.7 \\
31.7\end{array}$ & $\begin{array}{l}12.9 \\
20.7 \\
16.7\end{array}$ & $\begin{array}{l}25.8 \\
41.4 \\
33.3\end{array}$ & $\begin{array}{l}9.7 \\
3.4 \\
6.7\end{array}$ \\
\hline $\begin{array}{l}12.5 \\
10.7 \\
11.7\end{array}$ & $\begin{array}{l}21 \cdot 9 \\
42 \cdot 9 \\
31.7\end{array}$ & $\begin{array}{r}9.4 \\
25.0 \\
16.7\end{array}$ & $\begin{array}{l}43.8 \\
21.4 \\
33.3\end{array}$ & $\begin{array}{c}12.5 \\
0 \\
6.7\end{array}$ \\
\hline $\begin{array}{r}16.1 \\
6.9 \\
11.7\end{array}$ & $\begin{array}{l}25.8 \\
37.9 \\
31.7\end{array}$ & $\begin{array}{l}16.1 \\
17.2 \\
16.7\end{array}$ & $\begin{array}{l}35.5 \\
31.0 \\
33.3\end{array}$ & $\begin{array}{l}6.5 \\
6.9 \\
6.7\end{array}$ \\
\hline
\end{tabular}

19. How often do you ask other people to remind you of something? Age $-x^{2}(4)=8.02, p<.1 ; \operatorname{sex}-x^{2}(4)=11.2$, $\mathrm{p}<.05$.

\begin{tabular}{|c|c|c|c|c|c|}
\hline & $\begin{array}{r}0 . \\
\text { Never }\end{array}$ & $\begin{array}{l}\text { •..... } \\
\text { Rarely }\end{array}$ & $\begin{array}{l}\text {........... } \\
\text { sometimes }\end{array}$ & $\begin{array}{l}\cdots 3 \cdot \cdots \\
\text { often }\end{array}$ & $\begin{array}{l}\text {. } 4 \\
\text { Always }\end{array}$ \\
\hline Age & $\begin{array}{r}12.5 \\
3.1 \\
7.8\end{array}$ & $\begin{array}{l}40.6 \\
28.1 \\
34.4\end{array}$ & $\begin{array}{l}40.6 \\
31.5 \\
39.1\end{array}$ & $\begin{array}{r}6.3 \\
28.1 \\
17.2\end{array}$ & $\begin{array}{c}0 \\
3.1 \\
1.6\end{array}$ \\
\hline$E d$ & $\begin{array}{l}6.3 \\
9.4 \\
7.8\end{array}$ & $\begin{array}{l}34.4 \\
34.4 \\
34.4\end{array}$ & $\begin{array}{l}34.4 \\
43.8 \\
39.1\end{array}$ & $\begin{array}{l}21.9 \\
12.5 \\
17.2\end{array}$ & $\begin{array}{c}3.1 \\
0 \\
1.6\end{array}$ \\
\hline Sex & $\begin{array}{l}6.3 \\
9.4 \\
7.8\end{array}$ & $\begin{array}{l}18.8 \\
50.0 \\
34.4\end{array}$ & $\begin{array}{l}46.9 \\
31.3 \\
39.1\end{array}$ & $\begin{array}{r}28.1 \\
E .3 \\
17.2\end{array}$ & $\begin{array}{c}0 \\
3.1 \\
1.6\end{array}$ \\
\hline
\end{tabular}

20. How often do you mentally repeat something you are trying to remember?

$0 \ldots \ldots \ldots . \ldots \ldots \ldots . \ldots \ldots . \ldots . \ldots . \ldots 4$

$\begin{array}{ccccc}\text { Never } & \text { Rarely } & \text { Sometimes } & \text { Often } & \text { Always } \\ 3.1 & 21.9 & 31.3 & 37.5 & 6.3 \\ 0 & 18.8 & 37.5 & 40.6 & 3.1 \\ 1.6 & 20.3 & 34.4 & 39.1 & 4.7 \\ 3.1 & 12.5 & 34.4 & 43.8 & 6.3 \\ 0 & 28.1 & 34.4 & 34.4 & 3.1 \\ 1.6 & 20.3 & 34.4 & 39.1 & 4.7 \\ 3.1 & 25.0 & 28.1 & 37.5 & 6.3 \\ 0 & 15.6 & 40.6 & 40.6 & 3.1 \\ 1.6 & 20.3 & 34.4 & 39.1 & 4.7\end{array}$


21. How often to you particularly try to concentrate on something you want to remember later? Age $-x^{2}(3)=6.46$, $p<\cdot 1$.

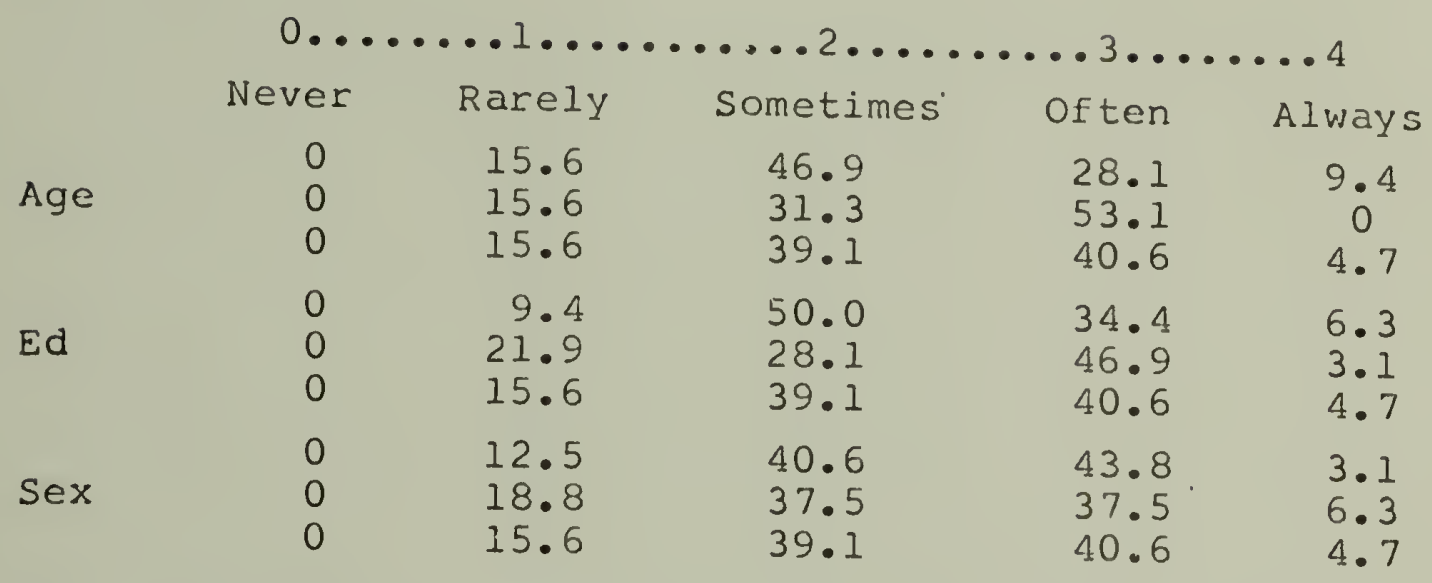

22. How often do you try to relate something you want to remember to something else, thinking this will increase the likelihood of your remembering later?

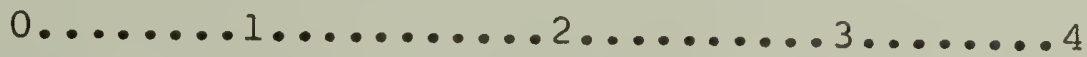

\begin{tabular}{cccccc} 
& Never & Rarely & Sometimes & Often & Always \\
Age & 6.3 & 12.5 & 34.4 & 40.6 & 6.3 \\
& 3.1 & 21.9 & 37.5 & 37.5 & 0 \\
& 4.7 & 17.2 & 35.9 & 39.1 & 3.1 \\
\multirow{3}{*}{ Ed } & 3.1 & 15.6 & 34.4 & 46.9 & 0 \\
& 6.3 & 18.8 & 37.5 & 31.3 & 6.3 \\
& 4.7 & 17.2 & 35.9 & 39.1 & 3.1 \\
Sex & 6.3 & 15.6 & 40.6 & 34.4 & 3.1 \\
& 3.1 & 18.8 & 31.3 & 43.8 & 3.1 \\
& 4.7 & 17.2 & 35.9 & 39.1 & 3.1
\end{tabular}

23. How often do you deliberately try to fix something in memory by relating it to other information?

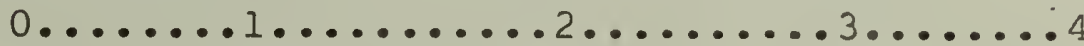

\begin{tabular}{crcccc} 
& Never & Rarely & Sometimes & Often & Always \\
& 9.4 & 15.6 & 34.4 & 34.4 & 6.3 \\
Age & 6.5 & 19.4 & 35.5 & 32.3 & 6.5 \\
& 7.9 & 17.5 & 34.9 & 33.3 & 6.3 \\
\multirow{3}{*}{ Ed } & 6.5 & 12.9 & 38.7 & 38.7 & 3.2 \\
& 9.4 & 21.9 & 31.3 & 28.1 & 9.4 \\
& 7.9 & 17.5 & 34.9 & 33.3 & 6.3 \\
Sex & 6.3 & 25.0 & 34.4 & 28.1 & 6.3 \\
& 9.7 & 9.7 & 35.5 & 38.7 & 6.5 \\
& 7.9 & 17.5 & 34.9 & 33.3 & 6.3
\end{tabular}


24. How often do you consciously attempt to reconstruct the days events in order to remember something?

\begin{tabular}{|c|c|c|c|c|c|}
\hline & Never & Rarely & Sometimes & Often & Always \\
\hline Age & $\begin{array}{c}0 \\
6.3 \\
3.1\end{array}$ & $\begin{array}{l}37.5 \\
21.9 \\
29.7\end{array}$ & $\begin{array}{l}40.6 \\
50.0 \\
45.3\end{array}$ & $\begin{array}{l}15.6 \\
21.9 \\
18.8\end{array}$ & $\begin{array}{c}6.3 \\
0 \\
3.1\end{array}$ \\
\hline $\mathrm{Ed}$ & $\begin{array}{c}0 \\
6.3 \\
3.1\end{array}$ & $\begin{array}{l}21.9 \\
37.5 \\
29.7\end{array}$ & $\begin{array}{l}56.3 \\
34.4 \\
45.3\end{array}$ & $\begin{array}{l}15.6 \\
21.9 \\
18.8\end{array}$ & $\begin{array}{c}6.3 \\
0 \\
3.1\end{array}$ \\
\hline Sex & $\begin{array}{l}3.1 \\
3.1 \\
3.1\end{array}$ & $\begin{array}{l}34.4 \\
25.0 \\
29.7\end{array}$ & $\begin{array}{l}37.5 \\
53.1 \\
45.3\end{array}$ & $\begin{array}{l}21.9 \\
15.6 \\
18.8\end{array}$ & $\begin{array}{l}3.1 \\
3.1 \\
3.1\end{array}$ \\
\hline
\end{tabular}

25. How often do you try to think of things that relate to something you forgot, hoping it will bring it to mind?

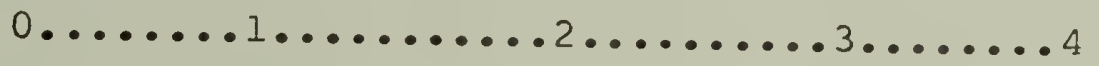

$\begin{array}{ccccc}\text { Never } & \text { Rarely } & \text { Sometimes } & \text { Often } & \text { Always } \\ 0 & 9.4 & 50.0 & 27.5 & 3.1 \\ 6.5 & 22.6 & 32.3 & 35.5 & 3.2 \\ 3.2 & 15.9 & 41.3 & 36.5 & 3.2 \\ 0 & 18.8 & 40.6 & 37.5 & 3.1 \\ 6.5 & 12.9 & 41.9 & 35.5 & 3.2 \\ 3.2 & 15.9 & 41.3 & 36.5 & 3.2 \\ 3.1 & 12.5 & 50.0 & 28.1 & 6.3 \\ 3.2 & 19.4 & 32.3 & 45.2 & 0 \\ 3.2 & 15.9 & 41.3 & 36.5 & 3.2\end{array}$

26. How often do you engage in deliberate attempts to remember information?

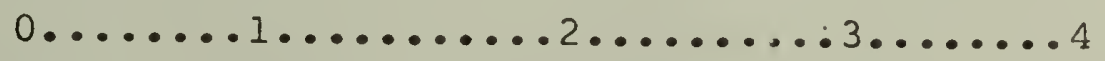

Never

Rarely

Sometimes

Often

Always

Age

$0 \quad 12.5$

$3.1 \quad 25.0$

$1.6 \quad 18.8$

$\begin{array}{ccc} & 0 & 25.0 \\ \text { Ed } & 3.1 & 12.5 \\ & 1.6 & 18.8 \\ & 0 & 18.8 \\ \text { Sex } & 3.1 & 18.8 \\ & 1.6 & 18.8\end{array}$

37.5
34.4
35.9
28.1
43.8
35.9
37.5
34.4
35.9

43.8

37.5

6.3

40.6

3.1

40.6

40.6

6.3

40.6

40.6

40.6

40.6 
27. How often do you look up things you can not remember? Ed $-x^{2}(4)=7.82, p<.1$.

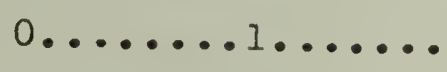

\begin{tabular}{cccccc} 
& Never & Rarely & Sometimes & Often & Always \\
Age & 0 & 25.0 & 40.6 & 25.0 & 9.4 \\
& 3.2 & 9.7 & 38.7 & 41.9 & 6.5 \\
\multirow{4}{*}{ Ed } & 1.6 & 17.5 & 39.7 & 33.3 & 7.9 \\
& 0 & 22.6 & 51.6 & 19.4 & 6.5 \\
& 3.1 & 12.5 & 28.1 & 46.9 & 9.4 \\
\multirow{4}{*}{ Sex } & 1.6 & 17.5 & 39.7 & 33.3 & 7.9 \\
& 0 & 15.6 & 46.9 & 28.1 & 9.4 \\
& 3.2 & 19.4 & 32.3 & 38.7 & 6.5 \\
& 1.6 & 17.5 & 39.7 & 33.3 & 7.9
\end{tabular}

28. How often do you ask other people things you can not remember? Age $-\mathrm{x}^{2}(3)=6.30, \mathrm{p}<.1$.

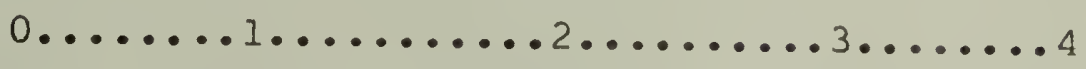

$\begin{array}{ccccc}\text { Never } & \text { Rarely } & \text { Sometimes } & \text { Often } & \text { Always } \\ 0 & 34.4 & 21.9 & 34.4 & 9.4 \\ 0 & 19.4 & 41.9 & 38.7 & 0 \\ 0 & 27.0 & 31.7 & 36.5 & 4.8 \\ 0 & 25.0 & 28.1 & 43.8 & 3.1 \\ 0 & 29.0 & 35.5 & 29.0 & 6.5 \\ 0 & 27.0 & 31.7 & 36.5 & 4.8 \\ 0 & 16.1 & 38.7 & 38.7 & 6.5 \\ 0 & 37.5 & 25.0 & 34.4 & 3.1 \\ 0 & 27.0 & 31.7 & 36.5 & 4.8\end{array}$


Please circle the number above the response that is closest to your choice.

29. Do you remember things well -- are you a good rememberer? Ed $-\mathrm{x}^{2}(2)=5.15, \mathrm{p}<.08$.

\begin{tabular}{|c|c|c|c|}
\hline & \multicolumn{3}{|c|}{$0 \ldots \ldots \ldots \ldots \ldots \ldots \ldots \ldots \ldots$} \\
\hline & No & Unsure & Yes \\
\hline Age & $\begin{array}{r}6.3 \\
18.8 \\
12.5\end{array}$ & $\begin{array}{l}25.0 \\
25.0 \\
25.0\end{array}$ & $\begin{array}{l}68.8 \\
56.3 \\
62.5\end{array}$ \\
\hline Ed & $\begin{array}{r}21.9 \\
3.1 \\
12.5\end{array}$ & $\begin{array}{l}21.9 \\
28.1 \\
25.0\end{array}$ & $\begin{array}{l}56.3 \\
68.8 \\
62.5\end{array}$ \\
\hline Sex & $\begin{array}{r}9.4 \\
15.6 \\
12.5\end{array}$ & $\begin{array}{l}28.1 \\
21.9 \\
25.0\end{array}$ & $\begin{array}{l}62.5 \\
62.5 \\
62.5\end{array}$ \\
\hline
\end{tabular}

30. Can you remember better than your friends?

\begin{tabular}{|c|c|c|c|}
\hline \multicolumn{4}{|c|}{$0 \ldots \ldots \ldots \ldots \ldots 1 \ldots \ldots \ldots \ldots}$. \\
\hline & No & Unsure & Yes \\
\hline Age & $\begin{array}{l}12.5 \\
21.9 \\
17.2\end{array}$ & $\begin{array}{l}43.8 \\
56.3 \\
50.0\end{array}$ & $\begin{array}{l}43.8 \\
21.9 \\
32.8\end{array}$ \\
\hline$E \mathrm{~d}$ & $\begin{array}{r}25.0 \\
9.4 \\
17.2\end{array}$ & $\begin{array}{l}46.9 \\
53.1 \\
50.0\end{array}$ & $\begin{array}{l}28.1 \\
37.5 \\
32.8\end{array}$ \\
\hline Sex & $\begin{array}{l}15.6 \\
18.8 \\
1.7 .2\end{array}$ & $\begin{array}{l}59.4 \\
40.6 \\
50.0\end{array}$ & $\begin{array}{l}25.0 \\
40.6 \\
32.8\end{array}$ \\
\hline
\end{tabular}

31. When you can not remember something do you find it upsetting?

$\begin{array}{cccc} & \text { No } & \text { Unsure } & \text { Yes } \\ \text { Age } & 15.6 & 6.3 & 78.1 \\ & 28.1 & 6.3 & 65.6 \\ & 21.9 & 6.3 & 71.6 \\ \text { Ed } & 15.6 & 3.1 & 81.3 \\ & 28.1 & 9.4 & 62.5 \\ & 21.9 & 6.3 & 71.9 \\ \text { Sex } & 28.1 & 9.4 & 62.5 \\ & 15.6 & 3.1 & 81.3 \\ & 21.9 & 6.3 & 71.9\end{array}$


32. Do you have more difficulty remembering things when you are tired? Age $-\mathrm{x}^{2}(3)=4.73, \mathrm{p}<. .1$.

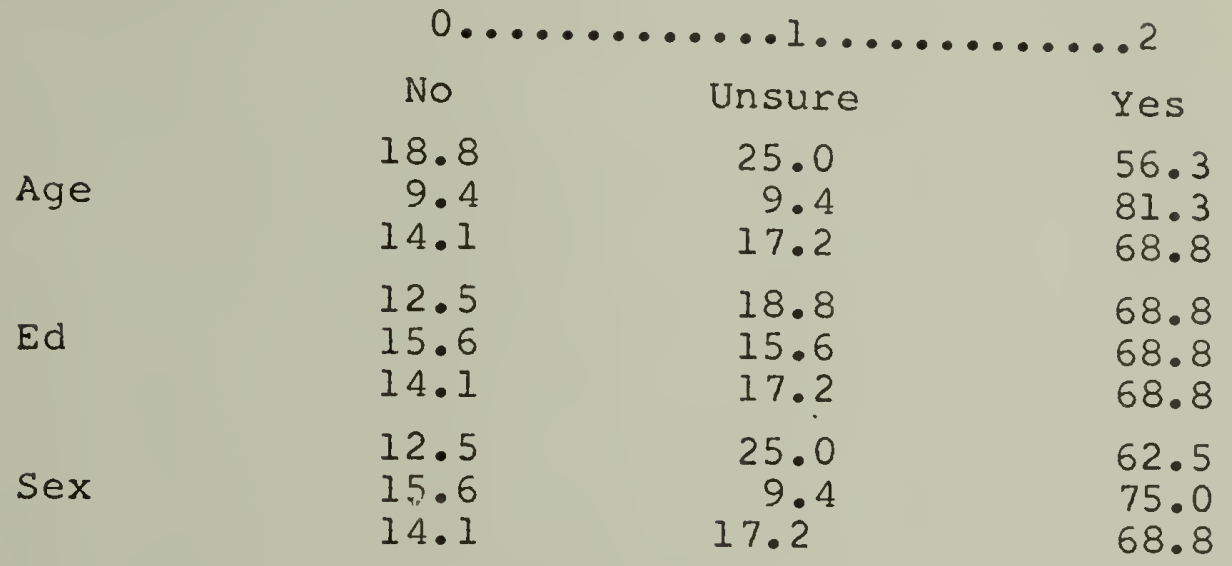

33. Do you have more difficulty remembering things when you are pressured? $E d-x^{2}(2)=7.22, p<.05$.

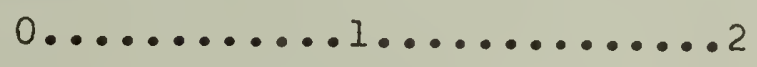

$\begin{array}{cccl} & \text { No } & \text { Unsure } & \text { Yes } \\ \text { Age } & 25.0 & 25.0 & 50.0 \\ & 31.3 & 12.5 & 56.3 \\ & 28.1 & 18.8 & 53.1 \\ \text { Ed } & 28.1 & 6.3 & 65.6 \\ & 28.1 & 31.3 & 40.6 \\ & 28.1 & 18.8 & \\ \text { Sex } & 21.9 & 18.8 & 59.4 \\ & 34.4 & 18.8 & 46.9 \\ & 28.1 & 18.8 & 53.1\end{array}$

34. Do you have more difficulty remembering things when you are anxious? Sex $-\mathrm{x}^{2}(2)=4.62, \mathrm{p}<.1$.

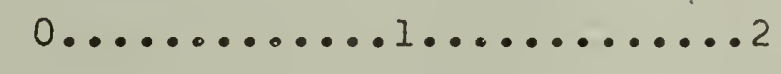

$\begin{array}{cccc} & \text { No } & \text { Unsure } & \text { Yes } \\ \text { Age } & 31.3 & 15.6 & 53.1 \\ & 22.6 & 29.0 & 48.4 \\ & 27.0 & 22.2 & 50.8 \\ \text { Ed } & 25.0 & 15.6 & 59.4 \\ & 29.0 & 29.0 & 41.9 \\ & 27.0 & 22.2 & 50.8 \\ \text { Sex } & 25.0 & 12.5 & 62.5 \\ & 29.0 & 32.3 & 38.7 \\ & 27.0 & 22.2 & 50.8\end{array}$


35. Are you more likely to forget things when your mind is preoccupied?

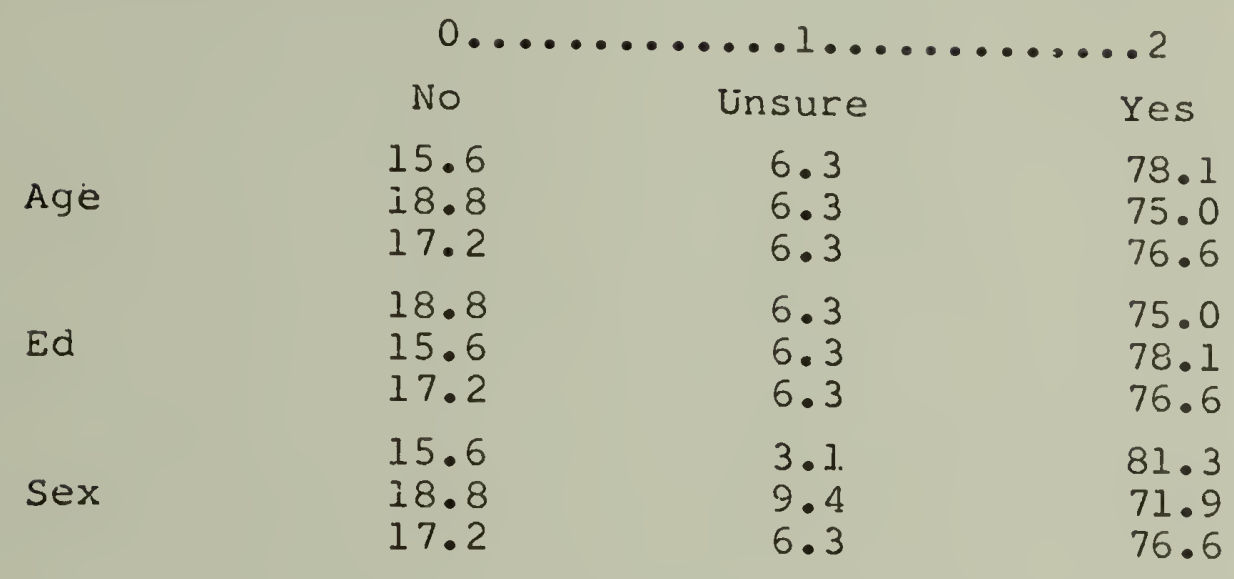

36. Over the years have you become more aware of your memory?

$\begin{array}{cccc} & 0 \ldots \ldots \ldots \ldots \ldots \ldots .2 \\ & \text { No } & \text { Unsure } & \text { Yes } \\ \text { Age } & 16.1 & 16.1 & 67.7 \\ & 6.5 & 6.5 & 87.1 \\ \text { Ed } & 11.3 & 11.3 & 77.4 \\ & 10.0 & 10.0 & 80.0 \\ \text { Sex } & 12.5 & 12.5 & 75.0 \\ & 11.3 & 11.3 & 77.4 \\ & 16.7 & 6.7 & 76.7 \\ & 6.3 & 15.6 & 78.1 \\ & 11.3 & 11.3 & \end{array}$

37. Over the years have you noticed any changes in your memory? Age $-x^{2}(2)=6.94, p<.05 ; \operatorname{sex}-x^{2}(2)=7.47$, $\mathrm{p}<.05$.

\begin{tabular}{cccc} 
& \multicolumn{2}{c}{$0 \ldots \ldots \ldots \ldots . . \ldots \ldots \ldots .2$} \\
& No & Unsure & Yes \\
Age & 34.4 & 12.5 & 53.1 \\
& 12.9 & 3.2 & 83.9 \\
Ed & 23.8 & 7.9 & 68.3 \\
& 25.8 & 6.5 & 67.7 \\
Sex & 21.9 & 9.4 & 68.8 \\
& 23.8 & 7.9 & 68.3 \\
& 29.0 & 16.1 & 54.8 \\
& 18.8 & 0 & 81.3 \\
& 23.8 & 7.9 & 68.3
\end{tabular}


38. Do you use memory aids more often than you previous ly did?

$\begin{array}{cccc} & & & \\ & & & \\ & \text { No } & \text { Unsure } & \text { Yes } \\ \text { Age } & 46.9 & 9.4 & 43.8 \\ & 43.3 & 6.7 & 50.0 \\ \text { Ed } & 45.2 & 8.7 & 46.8 \\ & 38.7 & 3.2 & 58.1 \\ \text { Sex } & 51.6 & 12.9 & 35.5 \\ & 45.2 & 8.1 & 46.8 \\ & 45.2 & 9.7 & 45.2 \\ & 45.2 & 6.5 & 48.4 \\ & 45.2 & 8.1 & \end{array}$

39. Over the years has your memory improved? Age $x^{2}(2)=4.84, p<.09$.

$\begin{array}{ccc}0 \ldots \ldots \ldots \ldots \ldots . . \ldots \ldots \ldots & \text { Yes } \\ \text { No } & \text { Unsure } & 31.3 \\ 8.1 & 40.6 & 15.1 \\ 4.8 & 29.0 & 23.8 \\ 1.3 & 34.9 & 22.6 \\ 5.2 & 32.3 & 25.0 \\ 7.5 & 37.5 & 23.8 \\ 41.3 & 34.9 & 19.4 \\ 45.2 & 35.5 & 28.1 \\ 37.5 & 34.4 & \end{array}$

40. Over the years has your memory become worse? Age $x^{2}(2)=11.90, p<.01$.

$\begin{array}{ccc}0 \ldots \ldots \ldots \ldots \ldots .2 \\ \text { No } & \text { Unsure } & \text { Yes } \\ 65.6 & 25.0 & 9.4 \\ 29.0 & 25.8 & 45.2 \\ 47.6 & 25.4 & 27.0 \\ 48.4 & 29.0 & 22.6 \\ 46.9 & 21.9 & 31.3 \\ 47.6 & 25.4 & 27.0 \\ 41.9 & 32.3 & 25.8 \\ 53.1 & 18.8 & 28.1 \\ 47.6 & 25.4 & 27.0\end{array}$


41. Do you think your memory will change as you get older? Ed $-x^{2}(2)=5.69, p<.06$.

\begin{tabular}{|c|c|c|c|}
\hline \multicolumn{4}{|c|}{$0 \ldots \ldots \ldots \ldots \ldots \ldots \ldots \ldots$} \\
\hline & No & Unsure & Yes \\
\hline Age & $\begin{array}{r}12.5 \\
6.5 \\
9.5\end{array}$ & $\begin{array}{l}37.5 \\
32.3 \\
34.9\end{array}$ & $\begin{array}{l}50.0 \\
61.3 \\
55.6\end{array}$ \\
\hline Ed & $\begin{array}{r}16.1 \\
3.1 \\
9.5\end{array}$ & $\begin{array}{l}41.9 \\
28.1 \\
34.9\end{array}$ & $\begin{array}{l}41.9 \\
68.8 \\
55.6\end{array}$ \\
\hline Sex & $\begin{array}{l}9.7 \\
9.4 \\
9.5\end{array}$ & $\begin{array}{l}29.0 \\
40.6 \\
34.9\end{array}$ & $\begin{array}{l}61.3 \\
50.0 \\
55.6\end{array}$ \\
\hline
\end{tabular}

42. Do you think your memory will get better when you get older? Age $-x^{2}(2)=10.33, p<.01 ; \operatorname{sex}-x^{2}(2)=$ $6.51, \mathrm{p}<.05$.

\begin{tabular}{|c|c|c|c|}
\hline & \multicolumn{3}{|c|}{$0 \ldots \ldots \ldots \ldots \ldots \ldots \ldots \ldots \ldots \ldots$} \\
\hline & No & Unsure & Yes \\
\hline Age & $\begin{array}{l}37.5 \\
77.4 \\
57.1\end{array}$ & $\begin{array}{l}50.0 \\
19.4 \\
34.9\end{array}$ & $\begin{array}{r}12.5 \\
3.2 \\
7.9\end{array}$ \\
\hline Ed & $\begin{array}{l}54.8 \\
59.4 \\
57.1\end{array}$ & $\begin{array}{l}35.5 \\
34.4 \\
34.9\end{array}$ & $\begin{array}{l}9.7 \\
6.3 \\
7.9\end{array}$ \\
\hline $\operatorname{sex}$ & $\begin{array}{l}71.0 \\
43.8 \\
57.1\end{array}$ & $\begin{array}{l}19.4 \\
50.0 \\
34.9\end{array}$ & $\begin{array}{l}9.7 \\
6.3 \\
7.9\end{array}$ \\
\hline
\end{tabular}

43. Do you thank your memory will get worse when you get older? Age $-x^{2}(2)=8.62, p<.05$; Ed $-x^{2}(2)=$ $4.95, \mathrm{p}<.09$.

$\begin{array}{cccc} & 0 \ldots \ldots \ldots \ldots \ldots . \ldots \ldots \\ & \text { No } & \text { Unsure } & \text { Yes } \\ \text { Age } & 25.0 & 53.1 & 21.9 \\ & 12.9 & 29.0 & 58.1 \\ & 19.0 & 41.3 & 39.7 \\ \text { Ed } & 29.0 & 41.9 & 29.0 \\ & 9.4 & 40.6 & 50.0 \\ \text { Sex } & 19.0 & 41.3 & 39.7 \\ & 16.1 & 35.5 & 48.1 \\ & 21.9 & 46.9 & 31.3 \\ & 12.0 & 41.3 & 39.7\end{array}$


44. Do you think your memory will always stay about as it is now?

$\begin{array}{cccc} & 0 \ldots \ldots \ldots \ldots \ldots \\ & \text { No } & \text { Unsure } & \text { Yes } \\ \text { Age } & 37.5 & 46.9 & 15.6 \\ & 58.1 & 29.0 & 12.9 \\ \text { Ed } & 46.6 & 38.1 & 14.3 \\ & 41.9 & 41.9 & 16.1 \\ & 53.1 & 34.4 & 12.5 \\ \text { Sex } & 47.6 & 38.1 & 14.3 \\ & 58.1 & 32.3 & 9.7 \\ & 37.5 & 43.8 & 18.8 \\ & 47.6 & 38.1 & 14.3\end{array}$

45. Do you think you will forget things more easily when you get older? Age $-x^{2}(2)=10.12, p<.01$.

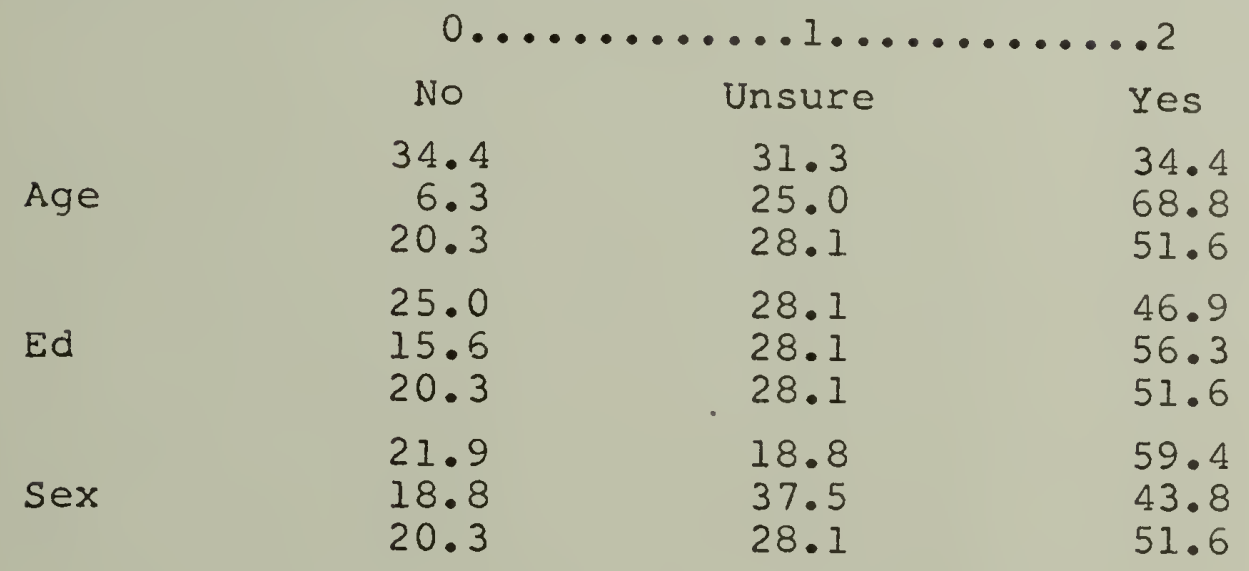

46. Do you think you will remember more details as you get older?

\begin{tabular}{cccc} 
& \multicolumn{2}{c}{$0 \ldots \ldots \ldots \ldots \ldots . \ldots \ldots$} \\
& No & Unsure & Yes \\
Age & 46.9 & 31.3 & 21.9 \\
& 62.5 & 21.9 & 15.6 \\
Ed & 54.7 & 26.6 & 18.8 \\
& 56.3 & 21.9 & 21.9 \\
Sex & 53.1 & 31.3 & 15.6 \\
& 54.7 & 26.6 & 18.8 \\
& 53.1 & 28.1 & 18.8 \\
& 56.3 & 25.0 & 18.8 \\
& 54.7 & 26.6 & 18.8
\end{tabular}


47. Do you consciously try to memorize things more than you used to?

$\begin{array}{cccc} & 0 \ldots \ldots \ldots \ldots \ldots \ldots .2 \\ & \text { No } & \text { Unsure } & \text { Yes } \\ \text { Age } & 56.3 & 12.5 & 31.3 \\ & 53.1 & 12.5 & 34.4 \\ \text { Ed } & 54.7 & 12.5 & 32.8 \\ & 43.8 & 12.5 & 43.8 \\ \text { Sex } & 65.6 & 12.5 & 21.9 \\ & 54.7 & 12.5 & 32.8 \\ & 53.1 & 15.6 & 31.3 \\ & 56.3 & 9.4 & 34.4 \\ & 54.7 & 12.5 & 32.8\end{array}$

48. Do you remember some kinds of things better than others?

$\begin{array}{cccc} & 0 \ldots \ldots \ldots \ldots \ldots .2 \\ & \text { No } & \text { Unsure } & \text { Yes } \\ \text { Age } & 6.3 & 3.1 & 90.6 \\ & 3.1 & 3.1 & 93.8 \\ \text { Ed } & 4.7 & 3.1 & 92.2 \\ & 3.1 & 0 & 96.9 \\ \text { Sex } & 6.3 & 6.3 & 87.5 \\ & 4.7 & 3.1 & 92.2 \\ & 3.1 & 0 & 96.9 \\ & 6.3 & 6.3 & 87.5 \\ & 4.7 & 3.1 & 92.2\end{array}$

49. Do you find it easier to remember organized things than unorganized things? Age $-x^{2}(2)=7.28, p<.05$.

$\begin{array}{cccc} & 0 \ldots \ldots \ldots \ldots \ldots . . \ldots \ldots \ldots \\ & \text { No } & \text { Unsure } & \text { Yes } \\ \text { Age } & 21.3 & 12.5 & 56.3 \\ & 6.3 & 9.4 & 84.4 \\ \text { Ed } & 18.8 & 10.9 & 70.3 \\ & 25.0 & 12.5 & 62.5 \\ & 12.5 & 9.4 & 78.1 \\ \text { Sex } & 18.8 & 10.9 & 70.3 \\ & 15.6 & 9.4 & 75.0 \\ & 21.9 & 12.5 & 65.6 \\ & 18.8 & 10.9 & 70.3\end{array}$


50. Is it easier to remember visual things than verbal things? Sex $-x^{2}(2)=4.74, p<.1$.

\begin{tabular}{|c|c|c|}
\hline No & Unsure & Yes \\
\hline $\begin{array}{l}1.9 \\
8.8 \\
0.3\end{array}$ & $\begin{array}{r}21.9 \\
6.3 \\
14.1\end{array}$ & $\begin{array}{l}56.3 \\
75.0 \\
65.6\end{array}$ \\
\hline $\begin{array}{l}5.6 \\
5.0 \\
0.3\end{array}$ & $\begin{array}{l}15.6 \\
12.5 \\
14.1\end{array}$ & $\begin{array}{l}68.8 \\
62.5 \\
65.6\end{array}$ \\
\hline 0.3 & $\begin{array}{l}15.6 \\
12.5 \\
14.1\end{array}$ & $\begin{array}{l}75.0 \\
56.3 \\
65.6\end{array}$ \\
\hline
\end{tabular}

51. Do you find it easier to remember bizzare things than usual things?

$\begin{array}{cccc} & 0 . \ldots \ldots \ldots \ldots \ldots \ldots . . \ldots \ldots \ldots \\ & \text { No } & \text { Unsure } & \text { Yes } \\ \text { Age } & 21.9 & 15.6 & 62.5 \\ & 18.8 & 25.0 & 56.3 \\ \text { Ed } & 20.3 & 20.3 & 59.4 \\ & 28.1 & 15.6 & 56.3 \\ \text { Sex } & 12.5 & 25.0 & 62.5 \\ & 20.3 & 20.3 & 59.4 \\ & 15.6 & 18.8 & 65.6 \\ 52.0 & 21.9 & 53.1 \\ & 25.0 & 20.3 & 59.4\end{array}$

52. Do you find it easier to remember things you are most interested in?

$\begin{array}{cccc} & 0 \ldots \ldots \ldots \ldots \ldots .1 \ldots \ldots \ldots \ldots & \\ & \text { No } & \text { Unsure } & \text { Yes } \\ \text { Age } & 6.3 & 12.5 & 81.3 \\ & 0 & 3.1 & 96.9 \\ \text { Ed } & 3.1 & 7.8 & 89.1 \\ & 0 & 3.1 & 96.9 \\ \text { Sex } & 6.3 & 12.5 & 81.3 \\ & 3.1 & 7.8 & 89.1 \\ & 3.1 & 6.3 & 90.6 \\ & 3.1 & 9.4 & 87.5 \\ & 3.1 & 7.8 & 89.1\end{array}$


53. Do you find it easier to remember concrete things than abstract things? Age $-x^{2}(2)=9.35, p<.01$.

$\begin{array}{ccc}0 \ldots \ldots \ldots \ldots \ldots . . \ldots \ldots \ldots \\ \text { No } & \text { Unsure } & \text { Yes } \\ 46.9 & 25.0 & 28.1 \\ 18.8 & 15.6 & 65.6 \\ 32.8 & 20.3 & 46.9 \\ 25.0 & 18.8 & 56.3 \\ 40.6 & 21.9 & 37.5 \\ 32.8 & 20.3 & 46.9 \\ 21.9 & 28.1 & 50.0 \\ 43.8 & 12.5 & 43.8 \\ 32.8 & 20.3 & \end{array}$

54. Do you find it easier to remember things that are related to each other than things that are not related to each other?

\begin{tabular}{crcc} 
& \multicolumn{2}{c}{$0 \ldots \ldots \ldots \ldots \ldots \ldots . \ldots \ldots \ldots$} \\
& No & Unsure & Yes \\
Age & 12.5 & 25.0 & 62.5 \\
& 6.3 & 15.6 & 78.1 \\
& 9.4 & 20.3 & 70.3 \\
Ed & 12.5 & 21.9 & 65.6 \\
& 6.3 & 18.8 & 75.0 \\
Sex & 9.4 & 20.3 & 70.3 \\
& 3.1 & 21.9 & 75.0 \\
& 15.6 & 18.8 & 65.6 \\
& 9.4 & 20.3 & 70.3
\end{tabular}

55. Are there some kinds of things that are really hard to remember?

\begin{tabular}{cccc} 
& \multicolumn{2}{c}{$0 \ldots \ldots \ldots \ldots \ldots . \ldots \ldots$} \\
& No & Unsure & Yes \\
Age & 6.3 & 15.6 & 78.1 \\
& 6.3 & 12.5 & 81.3 \\
Ed & 6.3 & 14.1 & 79.7 \\
& 6.3 & 6.3 & 87.5 \\
Sex & 6.3 & 21.9 & 71.9 \\
& 6.3 & 14.1 & 79.7 \\
& 0 & 15.6 & 84.4 \\
& 12.5 & 12.5 & 75.0 \\
& 6.3 & 14.1 &
\end{tabular}


56. Are you especially likely to forget unpleasant things?

\begin{tabular}{|c|c|c|c|}
\hline \multicolumn{4}{|c|}{$0 \ldots \ldots \ldots \ldots \ldots \ldots \ldots \ldots \ldots$} \\
\hline & No & Unsure & Yes \\
\hline Age & $\begin{array}{l}78.1 \\
68.8 \\
73.4\end{array}$ & $\begin{array}{l}12.5 \\
15.6 \\
14.1\end{array}$ & $\begin{array}{r}9.4 \\
15.6 \\
12.5\end{array}$ \\
\hline $\mathrm{Ed}$ & $\begin{array}{l}75.0 \\
71.9 \\
73.4\end{array}$ & $\begin{array}{r}9.4 \\
18.8 \\
14.1\end{array}$ & $\begin{array}{r}15.6 \\
9.4 \\
12.5\end{array}$ \\
\hline Sex & $\begin{array}{l}71.9 \\
75.0 \\
73.4\end{array}$ & $\begin{array}{l}12.5 \\
15.6 \\
14.1\end{array}$ & $\begin{array}{r}15.6 \\
9.4 \\
12.5\end{array}$ \\
\hline
\end{tabular}

57. Do you have more difficulty remembering details than generalities? Ed $-x^{2}(2)=9.17, p<.05$; sex $x^{2}(2)=7.30, p<.05$.

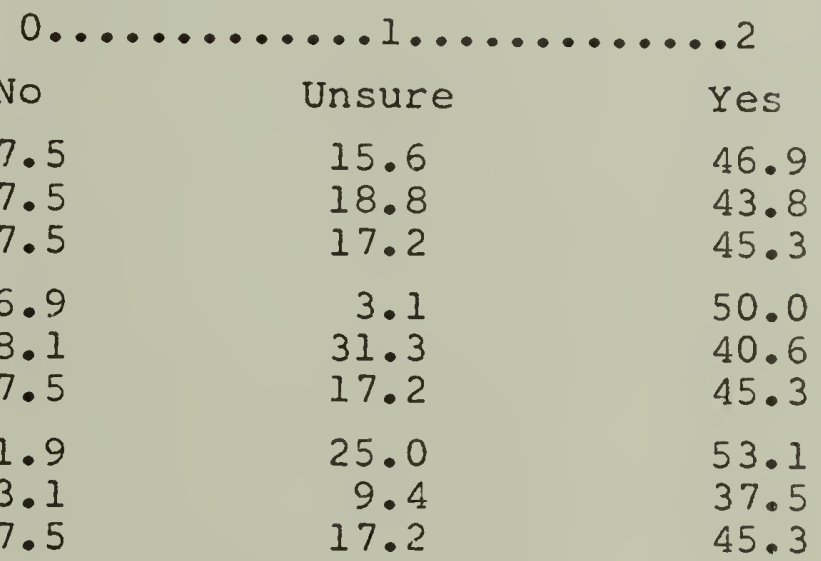

58. Do you find it more difficult to remember things you are not interested in? Age $-x^{2}(2)=5.20, p<.08$; sex $-x^{2}(2)=9.59, p<.01$.

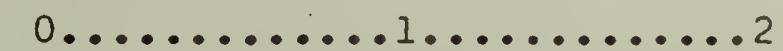

$\begin{array}{cccc} & \text { No } & \text { Unsure } & \text { Yes } \\ \text { Age } & 12.5 & 18.8 & 68.8 \\ & 6.3 & 3.1 & 90.6 \\ & 9.4 & 10.9 & 79.9 \\ \text { Ed } & 6.3 & 12.5 & 81.3 \\ & 12.5 & 9.4 & 78.1 \\ & 9.4 & 10.9 & 79.7 \\ \text { Sex } & 0 & 18.8 & 81.3 \\ & 18.8 & 3.1 & 78.1 \\ & 9.4 & 10.9 & 79.7\end{array}$


59. Do you find it more difficult to remember unfamiliar than familiar things? Ed $-x^{2}(2)=7.79, p<.05$.

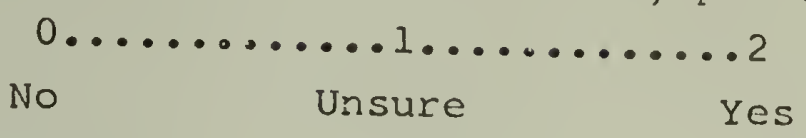

$\begin{array}{llll} & 37.5 & 21.9 & 40.6 \\ \text { Age } & 21.9 & 21.9 & 56.3 \\ & 29.7 & 21.9 & 48.4 \\ \text { Ed } & 21.9 & 12.5 & 65.6 \\ & 37.5 & 31.3 & 31.3 \\ & 29.7 & 21.9 & 48.4 \\ \text { Sex } & 21.9 & 21.9 & 56.3 \\ & 37.5 & 21.9 & 40.6 \\ & 29.7 & 21.9 & 48.4\end{array}$

60. Do you find it more difficult to remember things you do not really understand?

$0 \ldots \ldots . . \ldots . . . . . . . . .2$

$\begin{array}{cccc} & \text { No } & \text { Unsure } & \text { Yes } \\ \text { Age } & 9.4 & 9.4 & 81.3 \\ & 3.1 & 0 & 96.9 \\ & 6.3 & 4.7 & 89.1 \\ \text { Ed } & 12.5 & 3.1 & 84.4 \\ & 0 & 6.3 & 93.8 \\ \text { Sex } & 6.3 & 4.7 & 89.1 \\ & 9.4 & 6.3 & 84.4 \\ & 3.1 & 3.1 & 93.8 \\ & 6.3 & 4.7 & 89.1\end{array}$




$$
-399-
$$

Appendix C

Prediction, Confidence, Performance Correlations 


$$
-400-
$$

Correlations for Average Episodic and Semantic Memory Performance, Prediction, and Confidence For the Two Age Groups

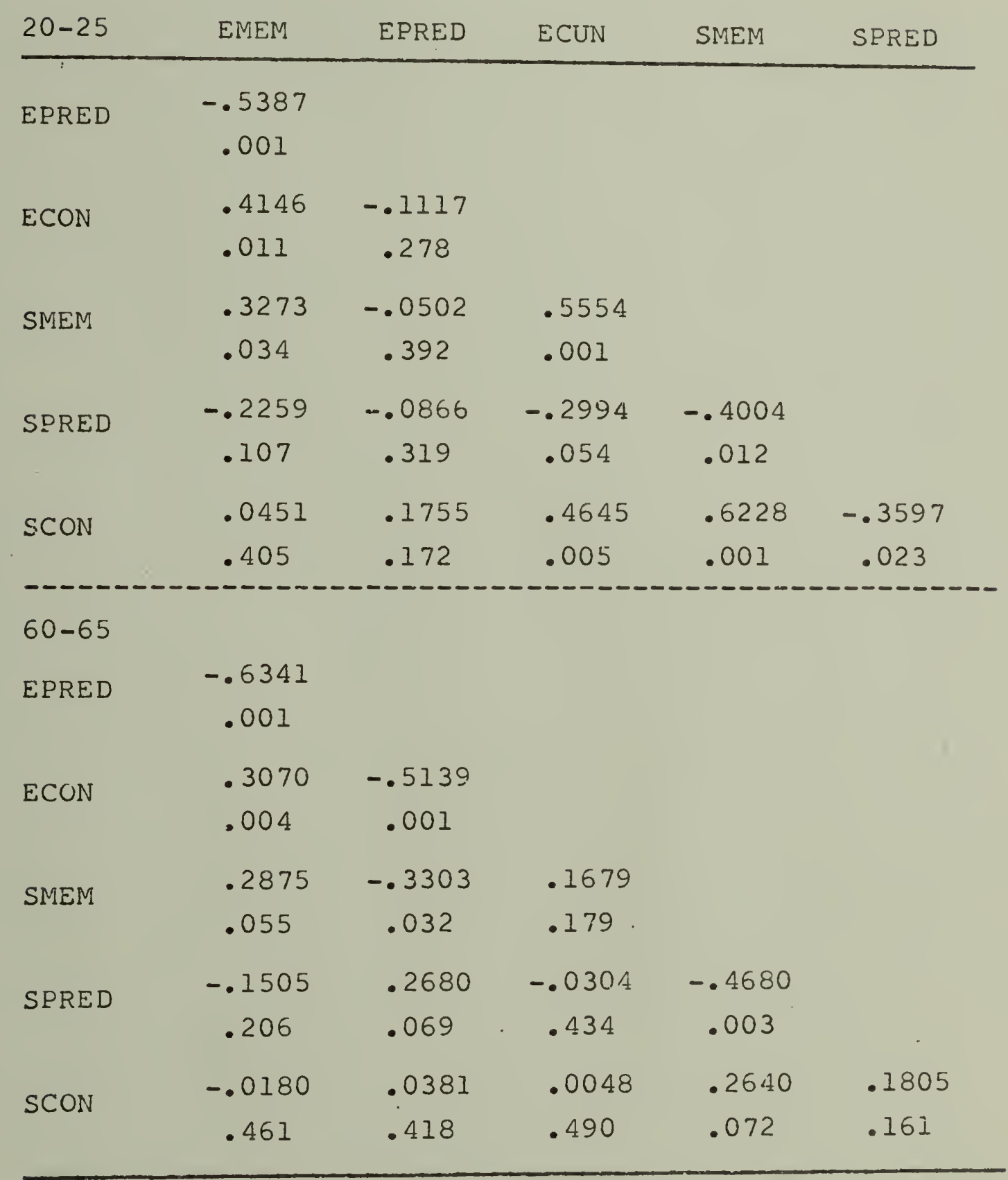


Correlations for Average Episodic and Semantic Memory Performance, Prediction, and Confidence For the Two Education Groups

\begin{tabular}{|c|c|c|c|c|c|}
\hline HS & EMEM & EPRED & ECUN & SMEM & SPRED \\
\hline \multirow{2}{*}{ EPRED } & -.6328 & & & & \\
\hline & .001 & & & & \\
\hline \multirow[t]{2}{*}{ ECON } & .2472 & -.3783 & & & \\
\hline & .090 & .018 & & & \\
\hline \multirow[t]{2}{*}{ SMEM } & -.0397 & .0821 & .1824 & & \\
\hline & .415 & .327 & .160 & & \\
\hline \multirow[t]{2}{*}{ SPRED } & .0531 & -.0310 & -.0265 & -.3091 & \\
\hline & .386 & .433 & .444 & .043 & \\
\hline \multirow[t]{2}{*}{ SCON } & -.0622 & .2391 & -.0221 & .3815 & .0872 \\
\hline & .370 & .098 & .453 & .017 & .320 \\
\hline \multicolumn{6}{|l|}{$\mathrm{Ph} \cdot \mathrm{D}$. } \\
\hline \multirow[t]{2}{*}{ EPRED } & -.5402 & & & & \\
\hline & .001 & & & & \\
\hline \multirow[t]{2}{*}{ ECON } & .4957 & -.4087 & & & \\
\hline & .002 & .011 & & & \\
\hline \multirow[t]{2}{*}{ SMEM } & .2399 & -.2482 & .2393 & & \\
\hline & .093 & .085 & .094 & & \\
\hline \multirow{2}{*}{ SPRED } & -.2534 & .0860 & -.709 & -.5061 & \\
\hline & .081 & .320 & .179 & .002 & . \\
\hline \multirow[t]{2}{*}{ SCON } & -.0009 & .0740 & .2790 & .0957 & -.0631 \\
\hline & .498 & .344 & .064 & .299 & .366 \\
\hline
\end{tabular}




$$
-402-
$$

Correlations for Average Episodic and Semantic Memory Performance, Prediction, and Confidence For the Two Sex Groups

\begin{tabular}{|c|c|c|c|c|c|}
\hline Males & EMEM & EPRED & ECON & SMEM & SPRED \\
\hline \multirow[t]{2}{*}{ EPRED } & -.6265 & & & & \\
\hline & .001 & & & & \\
\hline \multirow[t]{2}{*}{ ECON } & .4514 & -.2974 & & & \\
\hline & .005 & .052 & & & \\
\hline \multirow[t]{2}{*}{ SMEM } & .2277 & -.2072 & .2550 & & \\
\hline & .105 & .128 & .083 & & \\
\hline \multirow[t]{2}{*}{ SPRED } & -.2150 & .1411 & -.0230 & -.5352 & \\
\hline & .119 & .221 & .451 & .001 & \\
\hline \multirow[t]{2}{*}{ SCON } & .1160 & .0796 & .3229 & .3562 & -.0304 \\
\hline & .267 & .335 & .038 & .025 & .435 \\
\hline \multicolumn{6}{|c|}{ Females } \\
\hline \multirow[t]{2}{*}{ EPRED } & -.5636 & & & & \\
\hline & .001 & & & & \\
\hline \multirow[t]{2}{*}{ ECON } & .2638 & -.5382 & & & \\
\hline & .076 & .001 & & & \\
\hline \multirow[t]{2}{*}{ SMEM } & .1921 & -.2448 & .3740 & & \\
\hline & .146 & .088 & .019 & & \\
\hline \multirow{2}{*}{ SPRED } & -.1227 & .1127 & -.2803 & -.3623 & \\
\hline & .252 & .270 & .063 & .021 & \\
\hline \multirow[t]{2}{*}{ SCON } & -.0091 & -.0188 & .0534 & .3642 & -.0793 \\
\hline & .480 & .459 & .384 & .020 & .333 \\
\hline
\end{tabular}




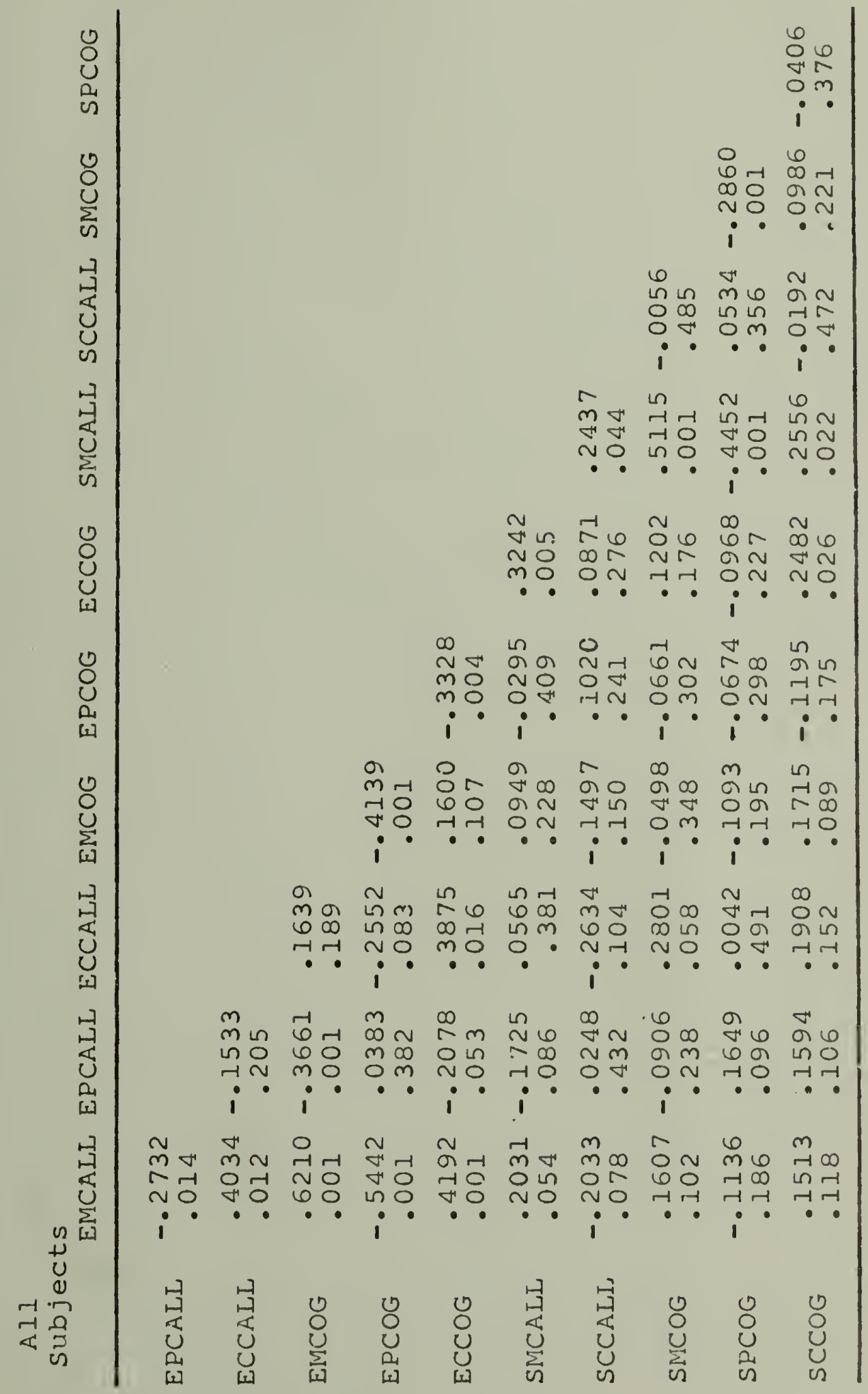




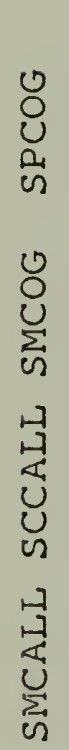

U

บั

نั

是

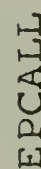

突

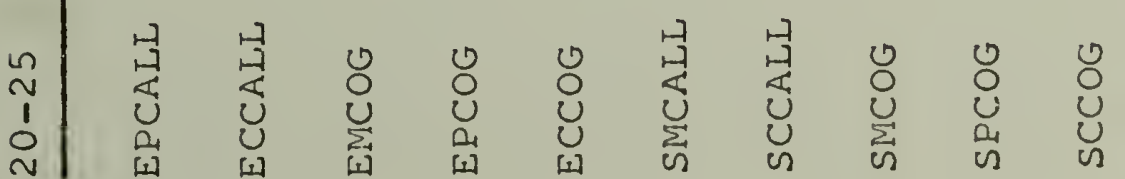

당요

i

$0 \sim \infty$

$\rightarrow \sim \infty r$

- mo

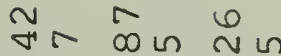

กN $\sim$ in $\mathrm{ON}$

? $-1 \sim r m$

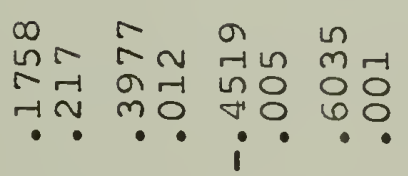

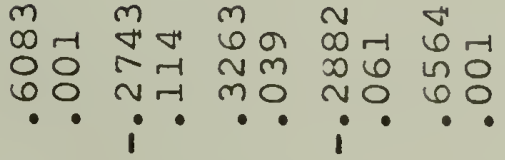

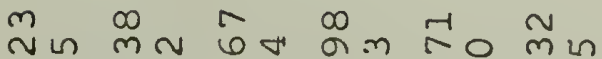
ชก 0 \% mo o $\pi$ ro on

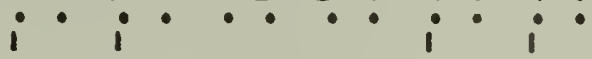

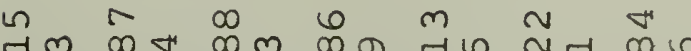

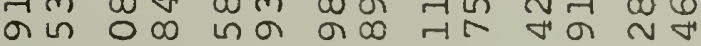

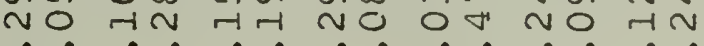

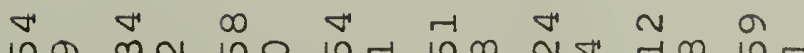
ind $\infty$ भก

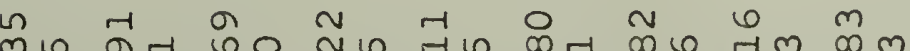

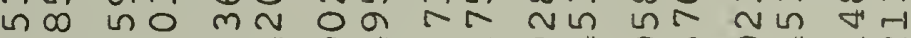
$\because ?$ ก 0 ?

שo

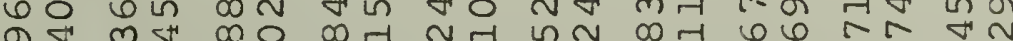
न

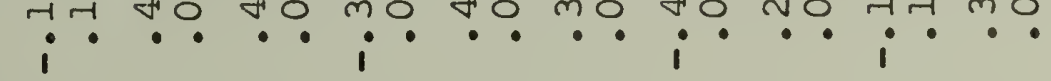




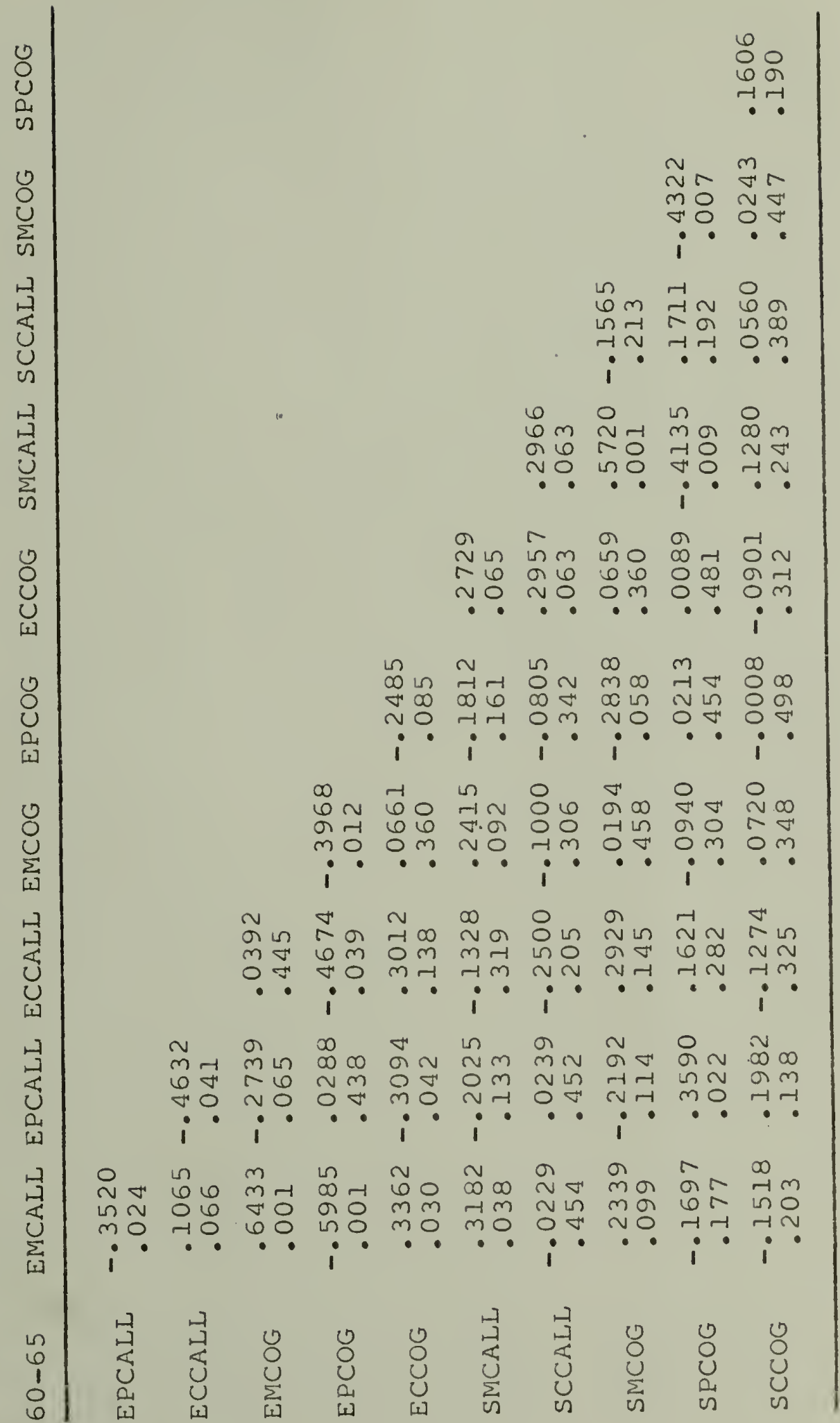




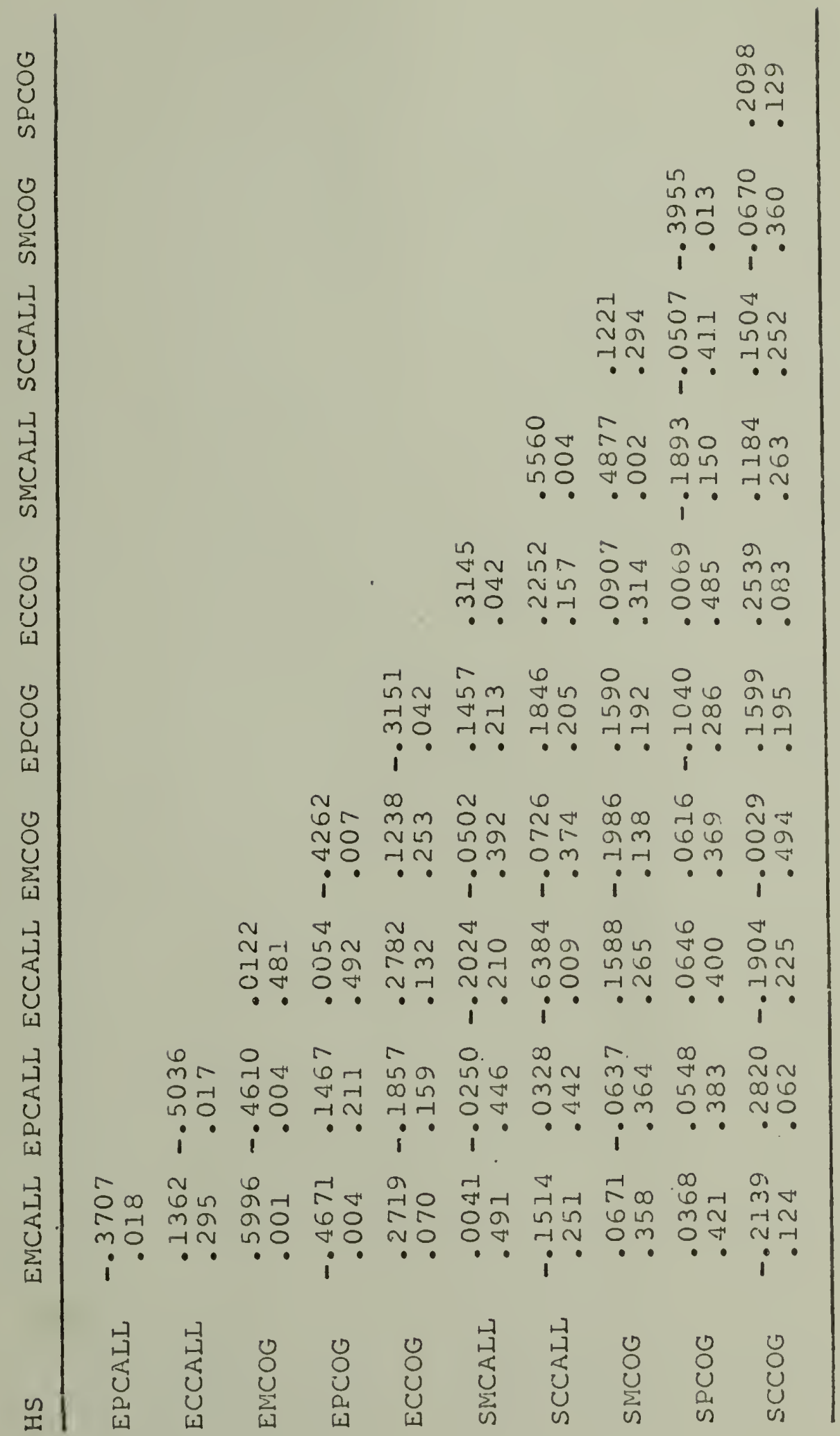




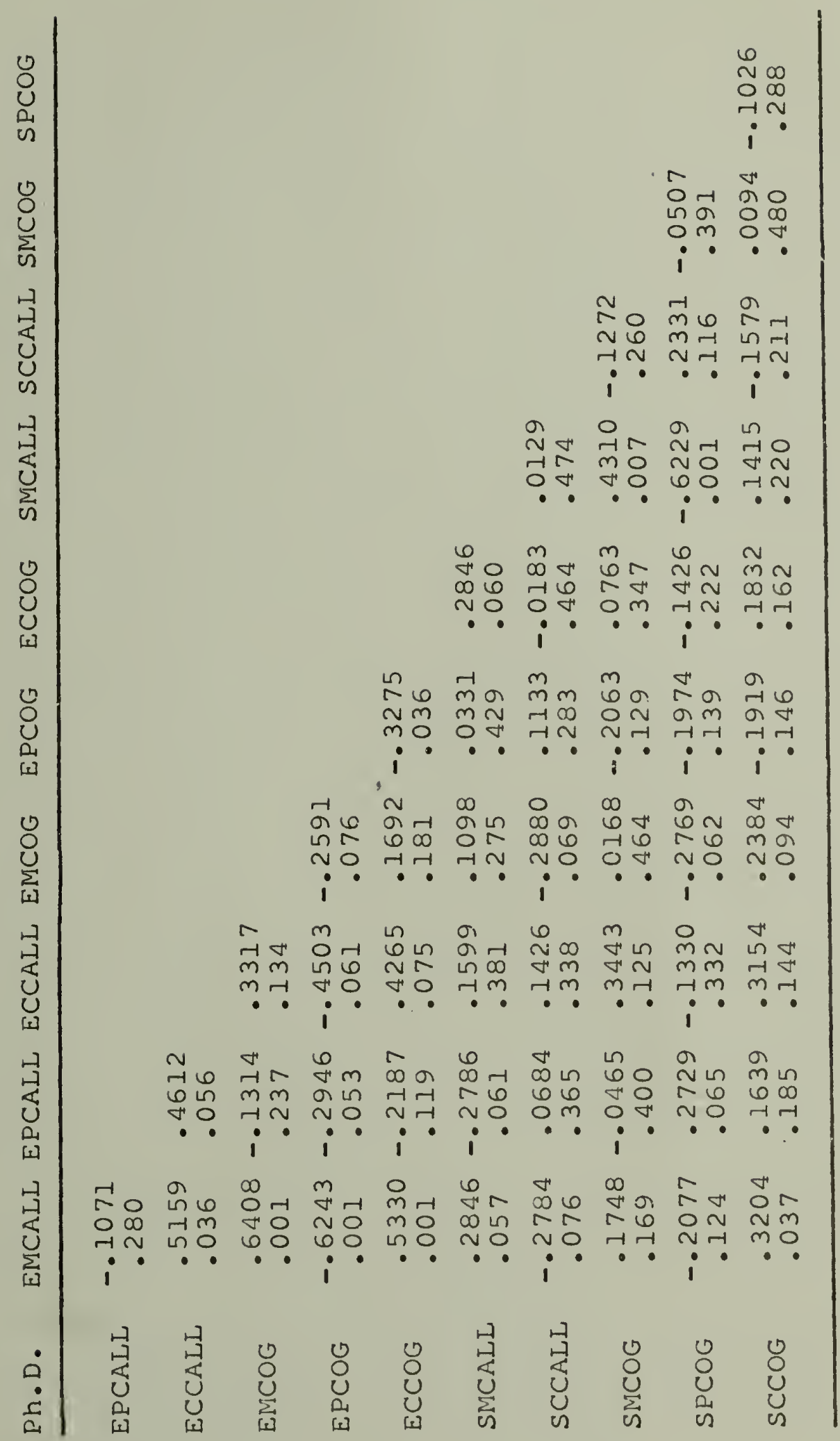




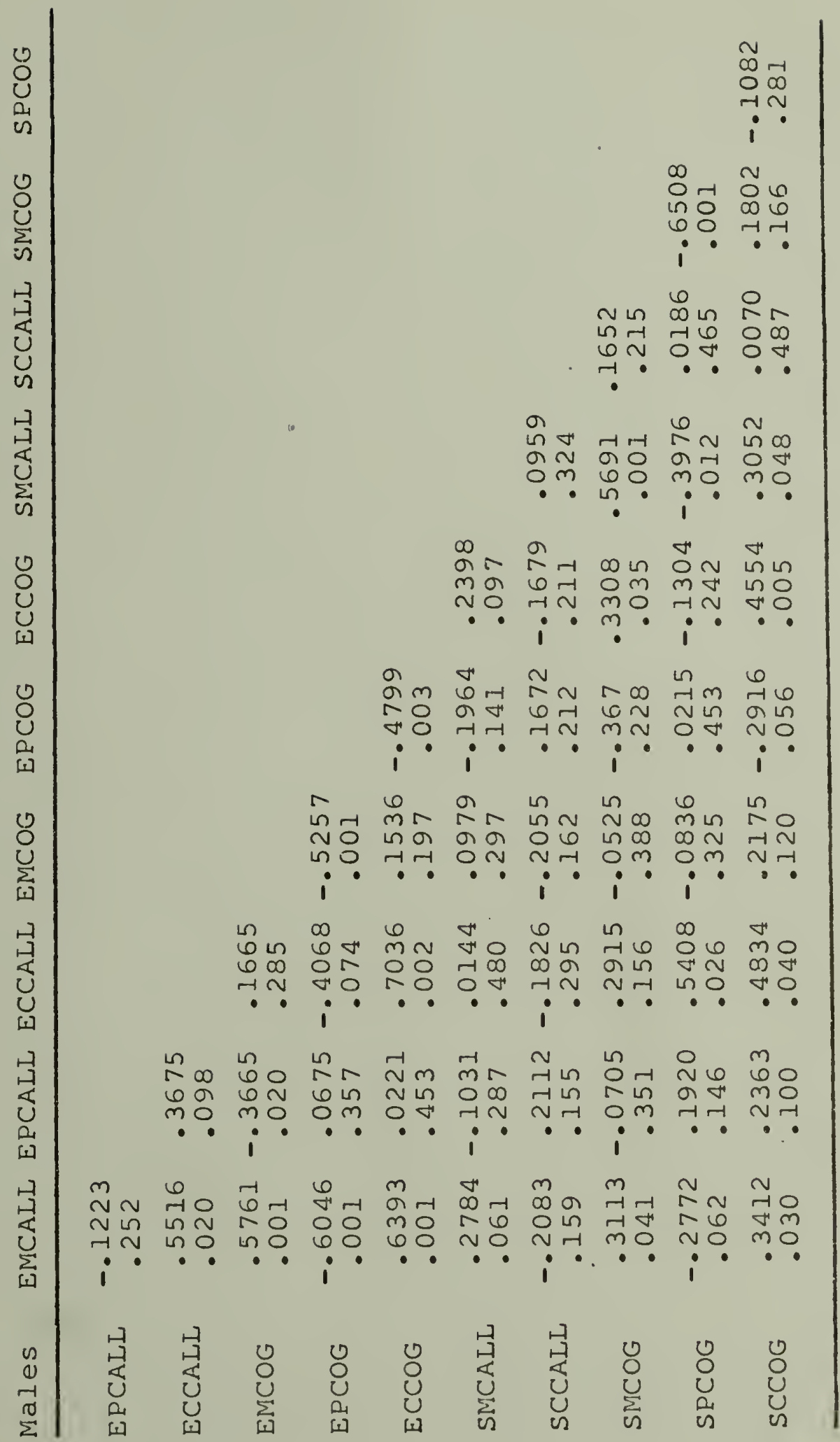




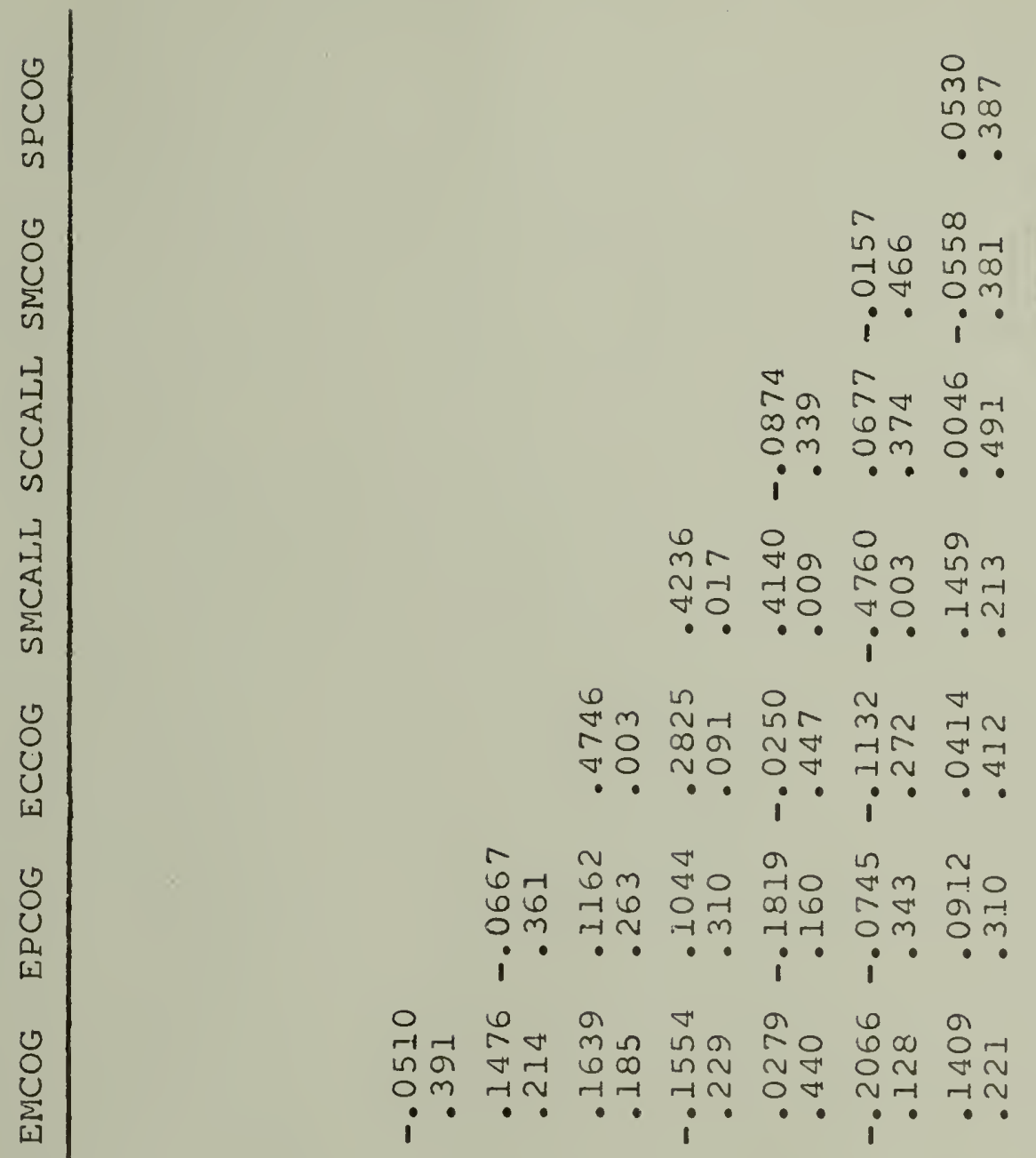

㳫

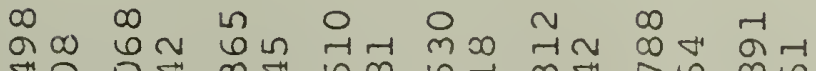
$\neg N$ I

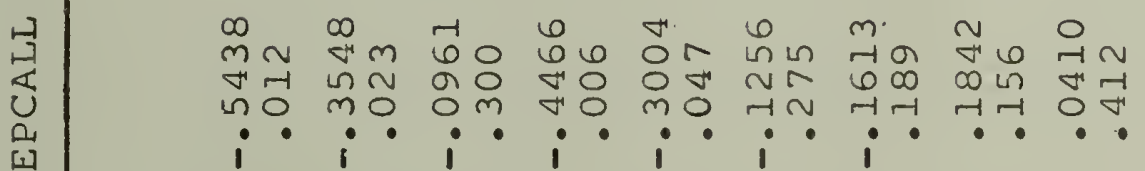

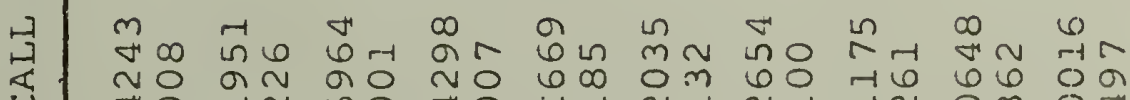

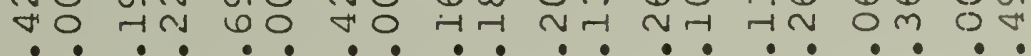


